$\underline{\mathrm{AK}} \mathrm{WI}$

Herausgeber

Thomas Barton Frank Herrmann Vera G. Meister Christian Müller Christian Seel

Angewandte Forschung in der Wirtschaftsinformatik

\title{
Prozesse, Technologie,
} Anwendungen, Systeme und Management 2017

unterstützt durch: 
Arbeitskreis Wirtschaftsinformatik an Fachhochschulen (AKWI) www.akwi.de

Angewandte Forschung in der Wirtschaftsinformatik

\title{
Prozesse, Technologie, Anwendungen, Systeme und Management 2017
}

\author{
Tagungsband zur 30. AKWI-Jahrestagung \\ vom 17.09.2017 bis 20.09.2017 an der \\ Hochschule Aschaffenburg \\ herausgegeben von \\ Thomas Barton, Frank Herrmann, Vera G. Meister, \\ Christian Müller, Christian Seel
}

unterstützt durch

Information Management Institut (IMI), Hochschule Aschaffenburg

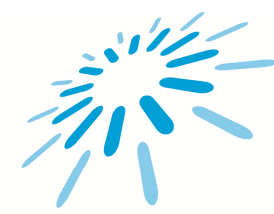

hochschule aschaffenburg

university of applied sciences

mana-Buch, Heide 
Bibliografische Information der Deutschen Nationalbibliothek:

Die Deutsche Nationalbibliothek verzeichnet diese Publikation in der Deutschen Nationalbibliografie, detaillierte bibliografische Daten sind im Internet über www.dnb.de abrufbar.

\section{Angewandte Forschung in der Wirtschaftsinformatik \\ Prozesse, Technologie, Anwendungen, Systeme und Management \\ 2017}

Tagungsband zur wissenschaftlichen Fachtagung am 18.09.2017 anlässlich der 30. Jahrestagung des Arbeitskreises Wirtschaftsinformatik an Fachhochschulen (AKWI) vom 17.09.2017 bis 20.09.2017 an der Technischen Hochschule Brandenburg

\section{Herausgeber:}

Thomas Barton, Hochschule Worms, barton@hs-worms.de

Frank Herrmann, Ostbayerische Technische Hochschule Regensburg, frank.herrmann@oth-regensburg.de Vera G. Meister, Technische Hochschule Brandenburg, vera.meister@th-brandenburg.de

Christian Müller, Technische Hochschule Wildau [FH], christian.mueller@th-wildau.de

Christian Seel, Hochschule für Angewandte Wissenschaften Landshut, christian.seel@haw-landshut.de

\section{Mitglieder des Programmkomitees:}

Thomas Barton (HS Worms)

Frank Herrmann (OTH Regensburg)

Christian Müller (TH Wildau)

Christian Seel (HAW Landshut)

Vera Meister (TH Brandenburg)

Wolfgang Alm (HS Aschaffenburg)

Carsten Reuter (HS Aschaffenburg)

\section{Gutachter:}

Thomas Barton (HS Worms)

Frank Herrmann (OTH Regensburg)

Christian Müller (TH Wildau)

Christian Seel (HAW Landshut)

Vera Meister (TH Brandenburg)

Georg Rainer Hofmann (HS Aschaffenburg)

Carsten Reuter (HS Aschaffenburg)

Carlo Simon (HS Worms)

\section{Redaktion:}

Wolfgang Alm

Redaktionsschluss: 09.07.2017

Erscheinungstermin: 17.09.2017

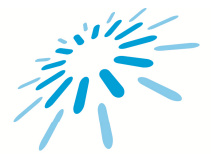

\section{hochschule aschaffenburg}

university of applied sciences

Die Herstellung dieses Tagungsbandes erfolgte mit freundlicher Unterstützung durch: Information Management Institut (IMI), Hochschule Aschaffenburg

Leitung und Verwaltung der Hochschule Aschaffenburg

Verlag: mana-Buch, Feldblick 24, 25746 Heide, Germany, www.mana-Buch.de

Druck: createSpace, North Charleston, USA

ISBN: 978-3-944330-56-3 


\section{Inhaltsverzeichnis}

Geleitwort des Sprechers des Arbeitskreises Wirtschaftsinformatik an Fachhochschulen im deutschsprachigen Raum.

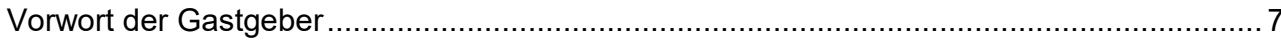

\section{Informationssysteme - IT-Management}

Berechnung von finanzwirtschaftlichen Modellen in einer integrierten R-Umgebung........... 10

Ein adaptives Vorgehensmodell für hybrides Projektmanagement ................................... 20

Konfiguration des hybriden Projektmanagements für die Entwicklung technischer, physischer Produkte.

\section{Geschäftsprozesse}

Metamodellbasierte Erweiterung der BPMN zur Modellierung und Generierung von Prozessvarianten

Erweiterung des Referenzprozessmodells eTOM um die Anforderungen des deutschen IT-Sicherheitsgesetzes

Eine Petri-Netz-Programmiersprache und Anwendungen in der Produktion.

Studienbasierte Entwicklung eines Frameworks zur Ableitung von Strategieempfehlungen Digitaler Service Transformationen für produzierende Unternehmen

Prozessoptimierung in „smarten“ Unternehmen durch Condition Monitoring ........................ 80

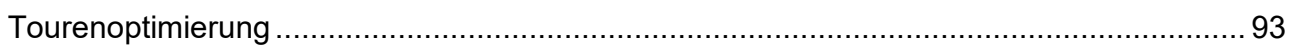

\section{Datenmanagement - Softwareengineering}

Einsatz einer NoSQL-Datenbank zur Analyse von Reiseblogs: Konzept und Integration .. 104 Aufwärtskompatibilität von Modellierungstechniken für Data Warehouses anhand von 3NF, Dimensional Modeling und Data Vault............................................................. 113

Modellgetriebene Entwicklung - Vor- und Nachteile in der Test- und Einführungsphase .. 123

Cloud - Mobile - Security

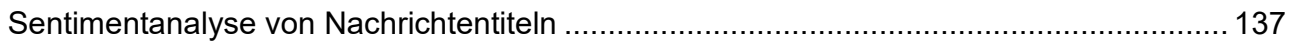

Erlösmodelle von mobilen Anwendungen - Eine Analyse ausgewählter Modelle .............. 146

Medizinische Therapiebegleitung und Compliancesteigerung mittels gamifizierter,

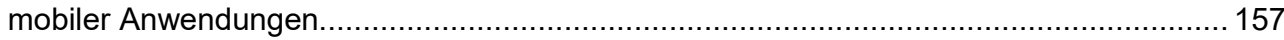

iScaleIT - Automatische Skalierung von Webservern in der Cloud ................................... 164

Sicherheitsüberlegungen für spezielle Einsatzbereiche des Internet of Things.................. 174 
Qualitative Wertschöpfungsanalyse von Anwendungsfällen des Narrowband Internet of Things

Wissensmanagement - Wissenstransfer

Kompetenzerwerb in angewandter Wissensmodellierung durch Lernen aus Fehlern 195

Elemente eines Referenzmodells für Wissenstransfereinrichtungen - ein Meta-

Wissenstransfer?

Datenerhebung für die CDG-Forschung

\section{Didaktik}

Der Arbeitsmarkt für Wirtschaftsinformatiker - Berufsbilder und Kompetenzen im Spannungsfeld der digitalen Transformation

Terminologiearbeit als Inhalt und Methode der Wirtschaftsinformatik

Steigerung der Attraktivität von Studiengängen der Wirtschaftsinformatik für Frauen und Männer durch geschlechtersensitives Branding...

\section{Industrie 4.0}

Industrie-4.0-Reifegradmodell

Schlüsselkompetenzen für die Entwicklung digital-vernetzter Lösungen

Industrie-4.0-Reifegradbestimmung produzierender Unternehmen im deutschsprachigen

Raum und Identifikation bedeutender Industrie-4.0-Einflussfaktoren

Autoren. 


\title{
Geleitwort des Sprechers des Arbeitskreises Wirtschaftsinformatik an Fachhochschulen im deutschsprachigen Raum
}

\author{
Liebe Teilnehmerin, lieber Teilnehmer,
}

herzlich willkommen zur 30. Jahrestagung des Arbeitskreises Wirtschaftsinformatik an Fachhochschulen (AKWI) an der Hochschule Aschaffenburg. Ich freue mich sehr, dass wir in diesem Jahr die 30. Jahrestagung durchführen können! Unsere wissenschaftliche Fachtagung mit dem jährlich erscheinenden Tagungsband hat zur Sichtbarkeit der Hochschulen für angewandte Wissenschaften einen großartigen Beitrag geleistet. Und unsere Jahrestagung ist zu einem festen Bestandteil der Tagungen auf dem Gebiet der Wirtschaftsinformatik geworden. Darauf können wir stolz sein! Daher ist es nur folgerichtig, dass zu unserer 30. Jahrestagung Persönlichkeiten wie z.B. Prof. Dr. Oliver Thomas (Universität Osnabrück, stellvertretender Sprecher des Fachbereichs Wirtschaftsinformatik der Gesellschaft für Informatik (GI)) und Prof. Dr. Klaus Lang (TH Bingen, Vorsitzender des Fachbereichstags Informatik (FBTI)) als Redner gewonnen werden konnten.

Ich möchte zunächst kurz einige wichtige AKWI-Aktivitäten seit der letzten Jahrestagung in Brandenburg an der Havel auflisten:

- Die GI-Rahmenempfehlung für die Ausbildung in Wirtschaftsinformatik an Hochschulen ist unter Beteiligung der Kollegen Prof. Dr. Christian Müller (TH Wildau) und Prof. Dr. Martin Wolf (FH Aachen) fertiggestellt worden. Im Rahmen der Fertigstellung konnte Paragraph 8.2 mit unseren Mitgliedern abgestimmt werden. Die ursprüngliche Version von Paragraph 8.2 ist aufgrund des Feedbacks gemeinsam von Christian Müller, von Martin Wolf und von mir überarbeitet worden.

- Erfreuliches gibt es auch wieder von unserem E-Journal „Anwendungen und Konzepte der Wirtschaftsinformatik" (AKWI) zu berichten. So ist in diesem Jahr die Ausgabe Nr. 5 erschienen. Besten Dank an alle Autoren und Gutachter sowie insbesondere an unsere Herausgeber Prof. Dr. Frank Herrmann (OTH Regensburg), Prof. Dr. Nobert Ketterer (Hochschule Fulda), Prof. Konrad Marfurt (Hochschule Luzern) und Prof. Dr. Christian Müller (TH Wildau)!

- In der Buchreihe Angewandte Wirtschaftsinformatik ist Band 2 mit dem Titel Geschäftsprozesse - Von der Modellierung zur Implementierung - erschienen. Als Autoren sind u. a. eine Kollegin und einige Kollegen beteiligt, die an Hochschulen für angewandte Wissenschaften tätig sind. Besten Dank an alle Autoren und an meine Mitherausgeber Prof. Dr. Christian Müller (TH Wildau) und Prof. Dr. Christian Seel (HAW Landshut).

Unsere Community wächst weiterhin. Nachdem im letzten Jahr die Fachhochschule Aachen und die HWR Berlin in unseren Kreis aufgenommen wurde, steht in diesem Jahr mit der Hochschule Osnabrück eine weitere Hochschule bereit, bei uns aktiv zu werden. Es ist sehr erfreulich, dass Sie sich so aktiv in unsere Community einbringen als Autorin oder als Autor in der wissenschaftlichen Fachtagung, als Vortragende oder Vortragender im hochschulpolitischen Teil unserer Tagung oder als Diskutantin und Diskutant. 
Die wissenschaftliche Tagung hat 27 Beiträge aufzuweisen, die in einem Doppelblindverfahren von mindestens zwei Gutachtern begutachtet wurden. Es sind wieder Beiträge von Hochschulen hinzugekommen, die noch nicht auf unserer Tagung vertreten waren. Durch unsere wissenschaftliche Fachtagung wird nicht nur die Vielfalt und Aktualität der Wirtschaftsinformatik aufgezeigt. Genauso wichtig ist, dass in der angewandten Forschung die Kompetenzen der Hochschulen vom Typ Fachhochschule sichtbar gemacht werden. Dafür und für die hohe Qualität der Beiträge meinen herzlichen Dank!

Herzlichen Dank auch an das Gutachterteam, in dem in alphabetischer Reihenfolge Prof. Dr. Wolfgang Alm (Hochschule Aschaffenburg), Prof. Dr. Thomas Barton (Hochschule Worms), Prof. Dr. Frank Herrmann (OTH Regensburg), Prof. Dr. Georg-Rainer Hofmann (Hochschule Aschaffenburg), Prof. Dr. Vera G. Meister (TH Brandenburg), Prof. Dr. Christian Müller (TH Wildau), Prof. Dr. Carsten Reuter (Hochschule Aschaffenburg), Prof. Dr. Christian Seel (HAW Landshut) und Prof. Dr. Carlo Simon (Hochschule Worms) aktiv waren. Ich freue mich mit Innen auf eine interessante Konferenz und bin mir sicher, dass diese den Austausch unter uns unterstützen und zu neuen Erkenntnissen führen wird. Daher bin ich sehr zuversichtlich, dass der Tagungsband sowohl von Kolleginnen und Kollegen aus Hochschulen als auch von Praktikerinnen und Praktikern in Unternehmen sowie von Studierenden der Wirtschaftsinformatik angenommen wird.

Die größte Arbeit obliegt stets dem Organisationsteam an der ausrichtenden Hochschule vor Ort. Besten Dank an das Team der Hochschule Aschaffenburg. Stellvertretend möchte ich mich hier insbesondere bei Prof. Dr. Wolfgang Alm bedanken!

Ich bin sehr zuversichtlich, dass wir in diesen Tagen eine sehr gut organisierte Jahrestagung erleben dürfen mit fachlich fundierten Vorträgen, erkenntnisreichen Diskussionen, interessanten Anregungen und tollen Begegnungen in einer sehr angenehmen Atmosphäre.

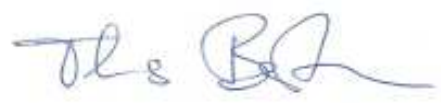

Prof. Dr. Thomas Barton (Hochschule Worms, Sprecher des AKWI) 


\section{Vorwort der Gastgeber}

Der vorliegende Tagungsband dokumentiert die wissenschaftlichen Beiträge zur 30 . Jahrestagung des Arbeitskreises „Wirtschaftsinformatik der deutschsprachigen Fachhochschulen (AKWI)“ der Gesellschaft für Informatik e. V., die vom Sonntag, 17. bis Mittwoch, 20. September 2017 an der Hochschule für Angewandte Wissenschaften in Aschaffenburg am Bayerischen Untermain stattfindet.

Das Information Management Institut (IMI) an der Hochschule Aschaffenburg wurde im Jahr 2009 gegründet. Das IMI hat heute mit 5 Professoren und 10 drittmittelfinanzierten Mitarbeitern eine im Hochschulkontext Aschaffenburg nennenswerte Personalstärke und umfangreiche Projekttätigkeit erreicht.

Die Hochschule in Aschaffenburg hat nicht verkannt, dass Fragen der Wirtschaftsinformatik („Information Management“) für die akademische und wirtschaftliche Entwicklung von elementarer Wichtigkeit sind. Die Abhängigkeit der hiesigen Wirtschaft von funktionierenden IT-Systemen und Netzwerken ist direkt evident. Ebenso evident sind der Wandel der Hochschullandschaft durch Digitale Bildung und die gesellschaftlichen Nebenwirkungen der Digitalisierung.

Akademischer Fortschritt soll zur Wahrung und Verbesserung von Wohlstand und Lebensqualität sinnvoll umgesetzt werden. Die Hochschulen sind aufgerufen, Forschungsergebnisse per Wissenstransfer der gesamten Gesellschaft zu Gute kommen zu lassen. Dies hat einen guten Grund, denn unsere Gesellschaft steht permanent vor der enormen Aufgabe, akademischen Fortschritt verantwortungsvoll umzusetzen. Hierfür müssen geeignete offene Kooperationsformen gefunden werden: Forschungsergebnisse sollen per Transfer der gesamten Gesellschaft zu Gute kommen.

Im Rahmen der 30. Tagung des AKWI wurden diverse, akademisch geschätzte und politisch erwünschte Beiträge zum permanenten wissenschaftlichen Dialog mit der Praxis dargelegt durch die Nutzbarmachung des Innovationspotenzials der Hochschulen für den Öffentlichen Sektor, die Unternehmen und deren Personal.

Ziel der 2017-er Tagung des AKWI in Aschaffenburg ist der Erfahrungsaustausch und ein gegenseitiges Weitergeben einschlägiger Erfahrungen. Dieses Ziel wurde mit und für die Teilnehmenden erreicht; wir bedanken uns sehr gerne bei der Kollegin und den Kollegen

\section{Thomas Barton, Frank Herrmann, Vera Meister, Christian Müller, Christian Seel und Carlo Simon,}

die als auswärtige Gutachter für die Aschaffenburger Veranstaltung die Auswahl der Beiträge vorgenommen haben. Unser Dank gilt auch der Aschaffenburger Hochschulleitung, den hiesigen Mitarbeiterinnen und Mitarbeitern, sowie den hiesigen Kolleginnen und Kollegen, die uns und die AKWI-Tagung unterstützt haben. 
Gerne haben wir die Gastgeberrolle für die 30. Tagung des AKWI übernommen. Wir bedanken uns bei allen Kolleginnen und Kollegen des AKWI für Ihr Vertrauen und Ihre Teilnahme und dass sie diese 30. Tagung nach Aschaffenburg vergeben haben.

Weiteren Veranstaltungen des AKWI dieses Formats in den nächsten Jahren kann mit gespannten Erwartungen entgegen gesehen werden.

Aschaffenburg im Frühherbst 2017

Prof. Dr. Georg Rainer Hofmann, Direktor

Prof. Dr. Wolfgang Alm, Direktor

Prof. Dr. Carsten Reuter

Information Management Institut (IMI)

Hochschule Aschaffenburg 


\section{Informationssysteme -}

\section{IT-Management}




\title{
Berechnung von finanzwirtschaftlichen Modellen in einer integrierten R-Umgebung
}

\author{
Natascha Nitzl, Christian Schulze, Michael Guckert
}

\section{Zusammenfassung}

Im Rahmen des von der Hessen-Agentur geförderten LOEWE3-Projektes ADOMIS (Ambient Delivery of Multiple Information and Statistics) wurde in Zusammenarbeit des Kompetenzzentrums KITE der THM mit der Content Software GmbH Bad Homburg eine Datenbank mit volks- und finanzwirtschaftlichen Informationen aufgebaut. Die Datenbank speichert u. a. Basisdaten wie zum Beispiel Zeitreihen, die für weitergehende Analysen verwendet werden können. Der Zugriff auf die Daten erfolgt über ein Web-Frontend, in das eine kontextsensitive Entwicklungsumgebung für die Programmiersprache $R$ integriert ist. Diese Umgebung erlaubt es zum einen R-basierte finanzwirtschaftliche Modelle interaktiv zu entwickeln und zum anderen komplette in $\mathrm{R}$ implementierte Modelle auf Basis der Daten der Datenbasis zu berechnen. Die Ergebnisse können dann wieder direkt in die Datenbasis gespeichert und in weiteren Berechnungen genutzt werden. Beispielhaft wurde zum Zwecke der Demonstration der Möglichkeiten im Rahmen des Projektes das klassische Merton-Modell zur Abschätzung der Ausfallwahrscheinlichkeit eines Unternehmens berechnet. Die Implementierung erfolgt einmal mit Hilfe des Newton-Verfahrens zur Berechnung der PD (Probability of Default) für ein Intervall und alternativ auf Basis einer Intervallzerlegung unter Anwendung des EulerMaruyama-Verfahrens. Die Ergebnisse werden für typische Fälle in Relation zu Einschätzungen von Rating-Agenturen gesetzt. Das vorliegende Papier zeigt Stärken und Schwächen des Merton-Modells für die Risikoabschätzung und fokussiert sich auf die beispielhafte Umsetzung in der integrierten R-Umgebung des ADOMIS-Systems.

\section{$1 \quad$ Einleitung}

Zu den zentralen Aufgaben von Kreditinstituten zählt die Bewertung des Kreditrisikos ihrer Kreditnehmer. Dazu ist die Auswertung zahlreicher Finanzdaten und Bestimmung von finanzmathematischen Kennzahlen notwendig.

Hierzu benötigen Kreditinstitute neben einer umfangreichen und aktuellen Datenbasis, auch entsprechende Programme zur Bearbeitung dieser Daten. Zugleich ist eine einfache Umsetzung finanzmathematischer Modelle auf dieser Datenbasis nötig.

Im Rahmen des ADOMIS-Projektes wurde eine Plattform entwickelt, die genau diese Komponenten vereint. Als Grundlage dient eine gut strukturierte Datenbasis, wobei durch eine Laderoutine verschiedene Datenquellen in der Datenbank zusammengefasst werden können. Das Datenmodell eignet sich für Zeitreihen aus dem finanzmathematischen Umfeld, welche in dem sogenannten ADOMIS-Portal abgefragt oder anschaulich dargestellt werden können. Mit Hilfe dieser Datenbasis können finanzmathematische Modelle in der Statistiksprache $\mathrm{R}$ berechnet werden, welche vom Anwender individuell implementiert oder vorgefertigt sein können. Eigene Modelle sowie deren Ergebnisse können gespeichert und erneut abgerufen werden. 
Ein in der ADOMIS-R-Umgebung hinterlegtes Modell ist unter anderem das sogenannte Merton-Modell, welches das Kreditrisiko von Unternehmen bewertet [Mart14]. Das Modell verwendet das Optionspreismodell von Fischer S. Black und Myron S. Scholes. 1997 erhielten Merton und Scholes dafür den Nobelpreis [Seyd00].

Im Folgenden werden die verschiedenen Komponenten der Plattform, die unter anderem für die Berechnung des Merton-Modells zusammenarbeiten, vorgestellt. Auf die allgemeine Softwarearchitektur von ADOMIS und die verwendeten Programme wird in Kapitel zwei eingegangen. In Kapitel drei wird das verwendete Datenmodell erläutert. Zu der Bewertung von Kreditrisiken wird das sogenannte Merton-Modell in Kapitel vier eingeführt. In Kapitel fünf runden ein Fazit und der Ausblick diese Arbeit ab.

\section{Architektur}

Die Softwarearchitektur von ADOMIS basiert auf dem Client-Server-Konzept. Als „ADOMISClients“ können Anwender interaktiv die ADOMIS-Services über ein Webportal, ein lokal installiertes R-Statistics Programm oder einen REST Service nutzen. Zu diesen ADOMISServices zählen unter anderem die Möglichkeit, Daten aus verschiedenen Quellen abzurufen, R-Modelle zu berechnen, oder eigene berechnete Daten wieder in der Datenbank bereitzustellen.

Auf der Server-Seite sind die drei logischen Schichten „ADOMIS-Services“, „ADOMIS API“ und „ADOMIS Storage“ vorhanden. Auf die verschiedenen Komponenten, deren Zusammenhänge und Aufgaben wird im Folgenden eingegangen. In Abbildung 1 ist dieses Zusammenspiel anschaulich dargestellt.

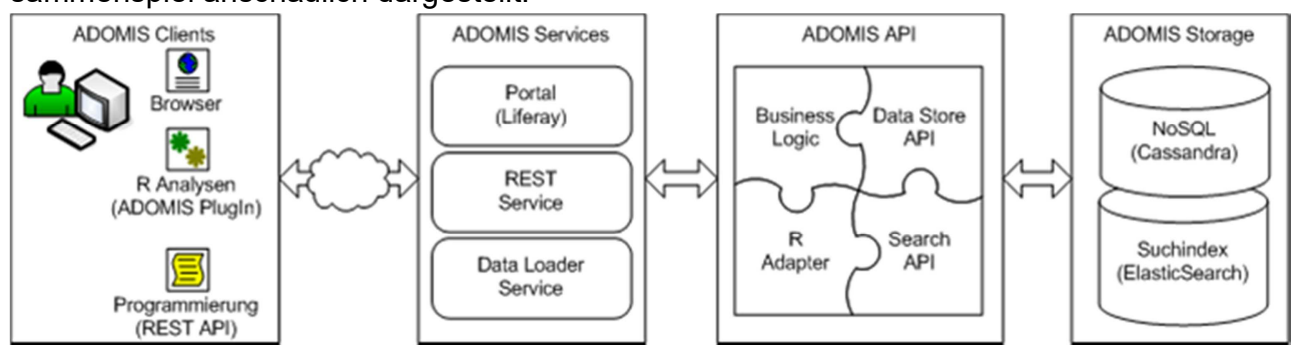

Abbildung 1: Softwarearchitektur von ADOMIS

\subsection{ADOMIS-Clients}

Der Anwender greift über die Clients auf die verschiedenen Services zu. Für den Zugriff bieten sich dem Benutzer drei verschiedene Möglichkeiten.

Zum einen kann der Anwender hierfür den Browser verwenden und in dem Portal alle Funktionen online nutzen. In Abbildung 2 ist das Portal zu erkennen. Es sind beispielhaft drei verschiedene Zeitreihen abgefragt und in der Oberfläche graphisch dargestellt worden.

Alternativ kann eine lokale R-Statistics-Session um die projektbezogenen R-Packages „adomisData“ und „adomisStatistics“ erweitert werden. Das Package „adomisData“ enthält Funktionen für die Basiszugriffe auf die Daten selbst und „adomisStatistics“ bietet analytische Funktionen auf der Datenbasis, wie zum Beispiel das Merton-Modell.

Es besteht zusätzlich die Möglichkeit, programmatisch auf die Dienste zuzugreifen, indem eine Client-Anwendung direkt den REST-Service anspricht. Hierbei kann eine beliebige Programmiersprache verwendet werden. 


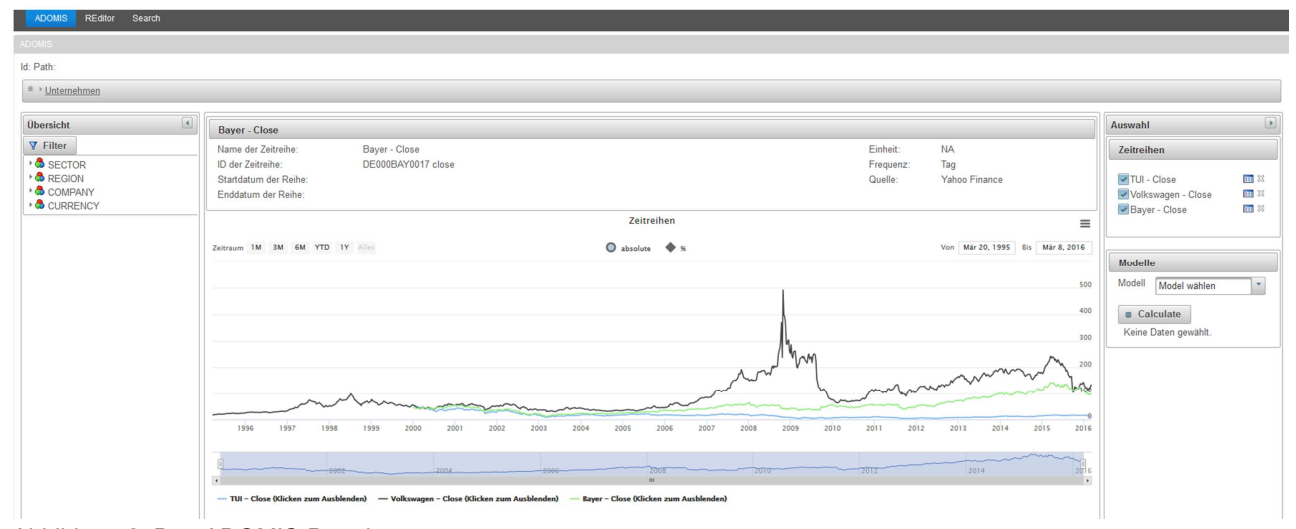

Abbildung 2: Das ADOMIS-Portal

\subsection{ADOMIS-Server}

In Abbildung 1 ist zu erkennen, dass sich die ADOMIS-Serverwelt aus den drei Schichten „ADOMIS-Services“, der „ADOMIS API“ und dem „ADOMIS Storage“ zusammensetzt, welche im Folgenden genauer erklärt werden.

Unter „ADOMIS-Services“ sind die für Clients erreichbaren Schnittstellen zusammengefasst. Als Schnittstelle bietet ADOMIS ein Web-Portal, einen REST-Service und einen DataLoader-Service an.

Das ADOMIS-Portal kann mit einem Browser verwendet werden. Hier können Daten direkt abgerufen, finanzmathematische Modelle in der Statistiksprache R gerechnet oder Daten in $\mathrm{R}$ analysiert werden. Über das Portal können die gesuchten Zeitreihen anschaulich dargestellt werden.

Der vom Portal verwendete REST-Service ist auch extern nutzbar. Hiermit können Daten dann in eigenen Programmen verwendet werden.

Ein weiterer Service ist der DataLoader, der über eine Weboberfläche Schnittstellen zu den Datenlieferanten verwaltet. Hier können ETL-Prozesse workflow-basiert konfiguriert und durchgeführt werden, sodass die Daten aus verschiedensten Quellen in der ADOMIS-Datenbank zusammengeführt werden können. Bestimmte Datenanbieter können standardmäßig verwendet werden. Zusätzlich kann der Anwender den DataLoader auch mit neuen Datenquellen erweitern.

In der zweiten Schicht der Server-Seite, der „ADOMIS-API“, versorgt die „Business Logic“ die verschiedenen Komponenten, wie DataLoader, Portal und REST-Service mit den Daten aus der Datenbank und dem Suchindex.

Durch die „Search-API“ kann innerhalb der Datenbank nach Schlagwörtern gesucht werden. Zugrunde liegt die dokumentenbasierte Suchmaschine Elasticsearch [Gorm15]. In dem sogenannten Suchindex werden die Metadaten zu den Zeitreihen gespeichert. Dieser wird von Elasticsearch verteilt gespeichert und kann optimiert abgefragt werden. Über das Portal sind Abfragen nach einem Schlagwort oder bestimmten Feldern möglich.

Über den „R-Adapter“ wird im Portal eine R-Schnittstelle bereitgestellt. Es können R-Skripte, die im Portal implementiert werden, an $\mathrm{R}$ weitergeleitet und dort ausgeführt werden, wobei die benötigten Daten aus der ADOMIS-Datenbank genommen werden können.

Die dritte Schicht der Server-Seite ist die Datenschicht, der "ADOMIS Storage". Die verwendeten Datenbanken wurden hinsichtlich einfacher skalierbarer verteilten Umgebungen ausgewählt. Man hat MySql- und NoSql-Datenbanken untersucht. Für die Basisdaten wird die 
dokumentenorientierte Datenbank Cassandra der Apache Software Foundation verwendet, welche sich auch zum Speichern von Zeitreihen eignet, worauf im nächsten Kapitel eingegangen wird.

Neben der Datenbank befindet sich im "ADOMIS Storage" noch der bereits erwähnte Suchindex von Elasticsearch.

\section{Das ADOMIS-Datenmodell}

\subsection{Die Datenlage am Finanzmarkt}

ADOMIS soll möglichst flexibel und gleichzeitig vollständig die Datenlage am Finanzmarkt abbilden. Die Datenbank muss Daten aus unterschiedlichen Quellen und Formaten aufnehmen können. Wegen der Vielfalt der Finanzierungsinstrumente und der mit innen verbundenen spezifischen Attribute und Dimensionen ist ein komplexes relationales Datenmodell entstanden. Viele der aus dem Datenmodell resultierenden Tabellen dienen dazu, die Recherche nach Zeitreihen zu vereinfachen beziehungsweise Attribute und hierarchische Abhängigkeiten ergänzen zu können, ohne eine grundlegende Veränderung des Datenmodells vornehmen zu müssen. Diese Tabellen sind für die vorliegende Arbeit von untergeordneter Bedeutung, so dass hier nur der für die Zeitreihen-Analyse und Modellberechnung relevanter Teil des Datenmodells vorgestellt wird. Für die Berechnung des Merton-Modells, auf das in Kapitel 4 näher eingegangen wird, werden beispielsweise Aktienkurszeitreihen benötigt, weshalb im Folgenden besonders auf deren Abbildung im Datenmodell eingegangen wird.

\subsection{Tabellen für die Abbildung von Zeitreihen}

Abbildung 3 zeigt die wesentlichen Tabellen, in denen die Zeitreihen und ihre wichtigsten Eigenschaften abgelegt sind.

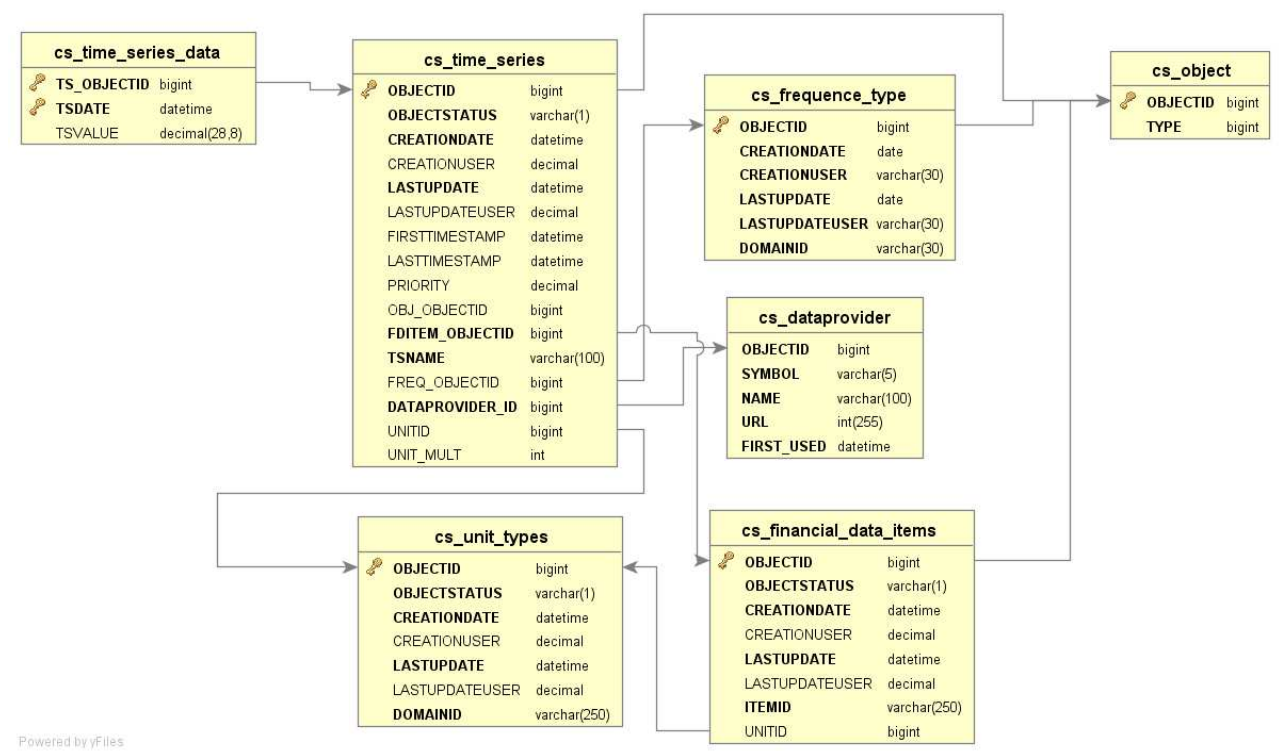

Abbildung 3: Auszug aus dem ADOMIS-Datenmodell: Zeitreihen 
Im Folgenden werden kurz die Tabellen und ihre Beziehungen erläutert:

Die wichtigsten Tabellen sind cs_time_series und cs_time_series_data, die die zentralen Eigenschaften der Zeitreihen und die eigentlichen Datenwerte enthalten.

\subsection{Die Tabelle cs_time_series}

In dieser Tabelle sind alle Zeitreihen durch genau einen Datensatz mit dem Primärschlüssel OBJECTID repräsentiert. Neben einigen Informationen zum Bearbeitungsverlauf der Zeitreihe (Erstellungsdatum, letztes Update, Nutzer etc.) geben die Felder OBJ_OBJECTID, FDITEM_OBJECTID und DATAPROVIDER_ID Aufschluss über die Zugehörigkeit der Zeitreihe zu einem Finanzinstrument und ihre Datenherkunft.

OBJ_OBJECTID verweist dabei auf ein Finanzinstrument, das an anderer Stelle in der Datenbank liegt. Ein solches Finanzinstrument kann beispielsweise eine spezielle Aktie oder ein Derivat sein, zu der die Zeitreihe gehört. Verschiedene Zeitreihen können dabei durchaus zum gleichen Finanzinstrument gehören, beispielsweise wenn eine Zeitreihe die Eröffnungskurse und eine weitere die Schlusskurse enthält.

FDITEM_OBJECTID beschreibt, um welchen Typ einer Zeitreihe es sich handelt, ob die Zeitreihe beispielsweise Tageshöchstwerte, Eröffnungs- oder Schlusskurse beinhaltet. Das Feld zeigt auf die Tabelle cs_financial_data_items, die die möglichen Typen enthält.

DATAPROVIDER_ID repräsentiert die Datenquelle, aus der die Zeitreihe stammt. Das Feld zeigt auf die Tabelle cs_dataprovider, die die potenziellen Datenquellen einer Zeitreihe enthält. Hier können zum Beispiel Verweise auf öffentliche Datenquellen stehen (Bundesbank, statistische Bundes- und Landesämter, Europäische Zentralbank o. ä.), aber auch Verweise auf kommerzielle Quellen (Reuters, Bloomberg o. ä.).

Mit einem einzigen Finanzinstrument, das über das Feld OBJ_OBJECTID referenziert wird, kann somit eine Vielzahl von Zeitreihen verknüpft sein. Für jede dieser Zeitreihen existiert ein Eintrag in der Tabelle cs_time_series. Es ist sogar denkbar, dass die gleiche Zeitreihe mehrfach aus unterschiedlichen Datenquellen vorliegt, was dann zu weiteren Einträgen in der Tabelle cs_time_series führt.

Das Feld FREQ_OBJECTID verweist auf die Tabelle cs_frequence_type, die die möglichen Wiederholungsraten einer Zeitreihe beschreibt. Hiermit ist die Frequenz gemeint, mit der die Datenerfassung erfolgt (täglich, wöchentlich, Quartal o. ä.). Das Feld UNITID zeigt auf die Tabelle cs_unit_types, die die Einheit einer Zeitreihe beschreibt (Währung, Stückzahl o. ä.).

\subsection{Die Tabelle cs time_series data}

Die Tabelle enthält die Werte der Zeitreihen. Die Werte finden sich im Feld TSVALUE. Der Fremdschlüssel TS_OBJECTID referenziert die Tabelle cs_time_series (siehe 3.3) und beschreibt, zu welcher Zeitreihe der Datensatz gehört. Der Primärschlüssel der Tabelle setzt sich zusammen aus diesem Fremdschlüssel und dem Feld TSDATE, in dem der Zeitpunkt festgehalten wird, zu dem der entsprechende Wert gehört.

\subsection{Die Tabelle cs_object}

Von den in Abbildung 3 gezeigten Tabellen fehlt nur noch eine Erläuterung für die Tabelle cs_object. Dies ist eine globale Tabelle, in der letztlich jedes Objekt, das in der Datenbank existiert, über seine OBJECTID repräsentiert wird, also beispielsweise auch die Zeitreihen, die Finanzinstrumente etc. Das Feld OBJECTID existiert in zahlreichen Tabellen und die Datenbank ist so angelegt, dass in der gesamten Datenbank jeder Wert nur einmal vergeben wird. Man kann das Feld als globalen Primärschlüssel für die gesamte Datenbank auf- 
fassen. Unter dem Feld TYPE kann man dann nachschlagen, welche Art von Objekt sich hinter der OBJECTID verbirgt (Zeitreihe, Aktie, Region etc.).

\subsection{Recherche}

Im Falle einer Recherche über die ADOMIS-Oberfläche wird über Suchen und Filtern eine Zeitreihe ermittelt, die einen eindeutigen Datensatz in der Tabelle cs_time_series referenziert. Zu den Recherche- und Filterkriterien gehören auch Felder aus der Tabelle selbst. So legt der Benutzer (über eine grafische Oberfläche) unter anderem fest, zu welchem Finanzinstrument seine Zeitreihe gehören soll (OBJ_OBJECTID), ob er Anfangs-, Schlusskurse oder Tageshöchstwerte recherchieren möchte (FDITEM_OBJECTID) und welche Datenquelle er nutzen möchte (DATAPROVIDER_ID). Damit steht fest, welche Zeitreihe abgefragt werden soll. Der Primärschlüssel der gefundenen Zeitreihe aus cs_time_series wird dann genutzt, um alle Werte der Zeitreihe aus der Tabelle cs_time_series_data abzufragen oder weiter zu filtern.

\section{Das Merton-Modell in der integrierten R-Umgebung}

Innerhalb der R-Umgebung können eigene finanzmathematische Modelle implementiert werden, aber auch bereits umgesetzte Modelle aus veröffentlichten R-Packages genutzt werden. Diese können auf der vorliegenden Datenbasis, wie in Kapitel 3 vorgestellt, durchgeführt und Ergebnisse anschließend hier gespeichert werden.

Beispielhaft ist an dieser Stelle das klassische Merton-Modell in R implementiert und auch als Funktion innerhalb des adomisStatistics-Packages gespeichert, wobei der Zugriff auf die Datenbasis mit Hilfe von Funktionen aus dem adomisData-Package erfolgt.

Im Rahmen des Projektes ADOMIS wurde eine Masterarbeit betreut, in der das MertonModell unter verschiedenen und vor allem numerischen Aspekten untersucht worden ist [Nitz16]. Diese Arbeit ist Grundlage dieses Kapitels.

\subsection{Das Merton-Modell}

Das Merton-Modell trifft durch die sogenannten Ausfallwahrscheinlichkeit (kurz PD für Probability of Default) eine Einschätzung darüber, wie wahrscheinlich es ist, dass ein Kreditnehmer seinen Zahlungsverpflichtungen nicht mehr fristgerecht nachkommen kann [Mart14, S. 1].

Für die Berechnung der Ausfallwahrscheinlichkeit eines Unternehmens werden die historische Volatilität, das Fremd- und Eigenkapital eines Unternehmens sowie der Unternehmenswert und seine Volatilität benötigt. Außerdem wird eine Driftrate verwendet, wofür meistens die risikoneutrale Zinsrate benutzt wird. Die erforderlichen Daten werden aus der Datenbank genommen, der Zugriff erfolgt über Funktionen aus dem adomisData-Package. Für die Bestimmung der historischen Volatilität werden zum Beispiel Aktienkurszeitreihen der jeweiligen Unternehmen verwendet, die wie in Kapitel drei geschildert in dem Datenmodell integriert sind.

Der Unternehmenswert und seine Volatilität sind nicht am Markt beobachtbar [Mart14]. Um diese zu bestimmen, wird unter anderem mit Hilfe der Optionsbewertung des Black-ScholesModells und der Annahme, dass der Unternehmenswert einer geometrischen Brownschen Bewegung folgt, ein zweidimensionales Gleichungssystem aufgestellt [Mart14, S. 70]. Das Newton-Verfahren bestimmt im Allgemeinen eine Näherungslösung von nichtlinearen Glei- 
chungen im Eindimensionalen, kann aber auch unter der Nutzung der Inversen der JacobiMatrix für mehrdimensionale Gleichungen angewendet werden. In der hier vorliegenden Problemstellung können also der Unternehmenswert und seine Volatilität mit Hilfe des mehrdimensionalen Newton-Verfahrens approximativ bestimmt werden.

Obwohl sich dieses numerische Verfahren im Allgemeinen als stabil erwiesen hat, ist das Ergebnis häufig nicht zufriedenstellend, da das Modell zu viele historische Werte verwendet und auf einzelne Parameter sehr sensitiv reagiert [Nitz16].

\subsection{Das Euler-Maruyama-Verfahren}

Der Unternehmenswert kann alternativ durch das Euler-Maruyama-Verfahren approximiert werden. Hierbei wird ebenfalls die allgemeine Annahme verwendet, dass der Unternehmenswert einer geometrischen Brownschen Bewegung folgt [Mart14, S. 70]. Mit Hilfe des Euler-Maruyama-Verfahrens kann dann an diskreten Zeitpunkten eine numerische Näherungslösung der stochastischen Differentialgleichung ermittelt werden [Günt10]. Diese Näherungslösung weist ein gewisses Konvergenzverhalten gegenüber der analytischen Lösung auf [Seyd00].

Durch eine große Anzahl an Simulationen für den Unternehmenswert kann durch die MonteCarlo-Methode ebenfalls eine Ausfallwahrscheinlichkeit bestimmt werden.

Da diese Herangehensweise deutlich aufwändiger ist, wird sie in der Praxis weniger verwendet. Trotzdem wird an dieser Stelle auf sie eingegangen, da Erfahrungen aus dem Vergleich beider Verfahren auf stochastische Differentialgleichungen übertragen werden können, bei denen keine analytische Lösung existiert und diese lediglich numerisch approximiert werden kann [Nitz16]. Diese Erfahrungen fließen unter anderem auch in verschiedene Erweiterungen des Merton-Modells ein.

\subsection{Umsetzung und Bewertung des Merton-Modells}

Das Merton-Modell kann rückwirkend über einen längeren Zeitraum monatlich berechnet werden. Diese Entwicklung der Ausfallwahrscheinlichkeit kann schließlich durch eine Kurve dargestellt werden, welche in der Oberfläche des ADOMIS-Systems analog zu Abbildung 2 graphisch abgebildet wird. Beispielsweise ist diese zeitliche Entwicklung für drei Unternehmen in Abbildung 4 zu sehen, wobei die zugrundeliegenden Daten von der EURIBOR, einem Reuters Datastream Testzugang und der Yahoo Finance-Schnittstelle stammen.

In dieser Abbildung können die zeitliche Entwicklung der Ausfallwahrscheinlichkeit der Bayer AG, TUI AG und Volkswagen AG einzeln betrachtet und untereinander verglichen werden. Alle drei Unternehmen haben zu den gleichen Zeitpunkten eine geringe bis gar keine Ausfallwahrscheinlichkeit und in den gleichen Zeiträumen eine erhöhte Ausfallwahrscheinlichkeit. Die der Bayer AG hat den geringsten Anstieg, die der TUI AG unterliegt etwas größeren Schwankungen und die der Volkswagen AG steigt 2009 am meisten an.

Der Anstieg der Ausfallwahrscheinlichkeit der Volkswagen AG im Jahr 2008 liegt darin begründet, dass sich durch äußere Einflüsse der Aktienkurs innerhalb von drei Börsentagen vervierfacht hat und innerhalb der nächsten vier Tage wieder gesunken ist. Dadurch ist die historische Volatilität, welche die Schwankungsintensität der Aktienkurse misst, stark angestiegen und hat das Ergebnis des Merton-Modells verfälscht [Nitz16]. Das Modell reagiert also sehr sensitiv auf Schwankungen der Aktienkurse. 


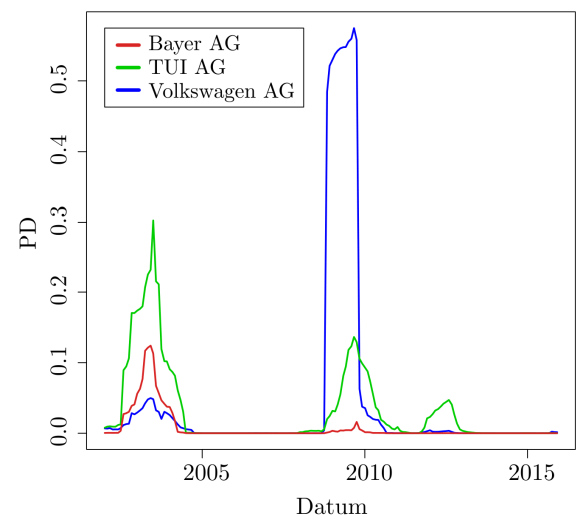

Abbildung 4: Zeitliche Entwicklung der Ausfallwahrscheinlichkeit der Bayer AG, TUI AG und Volkswagen AG (vgl. [Nitz16, S.24])

Die über das Merton-Modell bestimmte Ausfallwahrscheinlichkeit eines Unternehmens kann den von Ratingagenturen vergebenen, externen Ratings gegenübergestellt werden. Dieser Vergleich wurde unter anderem für die in Abbildung 4 verwendeten Unternehmen durchgeführt, wobei die externen Ratings den Geschäftsberichten der jeweiligen Unternehmen entnommen wurden.

Hieraus geht hervor, dass lediglich Tendenzen in eine ähnliche Richtung gehen, diese jedoch zum Teil zeitversetzt sind [Nitz16]. In anderen Fällen verlaufen die Entwicklungen der Einschätzungen ähnlich, liegen allerdings einige Ratingstufen auseinander.

Zudem beurteilt das Merton-Modell einen Ausfall schnell zu positiv oder negativ [Sobe00]. In den Zeiträumen, in denen die Ausfallwahrscheinlichkeit der drei Unternehmen in Abbildung 4 steigt, sind die zeitgleichen Einschätzungen der Ratingagenturen zum Beispiel deutlich weniger negativ als die Prognosen durch das Merton-Modell.

Ein weiterer Kritikpunkt des Modells ist seine starke Sensitivität bezüglich Schwankungen in den Aktienkursen eines Unternehmens, wie am Beispiel der Volkswagen AG zu erkennen ist.

\subsection{Ausblick auf weitere Kreditrisikomodelle}

Auf der Grundlage des Merton-Modells wurden weitere Modelle entwickelt, die auf diesem aufbauen und seine Schwächen minimieren. Hierzu zählt unter anderem das KMV-Modell der Ratingagentur Moody's [Albr05]. Da ein Unternehmen selten tatsächlich ausfällt, wird beispielsweise statt der absoluten Ausfallwahrscheinlichkeit, eine Wahrscheinlichkeit betrachtet, die angibt, wie wahrscheinlich es ist, dass sich das Rating eines Unternehmens verändert [Mart14].

Die Bewertung von Kreditrisiken hat durch die Finanzkrise an Bedeutung gewonnen und bleibt ein aktuelles Thema. Auch immer wieder neue Auflagen des Baseler Ausschuss für Bankenaufsicht stellen die Kreditinstitute vor weitere Herausforderungen [Mart14]. 


\section{$5 \quad$ Fazit und Ausblick}

\subsection{Fazit}

Es wurden die Struktur und Architektur von ADOMIS in den vorangegangenen Kapiteln aufgezeigt sowie auf das verwendete Datenmodell eingegangen. Finanzmathematische Anwendungen in der integrierten R-Umgebung wurden am Beispiel des Merton-Modells demonstriert.

Durch das Portal ist ADOMIS für den Anwender einfach in der Handhabung. Es bietet einen schnellen Zugriff auf Daten und die Möglichkeit Zeitreihen, anschaulich darzustellen. Finanzmathematische Modelle können auf der Datenbasis in der Statistiksprache R implementiert und berechnet werden. Zur Demonstration wurde das Merton-Modell umgesetzt.

Es wurden moderne Technologien, wie die NoSql-Datenbank Cassandra oder der Suchindex Elasticsearch verwendet. Das Datenmodell könnte allerdings auch auf andere Datenbanken, wie zum Beispiel Neo4j übertragen werden.

Über den DataLoader können einfach weitere Datenquellen einbezogen und die Datenbasis so schnell erweitert werden. Langfristig könnten auch Daten aus sozialen Netzwerken verwendet werden. ADOMIS hat somit den großen Vorteil, das verschiedene Datenquellen in einem System vereint sind.

\subsection{Ausblick}

In den letzten Jahren sind die Erwartungen an die Leistungsfähigkeit und Rechenleistung von Algorithmen und Systemen auch gerade im Finanzbereich gestiegen. Als Beispiel sei hier der Hochfrequenzhandel genannt, womit der Handel an den Börsen gemeint ist, der automatisiert und ohne menschliche Interaktion abläuft [Hofs14]. Damit in kürzester Zeit entschieden werden kann, welches Finanzinstrument gekauft oder verkauft wird, sind performante Systeme sehr wichtig.

Am Merton-Modell wird unter anderem kritisiert, dass es im Allgemeinen wenige Parameter und hauptsächlich historische Werte verwendet. Politische und psychologische Faktoren werden hier nur durch damit einhergehende Schwankungen in den Aktienkursen einbezogen, sind allerdings für die Kreditrisikomodellierung auch entscheidend. Die sogenannte „Verhaltensökonomie“ beschäftigt sich zum Beispiel mit solchen psychologischen Einflüssen [Hofs14, S. 171]. Eine umfassende und zugängliche Datenbasis erleichtert daher die Einbindung solcher Faktoren in die Modellbildung.

Es bleibt eine Herausforderung das Kreditrisiko und das Geschehen am Finanzmarkt mit geeigneten Modellen und Systemen zu modellieren und zu bewerten. ADOMIS bietet eine Möglichkeit diese verschiedenen Komponenten zu vereinen.

\section{Literaturverzeichnis}

[Albr05] Albrecht, Peter: Kreditrisiken - Modellierung und Management: Ein Überblick. Lehrstuhl für ABWL, Risikotheorie, Portfolio Management und Versicherungswirtschaft, Universität Mannheim, 2005.

[Gorm15] Gormley, C. und Tong, Z.: Elasticsearch: The Definitive Guide. O'Reilly and Associates, Sebastopol, 1. Auflage, 2015. 
[Günt10] Günther, M. und Jüngel, A.: Finanzderivate mit MATLAB: Mathematische Modellierung und numerische Simulation. Vieweg+Teubner, Wiesbaden, 2. Auflage, 2010.

[Hofs14] Hofstetter, Y.: Sie wissen alles: Wie intelligente Maschinen in unser Leben eindringen und warum wir für unsere Freiheit kämpfen müssen. Bertelsmann, München, 3. Auflage, 2014.

[Mart14] Martin, M. R. W.; Reitz, S. und Wehn, C. S.: Kreditderivate und Kreditrisikomodelle: Eine mathematische Einführung. Springer Spektrum, Wiesbaden, 2. Auflage, 2014.

[Nitz16] Nitzl, N.: Das Merton-Modell und seine numerische Simulation. Masterarbeit, Johannes Gutenberg-Universität Mainz, Mainz, 2016.

[Seyd00] Seydel, R.: Einführung in die numerische Berechnung von Finanz-Derivaten: Computational Finance. Springer-Lehrbuch. Springer, Berlin, Heidelberg, 2000.

[Sobe00] Soberhart, J. R. et al.: Moody's Public Firm Risk Model: A Hybrid Approach to Modeling Short Term Default Risk. Number Report \#53853, Moody's Investors Service, New York. 2000.

\section{Kontakt}

Prof. Dr. Christian Schulze, Prof. Dr. Michael Guckert, Natascha Nitzl

KITE Kompetenzzentrum für Informationstechnologie

Technische Hochschule Mittelhessen (THM), Fachbereich MND

Wilhelm-Leuschner-Str. 13, 61169 Friedberg

T +49 6031 604-4751, -452

\{christian.schulze, michael.guckert, natascha.nitzl\}@mnd.thm.de 


\title{
Ein adaptives Vorgehensmodell für hybrides Projektmanagement
}

\author{
Christian Seel, Holger Timinger
}

\section{Zusammenfassung}

Standardisierte Projektmanagementprozessen und Vorgehensmodelle helfen bei der Planung und Durchführung von Projekten. Durch die Standardisierung bieten sie Orientierung und helfen bei der Erfahrungssicherung und der Weiterentwicklung des Projektmanagements. Aufgrund des Einmaligkeitscharakters von Projekten müssen die standardisierten Prozesse an die jeweilige Projektsituation mit ihren Rahmenbedingungen angepasst werden.

Diese Anpassung, auch Tailoring genannt, geschieht meist manuell auf Basis der Erfahrung einzelner Individuen. Dieses Vorgehen erschwert strukturiertes Lernen und Verbessern. Das Tailoring ist aufwändig, subjektiv und rückblickend schwer nachvollziehbar. In diesem Beitrag wird ein adaptives Referenzmodell vorgestellt, welches traditionelle und agile Prozesse des Projektmanagements beinhaltet. Auf Basis projektspezifischer Parameter erfolgt ein automatisiertes Tailoring. Dieses kann zu Projektbeginn durchgeführt und während des Projektverlaufs aktualisiert werden. Durch den hohen Automatisierungsgrad ist das Tailoring schnell, objektiv, reproduzier- und nachvollziehbar.

\section{$1 \quad$ Einleitung}

In der Mitte des 20. Jahrhunderts setzte die Professionalisierung des Projektmanagements ein. Organisationen, wie das Project Management Institute und die International Project Management Association wurden gegründet und schufen ihre bis heute weiterentwickelten Standards. Mit dem Wasserfall- (vgl. [Royc70].)und dem V-Modell (vgl. [Boeh79].) wurden Vorgehensmodelle geschaffen, die die Qualität des Projektergebnisses sicherstellen sollen. Seit den 1990er Jahren kamen neue, sogenannte agile Vorgehensmodelle hinzu, die sich durch eine stärkere Kundenorientierung und eigenverantwortlich handelnde Teams auszeichnen. Anstelle von langfristigen Plänen treten iterative Arbeitszyklen. Typische Vertreter agiler Vorgehensmodelle sind Scrum und Kanban.

Diese Vorgehensmodelle werden in der Regel nicht ohne vorherige Anpassungen an unternehmensspezifische Rahmenbedingungen eingesetzt (vgl. [Whit14].)(vgl. [Komu17].). Dies gilt ganz besonders für kleine und mittlere Unternehmen (KMU) (vgl. [TuLK12].). Das Tailoring erfolgt unter Berücksichtigung der Projektart, der Komplexität, der Präferenzen der Auftraggeber, der Qualifikation der Mitarbeiter und weiterer Parameter. Am Ende des Tailoring steht ein angepasstes traditionelles, agiles oder hybrides Vorgehensmodell zur Verfügung. Letztgenanntes kombiniert traditionelle und agile Bestandteile eines Vorgehensmodells. Der Chance durch Tailoring ein auf die eigenen Bedürfnisse maßgeschneidertes Vorgehensmodell zu generieren, steht das Risiko entgegen, dass ein Vorgehensmodell entsteht, das wichtige Stärken der Eltern-Vorgehensmodelle verwässert oder gar eliminiert. Tailoring muss folglich immer mit entsprechender Sorgfalt geschehen. Gleichzeitig ist Tailoring nichttrivial. Die Menge an möglichen Kombinationen von Vorgehensbestandteilen über- 
steigt das, was realistischer Weise ohne Softwareunterstützung analysiert und optimiert werden kann.

In diesem Beitrag wird ein Vorgehensmodell für hybrides Projektmanagement (HyProMM) auf Basis eines adaptiven Referenzmodells vorgestellt. Die Adaption, d. h. das Tailoring erfolgt anhand definierter Parameter und ist schnell, objektiv, reproduzierbar und dokumentiert. Das Vorgehensmodell basiert auf einem adaptiven Referenzmodell, das in Abhängigkeit der vom Anwender gewählten Parameter zu einem traditionellen, agilen oder eben hybriden Vorgehensmodell konfiguriert wird. Regulatoren verhindern die Kombination von nicht sinnvollen Vorgehensmodellen und stellen die Konsistenz des adaptierten Modells sicher. Maßnahmen zur Konsistenzsicherung sind wichtig, um zu verhindern, dass einzelne Prozessbestandteile nicht zueinander passen, beispielsweise die Planung eines Sprint Backlog in einem rein traditionell arbeitenden Wasserfallprojekt. Konkret sollen in dem vorliegenden Beitrag folgende Forschungsfragen untersucht werden:

- $\quad$ RQ.1: Wie muss ein adaptives Referenzmodell strukturiert und modelliert werden, um Tailoring hybrider Projektmanagementprozesse zu ermöglichen?

- $\quad$ RQ.2: Wie kann ein Projektmanagementprozess mithilfe des Referenzmodells adaptiert werden?

In den folgenden Abschnitten wird zunächst der Stand der Wissenschaft hinsichtlich des Tailoring hybrider Projektmanagementprozesse skizziert und die Forschungsmethodik erläutert. Im Anschluss erfolgt die Beschreibung des Referenzmodells für adaptive hybride Projektmanagementprozesse (RQ.1). Im darauf folgenden Abschnitt wird eine beispielhafte Umsetzung anhand der Projektsteuerungsphase skizziert (RQ.2). Der Beitrag schließt mit einem Fazit und einem Ausblick, der den weiteren Forschungsbedarf skizziert.

\section{Stand der Wissenschaft und Forschungsmethode}

\subsection{Stand der Wissenschaft}

Vorgehensmodelle sind in der Regel starr. Sie geben je nach Modell mehr oder weniger detailliert Auskunft über den Ablauf eines Projekts, einzusetzende Methoden und ausführende Rollen. Manche Vorgehensmodelle weisen explizit auf Möglichkeiten des Tailoring hin, beispielsweise das V-Modell XT (vgl. [Bund05].). Auch der PRINCE2 Standard ermöglicht und fördert Tailoring (vgl. [Axel17].). Scrum als agiles Vorgehensmodell sieht kein Tailoring im engeren Sinne vor (vgl. [ScSu16].). Allerdings berücksichtigt Scrum in seinem Ablauf regelmäßige Retrospektiven zur Verbesserung der eigenen Arbeitsweise, Abläufe und Kommunikationswege. Die Adaption erfolgt also im Prozess selbst. Die scrumtypischen Rollen, Elemente und Artefakte wie Sprints, Daily Scrum und Product Backlog etc. bleiben jedoch bestehen.

Das Tailoring geschieht üblicherweise manuell und nicht notwendigerweise in einer systematischen, reproduzierbaren Art und Weise. In der Folge werden häufig subjektive Entscheidungen getroffen, die zudem nicht dokumentiert werden. Das Tailoring hängt stark von den das Tailoring ausführenden Individuen und deren Erfahrung ab. Damit geht die Nachvollziehbarkeit sowie die Erfahrungssicherung und Optimierung für künftige Projekte verloren. Regeln können dabei helfen, den Kern des Vorgehensmodells zu erhalten. So müssen bei PRINCE2 alle sieben Themen bestehen bleiben. Anpassungen dürfen auf Ebene der Themen, Produkte, Rollen und Prozesse sowie der Terminologie geschehen. 
Versuche, Prozesse strukturiert und automatisiert an Ziele und Rahmenbedingungen anzupassen, reichen weit zurück. Einige Aufmerksamkeit erregte das TAME Projekt zum Tailoring von Softwareprozessen [BR87]. Bis heute wird versucht das Tailoring zu strukturieren und zu systematisieren [Wh14]. Dennoch gibt es bisher kein kohärentes, digital realisiertes hybrides Prozessmodell für Projektmanagement, das sich anhand nachvollziehbarer Kriterien automatisiert adaptiert.

\section{$2.2 \quad$ Forschungsmethode}

Die Wahl der Forschungsmethode hängt von der zu beantwortenden Forschungsfrage ab. Diese lassen sich in zwei Arten unterscheiden (vgl. [Seel10].). Zum einen sind dies Forschungsfragen, die auf die Untersuchung des aktuellen Zustandes abzielen, wie beispielsweise die Frage, welche Projektmanagementmethoden in Unternehmen aktuell eingesetzt werden. Hier kommen Methoden der empirischen Sozialforschung zum Einsatz. Zum anderen gibt es Forschungsfragen, die auf die Entwicklung neuer Methoden abzielen, die mit dem Paradigma der Design Science bearbeitet werden.

Das hier vorgestellte adaptive Vorgehensmodell für hybrides Projektmanagement ist ein neu konzipierter Beitrag zur Digitalisierung des Projektmanagements. Dieser wird mit Hilfe der Design Science bearbeitet. Hevner et al. (vgl. [HMPR04].) postulieren dafür sieben Richtlinien, die einzuhalten sind: Artefakte als Ergebnis des Design Science Prozesses, Problemrelevanz, Evaluation, Forschungsbeitrag, Stringenz der Forschungsmethode, Forschung als Suchprozess sowie Kommunikation der Forschungsergebnisse.

An diesen Richtlinien orientiert sich der Aufbau dieses Beitrages. Als Artefakt wird ein Referenzmodell für hybrides Projektmanagement vorgestellt. Problemrelevanz und Evaluation werden in der Motivation bzw. im Rahmen der beispielhaften Umsetzung adressiert. Forschungsbeitrag, Stringenz und Forschung als Suchprozess werden in diesem Abschnitt berücksichtigt, da der Beitrag auf einen klar definierten Stand der Forschung aufbaut und diesen erweitert. Ferner ist die Forderung nach Kommunikation der Forschungsergebnisse durch die vorliegende Veröffentlichung abgedeckt.

\section{$3 \quad$ Adaptive Referenzmodellierung}

\subsection{Einführung}

In ihrem Beitrag zur Referenzmodellierungsforschung schlagen Fettke und Loos (vgl. [FeLo04].) die vier Phasen Problemdefinition, Konstruktion, Bewertung, Pflege für die Entwicklung eines Referenzmodells vor. Das hier vorgestellte adaptive Referenzmodell für automatisiertes Tailoring hybrider Projektmanagementprozesse nutzt den im Jahr 2016 vorgestellten Ordnungsrahmen für hybrides Projektmanagement nach Timinger und Seel als Basis (vgl. [TiSe16].). Der Ordnungsrahmen (vgl. [Meis01].) stellt dabei die oberste Ebene eines Referenzmodells zum hybriden Projektmanagement dar. Das Referenzmodell beinhaltet verschiedene traditionelle und agile Abläufe, die mit Parametern versehen werden. Bei Anwendung des adaptiven Referenzmodells werden die für das jeweilige Projekt zutreffenden Parameter ausgewählt und instanziiert. Die bei der Instanziierung vergeben Parameterwerte werden zur automatisierten Anpassung des Referenzmodells verwendet. Die Anpassung stellt sicher, dass das Vorgehen zum zugrundeliegenden Projekt, dem Kunden sowie den Projektmitarbeitern passt. Das adaptierte Referenzmodell enthält dann entweder 
nur traditionelle, nur agile oder traditionelle und agile Prozessbestandteile, die zu hybriden Projektmanagementprozessen zusammengefügt werden.

Nachfolgend werden das Referenzmodell und eine beispielhafte Umsetzung zur Beantwortung der Forschungsfragen vorgestellt und diskutiert.

\subsection{Ordnungsrahmen}

Das adaptive Referenzmodell basiert auf dem 2016 vorgestellten Ordnungsrahmen für hybrides Projektmanagement (vgl. [TiSe16].), siehe Abbildung 1.

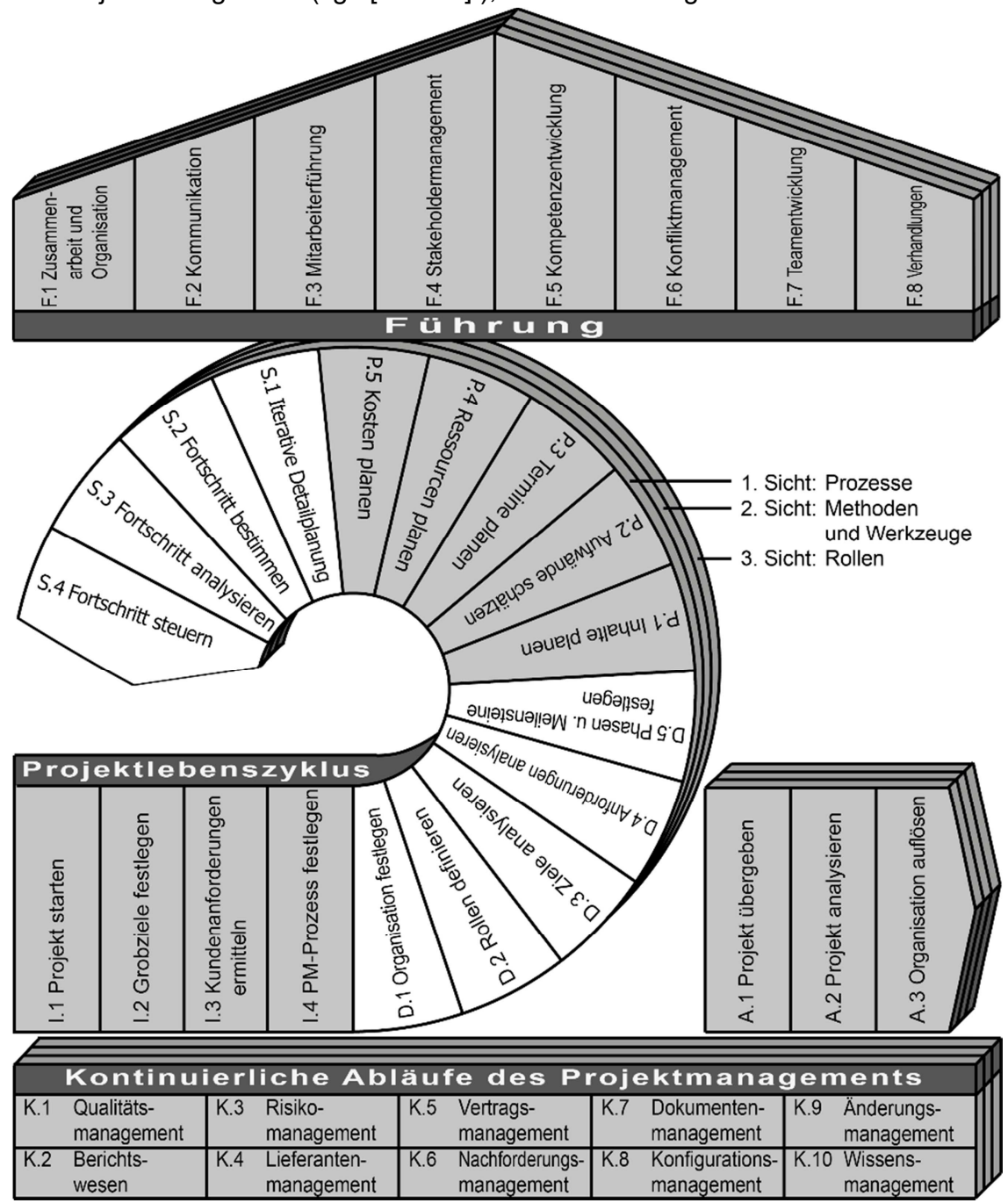

Abbildung 1: Ordnungsrahmen für hybrides Projektmanagement (HyProMM) (vgl. [TiSe16].)(vgl. [Timi17].)

Der Ordnungsrahmen hat die drei Funktionen, einen Überblick über das Referenzmodell zu ermöglichen, das Referenzmodell in inhaltlich sinnvolle Teilbereiche zu gliedern sowie als Markenzeichen des Referenzmodells eine rasche Wiedererkennung zu ermöglichen (vgl. [Meis01]). Der hier eingesetzte Ordnungsrahmen strukturiert wichtige Bausteine für erfolgreiches Projektmanagement aus den Bereichen Führung, dem eigentlichen Projektlebens- 
zyklus und die diesen begleitenden kontinuierlichen Abläufe, wie das Risiko- und Stakeholdermanagement. Der Ordnungsrahmen besteht aus drei Sichten: der Prozesssicht (Sicht 1), der Methoden- und Werkzeugsicht (Sicht 2) und der Rollensicht (Sicht 3).

In seiner jetzigen Ausbaustufe beschränkt sich das adaptive Referenzmodell auf den Bereich des Projektlebenszyklus, der in der Mitte des Ordnungsrahmens dargestellt ist. Dessen Gliederung orientiert sich an der DIN 69901 mit den Phasen Initialisierung, Definition, Planung, Steuerung und Abschluss des Projekts. Die Zuordnung zu diesen Phasen ist anhand der den einzelnen Elementen des Ordnungsrahmens voranstehenden Buchstaben I, $D, P, S$ und $A$ zu entnehmen.

Die runde Form des den Projektlebenszyklus beschreibenden Bereichs des Ordnungsrahmens deutet an, dass die einzelnen Phasen mehrfach durchlaufen werden können und somit nicht nur sequenzielle, sondern auch iterative Vorgehensmodelle abgebildet werden können. Generell gilt jedoch, dass der Ordnungsrahmen die Prozessbestandteile, Methoden und Werkzeuge sowie Rollen für hybride Vorgehensmodelle beschreibt, diese aber noch nicht zu Abläufen verbindet.

Das folgende Beispiel soll den hybriden Charakter des Ordnungsrahmens verdeutlichen: Das Prozesselement P.1 Inhalte planen (1. Sicht) des Ordnungsrahmens kann entweder traditionell als formlose Aufgabenliste oder als Projektstrukturplan methodisch umgesetzt werden oder aber agil anhand der Aufgaben eines Kanbanboards oder als Sammlung von User Stories in einem Product Backlog. Je nach Ausprägung werden die Methoden (2. Sicht) von unterschiedlichen Rollen angewendet, beispielsweise dem Projektmanager, dem Projektteam oder dem Product Owner (3. Sicht).

Über alle Phasen und deren Prozesselemente hinweg erlaubt der Ordnungsrahmen die Kombination von traditionellen und agilen Prozessen, Methoden und Werkzeugen sowie Rollen. Damit am Ende sinnvolle Abläufe entstehen, werden sogenannte Konsistenzparameter eingesetzt. Diese verhindern, dass beispielsweise der Product Owner zur Projektstrukturplanung eingesetzt wird. Der Ordnungsrahmen selbst gibt keine Auskunft darüber, wann in einem Projekt besser mit einem Projektstrukturplan, mit einem Product Backlog oder mit einer formlosen Aufgabenliste gearbeitet werden sollte. Der Ordnungsrahmen listet diese lediglich als Möglichkeiten der Inhaltsplanung eines Projekts. Die Auswahl und Verbindung zum optimalen Gesamtprojektablauf erfolgt durch Adaptionsmechanismen im Referenzmodell.

\subsection{Adaptives Referenzmodell im Überblick}

Der Aufbau des Referenzmodells erfolgt auf Basis der Elemente des Projektlebenszyklus des Ordnungsrahmens. Der Zusammenhang zwischen Ordnungsrahmen, Referenzmodell und Adaptionsparameter ist in Abbildung 2 skizziert. Der Anwender konfiguriert auf Basis seines Projekts und dessen Rahmenbedingungen die Parameter des adaptiven Referenzmodells.

Diese Konfiguration bestimmt, welche Prozesse gewählt werden und wie diese zu einem konsistenten Vorgehensmodell verbunden werden.

Außerdem kann auf Basis der gewählten Parameter eine getailorte Detailmodellierung der Prozesse inklusive anzuwendender Methoden und Werkzeuge erfolgen.

Für die Struktur und die Umsetzung des Referenzmodells ergeben sich drei Hauptaufgaben:

1. Die Parameter, die die Auswahl und Verbindung von Prozessen beeinflussen, müssen festgelegt und Konfigurationsmöglichkeiten definiert werden. 
2. Das aus verschiedenen Varianten traditioneller und agiler Projektmanagementprozesse bestehende Referenzmodell ist zu konstruieren.

3. Das Referenzmodell ist um Adaptionsmöglichkeiten zu erweitern. Dies geschieht durch Verknüpfung der Prozessbestandteile mit Parametern zur Konfiguration.

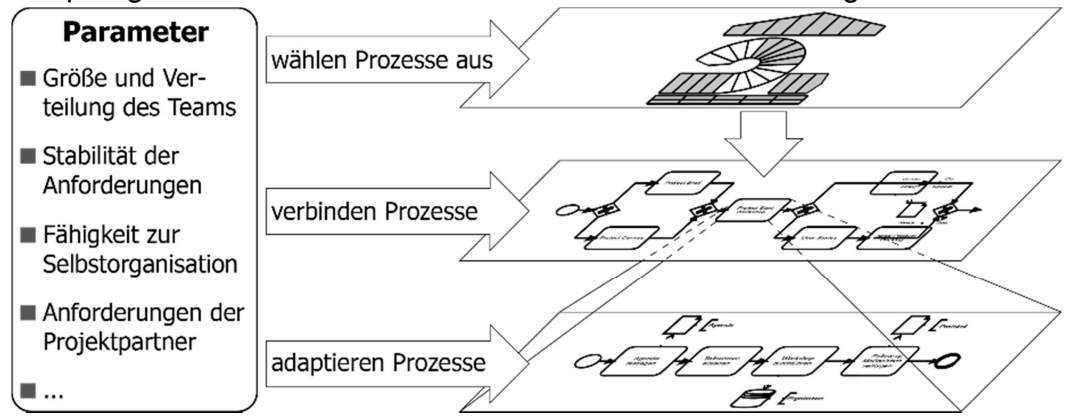

Abbildung 2: Die vom Anwender konfigurierten Parameter (links) legen fest, welche Prozesse aus dem Ordnungsrahmen (rechts oben) ausgewählt werden und zu stimmigen Abläufen verbunden werden (rechts in der Mitte). Die Prozesse können weiter detailliert und adaptiert werden (rechts unten). Die Modellierung der Prozesse erfolgt in BPMN 2.0.

\subsection{Gestaltung der Adaptionsparameter und -mechanismen}

Die Adaption des Referenzmodells geschieht automatisiert auf Basis der vom Anwender gewählten Parameter. Boehm und Turner (vgl. [BoTu04].) nennen beispielsweise die fünf Parameter Teamgröße, Stabilität der Anforderungen, Gefährdungspotenzial, Kultur und Qualifikationsgrad relevanter Stakeholder als Einflussgrößen auf ein Projekt. Diese lassen sich um weitere Parameter, wie Projektart, Komplexität, Budget, Festpreis, Risiko und Risikopolitik etc. ergänzen. Die Parameter können von unterschiedlichem Typ sein, beispielsweise numerische Parameter für die Teamgröße oder boolesche Parameter für die Festlegung, ob ein Festpreis vereinbart wurde. Die Ausgestaltung des Referenzmodells muss die spätere Integration weiterer Parameter ermöglichen.

Der aktuelle ACM-Survey von La Rosa et al. (vgl. [LvDM17].) strukturiert die Möglichkeiten zur Adaption von Prozessen in die vier Ansätze: node configuration, element annotation, activity specialization and fragment customization. Dabei ist element annotation auf der fachlichen Ebene universell einsetzbar (vgl. [LvDM17].) und kann daher auch zur Adaption der Methoden (2. Sicht) und Rollen (3. Sicht) verwendet werden. Konkrete Ansätze dazu stammen u. a. von [Delf06] oder [RiSe07]. Allerdings sind diese Ansätze bisher technisch nur unzureichend implementiert worden, da sie mit Meta-Metamodellen arbeiten und auf komplexen Mechanismen zur Konsistenzwiederherstellung zwischen Meta-Metamodell, Metamodell und Modell basieren. Daher wird an dieser Stelle ein ähnlicher, aber vereinfachter und besser zu implementierender Ansatz für Adaptionsparameter und darauf basierende Konfigurationsterme eingeführt.

Ein Konfigurationsterm setzt sich aus einem oder mehreren, verknüpften Parametern zusammen. Jeder Term lässt sich, sobald alle Parameter mit konkreten Werten instanziiert sind, entweder zu true oder false auswerten. Wird der Adaptionsterm zu false ausgewertet wird das Modellelement an dem er annotiert ist entfernt. Ansonsten verbleibt es im Modell. Die Konfigurationsterme werden als kontextfreie Grammatik in erweiterter Backus-NaurForm (vgl. [BW60].) beschrieben. 


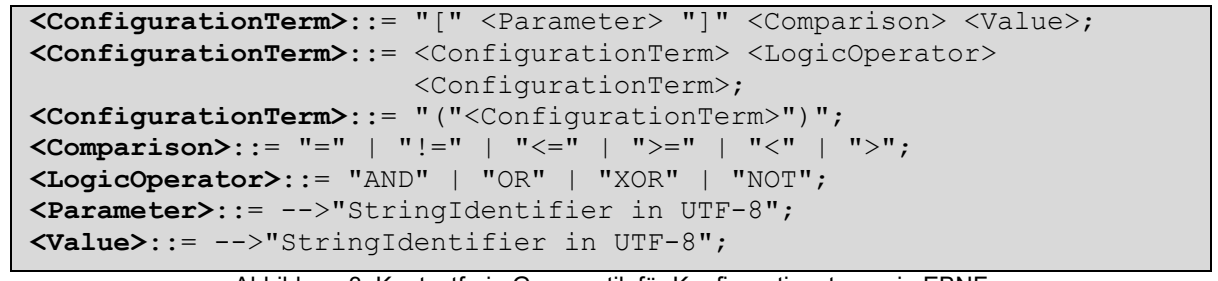

Abbildung 3: Kontextfreie Grammatik für Konfigurationsterme in EBNF

\subsection{Beispielhafte Umsetzung des Referenzmodells}

Das Referenzmodell für hybrides Projektmanagement wurde im Open Source Modeler Camunda BPM modelliert. Damit an jedes Prozesselement Konfigurationsterme annotiert und ausgewertet werden können, wurde die Software entsprechend erweitert. Jedem Prozesselement können nun beliebig umfangreiche Konfigurationsterme zugewiesen werden. Der Anwender kann im Anschluss die für inn wichtigen Parameter konfigurieren. Auf Basis dieser Konfiguration wird das Referenzmodell automatisch adaptiert und nicht benötigte Prozesselemente entfernt bzw. die verbleibenden Prozesselemente korrekt miteinander verbunden.

Im Beispiel der Abbildung 4 wird ein Auszug des Referenzmodells gezeigt, der sich auf die Elemente D.4, D.5 und P.1 des Ordnungsrahmens bezieht. Sowohl für agiles als auch für traditionelles Vorgehen kann es je Element mehrere Ausprägungen geben. So können die Inhalte des Projekts im Sprint Backlog beziehungsweise auf dem Taskboard nach Scrum geplant werden oder es kann ein Kanbanboard eingesetzt werden. Alternativ kann in kleinen traditionellen Projekten mit einer formlosen Aufgabenliste (To-Do-Liste) gearbeitet werden oder ein formalisierter Projektstrukturplan erstellt werden.

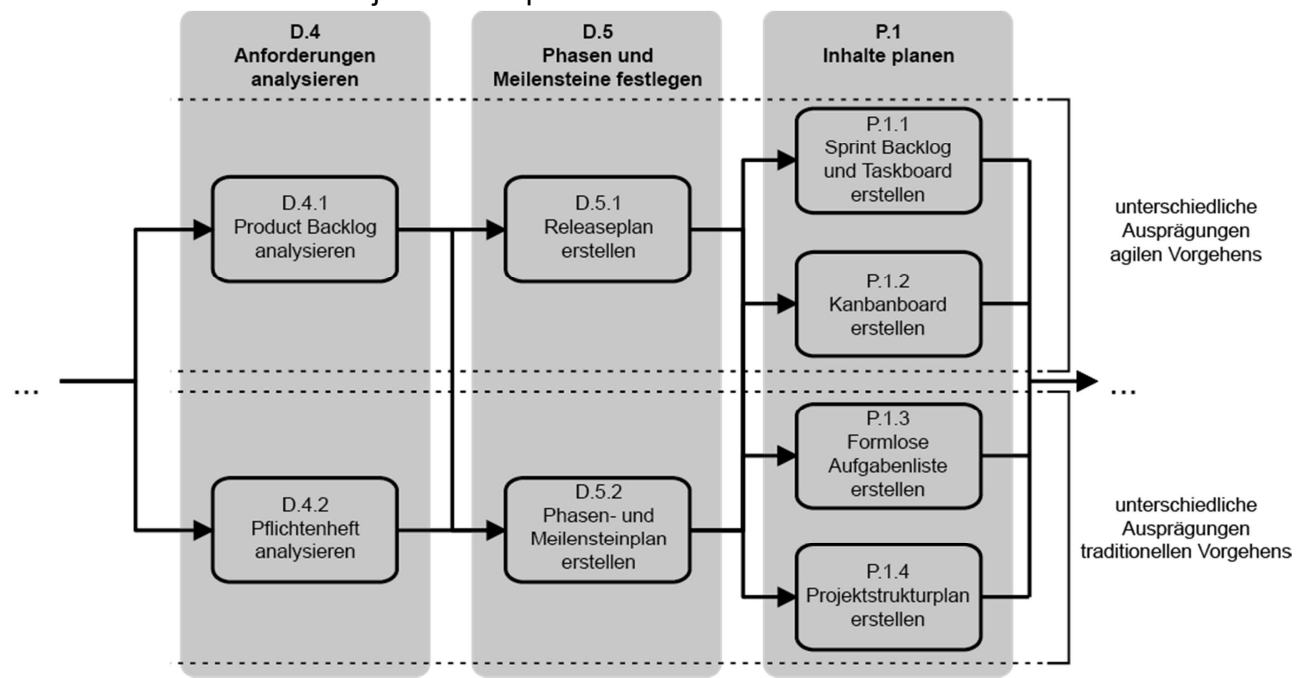

Abbildung 4: Auszugsweise Umsetzung der Elemente D.4 bis P.1 des Ordnungsrahmens im Referenzmodell

Für jede agile und traditionelle Ausprägung des Elements wird ein Konfigurationsterm annotiert, dessen Zustand (true oder false) von den vom Anwender konfigurierten Parametern abhängt. So kann beispielsweise die Festlegung, ob das Vorgehen grundsätzlich agil oder traditionell sein soll, von den Parametern Teamgröße (Variable iTeamSize) und von der Eignung des Projektgegenstands für agiles bzw. iteratives Vorgehen (Variable bltera- 
tionPossible) abhängig gemacht werden. Der Konfigurationsterm für agiles Vorgehen lautet dann:

$[$ iTeamSize $]<10$ AND [blterationPossible] == true

Der Konfigurationsterm für traditionelles Vorgehen lautet umgekehrt:

[iTeamSize $]>=9$ OR [blterationPossible $]==$ false

Das Referenzmodell erlaubt auch die gleichzeitige Nutzung agiler und traditioneller Vorgehenselemente. Abbildung 5 zeigt mehrere Beispiele, wie rein agiles, rein traditionelles und hybrides Vorgehen für den in Abbildung 4 vorgestellten Auszug des Referenzmodells nach erfolgter Konfiguration durch den Anwender aussehen kann.

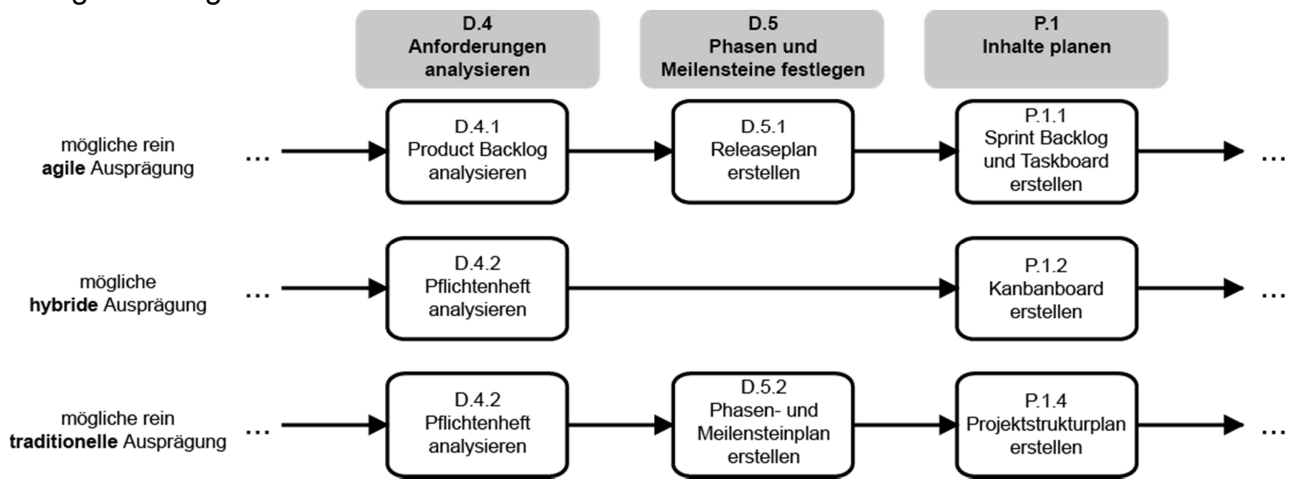

Abbildung 5: Mögliche agile, hybride und traditionelle Ausprägungen des auszugsweise dargestellten Referenzmodells aus Abbildung 4.

In einer rein agilen Ausprägung kann das Projekt beispielsweise nach Scrum durchgeführt werden. Dann wird das Product Backlog erstellt, ein Releaseplan angefertigt und anschließend der nächste Sprint geplant. In einem rein traditionell organisierten Projekt wird zunächst das Pflichtenheft erstellt und analysiert, ein grober Phasen- und Meilensteinplan erstellt und dann die inhaltliche Detailplanung mit Hilfe eines Projektstrukturplans durchgeführt. Im Beispiel des hybriden Projekts wird ein vom Kunden gewünschtes traditionelles Pflichtenheft analysiert. Anschließend werden daraus Aufgaben für eine agile Projektdurchführung unter Nutzung des Kanbanboards abgeleitet.

\section{$4 \quad$ Zusammenfassung}

In diesem Beitrag wurde das adaptive Vorgehensmodell für hybrides Projektmanagement „HyProMM“ vorgestellt. Das Vorgehen ist als Referenzmodell in BPMN 2.0 modelliert und die einzelnen Modellbestandteile mit Konfigurationstermen annotiert. Der Anwender konfiguriert die Parameter der Konfigurationsterme anhand projektspezifischer Gegebenheiten. Auf Basis dieser Konfiguration wird ein auf diese Gegebenheiten optimiertes Vorgehensmodell automatisiert erstellt. 
Die Adaption des Vorgehensmodells geschieht schnell, objektiv, reproduzierbar und ist dokumentiert. Erfahrungen mit dem Vorgehen können in eine Weiterentwicklung der Adaptionsmechanismen einfließen und so zum strukturierten Lernen beitragen.

Das Referenzmodell basiert auf einem Ordnungsrahmen. Die Modellierung des Modells erfolgte in einer speziell für diesen Beitrag erweiterten Version des Open Source Modelers Camunda BPM.

Künftige Forschungsfragen zielen auf die Erfassung weiterer relevanter Parameter ab, die das Projektmanagement beeinflussen. Ferner ist die Beschreibung des Zusammenhangs zwischen den Konfigurationsparametern und deren Auswirkung auf das Modell in Form von weiteren Konfigurationstermen notwendig. Als letzte weitere noch zu lösende Forschungsfrage ist der praktische Einsatz des Referenzmodells in der Praxis zu eruieren.

\section{Literaturverzeichnis}

[Axel17] Axelos Ltd.: PRINCE2, 2017. URL: https://www.axelos.com/best-practice-solutions/prince2. Abruf am 2017-03-27.

[BW60] Backus, J. W.; Wegstein, J. H.; van Wijngaarden, A.; Woodger, M., u.a.: Report on the algorithmic language ALGOL 60. In: Commun. ACM 3(5), 1960, S. 299-314.

[Beck07] Becker, Jörg (Hrsg.): Reference modeling: Efficient information systems design through reuse of information models ; with 5 tables, Heidelberg. Physica-Verl., 2007.

[Boeh79] Boehm, Barry: Guidelines for verifying and validating software requirements and design specifications: EURO IFIP 79, North Holland, 1979, S. 711-719.

[BoTu04] Boehm, Barry W.; Turner, Richard: Balancing agility and discipline: A guide for the perplexed. Addison-Wesley, Boston, 2004.

[Bund05] Bundesverwaltungsamt: V-Modell XT: Tailoring und projektspezifisches V-Modell, 2005. Abruf am 2017-03-23.

[Delf06] Delfmann, Patrick: Adaptive Referenzmodellierung: Methodische Konzepte zur Konstruktion und Anwendung wiederverwendungsorientierter Informationsmodelle. (Advances in information systems and management science, 25). Logos-Verl., Berlin, 2006.

[FeLo04] Fettke, Peter; Loos Peter: Systematische Erhebung von Referenzmodellen: Ergebnisse der Voruntersuchung. In: Working Papers of the Research Group ISYM(19), 2004.

[HMPR04] Hevner, Alan R.; March, Salvatore T.; Park, Jinsoo; Ram, Sudha: Design Science in Information Systems Research. In: MIS Q 28(1), 2004, S. 75-105.

[Komu17] Komus, Ayelt: Abschlussbericht: Status Quo Agile 2016/2017: 3. Studie über Erfolg und Anwendungsformen von agilen Methoden, 2017. BPM Labor Hochschule Koblenz. URL: https://www.hs-koblenz.de/index.php?id=1932. Abruf am 2017-04-20.

[LvDM17] La Rosa, Marcello; van der Aalst, Wil M.; Dumas, Marlon; Milani, Fredrik P.: Business Process Variability Modeling. In: ACM Comput. Surv. 50(1), 2017, S. 1-45.

[Meis01] Meise, Volker: Ordnungsrahmen zur prozessorientierten Organisationsgestaltung. Modelle für das Management komplexer Reorganisationsprojekte. (Studien zur Wirtschaftsinformatik, Bd. Band 10Bd). Verlag Dr. Kovac GmbH, Hamburg, 2001.

[RiSe07] Rieke, Tobias; Seel, Christian: Supporting Enterprise Systems Introduction by Controlling-Enabled Configurative Reference Modeling. In: Becker, Jörg (Hrsg.): Reference 
modeling: Efficient information systems design through reuse of information models ; with 5 tables. Physica-Verl., Heidelberg, 2007, S. 79-102.

[Royc70] Royce, Winston: Managing the Development of Large Software Systems: Proceedings IEEE WESCON, 1970, S. 328-338.

[ScSu16] Schwaber, Ken; Sutherland, Jeff: Scrum Guide, 2016. URL: http://www.scrumguides.org. Abruf am 2017-03-26.

[Seel10] Seel, Christian: Reverse Method Engineering: Methode und Softwareunterstützung zur Konstruktion und Adaption semiformaler Informationsmodellierungstechniken. (Wirtschaftsinformatik - Theorie und Anwendung, 20). Logos, Berlin, 2010.

[Timi17] Timinger, Holger: Modernes Projektmanagement: Mit traditionellem, agilem und hybridem Vorgehen zum Erfolg. 1. Auflage. Wiley-VCH, 2017.

[TiSe16] Timinger, Holger; Seel, Christian: Ein Ordnungsrahmen für adaptives hybrides Projektmanagement. In: GPM-Magazin PMaktuell 2016(4), 2016, S. 55-61.

[TuLK12] Turner, Rodney; Ledwith, Ann; Kelly, John: Project management in small to mediumsized enterprises. In: Management Decision 50(5), 2012, S. 942-957.

[Whit14] Whitaker, Sean: The benefits of tailoring - makeing a project management methodology fit, 2014.

\section{Kontakt}

Prof. Dr. Christian Seel, Prof. Dr. Holger Timinger

Hochschule Landshut

Institut für Projektmanagement und Informationsmodellierung (IPIM)

Am Lurzenhof 1, 84036 Landshut

christian.seel@haw-landshut.de, holger.timinger@haw-landshut.de 


\title{
Konfiguration des hybriden Projektmanagements für die Entwicklung technischer, physischer Produkte
}

\author{
Lars Brehm, Dorothee Feldmüller, Tobias Rieke
}

\begin{abstract}
Die Digitalisierung und die Dynamisierung der Märkte erfordern kurze Innovationszyklen. Die punktgenaue und zügige Erfüllung der Kundenanforderungen wird zum zentralen Erfolgsfaktor.

Agile Methoden ermöglichen im Projektmanagement, flexibel auf sich verändernde Rahmenbedingungen und Kundenanforderungen zu reagieren. Agile Methoden - in der Softwareentwicklung entstanden und zunehmend etabliert - können in der Regel nicht 1:1 auf die Entwicklung technischer Produkte übertragen werden. Dies bleibt auch bestehen, trotz des steigenden Anteils an Softwarekomponenten am Gesamtprodukt. Umgekehrt treibt ein steigender Anteil an Software auch das Interesse an der Nutzung agiler Methoden. In der Praxis finden sich bereits Ansätze, dass Teilprojekte agil (z. B. nach Scrum) und die technisch orientierten Produktbestandteile traditionell nach V-Modell oder Wasserfall entwickelt werden. Offen bleibt die Frage, wie das Projektmanagement an sich gestaltet werden kann, um stärker agile Aspekte auch in die technische Produktentwicklung einzubeziehen und eine bessere Vereinbarkeit unterschiedlicher Managementansätze in einem Projekt herzustellen. Hierzu sind die zentralen Aspekte des Produkts und dessen Lebenszyklus in die Gestaltung des Projektmanagementansatzes einzubeziehen.

Ziel des Beitrags ist, die wesentlichen Unterscheidungsmerkmale des Produkts und dessen Lebenszyklus, die eine Auswirkung auf die Wahl des Projektmanagementansatzes besitzen, darzustellen. Die um eine Produktperspektive erweiterten Kriterien bzw. Treiber von BOEHM/TURNER ermöglichen den Aufbau einer Klassifizierung von Projekten in Bezug auf ihr Agilitätspotenzial. Hierzu wird ein Ansatz vorgestellt, der als Bewertungsgrundlage eines hybriden Projektmanagementansatzes (z.B. nach TIMINGER/SEEL) dienen kann, und an einem Beispiel verdeutlicht.

Stichworte: Digitalisierung, Agilität, Hybrides Projektmanagement, Technische Produktentwicklungen
\end{abstract}

\section{$1 \quad$ Einleitung}

Die Entwicklung technischer Produkte folgt Vorgehensmodellen, die häufig auf ihre einzelnen Fachdisziplinen ausgerichtet sind (z. B.: nach Entwicklungsmodell des VDI [VDI93] oder für den Bereich Automobilbranche [HaWa13], Bauingenieurwesen [BMJ13]). Auch die Entwicklungszeit von marktreifen Produkten umfasst - je nach Größe/Komplexität des Produktes - mehrere Jahre. Die benannten Vorgehensmodelle gehen von einem geradlinigen Gesamtplan aus, der die einzelnen Stationen der Produktentwicklung konkret skizziert. Nachträgliche Änderungen an Produkten erfordern jedoch in vielen Fällen eine Anpassung des Vorgehens oder auch Rücksprünge zu vorherigen, bereits abgeschlossenen Projektphasen und -tätigkeiten, inkl. Freigabeentscheidungen durch das Lenkungsgremium. Durch dieses 
grundsätzliche Vorgehen sollen nachträgliche Änderungen überwacht und bewusst beschritten werden. Dabei werden die Konsequenzen der Veränderung auf sämtliche folgenden Phasen überprüft und in einen neuen Plan eingearbeitet. Dies ist zum einen aufwändig und kann zum anderen zu Fehlern führen.

Nun werden im Rahmen der Digitalisierung und Dynamisierung der Märkte Innovationszyklen immer kürzer. Daher werden dynamischere agile Vorgehensmodelle wie Scrum immer populärer. Im Kern dieser Modelle steht der Kunde bzw. Nutzer, der fortlaufend durch ein kleines, aus Generalisten bestehendes Entwicklerteam einbezogen wird. Neue Erkenntnisse werden im Laufe der Produktentwicklung aufgegriffen und flexibel berücksichtigt. Hier wird die Kundenorientierung höher priorisiert als das Verfolgen des ursprünglich formulierten Plans [Agil01]. Im Rahmen der Softwareentwicklung fällt dieser Ansatz auf fruchtbaren Boden, da Programmcode eine gute Adaptionsfähigkeit besitzt und dieses zusätzlich durch Softwaretools unterstützt wird.

Im Kontext der Digitalisierung werden Ansätze wie Industrie 4.0 oder Fabrik 4.0, Predictive Maintenance, Smart Products und Smart Services sowie Augmented Reality diskutiert und entwickelt. Diese Ansätze erfordern das gute Zusammenspiel zwischen Software und physischen Produkten bzw. Maschinen. Die historisch bedingten unterschiedlichen Vorgehensmodelle treffen hier aufeinander. In einem Projekt können sich somit klassisches und agiles Projektmanagement entweder (1) zeitlich gestuft oder (2) objektbezogen parallel existieren man spricht in diesem Fall auch von hybriden Projektmanagement [Hüss14].

Neben den objektbezogenen parallelen Ansätzen, die sich z. B. auf Hard- und Software oder andere Zuschnitte über Teilprojekte beziehen, ist auch eine Integration beider Vorgehensweisen und/oder Auflockerung entsprechender Regeln möglich, sodass einzelne Elemente oder Ansätze übernommen werden. Als Beispiel sei hier die Einbindung von Linienmitarbeitern im Team genannt, die in Teilzeit am Projekt arbeiten, was in dieser Form in der agilen Methode Scrum nicht vorgesehen ist.

Die agile Entwicklung technischer Produkte ist mit besonderen produkt- und organisationsbezogenen Herausforderungen verbunden. An dieser Stelle sind z. B. die schlechte Adaptionsfähigkeit bereits produzierter Produktbestandteile oder die Einbindung einer deutlich höheren Anzahl von häufig spezialisierten Personen zu nennen. Hier stellt sich die Frage, welchen Einfluss die Organisation, das Produkt und der Kunde auf die Konfiguration des hybriden Projektmanagementansatzes besitzen.

Entsprechend dem Design Science Ansatz werden im Folgenden zunächst die grundlegenden Unterschiede zwischen virtuellen Softwareprodukten und physischen Produkten herausgearbeitet und in einer Übersicht zusammengeführt. Die aktuellen, insbesondere agilen Projektmanagementansätze werden anschließend dargestellt. Danach werden die beiden Ansätze vereint und ein Modell vorgestellt, wie die Nutzung des Modells zur Konfiguration des Projektmanagementansatzes beitragen kann.

\section{Unterschiede zwischen Softwareentwicklungsprojekten und Entwicklungsprojekten für technische Produkte}

Die Softwareindustrie und die produzierenden Branchen sehen sich ähnlichen Herausforderungen gegenüber: Steigende Komplexität der Produkte, zunehmender Kostendruck, Forderung nach kürzerer Time-to-Market sowie der stärkere Wunsch nach Personalisierung der Produkte für den Kunden. 
Hinsichtlich der Produkte unterscheiden sich Software und technische Produkte aber erheblich. So sind bei digitalen Produkten die variablen Kosten - insbesondere die Kosten für die Produktion des Produktes, sprich die Kopie des digitalen Produktes - gleich Null [BuDH15]. Bei technischen Produkten sind Kosten und Zeitaufwand für Prozessplanung und Produktion, vor allem auch durch die einfließenden Materialkosten, hingegen erheblich [EiSt09].

Die Abbildung 1 gibt einen Überblick über die Kosten pro Phase im Produktlebenszyklus. Die Phasen sind an die Wertschöpfungskette in der Softwareindustrie nach Buxmann et. al. ([BuDH15], S. 142) und dem Produkt-Lifecycle-Management nach Eigner, Stelzer ([EiSt09], S. 8) angelehnt.

\begin{tabular}{|c|c|c|c|c|c|c|c|}
\hline & $\begin{array}{c}\text { Idee } \\
\text { Anforderung }\end{array}$ & Entwicklung & $\begin{array}{l}\text { Prozeß- } \\
\text { planung }\end{array}$ & Produktion & $\begin{array}{l}\text { Implemen- } \\
\text { tierung }\end{array}$ & Betrieb & $\begin{array}{l}\text { Recycling/ } \\
\text { Replacement }\end{array}$ \\
\hline Software & & & & & & & C \\
\hline $\begin{array}{l}\text { Tech. } \\
\text { Produkt }\end{array}$ & & & & & D & & D \\
\hline
\end{tabular}

Abbildung 1: Vergleich der Kosten pro Phase für Software und technische Produkte

Darüber hinaus gibt es mehrere relevante Unterschiede zwischen Software und technischen Produkten, welche in Tabelle 1 aufgeführt sind:

\begin{tabular}{|l|l|l|}
\hline Kriterium & $\begin{array}{l}\text { „Virtuelle Produkte“ - Software } \\
\text { mit begleitenden Dienstleistun- } \\
\text { gen }\end{array}$ & $\begin{array}{l}\text { Technische Produkte mit beglei- } \\
\text { tenden Dienstleistungen }\end{array}$ \\
\hline Losgröße & $\begin{array}{l}\text { Spezial- vs. Standardsoftware } \\
\text { Losgröße spielt keine Rolle }\end{array}$ & $\begin{array}{l}\text { Einzel / Serienfertigung / Massenfer- } \\
\text { tigung - Trend zur Losgröße 1 / Mass } \\
\text { Customization }\end{array}$ \\
\hline $\begin{array}{l}\text { Adaptions- } \\
\text { fähigkeit }\end{array}$ & $\begin{array}{l}\text { einfach möglich (nur Personal- } \\
\text { kosten) }\end{array}$ & $\begin{array}{l}\text { beschränkt möglich (v.a. hohe Mate- } \\
\text { rialkosten) }\end{array}$ \\
\hline Normen & wenig, weiche Standards & $\begin{array}{l}\text { viele Standards (z. B. DIN ISO, VDI, } \\
\text { VDE) }\end{array}$ \\
\hline Schutz & Code: Urheberrecht, Patente (US) & Patente \\
\hline $\begin{array}{l}\text { Produkt- } \\
\text { betrieb }\end{array}$ & Kein Verschleiß, nur „Alterung“ & $\begin{array}{l}\text { echter Verschleiß/Verbrauch, mäßige } \\
\text { Alterung }\end{array}$ \\
\hline Updates & $\begin{array}{l}\text { regelmäßig in kürzeren Abständen } \\
\text { einfach; schnell durch Hersteller } \\
\text { vorbereitet }\end{array}$ & $\begin{array}{l}\text { je nach Produkt nicht/erforderlich, } \\
\text { aufwändig und teuer }\end{array}$ \\
\hline
\end{tabular}

Tabelle 1: Unterschiede zwischen Software und technischen Produkten

\section{Aktueller Stand des Projektmanagements für die Entwicklung technischer, physischer Produkte}

Projektmanagement, insbesondere das, was wir heute den klassischen Ansatz nennen, hat sich bei der Entwicklung großer technischer physischer Produkte entwickelt: in der Luft- und Raumfahrt und bei großen Bauvorhaben.

Schon in den 80er Jahren des 20. Jahrhunderts beschreiben die Japaner Takeuchi und Nonaka [TaNo86], eine „neue“ Methode der Produktentwicklung, und verwenden dabei den 
Vergleich mit dem Sport: das Gedränge beim Rugby, wenn die Mannschaft nach dem Einwurf versucht den Ball zu gewinnen, ist ein Bild für gutes Zusammenspiel und heißt in der Sportart Scrum. Takeuchi und Nonaka adressieren in ihrer Arbeit auch "harte" physische Produkte. Was sie beschreiben, stimmt in hohem Maße mit dem überein, was in $2001 \mathrm{im}$ Agilen Manifest [Agil01] von prominenten Softwareentwicklern veröffentlicht wurde. „Agil“ kommt aus dem Lateinischen und bedeutet „beweglich“. Das agile Manifest beschreibt Werte und Prinzipien. Darauf aufbauend haben sich agile Methoden wie Scrum, IT-Kanban, XP, FDD u.a.m. in der Softwareentwicklung etabliert. Agile Methoden verwenden agile Praktiken wie z.B. ein tägliches nach bestimmten Spielregeln ablaufendes Meeting im Scrum, das Daily Scrum, oder die ebenfalls aus dem Scrum entstammende Praktik des Sprints, um ein Inkrement des Produktes zu entwickeln.

Zwischen Anhängern des klassischen und des agilen Projektmanagements ist es z.T. zur Polarisierung gekommen. Andere Schlüsselpersonen im Projektmanagement wie Boehm sehen Stärken und Schwächen beider Vorgehensweisen und plädieren dafür, diese je nach Situation und Projekt beide zu verwenden oder auch miteinander zu kombinieren [BoTu03] beschränken sich bei dieser Betrachtung aber auch auf den Bereich der Softwareentwicklung.

Von Boehm und Turner wurden auch Überlegungen entwickelt, wann in der Softwareentwicklung eher agil, wann eher klassisch gearbeitet werden sollte. Dort finden sich folgende Kriterien als begünstigend für den Einsatz agiler Methoden (vgl. [BoTu03], S. 55):

- Größe: Agiles Vorgehen eher für kleineres Vorhaben, kleineres Team

- Kritikalität: Agiles Vorgehen eher für unkritisches Vorhaben in Bezug auf Sicherheit / Gefährdungspotenzial

- Dynamik: Agiles Vorgehen eher bei mehr Dynamik / Unsicherheit / Instabilität in den Anforderungen

- Personal: Agiles Vorgehen eher für gut qualifizierte, intrinsisch motivierte Menschen („Theorie Y“ nach McGregor)

- Kultur: Agiles Vorgehen eher für junge/agile Unternehmenskultur, denen wir aus der Literatur noch folgende Kriterien hinzufügen möchten:

- Team an einem Standort (Co-Location)

- Teammitglieder arbeiten nur an einem Projekt (Dedication).

Im Kern verstehen wir in Anlehnung an [BoTu03], S. 17 unter agilen Vorgehensweisen in erster Linie den Einsatz von:

- $\quad$ Entwicklung in Iterationen und Inkrementen bei der Produktentwicklung

- Selbstorganisation des oder der Teams

- $\quad$ Permanente (Weiter-)Entwicklung der Prozesse und Strukturen anstelle vorheriger Festlegung.

Aufgrund zunehmender Erfolge agiler Methoden und Praktiken wird nun in jüngster Zeit auch in der Entwicklung technischer Produkte mit physischem Anteil die Frage gestellt, ob und wie agile Methoden und Praktiken hier erfolgreich eingesetzt werden können, und Beispiele des Einsatzes reflektiert [BeHa13, BGKS14, EHLB14, FeSt16, ÖzDr16] oder allgemeine Überlegungen angestellt [KIRe14].

Klein und Reinhardt ([KIRe14], vgl. S. 228)] empfehlen für ein physisches Produkt mehr Agilität, je kürzer der Produktlebenszyklus ist. In der Automobilindustrie mit Produktzyklen von vier bis sechs Jahren erscheint innen eine geeignete Mischung - ein hybrides Vorgehen - angemessen, bei der Luft- und Raumfahrt mit Produktzyklen von zehn bis zwanzig Jahren ist Agilität nach ihrer Einschätzung nicht hilfreich. 
Feste Entwicklungszyklen wie sie von Scrum bekannt sind, würden es ermöglichen, Fehlerkorrekturen für einen der nächsten Zyklen einzuplanen und weniger Wartezeiten sowie mehr Planbarkeit und Berechenbarkeit im Entwicklungsprozess zu erhalten. Die Einführung von festen kurzen Entwicklungszyklen für Iterationen wird mit horizontaler Agilität überschrieben (ebenda, S. 228f.). Unter vertikaler Agilität verstehen Klein und Reinhardt im Kontext der mechatronischen Produktentwicklung die Synchronisation der drei Disziplinen Mechanik, Elektronik und Informationstechnik (ebenda, S. 229), z.B. indem der IT-Anteil in kürzeren Zyklen entwickelt wird, und zum Abschluss eines jeden IT-Zyklus eine wenigstens informatorische Abstimmung mit dem in längeren Zyklen arbeitenden mechanischen bzw. elektronischen Anteil vorgenommen wird.

Diese Beispiele und Überlegungen verdichten sich in den statistischen Aussagen zum Einsatz agiler bzw. hybrider Vorgehensweisen in der Entwicklung technischer physischer Produkte: In den seit 2012 regelmäßig durchgeführten Studien „Status Quo Agile“ von Ayelt Komus in Zusammenarbeit mit der GPM Deutsche Gesellschaft für Projektmanagement e.V. findet sich in 2014 ein Anteil von 27\% der Teilnehmer der Studie, die agile Methoden bzw. agiles Projektmanagement auch für „Aktivitäten ohne besonderen IT-Bezug“ einsetzen ([KoKu15], in 2016 ist es ein Anteil von 34\% [KoKu17].

In der Automobilindustrie, die für ihre Prozessorientierung bekannt ist, und in der zudem viel verteilt entwickelt wird, ist man für ein agiles Vorgehen nicht gerade prädestiniert. Aber auch dort nimmt der Einsatz agiler Methoden zu. Während früher Agilität im Widerspruch zu den strengen Sicherheitsanforderungen im Automobil gesehen wurde, sieht heute die Mehrheit keinen Widerspruch mehr (vgl. [KuMa15], S. 4 und 41ff.). Allerdings findet der Einsatz agiler Methoden dort vor allem in der Softwareentwicklung statt (ebenda, S. 59 und 65). Es wird aber festgehalten, dass „die vollen Vorteile der Agilität erreicht werden können, wenn es jenseits der Softwareentwicklung angewendet wird und auch in der unternehmensübergreifenden Zusammenarbeit" (übersetzt nach [KuMa15], S. 65).

\section{$4 \quad$ Klassifizierung von Projekten und Bewertungsgrundlage für die Konfiguration eines hybriden Projektmanagementansatzes}

Im Folgenden wird ein Modell vorgestellt, das für ein konkretes Projekt eine Klassifizierung in Bezug auf sein Potential, agile Vorgehensweisen einzusetzen, anbietet.

\subsection{Aufbau einer Klassifizierung von Projekten in Bezug auf ihr Agilitätspotential}

Die bereits oben genannten von Boehm und Turner aufgezeigten Kriterien für die Bemessung des Agilitätspotenzials in der Softwareentwicklung nehmen wir als Ausgangspunkt ([BoTu03], S. 55). Dies sind Größe, Kritikalität, Dynamik, Personal, Kultur.

Weitet man das Spektrum der zu betrachtenden Entwicklungsprojekte auf solche mit physischen technischen Produkten aus, so ist den oben genannten fünf Kriterien aus unserer Sicht ein sechstes hinzuzufügen:

- $\quad$ Produkt: Agiles Vorgehen eher für Produkte mit kürzeren Lebenszyklen; hoher

Modularität und Kapselung; Inkremente mit geringerem Wert

Produkte mit kürzeren Produktentwicklungszeiten und/oder geringerem Wert eignen sich gut für die Anwendung agiler Vorgehensweisen. Entscheidender Engpass ist hier die Anwendbarkeit von Iterationen bzw. Inkrementen. In den Beispielen, die uns bekannt sind, finden die Anwender von Iterationen bei einer gesamten Entwicklungszeit von bis ca. zwei oder gar 
drei Jahren noch sinnvolle Möglichkeiten zu iterieren und halten Iterationsdauern von ca. 3 Monaten für machbar. Eine hohe Modularität und Kapselung von einzelnen Komponenten fördern ebenso die Anwendbarkeit von Inkrementen und damit den Einsatz von agilen Methoden. Eine weitere Restriktion sind die Kosten für eine Iteration bzw. ein Inkrement: steigen diese zu stark an, bringt diese keinen Mehrwert mehr im Vergleich zu dem Erkenntnisgewinn. Die Grenzen sind je Produkt, Projektsituation und Unternehmen festzulegen.

Die von Boehm und Turner gewählte Darstellung (vgl. [BoTu03 9, S. 56) erlauben wir uns wie folgt zu erweitern:

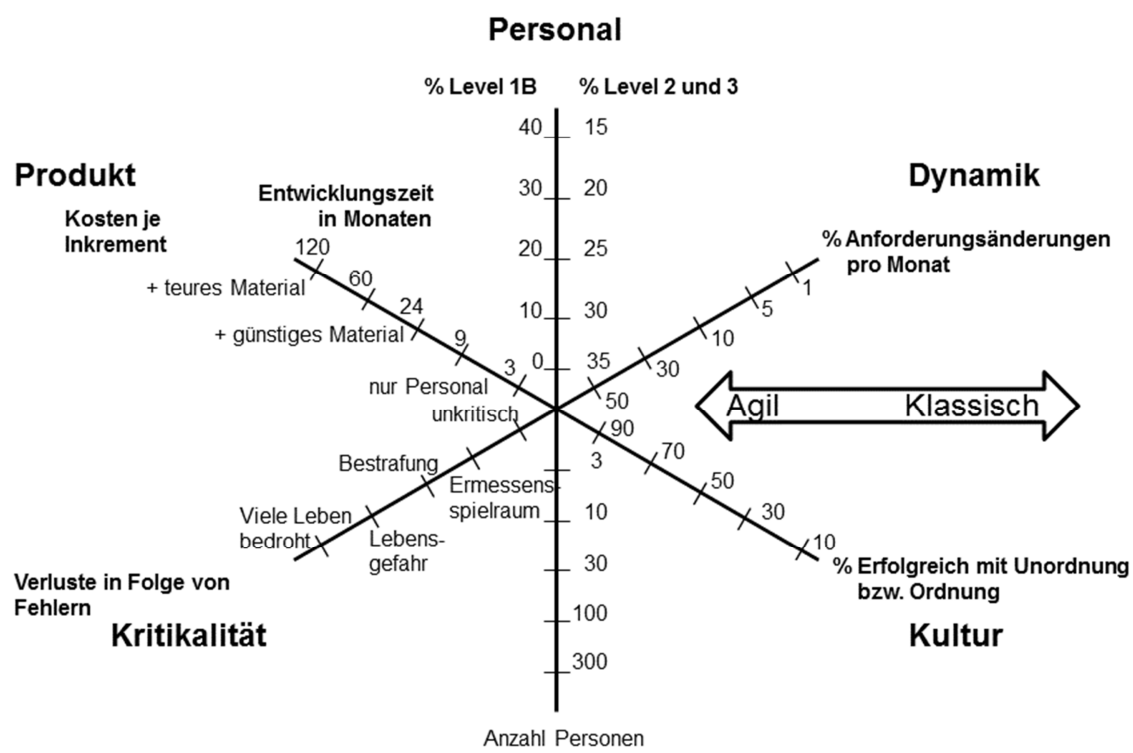

Größe

Abbildung 2: Agilitätspotential nach Boehm/Turner, erweitert um das Produkt.

\subsection{Bewertungsgrundlage für die Konfiguration eines hybriden Projektmanage- mentansatzes auf Basis des Ordnungsrahmens des hybriden Projektmanage- ments}

Anhand der sechs genannten Kriterien lassen sich nun das Unternehmen, das konkrete Projekt, die beteiligten Mitarbeiter und das Vorhaben bzw. das zu entwickelnde Produkt bewerten und daraus ableiten, in wieweit agile oder klassische Projektmanagementmethoden für ein konkretes Projekt geeignet sind. Dieses Vorgehen lässt sich analog auf einzelne Teilprojekte bzw. Projektphasen anwenden. So ergibt sich die Möglichkeit, dass die Entscheidung für klassisches vs. agiles Projektmanagement in einzelnen Teilprojekten bzw. Projektphasen unterschiedlich ausfällt. In diesem Zusammenhang sei darauf hingewiesen, dass die Art der Zerlegung des Projektgegenstands, z. B. anhand von „Gewerken“ wie Softwareentwicklung, Maschinenbau und Mechatronik im Gegensatz zu „interdisziplinären“ Modulen, einen wichtigen Einfluss auf die Bewertung der resultierenden Teilprojekte hat.

Timinger und Seel schlagen einen Ordnungsrahmen für die Konfiguration von hybridem Projektmanagement vor [TiSe16a]. Dazu definieren sie in den drei Bereichen Führung, Projektlebenszyklus und kontinuierliche Prozesse allgemein gültige Prozesse, Methoden und Werkzeuge sowie Rollen. In Abbildung 3 ist der Ordnungsrahmen wiedergegeben. 


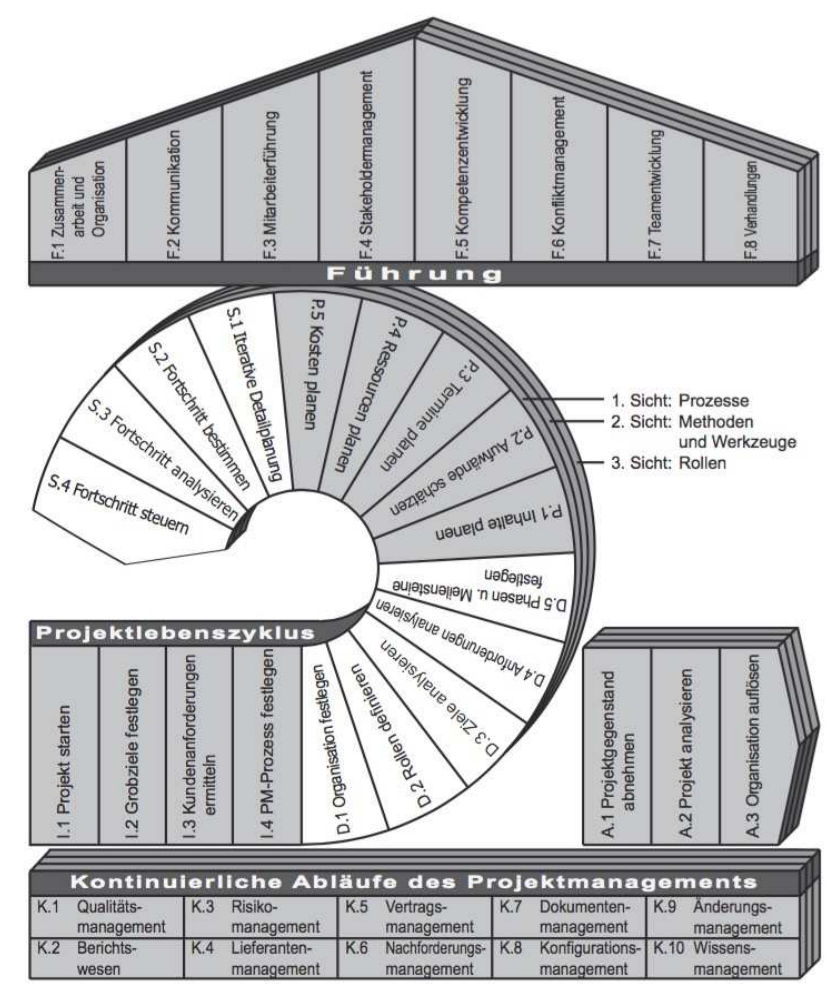

Abbildung 3: Ordnungsrahmen von Timinger / Seel [TiSe16a]

Für agiles bzw. klassisches Projektmanagementvorgehen sind jeweils nur bestimmte aller aufgeführten Prozesse in unterschiedlicher Ausprägung relevant.

Mit den sechs genannten Kriterien und deren Anwendung auf Projektphasen bzw. Teilprojekte lassen sich Gestaltungshinweise für die Auswahl der geeigneten Prozesse und der richtigen Ausprägung des Ordnungsrahmens finden.

Timinger und Seel forcieren eine Adaption des Ordnungsrahmens für verschiedene Ausgangssituationen auf Basis von Konfigurationsmechanismen, die relevante Elemente nach klaren Regeln aus- bzw. abzuwählen [TiSe16b]. Dieser Konfigurationsansatz ist vergleichbar mit den Arbeiten in den Projekten KOREAN [BDK04] und REFMOD06 [BDR06]. Einen ähnlichen Ansatz - im klassischen Projektmanagement - hatte auch bereits Thygs gewählt [Thyg07].

Der in diesem Beitrag verfolgte Ansatz soll keine festen Konfigurationsregeln aufbauen, sondern eine Entscheidungsunterstützung leisten, inwieweit klassische oder agile Ansätze vorteilhaft sind.

\subsection{Anwendung am Beispiel}

Über positive Erfahrungen aus der Industriepraxis auch mit vertikaler Agilität bei der Entwicklung technischer Systeme wird berichtet in [ÖzDr16] und [BGKS14] (S. 162ff.), in letzterem vom Automobilzulieferer Johnson Controls. Aufgrund der vorliegenden Informationen gehen wir von einer gemischten Situation in Bezug auf Teamgröße, Kritikalität, Dynamik und Personal aus, sowie einer eher traditionellen Unternehmenskultur. Die Produktentwicklungszeit beträgt ca. 15 Monate, bei vier bezahlbaren auch materialabhängigen Prototypen. 
Hierzu werden zu Beginn die Inkremente Software-, Elektrik-, Mechanik- und Zulieferkomponenten gebildet und geeignete Iterationen dafür geplant und synchronisiert und dann gesteuert (Prozesse I.4 bzw. P.1 und S.3).

Ein anderer Hersteller technischer Systeme, Schneider Electric, macht eine strikte Trennung von Software- und Hardwareentwicklung und arbeitet nur im Softwareanteil agil [BeHa13]. Auch hier gehen wir von einer vergleichbaren gemischten Situation in Bezug auf Teamgröße, Kritikalität, Dynamik, Personal und einer traditionellen Unternehmenskultur aus. In Bezug auf das Produkt lässt sich in der Hardwarekomponente kein geeignetes Inkrement finden, das sich rechnet: die Produktentwicklungszeit liegt typischerweise bei zwei bis drei Jahren, mit einem teuren Zertifizierungstest, der nicht mehrfach bezahlt werden kann. Die Betrachtung des Produktes führt zu der Auftrennung in Software- und Hardwareanteile, für die zeitlich parallel gearbeitet wird, einmal mit geplanten Inkrementen und Iterationen, einmal klassisch, auf ein gemeinsames Final Release hin ausgerichtet und gesteuert (Prozesse I.4 bzw. P.1 und S.3 mit anderen Ergebnissen).

Automobilzulieferer im Umfeld der Autoren versuchen sich an agilen Praktiken, indem in ihren Entwicklungsprozess Sprints und Daily Scrums eingeführt werden. Die Sprints erfolgen im Sinne von kurzfristigen Commitments ohne Umgestaltung des Prozesses im Sinne von Inkrementen aufgrund der Priorisierung eines Product Backlogs. Ohne große Änderungen am eigentlichen Prozess wird eine positive Auswirkung auf den Kommunikationsprozess im Projekt (F.2 im Ordnungsrahmen) berichtet.

\section{$5 \quad$ Ausblick / Fazit \& Limitationen}

Anhand der in der Literatur genannten Beispiele kann das von uns erweiterte Modell auf der Basis von Boehm und Turner als Grundlage für die Bewertung und Entscheidung des Agilitätspotenzials eines Projektes untermauert werden. Es leistet damit einen Beitrag zur Konfiguration des hybriden Projektmanagements bzw. zum heutzutage geforderten adaptiven Projektmanagement, und erweitert das Spektrum der Projektinhalte dabei von der Softwareentwicklung auf die Entwicklung technischer Produkte im Allgemeinen.

Es wird deutlich, dass die Modularisierbarkeit und Adaptionsfähigkeit eines technischen Produktes - im Entwicklungsprozess, im Fertigungsprozess (Eignung für Mass Customization) wie auch im Betrieb (spätere Updates), siehe Tabelle 1 - den wesentlichen Faktor für das Agilitätspotential darstellt. Uns erscheint diese am ehesten in (Entwicklungs-) Zeit und Kosten greifbar bzw. messbar. Die genannten Werte stellen nur eine erste Näherung dar. Sie können aufgrund der vorliegenden Beispiele und Erfahrungen noch als vollständig für ein finales Regelwerk im Sinne einer parametrisierbaren Konfiguration des Projektmanagementansatzes angesehen werden. Hierfür sind weitere Validierungen des Modells und eine quantitative Untersuchung sinnvoll.

\section{Literatur}

[Agil01] http://www.agilemanifesto.org/iso/de/manifesto.html, abgerufen am 13.05.2017, verabschiedet in 2001. 
[BDK04] Becker, J., Delfmann, P.; Knackstedt, R.: Konstruktion von Referenzmodellierungssprachen. Ein Ordnungsrahmen zur Spezifikation von Adaptionsmechanismen für Informationsmodelle. In Wirtschaftsinformatik 46 (2004) 4, S. 251-264.

[BDR06] Becker, Jörg, Delfmann, Patrick, Rieke, Tobia: RefMod06. Wiederverwendung fachkonzeptioneller Softwaremodelle für kleine und mittlere Softwareunternehmen durch adaptive, komponentenorientierte Referenzmodellierung. Münster 2006.

[BeHa13] Bergmann, E.; Hamilton, A.: Agile-Waterfall Hybrid: Living together in perfect harmony! Agile2013 Conference.

[BGKS14] Brandes, U.; Gemmer, P.; Koschek, H.; Schültken, L.: Management Y. Campus, Frankfurt 2014.

[BMJ13] Bundesministerium der Justiz und für Verbraucherschutz (BMJ). Verordnung über die Honorare für Architekten und Ingenieure (HOAI), in: Bundesgesetzblatt I, Nr. 37, Berlin 2013, S. 2276-2374.

[BoTu03] Boehm, B.; Turner, R.: Balancing Agility and Discipline - A Guide for the Perplexed. Addison Wesley, Boston 2003.

[BuDH15] Buxmann, P., Diefenbach, H., Hess, T., 2015. Die Softwareindustrie: Ökonomische Prinzipien, Strategien, Perspektiven, 3. Aufl., Springer Gabler, Berlin 2015.

[EHLB14] Eliasson, U.; Heldal, R.: Lantz, J.; Berger, C.: Agile Model-Driven Engineering in Mechatronic Systems - An Industrial Case Study, in: Lecture Notes in Computer Science, 8767, 2014, S. 433-449.

[EiSt09] Eigner, M., Stelzer, R., 2009. Product Lifecycle Management: Ein Leitfaden für Product Development und Life Cycle Management. Springer Science \& Business Media.

[FeSt16] Feldmüller, D.; Sticherling, N.: Agile Methoden in der Entwicklung mechatronischer Produkte, in: projekt MANAGEMENT aktuell 2/2016, Nürnberg 2016.

[HaWa13] Hab, G; Wagner, R: Projektmanagement in der Automobilindustrie - Effizientes Management von Fahrzeugprojekten entlang der Wertschöpfungskette. 4. Aufl., Wiesbaden 2013.

[Hüss14] Hüsselmann, C.: Agilität im Auftraggeber-/Auftragnehmer-Spannungsfeld - Mit hybridem Projektansatz zur Win-win-Situation. projektMANAGEMENT aktuell, 01/2014, S. 38-42, 2014.

[KIRe14] Klein, T.; Reinhart, G.: Approaches for Integration of Agile Procedures into Mechatronic Engineering of Manufacturing Systems., in: Zaeh, M.F. (Hrsg.): Enabling manufacturing competitiveness and economic sustainability, Springer, Cham Heidelberg New York Dordrecht London 2014, S. 225-230.

[KoKu15] Komus, A.; Kuberg, M.: Status Quo Agile, GPM Deutsche Gesellschaft für Projektmanagement e.V., Nürnberg 2015.

[KoKu17] Komus, A.; Kuberg, M.: Studie Status Quo Agile 2016/2017, Hochschule Koblenz, Koblenz 2017.

[KuMa15] Agile in Automotive -State of Practice 2015, www.kuglermaag.de/agile2015, abgerufen am 16.11.2016.

[ÖzDr16] Özcan, G.; Drescher, A.: Hybride Vorgehensmodelle und Lean Methoden in global verteilten Produktentwicklungsprojekten, in: Engstler, M.; Fazal-Baqaie, M.; Hanser, E.; Linssen, O.; Mikusz, M.; Volland, A. (Hrsg): Projektmanagement und Vorgehensmodelle 2016, Proceedings, Gesellschaft für Informatik, Bonn 2016. 
[TaNo86] Takeuchi, H.; Nonaka, I.: The new new product development game, in: Harvard Business Review 64 (1986) 1, S. 137 - 146.

[TiSe16a] Timinger, H., Seel, C., 2016. Ein Ordnungsrahmen für adaptives hybrides Projektmanagement. projektMANAGEMENT aktuell, S.55-61.

[TiSe16b] Timinger, H., Seel, C., 2016. Ein Ordnungsrahmen für die Entwicklung hybrider Vorgehensmodelle unter Nutzung agilen und traditionellen Projektmanagement. Vortrag im PM Forum am 18.10.2016 in Nürnberg.

[Thyg07] Thygs, M., 2007: Referenzdatenmodellgestütztes Vorgehen zur Gestaltung von Projektinformationssystemen: Adaptive Referenzmodellierung im Projektmanagement.

[VDI93] Verein deutscher Ingenieure (VDI): Methodik zum Entwickeln und Konstruieren technischer Systeme und Produkte. VDI-Richtlinie 2221:1993-05. (Bd. 6). Beuth, Berlin.

\section{Kontakt}

Prof. Dr. Lars Brehm

Hochschule München, Fakultät für Betriebswirtschaftslehre

Am Stadtpark 20, 81243 München

lars.brehm@hm.edu

Prof. Dr. Dorothee Feldmüller

Hochschule Bochum, Campus Velbert/Heiligenhaus

Kettwiger Straße 20, 42579 Heiligenhaus

dorothee.feldmueller@hs-bochum.de

Prof. Dr. Tobias Rieke

FH Münster, Institut für Technische Betriebswirtschaft

Bismarckstr. 11, 48565 Steinfurt

tobias.rieke@fh-muenster.de 


\section{Geschäftsprozesse}




\title{
Metamodellbasierte Erweiterung der BPMN zur Modellierung und Generierung von Prozessvarianten
}

\author{
Christian Seel
}

\section{Zusammenfassung}

Die Modellierung hat sich zentrales Instrument zur Planung, Gestaltung, Implementierung und zum Controlling von Geschäftsprozessen etabliert. In der Praxis tritt dort jedoch oft ein Problem beim Umgang mit Modellvarianten auf. Beispielsweise kann ein Prozess zum Leasing eines Fahrzeuges in einem Konzern je nach Land, in dem er stattfindet, verschieden ausgestaltet sein. Dennoch stimmen die überaus meisten Prozessschritte für alle Länder überein. Aktuelle Ansätze zum Umgang mit Prozessvarianten weisen jedoch zwei Defizite auf. Zum einen sind aktuelle Standards, wie BPMN 2.0 nicht ausreichend berücksichtigt. Zum anderen fehlt es an einer Softwareunterstützung für Prozessvarianten in BPMN-Modellen.

Aus den aufgezeigten Defiziten ergibt sich unmittelbar die Zielsetzung des Beitrages. Der Beitrag präsentiert eine BPMN-Erweiterung, die es erlaubt verschiedene Prozessvarianten in einem einzelnen Modell darzustellen. Diese Erweiterung wird um Mechanismen zur automatischen Konfiguration ergänzt, so dass man die jeweiligen Varianten in Abhängigkeit von Prozessparametern automatisch erzeugen kann. Um die Anwendbarkeit dieses Ansatzes zu demonstrieren, wird zudem eine prototypische Implementierung der Mechanismen auf Basis des Open-Source-Modellierungswerkzeuges Camunda vorgestellt.

\section{$1 \quad$ Einleitung}

Die Modellierung hat sich zentrales Instrument zur Planung, Gestaltung, Implementierung und zum Controlling von Geschäftsprozessen etabliert (vgl. [Seel10].). In der Praxis tritt dort jedoch oft ein Problem beim Umgang mit Modellvarianten auf (vgl. [Delf06].). Beispielsweise kann ein Prozess zum Leasing eines Fahrzeuges in einem Konzern je nach Land, in dem er stattfindet, verschieden ausgestaltet sein. Dort spielen Faktoren, wie gesetzliche Vorgaben, Besonderheiten des Marktes oder auch die Konkurrenzsituation eine Rolle. Dennoch stimmen die überaus meisten Prozessschritte für alle Länder überein.

Der Ansatz pro Land ein getrenntes Modell anzulegen, erweist sich bei der Pflege der Modelle aber schnell als nachteilig, da eine Änderung an einem Prozesselement, das alle länderspezifische Prozesse gemein haben, in jedem einzelnen Modell durchgeführt werden muss. Neben dem damit verbunden Aufwand besteht die Gefahr Inkonsistenzen zwischen den Prozessvarianten zu erzeugen.

Das Problem des Variantenmanagements von Prozessmodellen zeigt sich beispielsweise auch, bei der modellbasierten Anpassung von Software. Erstellt man hierbei für jeden Kunden ein eigenes unabhängiges Modell, obwohl die Modelle sich nur in wenigen Details unterscheiden, sieht man sich auch hier mit großem Aufwand bei der Modellpflege und Erweiterung sowie mit Inkonsistenzen konfrontiert. Abbildung 1 zeigt ein Beispiel für vier Varianten eines Prozess zum Leasing eines Fahrzeuges. Die blau dargestellten Modellelemente 
sind dabei bei allen Varianten identisch, während die rot dargestellten Modellelemente nicht in allen Varianten vorhanden sind.

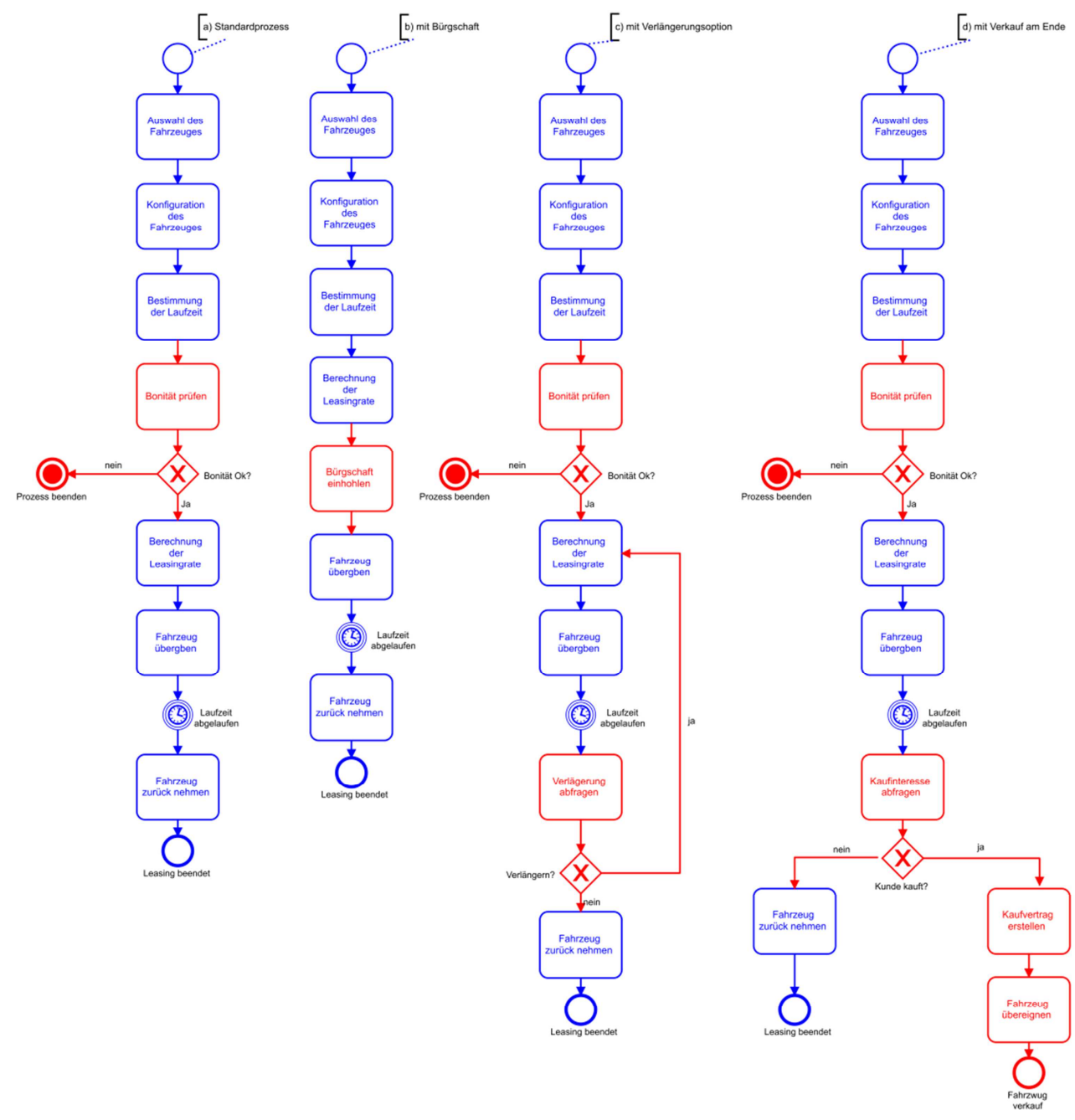

Abbildung 1: Prozessvarianten im Fahrzeugleasing

Da das Problem des Variantenmanagements bereits erkannt wurde, gibt es auch schon Lösungsvorschläge in Form der adaptiven Modellierung von Prozessvarianten. Eine Übersicht über bestehende Ansätze zur Modellierung von Prozessvarianten geben (vgl. [LvDM17].). Die bisherigen Ansätze, wie (vgl. [BDKK02]; [Rova07]; [LDtM09].) weisen jedoch in der praktischen Umsetzung Defizite auf.

Zum einen besitzen die wenigsten Ansätze eine Softwareunterstützung, die einen Einsatz in der Praxis erst sinnvoll ermöglicht. Zum anderen setzen die meisten Ansätze nicht auf standardisierte Modellierungssprachen. Aktuelle internationale Standards, wie die Business Process Model and Notation (BPMN) der OMG (vgl. [Obje11].) werden nicht berücksichtigt. Daher adressiert dieser Artikel die folgenden Forschungsfragen (RQ): 
- $\quad R Q .1$ : Wie kann die BPMN 2.0 erweitert werden, um Prozessvarianten darstellten zu können?

- $\quad$ RQ.2: Wie kann eine Softwareunterstützung zur Erstellung und Nutzung von

Prozessvarianten in BPMN-2.0-Modellen gestaltet werden?

Zur Klärung der Forschungsfragen, wird zunächst die eingesetzte Forschungsmethode dargelegt. Anschließend wird kurz auf bestehende Ansätze zur Modellierung von Prozessvarianten eingegangen. Dann erfolgt eine Erweiterung der BPMN 2.0 um einen Ansatz zur Modellierung von Prozessvarianten (RQ.1). Anschließend wird ein Softwarewerkzeug vorgestellt (RQ.2), das die BPMN-Erweiterung unterstützt und in der Praxis nutzbar macht.

\section{Forschungsmethode und Related Work}

\section{$2.1 \quad$ Forschungsmethode}

Grundsätzlich lassen sich in der Wirtschaftsinformatik zwei Arten von Forschungsfragen unterscheiden (vgl. [Seel10].). Zum einen sind dies Forschungsfragen, die auf die Untersuchung des aktuellen Zustandes abzielen, wie die Frage, welche Prozessmodellierungssprachen in Unternehmen aktuell eingesetzt werden. Zum anderen gibt es Forschungsfragen, die auf die Entwicklung neuer Methoden abzielen. Während sich erstere Gruppe mit dem Instrumentarium der empirischen Sozialforschung untersuchen lässt, existierte für letztere Gruppe das Paradigma der Design Science.

Da keine bestehenden Sachverhalte im Sinne einer Empirie untersucht werden, sondern eine Modellierungssprache erweitert und prototypisch umgesetzt werden soll, ist der Beitrag unter das Paradigma der Design Science zu subsumieren. [HMPR04] postulieren dafür sieben Richtlinien, die einzuhalten sind: Artefakte als Ergebnis des Design Science Prozesses, Problemrelevanz, Evaluation, Forschungsbeitrag, Stringenz der Forschungsmethode, Forschung als Suchprozess sowie Kommunikation der Forschungsergebnisse.

Dementsprechend orientiert sich der Aufbau dieses Beitrages an diesen sieben Richtlinien. Als Artefakt werden sowohl die Erweiterung der BPMN als auch das entwickelte Softwarewerkzeug vorgestellt. Problemrelevanz und Evaluation werden in der Motivation bzw. im Rahmen des Abschnittes „prototypische Implementierung“ adressiert. Forschungsbeitrag, Stringenz und Forschung als Suchprozess werden in diesem Abschnitt berücksichtigt, da der Beitrag auf einen klar definierten Stand der Forschung aufbaut und diesen erweitert. Ferner ist die Forderung nach Kommunikation der Forschungsergebnisse durch die vorliegende Veröffentlichung abgedeckt.

Eine forschungsmethodisch genauere Betrachtung verdient die BPMN-Erweiterung. [BrEs14] schlagen vor dazu zunächst für jede Erweiterung der BPMN zu prüfen, ob sie nicht durch bereits vorhandene Sprachelemente oder deren Nutzung für neue Inhalte realisiert werden kann. Nur falls dies nicht der Fall ist, soll die BPMN mit der Methode nach [StCV11] erweitert werden. Diese Methode postuliert ein Vorgehen in drei Schritten:

1. Modellierung der BPMN-Erweiterung als UML class diagram (CDME model)

2. Transformation des CDME in eine BPMN-Erweiterung (BPMN+X model). Dieser Schritt erfolgt, indem die erweiterten Sprachelemente mit bereits in der BPMN vorhandenen Sprachelementen in Beziehung gesetzt werden.

3. Transformation des $B P M N+X$ in eine $X M L$ Schema Extension Definition und ein $X M L$ Schema Extension Definition Document 


\subsection{Related Work}

Methoden zur Modellierung von Prozessvarianten erweitern Modellierungsstandards und erlauben es, verschiedene Prozessvarianten in einem Modell darzustellen. Darüber besitzen sie Parameter, die angeben, welche Variante in welchem Kontext verwendet werden soll. La Rosa et al. geben in ihrer ACM Survey (vgl. [LvDM17].) einen Überblick über bestehende Ansätze zur Modellierung von Prozessvarianten. Im Wesentlichen lassen sich vier Gruppen von Ansätzen unterscheiden: (1.) node configuration, (2.) element annotation, (3.) activity specialization und (4.) fragment customization.

Die node configuration basiert auf der Idee der konfigurierbaren Knoten, die eine Liste der Auswahlmöglichkeiten zur Verfügung stellen.

Element annotation basiert auf der Idee von Konfigurationstermen, die an Modellelemente annotiert werden können. Diese booleschen Konfigurationsterme werden entweder zu true oder false ausgewertet. Wenn der Term zur true ausgewertet wird, verbleibt das Modellelement im Modell, ansonsten wird es entfernt.

Activity specialization konzentriert sich auf ausführbare Modelle und ermöglicht eine Konfiguration, indem einzelne konfigurierbare Aktivitäten jeweils eine Liste von Varianten enthalten, durch die sie ersetzt werden können.

Fragment customization fügt entweder ganze vordefinierte Fragmente ein, oder löscht diese und erzeugt so verschiedene Prozessvarianten.

La Rosa et al. vergleichen alle vier Ansätze in ihrer Untersuchung. Dabei ist festzuhalten, dass die element annotation den größten Umfang an Einsatzgebieten aufweist. Der Nachteil der element annotation liegt jedoch in der mangelnden Unterstützung von Prozessvarianten in ausführbaren Prozessmodellen zur Laufzeit. Da dieser Beitrag sich aber auf Prozessmodelle zur Organisationsgestaltung und nicht auf ausführbare Workflowmodelle bezieht, kommt element annotation zum Einsatz. Die Einführung der element annotation in die BPMN erfolgt im folgenden Abschnitt.

\section{Metamodellbasierte Erweiterung der BPMN}

Um Prozessvarianten in der BPMN 2.0 mit der element annotation umzusetzen, ist die Erweiterung der BPMN um Konfigurationsterme (vgl. [BDKK02].) erforderlich. Allerdings beziehen sich die bisherigen Ansätze zur element annotation auf die eEPK (vgl. [KeNS92].) und sind technisch nur schwer umzusetzen, da sie mit Meta-Metamodellen arbeiten und auf komplexen Mechanismen zur Konsistenzwiederherstellung zwischen Meta-Metamodell, Metamodell und Modell basieren (vgl. [BDKK02].). Daher wird an dieser Stelle ein ähnlicher, aber vereinfachter Ansatz für Konfigurationsparameter und darauf basierende Konfigurationsterme eingeführt. Dabei ist das Ziel einen Konfigurationsterm an Elemente der BPMN $2.0 \mathrm{zu}$ annotieren, so dass über die Terme Prozessvarianten in einem Modell gekennzeichnet werden können. Ein Konfigurationsterm setzt sich aus einem oder mehreren, verknüpften Parametern zusammen. Jeder Term lässt sich, sobald alle Parameter mit konkreten Werten instanziiert sind entweder zu true oder false auswerten. Wird der Konfigurationsterm zu false ausgewertet, wird das Modellelement an dem er annotiert ist entfernt, ansonsten verbleibt es im Modell.

Gemäß des im Abschnitt „2.1 Forschungsmethodik“ vorgestellten Vorgehens ist daher zunächst zu prüfen, ob dazu vorhandene Sprachelemente der BPMN verwendet werden können. Da von der Konfiguration sowohl alle Elemente des Kontrollflusses, wie Tasks oder 
Gateways, aber auch Elemente zur Organisationsmodellierung, wie Lanes, betroffen sein können, muss ein potentielles Sprachelement zur Repräsentation der Konfigurationsterme an alle diese Sprachelement annotieret werden können.

Ein solches Element stellt die Text Annotation in der BPMN dar. Sie kann an Elemente vom Typ BaseElement annotiert werden (vgl. [OMG].). Da BaseElement die grundlegende abstrakte Superklasse für alle BPMN-Sprachelemente darstellt, ist damit auch die Text Annotation universell einsetzbar. Die Text Annotation besitzt die beiden Attribute text (Typ: String) und textFormat (Typ: String). Für eine Erweiterung der BPMN heißt das, dass die Text Annotation als bereits bestehendes Sprachelement wiederverwendet werden kann und lediglich ihre beiden Attribute definiert werden müssen.

Das Attribut text kann den Konfigurationsterm aufnehmen. Der Konfigurationsterm ist ein boolescher Term. Er muss mindestens Parameter unterstützen, die als ganze Zahlen, Fließkommawerte, Strings oder Aufzählungen instanziiert werden können. Um den Aufbau eines Konfigurationsterms zu standardisieren und inn automatisiert auswertbar zu machen, wird eine Sprache für Konfigurationsterme als kontextfreie Grammatik in erweiterter BackusNaur-Form (EBNF) (vgl. [BW60].) definiert. Abbildung 2 beschreibt den Aufbau eines Konfigurationsterms als kontextfreie Grammatik in erweiterter Backus-Naur-Form (EBNF):

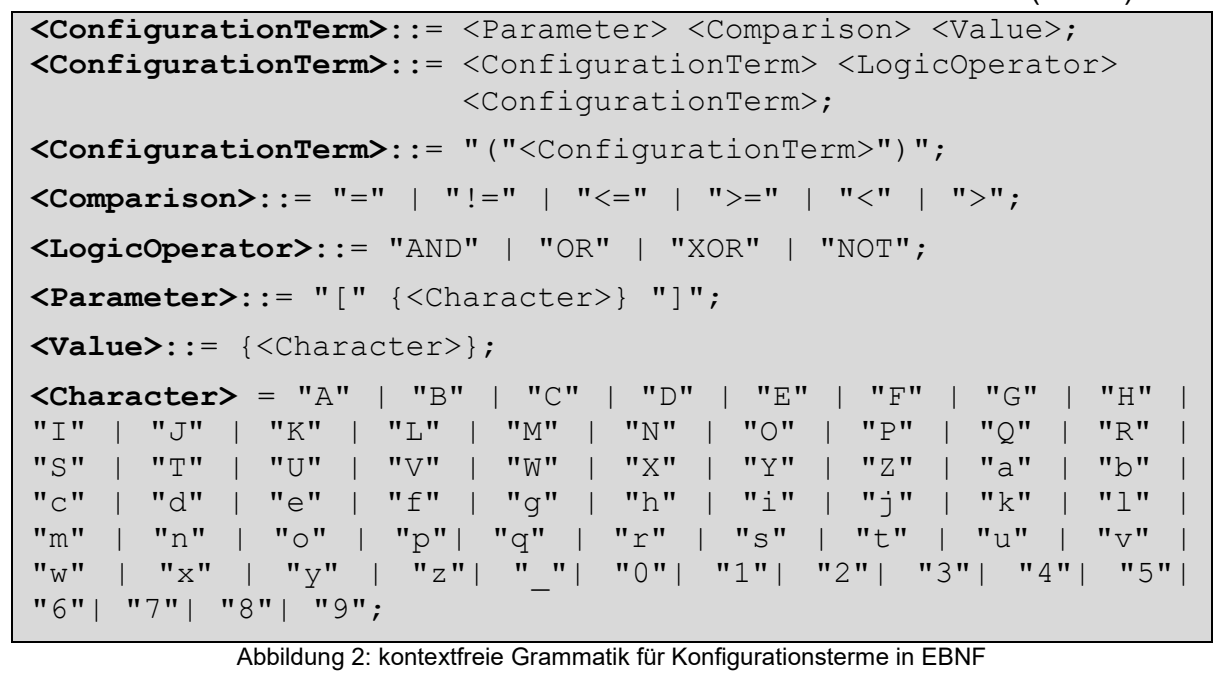

Als zweites Attribut ist das textFormat zu definieren. Als Wert enthält es einen MimeType. Um den Konfigurationsterm eindeutig als solchen zu kennzeichnen und von Kommentaren abzuheben, wird ein eigener Mime-Type definiert. Während für Kommentare in der Spezifikation der BPMN text/plain vorgeschlagen wird, wird für Konfigurationsterme text/confterm verwendet.

Zwei exemplarische Konfigurationsterme und ihre Verwendung im Modell sind in der folgenden Abbildung dargestellt. Der dargestellte Ausschnitt aus einem Prozess zum Fahrzeugleasing, enthält die Prozessvarianten „a) Standardprozess“ und „b) mit Bürgschaft“ aus Abbildung 1. Um das Beispiel an dieser Stelle übersichtlich zu gestalten, kommen lediglich die beiden Parameter „BürgschaftNötig“ und „AlterLetztePrüfung“ zum Einsatz. Während der erste boolesche Parameter angibt, ob eine Bürgschaft für das Leasing erforderlich ist oder 
nicht, gibt der zweite Parameter an, vor wie vielen Tagen die letzte Bonitätsprüfung des jeweiligen Kunden stattfand.

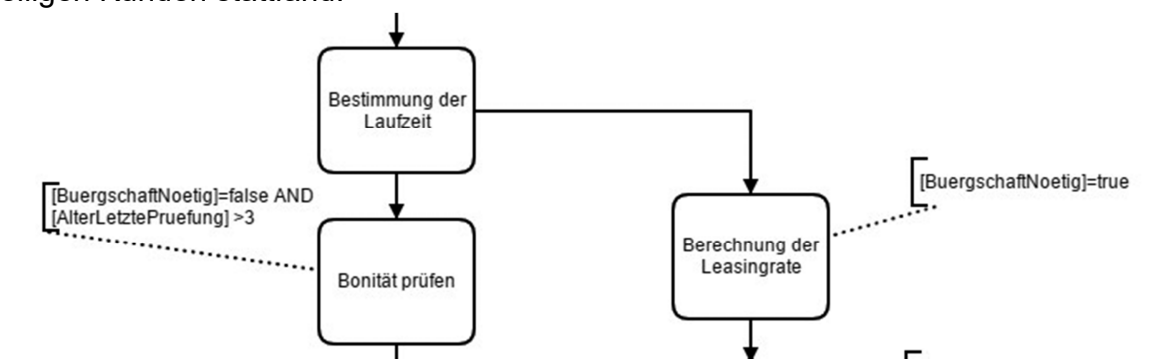

Abbildung 3: Exemplarischer Ausschnitt eines Modells mit mehreren Prozessvarianten zum Fahrzeugleasing

In diesem Beispiel wird die Bonität geprüft, wenn keine Bürgschaft vorhanden ist oder die letzte Bonitätsprüfung älter als 3 Tage ist.

Da die Auswertung der Konfigurationsterme für größere Modelle mit mehreren Prozessvarianten manuell sehr aufwändig wäre, wird im folgenden Abschnitt ein Softwarewerkzeug zur Unterstützung vorgestellt.

\section{$4 \quad$ Prototypische Umsetzung und exemplarische Anwendung}

\subsection{Prototypische Umsetzung}

Um sowohl die Konstruktion (Build-Time) des konfigurierbaren Modells mit mehreren Prozessvarianten als auch die Generierung einzelner Prozessvarianten aus inm (Run-Time) zu unterstützen, ist ein Softwarewerkzeug zur Unterstützung notwendig (vgl. [SDSS16].). Gerade bei großen Modellen, die viele Konfigurationsterme enthalten, wäre die manuelle Auswertung sowohl aufwändig als auch fehleranfällig.

Da als Modellierungssprache BPMN 2.0 zum Einsatz kommt und eine Neuimplementierung der Standardmodellierungsfunktionalität keine neuen Erkenntnisse verspricht, setzt der hier vorgestellte Prototyp auf bestehenden Open-Source-Modellierungswerkzeugen auf. Eine Übersicht über BPMN-Open-Source-Modellierungswerkzeuge als Grundlage für Forschungsprototypen findet sich u.a. bei (vgl. [SDSS16].). Aufgrund den expliziten bereits im Quellcode vorgesehenen Möglichkeiten zur Erweiterung setzt der im Folgenden vorgestellte Prototyp auf dem Camunda Open Source BPMN Modeler ${ }^{1}$ (vgl. [SD16].) auf. Die Funktionalität wurde in Java Script implementiert und erweitertet. In den beiden folgenden Abschnitten wird jeweils die erweiterte Funktionalität zur Build-Time und Run-Time vorgestellt.

\subsection{Build-Time}

Bevor die jeweils entwickelte Funktionalität vorgestellt wird, erfolgt zunächst eine kurze Anforderungsdefinition in Form von User Stories (vgl. [CoHe10].).

\footnotetext{
${ }^{1}$ Das Open-Source-Modellierungswerkzeuge von Camunda findet sich unter http://bpmn.io
} 


\section{User Story BT1: Effiziente Hinterlegung von Termen}

Als Modellersteller möchte ich bei einem oder mehreren Modellelementen gleichzeitig einen Konfigurationsterm annotieren können, um Prozessvarianten zu kennzeichnen.

\section{User Story BT2: Anzeige hinterlegter Terme}

Als Modellersteller möchte ich mir anzeigen lassen, welche Konfigurationsterme bei einem Modellelement hinterlegt sind, um die Konfigurationsterme verwalten zu können.

Zur Umsetzung der beiden User Stories wurde jeweils eine neue Funktionalität in den Camunda Modeler implementiert. Dazu wurde zunächst das Menü auf der linkten Seite erweitert. Abbildung 4 zeigt einen Screenshot des Modellierungswerkzeugs. Die Erweiterungen des Menüs zur Build-Time auf der linken Seite sind rot markiert. Zur Umsetzung von User Story $\mathrm{BT}_{1}$ wurde ein Pop-Up-Menü ergänzt, das die Eingabe von Konfigurationstermen erlaubt. Die eingegebenen Terme werden dann bei allen aktuell ausgewählten Modellelementen annotiert. Dafür wurde über die im Camunda Modeler vorgesehene Möglichkeiten eine neue Eigenschaft für Modellelemente namens IPIM_Term definiert, die den jeweiligen Konfigurationsterm als String aufnimmt.

Um Story $\mathrm{BT}_{2}$ umzusetzen wurde eine weitere Funktionalität ergänzt, die sich hinter dem Lupen-Icon verbirgt. Die Funktionalität führt eine Suche auf dem Modell durch und clustert dabei alle Modellelemente, an die derselbe Term annotiert ist. Dabei wird jedes Term-Cluster in einer unterschiedlichen Farbe eingefärbt, so dass sofort ersichtlich wird, welche Modellelemente denselben Konfigurationsterm besitzen und damit immer gemeinsam in einer Prozessvariante auftreten.

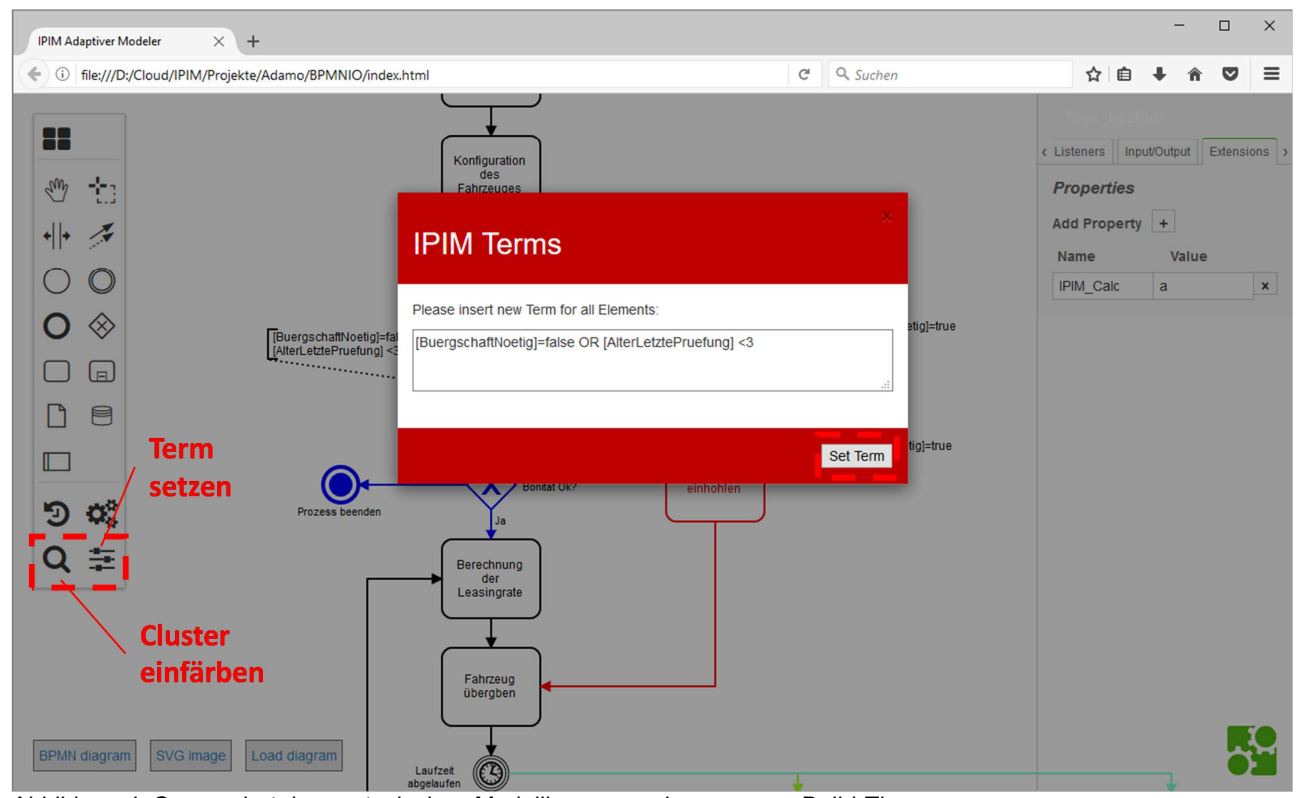

Abbildung 4: Screenshot des protypischen Modellierungswerkzeuges zur Build-Time

Nachdem bisher nur die Perspektive des Modellerstellers betrachtet wurde, erfolgt im nächsten Abschnitt ein Wechsel der Perspektive hin zum Modellnutzer, der mithilfe des Softwarewerkzeuges Prozessvarianten erzeugen möchte. 


\subsection{Run-Time}

Die Run-Time-Perspektive geht davon aus, dass ein BPMN-Prozessmodell vorhanden ist, das mehrere Modellvarianten enthält, die jeweils durch Terme gekennzeichnet sind. Analog zur Build-Time-Perspektive werden die Anforderungen in Form von User Stories dargestellt:

User Story RT1: Belegung der Konfigurationsparameter

Als Modellnutzer möchte ich alle Parameter, die in allen Konfigurationsparametern vorkommen in nur einem Menü mit Werten instanziieren, um Modellvarianten abzuleiten.

\section{User Story RT2: Generierung einzelner Prozessvarianten}

Als Modellnutzer möchte ich sämtliche Konfigurationsterme effizient auswerten lassen, um standardkonforme BPMN-Prozessvarianten zu erzeugen.

\section{User Story RT3: Zurücksetzen zum Ausgangsmodell}

Als Modellnutzer möchte ich die Konfiguration rückgängig machen und zum konfigurierbaren Ausgangsmodell, das alle Prozessvarianten enthält, zurückkehren können, um eine erneute Konfiguration mit veränderten Parametern durchzuführen.

Um die drei User Stories umzusetzen wurde das Menü auf der linkten Seite des Camunda Modelers erweitert. Abbildung 5 zeigt einen Screenshot des Modellierungswerkzeugs zur Run-Time. Die Erweiterungen des Menüs auf der linken Seite sind wiederum rot markiert. Zur Umsetzung von User Story $\mathrm{RT}_{1}$ wurde ein Pop-Up-Menü ergänzt, das die Belegung jedes Konfigurationsparameters mit Werten erlaubt. Dazu werden zunächst alle Konfigurationsterme geparst und eine Liste aller vorhandenen Parameter erstellt. Der Modellnutzer kann dann jeden Parameter mit Werten belegen.

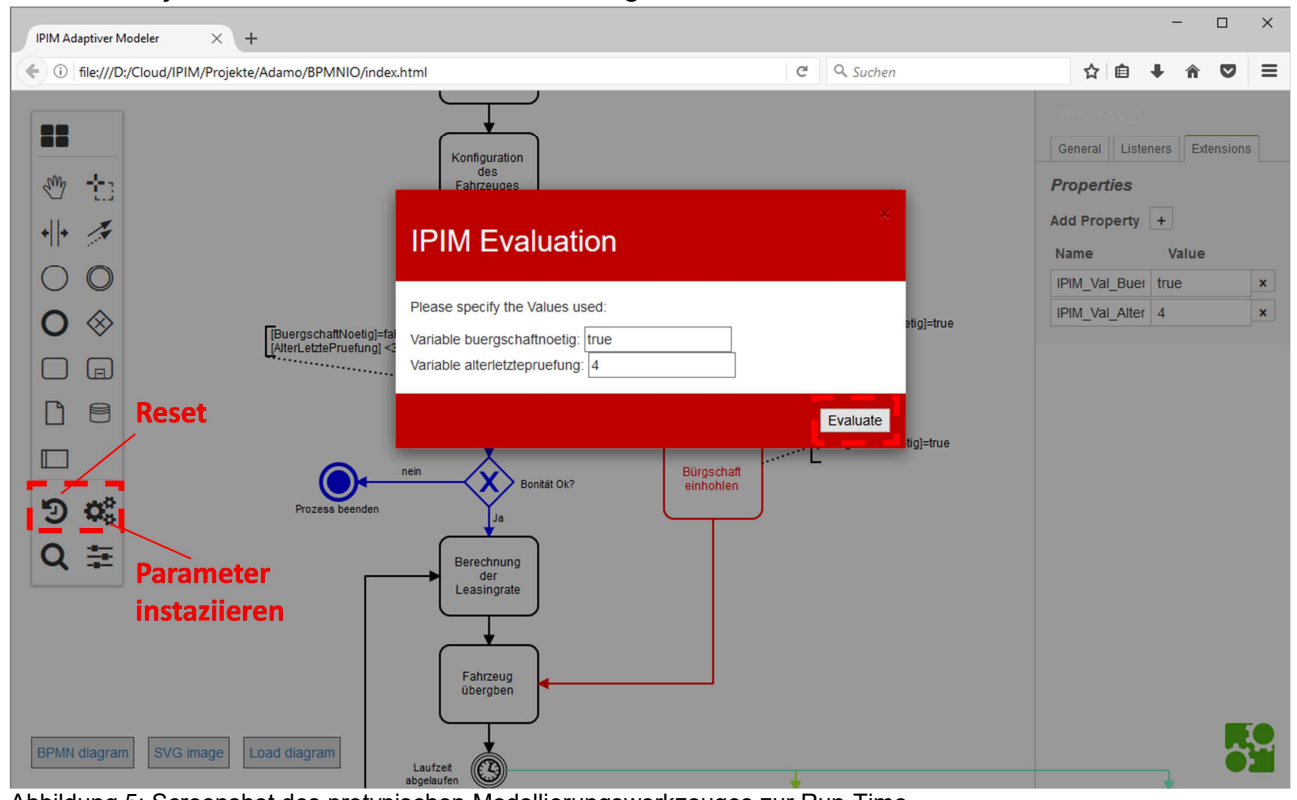

Abbildung 5: Screenshot des protypischen Modellierungswerkzeuges zur Run-Time

Zur Umsetzung von User Story $\mathrm{RT}_{2}$ bietet die Schaltfläche „Evaluate“ die Funktionalität alle Konfigurationsterme auszuwerten, wie in Abschnitt 3 beschrieben. Dabei werden alle nicht benötigen Modellelemente entfernt und auf diese Weise eine Prozessvariante erzeugt. 
Um User Story $\mathrm{RT}_{3}$ umzusetzen wurde im Menü eine Schaltfläche mit der Funktionalität zum zurücksetzen des konfigurierten Modells hinzugefügt. Diese Funktionalität lädt schlichtweg das konfigurierbare Modell mit allen Prozessvarianten erneut.

\section{$5 \quad$ Zusammenfassung}

Die Verwaltung von Prozessvarianten in einzelnen Modellen ist bei der Modellpflege aufwändig und fehleranfällig. Daher wurde eine Erweiterung der BPMN vorgenommen, die es auf Basis von Annotationen erlaubt mehrere Prozessvarianten in einem Modell darzustellen. Die Basis bilden dabei Konfigurationsterme. Um die Arbeit mit diesen Konfigurationstermen und ihren Parametern effizient zu gestalten, wurde ein Softwarewerkzeug vorgestellt, das sowohl die Konstruktion konfigurativer BPMN-Prozessmodelle unterstützt als auch ihre Anwendung. Dazu wurde der Open-Source-Modeler von Camunda um entsprechende Funktionalität erweitert. Die Implementierung zeigt die Umsetzbarkeit des zuvor vorgestellten Konzepts.

\section{Literaturverzeichnis}

[BW60] Backus, J. W.; Wegstein, J. H.; van Wijngaarden, A.; Woodger, M., u.a.: Report on the algorithmic language ALGOL 60. In: Commun. ACM 3(5), 1960, S. 299-314.

[BH16] Barton, Thomas; Herrmann, Frank; Meister, Vera; Müller, Christian, u.a. (Hrsg.): Prozesse, Technologie, Anwendungen, Systeme und Management 2016: Angewandte Forschung in der Wirtschaftsinformatik: Tagungsband zur 29. AKWI-Jahrestagung vom 11.09.2016 bis 14.09.2016 an der Technischen Hochschule Brandenburg, Heide. mana-Buch, 2016.

[Beck02] Becker, Jörg (Hrsg.): Wissensmanagement mit Referenzmodellen: Konzepte für die Anwendungssystem- und Organisationsgestaltung ; mit 13 Tab, Heidelberg. PhysicaVerl., 2002.

[BDKK02] Becker, Jörg; Delfmann, Patrick; Knackstedt, Ralf; Kuropka, Dominik: Konfigurative Referenzmodellierung. In: Becker, Jörg (Hrsg.): Wissensmanagement mit Referenzmodellen: Konzepte für die Anwendungssystem- und Organisationsgestaltung ; mit 13 Tab. Physica-Verl., Heidelberg, 2002, S. 25-144.

[BrEs14] Braun, Richard; Esswein, Werner: Extending BPMN for Modeling Resource Aspects in the Domain of Machine Tools. In: WIT Transactions on Engineering Sciences(87), 2014, S. $450-458$.

[CoHe10] Cohn, Mike; Hesse-Hujber, Martina: User Stories: [für die agile Software-Entwicklung mit Scrum, XP u.a.]. 1. Aufl. mitp, Heidelberg, 2010.

[Delf06] Delfmann, Patrick: Adaptive Referenzmodellierung: Methodische Konzepte zur Konstruktion und Anwendung wiederverwendungsorientierter Informationsmodelle. (Advances in information systems and management science, 25). Logos-Verl., Berlin, 2006.

[DiHK11] Dijkman, Remco M.; Hofstetter, Jörg; Koehler, Jana: Business Process Model and Notation - Third International Workshop, BPMN 2011, Lucerne, Switzerland, November 
21-22, 2011. Proceedings. (Lecture Notes in Business Information Processing, 95). Springer, 2011. URL: http://dx.doi.org/10.1007/978-3-642-25160-3.

[HMPR04] Hevner, Alan R.; March, Salvatore T.; Park, Jinsoo; Ram, Sudha: Design Science in Information Systems Research. In: MIS Q 28(1), 2004, S. 75-105.

[KeNS92] Keller, G.; Nüttgens, M.; Scheer, A.-W.: Semantische Prozeßmodellierung auf der Grundlage "Ereignisgesteuerter Prozeßketten (EPK)". (Veröffentlichungen des Instituts für Wirtschaftsinformatik, 89). Inst. für Wirtschaftsinformatik Univ, Saarbrücken, 1992.

[LDtM09] La Rosa, Marcello; Dumas, Marlon; ter Hofstede, Arthur H.; Mendling, Jan: Configurable multi-perspective business process models. In: Information Systems 2009(2), 2009, S. 313-340.

[LvDM17] La Rosa, Marcello; van der Aalst, Wil M.; Dumas, Marlon; Milani, Fredrik P.: Business Process Variability Modeling. In: ACM Comput. Surv. 50(1), 2017, S. 1-45.

[Obje11] Object Management Group (OMG): Business Process Model and Notation (BPMN), Version 2.0, 2011.

[OMG ] OMG BPMN 2.0 FTF: Business Process Model and Notation (BPMN), Version 2.0. URL: http://www.omg.org/spec/BPMN/2.0/PDF. Abruf am 2017-01-03.

[Rova07] Rosemann, M.; van der Aalst, W.M.P.: A configurable reference modelling language. In: Information Systems 32(1), 2007, S. 1-23.

[Seel10] Seel, Christian: Reverse Method Engineering: Methode und Softwareunterstützung zur Konstruktion und Adaption semiformaler Informationsmodellierungstechniken. (Wirtschaftsinformatik - Theorie und Anwendung, 20). Logos, Berlin, 2010.

[SDSS16] Seel, Christian; Dörndorfer, Julian; Schmidtner, Markus; Schubel, Alexander: Vergleichende Analyse von Open-Source-Modellierungswerkzeugen als Basis für Forschungsprototypen. In: Barton, Thomas; Herrmann, Frank, u.a. (Hrsg.): Prozesse, Technologie, Anwendungen, Systeme und Management 2016: Angewandte Forschung in der Wirtschaftsinformatik : Tagungsband zur 29. AKWI-Jahrestagung vom 11.09.2016 bis 14.09.2016 an der Technischen Hochschule Brandenburg. mana-Buch, Heide, 2016, S. $35-44$.

[SD16] Seel, Christian; Dörndorfer, Julian; Schmidtner, Markus; Schubel, Alexander, u.a.: Vergleichende Analyse von Open-Source-Modellierungswerkzeugen als Basis für Forschungsprototypen. In: Landshuter Arbeitsberichte zur Wirtschaftsinformatik (Lab WI)(5), 2016, S. 1-43.

[StCV11] Stroppi, Luis J.; Chiotti, Omar; Villarreal, Pablo D., 2011: Extending BPMN 2.0: Method and Tool Support BPMN 2011, Lucerne, Switzerland, November 21-22, 2011. Proceedings. In: Dijkman, Remco M.; Hofstetter, Jörg; Koehler, Jana (Hrsg.): Business Process Model and Notation - Third International Workshop, BPMN 2011, Lucerne, Switzerland, November 21-22, 2011. Proceedings. Springer. (Lecture Notes in Business Information Processing, 95), S. 59-73.

\section{Kontakt}

Prof. Dr. Christian Seel

Hochschule Landshut, Institut für Projektmanagement und Informationsmodellierung (IPIM)

Am Lurzenhof 1, 84036 Landshut

christian.seel@haw-landshut.de 


\title{
Erweiterung des Referenzprozessmodells eTOM um die Anforderungen des deutschen IT-Sicherheitsgesetzes
}

\author{
Tristan Eberhard Thomé, Christian Czarnecki, Gunnar Auth, Frank Bensberg
}

\section{Zusammenfassung}

Kritische Infrastrukturen sind primäre Ziele krimineller Hacker. Der Deutsche Bundestag reagierte darauf am 25. Juli 2015 mit einem Gesetz zur Verbesserung der Sicherheit von ITSystemen, dem IT-Sicherheitsgesetz. Dies verlangt von Betreibern kritischer Infrastrukturen, angemessene Mindeststandards für organisatorische und technische Sicherheit zu implementieren, um den Betrieb und die Verfügbarkeit dieser Infrastruktur zu gewährleisten. Telekommunikationsunternehmen sind einerseits von diesem Gesetz in besonderem Maße betroffen und verfügen andererseits mit dem Rahmenwerk enhanced Telecom Operations Map (eTOM) über ein international anerkanntes Referenzmodell zur Gestaltung von Geschäftsprozessen in dieser Branche. Da sämtliche Telekommunikationsunternehmen in Deutschland verpflichtet sind, das Gesetz innerhalb eines bestimmten Zeitrahmens zu implementieren, präsentiert dieser Beitrag einen Vorschlag zur Erweiterung von eTOM um die relevanten Anforderungen des deutschen IT-Sicherheitsgesetzes.

\section{$1 \quad$ Einleitung}

Mit der zunehmenden Cyberkriminalität rückt das Thema IT-Sicherheit immer stärker in den Vordergrund. So sind im Jahr 2013 allein in Deutschland 64.426 Fälle von Cyberkriminalität von der Polizei erfasst worden. Im Vergleich zum Jahr 2003 stellt dies einen Anstieg von über 200\% dar [Bund15]. Die Deutsche Telekom stellt auf ihrer Website sicherheitstacho.eu in Echtzeit ausgewerteten Daten zu weltweit beobachteten Cyber-Attacken bereit. Dabei werden mit sogenannten Honeypot-Systemen Angriffe provoziert, Muster identifiziert und das Verhalten der Angreifer aufgezeichnet, um mögliche Rückschlüsse zu ziehen [Deut16]. Die Statistik zeigt, neben dem erheblichen Anstieg der Anzahl von Angriffen, die primären Angriffsziele der Angreifer. Dabei stehen zum einen WWW-Dienste und zum anderen Netzwerkdienste im Fokus von Cyber-Attacken. Daraus wird deutlich, dass insbesondere die Telekommunikationsbranche (TK-Branche) als Betreiber von Netzwerkinfrastrukturen ein primäres Ziel der Angreifer darstellt.

Daher wurde im Jahr 2011 als Initiative des Bundesamts für Sicherheit in der Informationstechnik (BSI) und in Zusammenarbeit mit dem Bundesverband Informationswirtschaft, Telekommunikation und neue Medien e.V. (BITKOM) die sog. Cyber-Sicherheitsstrategie verabschiedet. Das Bündnis aus BSI, Partnern und Multiplikatoren verfolgt das Ziel, „die CyberSicherheit in Deutschland zu erhöhen und die Widerstandsfähigkeit des Standortes Deutschland gegenüber Cyber-Angriffen zu stärken" [BSIT15]. Aufbauend auf dieser Strategie verabschiedete die Bundesregierung am 12.06.2015 das Gesetz zur Erhöhung der Sicherheit informationstechnischer Systeme, kurz IT-Sicherheitsgesetz (IT-SiG). Das IT-SiG stellt wesentliche verpflichtende Anforderungen an die betroffenen Unternehmen wie auch 
an die TK-Branche. Diese steht als Dreh- und Angelpunkt der Digitalisierung im Fokus des IT-SiG und wird - neben anderen Branchen - als Kritische Infrastruktur (KRITIS) eingestuft. Nun stehen alle deutschen Unternehmen, die der Branche Telekommunikation im Sinne des IT-SiG angehören, vor der Aufgabe, die Anforderungen des IT-SiG gesetzeskonform binnen zwei Jahren umzusetzen. Da diese Anforderungen hauptsächlich die Geschäftsprozesse der Unternehmen beeinflussen, stehen prozessuale Anforderungen im Vordergrund. Die TKBranche pflegt in der Arbeitsgemeinschaft TM Forum, der über 900 Unternehmen aus mehr als 70 Ländern angehören [TMFO16], unter dem Namen enhanced Telecom Operations Map (eTOM) ein Referenzprozessmodell, das auch von der International Telecommunication Union (ITU) als Standard empfohlen wurde [ITUT07].

Zur Unterstützung einer effizienten und effektiven Umsetzung des IT-SiG in deutschen TKUnternehmen wird in diesem Beitrag eine Erweiterung von eTOM um die Anforderungen des IT-SiG vorgeschlagen. Dazu wird auf Basis von eTOM ein branchenspezifisches Referenzmodell entworfen, welches die Anforderungen des IT-SiG abbildet. Forschungsmethodisch ist diese Arbeit der gestaltungsorientierten Wirtschaftsinformatik zuzuordnen [Pico10] sowie dort dem Forschungsbereich der deduktiven Referenzmodellierung [BaPr10]: Es wird ein konkretes Artefakt (hier: Referenzprozessmodell) entwickelt, welches ein Problem löst (hier: fehlende Prozesse zur gesetzeskonformen Umsetzung der IT-SiG).

Entsprechend dieses Forschungsansatzes lehnt sich der Aufbau der Arbeit daher an die Design Science Research Methodology for Informations Systems Research an [HMPR04, PTRC07]. Da die Untersuchung problemzentriert ist, beginnt sie mit der Identifizierung des Problems und Beschreibung der Zielsetzung. Darauf folgen die Darstellung der Designmethode und des resultierenden Artefakts. Mit den Erweiterungen des Referenzprozessmodells eTOM wird eine neue Lösung für den Problembereich des IT-SiG vorgeschlagen, was eine originäre Aufgabe gestaltungsorientierter Forschung darstellt [GrHe13]. Eine ausführliche Diskussion der eTOM im Kontext der gestaltungsorientierten Forschung liefern [CzDi17b].

In Kapitel 2 werden die Grundlagen zum IT-SiG und Referenzprozessmodell eTOM erläutert. Kapitel 3 zeigt die angewendete Vorgehensweise und Kapitel 4 die Ergebnisse, die in Kapitel 5 kritisch diskutiert werden. Im letzten Kapitel werden die Ergebnisse zusammengefasst und mit der formulierten Forschungsfrage in Verbindung gebracht. Anschließend werden die Ergebnisse hinsichtlich ihrer Bedeutung beleuchtet und Folgeaktivitäten diskutiert.

\section{Konzeptionelle Grundlagen}

\section{$2.1 \quad$ IT-Sicherheitsgesetz}

Das am 24.07.2015 verabschiedete Gesetz zur Erhöhung der Sicherheit informationstechnischer Systeme ist eine Gesetzesverordnung der deutschen Bundesregierung und eine Folge der 2011 beschlossenen Cyber-Sicherheitsstrategie [Bund13] des Bundesinnenministeriums, welche wiederum an die KRITIS-Strategie anschließt [Bund09]. Das Gesetz zielt auf die Verbesserung der Sicherheit von IT-Systemen in KRITIS ab, also in „Organisationen und Einrichtungen mit wichtiger Bedeutung für das staatliche Gemeinwesen, bei deren Ausfall oder Beeinträchtigung nachhaltig wirkende Versorgungsengpässe, erhebliche Störungen der öffentlichen Sicherheit oder andere dramatische Folgen eintreten würden“ [Bund13]. Der erhöhte Schutz der IT-Systeme soll unter anderem durch eine engere Zusammenarbeit der Betreiber von KRITIS und des BSI erreicht werden. Eine zentrale Auf- 
gabe des BSI wird die Analyse gemeldeter IT-Sicherheitsvorfälle darstellen, sowie die Weiterleitung von Lösungsmöglichkeiten und ergänzender Hinweise an betroffene Betreiber.

Der Gesetzgeber hat den Betreibern von KRITIS eine Frist von zwei Jahren (bis zum 25.07.2017) gesetzt, um die Anforderungen des IT-SiG umzusetzen. Die Inhalte des IT-SiG umfassen folgende vier Themenfelder:

- Einhaltung eines Mindestniveaus an IT-Sicherheit;

- $\quad$ Auditierung der IT-Sicherheit;

- Melden kritischer Störungen;

- Betreiben einer Kontaktstelle.

Der Gesetzgeber verpflichtet KRITIS-Betreiber „angemessene organisatorische und technische Vorkehrungen zur Vermeidung von Störungen [...] ihrer informationstechnischen Systeme [...] zu treffen“ [DeBu15]. Dazu wird vom BSI ein Mindeststandard erarbeitet, welcher im Einvernehmen mit dem IT-Rat verbindlich einzuhalten sei. Dieses Mindestniveau an ITSicherheit haben „Betreiber Kritischer Infrastrukturen [...] mindestens alle zwei Jahre [...] nachzuweisen" [DeBu15]. Der Nachweis kann dabei über Audits, Prüfungen oder Zertifizierungen erfolgen, welche dem BSI zu übermitteln sind. Kommt es zu einer erheblichen Störung oder Beeinträchtigung der Funktionsfähigkeit der Kritischen Infrastruktur, so haben KRITIS-Betreiber diese „unverzüglich an das Bundesamt zu melden“ [DeBu15]. Des Weiteren sind KRITIS-Betreiber angehalten, eine Kontaktstelle zum BSI einzurichten und zu betreiben. Über diese erfolgen der Austausch der Störungsmeldungen sowie die Kommunikation des gegenwärtigen Lagebilds zur Sicherheit in der Informationstechnik durch das BSI.

\subsection{Referenzprozessmodell eTOM}

Das TM Forum ist ein internationales Unternehmenskonsortium zum Austausch von Erfahrungen und Lösungen in der TK-Branche [Czar13]. Unter der Bezeichnung Frameworx werden im TM Forum Methoden, Konzepte und Referenzmodelle zur Transformation von TKUnternehmen erarbeitet und anschließend veröffentlicht [Czar13]. Einer der Bereiche von Frameworx ist das Business Process Framework, welches unter dem Namen enhanced Telecom Operations Map (eTOM) ein Referenzprozessmodell für die TK-Branche enthält. Dieses bietet einen branchenbezogenen Ordnungsrahmen für Geschäftsprozesse [AABG16] und dient zur Identifikation und Systematisierung relevanter Prozesse von Telekommunikationsdienstleistern [BeCz17]. Eine Untersuchung von 184 Transformationsprojekten zeigt die umfangreiche Nutzung von eTOM [CzWS12].

Das TM Forum definiert eTOM als generalisiertes Modell, welches unabhängig von Organisation, Technologie und Service nutzbar ist [TeMa15]. Der Aufbau gestaltet sich hierarchisch in den Ebenen 0 bis 4 . Die Verknüpfung zwischen den einzelnen Ebenen erfolgt mit Hilfe von Relationen in der Kardinalität 1:1. Relationen innerhalb einer Ebene sind nicht vorgesehen. Dabei besitzen die Ebenen 0 bis 1 mit Domänen und Prozessgruppen den höchsten Abstraktionsgrad [CzDi17a]. Die Ebene 2 stellt eine abstrakte Gruppierung mehrerer Einzelprozesse dar, welche den Domänen der Ebene 0 fest zugeordnet sind. Auf der Ebene 3 sind die einzelnen Prozesse zugeordnet. Auf der untersten Ebene 4 werden einzelne Aktivitäten der Prozessebene 3 zugeordnet. Die Darstellung kann sowohl als Dekomposition in Form einer Baumstruktur, die den hierarchischen Zusammenhang aufzeigt, als auch als Referenzprozessmodell umgesetzt werden [CzDi17a]. Zur graphischen Repräsentation kommt die Business Process Model and Notation (BPMN) zum Einsatz, indem die einzelnen Aktivitäten in einen logischen Zusammenhang und im Rahmen der Notation in 
einen prozessualen Zusammenhang gebracht werden. Der vorliegende Beitrag basiert auf eTOM Version R15.0.

\section{Vorgehen}

Dieser Beitrag folgt dem Paradigma der gestaltungsorientierten Forschung [HMPR04, PTRC07]. Für das Problem fehlender Prozesse zur gesetzeskonformen Umsetzung des ITSiG wird ein Referenzprozessmodell als konkretes Artefakt entwickelt. Gemäß [BaPr10] sind dazu sowohl der Designprozess als auch das resultierende Artefakt in strukturierter Weise anhand intersubjektiv nachvollziehbarer Anforderungen des IT-SiG zu beschreiben. Zur Adäquanz eines prozessorientierten Ansatzes zur Gewährleistung der IT-Sicherheit siehe [Simi17].

Die Gesetzesverordnung des IT-SiG ist ein komplexes Werk zur Änderung und Erweiterung bereits bestehender Gesetze. Folglich ist ein systematisches Vorgehen notwendig, um relevante Abschnitte des Gesetzes zu identifizieren, zu interpretieren und Anforderungen abzuleiten. Zur Anforderungsanalyse wird in Anlehnung an Pohl und Rupp [PoRu15] das in Abb. 1 dargestellte Vorgehensmodell zugrunde gelegt.

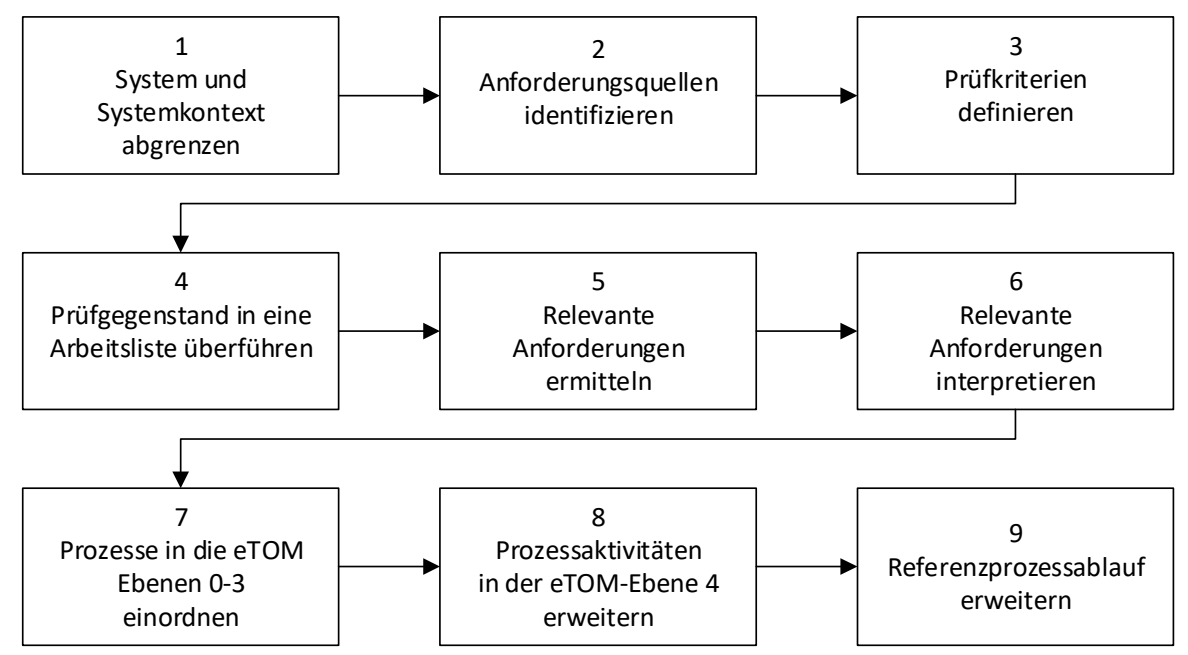

Abbildung 1: Vorgehen zur Erweiterung von eTOM um die relevanten Anforderungen des IT-SiG

Der erste Schritt sieht vor, das System von dessen Umgebung abzugrenzen und den Teil der Umgebung zu identifizieren, der die Anforderungen an das zu entwickelnde System bestimmt. Im zweiten Schritt werden die relevanten Anforderungsquellen identifiziert. Im dritten Schritt werden die Prüfkriterien, anhand des im ersten Schritt abgesteckten Systems und Systemkontexts, definiert. Im vierten Schritt wird der Prüfgegenstand in eine Arbeitsliste überführt. Im fünften Schritt werden relevante Anforderungen ermittelt, welche sämtliche Prüfkriterien erfüllen. Diese Anforderungen werden zusammengefasst und auf abstrakter Ebene tabellarisch konsolidiert. Im sechsten Schritt werden die identifizierten Anforderungen 
hinsichtlich prozessualer Bedeutung interpretiert, wobei auf relevante Literatur zurückgegriffen wird.

Anschließend werden die relevanten Anforderungen im eTOM-Referenzmodell ergänzt. Das dabei angewandte Vorgehen orientiert sich an Czarnecki et al. [CWS13]. Diese verwenden eine Top-Down-Strategie zur Erweiterung von eTOM. Dementsprechend wird im siebten Schritt der interpretierte Prozess in den eTOM-Ebenen 0 bis 3 eingeordnet. Anschließend werden im achten Schritt die betreffenden Aktivitäten auf der zugehörigen eTOM-Ebene 4 in logischer Reihenfolge hinzugefügt. Im neunten Schritt wird der Referenzprozessablauf (RPA) modelliert. Insofern die verwendete eTOM-Version kein Modell vorsieht, wird anhand der Aktivitäten ein Vorschlag erarbeitet.

\section{$4 \quad$ Erweiterung von eTOM um die Anforderungen des IT-SiG}

In diesem Abschnitt werden die konkreten Ergebnisse zur Erweiterung von eTOM dargestellt. Dazu werden die neun Schritte des Vorgehensmodells durchlaufen. Davon werden die Schritte 1 bis 6 ganzheitlich dargestellt. Die Schritte 7 bis 9 werden anhand eines Beispiels ausgeführt.

Im ersten Schritt wird der Systemkontext abgegrenzt. Anhand der Forschungsfrage beläuft sich der Systemkontext auf das Referenzprozessmodell eTOM. Da in diesem Rahmenwerk Unternehmensprozesse eingeordnet und beschrieben werden, sind Anforderungen an Geschäftsprozesse zu ermitteln (Kriterium K1). Das IT-SiG differenziert in dem IT-Sicherheitsgesetz zwischen Anforderungen an das BSI und an KRITIS-Betreiber. Im Rahmen dieser Untersuchung werden die Anforderungen an KRITIS-Betreiber betrachtet (Kriterium K2). Darüber hinaus beschreibt eTOM einen Vorschlag zur Standardisierung der Prozesse in der TK-Branche. Daher sind die Anforderungen zu ermitteln, die KRITIS-Betreiber aus dieser Branche betreffen (Kriterium K3).

Im zweiten Schritt werden die Anforderungsquellen festgelegt. Aufgrund der Forschungsfrage beläuft sich die Anforderungsquelle auf das IT-SiG, sodass ausschließlich dieses Dokument hinsichtlich der Anforderungen an das Referenzprozessmodell eTOM untersucht wird.

Im dritten Schritt werden die Prüfkriterien definiert und ihre Abhängigkeiten zueinander festgelegt. Grundlage dafür bieten die im ersten Schritt identifizierten Kriterien (s. Tab. 1).

\begin{tabular}{ll}
\hline Kriterium & Beschreibung \\
\hline K1 & Der Abschnitt ist eine prozessuale Anforderung \\
K2 & Der Abschnitt ist eine Prozessanforderung an KRITIS-Betreiber \\
K3 & Der Abschnitt ist eine Prozessanforderung an die TK-Branche \\
\hline & Tab. 1: Prüfkriterien zur Ermittlung relevanter Gesetzesabschnitte
\end{tabular}

Die drei Kriterien sind als aufeinander aufbauend zu betrachten. Somit kann das Kriterium K2 nur zutreffen, wenn auch das Kriterium K1 zutrifft bzw. das Kriterium K3 nur zutreffen, wenn auch die Kriterien K1 und K2 erfüllt sind. Anhand dieser drei Kriterien und der dargestellten Abhängigkeit können die relevanten Anforderungen identifiziert werden.

Im vierten Schritt wird der Prüfgegenstand in eine Arbeitsliste überführt. Das IT-Sicherheitsgesetz besteht aus 11 Artikeln mit 27 Abschnitten und 72 Absätzen, die zu analysieren sind. Anhand der Arbeitsliste können im folgenden Schritt die relevanten Anforderungen identifiziert werden. 
Im fünften Schritt werden die relevanten Anforderungen ermittelt. Grundlage hierfür sind die zuvor festgelegten Prüfkriterien. Umfang der Prüfung waren insgesamt 72 Gesetzesabschnitte. Nach der Untersuchung des IT-SiG verteilt sich das Ergebnis wie folgt auf die einzelnen Kriterien (s. Tab. 2).

\begin{tabular}{ll}
\hline Kriterium & Anzahl zutreffender Anforderungen \\
\hline K1 & 24 Absätze \\
K2 & 13 Absätze \\
K3 & 5 Absätze \\
\hline Tab. 2: Aufteilung der Analyseergebnisse nach Prüfkriterien
\end{tabular}

Im sechsten Schritt werden die identifizierten Anforderungen interpretiert. Zur Interpretation werden die Kommentare und Empfehlungen von Dolle und Geschonneck [DoGe14] sowie von Terhaag [Terh15] hinzugezogen. Dabei wird das Ziel verfolgt, entsprechende Prozesse und die zugehörigen Aktivitäten der Anforderung zu erarbeiten und eine Bewertung vorzunehmen, inwieweit die Anforderung für den Betreiber verpflichtend ist. Folgende Aufstellung stellt das Interpretationsergebnis über die fünf Anforderungen dar (s. Tab. 3).

\begin{tabular}{|c|c|c|c|c|}
\hline ID & $\begin{array}{l}\text { Referenz } \\
\text { IT-SiG }\end{array}$ & Typ & Anforderung & Identifizierte Aktivitäten \\
\hline$\overline{\mathrm{A} 1}$ & 1.7 .6 & Optional & $\begin{array}{l}\text { Potenzielle Sicher- } \\
\text { heitslücken ermitteln }\end{array}$ & $\begin{array}{l}\text { - Lagebericht analysieren } \\
\text { - Potenzielle Sicherheitslücke } \\
\text { untersuchen }\end{array}$ \\
\hline A2 & 1.7 .10 & Pflicht & $\begin{array}{l}\text { BSI fordert Unterneh- } \\
\text { men zur Mitarbeit an } \\
\text { der Beseitigung einer } \\
\text { Sicherheitslücke auf }\end{array}$ & $\begin{array}{l}\text { - Erstellen eines Berichts über die } \\
\text { Sicherheitslücke } \\
\text { - Analyse der Sicherheitslücke } \\
\text { - Schließen der Sicherheitslücke }\end{array}$ \\
\hline A3 & 5.3 .2 & Pflicht & $\begin{array}{l}\text { Durchführen des } \\
\text { IT-Sicherheitsaudits }\end{array}$ & $\begin{array}{l}\text { - Überprüfen der Umsetzung des } \\
\text { IT-Sicherheitskonzepts } \\
\text { - Erstellen des Berichts über den Stand } \\
\text { der Umsetzung } \\
\text { - Übermitteln des Berichts an die } \\
\text { Bundesnetzagentur }\end{array}$ \\
\hline A4 & 5.3 .3 & Pflicht & $\begin{array}{l}\text { Melden einer erhebli- } \\
\text { chen Störung an die } \\
\text { Bundesnetzagentur }\end{array}$ & $\begin{array}{l}\text { - Melden der erheblichen Störung } \\
\text { - Nachmelden neuer Erkenntnisse } \\
\text { - Erstellen eines detaillierten Berichts } \\
\text { - Übermitteln des Berichts an die } \\
\text { Bundesnetzagentur }\end{array}$ \\
\hline A5 & 5.4 .2 & Pflicht & $\begin{array}{l}\text { Benachrichtigung } \\
\text { eines störenden } \\
\text { Teilnehmers }\end{array}$ & $\begin{array}{l}\text { - Erstellen eines Konzepts zur } \\
\text { Erkennung u. Behebung der Störung } \\
\text { - Informieren des störenden } \\
\text { Teilnehmers }\end{array}$ \\
\hline
\end{tabular}

Tab. 3: Relevante Anforderungen des IT-Sicherheitsgesetzes an die TK-Branche

Für jede dieser Anforderungen wurden konkrete Lösungsmodelle als Erweiterung von eTOM erstellt. Dabei wurde gemäß des hierarchischen Aufbaus von eTOM mit einer groben Einordnung der Änderungen begonnen. Diese Einordnung wurde weiter detailliert bis zur Entwicklung konkreter Prozessabläufe (s. Abb. 2). Aufgrund des Umfangs der so entwickelten Lösungsmodelle werden die Schritte sieben bis neun beispielhaft anhand der Anforderung A4 „Melden einer erheblichen Störung an die Bundesnetzagentur“ erläutert.

Im siebten Schritt wird jede Anforderung, im Folgenden beispielhaft die Anforderung A4, in die eTOM-Ebenen 0 bis 3 eingeordnet. Die Anforderung A4 stellt einen Prozess dar, der beim Auftreten erheblicher Störungen eine Meldung an die Bundesnetzagentur absetzt. Außerdem sind Nachmeldungen und das Zusammenfassen eines Detailberichts bei Behebung der Störung Umfang der Anforderung. Da das Schließen der Störung im Interesse des 
Unternehmens liegt, wird dieser Prozess der Enterprise Domain zugeordnet. Die Störung spiegelt eine gegenwärtige Gefahrensituation für das Unternehmen wider. Daher wurde der Prozess der ersten Hierarchieebene dem Enterprise Risk Management und in der zweiten Ebene dem Security Management zugeordnet. Auf Ebene 3 existiert in der vorliegenden eTOM-Version kein Prozess, in den sich die Anforderung zum Meldeverhalten gegenüber dem zuständigen Bundesamt einordnen lässt. Daher wird Ebene 3 um den Prozess Report Considerable Incident to Responsible Federal Office erweitert (s. Tab. 4).

\begin{tabular}{lll}
\hline Ebene & Referenz & Einordnung \\
\hline Domäne & 1.7 & Enterprise Domain \\
Ebene 1 & 1.7 .2 & Enterprise Risk Management \\
Ebene 2 & 1.7 .2 .2 & Security Management \\
Ebene 3 & 1.7 .2 .2 .14 & Report Considerable Incident to Responsible Federal Office \\
Ebene 4 & 1.7 .2 .2 .14 .1 & Report Incident \\
Ebene 4 & 1.7 .2 .2 .14 .2 & Report Additional Findings \\
Ebene 4 & 1.7 .2 .2 .14 .3 & Create Detailed Incident Report \\
Ebene 4 & 1.7 .2 .2 .14 .4 & Report Problem Solving and Detailed Report \\
\hline \multicolumn{3}{r}{ Tab. 4: Einordnung der Anforderung A4 in den eTOM Ebenen 0 bis 4 }
\end{tabular}

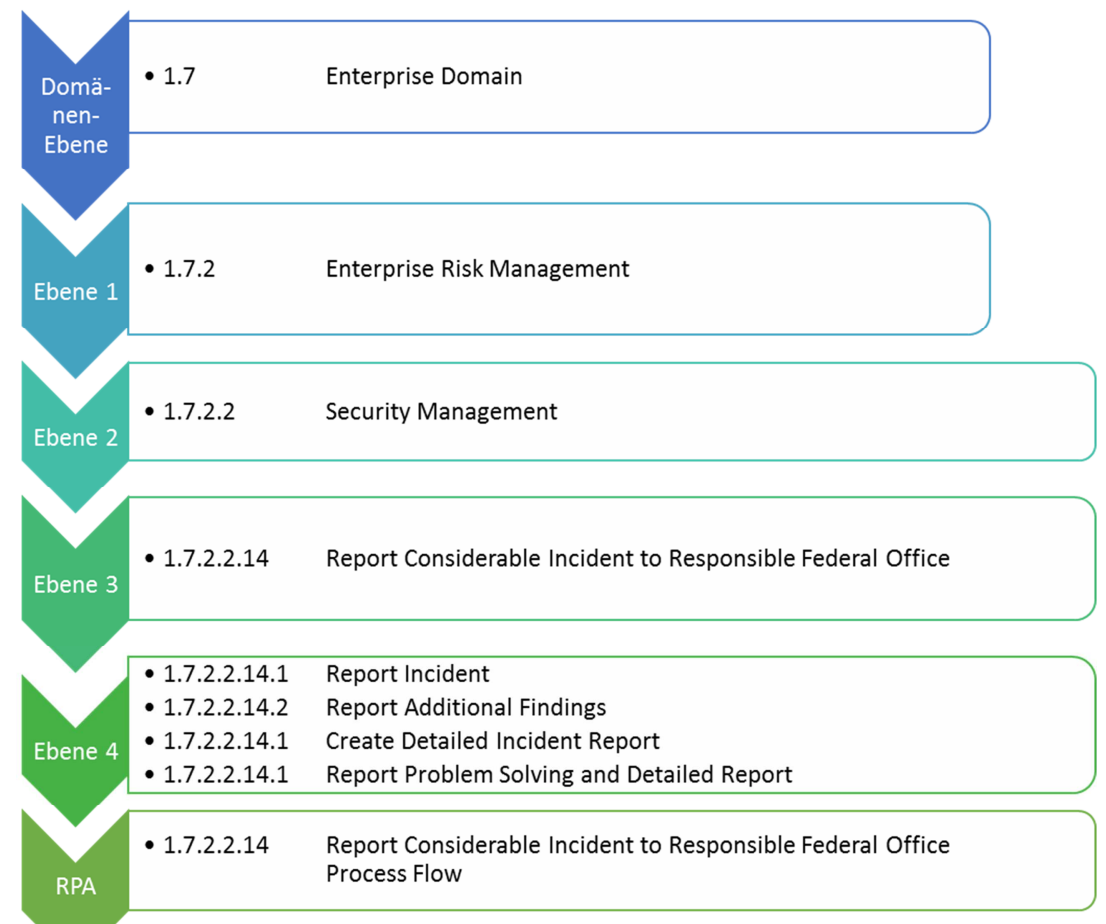

Abbildung 2: Hierarchischer Aufbau der eTOM mit Referenzprozessablauf (RPA)

Nachdem die Anforderung A4 dem Prozess Report Considerable Incident to Responsible Federal Office zugeordnet wurde, werden im achten Schritt die zugehörigen Aktivitäten (vgl. Tab. 4) auf Ebene 4 eingeordnet. Da die gegenwärtige eTOM-Version die vierte Ebene des benannten Prozesses nicht ausgeprägt hat, werden die vier Aktivitäten entsprechend alleinstehend hinzugefügt (s. Abb. 2). 
Nachdem der Prozess vollständig eingeordnet wurde, wird im letzten Schritt der zugehörige Referenzprozessablauf (RPA) erweitert. Dem Prozess Report Considerable Incident to Responsible Federal Office ist nach dem Dokument GB921E kein RPA zugeordnet [TMFo15]. Daher wird innerhalb dieses Abschnittes ein entsprechender Referenzprozessablauf vorgeschlagen (s. Abb. 3).

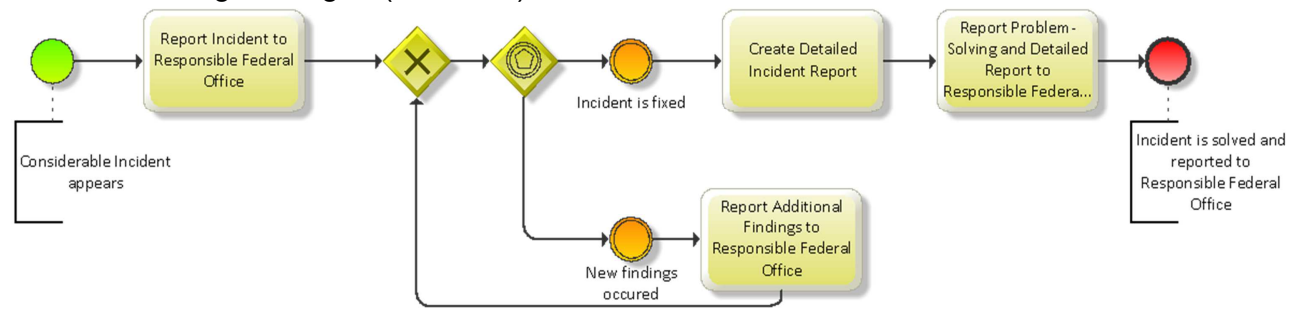

Abbildung 3: Process Flow Ebene 4 - Report Considerable Incident to Responsible Federal Office (BPMN)

Der Prozess beginnt bei dem Auftreten einer erheblichen Störung (Considerable Incident appears). Anschließend ist die Störung an das verantwortliche Bundesamt zu melden (Aktivität: Report Incident to Responsible Federal Office). Dann wird gewartet, bis entweder die Störung behoben wurde (Incident is fixed) oder neue Erkenntnisse vorliegen (New findings occurred). Insofern neue Erkenntnisse vorliegen, werden diese an das zuständige Bundesamt gemeldet (Aktivität: Report Additional Findings to Responsible Federal Office). Ist die Störung behoben, wird ein Detailbericht angefertigt (Create Detailed Incident Report) und mit der Abschlussmeldung an das Bundesamt übermittelt (Report Problem-Solving and Detailed Report to Responsible Federal Office). Anschließend endet der Prozess (Incident is solved and reported to Responsible Federal Office).

\section{$5 \quad$ Fazit}

Analog zur vorangehenden Darstellung wurden für sämtliche fünf Anforderungen aus Tab. 3 konkrete Lösungsmodelle zur Erweiterung von eTOM entwickelt. Entsprechend der Anforderungen des IT-SiG zielen die konstruierten Modelle auf die relevanten Inhalte für KRITISBetreiber der Telekommunikationsbranche ab. Damit wurde ein Modell gebildet, das aufgrund der Problemstellung und dem verwendeten Ausgangsmodell sowohl länder- als auch branchenabhängig ist. Die zu Beginn aufgestellte Forschungsfrage wurde durch Konstruktion des Artefakts zielgerichtet adressiert und bearbeitet. Das Artefakt entspricht dabei der Zielsetzung, einen ersten Ansatz zu präsentieren, der die Besonderheiten und Herausforderungen des IT-Sicherheitsgesetzes berücksichtigt. Ziel sollte es künftig sein, die Erweiterung aufzugreifen und an den jeweiligen Stand der Gesetzeslage zu adaptieren. Des Weiteren sollte die Erweiterung von eTOM aufgrund nationaler bzw. internationaler Rechtsnormen konsequent fortgeführt werden. So bildet das IT-Sicherheitsgesetz lediglich Erweiterungen bereits bestehender Gesetze, wie dem Telekommunikationsgesetz (TKG). Dieses sollte ebenfalls ganzheitlich auf relevante Anforderungen an die TK-Branche überprüft und im eTOM-Modell abgebildet werden. Das Verfahren dazu kann sich an dem hier vorgestellten Vorgehensmodell (Abb. 1) orientieren. Das resultierende Modell ist anschließend an konkreten Beispielen zu demonstrieren und als Erweiterungsvorschlag im TM Forum einzureichen. 
Vielversprechend erscheinen zudem die Potenziale, die das branchenabhängige Modell eTOM durch länder- bzw. staatenverbundabhängige (z. B. Europäische Union) Ausprägungen erschließen kann, insbesondere in Hinblick auf die Internationalisierung und länderübergreifende Regulierung von TK-Unternehmen. Beispielsweise könnte eine siebte Domäne Legal Domain eingeführt werden, welche die gesetzgebenden und regulatorischen Besonderheiten des jeweiligen Landes abbildet.

\section{Literaturverzeichnis}

[AABG16] Alpar, P.; Alt, R.; Bensberg, F.; Grob, H. L., Weimann, P., Winter, R.: Anwendungsorientierte Wirtschaftsinformatik. Strategische Planung, Entwicklung und Nutzung von Informationssystemen. Springer Vieweg, Wiesbaden, 2016.

[BaPr10] Baskerville, R.; Pries-Heje, J.: Erklärende Designtheorie. In: WIRTSCHAFTSINFORMATIK 5 (52), S. 259-271.

[BeCz17] Bensberg, F.; Czarnecki, C.: enhanced Telecom Operations Map. http://bit.ly/2bCaBAe. Abruf am 2017-04-05.

[BSIT15] Bundesamt für Sicherheit in der Informationstechnik: Fragen und Antworten zum Inkrafttreten des IT-Sicherheitsgesetzes. http://bit.ly/2bPE6Md. Abruf am 2016-02-03.

[Bund09] Bundesministerium des Inneren: Nationale Strategie zum Schutz Kritischer Infrastrukturen (KRITIS-Strategie). http://bit.ly/1PUiT3t. Abruf am 2016-02-03.

[Bund11] Bundesministerium des Inneren: Sektoren- und Brancheneinteilung Kritischer Infrastrukturen. http://bit.ly/2bnRMNK. Abruf am 2016-05-26.

[Bund13] Bundesministerium des Inneren: Friedrich stellt Wirtschaft IT-Sicherheitsgesetz vor. http://bit.ly/2bWILPH. Abruf am 2016-02-03.

[Bund15] Bundeskriminalamt: Polizeilich erfasste Fälle von Cyberkriminalität in Deutschland von 2000 bis 2014. http://bit.ly/2biR8z1. Abruf am 2016-05-26.

[CWS13] Czarnecki, C.; Winkelmann, A.; Spiliopoulou, M.: Referenzprozessabläufe für Telekommunikationsunternehmen. In: WIRTSCHAFTSINFORMATIK, 2 (55), S. 83-97.

[Czar13] Czarnecki, C.: Entwicklung einer referenzmodellbasierten Unternehmensarchitektur für die Telekommunikationsindustrie. Logos, Berlin, 2013.

[CzDi17a] Czarnecki, C.; Dietze C.: Reference Architecture for the Telecommunications Industry. Transformation of Strategy, Organization, Processes, Data, and Applications. Springer Berlin, Heidelberg, New York, 2017.

[CzDi17b] Czarnecki, C., Dietze, C.: Domain-Specific Reference Modeling in the Telecommunications Industry. In: Maedche, A., vom Brocke, J., Hevner, A. (Hrsg.): Designing the Digital Transformation. Springer International Publishing, S. 313-329, 2017.

[CzWS12] Czarnecki, C.; Winkelmann, A.; Spiliopoulou, M.: Transformation in Telecommunication - Analyse und Clustering von Real-life Projekten. In: Mattfeld, D. C; Robra-Bissantz, S. (Hrsg.): Multi-Konferenz Wirtschaftsinformatik 2012. GITO Verlag, Braunschweig, S. 985-998, 2012.

[DeBu15] Deutscher Bundestag: Gesetz zur Erhöhung der Sicherheit informationstechnischer Systeme (IT-Sicherheitsgesetz). 
[Deut16] Deutsche Telekom AG: Erfasste Versuche von Cyberangriffen von 01.01.2015 bis 01.04.2016. http://www.sicherheitstacho.eu/statistics. Abruf am 2016-05-26.

[DoGe14] Dolle, W.; Geschonneck, A.: IT-Sicherheit in Deutschland - Handlungsempfehlungen für eine zielorientierte Umsetzung des IT-Sicherheitsgesetzes. http://bit.ly/2biEOUu. Abruf am 2016-04-08.

[GrHe13] Gregor, S., Hevner, A.R.: Positioning and presenting design science research for maximum impact. MIS Q. 37, 337-356, 2013.

[HMPR04] Hevner, A.R., March, S.T., Park, J., Ram, S.: Design science in information systems research. MIS Q. 28, 75-105, 2004.

[ITUT07] ITU-T: Enhanced Telecom Operations Map (eTOM) - Introduction. ITU-T Recommendation M.3050.0.

[Pico10] Picot, A.: Richtungsdiskussionen in der Wirtschaftsinformatik. In: ZfbF 9 (62), S. 662 679.

[PoRu15] Pohl, K., Rupp, C.: Basiswissen Requirements Engineering - Aus- und Weiterbildung zum "Certified Professional for Requirements Engineering" - Foundation Level nach IREB-Standard. dpunkt-Verlag, Heidelberg, 2015.

[PTRC07] Peffers, K.; Tuunanen, T.; Rothenberger, M. A.; Chatterjee, S.: A Design Science Research Methodology for Information Systems Research. In: Journal of Management Information Systems 3 (24); S. 45-78.

[Simi17] Simic, D.: IT-Sicherheitsanalysen - ein prozessorientiertes Vorgehensmodell. De Gruyter Oldenbourg, Berlin, 2017.

[TeMa15] TM Forum: GB991 Core Frameworks Concepts and Principals. Business Process, Information and Application Frameworks.

[Terh15] Terhaag, M.: IT-Sicherheitsgesetz. Auswirkungen, Entwicklung und Materialien für die Praxis.

[TMFo15] TM Forum: GB921E End-to-End Business Flows R15.0.1. How To Guide. http://bit.ly/2bCb856. Abruf am am 2016-01-31.

[TMFO16] TM Forum: TM Forum Internetpräsenz. https://www.tmforum.org. Abruf am 2016-06-09.

\section{Kontakt}

Tristan Eberhard Thomé

Prof. Dr. Christian Czarnecki

Prof. Dr. Gunnar Auth

Hochschule für Telekommunikation Leipzig (HfTL)

Gustav-Freytag-Str. 43-45, 04277 Leipzig

T+4922818112769, s139109@hft-leipzig.de

T+493413062 227, czarnecki@hft-leipzig.de

T +49 3413062 235, auth@hft-leipzig.de

Prof. Dr. Frank Bensberg

Hochschule Osnabrück

Caprivistrasse 30a, 49076 Osnabrück

T +49 541969 3264, f.bensberg@hs-osnabrueck.de 


\section{Eine Petri-Netz-Programmiersprache und Anwendungen in der Produk- tion}

\section{Carlo Simon}

\section{Zusammenfassung}

Entlang der Forschungsmethode Design Science in Information Systems Research wird eine Programmiersprache für höhere Petri-Netze entwickelt, mit der sich komplexe Simulationsmodelle für die Produktionsplanung und -steuerung entwickeln lassen. Hierfür sind folgende Aspekte relevant: 1) Auftragsbezogene Daten müssen repräsentiert werden und Aufträge müssen sich entsprechend ihrer Priorisierung in einen simulierten Produktionsprozess einsteuern lassen. 2) Verschiede Produktionsstrategien wie Push- oder Pull-Prinzip müssen abgebildet werden können. 3) Die Simulation von Prozessen muss nicht nur die eigentlichen Arbeitszeiten abbilden, sondern auch Zeiten geplanten Stillstands, um so etwa den OEE-Index ermitteln zu können.

Für den genannten Aufgabenbereich wird eine Programmiersprache entwickelt und deren prototypische Implementierung in einer webbasierten Programmierumgebung demonstriert. Hierbei kommen Prinzipien des Compilerbaus zur Anwendung. Diese beziehen sich auf die Interpretation der Netzspezifikation, um das Petri-Netz als solches konstruieren zu können, aber auch auf die Interpretation der Netzstrukturen zur „Laufzeit“, also während der Simulation. Die Token sind hierfür Träger komplexer Datenobjekte, die die aus Datenbanken bekannten Datentypen unterstützen.

An einem Beispiel zur Produktionssteuerung wird die Anwendung des Forschungsansatzes demonstriert und veranschaulicht.

\section{$1 \quad$ Motivation und Zielsetzung}

Das Management von Prozessen ist eine der zentralen Themenstellungen in der Wirtschaftsinformatik (siehe hierzu die Ausführungen in [HaMeNe2015]). Weit verbreitet sind Prozess-Modellierungssprachen, durch die Prozesse veranschaulicht werden können, um auf dieser Basis Optimierungspotenziale zu erkennen, Arbeitsanweisungen für Mitarbeiter abzuleiten oder um sie als Grundlage für eine prozessorientierte Softwareentwicklung zu nutzen. Zu diesen Sprachen zählen Ereignisgesteuerte Prozessketten (siehe [St2006]), BPMN (siehe [AI2015]) oder entsprechende Sprachen der UML (siehe [VRBeFi2016]).

Petri-Netze unterscheiden sich hiervon signifikant durch die Möglichkeit, die erstellten Modelle zu simulieren und zu analysieren, wofür allerdings geeignete Werkzeuge benötigt werden. Doch genau hieran besteht ein Mangel. So sind unter https://www.informatik.unihamburg.de/TGI/PetriNets/tools/quick.html zwar insgesamt 91 Petri-Netz-Tools gelistet, allerdings ist bei 47 Tools die Homepage nicht mehr erreichbar oder enthält keinen Hinweis mehr auf das Tool. Bei weiteren 23 Werkzeugen ist die Entwicklung seit 2013 oder (viel) früher eingestellt, weswegen hier Wartung oder sonstiger Benutzersupport entfallen - zum Teil handelt es sich noch um MS-DOS-Projekte. Weitere 7 Projekte sind rein für die Forschung entwickelte Werkzeuge, die nur per Skripting oder als Eclipse-Plugin genutzt werden 
können und somit explizit Programmierkenntnisse voraussetzen, um sie benutzen zu können. Von den restlichen 14 Werkzeugen unterstützt lediglich TimeNet Datum und Uhrzeit als Datentypen (http://www.tu-ilmenau.de/sse/timenet/). Obwohl das Werkzeug noch bis 2015 weiter gewartet wurde, basiert es im Kern auf einer Version aus dem Jahr 2007, was auch am User-Interface erkennbar ist. Eine Umsetzung als Web-App, die auch auf mobilen Geräten eingesetzt werden könnte, ist wohl nicht geplant.

Für die Simulation von Produktionsprozessen muss ein geeignetes Werkzeug aber die folgenden Eigenschaften notwendig erfüllen:

- Simulation zeitbewerteter höherer Netze, bei denen die Token neben Zeit und Datum auch weitere, frei wählbare Attribute wie Chargengröße oder Priorität eines Auftrags als zusätzliche Informationen enthalten können.

- Möglichkeit zur Priorisierung von Schaltreihenfolgen, etwa mit Blick auf Prioritäten oder die Dauer, die ein (simulierter) Auftrag bereits in Bearbeitung ist.

- Kopplung der Stellen mit externen Datenquellen, um Simulationsläufe auf Basis von Realdaten durchspielen und Simulationsergebnisse exportieren zu können.

- Zeitgemäße Implementation als mobile Applikation, da Fragestellungen zur Optimierung von Abläufen oft vor Ort überprüft werden müssen.

- Schließlich sind Modelle, die Produktionsprozesse beschreiben, so komplex, dass der Versuch, sie mit einem graphischen Editor erstellen zu wollen, schnell scheitert. Solche Modelle müssen in einer flexiblen Programmierumgebung formal spezifiziert werden. Eine Visualisierung ist muss aber optional möglich sein.

Das vorliegende Paper dokumentiert den aktuellen Entwicklungsstand einer solchen Spezifikationssprache und einer passenden prototypischen Programmierumgebung. Die Forschungsarbeiten folgen dem gestaltungsorientierten Design-Science-Research-Ansatz [Hev+2004]. Die Sprache wird anhand von Beispielen erklärt, die in dieser Form vom Tool interpretiert, visualisiert und simuliert werden können. Bei den gezeigten Abbildungen der Netze und Symbolgraphen handelt es sich um Screenshots aus dem Tool heraus. Diese ist als Web-App in JavaScript implementiert.

\section{Forschungsmethode}

Nach [Hev+2004] gibt es sieben Guidelines bezüglich des Design Science Research. Im Folgenden werden diese kurz genannt und es wird gezeigt, wie sie im Rahmen des Vorhabens umgesetzt wurden:

- Der Design Science Research Prozess muss ein realisierbares Artefakt in Form eines Konstrukts, eines Modells, einer Methode oder einer Umschreibung erzeugen (Design as an Artifact): Das Artefakt in diesem Vorhaben ist eine Grammatik für die Spezifikationssprache sowie deren prototypische Umsetzung.

- Das Ziel des Design Science Research muss die Entwicklung einer auf Technik und/oder Methodik basierenden Lösung eines bedeutenden und einschlägigen Business Problems sein (Problem Relevance): Gespräche mit Praktikern aus Produktion und Logistik haben ergeben, dass diese oft improvisieren und etwa Lösungen mit Hilfe von Excel selber entwickeln. Der hier vorgestellte Ansatz ist dem gegenüber generisch und prozessorientiert.

- $\quad$ Die Nutzbarkeit, Qualität und Wirksamkeit eines Design Artefakts muss über Evaluations-Methoden nachweisbar sein (Design Evaluation): Nachdem die Anforderungen an 
eine Simulationsumgebung in Gesprächen mit Praktikern erhoben und eine Reihe von Prototypen entwickelt wurde, wird die Simulationsumgebung zwischenzeitlich in der Lehre eingesetzt. Hieraus ergeben sich neue Anforderungen an künftige Versionen.

- Design Science Research muss einen nachprüfbaren und wohlstrukturierten Beitrag in den Teilbereichen Design Artifact, Design Foundations sowie Design Methodologies liefern (Research Contributions): Diese Forderung wird durch die theoretische Fundierung auf Basis von Petri-Netzen erfüllt. Eine Einführung zum Thema Petri-Netze findet man in [Ba1996]. Die höheren Petri-Netz-Konzepte, die in der Spezifikationssprache und im Werkzeug genutzt werden, gehen zurück auf die Arbeiten [GeLa1981] und [LaSi1999].

- $\quad$ Das Ergebnis des Design Science Research ist stark von der Anwendung genauer und präziser Methoden zur Erstellung und Bewertung eines Design Artefakts abhängig $(R e-$ search Rigor): Spezifikationssprache und Simulationsumgebung werden im Rahmen von Lehrveranstaltungen komparativ zu alternativen Simulationsumgebungen eingesetzt und mit diesen verglichen.

- Die Suche nach einem wirksamen Artefakt erfordert die Verwendung verfügbarer Mittel, um das angestrebte Ziel unter Einhaltung festgelegter Richtlinien innerhalb des betrachteten Problem-Umfelds zu erreichen (Design as a Search Process): Bereits jetzt wurden in den letzten $11 / 2$ Jahren zwei Prototypen entwickelt (siehe [Be2016] und [SiBe2016]). Der aktuelle Stand kann als Basis für ein erstes Produktivsystem angesehen werden und wird derzeit mit Praxispartnern aus den Bereichen Logistik, Produktion und Automatisierung diskutiert.

- Design Science Research und auch die daraus hervorgehenden Ergebnisse müssen sowohl Technik orientierten Mitarbeitern als auch dem Management offengelegt und präsentiert werden (Communication of Research): Auch diese Forderung des Design Science Research Ansatzes ist hier erfüllt, da die vorgestellte Simulationsumgebung aufgrund differenzierter Abstraktionsebenen für unterschiedliche Anwendergruppen (Betriebsleiter, Produktionsplaner, mittleres Management und Berater) zugänglich und anwendbar ist.

\section{Sprachbasis und erste Beispiele}

Petri-Netze sind bipartite Graphen mit Stellen (engl. places) und Transitionen (engl. transitions) als Knoten, welche über gerichtete Kanten (engl. arcs) miteinander verbunden sind. Stellen sind Träger von Informationen und können markiert werden (engl. marking). Hieraus ergibt sich eine sehr einfache Möglichkeit zur Spezifikation entsprechender Graphen nach dem Schema in Abbildung 1.

Bei der Spezifikation der Petri-Netz-Elemente können deren Eigenschaften definiert werden. Im gezeigten Beispiel wird die Hintergrundfarbe des Netzes gesetzt und das automatische Layout ermöglicht. 


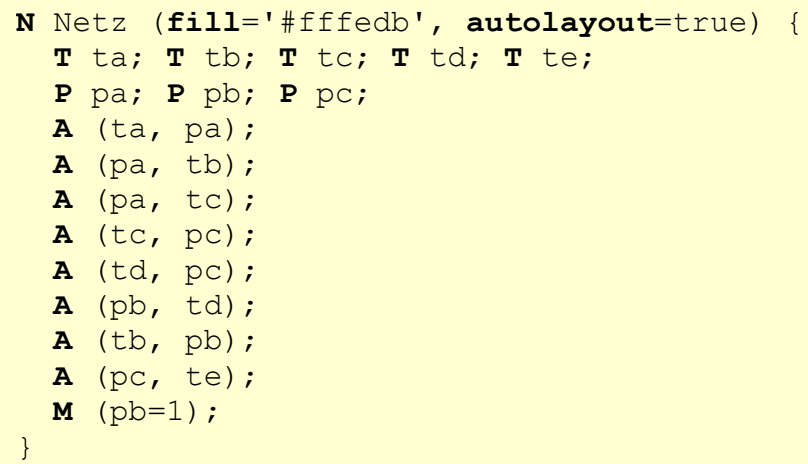

Abbildung 1: Spezifikation einer ersten, einfachen Netzstruktur

Das Ergebnis ist das in Abbildung 2 gezeigte Petri-Netz, dessen Layout automatisch berechnet wird. In dem Beispiel sind die Transitionen ta und td aktiviert - ta, da ihr Vorbereich leer ist und td, da inr Vorbereich markiert ist.

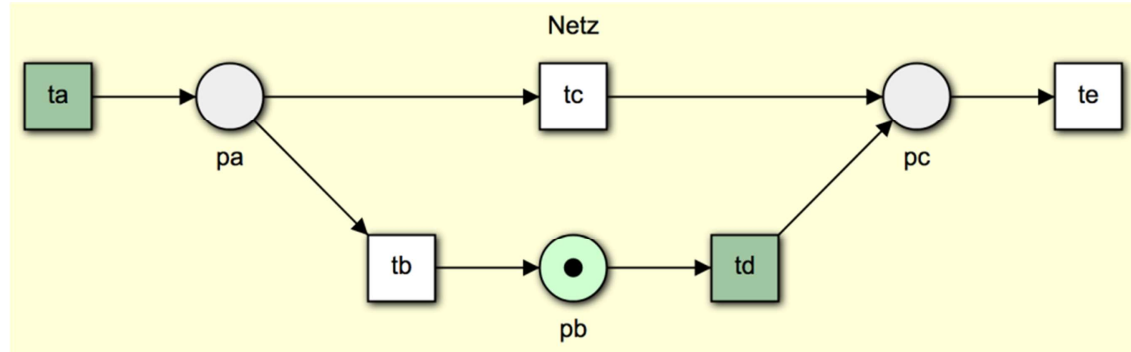

Abbildung 2: Petri-Netz-Modell zur Spezifikation aus Abbildung 1

Auch für die anderen Netzelemente können weitere Eigenschaften definiert werden. Einen Eindruck von den Möglichkeiten vermittelt die zweite Beispielspezifikation in Abbildung 3.

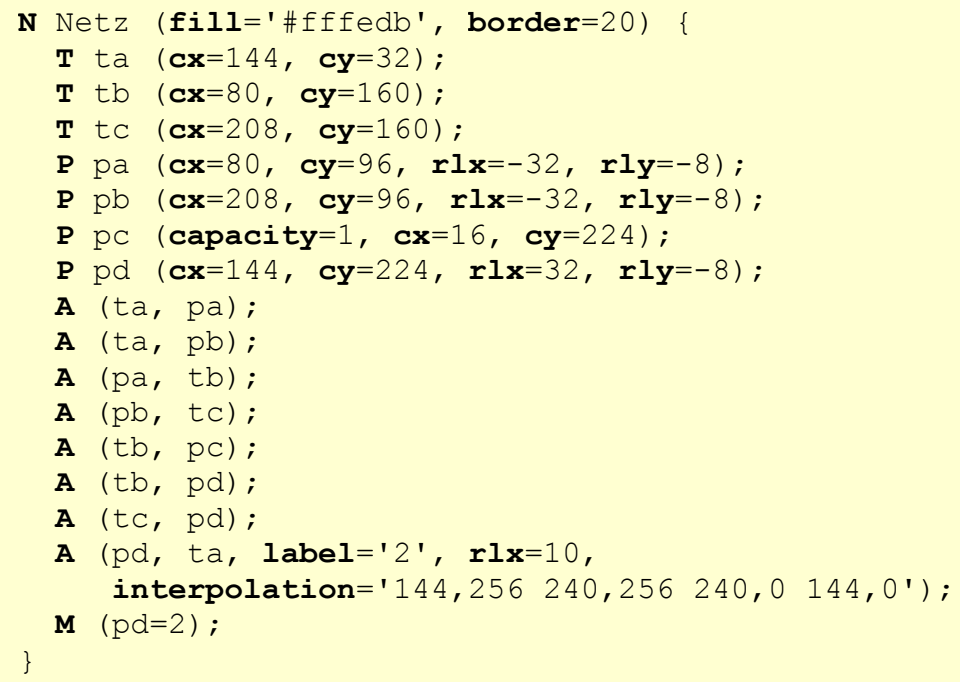

Abbildung 3: Spezifikation einer ersten, einfachen Netzstruktur 
Abbildung 4 zeigt das hierzu gehörende Petri-Netz. Mit den Attributen cx und cy werden die Zentralkoordinaten der jeweiligen Knoten spezifiziert (es wird nun also kein automatisches Layout generiert). Dabei gelten die folgenden Regeln: Der Nullpunkt ist oben links und für Knoten wird eine Ausdehnung von 32x32 (als Standardlängeneinheit in SVG) angenommen. Die Attribute $r l x$ und $r l y$ verschieben die Beschriftung von Knoten, wobei als Default für Transitionen das Zentrum eines Knotens angenommen wird, während Stellen per Default unterhalb des eigentlichen Knotens beschriftet werden. Die rückläufige Kante von Stelle pd zu Transition ta wird mittels Stützpunkten um das restliche Netz herum gelenkt. Schließlich wird der Rahmen um die Netzdarstellung auf 20 erhöht, damit die umlaufende Kante richtig dargestellt wird.

Die Stelle pc hat eine Kapazität von 1, so dass maximal ein Token auf ihr abgelegt werden kann. Die Kante von pd nach ta hat ein Kantengewicht von 2, d.h. beim Schalten von Transition ta werden gleich zwei Token von Stelle pd genommen. Da durch die Spezifikation Stelle pd mit zwei Token anfangsmarkiert wird, ist Transition ta aktiviert.

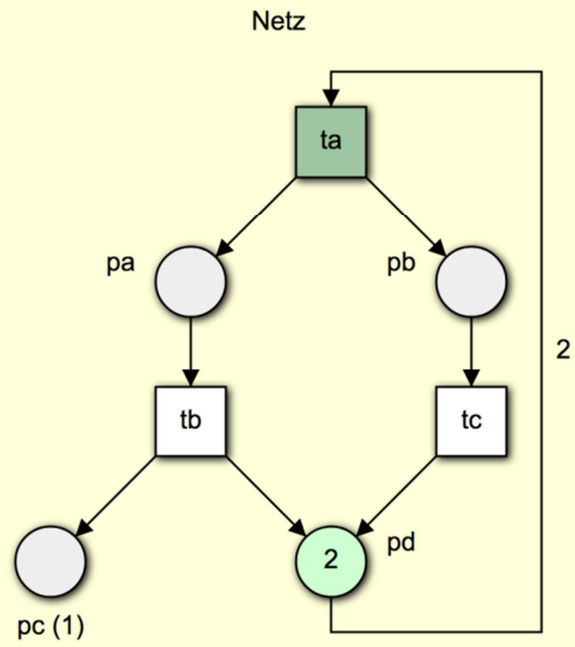

Abbildung 4: Petri-Netz-Modell zur Spezifikation aus Abbildung 2

Das Tool unterstützt schon jetzt zahlreiche weitere Layout-Optionen. Im Folgenden sollen aber vor allen Dingen die Möglichkeiten näher dargestellt werden, durch die komplexe Modelle und detaillierte Simulationsläufe möglich sind.

\section{$4 \quad$ Hierarchische Modellierung}

Entwickelt man Prozessmodelle mit dem Ziele, durch deren Simulation Aussagen über die Realität abzuleiten, die ansonsten nicht offensichtlich gelten, dann muss ein Tool Möglichkeiten zur Gruppierung und zur Abstraktion unterstützen [St1969].

Die in Abbildung 5 gezeigte Spezifikation verdeutlicht, wie diese Prinzipien in der Spezifikationssprache umgesetzt sind. Dabei sind in dem umgebenden Netz drei Teilnetze eingebettet. Abbildung 6 zeigt das hierarchische Modell. Die Teilnetze Input und Output enthalten 
dabei jeweils nur eine Stelle, das Netz Produktion eine Folge aus Transition, Stelle und Transition. Um die Teilnetze im Gesamtnetz positionieren zu können, wird deren obere linke Ecke durch die Attribute $x$ und $y$ spezifiziert. Koordinaten in den Teilnetzen verstehen sich relativ zum umgebenden Netz. Insofern ist das Netz Output eine Kopie von Input abgesehen von seiner x-Position im Gesamtnetz.

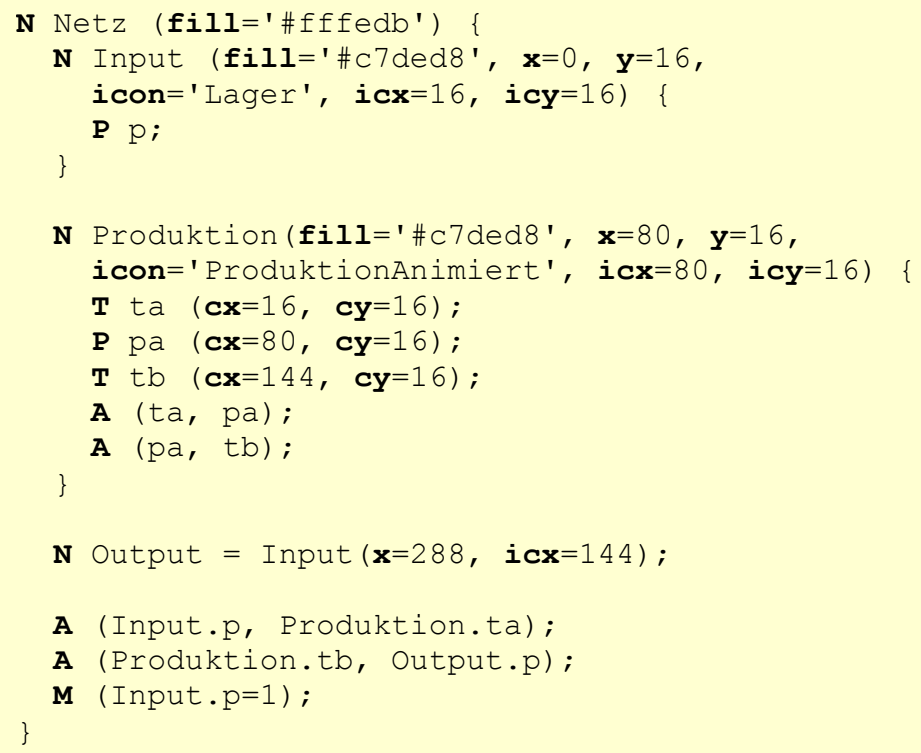

Abbildung 5: Hierarchische Netzstruktur mit alternativer Symboldarstellung

Netz

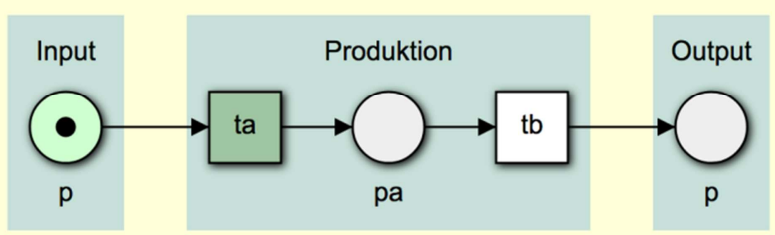

Abbildung 6: Hierachisches Petri-Netz-Modell zur Spezifikation aus Abbildung 5

Abbildung 7 zeigt schließlich eine alternative Darstellung des Netzes mit Hilfe von Symbolen. Hierzu ist im Tool eine erste Symbolbibliothek implementiert, durch die einem Netz mit Hilfe des Attributs icon ein alternatives Symbol zugewiesen werden kann. Die Attribute icx und icy sind die Zentralkoordinaten der Symbole.

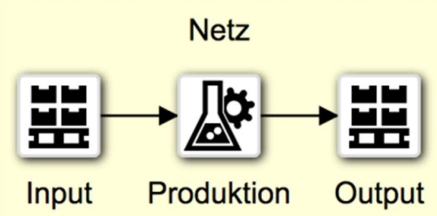

Abbildung 7: Alternative Symbolansicht zum Petri-Netz-Modell in Abbldung 6 
Entsprechend der Idee von Prädikat/Transitions-Netzen können Stellen nicht nur mit anonymen Token sondern mit individuellen Marken markiert sein. Die Umsetzung dieses Konzepts veranschaulichen die Spezifikation in Abbildung 8 und deren graphische Umsetzung in Abbildung 9. Das Petri-Netz beschreibt, wie Aufträge gemäß ihrer Priorisierung in einen Produktionsprozess eingesteuert werden. Dazu liegen zu Beginn auf der Stelle Auftraege die drei Marken (2, „VW“, 2), (7, „Volvo“, 3) und (9, „BMW“, 2), die wie Datensätze in einer Datenbanktabelle zu interpretieren sind. Das erste Attribut steht für die Auftragsnummer, das zweite für den Kundennamen und das letzte für die Priorität, die dem Auftrag zugeordnet wird. Von diesen Informationen sollen die Auftragsnummer und der Name in die Stelle PStart übertragen werden. Hierzu sind die beiden Stellen typisiert, wobei die Basistypen int für ganzzahlige Werte und char für Zeichenketten genutzt werden. Ferner gibt es einen Datentyp float für Kommazahlen sowie Datentypen für Datum- und Zeitinformationen. Letztere werden in einem weiteren Beispiel behandelt.

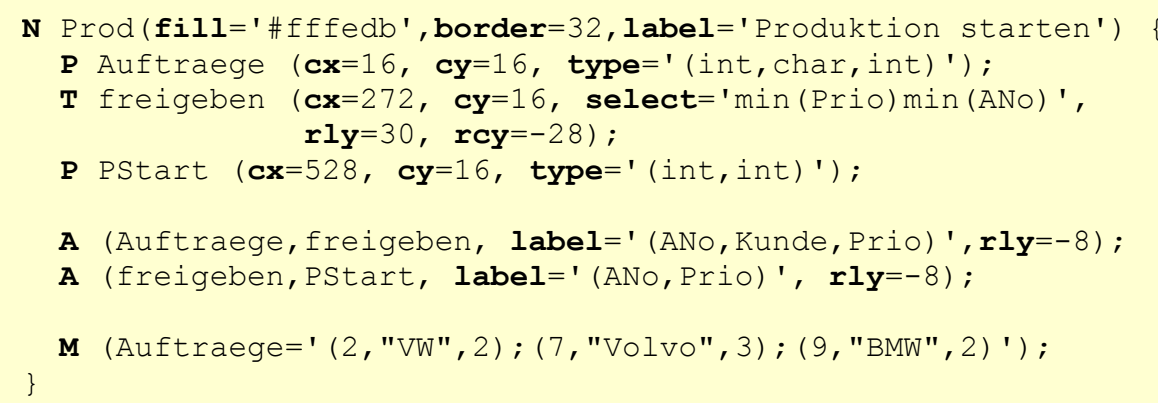

Abbildung 8: Netz-Spezifikation mit typisierten Stellen und definierter Schaltreihenfolge

Mit Blick auf die drei Marken steht die Transition freigeben zunächst im Konflikt zu sich selbst. Die Schaltbedingung select='min (PriO) $\min (A N O)$ ' löst diesen auf, indem die Datensätze mit der höchsten Priorität ausgewählt werden, und bei gleicher Priorität aufsteigend sortiert nach der Auftragsnummer. Im gewählten Beispiel werden die Aufträge also in der Reihenfolge 7, 2, 9 bezogen auf die Auftragsnummer freigegeben.

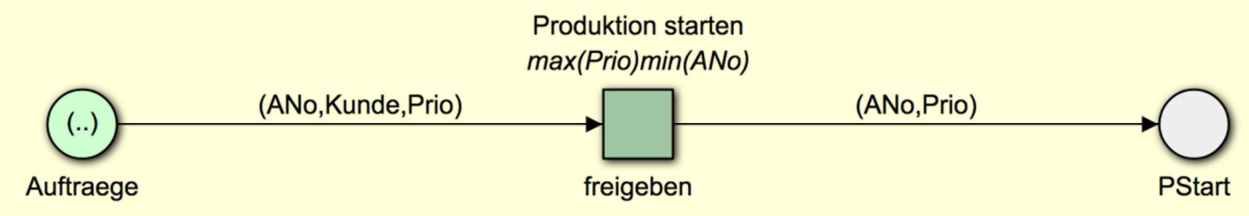

Abbildung 9: Petri-Netz-Modell zur Spezifikation aus Abbildung 8

Das letzte Beispiel demonstriert das Rechnen mit Zeit und schafft so die Voraussetzung, um ganze Produktionsabläufe abzubilden. Hierzu wird, anders als in vielen anderen Petri-NetzWerkzeugen, nicht mit einer Schaltdauer von Transitionen und einem abstrakten Zeitbegriff gearbeitet. Vielmehr sind Zeitinformationen gleich zu behandeln wie alle anderen Informationen. Hierzu wurden die Datentypen time, date und datetime eingeführt. 


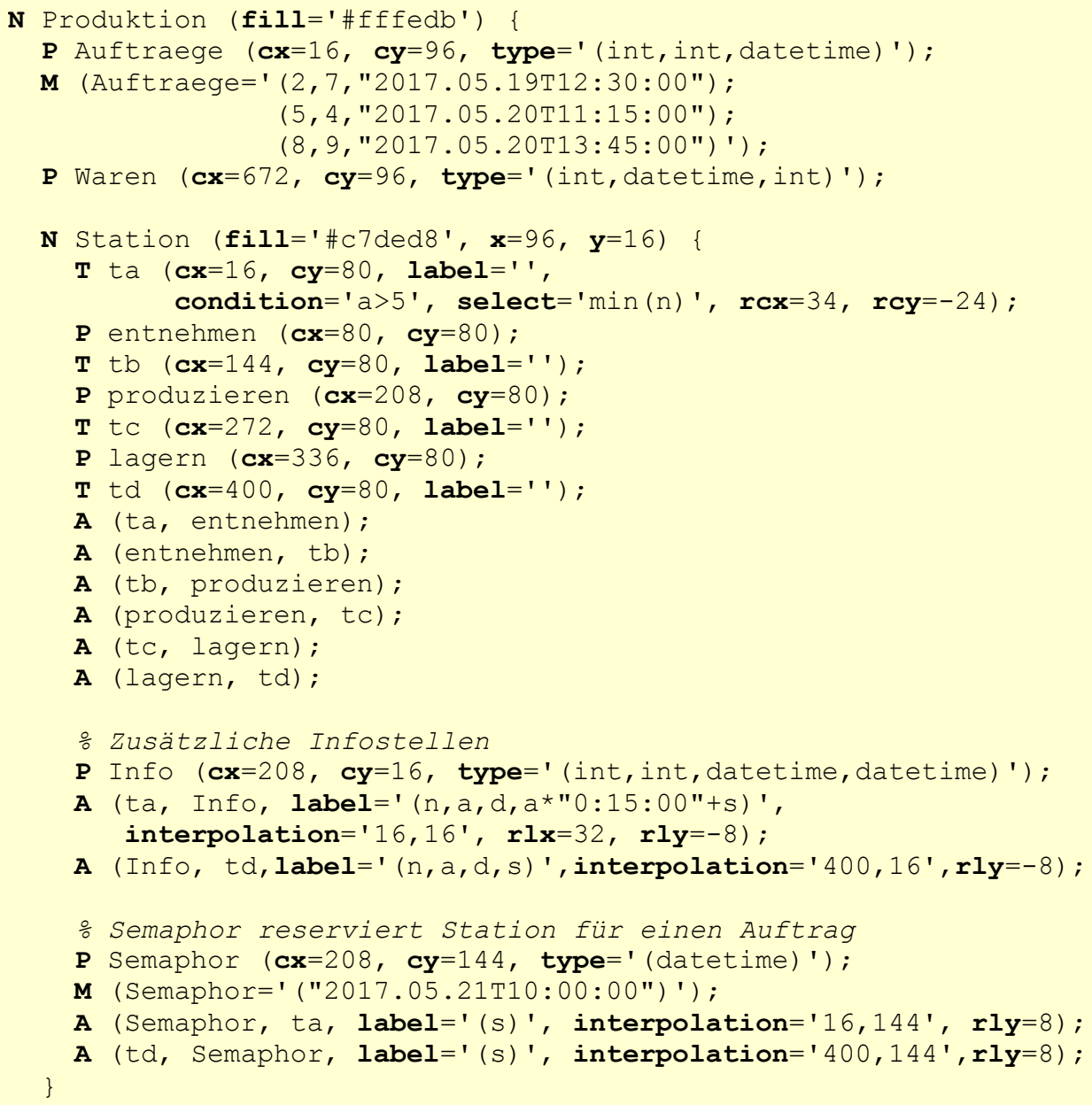

A (Auftraege, Station.ta, label=' (n,a,d)', rly=-8);

A (Station.td, Waren, label=' ( $\mathrm{n}, \mathrm{s}, \operatorname{diff}(\mathrm{s}, \mathrm{d}$, "minute")) ', rly=-8); \}

Abbildung 10: Netz-Spezifikation zu einem Produktionsprozess mit Zeitinformationen

Die Spezifikation in Abbildung 10 und das zugehörige Modell in Abbildung 11 beschreiben einen Produktionsprozess. Auf der linken Stelle Auftraege liegen zu Beginn die Marken (2,7,"2017.05.19T12:30:00"), (5,4,"2017.05.20T11:15:00") und (8, 9,"2017.05.20T13:45:00"), wobei das erste Attribut die Auftragsnummer ist, das zweite die Anzahl der zu produzierenden Güter und das dritte Datum und Uhrzeit des Auftragseingangs. Die Stelle Semaphor stellt nun sicher, dass in einem Schritt nur ein Auftrag durch die Arbeitsstation bearbeitet werden kann. Ferner enthält die Marke auf dieser Stelle die Information, ab wann die Produktion beginnen kann (im gezeigten Beispiel "2017.05.21T10:00:00").

Von der Arbeitsstation werden nur Aufträge bearbeitet, bei der die Anzahl der zu produzierenden Güter größer als 5 ist - für kleinere Chargen könnte ein vergleichbares Netz modelliert werde. Diese Aufträge werden nach der Auftragsnummer sortiert abgearbeitet. 


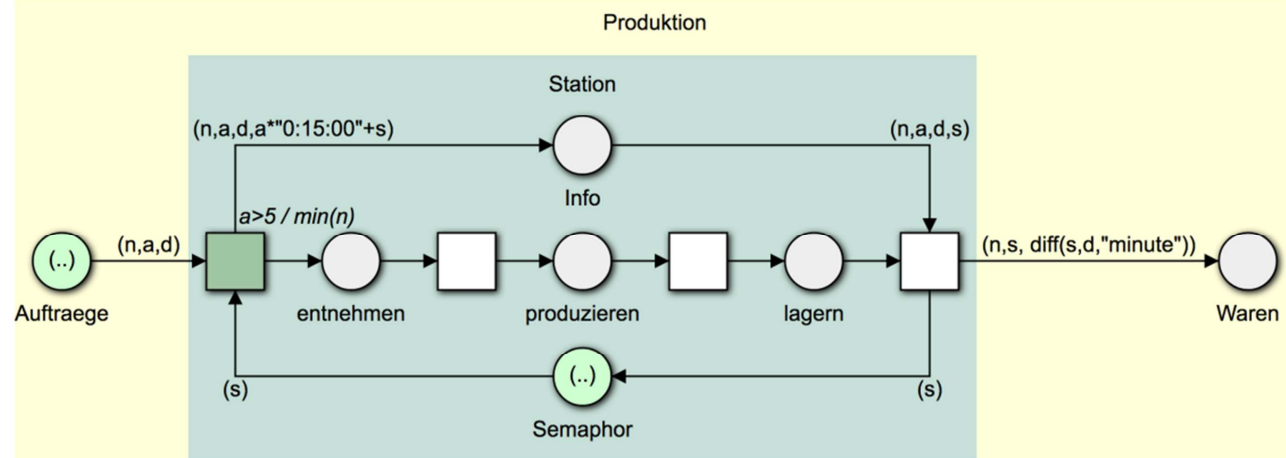

Abbildung 11: Petri-Netz-Modell zur Spezifikation aus Abbildung 10

Die Auftragsbearbeitung ist in drei Phasen unterteilt: Nach der Entnahme der Rohmaterialien erfolgt die eigentliche Produktion und abschließend werden die gefertigten Waren gelagert. Zeitgleich werden auf der Stelle Info die Daten zum Auftrag gespeichert sowie das voraussichtlich Fertigstellungdatum berechnet. Dabei wird unterstellt, dass die Produktion der einzelnen Güter je 15 min dauert. Abbildung 12 zeigt das Netz, während das Produzieren simuliert wird.

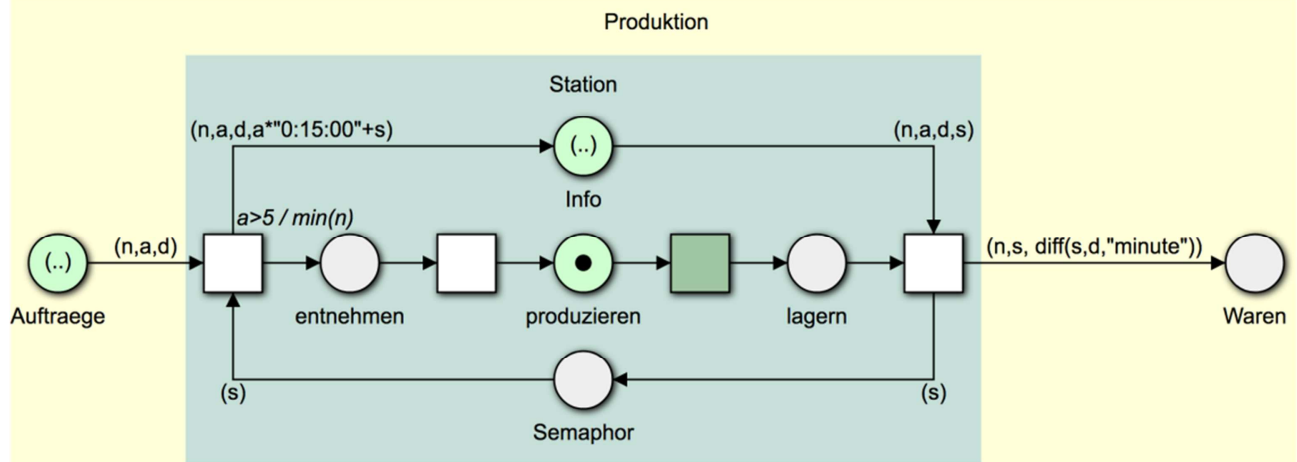

Abbildung 12: Zwischenstand zur Simulation des Netzes aus Abbildung 11

Nach der Produktion werden die Auftragsnummer, der Zeitpunkt der Fertigstellung sowie die Zeit in Minuten, in der der Auftrag auf der Stelle Auftraege gelegen hat, auf die Stelle Waren gespeichert, also (2,"2017.5.21T11:45:00",2835) und (8,"2017.5.21T14:00:00",1455). Durch Aufteilen der Produktion in die drei gezeigten Phasen könnte das Modell leicht erweitert werden, etwa mit Blick auf die verbrauchten Ressourcen oder die Gesamtlaufzeit der modellierten Arbeitsstation.

\section{Fazit und Ausblick}

Was als kleines Projekt gestartet war, um die Ideen der Theory of Constraints zu veranschaulichen, hat sich zwischenzeitlich zu einer Programmierumgebung für Petri-Netze ent- 
wickelt. Dabei ergeben sich gerade durch den Einsatz des Werkzeugs in der Lehre und den Austausch mit den Studierenden zahlreiche Ideen für künftige Weiterentwicklungen:

- Derzeit ist ein erster, einfacher Layout-Algorithmus implementiert. Dieser muss verbessert werden, denn im Augenblick müssen mehr Attribute für das Layout definiert werden, als für die Beschreibung des eigentlichen Problems.

- Schon jetzt lassen sich komplexe Produktionsabläufe darstellen und simulieren. Künftig wird es auch Möglichkeiten zum Import und Export von Daten aus der Umgebung geben, um Produktionsprozesse mit Realdaten simulieren zu können.

- $\quad$ Schließlich ist bereits jetzt die Möglichkeit gegeben, Kopien von bestehenden Netzstrukturen zu erstellen und wiederzuverwenden. Dieser Mechanismus soll nun genutzt werden, um eine Modellbibliothek zu definieren.

\section{Literaturverzeichnis}

[Al2015] Allweyer, T.: BPMN 2.0: Business Model and Notation. 3. Auflage, BOD, Noderstedt, 2015.

[Ba1996] Baumgarten, B.: Petri-Netze. Spektrum Akademischer Verlag, Heidelberg, 1996.

[Be2016] Behnert, T.: Simulator für höhere Petri-Netze. Master-Thesis, Provadis School of Management and Technology, 2016.

[GeLa1981] Genrich, H. J.; Lautenbach, K.: System Modelling with High-Level Petri Nets. Theoretical Computer Science, 13, 1981.

[HaMeNe2015] Hansen, H. R.; Mendling, J.; Neumann, G.: Wirtschaftsinformatik. 11. Auflage, De Gruyter, Oldenbourg, 2015.

[Hev+2004] Hevner, A.R.; March, S. T.; Park, J.; Ram, S.: Design Science in Information Systems Research. MIS Quarterly, Vol. 28(1), S. 75-105, 2004.

[LaSi1999] Lautenbach, K.; Simon, C.: Erweiterte Zeitstempelnetze. Fachberichte Informatik 03-99, Universität Koblenz-Landau, Institut für Informatik, 1999.

[vRBeFi2016] van Randen, H. J.; Bercker, C.; Fieml, J.: Einführung in UML: Analyse und Entwurf von Software, Springer-Vieweg, 2016.

[SiBe2016] Simon, C., Behnert, T.: Petri-Netz-Simulationen zur Theory of Constraints. AKWI Tagungsband 2016, Barton, T., Herrmann, F., Meister, V. G., Müller, C. Seel, C. (Hrsg), S. 78-87, 2016.

[St1969] Stachowiak, H.: Denken und Erkennen im kybernetischen Modell. 2. Auflage, SpringerVerlag, Wien, 1969.

[St2006] Staud, J. L.: Geschäftsprozessanalyse: Ereignisgesteuerte Prozessketten und objektorientierte Geschäftsprozessmodellierung für Betriebswirtschaftliche Standardsoftware. 3. Auflage, Springer, Berlin, 2006.

\section{Kontakt}

Prof. Dr. Carlo Simon

Hochschule Worms

Erenburgerstraße 19, 67549 Worms

T +49 6241 509-369, simon@hs-worms.de 


\title{
Studienbasierte Entwicklung eines Frameworks zur Ableitung von Stra- tegieempfehlungen Digitaler Service Transformationen für produzierende Unternehmen
}

\author{
Holger Klötzner, Martin Przewloka
}

\section{$1 \quad$ Einleitung}

Die Ausstattung und Erweiterung physischer Produkte mit digitalen Dienstleistungen oder die vollständige Umwandlung eines realen Produkts in einen Dienst unter Zuhilfenahme von Informationstechnologien wird als Digitale Service Transformation bezeichnet. Die Musikindustrie ist eine der ersten Branchen, dessen Struktur grundlegend durch diese Entwicklung verändert wurde. Digitale Musikdateien wurden über das Internet distributiert, um diese auf den neu entwickelten transportablen, kompakten Abspielgeräten verfügbar zu machen. Traditionelle Distributionskanäle, deren Geschäftsfeld den physischen Verkauf von Musik (maßgeblich über compact discs) adressierte, gerieten unter signifikanten Marktdruck. Schon wenige Jahre darauf wurden die ersten Streaming-Anbieter (zum Beispiel Spotify) gegründet, welche Musik als monatliches Abonnement vertrieben und damit das auf Einzeltransaktionen und Besitz basierte traditionelle Geschäftsmodell ablösten (vgl. auch [HAR14]). Entscheidend für den Erfolg der Digitalisierung war nicht die Transformation eines vormals analogen Produkts in ein digitales, gleichwertiges Produkt, sondern vielmehr die Schaffung signifikanter Mehrwerte für Anbieter und Kunde als Resultat der Digitalen Transformation ${ }^{2}$.

\section{Ziele der Arbeit}

Die fundamentalen Chancen der Service Transformation werden Unternehmen aller Branchen vor Herausforderungen stellen. Bestehende Geschäftsmodelle und Märkte müssen hinterfragt und schlussendlich ganzheitlich transformiert werden. Der anhaltende Konsolidierungstrend im produzierenden, maßgeblich mittelständischen Gewerbe wurde als ein wesentlicher Anhaltspunkt genommen, um im Rahmen einer Studie diesen Sektor hinsichtlich seiner Fähigkeiten und Bereitschaft zur digitalen Service Transformation zu untersuchen.

Im Rahmen der Fokussierung auf den produzierenden Sektor war es das Ziel der Studie, den Einfluss der Digitalisierungsentwicklung in dieser Branche empirisch zu erforschen und die von den repräsentativ ausgewählten Firmen genutzten Reaktionen, Transformationsansätze und -strategien zu beschreiben ${ }^{3}$. Die gewonnen Erkenntnisse sollten schlussendlich dazu führen, generalisierte Strategieempfehlungen für Unternehmen zu entwickeln, welche sich in verschiedenen Entwicklungsphasen befinden. Diese induktive Vorgehensweise sollte sowohl durch quantitative wie auch qualitative Erhebungsdaten gestützt werden.

\footnotetext{
${ }^{2}$ Beispielhaft sei genannt, dass die Digitalisierung es dem Kunden ermöglicht, an jeder Stelle, zu jeder Zeit, mit jedem beliebigen Abspielgerät, seine bevorzugte Musik abzuspielen.

${ }^{3}$ Bewusst adressieren die Autoren nur ansatzweise die Entwicklungen und Trendaktivitäten unter dem Stichwort „Industrie 4.0“ und lösen sich hiervon deutlich.
} 
Die Service Transformation beschreibt die Erweiterung physischer Produkte durch Dienstleistungen oder deren vollständige Ersetzung ${ }^{4}$. Der Informationstechnologie kommt hierbei eine fundamentale Bedeutung zu, da in vielen Fällen erst durch deren Einsatz dieser Übergang ermöglich wird. Man spricht hierbei auch von der Digitalen Service Transformation. In diesem Veränderungsprozess können disruptive Entwicklungen in Gang gesetzt werden, welche das Potenzial zur grundlegenden Veränderung eines Geschäftsmodells haben.

Im Rahmen der durchgeführten Studie werden drei Basistypen von Services als Resultat einer Transformation unterschieden:

1. Integrated Digital Services (IDS) sind Bestandteil eines hergestellten Produkts und werden den Kunden ohne weitere Kosten zur Verfügung gestellt, obwohl sie durchaus im Gesamtpreis einkalkuliert sein können. IDS sind komplementärer Natur und tendenziell mit der Hauptfunktionalität des Produkts verbunden.

2. Add-On Digital Services (ADS) werden als digitale Erweiterungen für hergestellte Produkte angeboten und separat abgerechnet. ADS sind ebenfalls komplementärer Natur, stellen aber im Gegensatz zu den IDS Funktionalitäten zur Verfügung, die unabhängiger vom Hauptzweck des Produkts sind.

3. Purely Digital Services (PDS) stellen digitale Dienstleistungen zur Verfügung, die unabhängig von dem hergestellten Produkt sind oder dieses substituieren.

Es gibt eine Vielzahl von Studien, die den Stand der Digitalen Transformation in der deutschen Industrie untersucht (vgl. bspw. [IDC17]). Dabei zeigt sich sehr deutlich, dass der Transformationsgrad basierend auf PDS nur zu einem sehr geringen Grad erreicht wird. Nicht zuletzt aus diesem Grund stellen die komplementären Services den größeren Anteil an Service-Typen.

Die folgenden komplementären (IDS/ADS) Service Typen wurden identifiziert:

1. Update Services haben die Funktion, Steuersoftware von Produkten zu aktualisieren.

2. Monitoring Services befähigen den Kunden dazu, bestimmte Aspekte von Produkten zu überwachen, wie beispielsweise deren Lokation (GPS), Effizienzrate, Betriebsstunden oder Wartungszyklen (nicht im Sinne von Predictive Maintenance).

3. Management Services werden in den meisten Fällen zusammen mit Monitoring Services eingesetzt. Ihre Aufgabe ist es, dem Kunden die Kontrolle eines Gerätes zu ermöglichen. Die Kommunikation zum Gerät erfolgt bidirektional.

4. Support Services stellen dem Kunden Informationen und Unterstützung zum Produkt zur Verfügung.

5. Analytics Services befähigen die Benutzer, vom Gerät zur Verfügung gestellte Daten mit Hilfe von vordefinierten oder selbst entwickelten Algorithmen zu analysieren. Das prominenteste Beispiel ist unter dem Namen Predictive Maintenance bekannt (vgl. bspw. [BUS16]).

6. Information Services erweitern die Funktionalität eines Produktes durch die Anreicherung mit nützlichen Daten, wie zum Beispiel Karten für ein Navigationssystem.

7. Upgrade Services haben eine große Schnittmenge mit Update Services, der Fokus liegt jedoch auf der Erweiterung der Funktionalität eines Produktes durch die Implementierung einer neuen Software oder der Aktivierung bestimmter Softwareteile.

\footnotetext{
${ }^{4}$ Im Folgenden werden in Abhängigkeit vom Transformationsgrad die Begriffe komplementär und substituierend gebraucht. In beiden Fällen sprechen wir aber von Service

Transformationen.
} 
8. Customization Services können als die interaktive Teilnahme des Kunden an den Fertigungsschritten des Produktes beschrieben werden. Dies stellt nicht notwendigerweise eine Vorverkaufsaktivität dar, sondern kann beispielsweise in Langzeitkontrakten angewendet werden, um bereits gekaufte Produkte anzupassen.

Des Weiteren wurden die folgenden Service-Typen kategorisiert, die sich zur vollständigen Substitution eines Produkts eignen (PDS):

9. Replacement Services substituieren ein physisches Produkt durch eine digitale Dienstleistung, welche die Kundenbedürfnisse gleichermaßen erfüllt. Die Notwendigkeit für das physische Produkt fällt weg, während die Funktionalität erhalten bleibt.

10. Manufacturing Shift Services sind digitale Dienstleistungen, mit denen der Herstellungsprozess eines Produktes über Technologien wie 3D-Druck zum Kunden hin verlagert werden kann. Das verkaufte Gut ist in diesem Fall die Herstellungslizenz für das Produkt.

11. Related Services haben ihren eigenen Verwendungszweck und erfüllen inn ohne ein physisches Produkt. Trotzdem war zu beobachten, dass in nachgelagerten Prozessen physische Produkte desselben Anbieters involviert sein können. Dies führt zu Synergieeffekten innerhalb des Produktportfolios.

12. Non-related Services haben ebenfalls ihren eigenen Verwendungszweck und erfüllen inn ohne ein physisches Produkt. Im Vergleich zu den Related Services haben sie jedoch keinen Einfluss auf ein physisches Produkt desselben Unternehmens, sondern sind komplett eigenständig.

\section{Empirische Studie und Ergebnisse}

Insgesamt beteiligten sich 129 Personen in relevanten Positionen an der Studie, welche $\mathrm{n}=104$ deutsche Unternehmen mit Niederlassungen in Spanien aus dem produzierenden Gewerbe repräsentierten. Ungefähr jeder fünfte Teilnehmer war Teil der Geschäftsleitung und damit unmittelbar strategieverantwortlich. Das Jahresumsatzspektrum der Unternehmen reichte von 20 Millionen bis 200 Milliarden Euro. 72 \% der teilnehmenden Unternehmen beschäftigten mehr als 1.000 Mitarbeiter. Die in dieser Studie am stärkesten vertretenen Branchen sind Maschinenbau, Metallindustrie, Chemie und Elektrotechnik.

Zur Gewinnung der Erkenntnisse wurden sowohl quantitative als auch qualitative Forschungsmethoden angewendet. Zunächst wurden anhand von drei Experten-interviews erste Erkenntnisse über den Stand und Umfang der Digitalen Service Transformation gewonnen. Anschließend wurde eine Online-Umfrage durchgeführt, mit der die zuvor erlangten Ergebnisse aus den Interviews tiefergehend untersucht wurden.

Neben allgemeinen Unternehmensdaten wurde konkret der Stand zum Einsatz von IDS, ADS und PDS erhoben sowie Details der jeweiligen Implementierungen. Weiterhin wurden die Teilnehmer zu Unternehmensstrategien bezüglich der digitalen Service Transformation sowie zu persönlichen Einschätzungen befragt.

\subsection{Einfluss von komplementären digitalen Dienstleistungen (IDS / ADS)}

Die Resultate zeigen, dass Unternehmen, welche ihre Produkte mit digitalen Dienstleistungen komplementieren, hieraus keine direkten Umsatzvorteile generieren können. Allerdings 
tragen diese Dienste maßgeblich dazu bei, die Verkaufsposition zu verbessern und die Kundenbasis zu verstärken (siehe hierzu ergänzend [CIO16]).

Die Auswirkungen von komplementären digitalen Dienstleistungen auf Schlüsselindikatoren in Unternehmen variierten stark, abhängig vom Typ der Dienstleistung. So konnten die kostenlosen IDS im Schnitt zwar alle Indikatoren mindestens leicht anheben, von einem großen Effekt kann jedoch nicht die Rede sein. Die einzige Ausnahme bildete die Kundenzufriedenheit, die allgemein stark zunahm. Im Gegensatz dazu konnten ADS einige der Indikatoren stark ansteigen lassen. Auch in diesem Fall gab es große Auswirkungen auf die Kundenzufriedenheit, aber auch auf die Profitabilität und den Zuwachs der Kundenbasis (vgl. Abbildung 1).

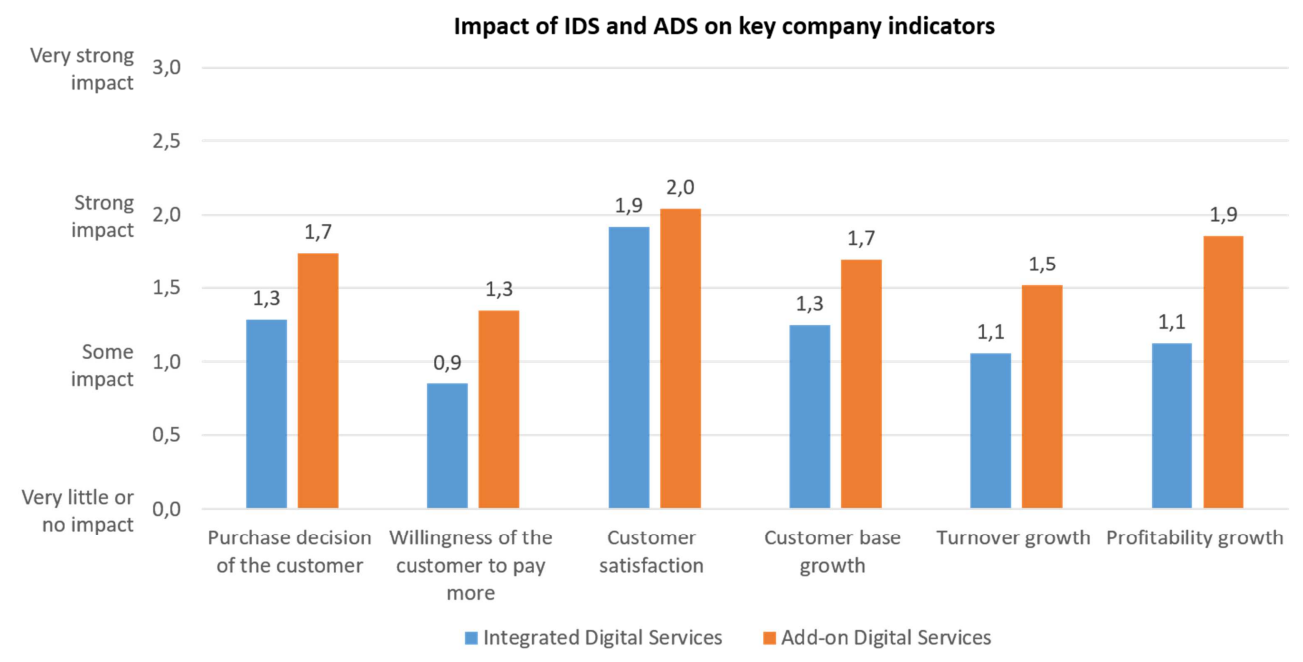

Abbildung 1: Einfluss von Integrated Digital Services (IDS) und Add-on Digital Services (ADS) auf

Schlüsselindikatoren

Einige der Ansätze wurden sowohl als integraler Bestandteil (IDS) als auch als kostenpflichtige Erweiterung angeboten (ADS). Jedoch ist bei den meisten Typen eine eindeutige Verwendungstendenz zu erkennen. So sind beispielsweise Update Services hauptsächlich als IDS implementiert worden, während Analytics Services überwiegend als ADS verfügbar waren (vgl. Tabelle 1).

Service-Typ

Update Services

Monitoring Services

Management Services

Support Services

Analytics Services

Information Services

Upgrade Services

Customization Services

Andere

\section{Implementiert als IDS}

$14 \%$

$15 \%$

$10 \%$

$13 \%$

$1 \%$

$2 \%$

$1 \%$

$0 \%$

$3 \%$

\section{Implementiert als ADS}

$5 \%$

$15 \%$

$7 \%$

$6 \%$

$10 \%$

$3 \%$

$2 \%$

$1 \%$

$0 \%$

Tabelle 1: Typen von komplementierten digitalen Dienstleistungen und deren Zugehörigkeit zu IDS und ADS 


\subsection{Einfluss von substituierenden digitalen Dienstleistungen (PDS)}

Nur $5 \%$ der teilnehmenden Firmen hatten bereits rein digitale Dienstleistungen (PDS) implementiert. Wie bei den Komplementärdiensten IDS und ADS konnte auch durch rein digitale Dienstleistungen die Kundenzufriedenheit signifikant gesteigert werden. Weiterhin wurde ein sehr starker Zuwachs der Kundenbasis beobachtet (vgl. Abbildung 2). Daraus kann die Hypothese abgeleitet werden, dass PDS neue Kundengruppen ansprechen, welche sich überwiegend für ein digitales Angebot interessieren.

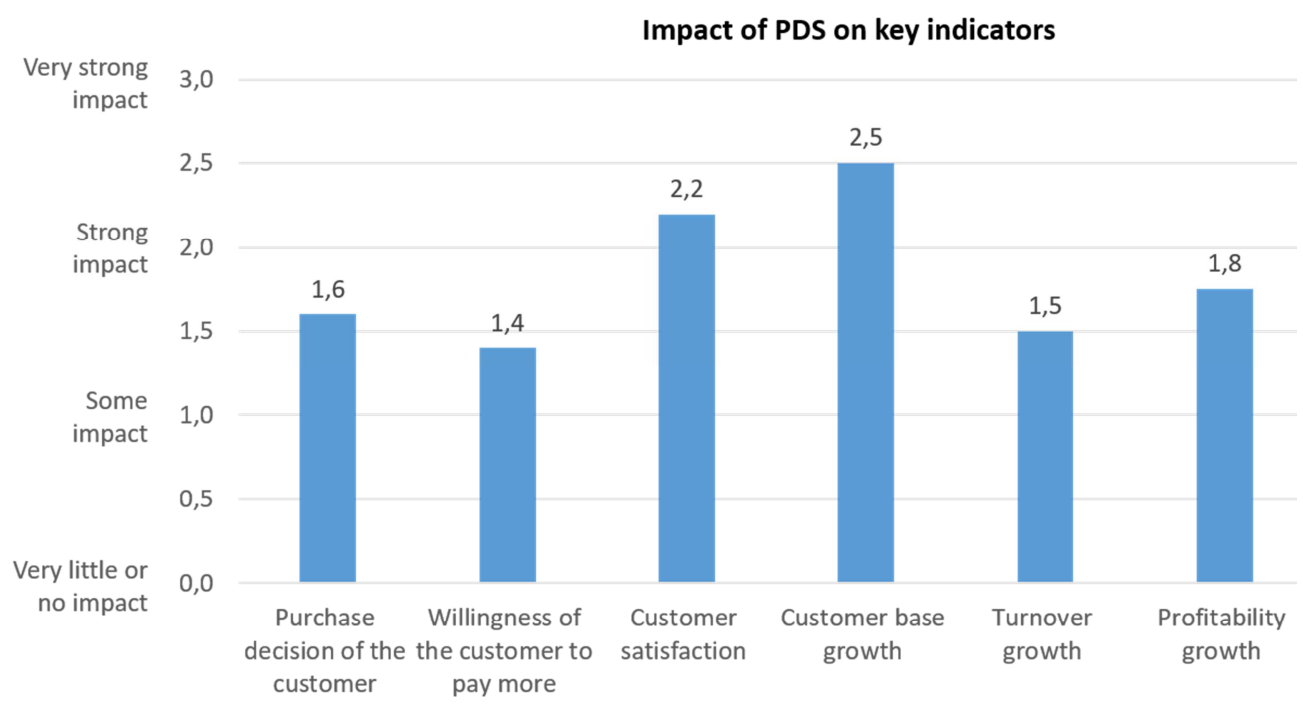

Abbildung 2: Einfluss von Purely Digital Servics (PDS) auf Schlüsselindikatoren

\section{Strategieempfehlungen und Strategieframework}

Um die passenden Ansätze zur Komplementierung oder Substituierung von physischen Produkten finden zu können, sollte im ersten Schritt eine Analyse der Kundenbedürfnisse durchgeführt werden. Dies sollte nach dem Input-Output Prinzip und damit unabhängig von bereits existierenden Produkten geschehen, um Möglichkeiten zur vollständigen Substitution frühzeitig zu erkennen.

Sobald die Kundenbedürfnisse evaluiert sind, können die technischen Möglichkeiten für eine Komplementierung oder Substitution geprüft werden. Dies kann mit Hilfe des „Technology Strategy Frameworks" geschehen, welches auf Basis dieser Forschungsergebnisse entwickelt wurde (vgl. Abbildung 3). Das Framework unterscheidet prinzipiell zwischen der Komplementierung von bereits bestehenden Produkten und substituierenden digitalen Dienstleistungen. Über den Pfad der bestehenden Produkte kann geprüft werden, welche grundlegenden technischen Voraussetzungen für den Einsatz bestimmter digitalen Dienstleistungen erfüllt werden müssen. Des Weiteren wurden die Unternehmen mit Hilfe eines Reifegradmodells in drei Gruppen aufgeteilt, für die verschiedene generalisierte Strategieansätze vorgeschlagen werden. 
Gruppe 1 repräsentiert Unternehmen, die bisher noch keine oder nur sehr oberflächliche Erfahrungen mit digitalen Dienstleistungen gesammelt haben. Sie zeichnen sich durch einen geringen oder keinen Umsatz mit digitalen Dienstleistungen und das Fehlen einer entsprechenden Strategie aus. Die Produkte der Unternehmen besitzen kein Alleinstellungsmerkmal, das von digitalen Diensten geschaffen wird.

Gruppe 2 besteht aus Unternehmen, die sich durch eine ausgeprägte Strategie gegenüber der Digitalen Service Transformation auszeichnen. Weiterhin setzen sie Produkte mit digitalen Diensten ein oder entwickeln diese zurzeit. Es wird davon ausgegangen, dass Unternehmen in dieser Gruppe bereits über fortgeschrittenes Know-How und weitere Ressourcen verfügen, welche im Zusammenhang mit den bisherigen Bestrebungen aufgebaut wurden.

Unternehmen in Gruppe 3 generieren bereits einen wesentlichen Teil ihres Umsatzes mit digitalen Dienstleistungen und verfolgen eine darauf fokussierte Strategie. Die Firmen verfügen weiterhin über erhebliche personelle und technologische Ressourcen in diesem Bereich.

Technology Strategy Framework
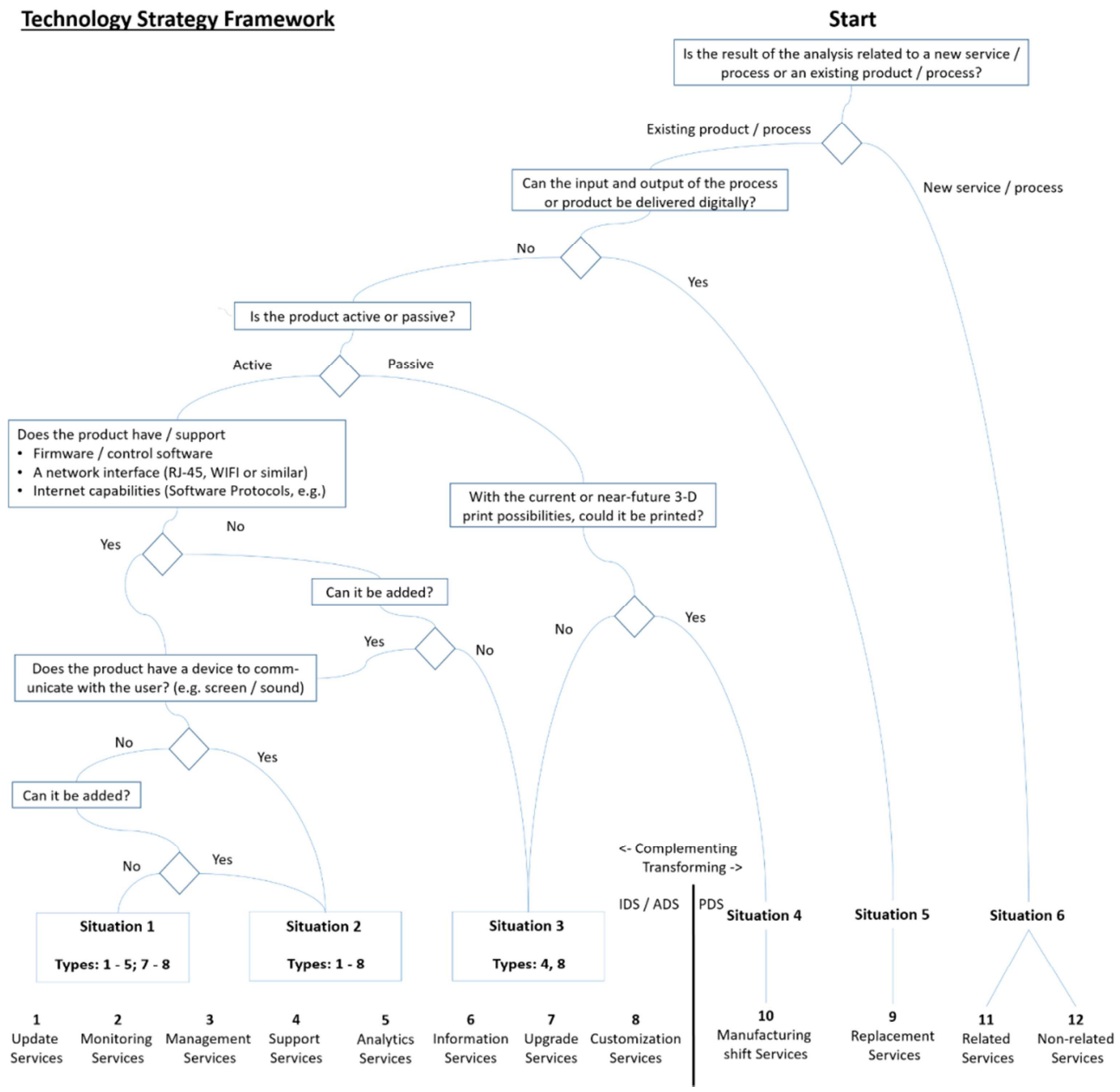

Abbildung 3: Technologie-Framework für die Findung von Komplementierungs- und Substituierungsansätzen 


\subsection{Strategieansatz für non-digitale Unternehmen (Gruppe 1)}

Der vorgeschlagene Strategieansatz für Unternehmen der Gruppe 1 kann als die Evaluierung von Möglichkeiten und Chancen mit Fokus auf die Komplementierung bestehender Produkte zusammengefasst werden. Bestehende Kunden, die bisher nicht mit digitalen Dienstleistungen konfrontiert wurden, sollen durch neue produktbezogene Technologien nicht verunsichert werden. Jedoch muss das Angebot gleichzeitig so offen kommuniziert werden, dass potenzielle Neukunden darauf aufmerksam werden. Der Strategieansatz sollte daher sowohl bestehende als auch potenzielle Kunden sowie die Geschwindigkeit der Veränderung berücksichtigen, die sich auf die Ergänzung bestehender Produkte mit digitalen Diensten ergibt. Darüber hinaus sollten Schlüsselkunden möglichst frühzeitig in den Prozess der Entwicklung neuer Lösungen eingebunden werden.

\subsection{Strategieansatz für fortgeschrittene Unternehmen (Gruppe 2)}

Der Strategieansatz für Unternehmen in Gruppe 2 fokussiert sich auf die Wandlung von vorhandenen digitalen Dienstleistungen in die nächsthöhere Stufe, um die Unabhängigkeit von einem bestimmten Produkt zu erhöhen. Eine größere Unabhängigkeit kann je nach Szenario zu einem besser diversifizierten Preismodell und damit höheren Erlösen, aber auch zur einer größeren Zielgruppe führen. IDS werden dem Kunden ohne sichtbare Mehrkosten angeboten. Die Entwicklung und die Umsetzung dieser Dienstleistungen erfordert jedoch Investitionen in Technologie, Software und Personal. Nützliche Zusatzfunktionen der Produkte sollten frühzeitig kommerzialisiert werden können und entsprechen damit maßgeblich dem Bereich der ADS. Vorhandene ADS sollten nach Möglichkeit gänzlich unabhängig vom Produkt vertrieben werden.

\subsection{Strategieansätze für digital transformierte Unternehmen (Gruppe 3)}

Der vorgeschlagene Strategieansatz für die Gruppe 3 kann als Halten und Ausbauen der Vorreiterposition und die verstärkte Orientierung in Richtung strategischer Allianzen beschrieben werden. Um dieses Ziel zu erreichen, sollten Unternehmen digitale Dienstleistungen zum Teil ihrer Kernstrategie machen, wenn dies nicht bereits der Fall ist. Es sollte weiterhin das Ziel sein, alle Entwicklungen beim Kunden und beim Wettbewerb durchgehend zu analysieren, um die Dienstleistungen anzupassen.

Strategische Allianzen können in sehr wettbewerbsorientierten Märkten eine wichtige Rolle spielen, in der sich Alleinstellungsmerkmale nur kurzzeitig halten lassen. Kunden kaufen nur selten von nur einem Hersteller und kombinieren stattdessen immer häufiger Produkte (vgl. bspw. [JAN16]). Die Schaffung von (servicebasierten) digitalen Plattformen wird sich zu starken und langfristigen Alleinstellungsmerkmalen entwickeln.

\subsection{Positionierung der digitalen Dienstleistungen (alle Gruppen)}

Sobald die Komplementierungs- oder Substituierungsmöglichkeiten ausgewertet worden sind, sollte der Fokus auf den Kunden gelegt werden. Dabei ist insbesondere die Bereitschaft der bestehenden Kunden für einen Wechsel hin zu digitalen Dienstleistungen zu evaluieren, um deren Absprung zu Mitbewerben zu verhindern (vgl. auch [KAN15]). Durch die Ausweitung der Produktpalette um digitale Dienstleistungen können jedoch auch neue Kundengruppen erschlossen werden, was ebenfalls berücksichtigt werden muss. Es ist davon auszugehen, dass die meisten Ansätze zur digitalen Service Transformation keinen Nachteil für den Kunden bedeuten, da sie zusätzliche Funktionen, Mehrwerte und Effizienzsteigerungen ermöglichen (vgl. auch [WEI16]). 


\section{Zusammenfassung und Ausblick}

Die durchgeführte Studie bestätigte eindrucksvoll, dass die Auseinandersetzung mit der Digitalen Servicetransformation in den Führungsetagen der deutschen produzierenden Industrie angekommen ist (siehe hierzu ergänzend [BUN17]). Bereits knapp die Hälfte (49) der 104 befragten Unternehmen setzt bereits auf digitale Dienstleistungen, welche fundamental für die sich ankündigenden Veränderungen sind. Eine noch größere Anzahl (70) hat zugehörige Strategien implementiert. Die Firmen setzen dabei heute noch zum Großteil auf die Komplementierung von Produkten des aktuellen Portfolios. Die Potenziale der Digitalen Transformation können wie in dieser Arbeit dargestellt so noch nicht gehoben werden. Die Studie zeigte aber auch, dass sich in den Unternehmen der nächste Transformationsschritt in Form eines schnellen Anstiegs von Substituierungsbestrebungen abzeichnet. Das Angebot an rein digitalen Dienstleistungen (PDS) wird basierend auf den erarbeiteten Unternehmensstrategien um mehr als das Dreifache zulegen (vgl. auch [HAR16]).

Das auf den Forschungsdaten basierende Strategieframework hilft Unternehmensentscheidern dabei, die richtigen Ansätze für die Transformation der eigenen Produkte zu finden. Noch wichtiger ist es jedoch, stets aus der Kundenperspektive zu denken. Digitale Dienstleistungen können ihr volles Potenzial nur dann entfalten, wenn sie ein für den Kunden brauchbares Ergebnis abliefern und sich möglichst nahtlos in die bestehenden Umgebungen einfügen.

Die digitale Service Transformation hat heute bereits einen großen Einfluss im produzierenden Gewerbe. Um zusätzlich die Transformationsgeschwindigkeit, d.h. die Veränderungen zu messen, bietet es sich an, diese Studie in Abständen von zwei Jahren zu wiederholen. Weiterhin bietet es sich an, die Studie auf weitere Branchen auszuweiten. Die Autoren empfehlen konkret die analoge Untersuchung des Handels, aufgrund seiner großen nationalen, aber auch internationalen Bedeutung.

\section{Literaturverzeichnis}

[BUN17] Bundesministerium für Wirtschaft und Energie: „Digitale Transformation in der Industrie“, bmwi.de (2016)

[BUS16] Businessdictionary.com: „Predictive Maintenance“, Businesdictionary.com (2016)

[CIO16] CIO.de: „Das Wunder der Digitalisierung - von CIO Martin Petry entmystifiziert.”, CIO.de (2016)

[HAR14] HarvardPolitics.com: „A Brave New World: Spotify and the Future of Music”, Harvard Politics (2014)

[HAR16] HBR.org: „German Manufacturing is leading a Digital Industrial Revolution“, Harvard Business Review (2016)

[IDC17] Hoop, L., Thorenz, L-K., Zacher, M.: "Zwischen Tradition und Wandel - Die Digitale Transformation in Deutschland“, IDC White Paper, IDC (2017)

[JAN16] Janrain Inc.: „Identity Trends Across the Web: 2016”, Janrain Inc. (2016)

[KAN15] Kane, Gerald C. et al.: „Strategy, not Technology, Drives Digital Transformation Becoming a digitally mature enterprise", Deloitte University Press (2015) 
[WEI16] Weinelt, B. et al.: "Digital Transformation of Industries: Digital Enterprise", World Economic Forum White Paper, World Economic Forum (2016)

Keywords

Digitale Transformation, Digitale Service Transformation, Digitale Dienstleistungen, Produzierendes Gewerbe, Online-Umfrage, Experteninterviews, Strategieframework, Purely Digital Services

\section{Kontakt}

Holger Kloetzner

GFFT Technologies GmbH

Niddastraße 6, 61118 Bad Vilbel

holger.kloetzner@gfft-technologies.de

Martin Przewloka

Technische Hochschule Mittelhessen

Wiesenstraße 14, 35390 Giessen

martin.przewloka@mnd.thm.de 


\section{Prozessoptimierung in „smarten“ Unternehmen durch Condition Monito- ring}

Frank Morelli, Jan-Felix Mehret, Thorsten Weidt, Lucas Zitterbart

\section{Zusammenfassung}

Ziel des "Condition Monitoring" (CM) ist es, für einen reibungslosen Fertigungsbetrieb durch Zustandsüberwachung zu sorgen. Traditionelle reaktive und proaktive CM-Verfahren greifen dabei zu kurz. Horizontale und vertikale IT-Integration bilden die Basis für zugehörige Prozessoptimierungen durch ein präskriptives und prädiktives Management. Digitale Konzepte der Industrie 4.0 ermöglichen darüber hinaus flexible und innovative Geschäftsprozesse bzw. -modelle.

\section{$1 \quad$ Einsatzpotenziale des Condition Monitoring}

\subsection{Anwendungsmöglichkeiten}

Der Wunsch nach ressourceneffizienteren Verfahren kommt insbesondere in Zusammenhang mit digitalen, disruptiven Technologien auf. Dabei werden in der Unternehmenspraxis sowohl innovative als auch etablierte Methoden berücksichtigt. Ein zukunftsorientierter Ansatz ist das "Condition Monitoring“ (Zustandsüberwachung). Dieses Konzept legt eine regelmäßige, ggf. sogar permanente Erfassung von Anlagen- bzw. Maschinendaten zugrunde. Dies erfolgt durch die Erfassung und Analyse verschiedener Größen wie beispielsweise Schwingung, Temperatur, Stückzahl und Abnutzung.

Die einfachste Anwendung des "Condition Monitoring“ (CM) basiert auf dem reaktiven Prinzip. Dabei ist die Maschine bzw. Anlage so lange produktiv einsetzbar, bis eine Komponente fehlerhaft arbeitet oder beschädigt wird. Diese repariert man dann oder wechselt sie aus. Somit wird nur ex post auf einen eingetretenen Schaden reagiert. Daraus resultieren Produktionsverzögerungen und geringere Ausbringungsmengen, ggf. Lieferverzögerungen und weitere Folgeschäden am Objekt. Als Grund für reaktives Verhalten wird in der Praxis oft der zu geringe Zeitrahmen genannt, um darüber hinausgehende Optimierungen anzugehen. Im Gegensatz zum reaktiven CM steht eine proaktive Vorgehensweise, die auf dem Grundsatz der Vorsorge basiert. Verschleißteile werden im Falle des proaktiven CMs nach Angaben des Herstellers oder der Erfahrung eines Instandhalters oder Werkers ggf. präventiv ausgewechselt. Dadurch soll der außerplanmäßige Stillstand eines Objekts sowie weitere, hieraus entstehende Folgekosten vermieden werden. Diese Methode erweist sich jedoch nur bedingt als ressourceneffizient: So kann z.B. das Auswechslungsintervall einer Komponente erreicht sein, obwohl diese eine hierfür eine zu geringe Abnutzung aufweist. Neben einem zu frühen Austausch von Verschleißteilen kommt es in solchen Fällen zum entbehrlichen Stillstand der Anlage bzw. Maschine.

Für „smarte“ Unternehmen, die auf eine digitale und vernetzte Fertigung im Rahmen von Industrie 4.0 setzen, bestehen über das reaktive und proaktive CM hinaus weitere Verbesserungspotenziale. Diese lassen sich insbesondere durch echtzeitbasierte, präskriptive und ggf. prädiktive IT-Lösungen umsetzen: Ein zugehöriger integrierter und flexibler Ansatz 
vermag es, Probleme und Einschränkungen von reaktivem und proaktivem $\mathrm{CM}$ zu beseitigen, die Ressourceneffizienz zu optimieren und gleichzeitig eine hinreichende Ausfallsicherheit zu gewährleisten. Zusätzlich können auf dieser Basis weitere Gestaltungsmöglichkeiten in den damit verbundenen Prozessen und ggf. auch in den Geschäftsmodellen erschlossen werden.

Durch den Einsatz von CM lassen sich bestimmte operative Aktivitäten im Umfeld von Fehlerentdeckung, Fehleridentifikation, Fehlerdiagnose, Prozess-Wiederherstellung/Intervention, Wartung und Fehlervermeidung bei der Entscheidung unterstützen [LaRP02, S. 14 ff] oder teilweise bzw. vollständig autonom übernehmen. Algorithmen realisieren dabei die repetitive (Routine-)Erfassung und Auswertung in Echtzeit. Ferner können IT-seitig zukünftige Zustände vorhergesagt sowie unterschiedliche Szenarien vorgeschlagen werden. Der Mensch wählt dann das optimale Szenario aus und passt die Parameter in Echtzeit zur Steuerung der Prozesse und zur Effizienzsteigerung sowie zur Zustandsverbesserung der Objekte an. Grundsätzlich ist auch eine durchgängige Steuerung durch CM-Systeme denkbar.

Auf der Ebene strategischer Entscheidungen lässt sich mittels „Smart Data“ eine höhere diagnostische Effektivität erzielen. [LaRP02, S. 11 f.] Hierbei geht es um die Planung, Steuerung und Kontrolle von Lebenszyklen in der Fabrik, die mit der Zustandsüberwachung von Produktionsmitteln und der Wartung verknüpft sind. [Hopp14, S. 250 f.]

\subsection{Chancen und Risiken}

Für jede CM-Implementierung und zugehörige Anwendungsszenarien sind zahlreiche Chancen und Risikopotenziale zu berücksichtigen. Tabelle 1 zeigt eine zugehörige Gegenüberstellung wichtiger Kategorien für ein Anwendungsunternehmen.

Bei der Zustandsüberwachung unterliegen Maschinen bzw. Anlagen generell einer andauernden Abnutzung, bis diese gewartet werden. Die Definition optimaler Wartungsintervalle erweist sich in der Praxis als äußerst komplex. Diese Festlegung bildet jedoch die Grundlage für eine intensive Abstimmung zwischen den Planungs-, Produktions- und Instandhaltungsbereichen im Unternehmen. Suboptimale Lösungen führen unausweichlich zu Konflikten zwischen der Auftragsplanung der Betriebsaufträge und dem damit verbunden Output einerseits und den festgelegten Wartungsintervallen andererseits. Eine Synergie aus den Erkenntnissen von Werkern, Instandhaltern sowie den Vorgaben des Herstellers stellt die zentrale Basis für eine Verbesserung dar.

\section{$2 \quad$ Prozessbasiertes Condition Monitoring}

\section{$2.1 \quad$ Traditionelle Zustandsüberwachung}

Der bestmögliche Zeitpunkt bzw. Zeitraum für durchzuführende Wartungen ist nicht präzise vorhersagbar und basiert bei der traditionellen Vorgehensweise insbesondere auf den Erfahrungswerten und der Einschätzung („Was passiert gerade“) der Instandhaltung. Diese ist für die Analyse des Zustandes und der Wartung eines Objekts verantwortlich. Unterstützt wird die Instandhaltung durch Fehlermeldungen von Werkern. Diese beiden Parteien bzw. Rollen zeichnen für die Wartung des Maschinen- bzw. Anlagenparks zentral verantwortlich, wenn bekannte Charakteristika einer Abnutzung wie beispielsweise Materialermüdungen auftreten. 


\section{Chancen}

- Möglichkeit zur horizontalen Integration prozessbeteiligter Maschinen/ Anlagen über zusätzliche Sensorik/Signale

- Ereignis-basierte Stör- und Fehlermeldungen

- Vertikale Datenintegration mit aggregierten Kennzahlen

- Verbesserte Grundlage für Analysen (z.B. Fehlerdiagnose, Durchlaufzeit-Effizienz)

- Potenzial für automatisiertes Advanced Analytics (prädiktiv und präskriptiv - z.B. im Hinblick auf Fertigstellungs- und Liefertermine)

- Erhöhte Maschinen- und Anlagen- sowie Prozesstransparenz auf der Basis von detaillierten Echtzeitinformationen

- Fundierte („smarte“) Datenbasis für Prozess-Entscheidungen

- Individualisierung steuernder Maßnahmen (Losgröße „1“)

- Selbststeuernde, dezentralisierte Prozesse auf der Basis von Algorithmen / loT und CPS

- Vereinfachung von Inbetriebnahme und Hochlauf/Anlauf von Maschinen/Anlagen (Datenanbindung)

- Verbesserte Möglichkeiten zur Simulation („Digital Twin“)

- Entlastung der Mitarbeiter (Anlagen-/Prozessüberwachung)

- Verbesserung der Entscheidungsgrundlage

- Interaktive, systematische Entscheidungen

- Flexible, rollenübergreifende Kooperation zwischen den Verantwortlichen

- Motivation durch qualitativ anspruchsvollere Aufgaben

Einhaltung von Kundenvorgaben (Normen, Standards)

- Erhöhte Kundenzufriedenheit (Qualität, Liefertreue)

- Einsparungspotenziale/Ressourceneffizienz

- Positive Effekte auf Amortisation und Rentabilität

- Zielgerichtete Optimierung (Order Management, Auslastung)

- Basis für Mehrwert-Services und neue Geschäftsmodelle

\section{Risiken}

- Gefahr von Datenredundanzen und -inkonsistenzen be heterogenen (IT-)Systemlandschaften („Big Data“ anstelle von "Smart Data")

- Interpretationsfehler durch fehlerhafte Signalanbindung ode durch unvollständige Algorithmen

- Verarbeitung personenbezogener Prozessdaten (Datenschutz)

- Hoher Aufwand durch eine detaillierte Vergabe von Zugriffsrechten

- Erhöhte IT-Systemabhängigkeit (z.B. Daten- und ProzessSynchronisation)

- Fehlerhafte automatisierte Diagnosen und/oder Entscheidungen aufgrund der Komplexität/Dynamik von Abläufen und Zusammenhängen

- Erhöhtes Risiko durch fehlerhafte Entscheidungen, welche die gesamte Prozesskette betreffen

- Überforderung der Benutzer bei der Mensch-MaschineKooperation und/oder der interaktiven Analyse

- Furcht vor Arbeitsplatzabbau durch „automatisierte“ Zustandsüberwachung

- Fehlende Akzeptanz durch potenzielle Leistungsüberwachung

- Ineffiziente Nutzung rollenübergreifender und erfahrungsbasierter Potenziale durch mangelnde Anpassung der Verantwortlichkeiten

- Gefahr durch Cyber-Angriffe

- Hohe Investitionserfordernisse in Hardware, Software und Netzwerke oder in Cloud-basierte Services

Tabelle 1: Chancen und Risiken des Condition-Monitoring-Einsatzes

Geringfügige Wartungen und Anpassungen an Maschinen bzw. Anlagen werden typischerweise manuell durch den jeweils zuständigen Werker durchgeführt. Der Erfolg der traditionellen Zustandsüberwachung hängt hierbei insbesondere von dessen Kompetenz und Erfahrungsspektrum ab. Im positiven Fall zieht dies einen Lernprozess mit einem reaktiven Diagnosecharakter („Was ist gerade passiert?“) nach sich. Dadurch lernt der Werker und kann sein Know-how weiter ausbauen.

Die Instandhaltung konzentriert sich bei der Zustandsanalyse i.d.R. auf eine Verflechtung von Erfahrungswerten in Verbindung mit punktuellen Stichproben. Sie stützt ihre Entscheidung im Hinblick auf das anzusetzende Wartungsintervall bzw. die Wartungsnotwendigkeit auf vorangegangene Analysen. Falls der optimale Abnutzungsgrad nicht exakt getroffen wird, resultieren daraus zumeist vermeidbare Wartungen bzw. eine zu geringe Laufzeit der Maschine/Anlage. Diese sind verbunden mit häufigeren Stillständen, die wiederum einen geringeren Fertigungsoutput nach sich ziehen. Weitere Umweltgegebenheiten wie beispielsweise Hitze oder Vibrationen können ebenfalls zu Abweichungen des optimal geplanten Wartungsintervalls führen.

Erfahrungswerte, welche die Werker und die Instandhaltung sich im Laufe der Zeit aneignen, sind nicht ohne weitere Maßnahmen auf anderes Personal transferierbar. Für den Fall der Nichtverfügbarkeit bzw. des Ausscheidens aus dem Unternehmen steht dieses Knowhow nicht (mehr) zur Verfügung, was eine Zustandsüberwachung durch Erfahrungswerte problematisiert. Ggf. können Unternehmen hierbei auf andere Teilbereiche/Werke zurückgreifen oder müssen den Herstellervorgaben für das zugehörige Wartungsintervall bedingungslos folgen. 
Zielsetzung einer systematischen Zustandsüberwachung im Unternehmen ist es, Werker, Instandhalter und weitere beteiligte Bereiche (z.B. Qualitätssicherung, Vertrieb, Einkauf und Personalmanagement) zu entlasten und als mögliche Fehlerquelle auszuschließen. Möglichkeiten zur Optimierung ergeben sich durch eine Orientierung am ITIL-Ansatz im Bereich Service Operation bzw. an der ISO/IEC 20000-1 innerhalb der "Resolution Processes“. Hierbei wird zwischen Incident- und Problem-Management differenziert:

- $\quad$ Ein „Incident“ charakterisiert eine ungeplante Unterbrechung bzw. Qualitätsminderung oder ein Ereignis, das in der Zukunft eine Beeinträchtigung bewirken könnte. Aufgabe des Incident-Managements ist eine Störungsbeseitigung in vorgegebener Zeit bzw. so schnell wie möglich. Es verwaltet alle Incidents über ihren gesamten Lebenszyklus.

- Der Terminus „Problem“ repräsentiert die Ursache für einen oder mehrere Incidents. Ein zugehöriger Prozess sorgt systematisch für vorbeugende Maßnahmen, um Incidents zu verhindern oder - falls dies nicht möglich ist - minimal zu halten. Das ITILProblem-Management umfasst dabei mehrere Teilprozesse: Proaktive Problemidentifikation, Problemkategorisierung und -priorisierung, Problemdiagnose und -lösung, Problem- und Errorüberwachung, Problemabschluss und -auswertung, einen Major Problem Review sowie ein Problem Management Reporting.

Das Unternehmen verfügt bei der traditionellen Zustandsüberwachung grundsätzlich über keine weiteren Indikatoren für den Abnutzungsgrad oder zu erwartende Ausfälle. Dies führt ggf. zu Komplikationen in der Produktion und Planung. Ferner kann der ungeplante Stillstand einer Maschine oder Anlage weitere Schäden an den Objekten verursachen. Daher erweist es sich für Unternehmen als komplexe Aufgabe, innerhalb dieses Rahmens eine Methodik zu implementieren, die näher an den optimalen Abnutzungsgrad eines Objekts heranführt. Hilfreich ist die Integration einer vertikalen und horizontalen IT-Landschaft, bei der die Informationstechnik die Betroffenen unterstützt. Dieser Ansatz wird im nachfolgenden Kapitel beschrieben.

\subsection{Optimierung durch zunehmende IT-Unterstützung}

Im Vergleich zur personenzentrierten, traditionellen Zustandsüberwachung mit gering vernetzten IT-Systemen haben in den vergangenen Jahren die Hersteller von Maschinen und Anlagen ihre Produkte mit zusätzlicher Sensorik ausgestattet. Hierbei ist eine dynamische Weiterentwicklung hinsichtlich Baugröße, Funktionalität und möglicher Anbindung/Vernetzung zu konstatieren. Standardisierungsinitiativen im Bereich der Schnittstellen haben die Möglichkeit zur Anbindung an „Manufacturing Execution"-Systeme (MES) deutlich verbessert.

Unter MES lassen sich IT-basierte Fertigungsleitsysteme verstehen, die Betriebsaufträge und Fertigungsressourcen verwalten. [Klet15, S. 20] Sie integrieren ggf. weitere Module wie das Qualitäts- und Personalmanagement und lassen sich an ERP- oder vergleichbare Systeme anbinden. Die MES-Entwicklung hat in der jüngeren Vergangenheit eine starke funktionale Verbesserung erfahren und die Integration fertigungsbeteiligter Bereiche ausgeweitet. Die unternehmens- und werksspezifische Konfiguration von MES-Komponenten wurden ebenfalls erweitert.

Diese Entwicklung und weitere digitale technologische Impulse ermöglichen es, verstärkt CM-Funktionalitäten einzusetzen: Durch Fernzugriffe auf die Maschinensteuerungen bzw. Steuerungsoberflächen (z.B. Mirroring), durch Parametrierung per Remote-Zugriff und durch zunehmende Vernetzung der produktionsnahen IT-Systeme können eigene oder an der 
Maschinen- und Anlagenüberwachung beteiligte externe Mitarbeiter in Echtzeit eine proaktive Zustandsüberwachung betreiben.

Das folgende Beispiel dient der Veranschaulichung aktueller Möglichkeiten des CM: In einem kunststoffverarbeitenden Betrieb werden mehrere Kunststoff-Spritzguss-Maschinen über ein MES bzw. Fertigungsmanagementsystem betrieben. Der nachfolgende Hallenspiegel (Abb. 1) zeigt alle Maschinen innerhalb einer ausgewählten Werkhalle mit ihrem Status, der durch die zugehörige Einfärbung (grün = läuft, rot =steht, gelb = Einfahrmodus) dargestellt wird. Die Anzeige von Ereignissen wie beispielsweise anstehende Maschinen- und Komponentenwartungen, Aktionen zur Qualitätssicherung und Grenzwertverletzungen erfolgt in Echtzeit durch das Einblenden von Symbolen.

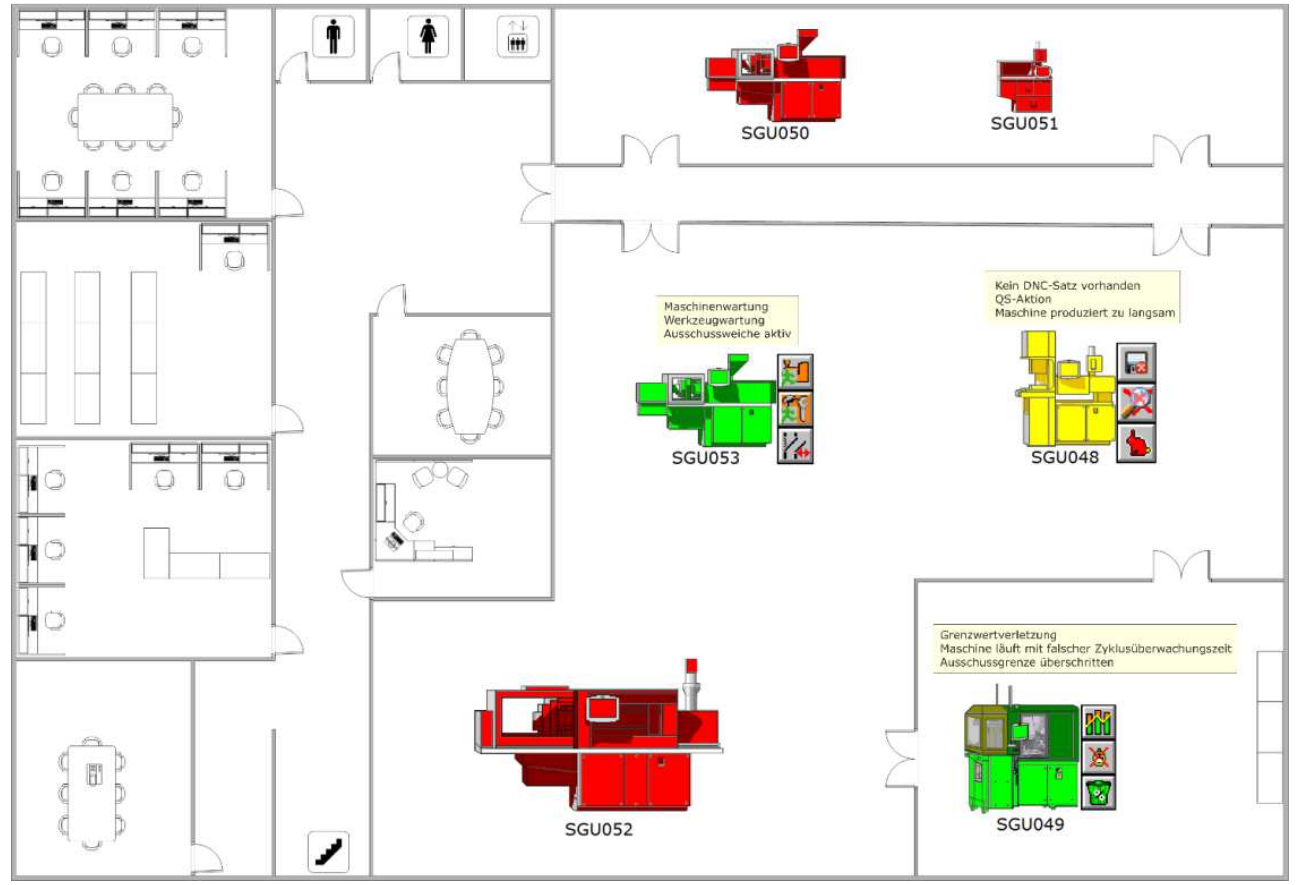

Abbildung 1: Hallenspiegel (Beispiel)

Im Rahmen der Betriebsauftragsplanung ist es über eine grafische Benutzungsoberfläche möglich, den Zustand mehrerer Maschinen mit ihren jeweiligen Betriebsaufträgen zu überwachen: Auf der linken Seite der nachfolgend dargestellten grafischen Planungsoberfläche (Abb. 2) werden die bereits zuvor ausgewählten Maschinen der Werkhalle mit ihrer Auslastung und ihrem aktuellen Status angezeigt. Die jeweils eingeblendete Prozentzahl unterhalb der Maschinennummer stellt die aktuelle Auslastung, d.h. das Verhältnis von Fertigungsstunden zu Kapazität innerhalb des Ansichtszeitraums, dar. Die hellblauen Spalten im Auftragsbereich zeigen Produktionszeiten und die dunkelblauen produktionsfreie Zeiten an. Die horizontalen Balken innerhalb des Betriebsauftragsbereichs repräsentieren die einzelnen geplanten Betriebsaufträge. Der hellgrüne Teil eines Betriebsauftragsbalkens gibt an, dass das Ende der Produktion voraussichtlich innerhalb der geplanten Frist erreicht wird. Dunkelgraue Balken beziehen sich auf die jeweilige Rüstzeit (Auf-/Abrüstzeit) der Maschine. Der gelbe Teil markiert, dass die Produktion des Betriebsauftrags voraussichtlich genau bis zum Solltermin fertiggestellt sein wird. Ein rot eingefärbter Teil eines Balkens signalisiert, dass 
der Betriebsauftrag über den geplanten Fertigstellungstermin hinausreicht. Ein lilafarbenes Rechteck oberhalb des Betriebsauftragsbalkens gibt an, dass Werkzeugüberlappungen existieren. Das orangene Quadrat innerhalb des Betriebsauftragsbalkens zeigt an, dass eine Maschinenwartung ansteht, wohingegen das grüne Quadrat für Werkzeugwartung steht.

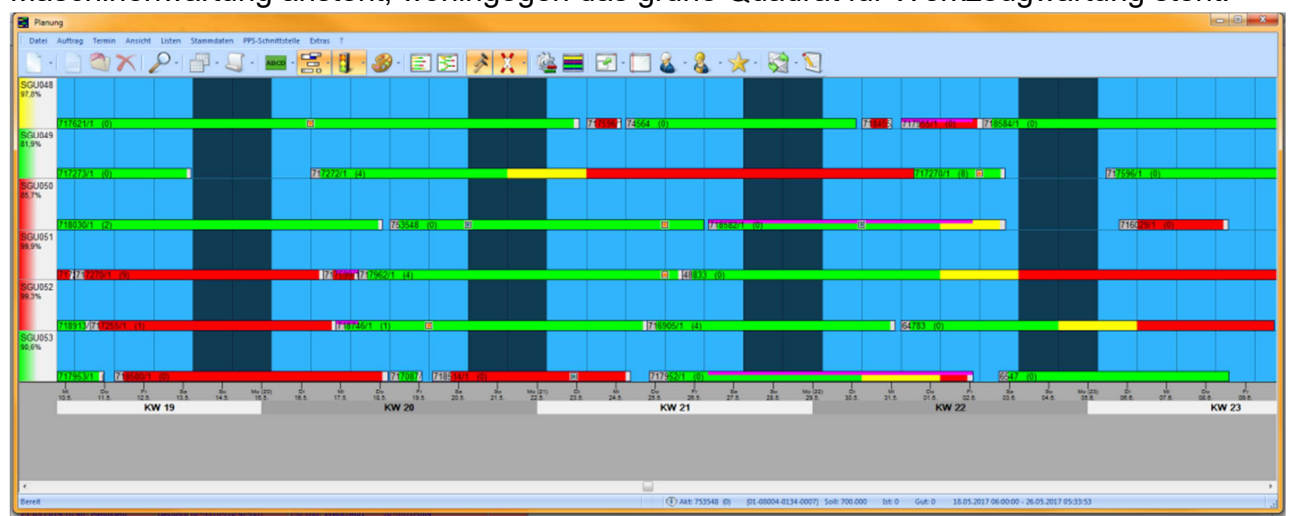

Abbildung 2: Grafische Planungsoberfläche (Beispiel)

Innerhalb der Betriebsauftragsplanung herrscht ein großes Spektrum an Optimierungsmöglichkeiten. Bei der traditionellen Zustandsüberwachung werden die Aufträge oft noch händisch berechnet und auf einer Tafel grafisch eingetragen. Abstimmungen zwischen den Abteilungen Planung, Produktion und Instandhaltung finden oft nur in begrenztem Umfang statt. Im Falle einer sich ständig ändernden Auftragslage unter Berücksichtigung von unvorhergesehenen Instandhaltungsaufgaben wird dieser Ansatz den Erfordernissen nicht gerecht: Als Konsequenz ergibt sich eine verzögerte Abarbeitung der Betriebsaufträge und ggf. gerät die Produktion ins Stocken. Der nachfolgende Use Case (siehe hierzu das BPMNDiagramm in Abb. 3) soll das Potenzial einer koordinierten Abstimmung aufzeigen. Er beschreibt die Betriebsauftragsplanung im Zusammenwirken mit der Instandhaltung für das Handling von Werkzeugen.

Das CM vermag es, bei der integrierten Planung unterstützend einzugreifen und eine Optimierung zu ermöglichen. [Klet15, S. 104] Nachdem Betriebsaufträge entweder manuell generiert oder vom führenden ERP-System übergeben wurden, lässt sich die Feinplanung der Betriebsaufträge durchführen. Diese findet meist auf einer grafischen Oberfläche statt, die alle Maschinen mit den jeweiligen Betriebsaufträgen über einen gewählten Zeitraum darstellt. Die Betriebsauftragsdauer wird in Echtzeit auf Grundlage der erfassten Sensordaten innerhalb der jeweiligen Maschinen automatisch berechnet. Zusätzlich erhält die Planungsabteilung bzw. die Arbeitsvorbereitung Daten über bestehende Ressourcenengpässe, Werkzeugsperren etc. In Kombination mit den geführten Stammdaten ist ein optimiertes Management möglich, um systemunterstützt Durchlaufzeiten, Lagerbestände und Rüstzeiten zu verringern. Somit lässt sich der Fertigstellungstermin des jeweiligen Betriebsauftrags genauer bestimmen und die Kapazitätsauslastung unter Berücksichtigung der erforderlichen Instandhaltungsaufgaben erhöhen.

Parallel zur grafischen Planung anhand der Betriebsauftragsbalken im Rahmen der Arbeitsvorbereitung werden anstehende Wartungen in einem separaten System geplant. Hierbei stehen kleine und große Wartungen sowie Reinigungen und Reparaturen im Vordergrund. Durch die horizontale Integration bzw. Synchronisierung der Daten zwischen den Systemen 
lassen sich die Aktivitäten aufeinander abstimmen bzw. harmonisieren. Bei einer traditionellen Zustandsüberwachung würde dies typischerweise nicht erfolgen.

Die Systemintegration führt zu einer stärkeren Vernetzung und intensiveren Kooperation: So erhält die Abteilung Planung bei erforderlichen Umplanungsaufgaben für einen Betriebsauftrag automatisch eine Nachricht, wenn es zu Verfügbarkeitskonflikten kommt. Dies ist beispielsweise der Fall, wenn das Werkzeug innerhalb des angedachten Zeitraumes durch eine Wartung gesperrt ist. Handelt es sich um einen höher priorisierten Betriebsauftrag, kann die Abteilung Planung sofort die Abteilung Instandhaltung kontaktieren, um die Wartung kurzfristig zu verschieben. Bei unvorhergesehenen Reparaturen erhält die Abteilung Planung in Echtzeit Kenntnis davon, damit notwendige Veränderungen ggf. unmittelbar vorgenommen werden können.

Das geschilderte Beispiel veranschaulicht, dass mit CM eine gleichzeitige Planung, Steuerung und Überwachung des Zustands zahlreicher Maschinen auf Basis von Echtzeitdaten möglich ist. Allerdings erfolgen die zugehörigen Managementaktivitäten derzeit nach wie vor stark menschen- bzw. rollenzentriert. Zudem werden zwar zahlreiche Kenngrößen in Bezug auf Grenzwertüber- bzw. -unterschreitungen überwacht, allerdings erfolgt keine (teil-) automatisierte Optimierung des Betriebszustands. Hierin liegt ein wichtiges Potenzial für die Ausweitung der Zustandsüberwachung zu einem zukunftsorientierten CM.

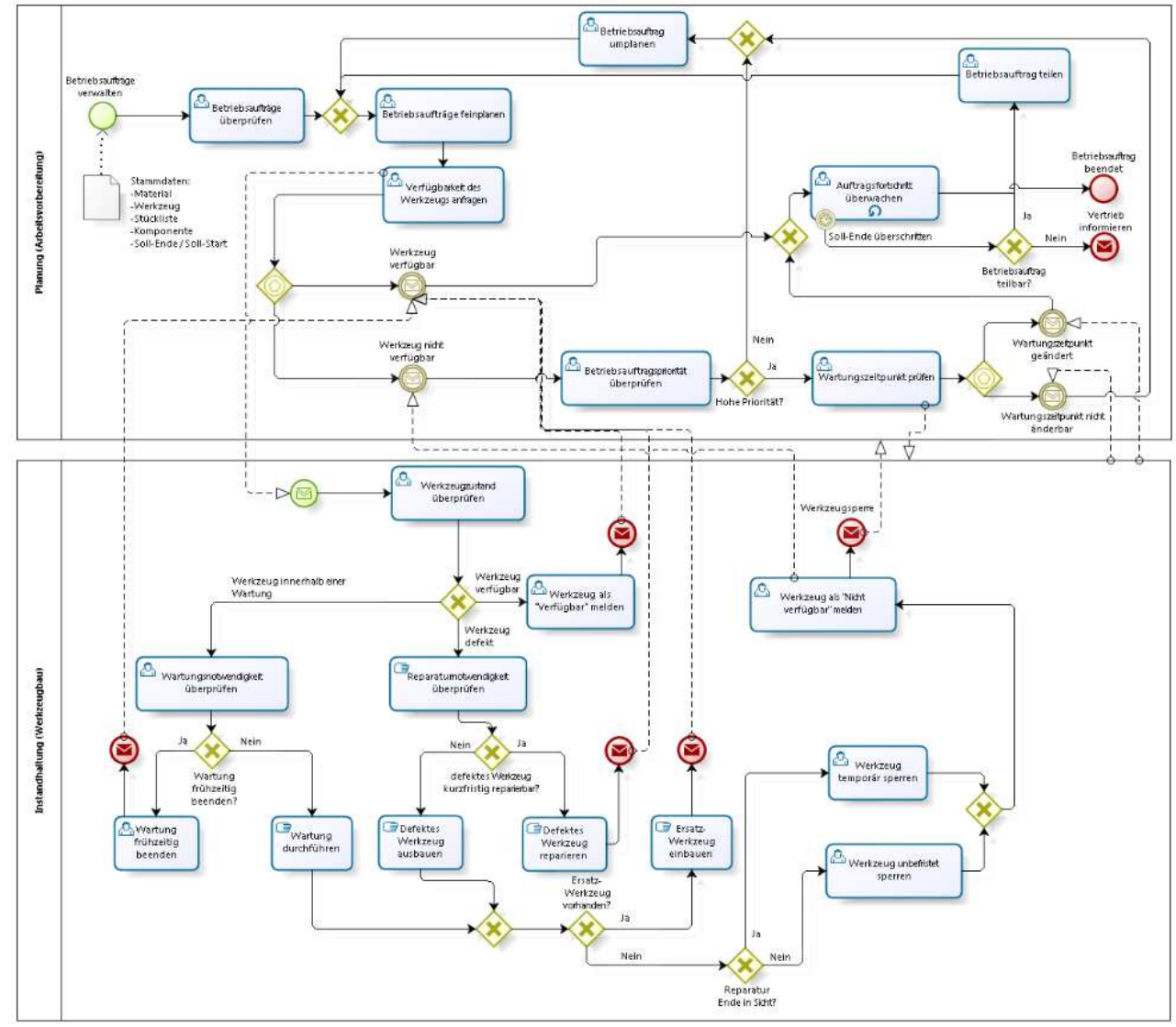

Abbildung 3: Koordination von Planungs- und Instandhaltungsaktivitäten (Beispiel) 


\subsection{Zukunftsorientierte Gestaltungspotenziale}

Geht das konventionelle Condition Monitoring von deskriptiver Analyse („Was ist passiert?") und Echtzeitdiagnose („Warum ist es passiert?") aus, ermöglicht ein erweitertes Verständnis die Einbeziehung von prädiktiven und präskriptiven Fragestellungen. Weiterhin erweist sich die Ausgestaltung von Geschäftsregeln („Business Rules“) zur Unterstützung von Echtzeitentscheidungen („Real Time Decisioning“) als richtungsweisende Option zur optimierten Interaktion zwischen Mensch und Maschine. Personell erfordert dies eine hohe Qualifikation in der strategischen Planung und der operativen Steuerung der Abläufe sowie eine durchgängige und kongruente Zuweisung von Verantwortung. In Abhängigkeit von der Komplexität und Tragweite lassen sich in diesem Kontext aber auch bestimmte Bereiche automatisieren.

Prädiktive Analyse („Was wird passieren bzw. was könnte geschehen?“) erfolgt auf der Basis eines geeigneten mathematischen Modells, um zukunftsrelevante Vorhersagen treffen zu können. Aus quantitativen Datensätzen werden Muster und Trends mit Hilfe induktiver statistischer Methoden generiert. Im Fokus steht dabei die Analyse der Beziehungen zwischen bereits bekannten und vorhergesagten Variablen mit Hilfe von vergangenen Ereignissen. Das Ergebnis wird dann für die Prognose verwendet. Entsprechend haben die Qualität der Datenanalyse sowie die Auswahl der getroffenen Prämissen direkte Auswirkungen auf die Genauigkeit und den Nutzen einer Vorhersage.

Barrieren für die Nutzung von prädiktiven Analysen bestehen u.a. durch folgende Gegebenheiten: [Ecke07, S. 20]

- Inhaltliche Komplexität: Die Entwicklung von realitätsnahen Modellen erweist sich typischerweise als langsamer, iterativer und arbeitsintensiver Prozess.

- Datenqualität: Fehlerhafte oder nicht verfügbare Daten erweisen sich als zentrales Problem für echtzeitbasierte Entscheidungen.

- IT-Performance: Komplexe analytische Abfragen und Auswertungen können sich negativ auf die Netzwerk- und Datenbankleistung auswirken. Dabei spielen auch Schnittstellen zwischen unterschiedlichen IT-Systemen eine wichtige Rolle.

- Gesamtkosten: Es sind direkte und indirekte Kosten zu berücksichtigen, sowohl im personellen Bereich als auch innerhalb der IT.

Prognosen liefern begründete Aussagen über Ereignisse, Zustände oder Entwicklungen in der Zukunft. Dies kann beispielsweise in Form einer Simulation mit „What-If“- bzw. „How-toachieve“-Fragen erfolgen. Projektionen lassen sich dadurch charakterisieren, dass sie nicht ausschließlich auf Vergangenheitsdaten basieren, sondern auch subjektive Einschätzungen mitverwenden.

Eng verbunden mit prädiktiver Analyse ist der Begriff des Data Mining. Hierunter versteht man die systematische Anwendung von deskriptiven Verfahren oder induktiven Testmethoden auf einen Datenbestand, um neue Muster zu erkennen. Bei der Mustererkennung geht es primär darum, in einer Menge von Daten Regelmäßigkeiten, Wiederholungen, Ähnlichkeiten oder Gesetzmäßigkeiten zu erkennen.

Präskriptive Modelle bzw. Analysen („Was soll passieren?") zielen demgegenüber darauf $a b$, durch logische Durchdringung der mit einer Entscheidungsfindung verbundenen Implikationen Orientierungshilfen für die praktische Abwicklung zu vermitteln. Sie sollen Empfehlungen generieren, die ggf. vorhergesagte Ereignisse verändern, unterbinden oder in eine gewünschte Richtung lenken. Entsprechende entscheidungslogische Analysen werden typischerweise durch Entscheidungsmodelle vorgenommen. Ein solches Modell beschreibt typischerweise folgende Faktoren: 
- $\quad$ Eine definierte Menge an Handlungs- bzw. Entscheidungsmöglichkeiten

- $\quad$ Eine Zielvorgabe oder Kennzahlen zur Erfolgsmessung

- $\quad$ Eine Menge an Regeln, die definieren, welche Auswahl erlaubt ist.

Entsprechende Modelle eignen sich für das „Real Time Decisioning“, welches einen analytischen Prozess charakterisiert: [Pani07, S. 29] Ziel hierbei ist es zum einen, eingetretene Ereignisse zu analysieren und daraus Handlungen vorzuschlagen, die das vorab definierte Ziel mit höchstmöglicher Wahrscheinlichkeit erreicht. Zugehörige Zwischen- und Endergebnisse werden systematisch erfasst und analysiert, um zukünftige Empfehlungen zu verbessern. IT-seitig lassen sich dafür „Decision-Management"-Systeme einsetzen. Ein Anwendungsgebiet in diesem Bereich sind sogenannte Geschäftsregeln („Business Rules“), die aus einer Sammlung von Wenn-Dann-Bedingungen bestehen. Diese überprüfen Attribute auf die jeweiligen Werte und führen entsprechende Aktionen aus, wenn die Bedingungen erfüllt sind.

In diesem Kontext lässt sich auch maschinelles Lernen („Machine Learning“) einsetzen: Ziel dabei ist es, Verfahren anzuwenden, durch die IT-Systeme befähigt werden, eigenständig Wissen aufzunehmen und zu erweitern. Die eingesetzten Algorithmen lernen aus Trainingsdaten bzw. aus Testfällen der Vergangenheit, um korrekte Hypothesen zu finden und damit einen Transfer auf neue Datenkonstellationen leisten zu können. Dies kann durch überwachtes, teilüberwachtes, unüberwachtes oder bestärkendes Lernen erfolgen. Ferner gibt es die Möglichkeit eines aktiven Lernens, bei dem der Algorithmus auf der Basis vorgegebener Eingabewerte Fragen nach korrekten Ausgaben generiert. Maschinelles Lernen eignet sich typischerweise für unbekannte, schwer beschreibbare oder schwer berechenbare Probleme.

Ein Beispiel für zukunftsorientiertes Condition Monitoring auf Basis digitaler Vernetzung liefert der Bereich Instandhaltung, beispielsweise durch Fernüberwachung und -wartung. Instandhaltung und Wartung gewinnen im Kontext von Industrie 4.0, „Internet of Things (loT)" und dem Einsatz von "Cyber-physical Systems (CPS)“ zunehmend zur Optimierung der Gesamtkosten an Bedeutung. Mit Industrie 4.0 wird die Individualisierung bzw. Hybridisierung von Produkten sowie die Integration von Kunden und Geschäftspartnern in die Geschäftsprozesse angesprochen. loT lässt sich in diesem Zusammenhang als eine dynamische, globale Netzwerkinfrastruktur charakterisieren, die auf standardisierten, interoperablen Kommunikationsprotokollen basiert und Fähigkeiten zur Selbstkonfiguration besitzt. Die eingesetzten „Dinge“ besitzen dabei eindeutige Kennungen sowie Attribute und „intelligente“ Schnittstellen. CPS bezeichnen integrierte Computersysteme und -netzwerke in Verbindung mit einem oder mehreren physischen Prozessen, in denen der Informationsaustausch stattfindet. Die Steuerung erfolgt in diesem Zusammenhang dezentral und ist durch Teilautonomie und -automatisierung geprägt.

Das zukunftsorientierte Konzept bewegt sich weg von ausfall- und zeitabhängiger Wartung, hin zu einer zustandsorientierten Instandhaltung. Hierbei spielen neben der eigentlichen Produktion, die sich in vielen Branchen auf eine Losgröße „1“ zubewegt, zunehmend auch vernetzte Logistikprozesse eine zentrale Rolle für den Wertschöpfungsprozess. Im Falle von Störungen bedeutet dies die Notwendigkeit eines echtzeitnahen Umplanens. Zielsetzung ist die Optimierung von Aufwand, Materialeinsatz, Lebensdauer und Zuverlässigkeit des Gesamtsystems.

Für eine prognostische Wartung müssen Daten über in der Vergangenheit aufgetretene Störungen systematisch gesammelt, analysiert und mit historischen Messwerten von Senso- 
ren korreliert werden. IT-seitig sind hierzu mehrere Komponenten erforderlich, wie z.B. [SASW17, S.13]

- Integrierte Daten in hinreichender Qualität: Messeinrichtungen, Überwachungs- und Kontrollsysteme müssen zu einem Gesamtbild der Maschinen- und Anlagenleistung sowie der logistischen Prozesse konsolidiert werden. Hierzu zählen u.a. Auftragsdaten, Wartungspläne, Normen und gesetzliche Vorschriften sowie die Verfügbarkeit personeller und materieller Ressourcen.

- $\quad$ Automatisches Monitoring und eventbasierte Signale: Die permanente Überwachung in Echtzeit weist in Kombination mit der Auswertung des historischen Datenbestands auf mögliche Problemzustände bzw. Wartungsfälle hin und löst beim Überschreiten von Schwellenwerten einen Alarm aus. Die Erkennung von Fehlermustern kann mit einer automatischen Erzeugung und Einplanung eines Instandhaltungsauftrags bzw. einer Instandhaltungsmaßnahme gekoppelt werden.

- web- und kennzahlenbasierte Dashboards, Analysewerkzeuge und Augmented Reality: Diese fungieren als Grundlage für eine mobile und flexible Mensch-Maschine-Interaktion. Betroffen auf menschlicher Seite sind zusätzlich zu den Werkern, der Instandhaltung und der Planung bzw. Arbeitsvorbereitung beispielsweise der Kundendienst, der Ersatzteilvertrieb und die Produktionsmittelplanung. Entsprechend erweisen sich neben Telemetriedaten auch betriebswirtschaftliche Informationen wie z.B. Garantieleistungen und ggf. weitere Daten und Dienste (beispielsweise Wettervorhersagen) als relevant. In Abhängigkeit von den Stakeholdern sind unterschiedliche Cockpits bzw. Lösungen zu designen.

- Dynamische Modellentwicklung: Die automatische Erkennung von Fehlermustern und die Ableitung zugehöriger Toleranzintervalle muss im zeitlichen Verlauf überprüft und ggf. optimiert werden.

Die Etablierung eines zukunftsorientierten CM erfordert entsprechend Maßnahmen zur Optimierung der Datenbasis, zur Anbindung und Vernetzung der technischen Komponenten sowie zur Verbesserung der unterstützenden Analysetools. Damit ist eine vertikale IT-Integration zu bewerkstelligen, von der Feldebene mit CPS bis hin zu Management-Informationssystemen. Darüber hinaus muss eine organisatorische Anpassung der mitwirkenden Abteilungen an die betrieblichen Prozesse erfolgen. Das Konzept ist durch entsprechende Governance-Maßnahmen im Sinne der Übertragung von Verantwortlichkeit auf das Top Management zu flankieren.

\section{3 „Smartes“ Condition-Monitoring-Management}

Unternehmen, die Ansätze wie Lean Management erfolgreich implementiert haben und sich auf dem Weg in Richtung Industrie 4.0 befinden, können ihre Geschäftsprozesse durch ein IT-basiertes CM erweitern und daraus zusätzliche Services ableiten. Eine zentrale Herausforderung besteht darin, den „Markt der technischen Möglichkeiten“ aktiv zu beobachten und sowohl den technischen als auch den wirtschaftlichen Reifegrad der jeweiligen Leistungen zu bewerten.

Für „smarte“ Fabriken bietet sich die Chance, bisherige Systematiken zur Zustandsüberwachung von Technikkomponenten und Prozessen mit Hilfe von loT-Elementen zu erweitern: Die Entwicklung im Bereich der Sensortechnologie, in der Steuerung und Überwachung von Aktoren, in der Automatisierung von Prozessabläufen, in der Verarbeitung und Speicherung 
von Prozessdaten sowie in der Entscheidungsunterstützung durch benutzerfreundliche, anpassbare Analysetools ergeben zahlreiche Möglichkeiten zur Prozessoptimierung. Die technologische Machbarkeit geht typischerweise mit einem wirtschaftlich vertretbaren Aufwand einher und die Optimierungspotenziale lassen eine rasche Amortisationsdauer realistisch erscheinen. [Klet15, S. 266 f.]

Die Digitalisierung bietet Anwendungsunternehmen die Chance, den eigenen Kunden Mehrwertservices zu bieten, die wiederum deren Arbeitsprozesse verbessern: Beispielsweise lässt sich die „Remote Maintenance“ von Blockheizkraftwerken und Dampfgeneratoren vom Hersteller zu einem CM erweitern. Eine solche Lösung ermöglicht es dem Kunden, seine Anlagen effizient und ausfallsicher zu betreiben und ihre Anlageneffektivität (gemessen z.B. über die „OEE“, die Overall Equipment Effectiveness) zu verbessern. Der Einsatz von digitalen Datenbrillen optimiert die Möglichkeiten zur Fernunterstützung durch Wartungsexperten im Störungsfall und senkt gegebenenfalls die Kosten im weltweiten Produktsupport. Produktionsrelevante Container, deren Zustand und Füllstand lassen sich über Realtime-Location-Systeme und Behältersensorik monitoren, was das Risiko von Produktionsverzögerungen senkt. Die Überwachung des historischen, stundenaktuellen und zu erwartenden zukünftigen Zustands von Flugzeugtriebwerken ermöglicht eine „GroundTime“-optimale Durchführung von Triebwerkswartungen. Die Echtzeitüberwachung von Gebäudeaufzügen bildet die Grundlage für einen sicheren und wirtschaftlichen Betrieb. Eine automatisierte Ferndiagnose von Haushalts- und Bürogeräten eröffnet Potenziale für die frühzeitige Erkennung zukünftiger Störungen sowie eine automatisierte Ersatzteil- bzw. Verbrauchsmaterialbeschaffung.

Es stellt sich die Frage, ob diese Entwicklung zu einer Reduktion oder zu einer Polarisierung der zugehörigen Arbeitsplätze führen wird. Die Diskussion der Folgen einer digitalen Transformation erfolgt in verschiedenen Disziplinen und Bereichen ambivalent: Grundsätzlich ergibt sich durch einen höheren Automatisierungsgrad z.B. die Möglichkeit, die Aufgabengebiete der Werker weiter auf wesentliche, qualitätssichernde bzw. überwachende Arbeiten zu verlagern und ggf. flexibel ad hoc angelernte Arbeitskräfte zu beschäftigen. Tendenziell werden die neuen Arbeitsprofile aber höhere Anforderungen an die Mitarbeiter stellen und Auswirkungen auf die damit verbundenen, integrierten Geschäftsprozesse haben. [WISU17, S. 350f.] Eine wichtige Fragestellung in diesem Kontext zielt auf das zukünftige Zusammenspiel zwischen Menschen und Maschinen ab. Im Sinne einer optimierten Verteilung bzw. Koordination geht es darum, wer bei der Mensch-Maschine-Interaktion welche Aufgaben und Rollen einnehmen wird.

Im Rahmen des CM-Management geht es aus Sicht der Autoren insbesondere um evolutionäre, weniger um disruptive Veränderungen: In der Unternehmenspraxis findet CM seine Grenzen, wenn die technischen Möglichkeiten sowie die angebotenen Services die Erwartungshaltung an eine effiziente und effektive Prozessüberwachung bzw. Entscheidungsunterstützung nicht erfüllen. Um den Ansatz einer automatisierten Zustandsüberwachung durch eine algorithmenbasierte Prozessautomatisierung zu erreichen, müssen alle relevanten Parameter vollständig mit einbezogen und das angestrebte CM-System zunehmend durch den Einsatz von künstlicher Intelligenz automatisiert werden. Dies erscheint aufgrund der Komplexität und Dynamik der Einflussfaktoren sowie aus Investitionssicht derzeit wenig realistisch. Der Mensch als Regulativ bzw. als wesentliche operative Planungs-, Steuerungs- und Kontrollinstanz ist auf absehbare Zeit nicht aus der Zustandsüberwachung von Maschinen, Anlagen und Prozessen wegzudenken. Im strategischen CM sind kreative Ideen für innovative Geschäftsmodelle gefragt, die sich nur durch hochqualifizierte und motivierte 
Beteiligte erzielen lassen. Hierzu sind die aktuellen Managementpraktiken in den Kern- und Support-Geschäftsprozessen zu ergänzen. Gezielte Investitionen in erweiterte CM-Funktionalitäten können dabei die Basis zur Platzierung von Mehrwert-Services bilden, welche die Beziehungen und Interaktionen mit Geschäftspartnern und Drittparteien im Sinne eines „Business Ecosystems“ intensivieren. Die „smarte Fabrik“ wird hierdurch erweitert zum „smarten“ Unternehmen.

\section{Literaturverzeichnis}

[Ecke07] Eckerson, Wayne W.: Predictive Analytics- Extending the Value of Your Data Warehousing Investment. TDWI Best Practice Report, Chatsworth (CA) USA, 2007

[Hopp14] Hoppe, Gerd: High-Performance Automation verbindet IT und Produktion. In: Bauernhansl, Thomas; ten Hompel, Michael; Vogel-Heuser, Birgit (Hrsg.): Industrie 4.0 in Produktion, Automatisierung und Logistik. Anwendung - Technologien - Migration, Springer Vieweg Verlag, Wiesbaden 2014, S. 249 - 276

[Klet15] Kletti Jürgen: MES als Werkzeug für die perfekte Produktion. In: Kletti, Jürgen (Hrsg.): MES - Manufacturing Execution System, 2. Auflage, Springer-Vieweg-Verlag, Berlin und Heidelberg, 2015

[LaRP02] Laakso, Kari; Rosqvist, Tony; Paulsen, Jette L.: The Use of Condition Monitoring. Information for Maintenance Planning and Decision-Making, Nordic Nuclear Saftey Research, NKS-80, NKS/SOS-2.2 Project, Technical Report,Roskilde (Dänemark), 2002

[Pani07] Panian, Zeljko: Just-In-Time Business Intelligence and Real-Time Decisioning. In: International Journal of Applied Mathematics and Informatics 1 (2007), S. 28-35

[SASW17] SAS: Predictive Maintenance: höhere Produktivität durch optimale Wartung. Analyse von Anlagen- und Maschinendaten ermöglicht längere Laufzeiten. SAS White Paper, Heidelberg, 2017

[WISU17] WISU-Forum: Wie Industrie 4.0 die Arbeitswelt verändert. In: WISU 4 (2017), S. 350351

\section{Kontakt}

Prof. Dr. Frank Morelli

HS Pforzheim

Tiefenbronnerstr. 65, 75175 Pforzheim

T +49 7231 28-6697, frank.morelli@hs-pforzheim.de

Jan-Felix Mehret

ProSeS BDE GmbH

Richard-Wagner-Allee 10c, 75179 Pforzheim

T +49 723114737 - 64, j.mehret@proses.de 
Thorsten Weidt

BridgingIT GmbH

Marienstr. 17, 70178 Stuttgart

T +49 151526693 98, thorsten.weidt@bridging-it.de

Lucas Zitterbart

HS Pforzheim

Tiefenbronnerstr. 65, 75175 Pforzheim

T+49 7231 28-6697, zitterba@hs-pforzheim.de

Wir bedanken uns für die Unterstützung von der Firma Kutterer Mauer AG, vertreten durch Herrn Sven Brenner. 


\section{Tourenoptimierung}

Johanna Schwab, Michael Guckert, Matthias Willems

\section{Zusammenfassung}

Eine optimale Planung von Transportnetzwerken ist für viele Unternehmen notwendig, um Ressourcen effizient einzusetzen und damit wettbewerbsfähig zu bleiben. Zur Bewältigung dieser Aufgabe bedarf es der Unterstützung einer maßgeschneiderten Softwarelösung, die insbesondere alle relevanten externen und internen Einflussfaktoren optimal berücksichtigt. Mit dem Transport Management System der inconso AG, einem europaweit führenden Consulting- und Softwareunternehmen für Logistiklösungen, steht eine solche Softwarelösung zur Verfügung, die u.a. einen Algorithmus zur Optimierung von Routen innerhalb komplexer Transportnetzwerke nutzt. Die grundlegende Herausforderung dieses Algorithmus ist die Suche nach der optimalen Reihenfolge aus einer Menge von Anfahrtspunkten (TravelingSalesman-Problem), bei der jedoch auch Restriktionen berücksichtigt werden müssen. Der im Folgenden beschriebene Algorithmus bezieht diese Restriktionen ein und liefert angesichts der Tatsache, dass die Suche nach der optimalen Route u.U. viel Rechenzeit beanspruchen kann, eine approximative, trotzdem aber hinreichend gute Lösung. Hierzu wird eine vorgegebene Anfahrtsreihenfolge unter Berücksichtigung vorliegender Restriktionen, wie Verfügbarkeitszeiten, Haltepunktterminen oder des Beladungsvorgangs des Fahrzeugs, optimiert. Der verwendete Algorithmus ist flexibel konfigurierbar. Somit können Optimierungskriterien, wie Durchlaufzeit, Streckenlänge, Emissionswerte oder Gesamtkosten, vorgegeben werden. Als Grundlage für den angewendeten Optimierungsalgorithmus dient ein Ameisenalgorithmus, der auf dem Verhalten einer Ameisenkolonie auf Futtersuche basiert. Es hat sich gezeigt, dass Ameisen auch ohne zentrale Steuerungsinstanz den effizientesten Weg zwischen Nest und Futterquellen finden können. Jede Ameise hinterlässt dafür auf ihrem Laufweg eine Pheromonspur und bevorzugt Wege, auf denen sich bereits eine hohe Pheromonkonzentration angesammelt hat. Testdurchläufe haben gezeigt, dass mit diesem Algorithmus eine Routenoptimierung in kurzer Zeit mit einem sehr guten Ergebnis erzielt werden kann.

\section{$1 \quad$ Einleitung}

Das steigende Volumen der Logistikleistungen wird auch an den steigenden Transportleistungen deutlich [STA16]. Im Folgenden wird speziell der Transport von Gütern innerhalb eines logistischen Netzwerks näher betrachtet. Die daraus resultierenden Transportnetzwerke stellen die Beförderungsstrecken von Waren zwischen festgelegten Anfahrtspunkten dar. Der grundlegende Aufbau dieser Netzwerke ist in der Regel firmenspezifisch festgelegt und an die Unternehmensziele angepasst. Nur dann können Wartezeiten verringert, Kosten eingespart, ökologische Vorteile erzielt und die Kundenzufriedenheit gesteigert werden [BRE08].

Aufgrund der Komplexität der Transportnetzwerke ist es oft nicht möglich, oder sinnvoll eine optimale Lösung für deren Aufbau zu suchen. Bestenfalls können bestehende Lösungen durch die Optimierung von Teilproblemen verbessert werden. Die Tourenoptimierung ist 
dabei ein Teil des Gesamtprozesses der Netzwerkoptimierung, wobei eine Tour eine Fahrtstrecke mit beliebig vielen Anfahrtspunkten darstellt. Gute Ergebnisse der Tourenoptimierung können selbstverständlich nur mit Hilfe von Softwareunterstützung erzielt werden. Als Europas führendes Consulting- und Softwareunternehmen für Logistiklösungen bietet die inconso AG bereits die Möglichkeit zur Abbildung solcher Netzwerke durch Produkte wie das Transport-Management-System inconsoTMS. Das inconsoTMS wird hauptsächlich für die Planung und Steuerung von Transporten in komplexen Logistiknetzwerken eingesetzt, um die Effizienz und Flexibilität des jeweiligen Transportwesens zu erhöhen. Das inconsoTMS optimiert Transportnetzwerke mit Hilfe eines naturanalogen Optimierungsverfahrens, das im Rahmen einer Masterarbeit in Kooperation mit dem Kompetenzzentrum KITE der THM entwickelt wurde. Grundlage der Tourenoptimierung ist dabei zunächst das klassische Traveling-Salesman-Problem (TSP), welches der Komplexitätsklasse der NP-vollständigen Probleme angehört. Hier wird eine optimale Rundreise durch eine variable Anzahl von Städten gesucht, wobei jede Stadt nur einmal besucht werden darf [EP08].

Ergänzt man das TSP mit Zeitfenstern für das Erreichen der Zielorte, spricht man von einem TSP mit Zeitrestriktion (TSPTW). Die Zeitfenster geben an, wann ein Knoten besucht werden kann und wann mit Wartezeiten zu rechnen ist. Auch dieses Problem gehört der Komplexitätsklasse NP an [SAV85]. Bei Problemen der Komplexitätsklasse NP wird häufig eine Heuristik angewendet um eine gute Lösung in akzeptabler Zeit zu erhalten. Dabei haben sich naturanaloge Verfahren bewährt und es hat sich gezeigt, dass sich diese erfolgreich in eine Standardsoftware integrieren lassen. Anforderungen an den Optimierungsalgorithmus sind zudem Effizienz und Konfigurierbarkeit, um eine flexible Integration in die Software zu ermöglichen.

\section{Ameisenalgorithmus und vergleichbare Ansätze}

Es gibt unterschiedliche Ansätze zur Lösung eines TSPTW. Neben kombinatorisch-heuristischen Verfahren (etwa Branch-And-Bound) werden häufig naturanaloge Vorgehen angewendet (etwa Simulated Annealing, Genetische Algorithmen oder der hier untersuchte Ameisenalgorithmus (ACO)).

Bei der Auswahl eines geeigneten Verfahrens wurden Kriterien für Effizienz und Konfigurierbarkeit untersucht. Unter anderem sollte gewährleistet werden, dass der Algorithmus auch während der Laufzeit Änderungen an Parametern für mögliche Wegebestimmungen einbeziehen kann. Bei einigen Algorithmen ist dies nur bedingt möglich, da sie deterministisch auf Grundlage der vorherigen Lösung eine weitere finden. Bei diesen Algorithmen kommt es deshalb vor, dass es sich bei der gefundenen Lösung nur um ein lokales Optimum handelt und eine existierende globale beste Lösung nicht mehr gefunden werden kann. Tabelle 1 zeigt die bei der Wahl des Verfahrens untersuchten Algorithmen und deren Ergebnisse für die im Vorfeld definierten Kriterien.

In Zusammenarbeit mit der inconso AG wurde durch Gegenüberstellung von alternativen Algorithmen und durch eine zusätzliche Marktanalyse von bereits etablierter Tourenoptimierungssoftware, der ACO als ein geeigneter Algorithmus zur Tourenoptimierung identifiziert. Dieser Algorithmus wurde erstmals 1991 von Colorni für die Lösung von Optimierungsproblemen vorgestellt [CDM91]. Er basiert auf dem Verhalten einer Ameisenkolonie auf Futtersuche, da die Ameisen in der Lage sind den effizientesten Weg zwischen ihrem Nest und der Futterquelle zu finden. Daraus resultiert, dass Ameisen in der Lage sind kürzeste Wege 
in einem Netzwerk zu finden, ohne dabei über eine zentrale Steuerungsinstanz zu verfügen. Der ACO gewichtet jede Kante neben ihren Kosten mit einem festgelegten Pheromonwert und erstellt daraus einen Graphen, in dem der optimale Weg gefunden werden soll. Zur Durchführung des Algorithmus werden mehrere Ameisen losgeschickt, um den idealsten Weg im Graphen zu finden. Ideal bedeutet hierbei hinreichend gut und wird nicht als Synonym für optimal verwendet.

\begin{tabular}{|c|c|c|c|c|}
\hline Algorithmus & $\begin{array}{c}\text { Parameter- } \\
\text { änderung }\end{array}$ & Parallelisierbar & Komplexität & $\begin{array}{c}\text { Optimale } \\
\text { Lösung }\end{array}$ \\
\hline $\begin{array}{c}\text { Ameisenalgorithmus } \\
\text { (ACO) }\end{array}$ & Ja & Ja & Mittel & Ja \\
\hline Branch\&Cut & Nein & Ja & Mittel & Nein \\
\hline Constraint Programming & Nein & Ja & Hoch & Nein \\
\hline $\begin{array}{c}\text { Dynamische Program- } \\
\text { mierung }\end{array}$ & Nein & Ja & Hoch & Nein \\
\hline Simulated Annealing & Ja & Nein & Niedrig & Ja \\
\hline Generische Algorithmen & Ja & Ja & Hoch & Ja \\
\hline
\end{tabular}

Tabelle 1: Evaluation von Algorithmen zur Lösung des TSPTW

Während der Wegfindung muss sich jede Ameise an einem Knoten für den weiteren Wegverlauf entscheiden. Der Grundlegende Ansatz des ACO verwendet für diese Entscheidung eine stochastische Erkundungsregel. Für die Übergangswahrscheinlichkeit $p$ lautet diese:

$$
p_{i, j}=\frac{\left[\tau_{i, j}(t)\right]^{\alpha}\left[\eta_{i, j}\right]^{\beta}}{\sum_{l \in N_{i}}\left[\tau_{i, l}(t)\right]^{\alpha}\left[\eta_{i, l}\right]^{\beta}}
$$

Dabei gibt $\tau_{i, j}(t)$ den Pheromonwert zum Zeitpunkt $\mathrm{t}$ an, $\eta_{i, j}=\frac{1}{d_{i, j}}=\frac{1}{\text { Kosten für Kante }(i, j)}$ und $N_{i}$ beschreibt alle restlichen Nachbarknoten. Mit dem Wert für $\alpha$ kann die Gewichtung der Kosten und mit $\beta$ die Gewichtung der Pheromone gesteuert werden.

Der allgemeine ACO führt mit jedem beendeten Ameisendurchlauf eine Anpassung des Graphen durch. Sobald eine Ameise ihre Tour beendet und einen möglichen Weg durch den Graphen gefunden hat wird dieser mit den Lösungswegen der anderen Ameisen verglichen. Ist der gefundene Weg besser als bisherige, muss der Pheromonwert jeder Wegkante im Graph, aus dem Weg der Ameise, aktualisiert werden. Bei der Aktualisierung verbessert sich der Pheromonwert der Kante um einen im Vorfeld festgelegten, konstanten Wert. Jede Ameise, die nach der Aktualisierung noch auf Wegfindung ist, wird auf Basis der neuen Pheromonwerte ihren weiteren Laufweg entscheiden. Erst wenn alle Ameisen einen Weg gefunden haben, werden sie erneut für einen Zyklus auf Wegsuche geschickt. Wie viele Ameisen dafür eingesetzt werden und wie oft dieser Zyklus wiederholt wird, ist anwendungsabhängig.

Um bessere Ergebnisse des Algorithmus zu erhalten, die näher an einer optimalen Lösung liegen, modifiziert Colorni den ACO und stellt drei Ansätze vor [CDM91]: Ant-Density-, AntQuantity- und Ant-Cycle-Algorithmus.

Unter den drei genannten stellte sich der Ant-Cycle-Algorithmus It. Colorni als effizienteste Variante heraus. Dabei wird mit dem Setzen der neuen Pheromonspur der Graphkanten gewartet, bis alle Ameisen eines Zyklus eine Lösung gefunden haben und nur der daraus 
resultierende beste Weg wird aktualisiert. Anschließend werden alle Ameisen erneut auf die Suche geschickt.

Aufbauend auf dem Ant-Cycle-Algorithmus wurde eine weitere Verbesserung zur Lösungsfindung durch den Ant-Colony-System-(ACS)-Algorithmus von [CM06] beschrieben.

Eines der wesentlichen Unterscheidungsmerkmale zum Ant-Cycle-Algorithmus ist, dass bei diesem Ansatz die Übergangswahrscheinlichkeit sowohl eine deterministische Ausbreitungsregel, wie auch eine stochastische Erkundungsregel beinhaltet. Welche der beiden genannten Regeln für die Berechnung der Übergangswahrscheinlichkeit angewendet wird, hängt von einer Zufallszahl $q$ ab, mit $0 \leq q \leq 1$, und einer benutzerdefinierten Konstante $q_{0}$, mit $0 \leq q_{0} \leq 1$. Mit der Ausbreitungsregel wird immer die beste Kante gewählt, wo hingegen die stochastische Erkundungsregel $\mathrm{S}=p_{i, j}$ durch die Gleichung 2.1 beschrieben wird.

$$
j=\left\{\begin{array}{c}
\arg \max _{z \in N_{i}} \tau_{i, z}(t) \cdot\left(\eta_{i, z}\right)^{\alpha}, \text { wenn } q \leq q_{0}, \\
S, \text { sonst }
\end{array}\right.
$$

Neben der globalen Pheromonspurverbesserung wird bei der ACS Variante zusätzlich eine Verfallszeit für Pheromone eingeführt, die den Pheromonwert einer Kante bei jedem Ameisenübergang vermindert. Diese Verfallszeit ist notwendig, damit der Algorithmus nicht zu schnell gegen eine suboptimale Lösung konvergiert [MT09] und ein besseres Ergebnis erzielt werden kann. Außerdem kann so verhindert werden, dass alle Ameisen zu früh den gleichen Weg einschlagen. Diese globale Pheromonaktualisierung wird durchgeführt, sobald alle Ameisen einen Zyklus beendet haben. Die Aktualisierung eines Kantenpheromonwerts wird mit der Gleichung 2.3 beschrieben.

$$
\tau_{i, j}(t+1)=(1-\rho) \tau_{i, j}(t)+\sum g(s)
$$

Dabei gibt $\rho$ die Verdunstungsrate in den Grenzen von $(0,1]$ und $g(s)$ den Evaluationsfaktor an. Ein akzeptabler Wert für $\rho$ ist auf 0,1 zu setzen [GD05] und die Evaluationsfunktion wird angegeben durch $\sum g(s)=\rho \cdot \tau_{0}$. Wobei $\tau_{0}$ der initiale Pheromonwert einer Kante ist und durch $\tau_{0}=\frac{1}{n+L_{n n}}$ beschrieben wird. Hierbei steht $\mathrm{n}$ für die Anzahl aller Knoten im Graphen und $L_{n n}$ sind die Kosten der besten Tour. Diese Kosten können näherungsweise durch die Anwendung des Nearest-Neigbour-Algorithmus ermittelt werden.

Zusammenfassend müssen die Parameter aus den beschriebenen Formeln 2.1, 2.2 und 2.3 problemabhängig anhand von Tests evaluiert werden.

\section{$3 \quad$ Implementierung}

Der ACS Algorithmus muss flexibel für unterschiedliche Anwendungsfälle in der Praxis implementierbar sein. Als Konfigurationseinstellungen wurden im beschriebenen Anwendungsfall allgemeine Optimierungseinstellungen, spezielle Optimierungskriterien und Restriktionen umgesetzt.

Die allgemeinen Optimierungseinstellungen beziehen sich auf Start- und Endpunkte der Ameisendurchläufe. Hierbei kann für alle Ameisen festgelegt werden, ob diese an einem festgelegten Startpunkt beginnen oder diesen zufällig aus der Menge aller Knoten wählen werden. Neben dem Start kann dies auch für das Ende festgelegt werden. 
Die speziellen Optimierungskriterien definieren die Güte eines Weges der als optimal angesehen wird. Zu den Kriterien gehören Kosten, Zeit, Umweltfaktoren und Streckenlänge, wobei auch eine Mehrauswahl möglich ist. Bei der Optimierung nach den Kosten wird der Weg mit den geringsten Gesamtkosten aus Anfahrtspunkten und Fahrzeugkosten als optimal angesehen. Die Optimierung nach der Zeit gewichtet den Weg mit der kleinsten Gesamtdurchführungsdauer als optimal. Als Zeitwert wird die Fahrtzeit pro Strecke bestimmt, welche sich aus der Streckenlänge und der Durchschnittsgeschwindigkeit des Fahrzeugs errechnet. Bei der Auswahl der Umweltfaktoren wird die geringste Umweltbelastung gesucht. Dafür wird der $\mathrm{CO}_{2}$-Ausstoß des Fahrzeugs bestimmt und ein kleiner Wert als optimal betrachtet. Das letzte Kriterium der Streckenlänge bezieht sich auf die Distanzen zwischen zwei Anfahrtspunkten. Diese kann sowohl als Route, wie auch als Luftlinie berechnet werden, wobei die Strecke mit den geringsten Gesamtkilometer als Optimum angesehen wird.

Als optionale Restriktionen sind eine Überladungsprüfung für das Fahrzeug, Zusammenladungsverbote von Ware, die Berücksichtigung von Terminen und Öffnungszeiten von Anfahrtspunkten umgesetzt. Werden diese Restriktionen nicht ausgewählt, werden sie beim Durchlauf des Algorithmus nicht geprüft.

Im Allgemeinen gilt für die Anwendung in der Praxis, dass für den Warentransport (Auftrag) Termine für Zustellung oder Abholung in der optimierten Tour eingehalten werden müssen. Eine zu optimierende Tour kann mehrere Aufträge beinhalten, wobei ein einzelner Auftrag unzertrennbar ist und als Gesamtpaket auf das Fahrzeug passen muss. Zusätzlich muss jedem Anfahrpunkt für eine erfolgreiche Optimierung eine Adresse mit Geokoordinaten zugewiesen sein. Ansonsten wäre es nicht möglich, positionsabhängige Werte, wie Distanzen und die damit verbundenen Kosten, zu bestimmen.

Um bei der Umsetzung des ACS eine akzeptable Laufzeit erzielen zu können, wurden die Ameisenläufe parallelisiert. Der Graph, der den Ameisen pro Durchlauf als Grundlage dient, wurde als ein Singleton-Objekt implementiert. Somit ist genau eine Instanz des Graphen während des Optimierungsvorgangs jederzeit global verfügbar.

$\mathrm{Zu}$ Beginn des Algorithmendurchlaufs werden alle notwendigen Daten aus der Datenbank geladen und Änderungen der Daten im Nachhinein werden für den Durchlauf somit nicht mehr berücksichtigt. Durch diesen Ansatz wird die allgemeine Performance verbessert, da wiederholende Datenbankabfragen und damit verbundene Laufzeit eingespart werden.

Der Graph wird vorab initialisiert und Kanten entfernt, die niemals verwendet werden dürfen. Beispielsweise muss bei einem Auftrag zwangsläufig die Einladestelle a vor der Ausladestelle $b$ angefahren werden. Damit wird die rückwärtige Kante (b, a) aus dem Graphen entfernt und bei den Ameisendurchläufen nicht mehr berücksichtigt. Ebenso werden bei dem festgelegten Start- bzw. Zielpunkt alle eingehenden bzw. ausgehenden Kanten entfernt.

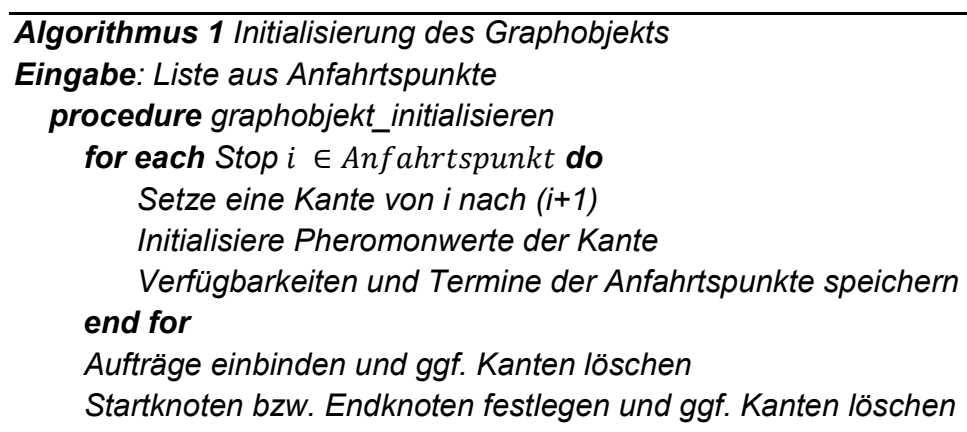


Die Parameterwerte für die Zyklen- und Ameisenanzahl sind zusätzlich entscheidend für die Laufzeit des Algorithmus, wobei große Werten eine längere Laufzeit zur Folge haben.

In einem Zyklendurchlauf sucht jede Ameise einen möglichen Weg im Graphen und gibt diesen als Ergebnis zurück. Beginnend bei einem Startknoten geht die Ameise über eine Kante zum nächsten Knoten und setzt so ihren Weg durch den Graphen fort bis alle Knoten besucht wurden. Erst wenn alle Ameisen einen Weg gefunden haben, werden die Pheromone auf den Graphkanten nach dem besten Weg aktualisiert und ein neuer Zyklus gestartet. Dieser Ablauf ist in Algorithmus 2 in Pseudocode dargestellt.

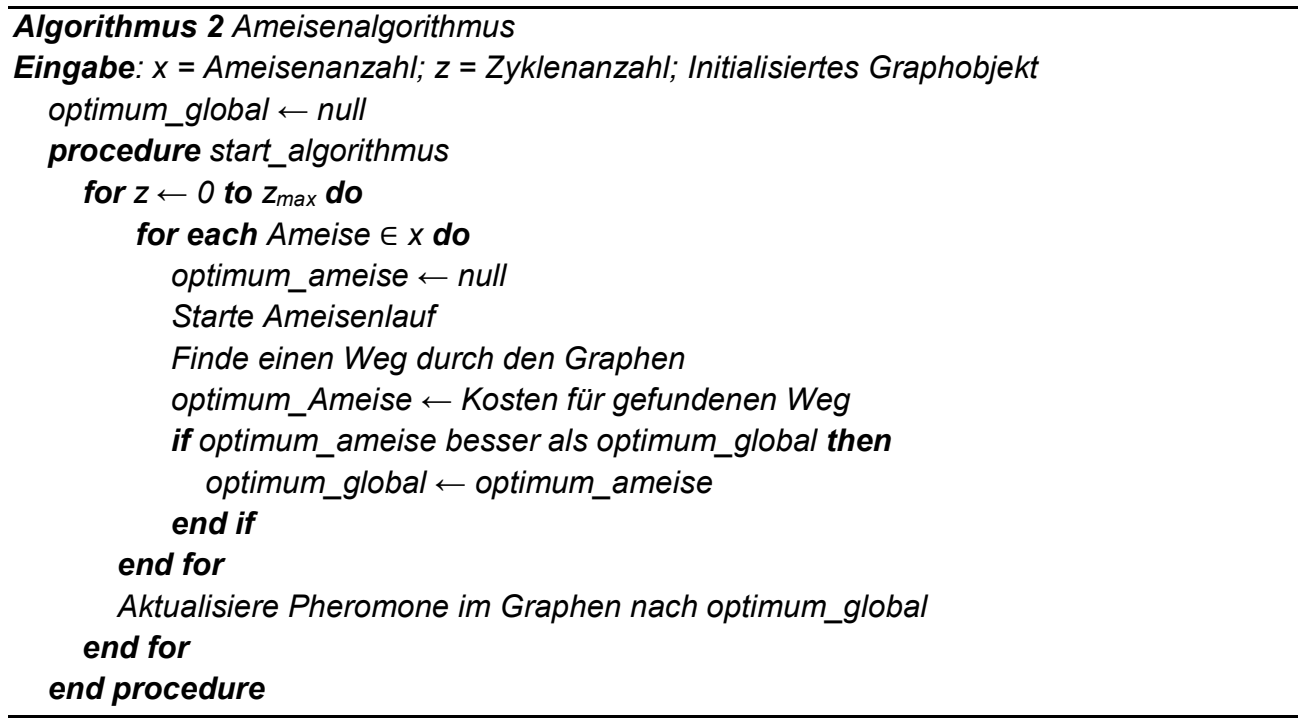

Als Übergangsfunktion zur Kantenauswahl werden sowohl der stochastische, wie auch der deterministische Ansatz verwendet. Die Auswahl erfolgt über eine Zufallszahl.

Für die Kantenauswahl müssen die, aus der Konfiguration vorgegebenen Restriktionen überprüft werden. Erst wenn es keine Restriktionsverletzungen gibt, darf die Kante als eine erlaubte Kante angesehen werden und ihr Endknoten als weiterer Punkt dem bisherigen Weg hinzugefügt werden. Bei der Kantenauswahl wird zusätzlich überprüft, ob jede Ware, die beim Endknoten der Kante abgeladen werden muss, auch bereits eingeladen wurde. Trifft dies nicht zu, kann die Kante nicht gewählt werden.

Wird eine erlaubte Kante gefunden, deren Endknoten noch nicht im aktuellen Weg vorkommt, erweitert sich der Weg um den Knoten. Die Kante wird einer Tabu-Liste hinzugefügt, welche alle Kanten enthält, die bereits besucht wurden um doppelte Wege zu vermeiden. Sind noch nicht alle Knoten im Weg enthalten, muss durch einen rekursiven Aufruf dieser Funktion der nächste Knoten gefunden werden.

Entspricht die Weglänge der Anzahl Knoten im Graphen und es wurde kein Knoten doppelt besucht, ist die Ameise am Ziel und gibt ihre gefundene Lösung aus.

Sind hingegen noch nicht alle Knoten besucht und es wird kein Weg mehr gefunden, dessen Kante noch nicht in der Tabu-Liste abgespeichert wurde, ist ein falscher Weg eingeschlagen 
worden und die Ameise muss wieder einen Knoten zurückgehen und erneut von diesem Knoten einen weiteren möglichen Weg finden. Sie kann dabei nicht den gleichen Weg wieder gehen, da die Kante bereits in der Tabu-Liste gespeichert wurde und es muss ein neuer, noch nicht besuchter Knoten, gefunden werden. Ist es nicht möglich, auf diese Weise alle Knoten zu besuchen, gibt es keinen Weg, den die Ameise gehen kann und sie muss ohne Ergebnis zurückkehren. Der Algorithmus ist so aufgebaut, dass sobald eine Ameise einen möglichen Weg gefunden hat, alle anderen Ameisen mindestens auch diesen Weg finden werden. Der Pseudocode eines Ameisendurchlaufs wird in Algorithmus 3 beschrieben.

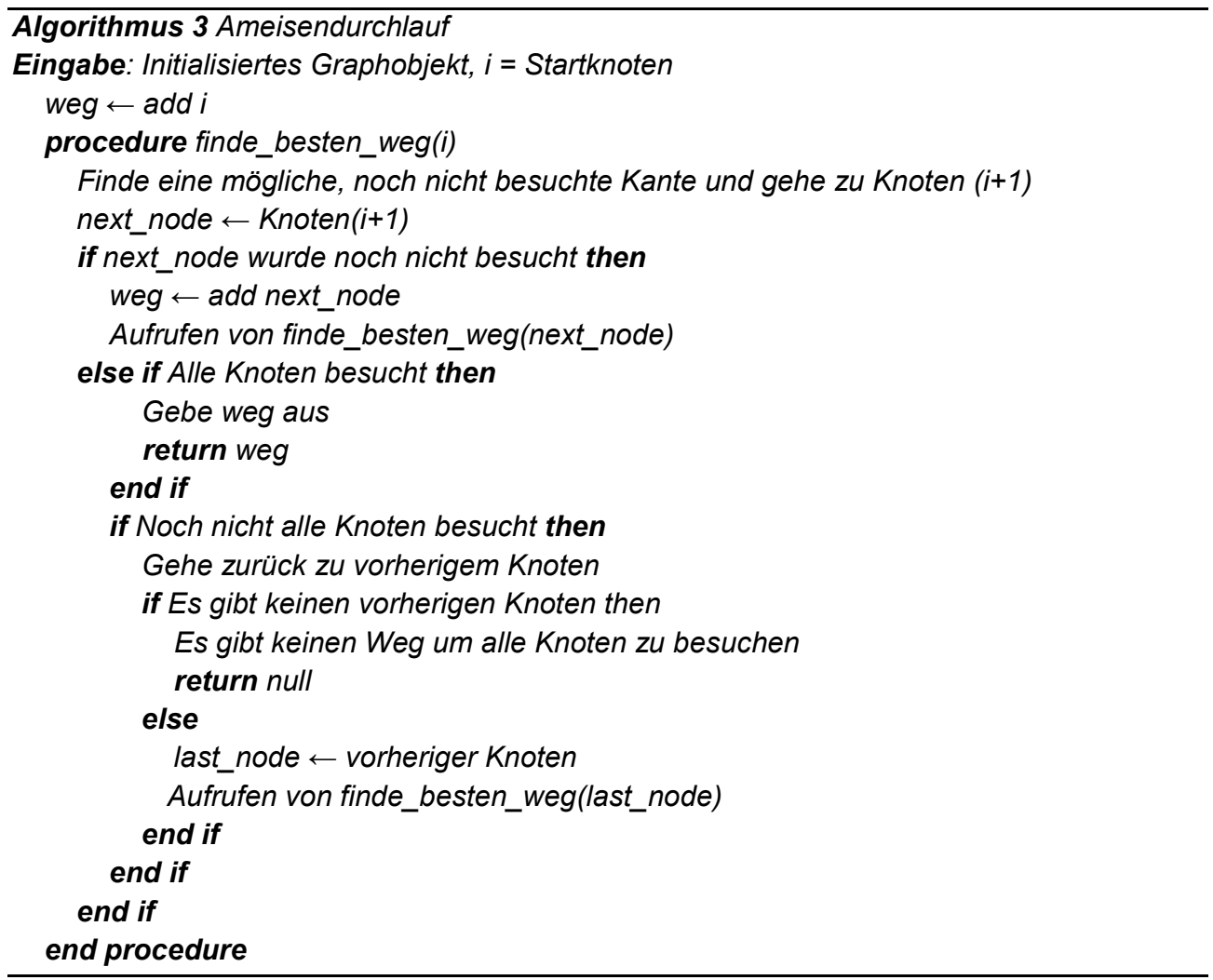

Aufgrund der Parallelisierung können alle Ameisen gleichzeitig starten und ihren eigenen Weg durch den Graphen finden. Da jeder neue Zyklendurchlauf auf einem aktualisierten Graphen basiert und dieser erst nach dem Beenden aller Ameisendurchläufe durchgeführt werden kann, wird an diesem Punkt gewartet, bis alle Ameisen ihren Durchlauf beendet haben. 


\section{$4 \quad$ Ergebnisse}

Die Güte eines Algorithmus hängt neben der gefundenen Lösung auch von seiner Laufzeit ab. Für den implementierten Ameisenalgorithmus sind hierbei die Knotenanzahl, die Parametereinstellungen und die zur Verfügung stehende Threadanzahl entscheidend.

Für die Laufzeitmessungen wurden sechs Versuchsdurchläufe festgelegt, die auf unterschiedlichen Parametereingaben operieren. Die Parameterwerte sind in Tabelle 2 beschrieben. Bestimmte Parameterwerte wurden bereits in anderen Quellen, darunter [GD05], als gut identifiziert. Diese wurden als Ausgangslage für den ersten Versuch gewählt und dienten den weiteren Parametereinstellungen als Grundlage.

\begin{tabular}{|c|l|l|l|l|l|l|l|}
\hline $\begin{array}{l}\text { Para- } \\
\text { meter }\end{array}$ & Beschreibung & Vers. 1 & Vers. 2 & Vers. 3 & Vers. 4 & Vers. 5 & Vers. 6 \\
\hline$\tau_{0}$ & $\begin{array}{l}\text { Initialer Phero- } \\
\text { monwert }\end{array}$ & 0,1 & 0,1 & 0,1 & 0,1 & 0,5 & 0,1 \\
\hline$m$ & Anzahl Ameisen & 10 & 50 & 10 & 0 & 15 & 100 \\
\hline$c$ & Anzahl Zyklen & 4 & 4 & 24 & 10 & 10 & 1 \\
\hline$q_{0}$ & $\begin{array}{l}\text { Konstante für } \\
\text { Übergangsfunktion }\end{array}$ & 0,5 & 0,5 & 0,5 & 0,5 & 0,7 & 0,5 \\
\hline$\rho$ & $\begin{array}{l}\text { Verfallszahl Phe- } \\
\text { romone }\end{array}$ & 0,1 & 0,1 & 0,1 & 0,1 & 0,2 & 0,1 \\
\hline$\alpha$ & $\begin{array}{l}\text { Gewichtung } \\
\text { der Kantenkosten }\end{array}$ & 1 & 1 & 1 & 1 & 1,5 & 1 \\
\hline$\beta$ & $\begin{array}{l}\text { Gewichtung } \\
\text { der Pheromone }\end{array}$ & 1 & 1 & 1 & 1 & 0,6 & 1 \\
\hline
\end{tabular}

Tabelle 2: Parametereinstellungen für Versuchsdurchläufe

Jeder Versuchsdurchlauf wurde auf drei Graphen angewandt, die 5, 25 und 50 Knoten besitzen. Jeder Versuch pro Graph, im Folgenden als Testtour beschrieben, wurde auf einem identischen Ausgangsgraphen begonnen und insgesamt zwei Mal mit verschiedener maximaler Threadanzahl durchgeführt. Es wurde dabei jeweils die Weglänge der gefundenen Tour und die Laufzeit des Algorithmus, inklusive der Graphinitialisierung, gemessen. Als Optimierungskriterium wurde die minimale Weglänge mit festem Startpunkt gewählt.

Bei der ersten Laufzeitanalyse des Graphen mit fünf Anfahrtspunkten wurde bei allen Versuchen, unabhängig von den verwendeten Parametern oder der Threadanzahl, dieselbe optimale Weglänge berechnet. Dabei ließen sich allerdings signifikante Unterschiede in der Laufzeit feststellen. Mit dem ersten Versuchsdurchlauf wurde die beste Laufzeit erzielt, eine niedrigere Threadanzahl auch eine deutliche Verbesserung bessere Laufzeit lieferte. Die Ergebnisse dieser Versuchsreihe sind in Tabelle 3 dargestellt.

\begin{tabular}{|l|l|l|l|l|l|l|}
\hline Ereignis & Vers. 1 & Vers. 2 & Vers. 3 & Vers. 4 & Vers. 5 & Vers. 6 \\
\hline Laufzeit (10 Threads) & $0,189 \mathrm{~s}$ & $2 \mathrm{~s}$ & $15 \mathrm{~s}$ & $1 \mathrm{~s}$ & $6 \mathrm{~s}$ & $1 \mathrm{~s}$ \\
\hline Weglänge Tour $(\mathrm{km})$ & 928,28 & 928,28 & 928,28 & 928,28 & 928,28 & 928,28 \\
\hline Laufzeit (20 Threads) & $0,517 \mathrm{~s}$ & $0,605 \mathrm{~s}$ & $6 \mathrm{~s}$ & $0,895 \mathrm{~s}$ & $3 \mathrm{~s}$ & $0,582 \mathrm{~s}$ \\
\hline Weglänge Tour $(\mathrm{km})$ & 928,28 & 928,28 & 928,28 & 928,28 & 928,28 & 928,28 \\
\hline
\end{tabular}

Tabelle 3: Laufzeitanalyse und Güte (Weglänge) bei fünf Knoten

Die Messungen auf Grundlage des Graphen bestehend aus 25 Knoten hatten bereits deutlich höhere Durchlaufzeiten zur Folge (Tabelle 4) als bei der ersten Testtour mit 5 Knoten. 
Bei maximal zehn Threads beträgt die Differenz zwischen der schnellsten und der langsamsten Laufzeit $62 \%$, wobei der gefundene Weg lediglich um 2,6\% kürzer ist. Daraus zeigt sich die Notwendigkeit einer vorher evaluierten Parametereinstellung.

\begin{tabular}{|l|l|l|l|l|l|l|}
\hline Ereignis & Vers. 1 & Vers. 2 & Vers. 3 & Vers. 4 & Vers. 5 & Vers. 6 \\
\hline Laufzeit (10 Threads) & $1 \mathrm{~s}$ & $16 \mathrm{~s}$ & $113 \mathrm{~s}$ & $62 \mathrm{~s}$ & $43 \mathrm{~s}$ & $9 \mathrm{~s}$ \\
\hline Weglänge Tour $(\mathrm{km})$ & 2434,27 & 2587,6 & 2417,38 & 2370,08 & 2541,28 & 2517,54 \\
\hline Laufzeit (20 Threads) & $3 \mathrm{~s}$ & $5 \mathrm{~s}$ & $38 \mathrm{~s}$ & $25 \mathrm{~s}$ & $20 \mathrm{~s}$ & $3 \mathrm{~s}$ \\
\hline Weglänge Tour $(\mathrm{km})$ & 2319,49 & 2404,1 & 2404,1 & 2404,1 & 2404,1 & 2477,8 \\
\hline
\end{tabular}

Tabelle 4: Laufzeitanalyse und Güte (Weglänge) bei 25 Knoten

Der letzte von drei Tests wurde mit einem Graphen aus 50 Knoten durchgeführt (Tabelle 5). Auch bei der dritten Testtour erwiesen sich die Parametereinstellungen des ersten Versuchs für die Auffindung der optimalen Weglänge im Verhältnis zur benötigten Laufzeit als akzeptabel. Bei dem Durchlauf mit maximal zehn Threads wurde lediglich in Versuch 6 eine kürzere Weglänge als in Versuch 1 gefunden. Hierbei bringt das Ergebnis bei einer $60 \%$ höherer Laufzeit lediglich eine Verbesserung von 6\%.

\begin{tabular}{|l|l|l|l|l|l|l|}
\hline Ereignis & Vers. 1 & Vers. 2 & Vers. 3 & Vers. 4 & Vers. 5 & Vers. 6 \\
\hline Laufzeit (10 Threads) & $6 \mathrm{~s}$ & $32 \mathrm{~s}$ & $225 \mathrm{~s}$ & $247 \mathrm{~s}$ & $86 \mathrm{~s}$ & $15 \mathrm{~s}$ \\
\hline Weglänge Tour $(\mathrm{km})$ & 3681,74 & 3785,05 & 3538,54 & 3704,28 & 4099,73 & 3458,24 \\
\hline Laufzeit (20 Threads) & $6 \mathrm{~s}$ & $17 \mathrm{~s}$ & $81 \mathrm{~s}$ & $108 \mathrm{~s}$ & $42 \mathrm{~s}$ & $8 \mathrm{~s}$ \\
\hline Weglänge Tour $(\mathrm{km})$ & 3886,99 & 4276,6 & 3737,6 & 3621,3 & 3984,36 & 3850.94 \\
\hline
\end{tabular}

Tabelle 5: Laufzeitanalyse und Güte (Weglänge) bei 50 Knoten

Allgemein konnte kein Zusammenhang zwischen der Threadanzahl und der Güte des Ergebnisses gezeigt werden. Allerdings hat eine höhere Threadanzahl oft auch eine Verbesserung der Laufzeit zur Folge, da diese die Anzahl von möglichen parallellaufenden Ameisen vorgibt. Zudem hat sich durch die Parametereinstellungen gezeigt, dass die Laufzeit stark von der Zyklenanzahl abhängt und eine kleine Zyklenanzahl sich bewährt. Das hängt mit der geforderten Wartezeit aller Ameisen am Ende eines jeden Zyklus zusammen, wodurch sich die Laufzeit bei vielen Durchläufen signifikant verschlechtern kann (siehe Versuch 3). Zusätzlich hat Versuch 6 allgemein gezeigt, dass auch ohne ein globales Graphupdate (ein Zyklendurchlauf) mit vielen Ameisen ein relativ gutes und teilweise sogar bestes Ergebnis in einer, noch akzeptablen Laufzeit, erzielt werden kann. Als Testumgebung diente eine virtuelle Maschine (VM) mit dem Betriebssystem Oracle Linux Server Release 2.6. Die VM verfügte über 4 virtuelle Kerne mit jeweils einer Intel(R) Xeon(R) CPU mit 2,60 $\mathrm{GHz}$ und $8 \mathrm{~GB}$ RAM.

\section{$5 \quad$ Fazit und Ausblick}

Für den Tourenoptimierungsalgorithmus hat sich bei einer Tour mit wenigen Stopps gezeigt, dass die Parametereinstellungen entscheidend die Laufzeit verändern, eine steigende Threadanzahl aber keine Verbesserung zur Folge hat. Eine allgemeingültige optimale Parametereinstellung ist in der Praxis nicht zu finden, da diese von vielen Faktoren einer Tour 
und des Optimierungsvorgangs abhängen. Eine gute Parametereinstellung zu finden ist nicht trivial und könnte zukünftig über eine computergestützte Berechnung erzielt werden.

Durch die Konfigurationstabelle, die dem Algorithmus zu Grunde liegt, ist dieser flexibel aufgebaut, lässt sich auch zu einem späteren Zeitpunkt erweitern und gewährt somit die dynamische Durchführung der Tourenoptimierung. Durch diesen Optimierungsvorgang kann der gesamte Tourenplan effizienter gestaltet werden. Allgemein kann mit der Tourenoptimierung immer eine gute Lösung in sehr kurzer Zeit gefunden und Kosten gespart werden.

\section{Literaturverzeichnis}

[BRE08] Bretzke, Wolf-Rüdiger: Logistische Netzwerke. Springer, Berlin, 2008

[CDM91] Colorni, Alberto; Dorigo, Marco; Maniezzo, Vittorio: Distributed Optimization by Ant Colonies. In: European Conference on Artificial Life, 1991, S. 134-142,

[EP08] Erk, Katrin; Priese, Lutz: Theoretische Informatik: eine umfassende Einführung. 3. erw. Aufl. Berlin: Springer, 2008

[SAV85] Savelsbergh, M.W.P.: Local search in routing problems with time windows. In: Annals of Operations-Research, Vol 4, Issue 1, December 1985, S. 285-305

[STA16] Statistisches Bundesamt: Transportleistung der Verkehrsträger im Bundesgebiet 1950 2040, 2016, http://www.bgl-ev.de/images/daten/verkehr/transportleistung_tabelle.pdf. Abruf am: 28.04.2017

[CM06] Cheng, Chi-Bin; Mao, Chun-Pin: A modified ant colony system for solving the travelling salesman problem with time windows. In: Mathematical and Computer Modelling. Vol.46, Issues 9-10, November 2007, S.1225-1235,

[MT09] Marco Dorigo; Thomas Stuetzle: Ant Colony Optimization: Overview and Recent Advances. vol. 146 of International Series in Operations Research \& Management Science, Springer, New York, NY, USA, 2010, S. 227-263

[GD05] Gilmour, Stephen; Dras, Mark: Understanding the Pheromone System Within Ant Colony Optimization. In: Zhang S., Jarvis R.: Advances in Artificial Intelligence. Al 2005. Springer, Berlin, Heidelberg, 2005, S. 786-789

\section{Kontakt}

Johanna Schwab

inconso AG

In der Hub 1-8, 61231 Bad Nauheim

T+49 6032 348-3017, jschwab@inconso.de

Michael Guckert, Matthias Willems

KITE - Kompetenzzentrum für Informationstechnologie

Technische Hochschule Mittelhessen

Wilhelm-Leuschner-Straße 13, 61169 Friedberg

michael.guckert@mnd.thm.de, matthias.willems@mnd.thm.de 


\section{Datenmanagement - Softwareengineering}




\title{
Einsatz einer NoSQL-Datenbank zur Analyse von Reiseblogs: Konzept und Integration
}

\author{
Marco Graf, Thomas Barton
}

\section{Zusammenfassung}

Dieser Beitrag behandelt die Anforderungen zur Analyse von großen Datenmengen am Beispiel einer bestehenden Reiseblogging-Anwendung auf Basis einer NoSQL-Datenbank. Hierzu müssen die im SQL-Format vorliegenden Daten in eine neue logische Struktur gebracht werden, welche Analysen auf bestimmte KPIs der Anwendung innerhalb einer NoSQL-Datenbank erlaubt.

This contribution deals with the requirements for the analysis of an existing travel blogging application based on a NoSQL database and shows its prototypical evaluation and implementation. For this purpose, a MongoDB database was chosen, which is available with an open-source license. MongoDB is classified as a NoSQL database, which stores data on a document-oriented way in a JSON-like format.

\section{$1 \quad$ Einführung}

Der Begriff Big-Data ist aktuell ein wichtiges Thema im IT-Umfeld. Tagtäglich entstehen enorme Mengen an Daten, die aus vielen verschiedenen Bereichen und unterschiedlichen Quellsystemen stammen können. Sowohl unternehmensinterne als auch -externe Quellen wie Social Media, Dokumente, Sensoren in der Produktion, Kommunikation, System-Logs und viele andere Bereiche, wie der Wandel hin zum Internet der Dinge, lassen die Datenmengen rasant wachsen. Diese Daten zu verwalten ist aufwendig und häufig auch mit hohen Kosten verbunden. Vor allem dann, wenn diese gesammelten Informationen schnell verfügbar sein müssen und zu Auswertungs- oder Monitoring-Zwecken eingesetzt werden. Zudem liegen diese Daten häufig unstrukturiert vor, sie folgen also keinem einheitlichen Schema, weshalb die Verwaltung zusätzlich verkompliziert wird [Roeb11]. Dies hat zur Folge, dass die Daten nicht ohne weiteres in das klassische Schema einer relationalen Datenbank passen. Um für dieses Szenario eine passende Lösung bereitstellen zu können, kommen NoSQL-Datenbanken (Not only SQL) zum Einsatz.

\subsection{SQL- und NoSQL-Datenbanken}

In der Welt der Datenbank-Technologien unterscheidet man zwischen zwei Haupttypen von Datenbanken: die sogenannten SQL- und NoSQL- oder relationale und nicht-relationale Datenbanken. Diese Datenbanken unterscheiden sich unter anderem in ihrem Aufbau, der Art an Informationen, die sie speichern und wie ebendiese Informationen in der Datenbank gespeichert und abgerufen werden. Vor allem in den vergangenen Jahren haben NoSQL oder nicht-relationalen Datenbanken in Bezug auf die Speicherung von riesigen Datenmengen und der einfachen Skalierbarkeit stark an Bedeutung gewonnen.

Relationale Datenbanken wie beispielsweise MySQL, PostgreSQL und Microsoft SQL repräsentieren und speichern Daten streng strukturiert in Tabellen, Spalten und Zeilen einer 
Datenbank. Diese RDBMS speichern somit ihre Relationen als Tabellen zeilenweise. Jede Spalte besitzt einen festen Datentyp. Felder einer Tabelle können zu Schlüsseln und Indices zum schnellen Zugriff definiert werden. Dieses Konzept der relationalen Datenbank wurde in den 70er Jahren entwickelt. Das wichtigste Merkmal aller relationalen Datenbanken ist die Unterstützung der ACID-Eigenschaften. ACID beschreibt die Verfügbarkeit der folgenden Merkmale: „Automicity“, „Consistency“, „Isolation“ sowie „Durability“. Somit wird sichergestellt, dass Transaktionen zuverlässig verarbeitet werden [Meie16].

Das Ziel der nicht-relationale Datenbanken war es, die riesigen Mengen an rasant wachsenden unstrukturierten Daten im Webumfeld in einer dementsprechenden Datenbank abzubilden und diese leicht skalierbar zu machen. NoSQL-Datenbanksysteme bieten daher eine flexible oder gar schemafreie Speicherung der Daten, ohne dass eine derartige "referentielle Integrität" vorliegt, wie es bei den relationalen Datenbanken der Fall ist. Die Daten innerhalb einer NoSQL-Struktur sind stark denormalisiert und ermöglichen in ihrer ursprünglichen Form keine SQL-Operationen wie JOIN oder GROUP-BY Anweisungen [Meie16]. Einige der Anbieter auf diesem Markt haben eigene Methoden entwickelt, um eine derartige Funktion auch für NoSQL-Strukturen bereitzustellen. Durch die Verteilung einer NoSQLDatenbank auf verschiedene Systeme müssen bestimmte Bedingungen sichergestellt werden. Diese werden durch das CAP-Theorem formuliert. Das CAP-Theorem beschreibt die Austauschbeziehung also die gegenläufige Abhängigkeit in verteilten Systemen. CAP setzt sich aus den englischsprachigen Begriffen Consistency (Konsistenz), Availability (Verfügbarkeit) und Partition Tolerance (Ausfalltoleranz). Wobei dieses Theorem besagt, dass ein verteiltes System jeweils nur zwei der drei Bedingungen erfüllen kann [Gaur13].

- Die "Consistency“ besagt, dass sichergestellt werden muss, dass nach dem Abschluss einer Transaktion auch alle Replikate des angesprochenen Datensatzes aktualisiert werden.

- Die „Availability“ meint die Verfügbarkeit in Form von Latenzzeiten der Antwort auf eine Anfrage. Das System ist verfügbar, wenn es auf alle Anfragen antwortet.

- Die „Partition Tolerance“ ist erfüllt, sofern das System bei einer Unterbrechung der Kommunikation mit einem Teil der Knoten noch funktionstüchtig bleibt.

Nicht-relationale Datenbanken lassen sich in verschiedene Arten und Ausprägungen gliedern, welche wiederum verschiedene Ansätze zum Repräsentieren und Speichern von Datensätzen verfolgen. Vereinfacht können sie in die folgenden vier Hauptkategorien unterteilt werden [Coro16]:

- Key-Value Datenbanken sind die am wenigsten komplexen NoSQL-Strukturen, welche Daten in einer schemalosen Weise speichern. Die Datensätze bestehen aus indizierten Schlüsseln und Werten. Jeder Wert ist hierbei mit einem eindeutigen Schlüssel verknüpft. Zum Beispiel: Cassandra, Azure, LevelDB und Riak.

- Column-Store oder Wide-Column-Store speichern Informationen in Datensätzen mit vielen dynamischen Spalten und daher oft als zweidimensionale Key-Value Stores bezeichnet. Zum Beispiel: HBase, BigTable, HyperTable.

- Dokumenten-Datenbanken zeichnen sich durch eine schemafreie Organisation der Daten aus. Eine Dokumenten-Datenbank speichert Informationen in verschiedenen Dokumenten meist im JSON-Format ab. Zum Beispiel: MongoDB, CouchDB.

- Graph-Datenbanken nutzen Graphen, bestehend aus Knoten und Kanten sowie den Verbindungen zwischen den Knoten, um stark vernetzte Informationen darzustellen und abzuspeichern. Zum Beispiel: Polyglot, Neo4J. 


\subsection{MongoDB}

Die schon zuvor, im Rahmen der dokumentenbasierten Datenbanken, genannte MongoDB stellt hier ein prominentes Beispiel für den Erfolg sogenannter NoSQL-Datenbaken dar. Diese Datenbank stellt eine teilweise schemalose dokumentenbasierte Verwaltung bereit. Beim Einsatz einer MongoDB sind die Datenbank an sich, Collections sowie die Dokumente einer Collection zu beachten. So kann ein MongoDB-Prozess mehrere Datenbanken verwalten, die wiederum individuell betrachtet werden und demensprechend konfigurierbar sind.

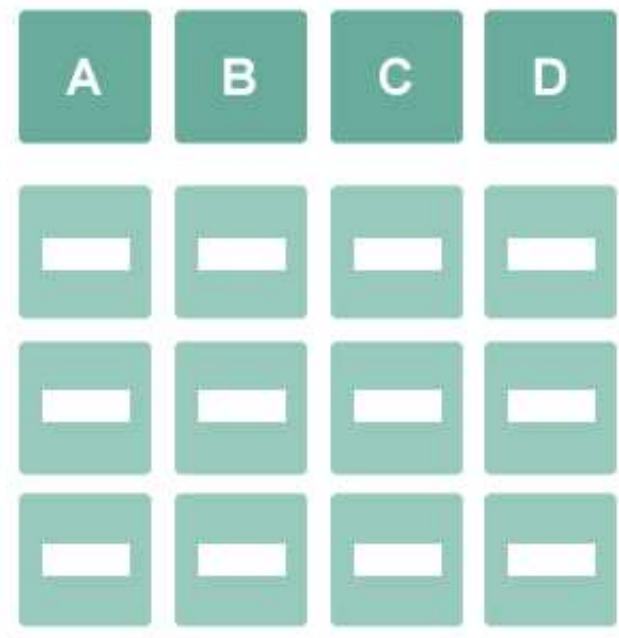

Relationales Datenmodel

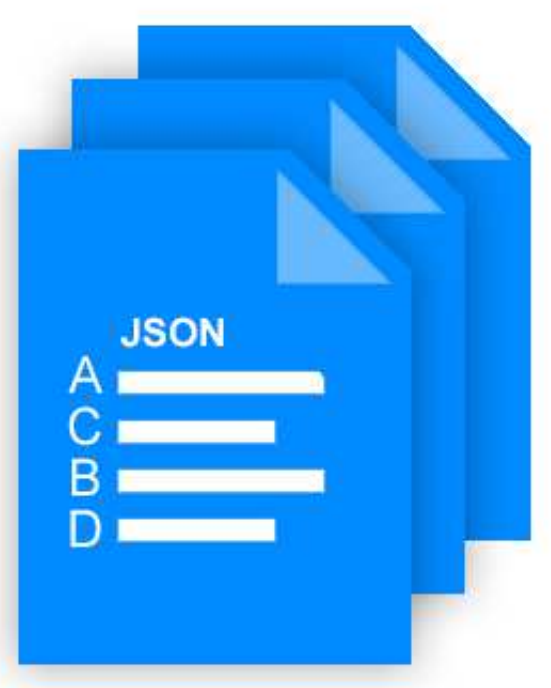

Dokumenten-basiertes Datenmodel

Abbildung 1: Schematischer Vergleich von SQL und NoSQL-Strukturen

Jede Datenbank besteht aus einer Menge an Collections. Eine Collection ist vergleichbar mit der Tabelle einer relationalen Datenbank. Im Gegensatz zu Tabellen werden Collections erst bei Bedarf angelegt und müssen nicht im Vorhinein definiert werden. Man unterscheidet hierbei zwischen allgemeinen Collections, sowie zwei Spezialformen, den System- und den Capped-Collections. Die System-Collections sind für die allgemeine Verwaltung der Datenbank zuständig und spielen bei der Datenhaltung keine Rolle. Capped-Collections wiederum ermöglichen es, einen größen- oder mengenbeschränkten Speicher, ähnlich dem Ringspeicher, zu erstellen.

Eine Collection besteht aus einer Vielzahl an Dokumenten (Siehe Abbildung 1). Ein Dokument ist vergleichbar mit der Zeile einer SQL-Struktur. Es enthält eine beliebige Anzahl an Key-Value-Paaren im JSON-Format. Technisch gesehen wird das Dokument im BSON (Binäres JSON) abgespeichert. Die Struktur eines JSON-Dokuments muss allerdings keinem einheitlichen Schema folgen. Das bedeutet, Werte desselben Schlüssels können verschiedene Datentypen besitzen. MongoDB gibt ein rudimentäres Schemadesign für JSONStrings vor und ermöglicht es folgende Datentypen festzulegen: Integer, Float, String, Array, Boolean, Date, Embedded-Doc, DBRef sowie die ObjectID. Die ObjectID eines Dokuments, ist für die eindeutige Identifizierung eines Datensatzes notwendig. Sie kann manuell für 
jeden Datensatz oder vom MongoDB-System automatisiert vergeben werden. Die ObjectID ist ein unique key, das bedeutet, dass jede ID nur einmalig vorkommen darf. Die Collection legt den Namensraum fest, in dem die Dokumente abgespeichert werden (z.B. user.student.informatik).

\begin{tabular}{|c|c|c|}
\hline Operation & MySQL & MongoDB \\
\hline Schreiben & $\begin{array}{c}\text { INSERT INTO users } \\
\text { (user_id, age, status) } \\
\text { VALUES }\left(, 1324^{\prime}, 45, \text { A' }^{\prime}\right)\end{array}$ & $\begin{array}{c}\text { db.users.insert(\{ user_id: ,1324', } \\
\text { age: } 45 \text {, status: ,A'\}) }\end{array}$ \\
\hline Lesen & SELECT * FROM users & db.users.find() \\
\hline Ändern & $\begin{array}{l}\text { UPDATE users SET status }=, C^{\prime} \\
\text { WHERE age }>25\end{array}$ & $\begin{array}{c}\text { db.users.update }( \\
\{\text { age: }\{\$ \text { gt: } 25\}\},\{\$ \text { set: }\{\text { sta- } \\
\text { tus: , C' }\}\},\{\text { multi: true }\} \\
\text { ) }\end{array}$ \\
\hline Löschen & DROP TABLE people & db.people.drop() \\
\hline Index anlegen & $\begin{array}{c}\text { CREATE INDEX } \\
\text { idx_user_id_ascON peo- } \\
\text { ple(user_id) }\end{array}$ & $\begin{array}{l}\text { db.people.createlndex( }\{\text { user_id: } \\
1\})\end{array}$ \\
\hline
\end{tabular}

Tab. 1: Vergleich der MySQL und MongoDB Syntax

Die Syntax (siehe Tabelle 1) der MongoDB basiert auf JavaScript basierten Funktionen und JSON-Strings, mithilfe derer bestimmte Key-Value-Paare übergeben werden. MongoDB benutzt nur wenige Query-Operatoren, um eine reibungslose Skalierung und Replikation zu gewährleisten. Jede Query kann einen Index nutzen, sofern dieser zuvor angelegt wurde. MongoDB erlaubt es, jedes einzelne Feld eines Dokuments abzufragen. Auch Bereichsabfragen, das Suchen nach regulären Ausdrücken und weitere Spezialabfragen anhand von benutzerdefinierte JavaScript-Funktionen sind möglich. Das Aggregation Framework und MapReduce queries ermöglichen zum Beispiel komplexe collectionübergreifende Abfragen, ähnlich einer SQL GROUP BY-Anweisung [Brad17].

\section{Use-Case}

Anhand von vorliegenden Datensätzen aus einer bestehenden MySQL-Datenbank einer Reiseblogging-Applikation namens Traveloca soll eine Migration und Verdichtung der vorliegenden Daten in eine MongoDB erfolgen. Traveloca (www.traveloca.com) ist ein Reiseblogging-Dienst, der es Reisenden ermöglicht, Reiseblogs zu erstellen und Reiseinhalte in Form von Beiträgen zu teilen.

Der erzeugte Datensatz wird anschließend der Auswertung und Analyse von vorher definierten KPIs der Blogging-Community dienen. Die neu erzeugten Datensätze sollen BIFunktionalität ermöglichen und auf einem separaten Server für Auswertungen bereitstehen. Der Prozess muss stetig wiederholbar sein, um aktuelle Daten aus dem Produktivsystem nachladen zu können. Hierzu sind mehrere Schritte nötig: Zunächst müssen die relevanten Informationen in der SQL-Datenbank definiert werden. Anschließend müssen diese Daten in eine entsprechende Form, bestehend aus Collections und Dokumenten sowie deren JSONStruktur gebracht werden, sodass eine spätere Analyse der Daten möglich ist. Dabei sind die Relationen zwischen den verschiedenen Datensätzen zu beachten und in die neue 
Struktur zu integrieren. Da MongoDB weder JOINS noch GROUP BY Anweisungen unterstützt, muss die Relation zwischen diesen Informationen auf eine andere Weise dargestellt werden.

\section{$2.1 \mathrm{KPIs}$}

Die Definition von KPIs dient der Identifizierung von relevanten Informationen und deren Relationen innerhalb der SQL-Datenbank. Die benötigten Informationen lassen sich in drei Kategorien gliedern: Informationen zu den Benutzern, den Blogs und deren Beiträge. Zu jeder dieser übergeordneten Kategorien lassen sich bestimmte Kennzahlen definieren (siehe Tabelle 2), die wiederum voneinander abhängig sein können.

\begin{tabular}{|c|c|c|}
\hline Benutzer & Blogs & Beiträge \\
\hline $\begin{array}{c}\text { Anzahl aktiver Nutzer nach } \\
\text { Datum }\end{array}$ & $\begin{array}{c}\text { Anzahl aktiver Blogs nach } \\
\text { Datum }\end{array}$ & $\begin{array}{c}\text { Anzahl der Beiträge nach } \\
\text { Region, Ort, Lat \& Lng }\end{array}$ \\
\hline $\begin{array}{c}\text { Nutzerentwicklung zeitlich } \\
\text { pro Blog }\end{array}$ & $\begin{array}{c}\text { Durchschittliche Beiträge } \\
\text { Beiträge oder Kommentare } \\
\text { nach Schlagwort }\end{array}$ \\
\hline $\begin{array}{c}\text { Nutzer nach Region und } \\
\text { Land }\end{array}$ & Anzahl der Follower pro Blog & $\begin{array}{c}\text { Anzahl der Beiträge nach } \\
\text { zeitlichen Kriterien }\end{array}$ \\
\hline $\begin{array}{c}\text { Durchschnittliche Anzahl } \\
\text { an Blogs pro Nutzer }\end{array}$ & Blogs nach Status & Anzahl Beiträge nach Art \\
\hline $\begin{array}{c}\text { Anzahl der Nutzer nach } \\
\text { Upgrade }\end{array}$ & Blogs nach Sprache & Anzahl Beiträge nach Spra- \\
che \\
\hline $\begin{array}{c}\text { Anzahl der Beiträge pro } \\
\text { Benutzer }\end{array}$ & Anzahl der Beiträge pro Blog & Beiträge nach Status \\
\hline & & $\begin{array}{c}\text { Anzahl an Kommentaren } \\
\text { oder Likes pro Beitrag }\end{array}$ \\
\hline
\end{tabular}

Tab. 2: Schematische Darstellung am Beispiel einiger KPIs

Um nun aus den festgelegten KPIs relevante Informationen aus der Datenbank zu identifizieren, muss zunächst die bisherige Struktur betrachtet werden. Die Ausgangsdatenbank ist auf mehrere Rechner verteilt. So liegen global benötigte Informationen zu Beiträgen, Blogs und Benutzern auf einer Datenbank über mehrere Tabellen verteilt bereit. Die einzelnen Blogs und deren spezifischen Metadaten, Beiträge und Kommentare sind auf zahlreiche Tabellen unterschiedlicher Server verteilt. Hierbei wird ein Hash-Verfahren angewendet, das jedem Blog auf Grundlage seiner ID eine eindeutige Datenbank zu weist welche alle Tabellen des Blogs beinhaltet (Siehe Abbildung 2). Jeder Datenbankserver kann nach diesem Verfahren eine Vielzahl an unterschiedlichen Tabellen von verschiedenen Blogs beinhalten. Die globale Tabelle „Blogs“ beispielsweise enthält alle Blogs mit ihrer ID und Key-Daten wie Status und Sichtbarkeit. Alle blogspezifischen Metadaten wie Titel, Beschreibung oder Nutzer befinden sich in der Metatabelle der entsprechenden Datenbank. Die globale Tabelle "Beiträge“ enthält ausschließlich Daten zur BlogID und BeitragsID und verweist damit auf den entsprechenden Beitrag, der innerhalb der Blog-Datenbank gespeichert wird. Inhalte können aus dieser Tabelle nicht gelesen werden.

Abbildung 2 zeigt die Relationen der Tabellen innerhalb der aktuellen MySQL-Struktur. Die Datenbank auf der linken Seite der Grafik (Global DB) enthält alle global gültigen Tabellen, wie zum Beispiel die Posts, die User sowie deren Metadaten und die Blogs. Die rechte Seite (Blog DB $<\mathrm{N}>$ ) beschreibt die einzelnen Datenbanken, welche blogspezifische Tabellen beinhalten. Die beiden Tabellen „Posts“ und „Blogs“ der globalen Datenbank enthalten lediglich Basis Informationen wie die ID oder den Status. Inhalte wie Text, Beschreibung und 
Metadaten zu den Beiträgen und Blogs werden in den Blogspezifischen Tabellen gespeichert. Zum Auslesen vieler Informationen müssen daher sowohl die globalen als auch speziellen Blog-Tabellen angesprochen werden.

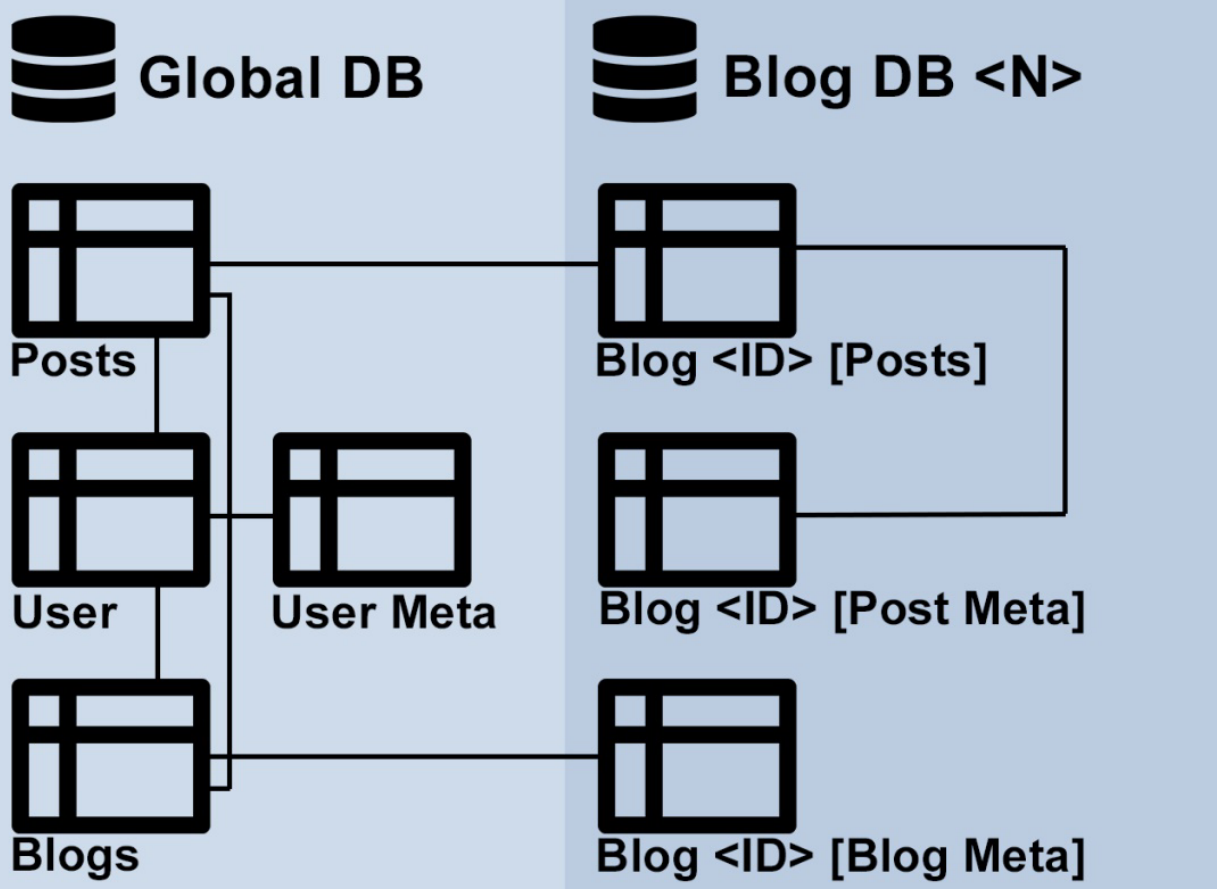

Abbildung 2: Relationen und Aufbau der SQL-Datenbank

Um beispielsweise die Beschreibungen aller Blogs des Users mit dem Namen Max Mustermann auszulesen, werden drei Tabellen angesprochen (User, Blogs und die Blog <ID> Meta Tabelle). Es wird die Benutzer-ID in der User Tabelle mit dem Namen Max Mustermann benötigt, welche in der Blogs Tabelle einem oder mehreren Blogs zugewiesen ist. Über die blogspezifische Metadaten-Tabelle können anschließend die Informationen wie die Beschreibung abgerufen werden. Es müssen also datenbankübergreifende INNER JOIN-Operationen vorgenommen werden.

\section{Migration}

Relevante Informationen für den zu erzeugenden Datensatz kommen in allen, in Abbildung 2, dargestellten Tabellen vor. Da MongoDB keine JOIN-Abfragen kennt, sollte die Komplexität der neuen Datenbankstruktur möglichst gering gehalten werden. Das Ziel ist es die Informationen aus vielen verschiedenen Tabellen in einigen wenigen Collections unterzubringen. Die Informationen lassen sich beispielsweise anhand der in Tabelle 2 definierten Kategorien in drei Collections unterteilen. Jede dieser Collection enthält JSON-Dokumente, die einen demensprechend kompletten Datensatz aller relevanten Informationen zu Benutzern, Blogs oder Beiträgen enthalten (siehe Abbildung 3). 


\section{MongoDB}

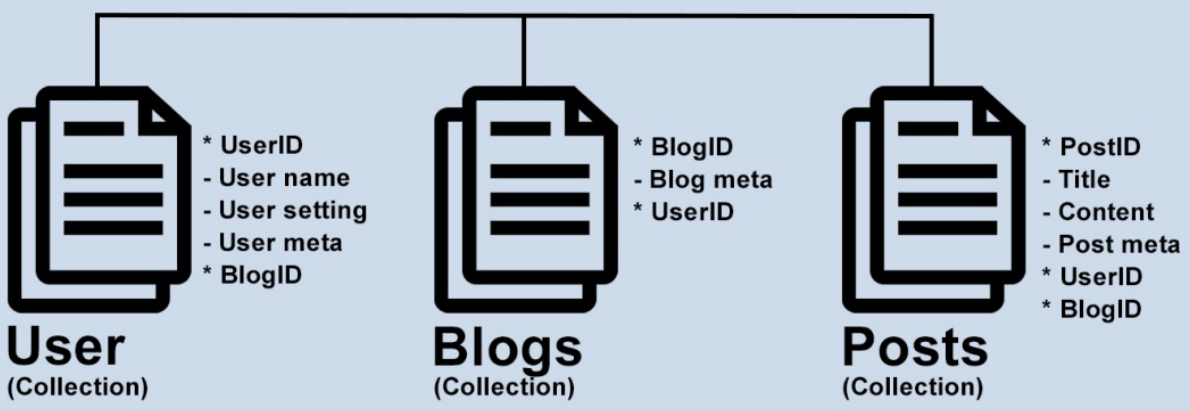

Abbildung 3: Aufbau der MongoDB

MongoDB stellte einige Methoden bereit, um Relationen zwischen Dokumenten herzustellen. Da jedoch keine Queries für diesen Fall existieren, müssen die Zugehörigkeiten von Dokumenten untereinander im Vorfeld definiert werden. Daher muss die Struktur der Dokumente unbedingt im Vornherein betrachtet werden. MongoDB kann solche Verweise durch Embedded-Documents, ObjectID sowie DBref realisieren.

- Embedded-Documents: Diese Methode ermöglicht es ein Dokument in ein anderes Dokument zu Integrieren. Durch die Integration von Teildokumenten eines Top-LevelDokuments, werden weniger Abfragen benötigt. Um zum Beispiel Informationen aus zwei verschiedenen Dokumenten zu erhalten, müssen im Regelfall zwei separate Abfragen durchgeführt werden. Sobald allerdings die Tiefe der Verschachtelung oder die Anzahl an Embedded-Documents steigt, wird ein Dokument schnell unübersichtlich.

- ObjectID: Der Client Driver bzw. die Datenbank generiert die ObjectID eines Dokuments automatisch sofern diese nicht nach einem eigenen Schema definiert wurde. Mithilfe der ObjectID. Innerhalb derselben Collection kann ein Dokument per ObjectID auf ein anderes Dokument verweisen. Somit lassen sich auch komplexere Relationen einfach darstellen.

- DBref: Sollten mehrere Dokumente auf ein Dokument in einer anderen Collection verweisen, kann DBref eingesetzt werden. Ähnlich dem Fremdschlüssel bei relationalen Datenbanken können anhand der ObjectID Verweise auf Dokumente einer bestimmten Collection gesetzt werden.

Jede der Methoden hat Vor- und Nachteile und ist für bestimmte User-Cases besser und für andere wiederum weniger gut geeignet. Hierbei spielen vor allem die späteren Abfragen eine Rolle.

\subsection{Dokumentenstruktur}

Nachdem eine Aufteilung der Daten in verschiedene Collections erfolgt ist, muss die Dokumentenstruktur der einzelnen Dokumente (siehe Abbildung 4) betrachtet werden. Um Relationen aus der SQL-Struktur darzustellen, werden verschiedene der zuvor genannten Methoden benötigt. Die ObjectID der Dokumente wird aus dem Schema der SQL-Datenbank übernommen und nicht vom System erzeugt. Dies vereinfacht später die Implementierung von Verweisen untereinander. Wie in Abbildung 2 zu sehen ist, sind die Metadaten zu allen 
Informationen jeweils in eine separate Tabelle ausgegliedert. Da ein Datensatz immer genau einem Metadatensatz zugeordnet ist, ist es sinnvoll diese Informationen in Form von Embedded-Documents im übergeordneten Datensatz darzustellen.

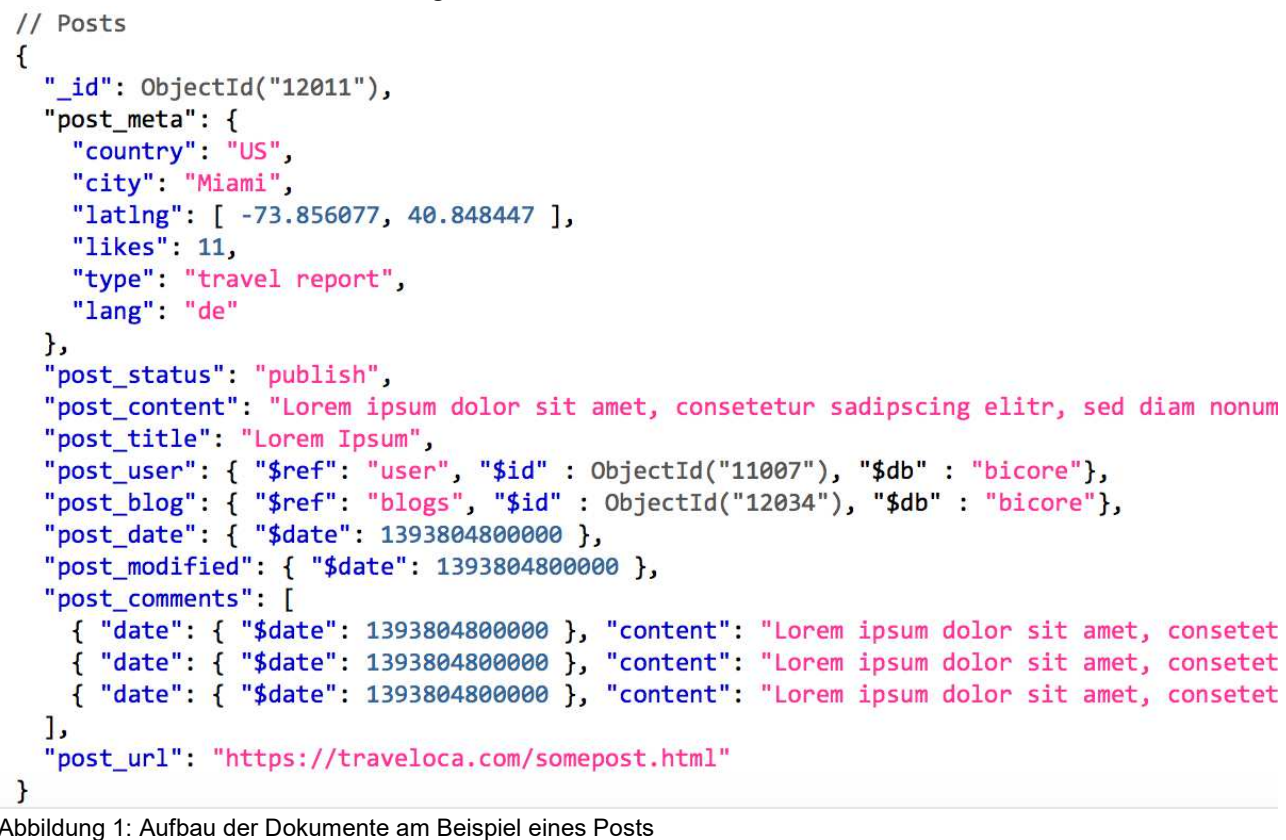

Nun müssen die Verweise zwischen Posts, User und Blogs hergestellt werden. Da jeder Datensatz für eine dieser Gruppen in einem Dokument der gleichnamigen Collection gespeichert ist, bietet es sich an, Verweise in Form von DBrefs zu bilden. Wie man in Abbildung 4 sehen kann werden dem Beitrag ein Autor (post_user) und ein Blog (post_blog) zugewiesen. Über den Schlüssel „post_user“ wird dazu eine Referenz auf das Dokument mit der angegebenen ID 11007 in der Collection „user“ gebildet. Bicore bezeichnet die Datenbank, auf der die Collections liegen.

\subsection{Implementierung}

Um nun die relevanten Daten aus der MySQL-Datenbank zu exportieren und in die MongoDB zu importieren, stehen verschiedene Methoden bereit. Es existieren mittlerweile einige Anbieter wie beispielsweise mongify die eine derartige Funktionalität bereitstellen. Diese sind allerdings häufig auf einmalige Migrationen ausgelegt und erfordern viele Anpassungen. Da der Prozess wie eingangs erwähnt ständig wiederholbar sein muss, um in bestimmten zeitlichen Intervallen aktuelle Daten aus dem Produktivsystem nachzuladen, bietet es sich in diesem Fall an, eine selbst implementierte Funktion zu nutzen. Somit kann der Prozess automatisiert werden, der an die Datenstruktur angepasst ist und ohne großen Aufwand ständig (durch beispielsweise einen Cron-Job) wiederholt werden kann. In diesem Fall wurde ein PHP-Script erstellt, das täglich die neuen Daten aus der Datenbank liest, in JSON-Format speichert und in die MongoDB einspielt. Durch einen MongoDB Driver verbindet sich das Script mit dem Server und lädt den erstellten Datensatz an JSON-Dokumenten in die Datenbank. 


\section{Abfragen am Beispiel des Aggregation Frameworks}

Anhand der Datensätze lassen sich anschließend Abfragen zu Analysezwecken durchführen. Neben einfachen Abfragen nach einzelnen schlüssel-werten Paaren oder Bereichen, ermöglicht MongoDB auch komplexere Abfragen mittels Map-Reduce oder dem Aggregation Framework.

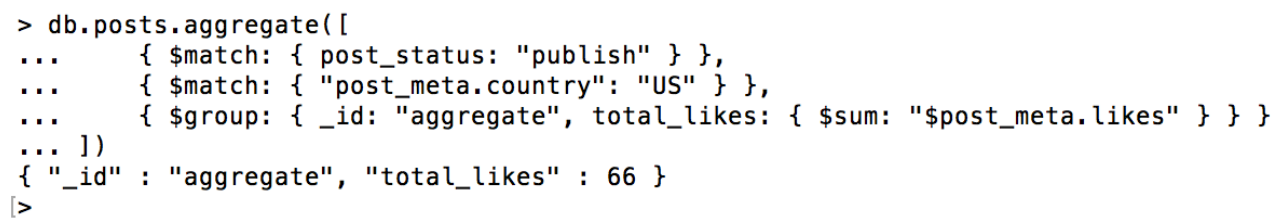

In Abbildung 5 wird eine Abfrage anhand des Aggregation Frameworks durchgeführt. Zunächst wird die Collection „posts“ ausgewählt. Mittels der „\$match“ Operatoren innerhalb der Aggregation Abfrage werden Datensätze anhand bestimmter Schlüssel-Wert-Paare ermittelt. In diesem Fall sollen alle Beiträge mit dem Status „publish“ und dem Standort „US“ ausgelesen werden. Die „\$group“-Anweisung gibt ein Dokument mit der ID „_id“ zurück und summiert in diesem Dokument die Werte "likes“ aus dem Embedded-Dokument „post_meta“, welches die beiden „\$match“ Bedingungen erfüllt. Dieses Dokument könnte nun in Verbindung mit anderen Dokumenten für weitere Abfragen dienen. Dieses Praxisbeispiel zeigt, welche Hürden bei der Migration von Datensätzen einer relationalen Datenbank hin zu einer NoSQL Datenbanken entstehen. Ein besonderes Augenmerk muss hier auf die Struktur der Daten gelegt werden. Anhand dieses Vorgehens lassen sich kostengünstige, skalierbare Möglichkeiten für die Verwaltung von riesigen Datenmengen schaffen auf Basis von NoSQL-Datenbanken schaffen.

\section{Literaturverzeichnis}

[Roeb11] Kubacki W. Mark: Storing and Managing Big Data - NoSQL, Hadoop and More: Highimpact Strategies - What You Need to Know. Tebbo, 2011.

[Meie16] Meier Andreas; Kaufmann Michael: SQL- \& NoSQL-Datenbanken. Springer Vieweg, Hidelberg, 2016.

[Gaur13] Vaish Gaurav: Getting Started with Nosql. Packt Publishing, Birmingham, 2013.

[Coro16] Coronel Carlos; Morris Steven: Database Systems: Design, Implementation, \& Management. Cengage Learning, Boston, 2016.

[Brad17] Bradshaw Shannon; Chodorow Kristina: Mongodb: The Definitive Guide: Powerful and Scalable Data Storage. O'Reilly Media, 2017.

\section{Kontakt}

Prof. Dr. Thomas Barton, Marco Graf

Hochschule Worms

Erenburgerstr. 19, 67549 Worms

barton@hs-worms.de,graf@hs-worms.de 


\section{Aufwärtskompatibilität von Modellierungstechniken für Data Warehouses anhand von 3NF, Dimensional Modeling und Data Vault}

Katja Kubitta, Harald Ritz

\section{Zusammenfassung}

Data Warehouses sind immer stärker gefordert auf neue Anforderungen in kürzester Zeit zu reagieren. Eine gewisse Flexibilität wird erreicht, indem Data Warehouses in mehreren Schichten aufgebaut werden. Dabei ist die zentrale Komponente das Core Data Warehouse (Core DW). Aufbauend auf diesem integrierten Datenbestand werden bspw. Data Marts zu Auswertungszwecken aufgesetzt.

Dieser Artikel untersucht die Aufwärtskompatibilität der drei bekanntesten Modellierungstechniken für Core Data Warehouses: 3NF, Dimensional Modeling und Data Vault. Mit Aufwärtskompatibilität sind dabei die Auswirkungen von Änderung im Core DW hinsichtlich nachgelagerter Data Marts gemeint. Es wird untersucht, ob sich eine Modellierungstechnik bzgl. Aufwärtskompatibilität besonders von den anderen Modellierungstechniken abhebt.

Zur Untersuchung der Aufwärtskompatibilität werden umfangreiche Szenarien von Änderungen an einem Core DW ermittelt. Diese Änderungen werden anhand der jeweiligen Modellierungstechnik durchgeführt und hinsichtlich der Auswirkungen auf bspw. nachgelagerte Data Marts bewertet. Trotz der verschiedenen Vorgehensweisen in den drei Modellierungstechniken zeigen sich hinsichtlich Aufwärtskompatibilität keine wesentlichen Unterschiede zwischen den Modellierungstechniken. Daraus folgt, dass Lösungen hinsichtlich weiterer Flexibilität eher nicht in der Aufwärtskompatibilität, sondern verstärkt in der Entkopplung von Core DW und Data Marts zu suchen sind, indem die Änderungen im Core DW vor Data Marts gekapselt werden.

\section{$1 \quad$ Einleitung}

Zur optimalen Unterstützung verschiedener Fachbereiche oder Prozesse eines Unternehmens wird auf spezialisierte Software zurückgegriffen. Daher liegen die Daten eines solchen Unternehmens in unterschiedlichen Speicherformaten vor. Entscheider benötigen zur Entscheidungsunterstützung allerdings konsistente und integrierte Daten übergreifend aus mehreren Softwaresystemen. Dafür wird eine zentrale Datenbasis benötigt, in der die Daten losgelöst von den unterschiedlichen operativen Systemen rein für vielfältige analytische Zwecke verwendet werden. Eine solche Datenbasis, die durch Integration und Konsolidierung von Daten aus unterschiedlichen operativen Systemen entsteht, wird Data Warehouse genannt (s. [GoRP09], S. 5).

Da Unternehmen heutzutage auf schnell wechselnde Anforderungen reagieren müssen, ist ein Data Warehouse so zu konzipieren, dass es die Flexibilität besitzt, die neuen Anforderungen schnell umzusetzen, damit das Unternehmen wettbewerbsfähig bleibt (s. [TrZi16], S. 1). Ein Konzept, diesen Anforderungen gerecht zu werden, ist der Aufbau eines Data Warehouse in mehreren Schichten. Die zentrale Komponente ist dabei ein Core DW, das den zur Auswertung benötigten Datenbestand eines Unternehmens integriert und historisiert 
speichert (s. [Hahn14], S. 16). Zur besseren Auswertung von Daten werden aufbauend auf dem Core DW weitere Schichten oder Data Marts mit Teildaten aufgesetzt.

Die wechselnden Anforderungen erfordern auch Änderungen im Core DW. Diese Änderungen können sich auf den Datenfluss vom Core DW zu nachgelagerten Schichten oder Data Marts auswirken.

Für die Modellierung eines Core DW existieren unterschiedliche Modellierungstechniken (s. [ArRi12], S.23-32). Der vorliegende Artikel gibt einen Überblick über gängige Modellierungstechniken für Core Data Warehouses und analysiert die Auswirkungen von Änderungen innerhalb dieser Modellierungstechniken auf nachgelagerte Komponenten. Dabei wird darauf geachtet, ein möglichst breites Spektrum an Anwendungsfällen von Änderungen zu betrachten.

Im zweiten Kapitel dieses Artikels werden grundlegend die unterschiedlichen Modellierungstechniken vorgestellt und einige Begriffe erläutert. Das dritte Kapitel analysiert und evaluiert die Anwendungsfälle und deren Änderungen in der Modellierung im Core DW sowie die Auswirkungen dieser Änderungen auf nachgelagerte Komponenten. Im abschließenden vierten Kapitel werden die Ergebnisse zusammengefasst sowie ein Ausblick auf mögliche weiterführende Untersuchungen und Methoden zum Kapseln von Änderungen vor abhängigen Komponenten gegeben.

\section{Grundbegriffe und Modellierungstechniken}

\subsection{Aufwärtskompatibilität}

Der Begriff Aufwärtskompatibilität kommt ursprünglich aus dem Softwarebereich. Der Begriff beschreibt, dass Funktionen, die in einer älteren Softwareversion nutzbar sind, auch in einer neueren Softwareversion weiterhin verwendbar bleiben (s. [LaSi01])

Im Zusammenhang mit dieser Arbeit ist unter dem Begriff Aufwärtskompatibilität folgendes zu verstehen. Vorausgesetzt wird, dass ein Data Warehouse aus mehreren Komponenten besteht. Der zentrale Bestandteil dabei ist ein Core DW. Ausgehend davon werden nachgelagerte Komponenten, wie bspw. Data Marts, mit den Daten des Core DW versorgt. Durch neue Anforderungen oder auch Änderungen wird das Core DW fortlaufend angepasst. Die Aufwärtskompatibilität ist dabei, dass die nachgelagerten Komponenten auch bei einer neuen Version des Core DW über die gleichen Schnittstellen unverändert die Daten erhalten. Damit ist gemeint, dass die nachgelagerten Komponenten keiner Anpassung bedürfen, obwohl sich das Core DW ändert.

\section{$2.2 \quad$ Änderungsszenario}

Ein Änderungsszenario ist eine neue Anforderung von Endbenutzern, die aktuell mit den Daten im Core DW nicht umgesetzt werden kann. Dabei gibt es viele verschiedene Szenarien, die ggf. nur Änderungen oder Erweiterungen an der Beladung des Core DW zur Folge haben, und andere Szenarien, die ggf. eine strukturelle Änderung des Core DW erfordern.

Darunter fallen sowohl die Anforderungen, neue Informationen ins Core DW zu bringen, als auch die Anpassung und Änderung schon vorhandener Informationen, wie bspw. eine Berechnungsformel. Zusätzlich gibt es technische Anforderungen, die Änderungen am Core DW zur Folge haben. Dazu zählen bspw. Namenskonventionen für Tabellen und Spalten. 
Dafür wurden mittels Experteninterviews Änderungsszenarien an einem Core DW ermittelt und kategorisiert. Es wurde darauf geachtet, ein breites Spektrum an Änderungsszenarien zu ermitteln, um eine möglichst umfassende Untersuchung zu erhalten.

\subsection{Normalisierte Modellierungstechnik nach Bill Inmon}

Inmon empfiehlt für das Core DW eine Modellierung, die nah an der dritten Normalform (s. [Geis14], S. 186-191) liegt, und für nachgelagerte Data Marts die dimensionale Modellierung nach Ralph Kimball (s. [InSN08], S.18ff und [Hahn14], S.13-15).

\subsection{Dimensionale Modellierungstechnik nach Ralph Kimball}

Kimball untergliedert seine Modellierungstechnik hauptsächlich in die zwei Bestandteile Faktentabellen und Dimensionstabellen, die im Folgenden erläutert werden.

\subsubsection{Faktentabellen}

In den Faktentabellen stehen die Daten, die zur Messung und Untersuchung eines Geschäftsprozesses relevant sind. So werden in einer Faktentabelle bspw. die Transaktionen von Verkäufen gespeichert, damit sind die Verkaufspositionen (Produkte mit jeweiligem Preis und Anzahl) gemeint. Faktentabellen bestehen zum einen aus Kennzahlen und zum anderen aus Fremdschlüsseln auf die Dimensionstabellen. Der Primärschlüssel der Faktentabelle besteht aus den Fremdschlüsseln auf die Dimensionstabellen oder aus einer Teilmenge von innen (s. [Beck06]).

Mit Kennzahlen werden die zugrunde liegenden Geschäftsprozesse quantifiziert und messbar. Daher liegen diese Kennzahlen in der Regel in numerischer Form vor. Jegliche beschreibenden Attribute, die nicht in direkter Abhängigkeit des einzelnen Faktums stehen, sind in Dimensionstabellen auszulagern (s. [KiRo13], S. 12).

Nahezu alle Faktentabellen haben eine oder mehrere Referenzen auf eine Dimension, die die Zeit beinhaltet. Die Inhalte einer Faktentabelle weisen einen Zeitbezug auf, nämlich die Information, wann das jeweilige Faktum stattgefunden hat (s. [Kimb04]).

Es ist wichtig, darauf zu achten, die Daten in der Faktentabelle möglichst auf atomarer Ebene und nicht aggregiert zu erfassen. Im Beispiel der Verkäufe bedeutet das, dass jede einzelne Verkaufsposition in der Faktentabelle gespeichert wird. Außerdem sind alle Daten in der Faktentabelle mit der gleichen Granularität zu speichern (s. [KiRo13], S. 11). Für jeden notwendigen Detailgrad sollte eine eigene Faktentabelle verwendet werden, um spätere Komplikationen zu vermeiden.

Die Faktentabellen sind die grundlegenden Tabellen für die nachfolgenden Analysen und Abfragen. Daher sind sie auch mit Abstand die größten Tabellen und enthalten meist mehrere Milliarden Datensätze. Über das gesamte Data Warehouse gesehen, machen die Faktentabellen ca. $90 \%$ des gesamten Speicherplatzes aus, während die Dimensionstabellen nur ca. $10 \%$ des Speicherplatzes benötigen (s. [KiRo13], S. 12).

\subsubsection{Dimensionstabellen}

Die Dimensionstabellen enthalten vor allem textliche Informationen zu den Objekten, mit denen die Fakten in Beziehung stehen. Im Beispiel des Verkaufs sind dies das Produkt und der Kunde, an den das Produkt verkauft wird. Diese beiden Objekte stehen mit Einträgen aus der Faktentabelle in Beziehung. Während die Anzahl an Attributen bei den Faktentabellen oft recht klein ist, können Dimensionstabellen eine große Menge an Attributen aufweisen, die vorwiegend Texte beinhalten (s. [KiRo13], S. 13). 
Dimensionstabellen haben als Primärschlüssel eine einzige Spalte, bspw. eine Surrogate ID (SID), die in den Faktentabellen referenziert wird. Die SID dient zur eindeutigen Identifizierung eines Datensatzes in der Dimension (s. [KiRo13], S. 46).

Neben der SID wird in den Dimensionen auch der fachliche Schlüssel gespeichert. Die Inhalte der Dimensionstabellen werden nicht zu Analysezwecken, sondern zur Gruppierung der Daten aus der jeweiligen Faktentabelle verwendet. Ein Beispiel dafür ist die Kundengruppe, die in der Dimensionstabelle Kunde enthalten ist.

Wesentlich ist die Entscheidung, ob die Dimensionen denormalisiert (Star-Schema) oder normalisiert (Snowflake-Schema) modelliert werden.

\subsubsection{Star- und Snowflake-Schema}

Im Star-Schema hat die Faktentabelle die zentrale Position. Die Dimensionstabellen werden um die Faktentabelle herum angeordnet. Der Umriss der Modellierung erinnert dabei an einen Stern, woher das Star-Schema seinen Namen hat. Die Besonderheit dieser Modellierung liegt in der Denormalisierung der Dimensionstabellen. Zu jeder Dimension gibt es nur eine Dimensionstabelle, in der alle Informationen abgelegt werden. So werden auch Hierarchien in den Dimensionstabellen abgebildet, weshalb eine gewisse Redundanz in den Daten der jeweiligen Dimensionstabelle entsteht (s. [BaGü13], S. 243-246).

Bei der normalisierten Form von Dimensionstabellen, auch Snowflake-Schema genannt, werden redundante Daten in eigene Tabellen ausgelagert und anschließend referenziert (s. [KiRo13], S. 15).

\subsection{Data Vault nach Dan Linstedt}

Als wichtigen Unterschied zur normalisierten und dimensionalen Modellierungstechnik werden Schlüssel, deskriptive Informationen und Beziehungen bei Data Vault in drei getrennten Typen von Tabellen abgelegt.

Diese Modellierungstechnik konzentriert sich auf die Modellierung des Core DW. Für Data Marts empfiehlt auch Dan Linstedt die dimensionale Modellierung (s. [Hult12], S. 45).

\subsubsection{Hub}

Ein Hub enthält mehrere gleichartige Objekte der Wirklichkeit, die für das jeweilige Unternehmen von Bedeutung sind. Beispiele dafür sind Kunde, Konto oder Produkt. Hubs enthalten keine beschreibenden Informationen oder Fremdschlüssel.

In Hubs werden die Business Keys (fachliche Schlüssel) der jeweiligen Objekte gespeichert. Beispiele hierfür sind Kundenummern, Kontonummern oder Produktnummern. Dabei können diese Business Keys auch aus mehreren Attributen bestehen. Ist dies der Fall, müssen alle diese Attribute im Hub abgebildet werden (s. [Hult12], S. 84-87).

Weiterhin enthält ein Hub standardmäßig folgende Felder:

- SID: Besteht der Business Key aus mehreren Attributen, so wird eine SID als Primärschlüssel verwendet.

- $\quad$ Load Date: Ein Zeitstempel, der angibt, wann der Business Key zum ersten Mal geladen wurde.

- Record Source: Gibt das Quellsystem an, aus dem der Eintrag stammt.

\subsubsection{Link}

Zur Speicherung von Beziehungen zwischen zwei oder mehreren Hubs oder Links werden Links verwendet. Genau wie ein Hub enthält auch ein Link keine beschreibenden Informationen. In einen Link werden die SIDs der beteiligten Hubs bzw. Links übernommen, wodurch 
die Verbindung zwischen den Hubs bzw. Links hergestellt wird. Diese Vorgehensweise, Verbindungen zu speichern, entspricht einer n:m-Beziehung. Jede Beziehung wird nach Data Vault gemäß einer n:m-Beziehung in einer Link-Tabelle gespeichert (s. [Hult12], S. 94104).

Zusätzlich zu den Fremdschlüsseln auf die SIDs der beteiligten Hubs und Links enthält ein Link die gleichen Felder, analog zum Hub.

\subsubsection{Satellit}

In Satelliten werden die deskriptiven (beschreibenden) Informationen gespeichert und historisiert. Satelliten gehören immer zu genau einem Hub oder Link. Sowohl Hubs als auch Links können beliebig viele Satelliten haben.

Der Primärschlüssel eines Satelliten besteht aus der SID des zugehörigen Hubs oder Links und einem Zeitstempel bzw. Ladedatum mit der Angabe, wann die Informationen in das Data Warehouse geladen wurden (s. [Hult12], S. 114). Dabei sind Satelliten die einzigen Komponenten, die ein zeitliches Attribut als Teil ihres Primärschlüssels verwenden.

Um die Gültigkeit eines Eintrags abzubilden, wird das Ladedatum und optional ein Enddatum verwendet. Jedes Mal, wenn eine Änderung an beschreibenden Informationen erfolgt, wie bspw. eine Änderung des Kundennamens, wird eine neue Zeile mit den geänderten Daten in den Satelliten geladen (s. [Hult12], S. 115).

Wie viele Satelliten ein Hub oder Link erhält, hängt von der Entscheidung ab, ob bzw. wie die deskriptiven Daten gruppiert werden. Eine mögliche und oft verwendete Gruppierung ist die nach der Änderungsrate, mit der sich die einzelnen Informationen ändern (s. [Lins11], S. 78-84).

\section{Untersuchung und Bewertung}

In diesem Kapitel werden die zuvor vorgestellten Modellierungstechniken hinsichtlich ihrer Aufwärtskompatibilität untersucht und bewertet. Grundlage der Untersuchung sind zum einen die ermittelten Änderungsszenarien und zum anderen die gewählten Kriterien zur Bewertung der Änderungsauswirkungen im Core DW sowie in Bezug auf die nachgelagerten Komponenten. Damit die Modellierungstechniken vergleichbar miteinander untersucht werden können, wurde vor der Untersuchung ein einheitliches Grundbeispiel erstellt. An diesem Grundbeispiel wurden anschließend die Änderungen aus den Szenarien jeweils durchgeführt und anhand der folgenden Kriterien untersucht.

\subsection{Bewertungskriterien}

Die Bewertungskriterien bilden die Grundlage für das Beurteilen der Auswirkungen, die die jeweilige Änderung auf die unterschiedlichen Modellierungstechniken hat. Die Kriterien sind so gewählt, dass eine umfangreiche Untersuchung der Änderungsauswirkungen stattfindet. Bei allen Kriterien werden Aufwand und Komplexität betrachtet. Ein hoher Aufwand kann dabei sowohl aus einer hohen Anzahl an Anpassungen als auch aus einer einzelnen großen Anpassung resultieren. Unter Komplexität wird in diesem Artikel die Schwierigkeit von Änderungen verstanden. Je höher die Komplexität einer Änderung ist, desto höher ist die Fehleranfälligkeit ihrer jeweiligen Realisierung und entsprechend der nötige Testaufwand. 


\subsubsection{Umfang Core-Strukturänderung}

Dieses Kriterium drückt aus, wie stark sich die Struktur der Daten im Core DW ändern muss, um die Änderung eines Szenarios abbilden zu können. Zu Strukturänderungen zählen bspw. Ergänzungen oder Änderungen von Tabellen, Attributen oder Fremdschlüsselbeziehungen. Je größer die für ein Szenario nötigen Strukturänderungen sind, desto größer ist der zu erwartende Einfluss, den das Szenario auf nachgelagerte Komponenten hat.

\subsubsection{Umfang Core-Datenänderung}

Dieses Kriterium bewertet, ob oder in welchem Umfang die im Core DW vorhandenen Daten für das Szenario geändert werden müssen. Je nach Szenario können bspw. Datenkorrekturen, Datenergänzungen oder Datentransfers nötig werden. Datenänderungen im Core DW haben meist Auswirkungen auf nachgelagerte Komponenten.

\subsubsection{Umfang Core-Beladungsänderung}

Mit diesem Kriterium wird beschrieben, wie umfangreich die ETL-Prozesse zur Beladung des Core DW angepasst werden müssen, um die Änderung des jeweiligen Szenarios abbilden zu können. Erstellungen von neuen ETL-Prozessen sowie Änderungen oder Beendigungen bestehender ETL-Prozesse können je nach Szenario erforderlich werden.

Bei manchen Szenarien hängt die Anzahl der anzupassenden ETL-Prozesse von Faktoren $a b$, die sich nicht aus dem Szenario selbst ergeben, so z.B. von der Anzahl der vorhandenen Fremdschlüsselbeziehungen zu betroffenen Tabellen. In solchen Fällen spiegelt dieses Kriterium den Mindestumfang an Anpassungen wider, und ein hochgestelltes Sternchen $\left(^{*}\right)$ hinter der Bewertung gibt an, dass der tatsächliche Umfang aufgrund äußerer Faktoren höher sein kann.

\subsubsection{Aufwärtskompatibilität}

Szenarien, die keine Auswirkungen auf nachgelagerte Komponenten haben, sind aufwärtskompatibel. Die Auswirkungen von Szenarien, die die Aufwärtskompatibilität nicht erfüllen, können unter Umständen vor nachgelagerten Komponenten verborgen werden, indem die ETL-Prozesse, die die nachgelagerten Komponenten beladen, so angepasst werden, dass sie die Auswirkungen kapseln. Der Umfang der zur Kapselung nötigen Anpassungen an den ETL-Prozessen wird durch dieses Kriterium bewertet.

Die Bewertung bezieht sich dabei stets auf genau eine nachgelagerte Komponente. Für jede weitere nachgelagerte Komponente muss die Anpassung erneut durchgeführt werden, wobei davon ausgegangen wird, dass der Umfang der Anpassungen zwischen verschiedenen nachgelagerten Komponenten jeweils ähnlich ist.

Kann eine Änderung vor einer nachgelagerten Komponente auch durch Anpassung eines ETL-Prozesses nicht gekapselt werden, so müssen die nachgelagerten Komponenten angepasst werden. Dieser Fall wird in der Bewertung gesondert berücksichtigt.

\subsection{Bewertungsskala}

Alle vier Bewertungskriterien nutzen eine gemeinsame Bewertungsskala. Dadurch sind die Bewertungen der Kriterien untereinander vergleichbar.

Insgesamt hat sich zur Bewertung eine gemeinsame Skala als zweckmäßig erwiesen, die Aufwand und Komplexität zusammenfasst. Dabei werden Aufwand und Komplexität jeweils in drei Stufen unterteilt (niedrig, mittel, hoch), wobei sich die Komplexität nur bei hohen Aufwänden in der Bewertung widerspiegelt. Auf diese Weise werden komplizierte Änderungen höher gewichtet als aufwendige aber einfache Änderungen. Dadurch werden bei kom- 
plizierten Änderungen die höhere Fehleranfälligkeit und der höhere Testaufwand berücksichtigt.

Die Skala verfügt zusätzlich über zwei Extremstufen, die die beiden Sonderfälle abbilden, dass eine Änderung einerseits unnötig oder andererseits unmöglich ist (Stufen 0 und 6).

\begin{tabular}{|c|c|}
\hline Bewertung & Beschreibung \\
\hline 0 & unnötig / direkt gegeben \\
\hline 1 & niedriger Aufwand, niedrige Komplexität \\
\hline 2 & mittlerer Aufwand, niedrige Komplexität \\
\hline 3 & hoher Aufwand, niedrige Komplexität \\
\hline 4 & hoher Aufwand, mittlere Komplexität \\
\hline 5 & hoher Aufwand, hohe Komplexität \\
\hline 6 & unmöglich \\
\hline
\end{tabular}

Tab. 1.: Bewertungsskala

\section{3 Änderungsszenarien}

In der folgenden Tabelle werden die untersuchten Änderungsszenarien aufgelistet. Die Szenarien sind mit einer Abkürzung versehen, um sie in der Gesamtübersicht (Tab. 3) wiederzufinden.

\begin{tabular}{|c|c|}
\hline Abkürzung & Szenario \\
\hline NA & Neues Attribut \\
\hline NE & Neuer Entitytyp \\
\hline VAn & Veränderung eines Attributnamens \\
\hline VEn & Veränderung eines Entitytypnamens \\
\hline AzE & Attribut wird zu neuem Entitytyp \\
\hline Kn:m & Kardinalität 1:n wird n:m \\
\hline EG & Erhöhung der Granularität \\
\hline VG & Verringerung der Granularität \\
\hline FSE & Fachlichen Schlüssel erweitern \\
\hline FSR & Fachlichen Schlüssel reduzieren \\
\hline NQE & Neue Quelle zu bestehendem Entitytyp \\
\hline DA & Datentyp eines Attributs ändern \\
\hline ÄH & Änderung der Historisierung \\
\hline DV:AS & Data Vault: Aufsplittung von Satelliten \\
\hline BA & Berechnung eines Attributs ändern \\
\hline ÄF & Änderung im Filter \\
\hline & Tab. 2.: Änderungsszenarien
\end{tabular}

Einige dieser Änderungsszenarien sind aus ihrem Namen heraus verständlich, die übrigen Szenarien werden im Folgenden kurz erläutert.

Unter dem Szenario Attribut wird zu neuem Entitytyp wird verstanden, dass ein Attribut in eine eigene Tabelle ausgelagert und über einen Fremdschlüssel mit der ursprünglichen Tabelle verbunden wird.

Bei der Erhöhung der Granularität wird der Fall untersucht, dass der Detailgrad der Daten im Core DW abnimmt. Damit ist bspw. gemeint, dass Daten bisher auf Tagesbasis ins Core DW geladen wurden und nach der Änderung kumuliert einmal pro Monat ins Core DW geladen werden. Die Verringerung der Granularität untersucht das Gegenteil.

Das Szenario Fachlichen Schlüssel erweitern untersucht den Fall, dass der bisherige fachliche Schlüssel nicht ausreicht, um ein Objekt in einem Unternehmen eindeutig zu identifizieren. Ein Beispiel dafür ist, dass ein Kunde nur durch die Kundennummer in Verbindung mit einer Mandantennummer eindeutig identifiziert werden kann, während der fachliche Schlüs- 
sel bisher nur aus der Kundennummer bestand. Das Szenario Fachlichen Schlüssel reduzieren untersucht das Gegenteil.

Im Szenario Neue Quelle zu bestehendem Entitytyp wird untersucht, was sich ändert, wenn Daten aus einer weiteren Quelle in eine bestehende Tabelle geladen werden.

Beim Szenario Änderung der Historisierung wird untersucht, welche Auswirkungen es hat, wenn ein Attribut in der Historisierung von SCD Typ 1 auf SCD Typ 2 (Slowly Changing Dimensions nach Kimball, s. [KiRo13], S. 53-56) geändert wird. Dieses Szenario kann für Data Vault nicht untersucht werden, da die Historisierung in der Modellierung vorgeben ist. Als Ersatz wurde das Szenario Data Vault: Aufsplittung von Satelliten untersucht. Dabei werden die Attribute eines Satelliten in zwei oder mehrere Satelliten aufgeteilt.

\subsection{Untersuchungsergebnisse}

Die folgende Tabelle zeigt die ermittelten Bewertungen der untersuchten Szenarien. Die Abkürzungen stehen für die Szenarien aus Tabelle 2.

\begin{tabular}{|c|c|c|c|c|c|c|c|c|c|c|c|c|}
\hline & \multicolumn{4}{|c|}{ Inmon } & \multicolumn{4}{|c|}{ Kimball } & \multicolumn{4}{|c|}{ Data Vault } \\
\hline & 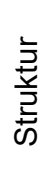 & $\begin{array}{l}\frac{c}{d} \\
\stackrel{\mathbb{N}}{0}\end{array}$ & $\begin{array}{l}\frac{1}{0} \\
\frac{0}{0} \\
\frac{\pi}{0} \\
\infty\end{array}$ & $\begin{array}{l}\dot{0} \\
\stackrel{2}{E} \\
\frac{0}{1} \\
\frac{1}{3} \\
\frac{3}{2}\end{array}$ & 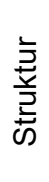 & $\begin{array}{l}\frac{c}{d} \\
\frac{\pi}{\pi} \\
\frac{0}{\sigma}\end{array}$ & $\begin{array}{l}\frac{c}{\Phi} \\
\frac{0}{0} \\
\frac{\pi}{\infty} \\
\infty\end{array}$ & 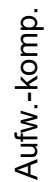 & 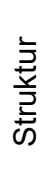 & $\begin{array}{l}\frac{c}{d} \\
\frac{ \pm}{\pi} \\
0\end{array}$ & $\frac{\frac{c}{d}}{\frac{d}{0}} \frac{\bar{d}}{\frac{D}{\infty}}$ & 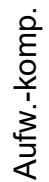 \\
\hline NA & 1 & 0 & 1 & 0 & 1 & 0 & 1 & 0 & 1 & 0 & 1 & 0 \\
\hline $\mathrm{NE}$ & 1 & 0 & 1 & 0 & 1 & 1 & 1 & 0 & $2^{*}$ & 0 & $2^{*}$ & 0 \\
\hline VAn & 1 & 0 & 1 & 1 & 1 & 0 & 1 & 1 & 1 & 0 & 1 & 1 \\
\hline VEn & 1 & 0 & $1^{*}$ & 1 & 1 & 0 & $1^{*}$ & 1 & 1 & 0 & $2^{*}$ & 1 \\
\hline AzE & 1 & 2 & 1 & 1 & 1 & 2 & 1 & 1 & 2 & 3 & 2 & 2 \\
\hline $\mathrm{Kn}: \mathrm{m}$ & 1 & 1 & 1 & 6 & 2 & 2 & 5 & 6 & 0 & 0 & 1 & 6 \\
\hline$E G$ & 1 & 0 & 4 & 6 & 1 & 0 & 4 & 6 & 1 & 0 & 4 & 6 \\
\hline VG & 2 & 0 & 2 & 4 & 1 & 0 & 1 & 4 & 3 & 0 & 3 & 4 \\
\hline FSE & 1 & 1 & $1^{*}$ & 6 & 0 & 5 & $1^{*}$ & 6 & 1 & 0 & 1 & 6 \\
\hline FSR & 0 & 5 & $1^{*}$ & 6 & 0 & 5 & $1^{*}$ & 6 & $2^{*}$ & $3^{*}$ & $2^{*}$ & 6 \\
\hline NQE & 0 & 0 & 1 & 1 & 0 & 0 & 1 & 1 & 0 & 0 & $2^{*}$ & 1 \\
\hline DA & 1 & 0 & 1 & 6 & 1 & 0 & 1 & 6 & 1 & 0 & 1 & 6 \\
\hline ÄH & 0 & 0 & 1 & 1 & 0 & 0 & 1 & 1 & - & - & - & - \\
\hline DV:AS & - & - & - & - & - & - & - & - & $2^{*}$ & 5 & $1^{*}$ & 1 \\
\hline BA & 0 & 0 & 1 & 1 & 0 & 0 & 1 & 1 & 0 & 0 & 1 & 1 \\
\hline ÄF & 0 & 0 & 1 & 1 & 0 & 0 & 1 & 1 & 0 & 0 & 1 & 1 \\
\hline
\end{tabular}

\section{$4 \quad$ Fazit und Ausblick}

Dieser Artikel beschäftigt sich mit der Frage, ob eine der gängigen Modellierungstechniken für Core DWs (Inmon, Kimball oder Data Vault) sich hinsichtlich der Aufwärtskompatibilität gegenüber nachgelagerten Komponenten besonders eignet. Denn Änderungen am Core DW sind nötig, um neuen Anforderungen gerecht zu werden. Dabei sollen bestehende nachgelagerte Komponenten, die nicht durch die Änderung beeinflusst werden sollen, nach Möglichkeit weiterhin ihre Daten ohne Anpassung erhalten können. Hat eine Änderung im 
Core DW keine Auswirkungen auf den Datenfluss zu nachgelagerten Komponenten, so ist diese aufwärtskompatibel.

Ist eine Änderung nicht aufwärtskompatibel, so können ihre Auswirkungen unter Umständen vor nachgelagerte Komponenten verborgen (gekapselt) werden. Der Umfang einer solchen Anpassung der ETL-Prozesse ist ein Maß für die Kapselbarkeit einer Änderung und wurde im Rahmen dieses Artikels ebenfalls untersucht und bewertet. Demgegenüber sind manche Änderungen weder aufwärtskompatibel noch kapselbar. Bei solchen Änderungen muss ggf. jede nachgelagerte Komponente separat bzgl. der Änderung angepasst werden.

Aus den Bewertungen geht hervor, dass bei den untersuchten Szenarien sowohl die Aufwärtskompatibilität als auch die Kapselbarkeit von der Modellierungstechnik des Core DW unabhängig sind. Lediglich der Kapselungsumfang variiert bei Änderungen, die nicht aufwärtskompatibel aber kapselbar sind, zwischen den verschiedenen Modellierungstechniken. Insgesamt ist damit von den untersuchten Modellierungstechniken keine als besonders geeignet bzgl. Aufwärtskompatibilität hervorzuheben. Lediglich bei Erweiterungen im Core DW haben sich alle Modellierungstechniken als vollständig aufwärtskompatibel erwiesen, weil die betreffenden Daten bisher nicht an die nachgelagerten Komponenten weitergegeben wurden.

Aus dieser Untersuchung folgt, dass die Aufwärtskompatibilität kein Kriterium bei der Wahl der Modellierungstechnik für ein Core DW ist. Denn für die Modellierung eines Core DW sind die spezifischen Eigenschaften der Modellierungstechniken, wie etwa die starke Ausrichtung auf Geschäftsprozesse bei der dimensionalen Modellierung nach Kimball, wesentlich relevantere Kriterien als die geringfügigen Unterschiede bzgl. des Kapselungsumfangs von Änderungen.

Weiterhin lässt das Ergebnis der Untersuchung die Vermutung zu, dass die Kapselbarkeit von Szenarien unabhängig von den Modellierungstechniken ist. Aus der Untersuchung geht hervor, dass bei einigen Szenarien die Kapselbarkeit daran scheitert, dass Aggregationen in nachgelagerten Komponenten nach der Änderung zu fehlerhaften Daten führen können. Daher ist eine der offenen Fragen, ob sich eine Modellierungstechnik finden lässt, bei der auch nach der Änderung solche Aggregationen in nachgelagerten Komponenten korrekte Ergebnisse liefern. Gegebenenfalls lässt sich aber auch zeigen, dass bestimmte Änderungen grundsätzlich bei keiner Modellierungstechnik gekapselt werden können.

Ein anderer Ansatz zur Reduktion des Kapselungsaufwands ist, das Core DW und die nachgelagerten Komponenten durch eine Zwischenschicht zu entkoppeln. Dabei müssen die Änderungen aus dem Core DW nur in der Zwischenschicht gekapselt werden und der Datenfluss Richtung Data Marts bleibt unverändert. Eine Idee, diese Kapselschicht aufzubauen, liegt in der Nutzung von Datenbanksichten. Ferner ergibt sich die Frage, ob weitere Möglichkeiten für den Aufbau einer solchen Kapselschicht existieren. Gegebenenfalls lassen sich Kapselungen bestimmter Änderungen automatisieren, sodass der Aufwand für die Kapselung weiter reduziert werden kann.

\section{Literaturverzeichnis}

[ArRi12] Arnold, Christoph und Ritz, Harald: Eignung unterschiedlicher Faktenmodellierungen in Data-Warehouse-Systemen, in: Barton, Thomas u.a. (Hrsg.): Herausforderungen an die Wirtschaftsinformatik: Management und IT, News \& Media, Berlin 2012, S.23-40. 
[Beck06] Becker, Bob: Design Tip \#81 Fact Table Surrogate Key, 2006, URL: http://www.kimballgroup.com/2006/07/design-tip-81-fact-table-surrogate-key/. Abruf am 2017-05-02.

[BaGü13] Bauer, Andreas und Günzel, Holger: Data-Warehouse-Systeme: Architektur, Entwicklung, Anwendung. dpunkt.verlag, Heidelberg, 2013.

[Geis14] Geisler, Frank: Datenbanken: Grundlagen und Design. mitp-Verlag, Heidelberg, 2014.

[GoRP09] Golfarelli, Matteo; Rizzi, Stefano und Pagliarani, Claudio: Data Warehouse Design: Modern Principles and Methodologies. McGraw-Hill, New York, 2009.

[Hahn14] Hahne, Michael: Modellierung von Business-Intelligence-Systemen: Leitfaden für erfolgreiche Projekte auf Basis flexibler Data-Warehouse-Architekturen. Edition TDWI. dpunkt.verlag, Heidelberg, 2014.

[Hult12] Hultgren, Hans: Modeling the Agile Data Warehouse with Data Vault. New Hamilton, Denver and Stockholm and NYC and Sydney, 2012.

[InSN08] Inmon, William H.; Strauss, Derek und Neushloss, Genia: DW 2.0 - The Architecture for the Next Generation of Data Warehousing. Morgan Kaufmann, Amsterdam, 2008

[Kimb04] Kimball, Ralph: Design Tip \#51: Latest Thinking On Time Dimension Tables, 2004, URL: $\quad$ http://www.kimballgroup.com/2004/02/design-tip-51-latest-thinking-on-time-dimension-tables/. Abruf am 2017-05-02

[KiRo13] Kimball, Ralph und Ross, Margy: The Data Warehouse Toolkit: The Definitive Guide to Dimensional Modeling. Wiley, Indianapolis, 2013.

[LaSi01] Lackes, Richard und Siepermann, Markus: Definition Aufwärtskompatibilität. 2001, URL: http://wirtschaftslexikon.gabler.de/Definition/aufwaertskompatibilitaet.html. Abruf am 2017-05-02.

[Lins11] Linstedt, Dan: Super Charge your Data Warehouse: Invaluable Data Modeling Rules to Implement Your Data Vault. Saint Albans, Vermont, 2011.

[TrZi16] Trahasch, Stephan und Zimmer, Michael (Hrsg.): Agile Business Intelligence: Theorie und Praxis. dpunkt.verlag, Heidelberg, 2016.

\section{Kontakt}

Prof. Dr. Harald Ritz, Katja Kubitta (B. Sc. Wirtsch.-Inform.)

Technische Hochschule Mittelhessen (THM)

Campus Gießen, Fachbereich MNI

Wiesenstraße 14, 35390 Gießen

T+49 641 309-2431, harald.ritz@mni.thm.de 


\title{
Modellgetriebene Entwicklung - Vor- und Nachteile in der Test- und Ein- führungsphase
}

\author{
Melanie Vanderpuye, Marco Richter, Michael Guckert
}

\section{Zusammenfassung}

Auf der AKWI Tagung 2015 stellten wir in dem Konferenzbeitrag "Modellgetriebene Entwicklung einer Eclipse RAP-Anwendung unter Verwendung des Eclipse Modeling Frameworks" das Projekt IsyPlus vor, in dem ein neues Campus-Management System für das duale Angebot StudiumPlus der Technischen Hochschule Mittelhessen entwickelt werden sollte. Die Software ist nun fertiggestellt und wurde nahezu vollständig modellgetrieben entwickelt. Neben der CRUD Funktionalität wurden insbesondere auch die GUI sowie das Berechtigungsmodell komplett aus entsprechenden Modellen generiert. Neben dem mit BIRT-Elementen umgesetzten Berichtswesen wurden einzelne Prozessabläufen gezielt konventionell implementiert. Diese Abläufe integrieren die generierten Komponenten und bilden eine Klammer um die Einzelfunktionen. In der ca. dreijährigen Entwicklungszeit hat sich der modellgetriebene Ansatz absolut bewährt. Im Laufe des nach einem evolutionären Ansatz durchgeführten Projekts wurde das Klassenmodell wiederholt überarbeitet. Kompliziertere Zusammenhänge machten ein wiederholtes Refactoring notwendig. Diese Modelländerungen konnten durch den im Projekt entwickelten Generator immer wieder mit vergleichsweise geringem Aufwand im lauffähigen Code berücksichtigt werden, so dass stets ein frühes Testen der gesamten Software möglich blieb. Der Artikel vergleicht die Funktionalität und den Aufwand der Entwicklung mit den Erfahrungen aus der Entwicklung der abgelösten Software und diskutiert Schwierigkeiten, die aus dem allgemeinen Ansatz entstanden sind (z.B. Performance) und deren Lösung durch ergänzende konventionelle Programmierung. Darüber hinaus werden generelle Schlüsse für ein Vorgehen in einem derartigen Projekt gezogen.

\section{Entscheidung für eine Neuentwicklung - die Vorphase des Projekts}

StudiumPlus ist das duale und berufsbegleitende Studienkonzept der Technischen Hochschule Mittehessen (THM). (Vgl. [StudP17]) Die speziellen Anforderungen des dualen Studiums an ein Campus Management System - insbesondere die Abbildung der Zusammenarbeit mit den Partnerunternehmen - werden in der angebotenen Standardsoftware nur unzureichend oder gar nicht erfüllt. Daher fiel im Jahr 2003 die Entscheidung für die hausinterne Entwicklung von IsyPlus.

IsyPlus ist ein Informationssystem zur Verwaltung von personenbezogene Hochschuldaten zum dualen Studium. Zusätzlich unterstützt die Anwendung die administrativ tätigen Mitarbeiter bei der Durchführung verschiedener Arbeitsprozesse, die im Rahmen des Studienbetriebs erforderlich sind. Da die Software nicht mehr den Anforderungen gerecht wurde und Prozesse zunehmend nicht mehr vollständig unterstützt wurden, musste das System ersetzt werden. Aus diesem Grund fiel 2014 der Startschuss für eine Neuentwicklung des Systems (Vgl. [RGVM15], S. 215ff.) mit der Entscheidung dabei ganz neue Wege zu gehen. 
Das zu modellierende Umfeld unterliegt sich stetig ändernden Gegebenheiten. So kamen seit Gründung von StudiumPlus neben zusätzlichen Studienorten und Studiengängen auch völlig neue Tätigkeitsfelder hinzu. Folglich wurde ein modellgetriebener Ansatz zur Umsetzung des neuen Systems gewählt. Dieses Vorgehen versprach die nötige Flexibilität für den Umgang mit einer sich stetig ändernden Umwelt.

Als begleitendes Ziel sollen die im Rahmen der Softwareentwicklung erarbeiteten Erkenntnisse in Lehrveranstaltungen einfließen. In den einschlägigen Veranstaltungen werden im Wesentlichen OpenSource-Produkte wie MySQL und Eclipse zur Vermittlung der Lehrinhalte verwendet. Aufgrund dessen lag die Verwendung von MySQL und Eclipse RAP für dieses Projekt nahe. Der im Projekt mittels der Sprache Xtend entwickelte Generator generiert aus angereicherten EMF Ecore-Modellen lauffähigen Java-Code für die Zielplattform.

\section{Die Entwicklungsphase der Anwendung IsyPlus}

Die Entwicklungsphase begann nach der Entscheidung für die Neuentwicklung im Jahr 2014 und endete mit dem Beginn des produktiven Betriebs im Januar 2017.

\section{$2.1 \quad$ Modellierung des Umfelds}

Zum Projektbeginn in 2014 wurden die Anforderungen an die Neuentwicklung von IsyPlus mit Hilfe von UML-Klassen- und Aktivitätsdiagrammen dokumentiert. Das Einbeziehen der Anwender in den gesamten Planungsprozess - in diesem Fall den Modellierungsprozess ist ein Faktor für eine erfolgreiche Einführung von Campus-Management-Systemen. [AuKün16, S. 927]. Daher wurden zunächst die bestehenden Arbeitsprozesse des Ist-Zustands aufgenommen und in Aktivitätsdiagrammen modelliert. In anschließenden Diskussionen mit ausgewählten Mitarbeiterinnen und Mitarbeitern aus dem Kreise der Nutzer wurde daraus der Soll-Zustand abgeleitet. In den nun definierten Prozessen wurden Klassen für erste Klassendiagramme identifiziert. Die entwickelten Klassenmodelle wurden wiederum im Anschluss mit Key-Usern besprochen und überarbeitet.

Die identifizierten Probleme des abzulösenden Systems lagen insbesondere in der unflexiblen Struktur. Beispielsweise wurden die Personendaten nach Ihrer Funktion - Student, Dozent oder Ansprechpartner - abgespeichert. Wurde ein Studierender nach Abschluss seines Studiums im Unternehmen zum Ansprechpartner für dual Studierende, so mussten die Personendaten erneut erfasst werden. Ein Bezug zwischen Studierendendaten und den Daten des Ansprechpartners herzustellen, war nicht möglich. Die Daten wurden redundant abgelegt.

Wie in Abbildung 1 dargestellt werden im neuen Klassenmodell die Personendaten in den Klassen Person und Personenrolle abgebildet. Allgemeingültige Personendaten - wie Vorname und Nachname - werden getrennt von den Personenrollen - wie Student oder Ansprechpartner - abgelegt. Kontaktinformationen - wie Adresse oder Telefonnummer - werden direkt der Person zugeordnet. Über die Person können so für jede Personenrolle die Kontaktdaten ermittelt werden, ohne diese mehrfach ablegen zu müssen.

Das Konzept ermöglicht im Besonderen eine einfache Erweiterung um zusätzliche Personenrollen. Weiterhin werden die Personendaten mit den zugehörigen Adressen, Telefonnummern und Emailadressen redundanzfrei abgelegt. 


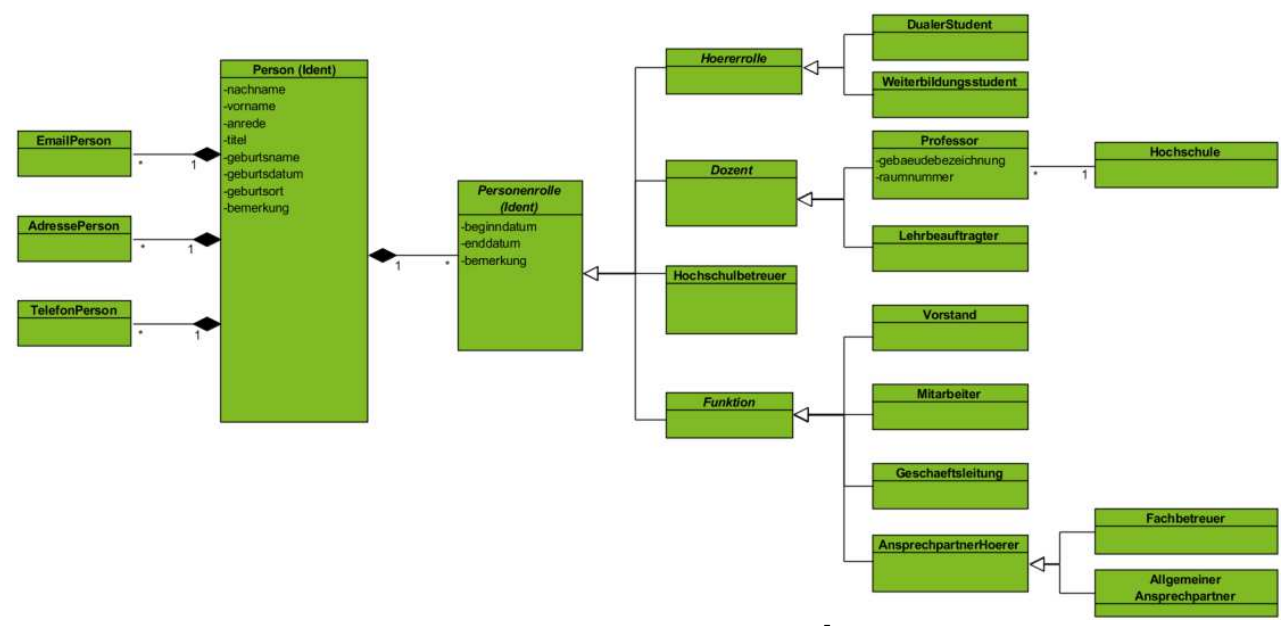

Abbildung 1: reduziertes Klassenmodell zur Verwaltung der Personendaten ${ }^{5}$

\subsection{Datenmigration}

Von Projektbeginn an wurde die Migration der Daten aus dem Altsystem parallel zur Entwicklung der Software bearbeitet. Die Information aus dem abzulösenden System musste verlustfrei und korrekt ins neue System übernommen werden. Dazu wurde zunächst die bestehende Datenbank des Altsystems von Microsoft SQLServer in MySQL überführt. Anschließend wurden die Daten mit SQL-Skripten in die neuen Tabellen migriert.

In einigen Bereichen stimmt die Datenstruktur der alten und neuen Datenbank weitgehend überein, sodass die Daten ohne Anpassungen übernommen werden konnten. In anderen Bereichen weicht die neue Datenstruktur vollständig von der alten ab. Zur Migration der Daten mussten bestehende Entitäten zerlegt und in einen neuen Zusammenhang, zum Teil nach Neuberechnungen oder geeigneter Extrapolation, gebracht werden. Zusätzlich wurden in der alten Anwendung einige Sonderfälle nur teilweise abgebildet und mussten bei der Migration über Hilfskonstruktionen transformiert werden. Wechselte beispielsweise ein Studierender während des Studiums das Partnerunternehmen, so musste das Studium als abgebrochen vermerkt und ein Studium im höheren Semester eingetragen werden. Das alte System sah einen Unternehmenswechsel während des Studiums nicht vor. Sonderfälle wie dieser stellten die Migration vor eine besondere Herausforderung, die eine genaue Kenntnis der alten Datenstrukturen sowie der Daten voraussetzte.

Der frühzeitige Beginn der Entwicklung der Migrationsskripte direkt zum Projektstart, war ein kritischer Erfolgsfaktor für die gesamte Entwicklung sowie Test- und Einführungsphase. Die Validierung der Anwendung durch die Mitarbeiter konnte nur anhand der übernommenen Daten durchgeführt werden, daher war die Bereitstellung von Echtdaten ab der ersten Testversion elementar. Dies konnte nur mit Hilfe der dann schon bereitstehenden SQL-Skripte sichergestellt werden. Alle Anpassungen an den Modellen wurden bis zum Tag der Einführung im Januar 2017 fortwährend unmittelbar im Migrationsverfahren nachgebildet, um die Testszenarien kontinuierlich ausführen zu können.

\footnotetext{
${ }^{5}$ Abbildung 1 zeigt wegen der Übersichtlichkeit ein reduziertes Modell.
} 


\subsection{Entwicklung des Generators}

Die Entwicklung des Generators wurde anhand eines reduzierten Referenzmodells mit Testdaten durchgeführt. Dieses Modell enthält bei einem Umfang von ca. 20 Klassen Repräsentanten aller relevanten Klassenstrukturen (abstrakte Klassen, Subklassen) und Beziehungen. Es wurde als UML-Klassenmodell entworfen und anschließend in ein EMFEcore-Modell überführt, das dann als Grundlage für die Realisierung des Generators diente. (Vgl. [RGVM15], S. 218.). Neben der Benutzeroberfläche, die das Navigations- und Reportmenü, sowie Views und Editoren umfasst, kann auch die zugehörige Datenbank automatisiert aus dem Modell erstellt werden. Zudem verfügt die Anwendung über ein ausgefeiltes System für die Verwaltung der Zugriffsrechte, welches Einschränkungen sowohl auf Tabellen- und Attributebene als auch auf Datensatzebene erlaubt.

Die Entwicklung des Generators erfolgte stets modellunabhängig, also nicht spezifisch für die Anwendung IsyPlus. Als Zieldomäne des Generators sehen wir stark CRUD-lastige Anwendungen, bei denen die manuelle Eingabe von Daten im Vordergrund steht. Das Testen des Generators bzw. der lauffähigen Anwendung erfolgte auf Basis eines eigens definierten Referenzmodells, welches alle relevanten Modellierungsfälle abdeckt und somit die korrekte Ausführbarkeit einer aus einem konkreten Modell generierten Software garantiert.

Anforderungen an die zu generierende Anwendung wurden zunächst auf deren Modellunabhängigkeit untersucht. Falls Anforderungen modellunabhängig in den Generator aufgenommen werden können, werden diese zunächst konkret implementiert („Durchstich“) und anschließend abstrahiert (somit modellunabhängig) in den Generator übernommen. Modellspezifische Anforderungen werden nicht im Generator berücksichtigt, sondern können als ebenfalls während der Generierung erzeugte Subklassen der generierten Basisklassen im Klassenmodell der erzeugten Software integriert werden.

\section{Die Testphase}

Zu Beginn der Testphase konnten bereits die für den Betrieb notwendigen Funktionen des Systems - wie Dialoge für die Eingabe, Ausgabe und Änderung von Daten, Filter- und Suchhilfen, Historienfähigkeit sowie ein umfangreiches Rechtesystem - generiert werden. Die Testphase startete mit allgemeinen Schulungen für alle Anwender. Zusätzlich wurden einerseits über das gesamte Jahr 2016 teambezogene Schulungen zu speziellen Funktionen durchgeführt und andererseits einzelne Prozesse teamübergreifend in Sondersitzungen besprochen. Parallel stand den Mitarbeitern das Testsystem mit zeitnah migrierten Daten aus dem noch laufenden Altsystem durchgehend zur Prüfung zur Verfügung. So konnten Prozesse in der Anwendung bereits weit vor der Einführung getestet werden. Das kontinuierliche Testen führte zu häufigen Modelländerungen, welche regelmäßig als Updates eingespielt wurden.

Im Rahmen der Tests wurde deutlich, dass es Prozesse gibt, in denen die modellierbaren Dialoge (Wizards) nicht ausreichen, um die zu erledigenden Schritte effizient abbilden zu können. Ab Mitte des Jahres wurden die für die Kernprozesse zusätzlich benötigten Wizards als CustomWizards hinzugefügt. Üblicherweise handelt es sich dabei um Wizards, die Massendaten oder Datensätze in mehreren Objekten zeitgleich anlegen und bearbeiten können. Bis zur Einführungsphase wurden 18 derartige CustomWizards auf konventionelle Weise implementiert. 
3.1 Modelländerungen und Anpassung der Datenmigration während der Testphase Modelländerungen und die Anpassung der Migrationsskripte liefen während der gesamten Testphase Hand in Hand. Die Notwendigkeit des Testens mit Echtdaten zog bei Änderung des Modells Anpassungen an den zugehörigen SQL-Skripten nach sich. Ein Vorteil dabei war, dass fehlerhaft übernommene Daten identifiziert werden konnten und die zugehörigen Skripte frühzeitig korrigiert wurden. Neue Testversionen wurden stets mit aktuellen Daten bereitgestellt, sodass auch Korrekturen an den SQL-Skripten in der Testphase einer stetigen Überprüfung unterlagen.

Bei den durchgeführten Modellmodifikationen handelte es sich typischerweise um Änderungen, die durch Annahmen bei der Modellierung entstanden, die nicht der Realität entsprachen. Beispielsweise wurde im alten System der Unternehmenswechsel eines Studierenden semesterweise eingegeben. Dieser Ansatz wurde im neuen Klassenmodell zunächst übernommen. In der Testphase stellte sich heraus, dass die Studierenden ein Partnerunternehmen zum Monatsende verlassen und das Studium zum Monatsersten bei einem neuen Partnerunternehmen fortsetzen können. Um dies abzubilden, mussten der Klasse Einschreibung zwei zusätzliche Attribute hinzugefügt werden. Kleinere Modelländerungen wie diese wurden während der Testphase zeitnah vorgenommen. In diesem Fall beschränkt sich der Aufwand für die Erzeugung einer neuen Testversion im Wesentlichen auf eine Neugenerierung der Software. Eine vollständige Migration der Daten ist hier nicht nötig. Die Datenbank wird dabei nicht neu erzeugt, sondern die neuen Datenfelder manuell in die Tabelle eingefügt. Die Daten konnten in diesem Fall mit einem nur für diesen Zweck erstellten SQLSkript ergänzt werden. Das eigentliche Migrationsskript wurde im Nachgang für die nächste Datenübernahme entsprechend angepasst.

In einigen Fällen wurden umfangreichere Modelländerungen notwendig, die Änderungen an den Referenzen der Entitäten und/oder das Hinzufügen neuer Entitäten notwendig machten. Hier ist im Vergleich zu den zuvor beschriebenen Änderungen der Zeitaufwand bei der Erzeugung einer Testversion höher. Nach der erneuten Generierung der Software wird in diesen Fällen beim ersten Starten der Anwendung die neue Datenbank erzeugt. Im Anschluss wurden die Migrationsskripte angepasst und vollständig ausgeführt. Allein das Ausführen der Migrationsskripte konnte dabei mehrere Stunden in Anspruch nehmen.

Bei einem konventionellen Ansatz der Entwicklung eines solchen Systems müssen alle Elemente der Benutzeroberfläche, wie Views, Editoren oder Wizards, mehr oder weniger manuell implementiert werden. Dies bietet einerseits Möglichkeiten der visuellen Gestaltung. Kommt es allerdings zu Modelländerungen, so müssen die daraus resultierenden Änderungen im Code wieder manuell durchgeführt werden. Im Falle von IsyPlus war eine sehr einheitliche Gestaltung der Oberfläche Ziel und durch den konsequenten Einsatz modellgetriebener Entwicklung konnten Testversionen zügig zur Verfügung gestellt werden, da Modelländerungen am EMF-Ecore-Modells lediglich eine Neugenerierung nach sich zogen. Manuelle Programmierung ist nur bei Änderungswünschen an den CustomWizards notwendig.

\section{2 Änderungen am Generator}

Während der Testphase wurde zunächst auf die Antwortzeiten des Systems fokussiert. Lange Ladezeiten erschwerten ein konsequentes Testen der Anwendung durch die Mitarbeiter. Daher stand Abhilfe bei lang laufenden Funktionen im Mittelpunkt. Die leistungssteigernden Maßnahmen sollen im Folgenden an einigen Beispielen verdeutlicht werden. 
In der ersten Testversion wurden bereits beim Öffnen eines Editors die Daten aller aus den Referenzen abgeleiteten Tabs geladen. Bei einzelnen Editoren konnte dies zu Ladezeiten von mehr als zehn Sekunden führen. Regelmäßig wurden die Rechte des eingeloggten Users ermittelt, um Informationen ein- und ausblenden zu können. Zudem wurden alle geöffneten Elemente der Benutzeroberfläche automatisch neu geladen, wenn aktualisierte Daten vorlagen. All dies führte zu Ladezeiten, die von den Anwendern als zu lang zurückgemeldet wurden.

Der Generator wurde im Laufe der Testphase in Bezug auf das Lade- und Aktualisierungsverhalten optimiert. So wird nun der Inhalte von Tabs erst bei Aufruf des Tabs geladen. Sind die GUI-Elemente nicht sichtbar oder haben keinen Fokus, wird auf eine sofortige Aktualisierung zugunsten der Performance verzichtet. Erst, wenn das GUI-Element sichtbar bzw. fokussiert wird, findet eine Aktualisierung des GUI-Elements statt. Durch Caching konnten die Rechteprüfung optimiert werden: bei Erststart der Anwendung werden die aggregierten Rechte eines jeden Users in einem dediziert dafür vorgesehenen Cache hinterlegt. Dies bedingt (zunächst), dass Rechteänderungen nicht zur Laufzeit möglich sind.

Zudem werden generell Hash-Sets anstatt Listen im Java-Code verwendet. Der Zugriff auf ein Element ist im Gegensatz zu einer Liste mit konstantem Aufwand möglich. Anders als Listen speichern Hash-Sets ihre Elemente unsortiert und müssen für die Anzeige gegebenenfalls von Komponenten der Oberfläche nachgelagert sortiert werden. Dieser (geringfügige) Nachteil wird bewusst in Kauf genommen.

Wie in Kapitel 0 beschrieben wurde der Generator mit Hilfe eines Referenzmodells entwickelt. Dies führt dazu, dass zwar die Dauer der Generierung sowie des Builds möglichst geringgehalten werden können, jedoch muss der Generator jeweils anhand des IsyPlusEcore-Modells zusätzlich validiert werden. Das IsyPlus-Ecore-Modell verfügt aktuell über ca. 250 Klassen, die in unterschiedlichster Form miteinander vernetzt sind. In der Testphase stellte sich heraus, dass im Referenzmodell nicht alle Modellierungsfälle berücksichtigt wurden, die im IsyPlus-Modell Anwendung finden. Diese Modellierungsfälle führten bei der Generierung zu fehlerhaften Java-Code, der dann korrigiert und in den Generator implementiert wurde. Zudem führte das Testen des IsyPlus-Ecore-Modells an einer Stelle dazu, dass die zulässige maximale Anzahl von Methoden (etwa 65000) überschritten wurde, sodass eine Aufteilung der generierten Klasse erfolgen musste.

\section{Einführungsphase}

Für die Einführung der Anwendung konnten zunächst verschiedene Migrationsstrategien in Betracht gezogen werden. Von den bei Brodie und Stonebraker [BroSto95] beschriebenen Vorgehensweisen kamen auf Grund der Gegebenheiten aber weder eine komponentenweise Ablösung (wie etwa bei chicken little) noch Ansätze, die auf einer zumindest zeitweisen Koexistenz von Teilen des neuen und des alten Systems basieren in Frage. Die Besonderheit von IsyPlus liegt in der starken Vernetzung des Systems durch Beziehungen der Entitäten untereinander (Vgl. [RGVM15], S. 216). Das neue System wurde neben dem weiter in Betrieb befindlichen Alt-System entwickelt und mit in kurzen Zyklen übernommen Echtdaten getestet. Ein vollständiger Parallelbetrieb nach Einführung war dabei nicht möglich, da Daten aus dem neuen System nicht in das alte System zurückgeführt werden konnten. Damit musste letztlich ein kompletter Austausch der Systeme (big bang/cold turkey) angestrebt werden. 
Die Inbetriebnahme der neuen Anwendung erfolgte am 02. Januar 2017. Zu diesem Zeitpunkt standen alle wichtigen Funktionen (komplette Eingabe und Ausgabe, wesentliche Auswertungen und Berichte) zur Nutzung bereit. Funktionen von geringerer Priorität (z.B. ausgewählte Berichte) wurden parallel fertiggestellt und sukzessive ausgeliefert

Die Einführungsphase wurde von regelmäßigen Schulungen und Fragerunden zur Anwendung begleitet, die auch jetzt noch in größeren Zeitabständen angeboten werden. Bei diesen Treffen sind typischerweise mehrere Entwickler anwesend, um direkt Lösungsansätze in Zusammenarbeit mit den Anwendern diskutieren zu können. Zunächst wurde Kritik am neuen System geäußert. Die Hauptkritikpunkte der Anwender am neuen System lagen während der frühen Einführungsphase darin, dass in den Editoren und Views mehr Daten als erwartet angezeigt wurden und die Anzahl der notwendigen Mausaktionen in der Oberfläche im Vergleich zur alten Anwendung gestiegen war. Diese Rückmeldungen werden entgegengenommen und nach Möglichkeit in der Anwendung berücksichtigt. Hier hat sich insgesamt die entscheidende Bedeutung des Changemanagements gezeigt: Bedürfnisse der Anwender müssen beim Wechsel der Systeme beachtet und moderiert werden.

\subsection{Modellerweiterungen in der Einführungsphase}

Nach der einjährigen Testphase waren nach der Einführung keine umfangreicheren Modelländerungen mehr notwendig. Die für die Kernprozesse benötigten Dialoge und Editoren wurden in der Testphase mehrfach validiert und angepasst. Daher konzentrierte sich die Modellierung nach der Einführung hauptsächlich auf ergonomische Verbesserungen der Benutzeroberfläche.

Bei der Erzeugung von Editoren und Views setzt der Generator die modellierten Entitäten sowie deren Attribute und Referenzen standardisiert um. Jede Entität erhält dabei eine View, die zum Aufruf des zugehörigen Editors dient und einen Editor für die Anzeige und zur Manipulation der Daten. In dem Editor werden die Attribute und zu-1-Referenzen der Entität auf der Registerkarte Stammdaten angezeigt. Für jede zu-n-Referenz wird eine eigene Registerkarte erzeugt und die Daten in einer Tabelle dargestellt. Die Historie und Eigenschaften - wie Erstellungs- und Änderungsdatum - einer Entität werden jeweils auf einer zusätzlichen Registerkarte einsehbar.

Das Klassenmodell umfasst zentrale Entitäten, die vergleichsweise viele zu-n-Referenzen besitzen. Beispielsweise weist die Entität Person zehn zu-n-Beziehungen auf, die im Editor jeweils als eigene Registerkarte dargestellt werden. Hinzu kommen noch Registerkarten für die Stammdaten, Historie und Eigenschaften, sodass der Personeneditor zunächst insgesamt 13 Registerkarten besitzt. Bei einer Anzeige des Editors kann daher nur ein Teil der Registerkarten sofort angezeigt werden. Die restlichen Registerkarten werden in eine Liste aufgenommen, welche über einen seitlichen Button erreichbar ist. Dies wurde von den Anwendern als unübersichtlich wahrgenommen.

Wie in Abbildung 2 dargestellt, zeigte Personeneditor anfangs drei eigenständige Tabs für Adressen, Telefonnummern und Emailadressen. Die Adressen, Telefonnummern und Emailadressen einer Person können unter dem Begriff Kontaktdaten subsummiert werden. Die Kontaktdaten müssen regelmäßig durch die Mitarbeiter überprüft werden. Dies führte dazu, dass ein Mitarbeiter drei Klicks benötigte, um diese Daten vollständig überprüfen zu können. Im Personeneditor wurden zudem Registerkarten angezeigt, die für die Mitarbeiter an dieser Stelle nur von geringer Bedeutung sind und daher nicht angezeigt werden sollten. 


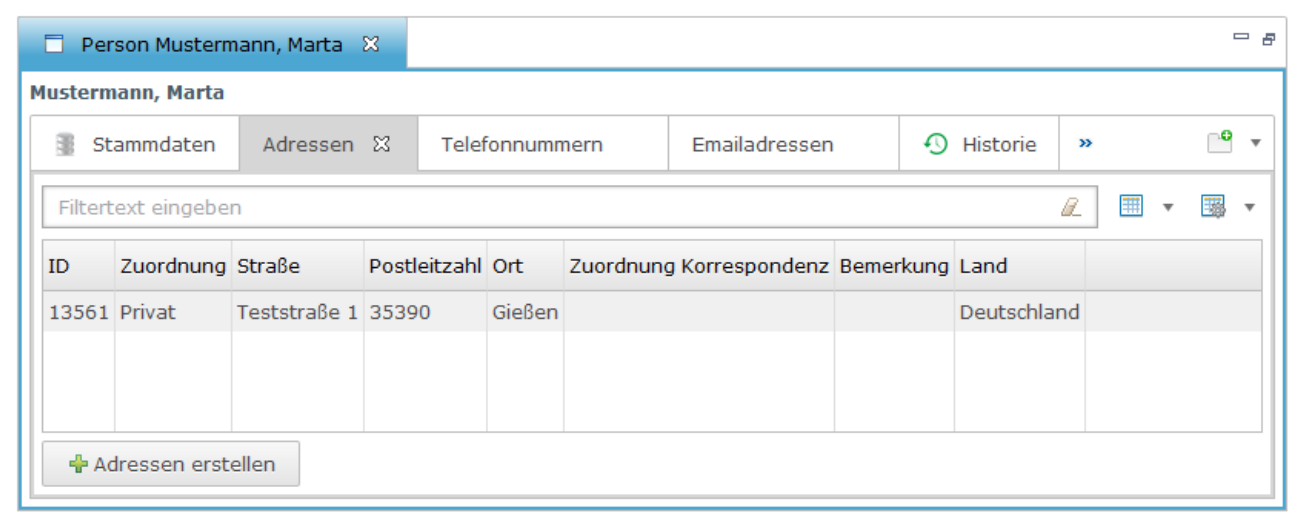

Abbildung 2: Editor Person vor der Zusammenführung der Kontaktdaten

Die Überprüfung der Kontaktdaten einer Person wird für gewöhnlich pro Personenrollen durchgeführt. Der Einstieg erfolgt dann nicht über den Personeneditor selbst, sondern über den Editor einer Personenrolle - z.B. Student, Dozent. Hier waren die Kontaktdaten nicht direkt verfügbar. Um die Adresse prüfen zu können, musste der Mitarbeiter von der Personenrolle aus den Personeneditor aufrufen und in die Registerkarte Kontaktdaten wechseln. Hierzu waren zwei Klicks notwendig.

Bei bestimmten Objekten ist eine Verminderung der Anzahl der Mauskationen möglich, indem transitive Referenzen direkt angezeigt werden und damit keine Navigation über die Masken einer weiteren Entität notwendig wird. Beispielsweise werden im Rahmen des Studienbetriebs die Abbildung von Studienorten, Gebäuden und Räumen benötigt. Wie in Abbildung 3 dargestellt können an einem Studienort mehrere Gebäude mit mehreren Räumen genutzt werden.

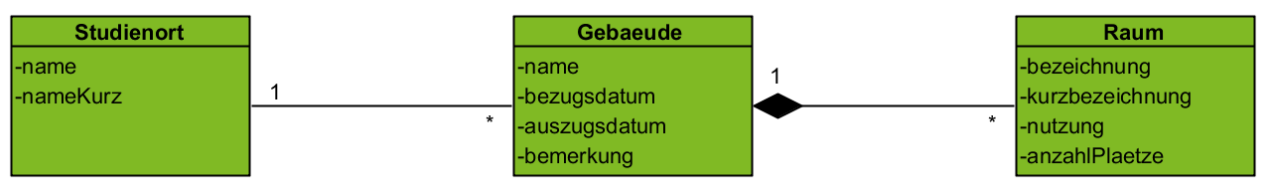

Abbildung 3: Modell zur Studienortsverwaltung

Standardmäßig werden im Editor des Studienorts nur die zugehörigen Gebäude in einem Tab angezeigt. Um die angehörenden Räume einsehen zu können, musste der jeweilige Editor des Gebäudes geöffnet und anschließend die Registerkarte der Räume angeklickt werden. Dies erforderte dann pro Gebäude zwei Klicks.

Um die Editoren übersichtlicher zu gestalten, sollte die Anzahl der Registerkarten soweit wie möglich reduziert werden. Dazu sollten zu-n-Referenzen auf Registerkarten zusammengefasst sowie sortiert oder ausgeblendet werden können. Zusätzlich sollten transitive Referenzen auf Registerkarten hinzugefügt werden können.

Um dies zu realisieren, wurden Erweiterungen am Generator in enger Abstimmung zwischen Modellierer und Generatorentwickler umgesetzt. Zunächst wurden in den Fragerunden mit den Anwendern möglichen Änderungen an der Benutzeroberfläche ermittelt. Anschließend wurden die notwendigen Ergänzungen im Projektteam diskutiert und so umgesetzt, dass sie modellweit zum Einsatz kommen konnten. Diese Vorgehensweise wurde 
dabei iterativ durchgeführt und damit eine schrittweise Annäherung an den Zielzustand erreicht.

\subsection{Umsetzung von GUI-Anforderungen im Generator}

Mit Ecore stellt EMF Klassendiagramme mit zu UML vergleichbarer Funktionalität bereit. Mit diesen können bereits vollständige fachliche Modelle definiert werden. Für spezielle, im Projekt benötigte Beschreibungen wie etwa das Aussehen der GUI oder die Definition von Zugriffsrechten ist Ecore zunächst nicht geeignet. Allerdings erlaubt EMF Instanziierungen des Metamodells, mit denen neue Metamodelle definiert werden können. Die dort definierten Elemente können dann speziell für zusätzliche Sachverhalte (Oberfläche, Rechteverwaltung) zugeschnitten werden. In einem konkreten Modell des neuen Metamodells wie z.B. dem View-Modell, stehen dann nur die dort definierten Elemente zur Verfügung. Dass sich im Hintergrund eigentlich Ecore-Instanzen befinden, bleibt dem Modellierer verborgen.

Das View-Modell wurde dahingehend erweitert, dass Spalten in bestimmten Situationen nicht sichtbar oder in einer anderen Reihenfolge angeordnet sind. Attribute und Referenzen eines Objektes können in Gruppen zusammengefasst werden. Diese werden in Tabs angesiedelt, die wiederrum (bei Bedarf oder standardmäßig) ein- und ausgeblendet werden können. Diese (und auch andere Anforderungen) können mit moderatem Aufwand ins ViewModell übernommen werden.

Ein Element des View-Modells ist die in Abbildung 4 dargestellte GroupConfiguration. Sie erlaubt, die in den Editoren verwendeten Registerkarten zusammenzufassen, zu sortieren und auszublenden.

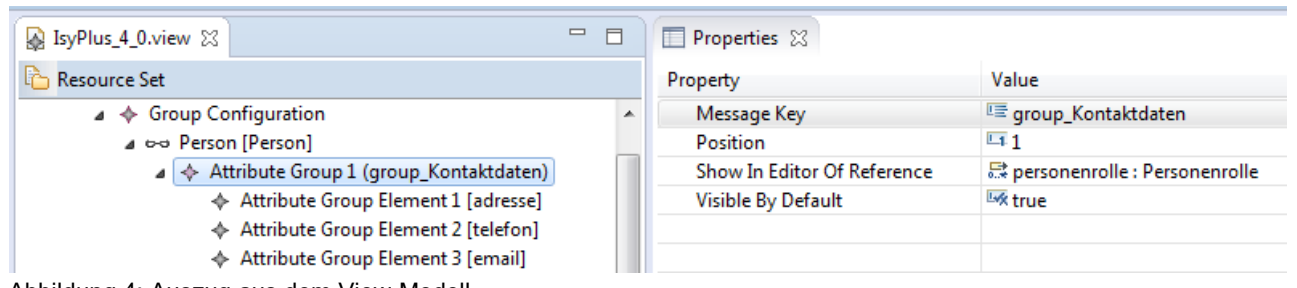

Abbildung 4: Auszug aus dem View-Modell

Durch die in Abbildung 4 dargestellte Zusammenfassung von Adress-, Telefon- und Emaildaten auf der Registerkarte ,group_Kontaktdaten', sind die Kontaktdaten mit einem Klick erreichbar. Das Ergebnis des mit diesem View-Modell generierten Codes ist in Abbildung 5 dargestellt.

Über die Eigenschaften Show in Editor Of Reference können ganze Registerkarten an eine zu-n-Referenz weitergegeben werden. Dies reduziert die Mausaktionen in dem oben beschriebenen Fall auf einen Klick. Wie in Abbildung 6 dargestellt werden die übergebenen Registerkarten kursiv bzw. fett hervorgehoben. 


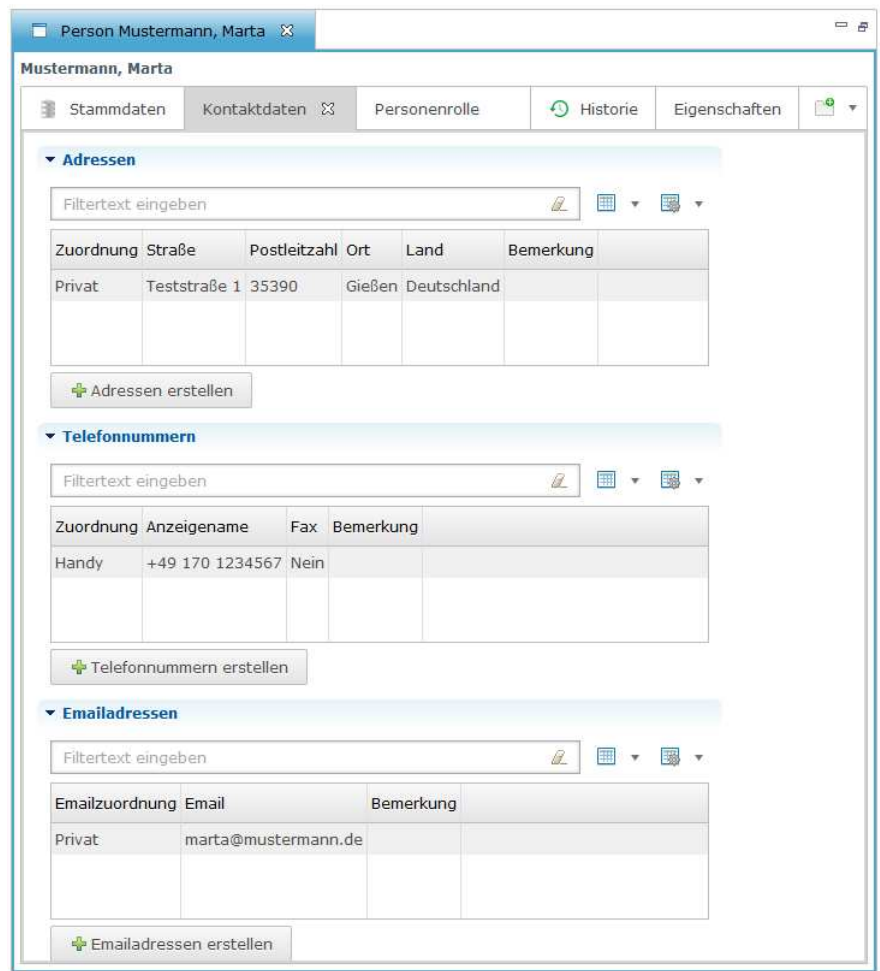

Abbildung 5: Editor Person nach der Zusammenführung der Kontaktddaten

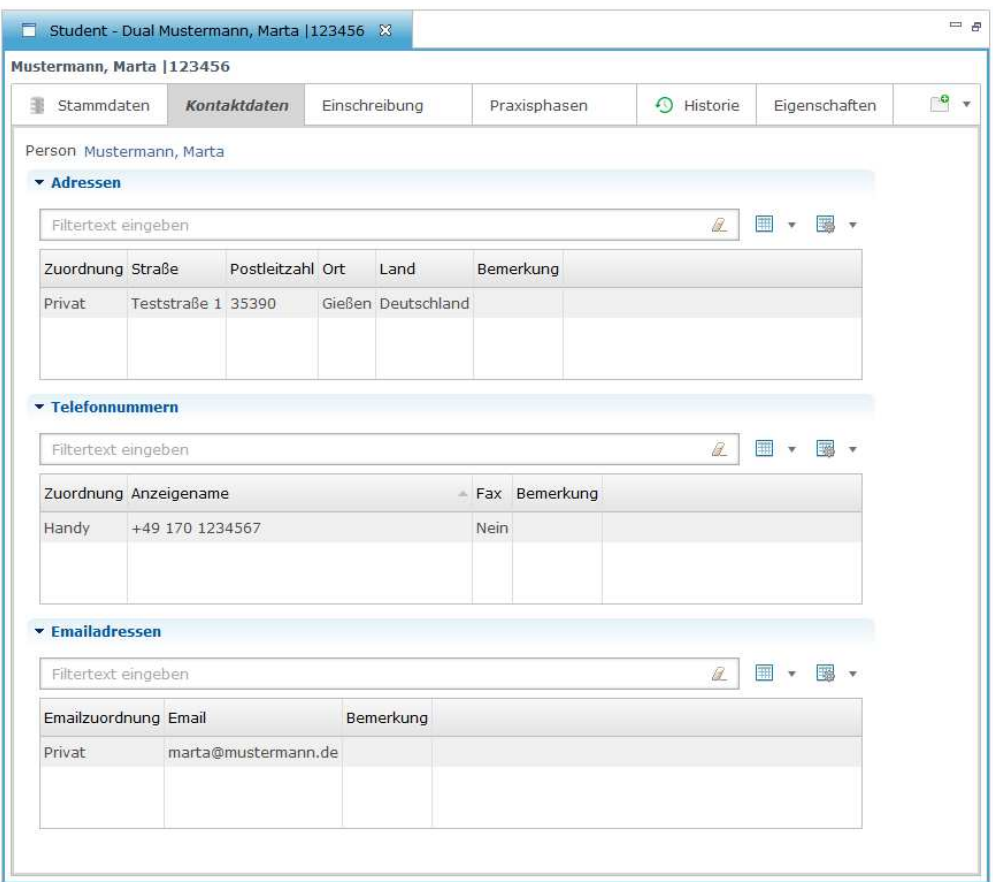

Abbildung 6: Editor Student mit übergebener Registerkarte ,Kontaktdaten“ 
Auch die Möglichkeit transitive Referenzen darzustellen, wurde in das View-Modell aufgenommen. Abbildung 7 zeigt die zur Darstellung von transitiven Referenzen entwickelte Erweiterung namens EditorAddition. Hier können transitive Referenzen modelliert werden, die dann auf den Tabs der direkten Referenz angezeigt werden.

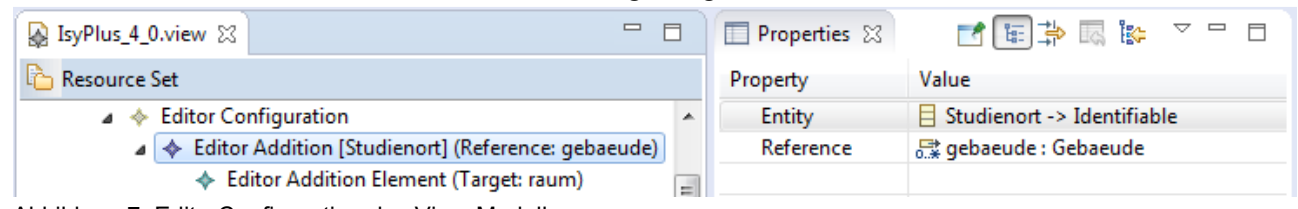

Abbildung 7: EditorConfiguration des View-Modells

Abbildung 8 zeigt die Registerkarte Gebäude des Editors Studienort nach dem die Referenz zum Raum eines Gebäudes hinzugefügt wurde. Wird ein Gebäude angeklickt, erscheinen in der darunterliegenden Tabelle die zugehörigen Räume. Um die Räume einzusehen, muss der Benutzer den Gebäudeeditor nicht mehr öffnen. Somit reduziert sich die Anzahl der Mausaktionen im Rahmen dieses Vorgangs um 50 Prozent.

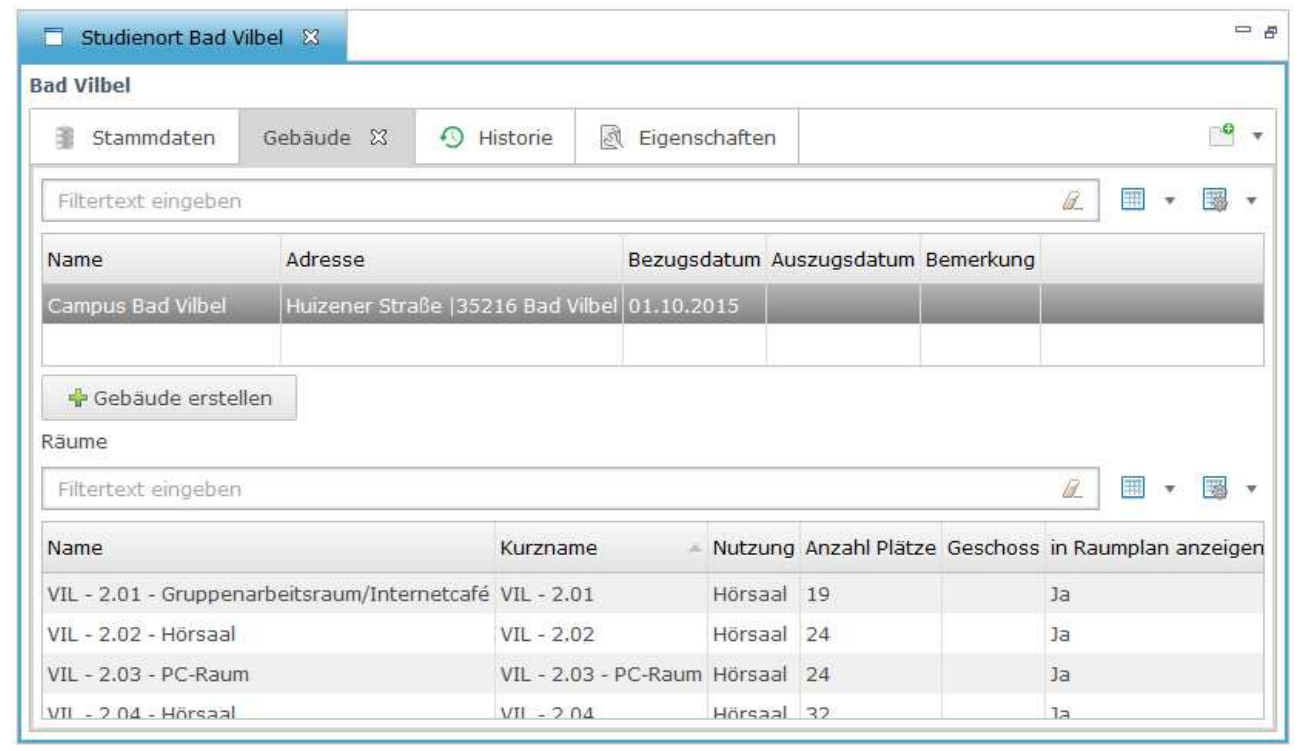

Abbildung 8: Editor Studienort nach Hinzufügen einer transitiven Referenz

\subsection{Manuelle Anpassung automatisch migrierter Daten}

Für die endgültige Portierung der Daten wurde die letzte Arbeitswoche im Dezember 2016 vorgesehen, da zu diesem Zeitpunkt keine Vorlesungen stattfanden. Hier wurden alle abschließenden Arbeiten an der Anwendung sowie an den Daten durchgeführt und das System für die Inbetriebnahme vorbereitet. Das Altsystem wurde zu Beginn der Portierung für Dateneingaben und -änderungen gesperrt. So konnte die Übernahme von inkonsistenten Daten ausgeschlossen werden.

Nach der Einführung mussten keine automatisierten Datenkorrekturen per SQL mehr vorgenommen werden, da bereits in der Testphase für die Integrität der SQL-Skripte und somit der übernommenen Daten gesorgt wurde. Jedoch waren - insbesondere bei den Personendaten - manuelle Korrekturen nötig. Die Daten der Personen wurden aus verschiedenen Tabellen der alten Datenbank in die neue Tabelle Person übernommen. Bei der Migration 
der Daten konnte nicht festgestellt werden, ob die Person bereits in der neuen Datenbank vorkommt, da nur Vorname und Nachname der Person zur Überprüfung zur Verfügung stand. Zur eindeutigen Identifizierung einer Person reicht dies nicht aus. Daher konnten die Daten nur redundant übernommen werden.

Nach der Einführung wurden namensgleiche Personen per Abfrage ermittelt und in Microsoft Excel ausgegeben. Diese Datei wird durch Mitarbeiter geprüft und bearbeitet. Die überprüften Datensätze können per SQL-Anweisung in der Datenbank korrigiert und zusammengeführt werden. Kann die Überprüfung und Vervollständigung der Personendaten nicht direkt aus den vorhandenen Daten erfolgen, so sind Nachforschungen oder individuelle Bewertungen des Sachverhalts notwendig. Für die Prüfung und Konsolidierung der Daten ist daher das gesamte Jahr 2017 vorgesehen.

\section{$5 \quad$ Vor- und Nachteile in der Test- und Einführungsphase und Fazit}

In einer rückblickenden Bewertung des Projekts können die folgenden Vorteile des modellgetriebenen Ansatzes gegenüber einem konventionellen Vorgehen identifiziert werden: Modelländerungen konnten den Anwendern sowohl in der Test- als auch in der Einführungsphase in Form von Updates regelmäßig und - vor allem - zeitnah zur Verfügung gestellt werden. Der Generator wurde kontinuierlich und den Wünschen der Anwender entsprechend weiterentwickelt. Durch die Abstraktion der konkreten Anwendungsfälle wurden Änderungen wie die Verbesserung der Benutzeroberfläche durch das View-Modell nicht nur partiell, sondern flächendeckend für das gesamte Modell verwendbar. Nur knapp 8 Prozent (18 von 224 Wizards) aller Wizards mussten konventionell programmiert werden. Die restlichen Elemente der Anwendung - Views, Editoren, usw. - wurden zu 100 Prozent generiert. Dies reduziert den wesentlichen Programmieraufwand auf die Weiterentwicklung des Generators.

Die standardisierte Umsetzung von UML-Modellen in eine Benutzeroberfläche sowie das im Vergleich zu dem Modell des vorangegangenen Systems - komplexere Klassenmodell führte zu Verständnisproblemen bei den Anwendern. Dies ließ sich nur durch Schulung der Anwender sowie die Erweiterung des View-Modells kompensieren.

Projekte diese Art stellen an die Abstraktionsfähigkeit des Generatorentwicklers spezielle Anforderungen. Die personelle Besetzung dieser Position ist deshalb von besonderer Bedeutung. Nicht zuletzt wegen der durchgängigen Einbindung der Anwender in die gesamte Entwicklung des Generators und dem kontinuierlichen Testen konnte die Anwendung erfolgreich eingeführt werden.

\section{Literaturverzeichnis:}

[AuKün16] Auth G., Künstler S.: Erfolgsfaktoren für die Einführung integrierter Campus-Management-Systeme - eine vergleichende Literaturanalyse mit praxisbezogener Evaluation in: Mayr H.C., Pinzger M. (Hrsg.): INFORMATIK2016 Lecture Notes in Informatics (LNI), Gesellschaft für Informatik, Bonn, 2016, s. 915ff.

[BroSto95] Brodie M., Stonebraker M.: Migrating Legacy Systems; Morgan Kaufmann Publishers Inc., 1995 
[RGVM15]: Richter M., Guckert M., Vanderpuye, M.: Modellgetriebene Entwicklung einer EclipseRAP-Anwendung unter Verwendung des Eclipse Modeling Frameworks in: Barton T., Erdlenbruch B., Herrmann F., Müller C., Marfurt K., Seel C. (Hrsg.): Angewandte Forschung in der Wirtschaftsinformatik, Prozesse, Technologie, Anwendungen, Systeme und Management 2015, mana-Buch, Heide, 2015, s. $215 \mathrm{ff}$.

[StudP17] www.Studiumplus.de , Abruf 08.05.2017

\section{Kontakt}

Dipl.BW.(FH) Melanie Vanderpuye

StudiumPlus

Charlotte-Bamberg-Straße 3, 35578 Wetzlar

T+49 6441 2041-223, melanie.vanderpuye@zdh.thm.de

MSc. Marco Richter

Technische Hochschule Mittelhessen

KITE-Kompetenzzentrum für Informationstechnologie

Wilhelm-Leuschner-Straße 13, 61169 Friedberg

T +496031604-4783, marco.richter@mnd.thm.de

Prof. Dr. Michael Guckert

Technische Hochschule Mittelhessen

KITE-Kompetenzzentrum für Informationstechnologie

Wilhelm-Leuschner-Straße 13, 61169 Friedberg

T +49 6031 604-452, michael.guckert@mnd.thm.de 
Cloud - Mobile - Security 


\title{
Sentimentanalyse von Nachrichtentiteln
}

\author{
Marcel Lang, Michael Guckert, Christian Schulze
}

\section{Zusammenfassung}

Im Rahmen des von der Hessen-Agentur geförderten LOEWE3-Projektes ADOMIS (Ambient Delivery of Multiple Information and Statistics) wurde eine Datenbank mit volks- und finanzwirtschaftlichen Informationen aufgebaut. Diese Datenbank enthält sowohl strukturierte Daten wie Zeitreihen der Kursentwicklung börsennotierter Unternehmen als auch semistrukturierte Daten wie Nachrichten zu den Unternehmen aus diversen Quellen. Klassische Ansätze des Financial Research nutzen bereits umfassende Daten für die Einschätzung der zukünftigen Entwicklung solcher Wertpapiere. Ergänzt werden diese Informationen künftig durch weitere Daten, beispielsweise aus Websites von Nachrichtenagenturen, Tageszeitungen und sozialen Netzwerken. Die Fülle an zur Verfügung stehender Information ist vom Menschen kaum mehr zu verarbeiten und kann nur mit Hilfe intelligenter Ansätze bewältigt werden. Im Rahmen des Projektes wurden daher Machine-Learning-Algorithmen für die Analyse von Nachrichten aus diversen Quellen untersucht. Dabei wurden sowohl Experimente mit kompletten Nachrichtentexten als auch Auswertungen, die nur deren Überschriften berücksichtigen, durchgeführt. Mit dem Einsatz Neuronaler Netze wurden zum einen Klassifikationen der Nachrichten nach deren Inhalt (etwa nach dem Bezug zu Branchen) vorgenommen und zum anderen Analysen mit einer wertenden Einschätzung der Nachricht durchgeführt. Die vorliegenden Texte wurden vor Anwendung der Netze mit Hilfe des Word2Vec-Modells in Vektorrepräsentationen übertragen. Das Verfahren liefert bei Anwendung auf die Überschriften der Nachrichten sehr gute Ergebnisse.

\section{$1 \quad$ Einleitung}

Das von der Hessen-Agentur geförderte LOEWE-3 Projekt ADOMIS ${ }^{6}$ (Ambient Delivery of Multiple Information and Statistics) hat eine einheitlich strukturierte und leicht erweiterbare Datenbasis für finanzwirtschaftliche Analysen und Modelle geschaffen, die es ermöglicht, Daten unterschiedlicher Struktur aus vielfältigen Quellen in der gemeinsamen Datenbasis zusammenzuführen. Darüber hinaus wurden modular aufgebaute Analysekomponenten zur Auswertung dieser Datenbestände entwickelt, sodass Analysten nun auf einen integrierten Datenbestand zugreifen können. Das Projekt wird vom Kompetenzzentrum für Informationstechnologie (KITE) der Technischen Hochschule Mittelhessen zusammen mit dem Industriepartner Content Software $\mathrm{GmbH}$ durchgeführt. Die ADOMIS-Datenbank speichert dabei primär Zeitreihen mit finanz- und volkswirtschaftlichen Kennzahlen und stellt diese über eine integrierte R-Umgebung für Analysen und für die Berechnung von Modellen zur Verfügung. Im Rahmen der Projektarbeiten wurde neben diesen quantitativen Aspekten darüber hinaus Experimente zu Ansätzen qualitativer Auswertungen gemacht.

\footnotetext{
${ }^{6}$ Fördernummer 474/15-16, siehe https://www.innovationsfoerderung-hessen.de/projektbeispiele
} 


\section{Motivation}

Neben Chart- und Fundamentalanalysen auf Basis quantitativer Daten, wie bspw. KursZeitreihen und Unternehmensdaten, sind prognostische Einschätzungen von Marktentwicklungen eine wichtige Entscheidungsgrundlage für den Handel mit Finanzinstrumenten. Der Analyst kann derartige Einschätzung aufgrund seiner Erfahrungen aus einer Vielzahl semioder schwachstrukturierter Informationen wie Nachrichten, sozialen Medien oder Twitter ableiten. In diesen Medien werden aktuelle Ereignisse wie etwa politische Entwicklungen diskutiert, deren Auswirkungen auf Märkte, Branchen und Unternehmen zu diesem Zeitpunkt nur erahnt werden können und die sich deshalb noch nicht in quantitativen Daten widerspiegeln. Gerade die Auswertung von Daten aus sozialen Medien bietet die Möglichkeit, frühzeitig aktuelle Trends und Stimmungsbilder zu erfassen. Da diese Daten sich in keiner strukturierten Form befinden, können sie kaum mit den üblichen Data-Mining-Methoden ausgewertet werden. Erst nach einer entsprechenden Vorbereitung werden sie üblichen Verfahren zugänglich. Neben klassischen Verfahren der semantischen Analyse stellen selbstlernende Ansätze auf Grundlage der sogenannten Distributional Semantics einen vielversprechenden und bereits etablierten Ansatz dar ${ }^{7}$. Zwar sind bereits kommerzielle Werkzeuge für die sogenannte Sentimentanalyse am Markt verfügbar ${ }^{8}$, eine Untersuchung der Relevanz derartigen Ansätze für die im Projekt ADOMIS entwickelte Datenbasis sollte trotzdem überprüft werden. Ziele waren dabei zum einen die Klassifikation von Nachrichten nach ihrer Relevanz für die Entwicklung von Zeitreihen (z.B. Nachrichten zu Branchen) und zum anderen eine Einschätzung, ob es sich bei einer Nachricht um eine grundsätzlich positive oder eine eher negative Aussage handelt. Zum Einsatz kommen dabei das von Google veröffentlichte Modell word2vec sowie das Framework deeplearning $4 j^{9}$.

\section{$3 \quad$ Neuronale Netze und Word2Vec}

Künstliche neuronale Netze (KNN) sind ein zentrales Werkzeug des maschinellen Lernens mit einer langen, durchaus wechselvollen Historie, die im Prinzip 1943 mit der Arbeit von Warren McCulloch und William Pitts ihren Anfang nahm [McCP43]. KNN sind am Aufbau natürlicher neuronaler Netze orientierte künstliche Strukturen, die in einem weit gefassten Sinne das biologische Vorbild von vernetzten Nervenzellen im Gehirn von Lebewesen nachempfinden. Miteinander verbundene Neuronen bilden eine komplexe Topologie, die nach einer Lernphase in der Lage ist, Fähigkeiten im Bereich der Mustererkennung und anderer Aufgaben zu lösen. KNN sind aber keine Nachbildung des menschlichen Gehirns und werden auch nicht als solche aufgefasst. Es handelt sich vielmehr um ein Instrument zur Ableitung von Zusammenhängen in gegebenen Informationen, die dann auf neue Gegebenheiten angewendet werden können. Abbildung 1 zeigt eine schematische Darstellung eines solchen Neurons.

\footnotetext{
${ }^{7}$ http://www.nytimes.com/2009/08/24/technology/internet/24emotion.html? r=1

${ }^{8} \mathrm{https}$ ://www.iprospect.com/en/ca/blog/10-sentiment-analysis-tools-track-sōocial-marketing-success/

${ }^{9}$ https://deeplearning4j.org/
} 


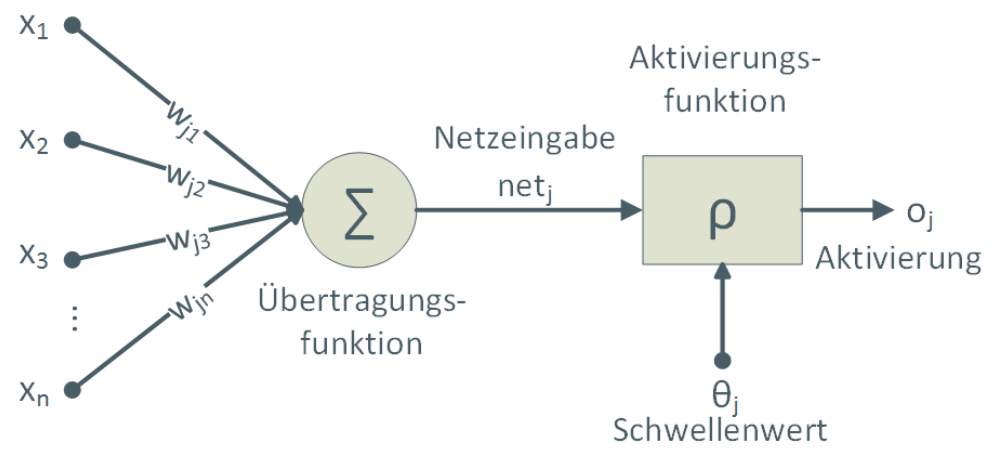

Abbildung 2: Schematische Darstellung eines Neurons [Lang16]

Die gewichteten Eingabekanäle - im natürlichen Vorbild Synapsen genannt - werden addiert. Mit der der Aktivierungsfunktion wird aus den Eingaben des Knoten die Ausgabe berechnet. Diese Ausgabe wird wiederum an weitere Neuronen weitergegeben. Das einzelne Neuron ist Teil eines größeren in Schichten aufgebauten Netzes, bei dem die Ausgabe einer Schicht die Eingabe der nächsten Schicht liefert. Abbildung 2 zeigt die Struktur eines neuronalen Netzwerks.

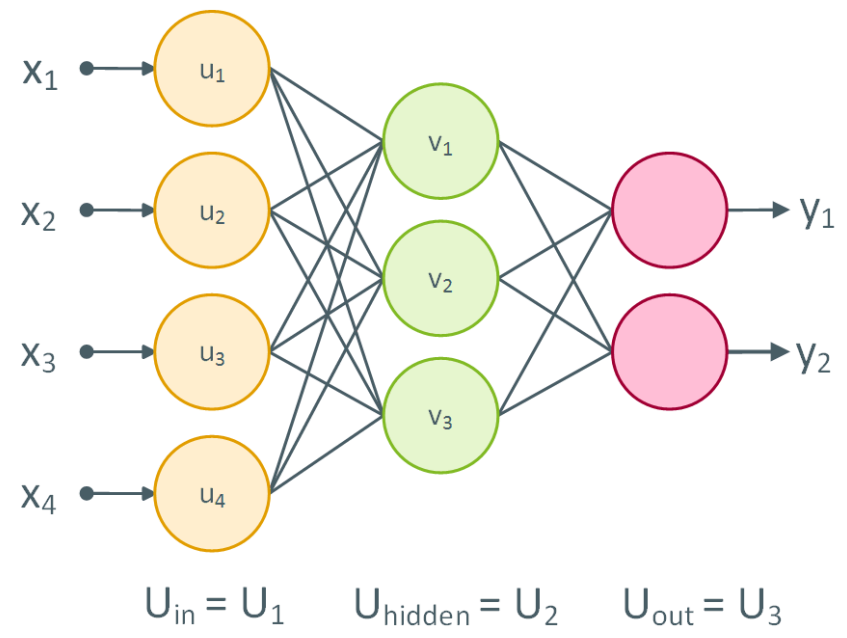

Abbildung 2: Neuronales Netz [Lang16]

Hierbei handelt es sich um ein Netz, das neben der Eingabe- und Ausgabeschicht eine verborgene Schicht besitzt (input layer, output layer, hidden layer). Im Beispiel werden die vier Eingaben über die Verschaltungen des Netzes in die Ausgabe $\mathrm{y}_{1}$ und $\mathrm{y}_{2}$ umgewandelt. Die Gewichte der Synapsen werden dabei zunächst in einem Lernprozess angepasst. Hierbei handelt es sich um sogenanntes überwachtes Lernen (supervised learning): Anhand einer Menge bewerteter Beispiele und dem Vergleich des berechneten Outputs mit der Bewertung werden die Gewichte angepasst. Dies erfolgt in einem umgekehrten Durchlauf des Netzes (Backpropagation) mittels eines Gradienten-Verfahrens, bei dem das Delta der Abweichung vom erwarteten Wert (der Fehler) gemäß einer vorgegebenen Lernrate schrittweise reduziert werden soll. Nach der Lernphase kann das Netz auf neue unbekannte Da- 
tensätze angewendet werden und diese bewerten. Bei rekurrenten Netzen fließt der letzte Zustand eines Knoten bzw. eines benachbarten Knotens mit in den neuen Zustand ein. Damit kann ein Knoten seinen Status speichern. Abbildung 3 zeigt die grundsätzliche Struktur eines Neurons aus einem derartigen Netz, bei dem $\mathrm{o}_{t}$ die Ausgaben zur nächsten Schicht, $x_{t}$ die Eingaben aus der vorherigen Schicht und $h_{t}$ die Rückkopplung zum eigenen Zustand darstellen.

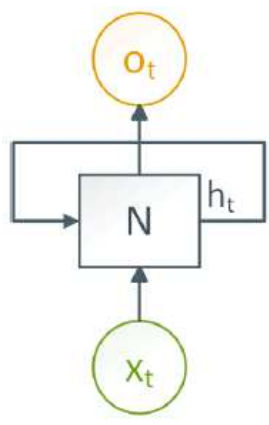

Abbildung 3: Neuron eines Rekurrentes Neuronales Netz [Lang16]

Die bereits 1997 von Hochreiter und Schmidhuber [HS97] beschriebenen LSTM-Netze (für Long short-term memory) sind rekurrente Netze, die in der Lage sind, Informationen über mehrere Schritte zu persistieren (siehe [Olah15]). Diese Eigenschaft macht sie für die Anwendung zur Klassifizierung von Texten interessant.

Ziel ist es nun, künstliche neuronale Netze die Bewertung der Nachrichtentext durchführen zu lassen. Hierzu müssen die Texte in eine geeignete Form gebracht werden, die über die Eingabe an ein geeignetes KNN übergeben werden kann. Das Netz soll dann zum Beispiel ausgeben, ob der Nachrichtentext einen eher positiven oder negativen Charakter hat.

Das von Tomas Mikolov bei Google entwickelte word2vec-Modell leitet aus einem Textkorpus eine vektorielle Darstellung der Wörter dieses Korpus ab. Anders als bei Modellen, die mit sogenannten One-Hot-Vektoren (ein Wort wird durch einen Eintrag in der dem Wort zugeordneten Komponente des Vektors repräsentiert) arbeiten und damit eine große Dimension besitzen, kann das das word2vec-Modell die Dimension der Vektoren reduzieren. Typische Dimensionen liegen zwischen 50 und 300 Komponenten. Die Vektoren stellen nun Wörter in einem durch den Korpus gegebenen Kontext dar und können zum Beispiel für Ähnlichkeitsbetrachtungen herangezogen werden. Die erzeugten Vektoren sind eine numerische Repräsentation der verschiedenen Wortmerkmale im Kontext der Verwendung des Wortes. Diese Wortvektoren können in einer Vielzahl von NLP und Machine-Learning-Aufgaben eingesetzt werden (vgl. [Mi++13a] S. 5).

Mikolov stellt zwei Modellansätze für die Berechnung der Vektoren vor: das Continuous Bag-of-Words Modell (CBOW) und das Skip-gram Modell (SG).

Während das CBOW-Modell auf die Vorhersage eines Wortes anhand des Kontexts zielt, schließt das Skip-gram-Modell vom Wort zum Kontext (siehe Abbildungen 4 und 5).

Dem word2vec-Modell liegt ein dreilagiges neuronales Netz zugrunde. Dieses neuronale Netz erhält als Eingabe die als One-Hot-Vektor codierten Wörter, die versteckte Schicht besitzt die Identitätsfunktion als Aktivierungsfunktion, d.h. die Eingabe in die einzelnen Neuronen entspricht der Ausgabe der Neuronen. 


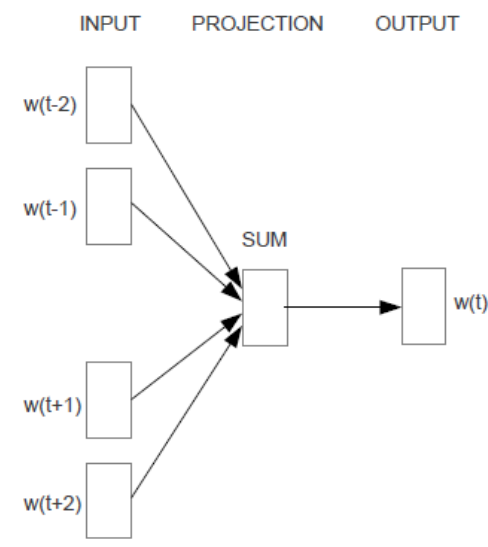

CBOW

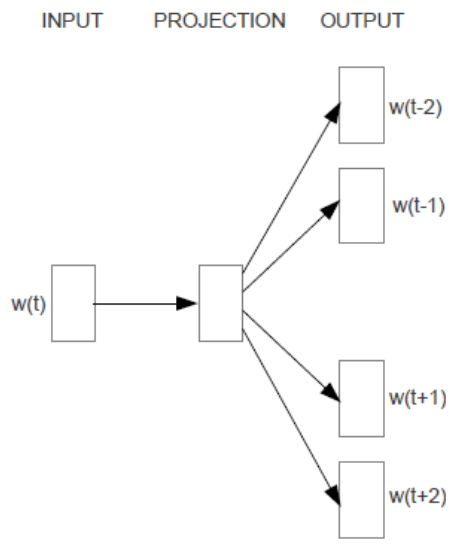

Skip-gram

Abbildung 4: CBOW vs. Skip gram ([Mi++13a])

\section{Input Output}

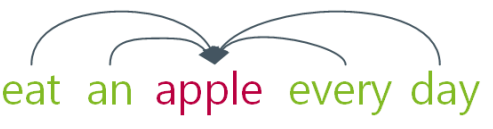

CBOW

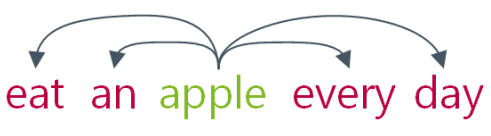

Skip-gram

Abbildung 5: Beispiel Schlussfolgerungen CBOW und Skip-gram

In der Ausgabeschicht wird als Aktivierungsfunktion die sogenannte Softmax-Funktion ${ }^{10}$ verwendet. Die Anzahl der Neuronen in der Input- und der Output-Schicht wird dabei durch die Größe des verwendeten Vokabulars $(V)$ festgelegt, die Anzahl der Neuronen in der mittleren Schicht ist frei wählbar und legt die Dimension $(N)$ der zu erzeugenden Wortvektoren fest. Die Gewichte der Verbindungen zwischen der Eingangs- und der versteckten Schicht als auch zwischen der versteckten und der Ausgabeschicht werden in den Matrizen $W$ und $W^{\prime}$ abgebildet (siehe Abbildung 6).

Die Wordvektoren, die das Modell als Resultat erzeugen soll, befinden sich in den beiden Gewichtsmatrizen $W$ und $W^{\prime}$, wobei bei der ersten Matrix die einzelnen Reihen die jeweiligen Wordvektoren abbilden, bei der zweiten Matrix werden diese durch die einzelnen Zeilen dargestellt. Somit existieren prinzipiell zwei verschiedene Repräsentationen für Wörter (vgl. [Rong14] S. 3).

Die mit dem word2vec-Modell erstellten Wortvektoren zeigen durchaus verblüffende Effekte. So gilt nach geeignetem Anlernen die Wortvektoren für „king“, „man“, „queen“ und „woman“ die folgende Gleichung:

$$
\overrightarrow{x_{\text {klng }}}-\overrightarrow{x_{\text {man }}} \cong \overrightarrow{x_{\text {queen }}}-\overrightarrow{x_{\text {woman }}}
$$

${ }^{10} p\left(x_{i}\right)=\frac{e^{x_{i}}}{\sum_{j} x_{j}}$. Vgl. [Bish09] S. 198 


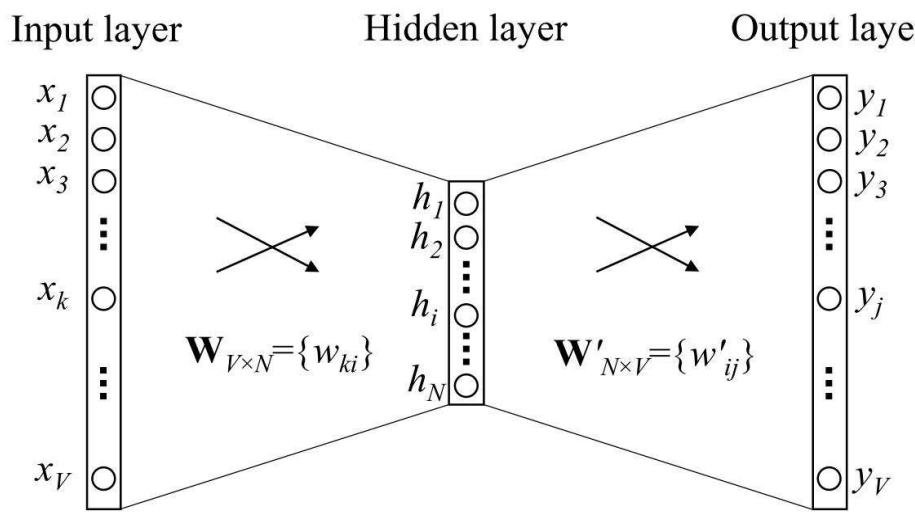

Abbildung 6: Vereinfachtes word2vec-Modell ([Rong14] S. 2)

Weitere semantische Beziehungen wie Meronymie und Antonymie lassen sich aus den Vektoren ebenfalls ablesen (siehe [Mi++13a]). Eine typische Anwendung ist die Bestimmung der Ähnlichkeit zweier Worte durch die Berechnung des Cosinus des durch die Vektoren eingeschlossenen Winkels mit Mitteln der Linearen Algebra: ein Wert von eins spricht für eine hohe Ähnlichkeit, ein Wert von Null für eine sehr geringe (die Vektoren sind in diesem Fall orthogonal).

Für die Bearbeitung mit word2vec und die Klassifizierung mit einem KNN müssen die Texte einer Vorverarbeitung unterzogen werden, der unter anderem Texte unter Berücksichtigung der Satzstruktur in einzelne Wörter zerlegt (Parsing und Tokenization), diese auf ihre Grundform abbildet (Stemming bzw. Lemmatization) und häufige Wörter (sogenannte Stopwords) ohne eigentlich Relevanz für die Aussage des Textes eliminiert. Diese Funktionen werden zum Beispiel von der Stanford-Core-NLP-Bibliothek (siehe [Ma++14]) zur Verfügung gestellt.

Im Projekt wurde die in Abbildung 7 dargestellte Architektur eingesetzt. Das Word2vec-Modell wurde mit Texten aus Wikipedia angelernt. Danach wurden Textnachrichten der britischen Zeitung The Guardian genutzt, um ein LSTM-Netzwerk anzulernen.

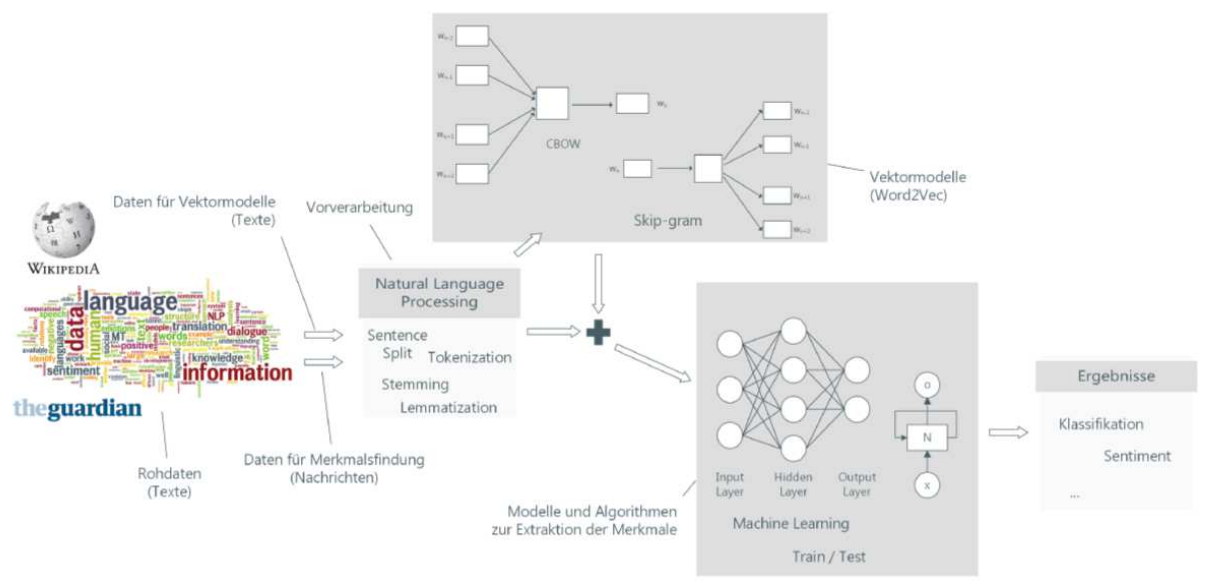

Abbildung 7: Projektumgebung [Lang16] 


\section{$4 \quad$ Ergebnisse}

In der im letzten Abschnitt beschriebenen Architektur wurden Experimente mit verschiedenen Ausprägungen der Komponenten vorgenommen und die jeweiligen Ergebnisse verglichen.

Zunächst wurden alternative Parametrisierungen des word2vec-Modells in Bezug auf die Nutzung von CBOW vs. Skip-gram, die Datenmenge, die Dimension der Vektoren der Kontextgröße und verschiedener Vorverarbeitungen der Texte untersucht. Die Bewertung erfolgt hier anhand der von Mikolov genannten Wortpaare (siehe [Mi++13b]). Die Kenngrößen der Bewertung sind in Abbildung 8 aufgelistet.

\begin{tabular}{|c|c|}
\hline Kennzahl & Bedeutung \\
\hline Semantic Accuracy (g) & Anteil an richtig beantworteten semantischen Paaren \\
\hline Syntactic Accuracy (g) & Anteil an richtig beantworteten syntaktischen Paaren \\
\hline $\begin{array}{l}\text { Overall Accuracy (1st) I } \\
(2 \mathrm{nd})(\mathrm{g})\end{array}$ & $\begin{array}{l}\text { Anteil an richtig beantworteten Paaren (das gesuchte Wort } \\
\text { befindet sich an der ersten Stelle / an einer der ersten bei- } \\
\text { den Stellen) }\end{array}$ \\
\hline Avg Top 100 (k) & $\begin{array}{l}\text { Durchsch. Position des gesuchten Wortes (nur Wörter, die } \\
\text { innerhalb der ersten } 100 \text { Treffer vorkommen) }\end{array}$ \\
\hline Avg Overall (k) & Durchsch. Position des gesuchten Wortes \\
\hline Not Top $100(k)$ & $\begin{array}{l}\text { Anteil der Wortpaare, deren Antwort nicht innerhalb der ers- } \\
\text { ten } 100 \text { Wörter zu finden ist }\end{array}$ \\
\hline Cosine Similarity $(g)$ & Ähnlichkeit zwischen berechneten und tatsächl. Vektor \\
\hline
\end{tabular}

Abbildung 8: Kennzahlen für die Modellbewertung ( $\mathrm{g}=$ größer besser, $\mathrm{k}=\mathrm{kleiner}$ besser)[Lang16]

Ein Vergleich von CBOW und Skip-gram zeigte, dass die erste Methode wesentlich schneller arbeitet - beobachtet wurde ein Faktor von vier - und im Prinzip in Bezug auf fast alle Kennzahlen bessere Ergebnisse lieferte, so dass im Folgenden stets ein CBOW-Modell genutzt wurde. Experimente mit zunehmender Dimension und Kontextgröße zeigten, dass hier zunächst Verbesserungen eintreten, die sich dann nach Erreichen bestimmter Werte nicht mehr steigern (siehe Abbildung 9).
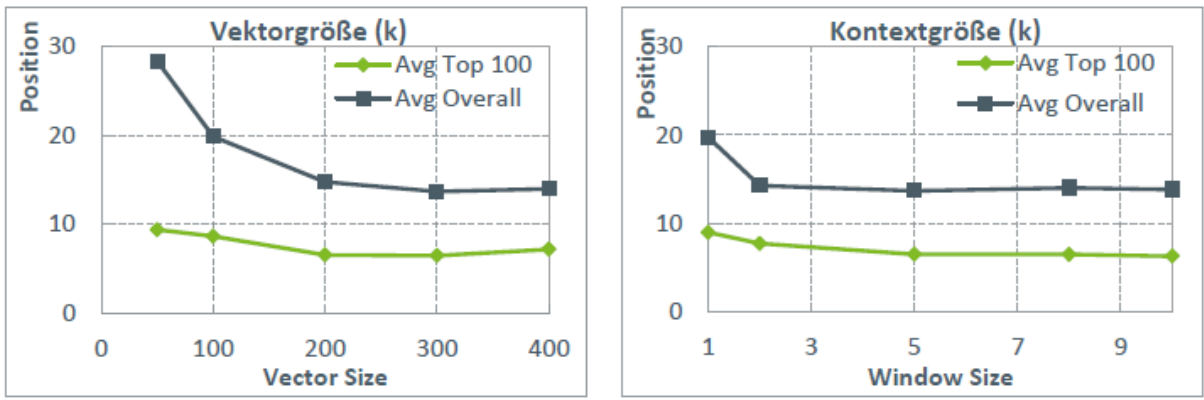

Abbildung 9: Qualität der Vergleiche nach Dimension und Kontextgröße [Lang16]

Es wird deutlich, dass eine zu geringe Dimension bzw. ein zu kleiner Kontext nicht ausreichen, um Aussagen zu treffen. Werden die Werte zu groß gewählt, werden die Ergebnisse bei größerem Aufwand - nicht besser oder sogar schlechter. Hier wird das Modell zu genau 
an die Trainingsdaten angepasst und ist nicht mehr in der Lage, Generalisierungen durchzuführen. Es zeigte sich weiterhin, dass die Ergebnisse bei wachsendem Korpus immer besser wurden und bei den betrachteten Werten noch kein Überlernen eingetreten ist.

Für den Test des gesamten Systems einschließlich des rekurrenten Netzwerks wurden zwei Szenarien betrachtet. Zum einen ein Test, ob das System eine Nachricht korrekt zu einer Branche bzw. Industrie zuordnen kann und zum anderen eine Einschätzung der grundsätzlichen Aussage (positive vs. negative Nachricht). Hierbei wurden die Konfigurationsparameter Lernrate, Anzahl der Neuronen der versteckten Schicht sowie die Aktivierungsfunktion der verstreckten Schicht des Netzes variiert und die jeweiligen Effekte beobachtet. Bei der Anzahl der Neuronen und bei der Lernrate liefern zu kleine Werte und zu große Werte schlechte Ergebnisse. Bei der Wahl zu großer Werte verliert das Netz seine Fähigkeiten der Verallgemeinerung der Sachverhalte, bei zu kleinen Werten ist die Anpassung zu langsam bzw. das Netz zu einfach. Bei der Aktivierungsfunktion zeigte die Sigmoid-Funktion insgesamt gute Ergebnisse. Dies gilt für beide Szenarien

Für die Sentimentanalyse lieferte das Anlernen des Netzes anhand kompletter Nachrichten keine guten Ergebnisse, da die komplette Nachricht in der Aussage zu viele Facetten aufweist und damit nicht eindeutig klassifiziert werden kann. Gleiches gilt für den Versuch des Anlernens mit Filmbewertungen, deren Zusammenhänge nicht ausreichend auf Nachrichtentexte übertragbar sind. Daraufhin wurde das Anlernen über die Titel der Nachrichten durchgeführt. Da der Titel eine wesentlich geringere Anzahl an Wörtern enthält und damit in diesem Sinne aussagekräftiger ist, ist das Anlernen hier effektiver und effizienter.

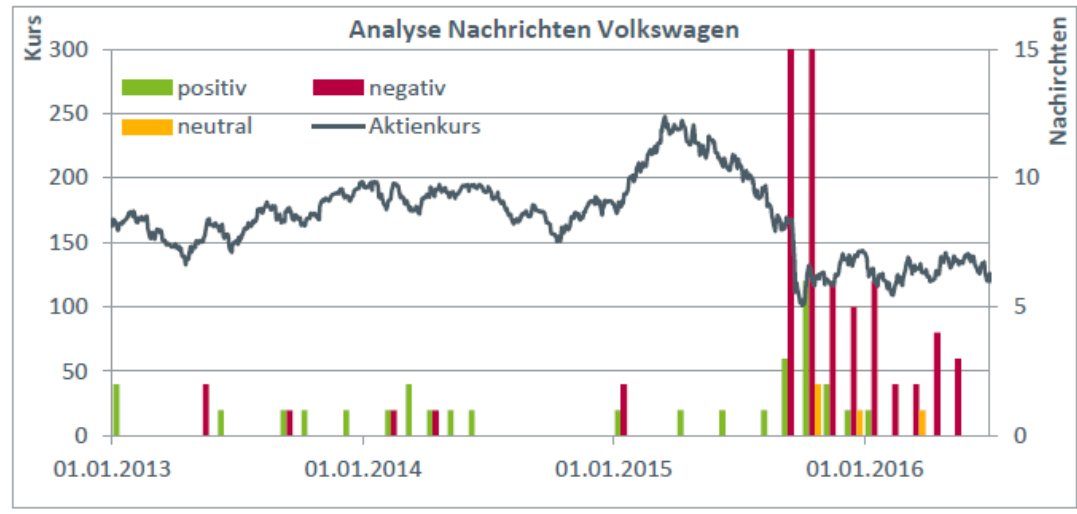

Abbildung 10: Korrelation Aktienkurs Sentiment von Nachrichten [Lang16]

Abbildung 10 zeigt, dass eine intuitiv erwartete Korrelation von schlechten Nachrichten und Aktienkurs tatsächlich beobachtet werden kann.

\section{$5 \quad$ Fazit und Ausblick}

Anwendungen des Text Minings und der automatischen Verarbeitung von Texten zur Gewinnung von Erkenntnissen spielen eine immer größere Rolle. Projekte wie IBM Watson oder die Aktivitäten von Google [Googl16] sind ein Belege dafür. Auch für die Analyse von Finanznachrichten gibt es bereits kommerzielle Produkte. So bietet das deutsche Unternehmen YUKKA Lab ein System an, das laut eigener Aussage des Anbieters ein "[...] 
Früherkennungssystem für die Aktien-Märkte auf Basis von Nachrichten-Bewertungen" zur Verfügung stellt. Bis auf die Aussage, dass zur Identifizierung des Sentiments eines Artikels eine Kombination von statistischen, computerlinguistischen und regelbasierten Verfahren angewendet wird und die Genauigkeit der Interpretation bei ca. $85 \%$ liegt, sind für ein kommerzielles System verständlicherweise keine weiteren Informationen veröffentlicht. Das System wird explizit als Black Box beschrieben .Die Arbeiten im Rahmen des Projektes ADOMIS haben einen Beleg der prinzipiellen Machbarkeit automatischer Textanalyse geliefert. Die hier entwickelte prototypische Architektur wird als Ausgangspunkt weiterer Experimente genutzt.

\section{Literaturverzeichnis}

[Bish09]: $\quad$ Bishop, C. M.: Pattern recognition and machine learning. Springer, New York, NY, 2009

[Goog16]: Google Inc.: Google Cloud Platform. Cloud Natural Language API. Online verfügbar unter https://cloud.google.com/natural-language/, zuletzt geprüft am 16.10.2016.

[HS97]: Hochreiter; Schmidhuber: Long Short-Term Memory, Journal of Neuro Computation, Volume 9 Issue 8, November 15, 1997

[Lang16]: Lang, Marcel: Text-Mining-Methoden zur Klassifikation und Bewertung von Textnachrichten, Masterthesis THM, 2016

[Ma++14]: Manning, C. D. et al.: The Stanford CoreNLP Natural Language Processing Toolkit. In (Association for Computational Linguistics Hrsg.): Association for Computational Linguistics (ACL) System Demonstrations, 2014; S. 55-60

[McCP43]: Warren McCulloch und William Pitts: A logical calculus of the ideas immanent in nervous activity. In: Bulletin of Mathematical Biophysics, Bd. 5 (1943), S. 115-133

[Mi++13a]: Mikolov, T. et al.: Distributed Representations of Words and Phrases and their Compositionality. In (Neural Information Processing Systems Foundation, Inc. Hrsg.): Proceedings of NIPS, 2013, 2013a.

[Mi++13b]: Mikolov, T. et al.: Efficient Estimation of Word Representations in Vector Space. In (International Conference on Learning Representations Hrsg.): International Conference on Learning Representations 2013, $2013 \mathrm{~b}$.

[Olah15]: Olah, C.: Understanding LSTM Networks. Online verfügbar unter http://colah.github.io/posts/2015-08-Understanding-LSTMs/, zuletzt geprüft am 13.07.2016.

[Rong14]: Rong, X.: word2vec Parameter Learning Explained. In CoRR, 2014, abs/1411.2738.

\section{Kontakt}

MSc Marcel Lang, Prof. Dr. Michael Guckert, Prof. Dr. Christian Schulze

KITE Kompetenzzentrum für Informationstechnologie

Technische Hochschule Mittelhessen, Standort Friedberg

Wilhelm-Leuschner-Straße 13, 61169 Friedberg/Hessen

\{marcel.lang, michael.guckert, christian.schulze\}@mnd.thm.de 


\title{
Erlösmodelle von mobilen Anwendungen - Eine Analyse ausgewählter Modelle
}

\author{
Julian Dörndorfer, Christian Seel
}

\section{Zusammenfassung}

Im Jahr 2017 feiert das iPhone sein zehnjähriges Jubiläum und mit inm der junge Markt der mobilen Anwendungen. Dieser Markt hat sich in den letzten Jahren stark entwickelt. Lag der Umsatz 2011 in Deutschland noch bei 211 Millionen Euro, ist er mittlerweile auf über 1,3 Milliarden Euro angewachsen. Jedoch verteilt sich dieser beträchtliche Umsatz nicht gleichmäßig unter den agierenden Entwicklern und Unternehmen auf, sondern es profitieren zumeist nur wenige Anbieter mobiler Anwendungen. Das Gros der restlichen Anbieter macht nur geringen oder gar keinen Profit. Deshalb ist es wichtig zu wissen, wie App-Entwickler mit ihrer mobilen Anwendung einen Gewinn erzielen können. Um diese Frage zu beantworten, verfolgt dieser Beitrag zwei Ziele. Erstens sollen ausgewählte Erlösmodelle betrachtet und analysiert werden. Somit soll geklärt werden, welche Vor- und Nachteile jedes spezifische Erlösmodell hat, für welche Kategorien es eingesetzt, und ob es mit anderen Erlösmodellen kombiniert werden kann. Zusätzlich wird betrachtet, welche Zielgruppe und Kategorie für ein Erlösmodell sinnvoll ist und welche juristischen Vorschriften beachtet werden müssen. Dies kann beispielsweise den Datenschutz und die Datenweitergabe bei mobilen Anwendungen und deren Erlösmodell betreffen. Zweitens wird der Mobile Revenue Catalogue vorgestellt und darin die in dem Beitrag analysierten Erlösmodelle eingetragen. Dieser ist frei zugänglich, womit er für die Anbieter von mobilen Anwendungen einsehbar ist. Er soll damit AppEntwickler sowie Startups und anderen Firmen eine Orientierungs- beziehungsweise Entscheidungshilfe geben, um das profitabelste Erlösmodell zu selektieren.

\section{$1 \quad$ Einleitung}

2017 jährt sich die Erscheinung des iPhones zum zehnten Mal und damit die Geburt des völlig neuen Marktsegments der mobilen Anwendungen. Dieser Markt wuchs innerhalb dieser Dekade von einem weltweiten Jahresumsatz von 100 Millionen US-Dollar (Vgl. ([Bern13].)) auf 36 Milliarden US-Dollar (Vgl. ([Stat17a].)). Auch in Deutschland hat der Markt für mobile Anwendungen bereits 2015 die eine Milliarden Euro Marke geknackt (Vgl. ([Bitk15].)). Der Markt wird auch weiterhin wachsen, da durch den weiteren Ausbau des Funknetzes und dem damit verbesserten Zugang zu breitbandintensive Technologien, mobile Anwendungen und Endgeräte immer mehr in den Alltag integriert werden (Vgl. ([AiSc14], S. 13.)). Jedoch macht es die Heterogenität bei den Betriebssystemen, den Endgeräten und den Vermarktungsmöglichkeiten schwer eine mobile Anwendung zum Erfolg zu führen. Zumal sich der beträchtliche Umsatz nicht gleichmäßig auf die Anbieter der mobilen Anwendungen, sondern nur auf die erfolgreichsten verteilt. Das Gros der Entwickler und Publisher macht keinen, beziehungsweise nur einen geringen Umsatz (Vgl. ([Loui13].)). $\mathrm{Zu}$ diesem Ergebnis kam auch eine Untersuchung unter 10.000 Entwicklern laut derer nur $1,6 \%$ der Befragten mehr als 500.000 US-Dollar verdienen (Vgl. ([Asay14].)). Eine andere 
Umfrage unter amerikanischen Entwicklern ergab, dass $80 \%$ der Teilnehmer nicht genug verdienen, um ein eigenes Geschäft zu unterhalten, 59\% verdienen nicht einmal die Selbstkosten und gar 68\% verdienen unter 5000 US-Dollar im Jahr (Vgl. ([App 12].)).

Um Entwicklern und Unternehmen einen klareren Einblick auf die verschiedenen Erlösmodelle von mobilen Anwendungen mit deren Vor- aber Nachteilen zu geben, soll ein Mobile Revenue Model Catalogue (MRMC) entwickelt werden. Dieser Katalog soll die gängigsten Erlösmodelle für mobile Anwendungen umfassen, sowie die verschiedenen Strategien für mobile Anwendungen, beispielsweise eine Marketing Anwendung rundum ein neues (physisches) Produkt eines Unternehmens, beleuchten. Darauf aufbauend soll letztendlich eine Selektionshilfe zur Wahl des richtigen Erlösmodells erstellt werden, das aufgrund von beantworteten Fragen eine geordnete Liste an Vorschlägen ausgibt.

Um dies zu ermöglichen, sollen in diesem Beitrag ausgewählte Erlösmodelle analysiert werden. Die Analyse soll mit Hilfe eines morphologischen Kastens erfolgen, da dieser es erlaubt mit Hilfe von Merkmalen und deren Merkmalsausprägungen die Untersuchungsobjekte zu klassifizieren. Dies erleichtert später bei der gezielten Befragung der Entwickler und Unternehmen Erlösmodelle zu selektieren, beziehungsweise auszuschließen.

Darauf aufbauen stellt und adressiert dieser Artikel die folgenden Forschungsfragen (RQ):

- $\quad R Q$ 1: Nach welchen Kriterien lassen sich Erlösmodelle von mobilen Anwendungen vergleichen?

- $\quad R Q$ 2: Welche Erlösmodelle sind für diese Klassifikation relevant?

- $\quad R Q$ 3: Wie fällt eine Klassifizierung von Erlösmodellen von mobilen Anwendungen anhand dieser Kriterien aus?

Zur Klärung der Forschungsfragen wird zunächst eine Klassifikation der Erlösmodelle von mobilen Anwendungen mit Hilfe eines morphologischen Kastens aufgezeigt (RQ 1). Im Anschluss werden erst Erlösmodelle, die in diesem Beitrag klassifiziert werden vorgestellt $(R Q 2)$ und anschließend eingeordnet $(R Q 3)$.

Das Gesamtziel des Beitrags besteht darin ausgewählte Erlösmodelle zu klassifizieren, um sie für die Verwendung im MRMC aufzubereiten, um somit Entwickler und Unternehmen bei der Selektion eines passenden Erlösmodells zu unterstützen. Der Beitrag ist wie folgt aufgebaut. Zuerst wird die Literatur analysiert und Beiträge zum Thema vorgestellt, danach werden die Kriterien, die für die Einordnung der Erlösmodelle notwendig sind vorgestellt. Anhand dieser Kriterien werden die Modelle anschließend eingeordnet und bewertet. Abschließend wird ein Ausblick auf zukünftige Arbeiten geben.

\section{Related Work}

Zuerst sollten die oft synonym verwendeten Begriffe Erlösmodell und Geschäftsmodell voneinander abgegrenzt werden (Vgl. ([MaTr12].)). Der Begriff Geschäftsmodell wird oftmals verwendet, um auszudrücken wie aus einem Gut ein Umsatz generiert werden kann. Jedoch umfasst der Begriff mehr als die reine Erlösquelle. Die häufige Verwechslung resultiert auch daraus, dass der Begriff nicht einheitlich definiert ist ((Vgl. ([MaTr12].); ([lbra06].); $([\mathrm{PaGi04}])$.$) und sowohl von der Seite der Praktiker, als auch von Seiten der Wissenschaft-$ ler gesehen werden kann. PATELI und GIAGLIS (VGL. ([PAGI04].)) stellen fest, dass Praktiker den Begriff Geschäftsmodell verwenden, um auszudrücken wie sie einen Erlös aus einem Gut erwirtschaften. Wissenschaftler aus dem Feld der Wirtschaftsinformatik hingegen sehen in dem Begriff die Schlüsselkomponente, welche die Geschäftsidee eines Unternehmens 
definiert, beispielsweise welche Akteure, Informationen, Rollen, Services, Produkte, Erlöse es gibt. Sie stellen auch weiter fest, dass viele Wissenschaftler das Konstrukt Geschäftsmodell als ein Bindeglied zwischen Strategie und Geschäftsprozesse halten.

Der Begriff Erlösmodell wird von AMIT und ZotT (Vgl. ([AmZo01].)) als Erlösquelle, sowie deren Umfang und Verteilung beschrieben. Einig sind sich Praktiker und Wissenschaftler darin, dass ein Erlösmodell integraler Bestandteil eines Geschäftsmodells ist ((Vgl. ([GrWe05].); ([GeBo11].)). TIMmeRS (Vgl. ([Timm98].)) definiert Erlösmodell als "die Organisation oder Architektur eines Produkt-, Service- oder Informationsflusses und die Quelle der Erlöse und Nutzen für Zulieferer und Kunden“. Somit ist das Erlösmodell die Quelle der Erlöse eines Produkts und deshalb entscheidend für ein Unternehmen. Zwar werden mobile Anwendungen bei Geschäftsmodellen eingeplant, die alleinige Betrachtung für den monetären Erfolg einer mobilen Anwendung fällt jedoch in die Kategorie der Erlösmodelle. Deshalb wurde der Begriff Erlösmodell für diesen Artikel, den damit verbundenen Nachforschungen und dem MRMC gewählt.

Es gibt bereits Arbeiten auf dem Gebiet der Erlösmodelle für mobile Anwendungen. Beispielsweise beschreibt WÄCHTER (VGL. ([WÄCH16].)) "den mobilen Tsunami“ und wie man auf ihn reagieren kann. Jedoch ist das Buch mehr eine Retrospektive und Erklärung des IstZustandes. Erst im letzten Teil des Buches werden Mechanismen von mobilen Anwendungen erklärt, jedoch liegt der Fokus auf Management und nicht auf Entwicklung oder Markteintritt. Tosıc (VGL. ([TosI15].)) beschreibt in seinem Buch, wie kleine und mittlere Unternehmen (KMU) mobile Anwendungen nutzen können. Er beschreibt dabei sehr anschaulich welche verschiedenen Einsatzgebiete für Apps in KMUs existieren und wie diese nutzbar gemacht werden können. Jedoch fehlen hier ein paar Erlösmodelle und eine generelle Übersicht über den Markt. Deshalb soll dieser Artikel dazu beitragen eine Übersicht über die verschiedenen Erlösmodelle zu erbringen, damit diese später in die Entwicklung des MRMC einfließen können.

Die Analyse der Erlösmodelle stützt sich auf Meta-Analysen verschiedener Studien und Experteninterviews, die zu dem Thema durgeführt wurden. In der Analyse der Erlösmodelle wurden nur die Betriebssysteme von Google und Apple und deren Marktplätze berücksichtigt, da sie zusammen einen weltweiten Marktanteil von 98,4\% (Vgl. ([GoFo17].)) haben.

\section{$3 \quad$ Kriterien für die Analyse von Erlösmodellen}

Um die einzelnen Erlösmodelle miteinander vergleichen zu können, sowie deren Vor- und Nachteile herauszuarbeiten, ist es zuerst notwendig eine Reihe von Merkmalen festzulegen und diese bei den einzelnen Modellen auf ihre Ausprägung hin zu prüfen. Um die Erlösmodelle möglichst detailliert zu klassifizieren und die Übersicht zu wahren, wurden zwei morphologische Kästen entwickelt. Da der Begriff Zielgruppe zu grob granular ist, wurde entschieden verschiedene Merkmale herauszuarbeiten und in einen eigenen morphologischen Kasten darzustellen (Tab. 1). Dabei soll festgestellt werden, welche Zielgruppe mit einem Erlösmodell erreicht werden kann. Der Fokus liegt dabei auf dem Alter, dem Einkommen und der Zielgruppe. Darüber hinaus werden das Geschlecht und der potenzielle Marktanteil des Erlösmodells betrachtet. Letzteres dient zur Einschätzung, wie viele Benutzer durch ein Erlösmodell erreicht werden können. 


\begin{tabular}{|c|c|c|c|c|}
\hline Merkmal & Merkmalsausprägung & & & \\
\hline Alter & $12-18$ & $25-34$ & $35-44$ & $45+$ \\
\hline Einkommen & Hoch & Mittel & & Gering \\
\hline Geschlecht & Frauen & & Männer & \\
\hline Zielgruppe & $\begin{array}{l}\text { Geschäfts- } \\
\text { leute/Firmen }\end{array}$ & Entwickler & $\begin{array}{c}\text { Spezielle } \\
\text { Gruppierung }\end{array}$ & $\begin{array}{c}\text { Early } \\
\text { Adopters }\end{array}$ \\
\hline $\begin{array}{l}\text { Pot. Markt- } \\
\text { anteil }\end{array}$ & Hoch & Mittel & & Gering \\
\hline
\end{tabular}

Der zweite morphologische Kasten (Tab. 2) fasst die Merkmale und Ausprägungen rund um die Monetarisierung von Erlösmodellen übersichtlich zusammen. Dazu werden die strategischen Firmenziele, die das Modell unterstützt, betrachtet, ob direkt ein Erlös erzielt werden kann, in welchen Kategorien es einsetzbar ist und ob juristische Hürden die Realisierung erschweren. Zudem wird die Beliebtheit des Modells bei den Entwicklern und passend dazu die Kundenakzeptanz betrachtet. Des Weiteren werden die Implementierungsmöglichkeit und Kombinierbarkeit mit den anderen vorgestellten Modellen untersucht.

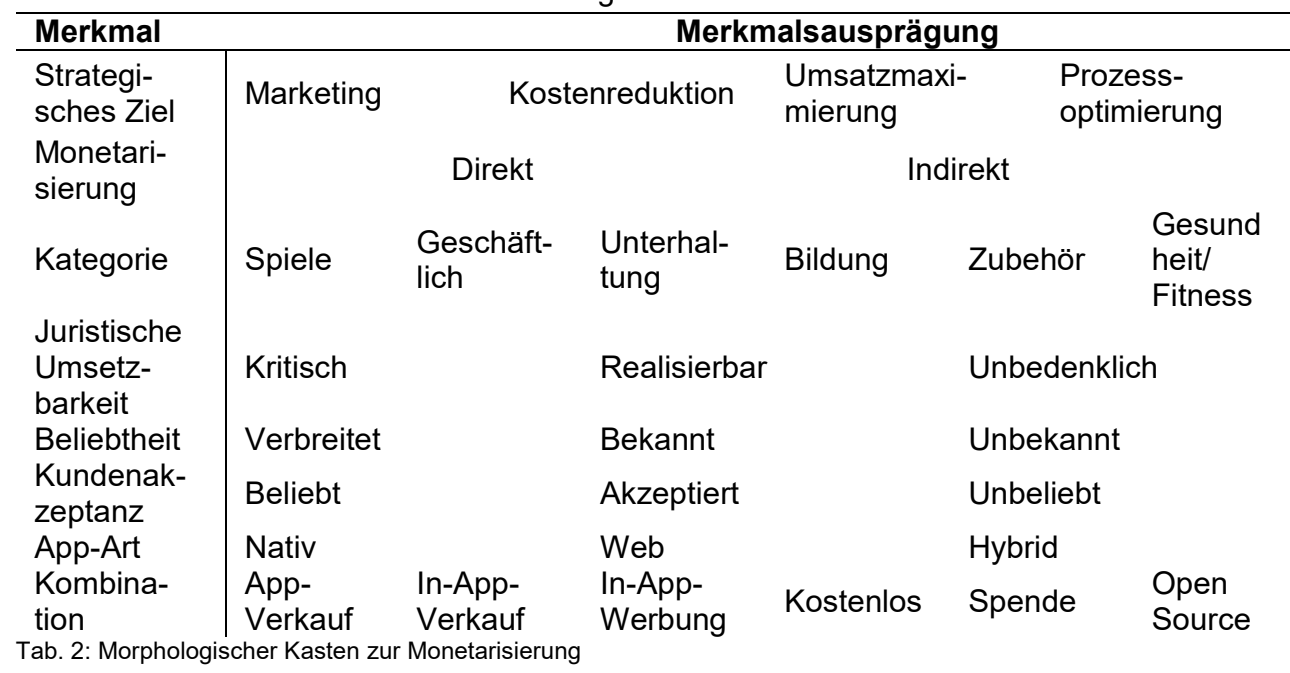

\section{Die Klassifizierung der Erlösmodelle}

Im Folgenden werden die Erlösmodelle App-Verkauf, In-App-Verkauf, In-App-Werbung, Kostenlos, Spende und Open Source kategorisiert. Basis hierfür waren die identifizierten Erlösmodelle von DÖRNDORFER und SEEL, die auch in deren Artikel näher beschreiben werden (Vgl. ([DöSe16].)). In Tab. 3 sind alle Modelle aufgelistet (Spalten) und wurden durch die Merkmalsausprägungen aus den zwei morphologischen Kästen in der jeweiligen Kategorie (Zeilen) klassifiziert. 


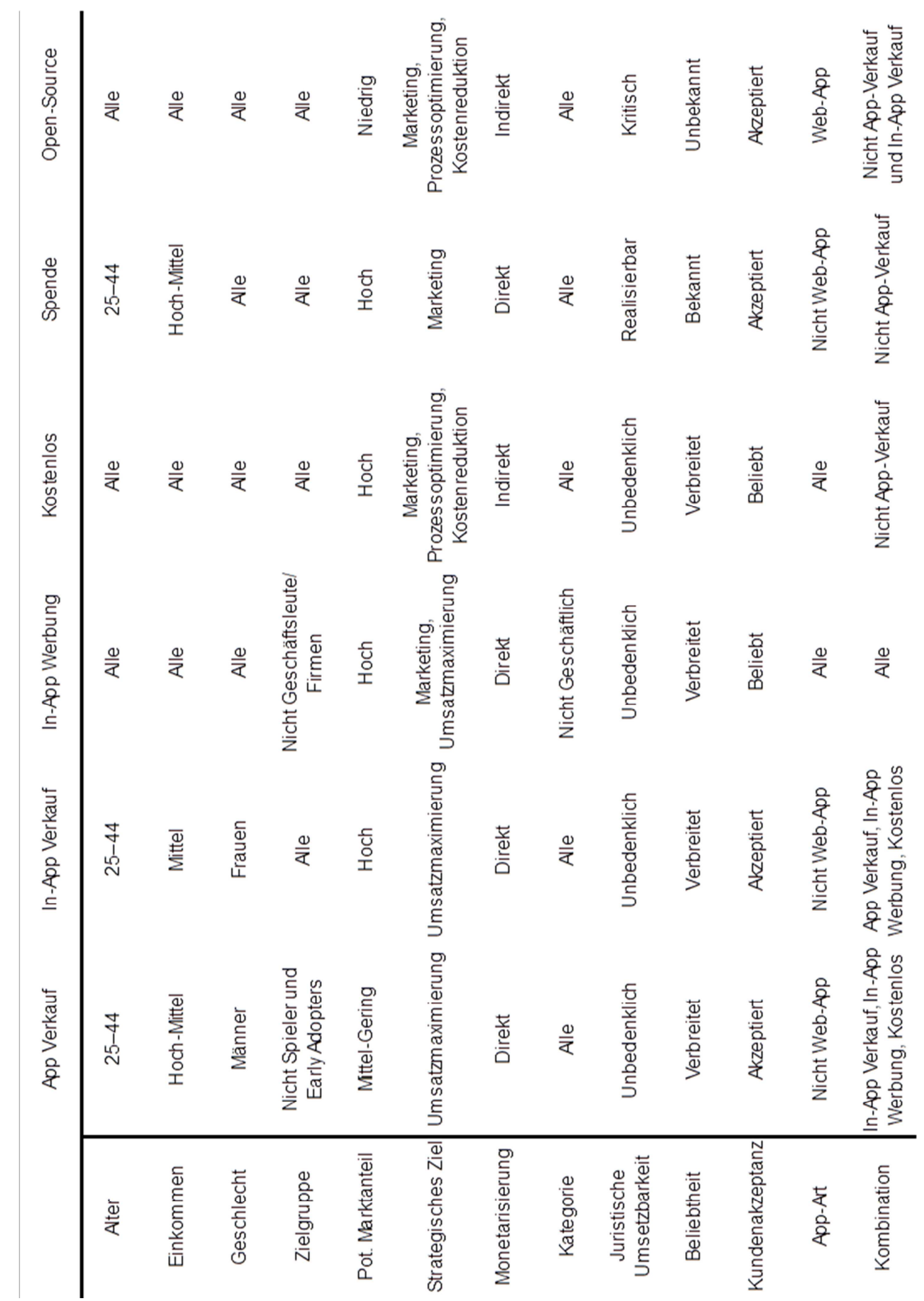

Tab. 3 Klassifizierung der betrachteten Erlösmodelle 


\subsection{App-Verkauf}

Bei diesem Erlösmodell wird die mobile Anwendung zum Verkauf in den jeweiligen Stores angeboten. Durch diese Barriere werden die meisten Nutzer unter 18 Jahren als Käufer ausgeschlossen, da sie meist nur in etwa $1 €$ pro Monat für mobile Anwendungen ausgeben (Vgl. ([HoSH14].)) und nur über begrenzte Zahlungsmöglichkeiten verfügen. Das meiste Geld hingegen geben die Leute in der Altersgruppe der 25- bis 34-Jährigen aus, gefolgt von den 34 bis 44-Jährigen (Vgl. ([Wood16].)). Dieses Modell zielt auf hohe bis mittlere Einkommen $a b$, da es sich um einen direkten Kauf handelt. Männer sind eher bereit Geld für eine mobile Anwendung zu zahlen als Frauen und kaufen eine Anwendung ohne sie vorher testen zu können (Vgl. ([Wood16].)). Bei der Zielgruppe sind nur bei Spielern und Early Adopters Einschränkungen zu erwarten, da Spieler meist jünger und somit nicht spendabel sind. Early Adopters werden ein Produkt eher ausprobieren, wenn es für sie erst einmal kostenlos ist. Der potenzielle Marktanteil wird bei einem reinen Erlösmodell Verkauf nur bei mittel bis gering liegen, da die Benutzer nur bei sehr ausgereiften und beliebten mobilen Anwendungen breit sind Geld auszugeben. Generell ist die Kaufbereitschaft relativ gering und liegt bei 7,40 US-Dollar in drei Monaten (Vgl. ([Wood16].)).

In dem direkt ein Erlös erzielt werden soll, dient das Modell primär der Umsatzmaximierung. Das Erlösmodell ist weit verbreitet, von den Kunden akzeptiert und wurde bei Android-Apps 2015 weltweit bei etwa 30\% der angebotenen Apps im Google-Play-Store verwendet (Vgl. ([Stat17b].)). Jedoch kann dieses Modell nur bei nativen oder hybriden Anwendungen angewendet werden, da sie in den jeweiligen App Stores angeboten und abgerechnet werden müssen. Zu beachten ist hierbei, dass die App-Store-Betreiber 30\% des Verkaufspreises einbehalten und auch eine Mehrwertsteuer von 19\% fällig wird. Kombinierbar ist dieses Modell mit In-App-Verkauf (Paidmium), Kostenlos (Freemium) und In-App-Werbung. Die letzte Kombination sollte gut überlegt werden, da viele Käufer nach einem Kauf keine beziehungsweise nur wenig Werbung erwarten dafür, dass sie die mobile Anwendung gekauft haben.

\subsection{In-App-Verkauf}

Das Erlösmodell In-App-Verkauf zielt darauf ab, virtuelle Güter (beispielsweise neue Funktionen) innerhalb einer Anwendung zu verkaufen, ohne dass die mobile Anwendung selbst etwas kostet. Wie auch bei reinen App-Verkäufen sollten auch hier bevorzugt die Leute ab 25 Jahren adressiert werden, da sie das meiste Geld ausgeben. Dadurch, dass die Anwendung erst einmal kostenlos ist, können zusätzlich Gruppen mit geringerem Einkommen die Adressaten sein. Da bevorzugt Frauen erst die Anwendung ausprobieren wollen und danach eher bereit sind Geld auszugeben, sind sie als Zielgruppe interessant (Vgl. ([Wood16].)). Durch den kostenlosen Download steht grundsätzlich keine Hürde bei der Verbreitung der Anwendung im Weg, somit kann theoretisch ein hohes Marktpotenzial abgeschöpft werden. Das belegt auch eine Studie, wonach 99\% der Top 200 Anwendungen im Apple-App-Store In-App-Verkäufe anbieten (Vgl. ([Spen16].)).

Der direkte Erlös durch die In-App-Käufe führt dazu, dass das Erlösmodell zur Umsatzmaximierung verwendet wird. Das Erlösmodell ist bei Entwicklern beliebt, da sie damit einen regelmäßigen Umsatz generieren können, denn die meisten virtuellen Güter können mehrmals gekauft werden. Bei den Benutzern ist dieses Erlösmodell akzeptiert. 


\subsection{In-App-Werbung}

Die Einblendung von Werbung innerhalb einer mobilen Anwendung wird In-App-Werbung (Advertising) genannt. Da bei diesem Modell keine Kosten für den Nutzer anfallen, ist in Bezug auf Alter, Einkommen, Geschlecht und dem potenziellen Marktanteil mit keinen Einschränkungen zu rechnen. Bei der Zielgruppe ist jedoch zu beachten, dass Geschäftsleute bzw. Firmen ungern Werbung in mobilen Anwendungen sehen, wenn diese für einen betrieblichen Zweck eingesetzt werden.

In-App-Werbung kann eingesetzt werden, um beispielsweise Kosten einer Anwendung für Marketingzwecke teilweise zu refinanzieren oder andere Produkte desselben Unternehmens zu bewerben. Darüber hinaus kann In-App-Werbung natürlich auch zur Umsatzmaximierung hergenommen werden. Durch die finanzielle Beteiligung an der Ausspielung von Werbung wird die mobile Anwendung direkt monetarisiert. Jedoch sollte man sich bewusst sein, dass hierfür meist nur Centbeträge ausgezahlt werden und für einen hohen Umsatz je nach Vergütungsmodell mehrere tausend Anzeigen geschaltet werden müssen (Vgl. ([Maye12], S. 281.)). Da für den Nutzer keine Kosten anfallen ist das Modell sehr beliebt, außer die Werbeeinblendungen werden als störend empfunden(Vgl. ([Wäch16].)) . Auch in Entwicklerkreisen ist dieses Modell beliebt, da es erst einmal keine Hürde für eine weite Verbreitung setzt und es für alle Arten von mobilen Anwendungen einsetzbar ist. Des Weiteren ist es mit allen anderen Erlösmodellen kombinierbar.

\subsection{Kostenlos}

Wenn eine mobile Anwendung kostenfrei in den App Stores zum Download bereitsteht, handelt es sich um das Erlösmodell Kostenlos (Free). Auch bei diesem Erlösmodell sind bei der Zielgruppenkategorisierung keine Einschränkungen zu erwarten.

Die Erlöse erfolgen bei diesem Modell immer indirekt. Das bedeutet, nicht mit der App selbst wird ein Umsatz erzielt, sondern durch andere Leistungen oder Produkte. Dabei kann die Anwendung als Promotion für ein neues Produkt dienen, beispielsweise einem neuen Auto, oder sie kann dazu dienen die Funktionen eines Produktes zu komplettieren, wie eine Fernbedienungsanwendung zu einem Soundsystem. Die Flexibilität dieses Modells spiegelt sich auch darin, dass sich alle strategischen Firmenziele, bis auf die Umsatzmaximierung, unterstützen lassen. Um die teilweise sehr hohen Entwicklungskosten zu refinanzieren ist es möglich dieses Erlösmodell mit allen anderen zu kombinieren. Eine der bekanntesten Kombinationen ist das Freemium-Modell, bei dem die App kostenlos angeboten wird und dann durch zusätzliche Funktionen kostenpflichtig erweitert werden kann (Vgl. ([Maye12].)).

\subsection{Spende}

Beim Modell Spende handelt es sich um eine mobile Anwendung, die kostenfrei heruntergeladen werden kann, aber den Benutzer auffordert dem Programmierer beziehungsweise der Entwicklerfirma einen Betrag zu spenden, um ihre Aufwände zu refinanzieren. Dabei sollte sich, genauso wie bei den Erlösmodellen Verkauf und In-App-Verkauf, auf eine Zielgruppe im Alter von 25-44 konzentriert werden, denn in diesem Altersbereich sind die Nutzer am ausgabefreudigsten (Vgl. ([Wood16].)). Dazu kommt, dass ältere Menschen eher bereit sind etwas zu spenden als jüngere (Vgl. ([Schi15].)). Dadurch, dass die Anwendung kostenfrei heruntergeladen werden kann, ist der potenzielle Marktanteil nicht eingeschränkt. Durch Spenden werden mit der mobilen Anwendung direkt Einnahmen erzielt und können für politische und karitative Vereine eine Möglichkeit sein, gleichzeitig Spenden zu sammeln und Werbung zu machen. Zu beachten ist dabei jedoch, dass Apple sowie Google bei 
Spenden in mobilen Anwendungen sehr restriktiv sind. Apple erlaubt Spenden nur außerhalb ihres App Stores und dann auch nur für von innen anerkannte Vereine (Vgl. ([Appl17a].)), ansonsten sind Spenden nur als In-App-Verkäufe möglich. Ähnlich verfährt auch Google, jedoch erlauben sie generell keine Spenden außerhalb ihres Marktplatzbezahlungssystems (Vgl. ([Goog17].)). Das Modell ist generell bei Anbietern und Nutzern bekannt, jedoch nicht sehr weit verbreitet, da es schwer kalkulierbare Einnahmen generiert (Vgl. ([Maye12].)). Zudem kann es aufgrund der Restriktionen von Google und Apple nicht für Web-Apps verwendet werden. Dafür kann es aber mit allen Erlösmodellen kombiniert werden, außer mit dem Verkaufsmodell, da Anwender erwarten nach dem Kauf einer Anwendung nicht noch zusätzlich dafür zu spenden.

\subsection{Open Source}

Eine mobile Anwendung deren Quellcode verfügbar und grundsätzlich veränderbar ist, nennt man Open-Source-Anwendung. Diese bei „normalen“ Desktop- und Webanwendungen verbreitete Form eines Erlösmodells ist bei mobilen Anwendungen bisher kaum vorhanden. Obwohl damit grundsätzlich eine breite Masse erreicht werden kann, da Open-SourceAnwendungen kostenlos zur Verfügung stehen (Vgl. ([AiSc14].)). Bei dem Modell wird versucht indirekt Geld zu verdienen, indem beispielsweise die Anwendung an die Kundenbedürfnisse angepasst wird oder zusätzliche Leistungen wie Serverkapazitäten vermietet werden.

Mit dem Modell können mobile Anwendungen erstellt werden, welche innerbetrieblich zur Prozessoptimierung oder Kostenreduktion beitragen. Genauso können sie zu Marketingzwecken eingesetzt werden, um die Bekanntheit eines Unternehmens zu steigern (Vgl. ([AiSc14].)). Beispielsweise kann eine Raumreservierungs-App in einem Unternehmen erfolgreich eingeführt worden sein, jedoch sind keine weiteren Ressourcen zur Wartung und Weiterentwicklung eingeplant. Um dennoch die Anwendung zu verbessern, kann dies durch Veröffentlichung als Open-Source-Projekt versucht werden. Juristisch kann die Verbreitung von mobilen Open-Source-Anwendungen bei Apple kompliziert werden. Denn Apple erlaubt es nicht die mobile Anwendung „über andere Vertriebskanäle an Dritte weiter zu vertreiben oder es Dritten zu ermöglichen oder zu gestatten“, wenn sie gleichzeitig im App Store zur Verfügung steht (Vgl. ([Appl17b].)). Die Lizenzauswahl bei Open-Source-Apps muss somit für den Apple-App-Store sorgfältig getroffen werden, damit Lizenzkonflikte vermieden werden. Google macht dahingegen keine Restriktionen. Aufgrund dieser Lage ist das Modell bei Entwicklern kaum verbreitet.

\section{$5 \quad$ Zusammenfassung und Ausblick}

Das richtige Erlösmodell für eine mobile Anwendung zu finden kann mitunter schwierig sein und sollte gut durchdacht werden, da sonst das Risiko groß ist mit seiner Anwendung keinen oder wenig Umsatz zu erzielen. Dieser Artikel hat dazu beigetragen eine Übersicht über ausgewählte Modelle zu geben. Dabei kam heraus, dass manche Modelle schwieriger umzusetzen sind als andere oder von bestimmten Zielgruppen bevorzugt werden. Letztendlich sollen die Ergebnisse in den MRMC einfließen und Entwicklern sowie Firmen eine Entscheidungshilfe für die Selektion eines Erlösmodells geben. 
Jedoch sind noch nicht alle Modelle, die in (Vgl. ([DöSe16].)) genannt werden, untersucht. Deshalb sollen auch die restlichen Modelle in der gleichen Weise analysiert und ausgewertet werden, damit der MRMC vervollständigt wird.

\section{Literaturverzeichnis}

[AiSc14] Aichele, Christian; Schönberger, Marius: App4U. Springer Fachmedien Wiesbaden, Wiesbaden, 2014.

[AmZo01] Amit, R.; Zott, C.: Value creation in E-business. In: Strategic Management Journal(22(67)), 2001, S. 493-520.

[App 12] App Promo: The Necessity of Mobile App Marketing, Toronto, 2012. URL: http://apppromo.com/wp-content/uploads/2012/04/AppPromo-TheNecessityofMobileAppMarketing.pdf. Abruf am 2015-12-21.

[Appl17a] Apple: Apple Guidelines, 2017. URL: https://developer.apple.com/appstore/review/guidelines/2016-06-13/\#charitiescontributions.

[Appl17b] Apple: Lizenzvertrag für das iOS-Entwicklerprogramm, 2017. URL: https://developer.apple.com/programs/terms/ios/standard/ios_program_standard_agree ment_20140909.pdf. Abruf am 2017-04-06.

[Asay14] Asay, Matt: Want To Make Money In Apps? Develop For Business, 2014. URL: http://readwrite.com/2014/07/22/app-developers-enterprise-opportunity/.

[Bern13] Bernau, Varinia, 2013: Großes Geschäft mit kleinen Helfern: Die neue App-Economy: Rund um Smartphones ist ein Milliardenmarkt für Anwenderprogramme entstanden. Die Entwickler können in kurzer Zeit viel Geld verdienen. Größte Profiteure sind aber Apple und Google. In: Süddeutsche Zeitung 12. März, S. 17.

[Bitk15] Bitkom: Deutscher App-Markt knackt Milliarden-Marke, Berlin, 2015. URL: http://www.pressebox.de/pressemitteilung/bitkom-bundesverband-

informationswirtschaft-telekommunikation-und-neue-medien-ev/Deutscher-App-Marktknackt-Milliarden-Marke/boxid/753590. Abruf am 2017-01-13.

[DöSe16] Dörndorfer, Julian; Seel, Christian: Mobile Revenue Model Catalogue: A guide through the diversity of mobile revenue models. In: Hofmann, Rainer; Alm, Wolfgang (Hrsg.): Wissenstransfer in der Wirtschaftsinformatik: Fachgespräch im Rahmen der MKWI 2016, Aschaffenburg, 2016, S. 10-18.

[GeBo11] George, G.; Bock, A. J.: The business model in practice and its implications forentrepreneurship research. In: Entrepreneurship Theory and Practice(35(1)), 2011, S. 83-111.

[GoFo17] Goasduff, Laurence; Forni, Amy A.: Gartner Says Worldwide Sales of Smartphones Grew 7 Percent in the Fourth Quarter of 2016, 2017. URL: https://www.gartner.com/newsroom/id/3609817. Abruf am 2017-04-04.

[Goog17] Google: Monetarisierung und Werbung, $2017 . \quad$ URL: https://play.google.com/intl/de/about/monetization-ads/. Abruf am 2017-04-05.

[GrWe05] Gruhn, Volker; Weber, Thorsten: From an E-Business Revenue Model to Its Software Reference Architecture. In: Grzech, Adam; Funabashi, Matohisa (Hrsg.): Challenges of expanding Internet: E-commerce, e-business, and e-government ; 5th IFIP Conference 
on e-Commerce, e-Business, and e-Government (13E'2005), October 28-30, 2005, Poznan, Poland. Springer, New York, N.Y, 2005, S. 33-47.

[GrFu05] Grzech, Adam; Funabashi, Matohisa (Hrsg.): Challenges of expanding Internet: Ecommerce, e-business, and e-government ; 5th IFIP Conference on e-Commerce, eBusiness, and e-Government (13E'2005), October 28-30, 2005, Poznan, Poland, New York, N.Y. Springer, 2005. (International Federation for Information Processing, 189).

[HoAl16] Hofmann, Rainer; Alm, Wolfgang (Hrsg.): Wissenstransfer in der Wirtschaftsinformatik: Fachgespräch im Rahmen der MKWI 2016, Aschaffenburg, 2016.

[HoSH14] Holdampf-Wendel, Adél; Shahd, Maurice; Hampe, Katja: Jung und vernetzt: Kinder und Jugendliche in der digitalen Gesellschaft 2014, Berlin, 2014. BITKOM - Bundesverband Informationswirtschaft, Telekommunikation und neue Medien e.V. URL: https://www.bitkom.org/noindex/Publikationen/2014/Studien/Jung-und-vernetzt-Kinderund-Jugendliche-in-der-digitalen-Gesellschaft/BITKOM-Studie-Jung-und-vernetzt2014.pdf. Abruf am 2017-04-03.

[Ibra06] Ibrahim, I. K.: Handbook of Research on Mobile Multimedia. Idea Group Inc (IGI), 2006.

[Loui13] Louis, Tristan, 2013: How Much Do Average Apps Make? In: Forbes 10. August. Online im Internet: URL: http://www.forbes.com/sites/tristanlouis/2013/08/10/how-much-doaverage-apps-make/.

[MaTr12] Marques Silva, Carlos; Trkman, Peter: Business Model: What It Is and What It Is Not, 2012.

[Maye12] Mayerhofer, Josef: Apps erfolgreich verkaufen. Carl Hanser Verlag GmbH \& Co. KG, München, 2012.

[PaGi04] Pateli, Adamantia G.; Giaglis, George M.: A research framework for analysing eBusiness models. In: Eur J Inf Syst 13(4), 2004, S. 302-314.

[Schi15] Schiemenz, Andreas: Das persönliche Gespräch: Fundraising durch Überzeugung Großspender und Unternehmer erfolgreich ansprechen. Springer Gabler, Wiesbaden Germany, 2015.

[Spen16] Spencer, Graham: Exploring the App Store's Top Grossing Chart, 2016. URL: https://www.macstories.net/stories/exploring-the-app-stores-top-grossing-chart/. Abruf am 2017-04-04.

[Stat17a] Statista: Umsatz im Apple App Store und Google Play Store weltweit in den Jahren 2014 bis 2016 (in Milliarden US-Dollar), 2017. URL: https://de.statista.com/statistik/daten/studie/180896/umfrage/apple-app-store-vs-googleplaystore-umsatz/.

[Stat17b] Statista: Verteilung der Android Apps nach kostenlosen und kostenpflichtigen Angeboten in den Jahren 2009 bis 2015, 2017. URL: https://statista.de/statistik/daten/studie/74370/umfrage/android-apps-nach-kostenlosenund-kostenpflichtigen-angeboten/. Abruf am 2017-04-04.

[Timm98] Timmers, Paul: Business Models for Electronic Markets. In: Elec. Markets 8(2), 1998, S. 3-8.

[Tosi15] Tosic, Marko: Apps für KMU. Springer Fachmedien Wiesbaden, Wiesbaden, 2015.

[Wäch16] Wächter, Mark: Mobile Strategy: Marken- und Unternehmensführung im Angesicht des Mobile Tsunami. (\#RideTheMobileTsunami). Springer Gabler, Wiesbaden, 2016. 
[Wood16] Woods, Viveca: Gartner Mobile App Survey Reveals 24 Percent More Spending on InApp Transactions Than on Upfront App Payments, 2016. URL: https://www.gartner.com/newsroom/id/3331117. Abruf am 2015-04-03.

\section{Kontakt}

Julian Dörndorfer

Hochschule Landshut

Am Lurzenhof 1, 84036 Landshut

T.: +49 (0) 871506 731, julian.doerndorfer@haw-landshut.de

Christian Seel

Hochschule Landshut

Am Lurzenhof 1, 84036 Landshut

T.: +49 (0) 871506 649, christian.seel@haw-landshut.de 


\title{
Medizinische Therapiebegleitung und Compliancesteigerung mittels gamifizierter, mobiler Anwendungen
}

\author{
Martin Przewloka, Katharina Dort
}

\section{$1 \quad$ Einleitung}

Behandlungs- und Verhaltensmaßnahmen bilden eine wesentliche Grundlage zur Umsetzung von Therapien. Letztere können präventiv, symptomatisch oder kurativ sein, um nur einige Formen zu nennen. Eines der zentralsten Probleme heute ist die Nachhaltigkeit in der Ausführung von verordneten Therapieplänen - man spricht auch von der Compliance bzw. Adhärenz - und dies umso mehr, je eigenständiger und eigenverantwortlicher der Patient die Therapie durchzuführen hat. Es ist heute unbestritten, dass die Kommunikation zwischen Therapeut und Patient einen wesentlichen Erfolgsfaktor der Therapiemaßnahmen einer evidenzbasierten Medizin darstellt. Werden die durchgeführten Maßnahmen, wie die Einnahme von Pharmazeutika, nicht mehr direkt durch den Therapeuten kontrolliert, verringert sich aufgrund eines Kommunikationsbruchs die Therapie-Compliance signifikant ${ }^{11}$.

Überträgt man die beschriebene Problematik auf den Bereich der physikalischen Therapien, und dabei insbesondere auf Anwendungen, die patientenseitig in vollständiger Eigenverantwortung sowie im Regelfall auch mit größerem Zeitaufwand durchgeführt werden müssen, so ist eine Kontrolle zur Vermeidung von Non-Compliance schon alleine aus Praktikabilitätsgründen kaum durchführbar. Bewegungs- und Atemtherapien seien beispielhaft genannt.

Die digitale Transformation wird die Entwicklung von Digitalen Assistenten forcieren ${ }^{12}$. Exakt an dieser Stelle setzt der Ansatz an, einen Therapieplan, verordnet von einem Therapeuten, intelligent in ein Spiel zu konvertieren ${ }^{13}$. Letzteres wird alle individuellen Parameter berücksichtigen, d.h. sowohl die spezifischen Merkmale des Patienten, als auch die konkreten Anforderungen an die Therapie, wozu beispielsweise Häufigkeit der Durchführung, Intensität und Erfolgskontrolle zählen.

Es liegt daher nahe, den Patienten kontinuierlich und spielerisch zur Therapieausführung zu motivieren. Der heutige Verbreitungsgrad von Smartphones, inklusive der darin verbauten Sensorik, sollte es möglich machen, innovative, spielerische Anwendungen zu entwickeln, die die Abbildung eines Therapieplans in Form des implementierten Spiels, dem Spieleplan, repräsentiert. Die medizinischen Parameter Therapieintensität, Wiederholungen, Therapiedauer erfahren hierdurch eine Übersetzung in die Spieleparameter direkte Erfolgsrückmeldung, Schwierigkeitsgrad, Spielelevel, Bonus/Malus, u.a.

\footnotetext{
${ }^{11}$ Unzählige Reports weisen die Problematik und wirtschaftlichen Schäden der nicht ausreichenden Therapie-Compliance aus. Siehe hierzu beispielsweise „Adherence to Long-Term Therapies - Evidence for Action“ WHO (2003) und Studie zu den Auswirkungen der non-compliance der Bertelsmann Stiftung und Booz\&Company (2012)

12 Siehe hierzu beispielsweise: Martin Przewloka: „10 Jahre GFFT, 10 Jahre neue

Informationstechnologien“, in: GFFT Jahresbericht 2015/2016 (2016)

${ }^{13}$ Hierunter ist eine Cloud-Anwendung zu verstehen, die Therapieparameter mittels selbstlernender Algorithmen in Spielparameter konvertiert. Auch hiermit haben sich die Autoren beschäftigt.
} 
Stichworte: Gamifizierung, Mobile Anwendungen, COPD, Cloud, Compliance, Künstliche Intelligenz, Machine Learning

\section{$2 \quad$ Entwicklung eines digitalen Atemtrainers}

Die Lungenerkrankung COPD ${ }^{14}$, neben der einfach chronischen Form vermehrt als chronisch-obstruktive Bronchitis auftretend, wird heute auf einen weltweiten Verbreitungsgrad von 600 Millionen Menschen geschätzt, Tendenz steigend. Alleine in Deutschland geht man davon aus, dass etwa 3 bis 5 Millionen Menschen betroffen sind. Zur einfachen Therapie dieser Krankheit wird heute oftmals ein nach aerodynamischen Prinzipien arbeitender Balltrainer eingesetzt. Die Aufgabe des Patienten ist es dabei, durch das Einatmen einen oder mehrere Bälle zum Schweben zu bringen. Dies erfordert eine konstante Atemleistung, und die positive Rückmeldung besteht einzig und allein in der im Sichtfeld des Patienten befindlichen schwebenden Kugel. Die Folge: Atemübungen mit dem Balltrainer werden sehr schnell aufgegeben, die Therapie-Compliance geht damit zügig zurück bzw. ist überhaupt nicht mehr gegeben.

Es wurde von den Autoren ein völlig neuartiger Atemtrainer, genannt MOPS ${ }^{15}$, entwickelt, der eine elektronische Komponente beinhaltet, welche folgende Parameter erfasst:

- Atemintensität

- Gradient der Atemintensität

- Atemdauer und Atemverlauf

- $\quad$ Art der Atmung (inspiratorisch vs. exspiratorisch)

Der akkubetriebene Atemtrainer (siehe auch Abbildung 1) ist vollständig digitalisiert und nutzt ein verschlüsseltes Low-Energy-Bluetooth-Protokoll zum Echtzeitdatenaustausch mit einem mobilen Endgerät (im Regelfall ein Smartphone). Die Daten werden von einer Applikation normiert und als Eingangsparameter für die Spieleanwendung genutzt. Der Atemwiderstand kann mechanisch über eine Verjüngung der Ansaugöffnung verstellt werden. Die Geometrien des Atemtrainers wurde turbulenzmindernd optimiert, so dass sich zu jeder Zeit und in jeder Betriebsform stabile und reproduzierbare Messwerte ergeben. Die Messfrequenz ist softwareseitig einstellbar und kann oberhalb $10 \mathrm{kHz}$ liegen $^{16}$.

Die Autoren haben sich schon zu Beginn des Projekts intensiv mit den Anforderungen an medizinische Geräte auseinandergesetzt. Um den Einsatz an Patienten im Rahmen einer Studie und in einem vorgesehen Routinebetrieb vornehmen zu können, war eine Zertifizierung des Geräts notwendig. Es wurde daher bereits in der frühen Phase des Projekts die Zertifizierung in Angriff genommen, welche 2016 erfolgreich durchgeführt wurde. Der Atemtrainer MOPS ist vollständig EG-konform als Medizinprodukt der Klasse 2 zertifiziert.

\footnotetext{
${ }^{14}$ COPD = Chronic Obstructive Lung Deseases / Chronisch Obstruktive Lungenerkrankung

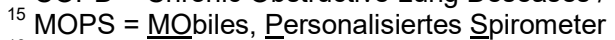

${ }^{16}$ Eine Messfrequenz von $10 \mathrm{~Hz}$ hat sich als völlig ausreichend für die Anwendung herausgestellt. Die technische Konzeption des Geräts erlaubt aber deutlich höhere Frequenzen.
} 
Die Digitalisierung der Medizin und insbesondere der Therapie ist in aller Munde. Neben der personalisierten Medizin wird die Therapiegamifizierung bereits als wichtiger, nächster Schritt erkannt, allerdings aktuell in der Kombination therapeutisches Hilfsmittel und Kommunikationseinheit nur wenig verfolgt. Wesentlich hierbei ist, dass viele Ansätze primär Kinder und Jugendliche adressieren, dabei aber nicht erkannt wird, dass Alter und Erfahrung im Umgang mit digitalen Technologien, nicht notwendigerweise Akzeptanzentscheidend sein müssen. Der Spieletrieb ist in jeder Person vorhanden. Entscheidend ist, dass die Spieleanwendung sich den Anforderungen des Spielers anpasst, d.h. die Entwicklung einer universellen Anwendung wird nicht zielführend werden. So sind für die Akzeptanz eines Spiels neben der Art des Spiels (wie bspw. Abenteuerspiele versus Konstruktionsspiele) insbesondere die Parameter Schwierigkeitsgrad, Spieledauer und Komplexität der Bedienung für den Endbenutzer entscheidend:

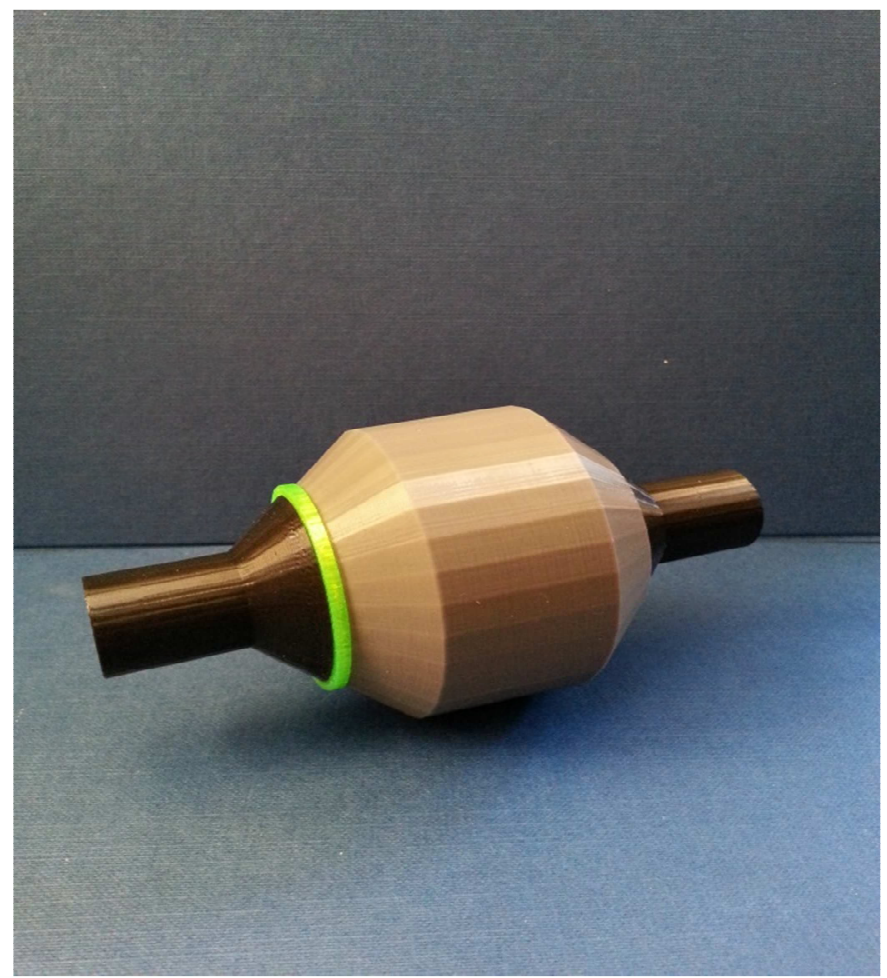

Abbildung 1: Neu entwickelter Atemtrainer MOPS. Alle Komponenten sind durch einfache Verschraubungen von Hand lösbar, damit jederzeit eine vollständige Desinfektion des Trainers erfolgen kann. Die Elektronik im Innern ist vollständig verkapselt.

In einem ersten Schritt wurde die Spieleanwendung "Catch the Flower" für das digitale Spirometer MOPS als erste Implementierungsvariante entwickelt. Dieses Spiel benötigt keine Anlernphase und kann sofort ausgeführt werden. Ein- und Ausatmen wirken sich wie eine Maus- oder Joystickbewegung auf eine Biene aus, deren Aufgabe es ist, so viele Blumen wie möglich zu sammeln. Der von einem Arzt oder einer Fachkraft verordnete, individuelle 
Therapieplan hat direkten Einfluss auf die konkrete Implementierung des Spiels in Form von:

- Trainingsdauer,

- Trainingsintensität,

- Variabilität des Trainings, d.h. schnelle bzw. langsame Veränderungen der geforderten Atemintensitäten,

- Vermeidung von Ausreißern, die bspw. eine Überbeanspruchung der Lunge explizit ausschließen,

- Incentivierung,

- $\quad$ das Erreichen von Levels mit der damit verbundenen Steigerung oder ggf. auch Abschwächung von Trainingsintensitäten und -dauern.

In der aktuellen Version wird das Spiel noch in den Einstellungen parametrisiert und damit ein konkreter Therapieplan eingestellt. Zusätzlich werden alle Übungen des Patienten lokal aufgezeichnet. Um sämtliche Anforderungen des Datenschutzes zu erfüllen, entscheidet der Patient eigenständig, wem er diese Daten zur Verfügung stellen möchte. Es kann sowohl eine einfache Auswertung direkt auf dem Smartphone erfolgen, wie auch ein vollständiger Datenexport in Form einer standardisierten Datei. Letzteres erlaubt dann bspw. dem Arzt das Einverständnis des Patienten vorausgesetzt - mittels eines geeigneten Auswerteprogramms detailliertere Untersuchungen anzustellen.

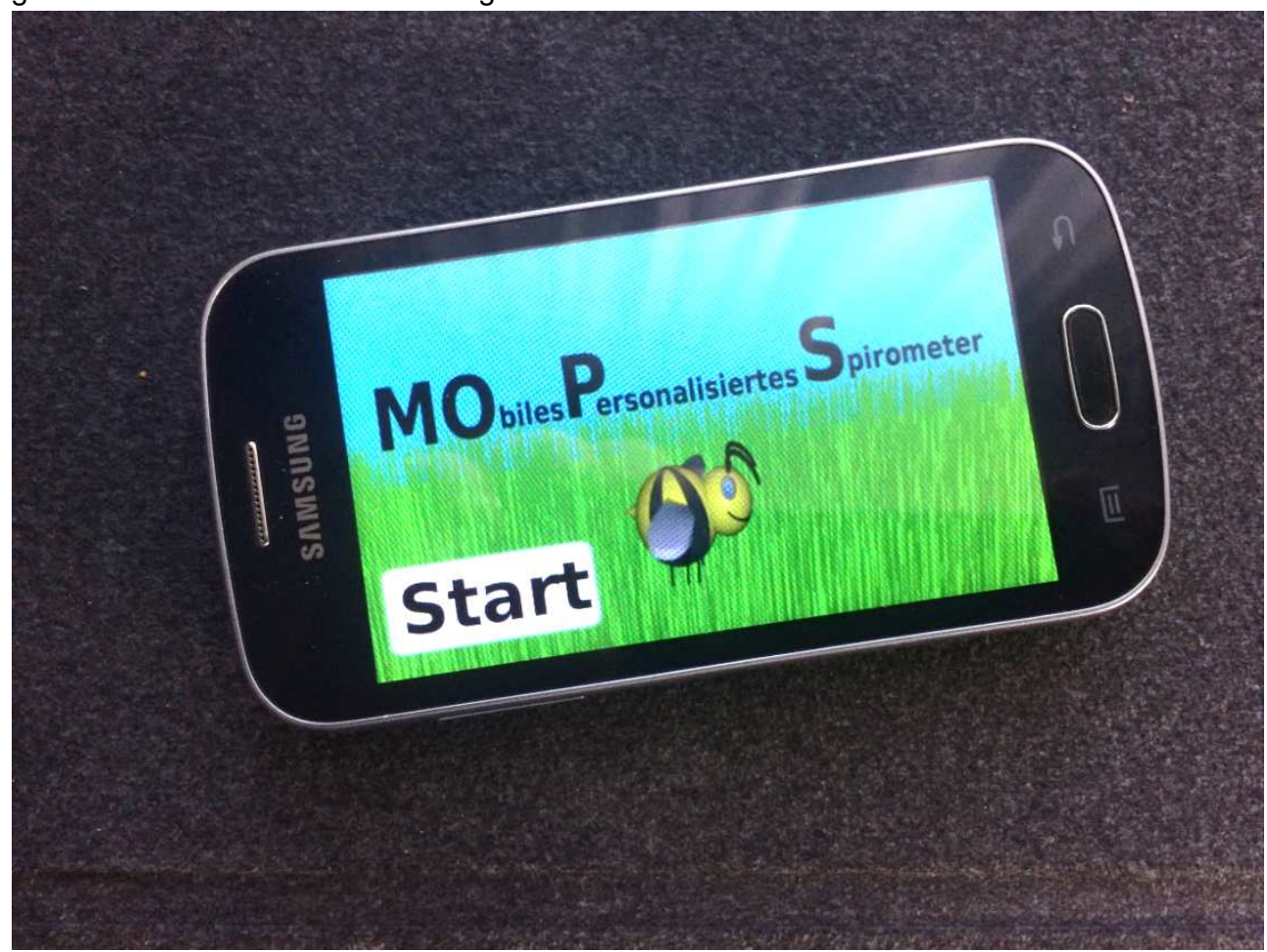

Abbildung 2a: Spieleanwendung „Catch the Flower". Diese bewusst einfache Anwendung besitzt eine besonders hohe Variabilität in Form der Schwierigkeitsgradgestaltung und kann daher für unterschiedlichste Patientengruppen eingesetzt werden. 


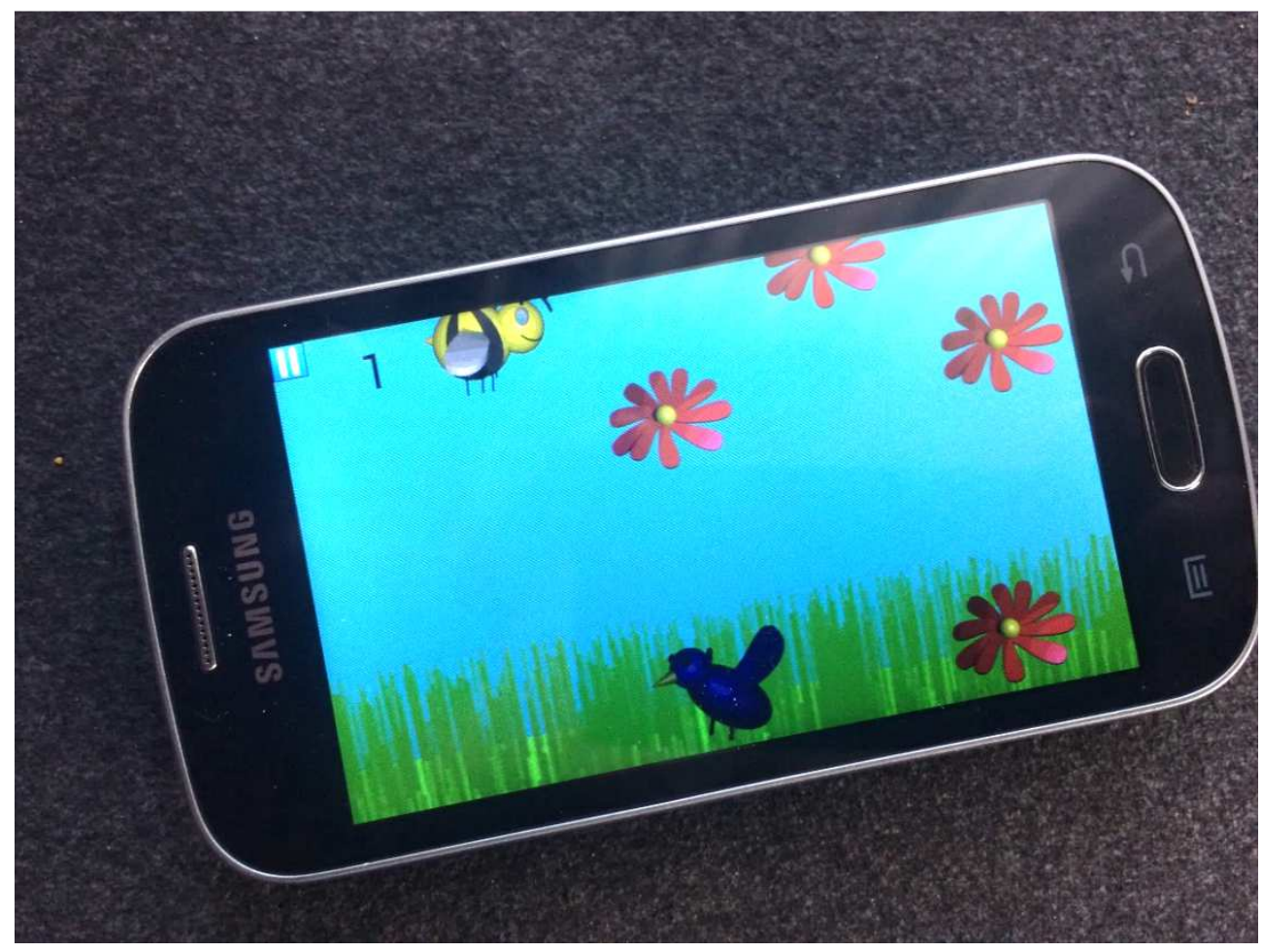

Abbildung 2b: Spieleanwendung „Catch the Flower“ im Spielmodus.

\section{Der nächste Schritt: „Der Therapieplan versteckt sich im Spiel“}

Aktuell erfolgt die Parametrisierung, d.h. das Vorgeben eines Therapieplans noch in Form von „klassischen“ Einstellungen. Dies sind bspw. Werte wie Steigerungsrate der Geschwindigkeiten der zu fangenden Objekte, Anzahl der Objekte pro Zeiteinheit, Veränderung der vertikalen Bienenbewegung in Abhängigkeit von der Atemintensität u.v.m.

Es liegt sehr nahe, diesen Therapieplan künftig von einem Digitalen Assistenten erstellen zu lassen. Dieser selbstlernende Assistent nutzt die Methoden des Machine Learning, um basierend auf medizinischen Vorgaben (durch den Arzt oder Therapeuten) vollständig automatisiert das Spiel zu parametrisieren. Dabei wird ein weiterer wesentlicher Punkt erreicht, der für den Erfolg und Misserfolg gamifizierter Lösungen entscheidend ist: die Parametrisierung des Spiels passt sich dynamisch und hochflexibel dem aktuellen Zustand des Patienten an, damit sich die Anwendung permanent auf dem sogenannten "flow path" bewegt ${ }^{17}$. Die Autoren erweitern damit ihre primäre Hypothese der Therapieverbesserung durch Spieleanwendungen um eine weitere Hypothese: gamifizierte Anwendungen in der Therapie

\footnotetext{
${ }^{17}$ Der flow path beschreibt den schmalen Grad eines Spiels zwischen Verdruss/Langeweile und Angst/Unerreichbarkeit, den ein Spiel gewährleisten muss, damit der Spieler diesem Spiel treu bleibt. Wird der Pfad verlassen, da bspw. das Spiel einen Schwierigkeitsgrad erreicht hat, der es dem Spieler nicht mehr erlaubt, Erfolge einzufahren, so wird er schlussendlich dieses Spiel verwerfen. Analog verhält sich der Spieler bei einem zu geringen Schwierigkeitsgrad. Dieser flow path ist spielerabhängig und für jede Person individuell.
} 
benötigen adaptive Spiele. Dieser zweite Baustein ist fundamental für die nachhaltige Ausführung der im Spiel versteckten Therapie.

Aktuell sind bereits die Entwicklungen zu diesem Modell im Gange.

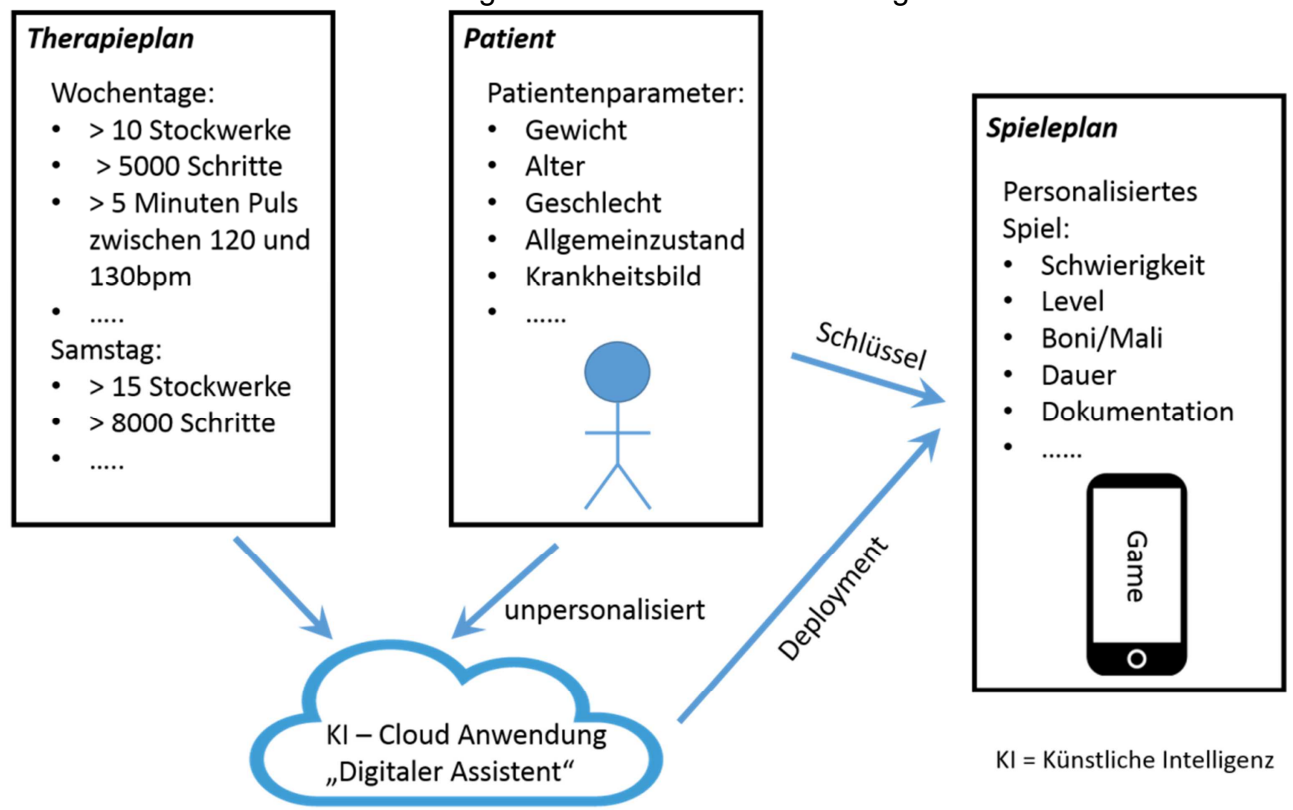

Abbildung 3: High-Level-Architektur eines digitalen Assistenten, der flexibel die Parametrisierung des Spiels vornimmt Auch hierbei ist es wichtig, dass insbesondere den Anforderungen des Datenschutzes genüge geleistet wird, wenn cloud-basierte Systeme zum Einsatz kommen. Der Digitale Assistent verfügt zu keiner Zeit über personalisierte Informationen des Patienten, der Patient erhält aber ein für inn personalisiertes Spiel.

\section{$5 \quad$ Zusammenfassung und Ausblick}

Es wurde ein völlig disruptiver Ansatz entwickelt, Therapiemaßnahmen und digitale Anwendungen so miteinander zu verknüpfen, dass Patienten hochgradig effizient und individuell eigenverantwortlich Behandlungsaktivitäten durchführen können. Alle bis jetzt durchgeführten Labortests bestätigen, dass die Kombination aus individuell konfigurierbarem Lungentrainingsgerät und spielerische Anwendung zu einer nachhaltigen Durchführung der Übungen bzw. Therapien führen.

Die vorgestellte COPD-Anwendung hat zusätzlich erfolgreich alle Hürden der Medizinproduktezertifizierung absolviert. Ebenso erfolgte eine Freigabe durch die Ethik-Kommission. Damit wird in Kürze eine wissenschaftliche Studie am Patienten gestartet werden können, welche durch das Universitätsklinikum UKGM bereits zugesagt wurde. Hierbei ist das Ziel, die Primärhypothese, dass die Compliance durch den Einsatz gamifizierter Anwendungen signifikant gesteigert werden kann, zu überprüfen. Ebenso soll der Therapieerfolg ermittelt und verglichen werden.

Weitere Anwendungen sind in der Diskussion, wobei in zwei weiteren Schwerpunkten Bewegungstherapien und die Therapierung koronarer Herzerkrankungen verfolgt werden sollen. Auch diesbezüglich laufen bereits Gespräche mit bundesdeutschen Kliniken. 
Der von den Autoren entwickelte Ansatz erzeugte auch informatikseitig eine sehr große Aufmerksamkeit. Die offene Bereitstellung der Geräte-API führte dazu, dass die PhilipsUniversität Marburg an zwei Informatiklehrstühlen projektseitig Spieleanwendungen entwickelt. Hierbei werden neue Wege der Spieleentwicklung untersucht, da gerade im Falle von Therapien das Erfahrungsspektrum der Patienten im Umgang mit digitalen Endgeräten und Spielen sehr heterogen gefächert ist. Ziel dieser Projekte ist es, neue Möglichkeiten der User-Interaktion in die Anwendungen einzubringen, so dass die Spiele im Rahmen der Therapieanwendung stets eine motivierende Wirkung erzeugen. Auch dies soll nun systematisch erforscht werden.

\section{Literaturverzeichnis}

Deutscher Ethikrat: „Die Medizin nimmt's persönlich. Möglichkeiten und Grenzen der Individualisierung von Diagnose und Therapie“, Forum Bioethik, 24.6.2009 (2009)

Hinderer, K.-U., Friedrich; P., Wolf, B.: Biofeedback: Gamification im Vergleich zu klassischem Feedback, $\quad$ Konferenz: Zukunft Lebensräume - $\quad$ Kongress 20.4.2016, Frankfurt/Main (2016)

Papandrinopoulou, D., Tzouda, V., Tsoukalas, G.: "Lung Compliance and Chronic ObstructivePulmonary Disease", in: Pulmonary Medicine, Volume 2012, Article ID 542769 (2012)

Scheen A.J., Giet D.: "Non compliance to medical therapy; causes, consequences, solutions", Rev Med Liege. 65(5-6), S. 239-45. (2010)

Przewloka, M.: „10 Jahre GFFT, 10 Jahre neue Informationstechnologien“, in: GFFT Jahresbericht 2015/2016 (2016)

World Health Organization: Adherence to Long-Term Therapies - Evidence for Action, WHO (2003)

Zichermann, G., Cunningham, Chr.: "Gamification by Design: Implementing Game Mechanics" in: Web and Mobile Apps. O'Reilly Media, 1. Auflage, (2011)

\section{Kontakt}

Martin Przewloka

Technische Hochschule Mittelhessen

Wiesenstraße 14, 35390 Giessen

martin.przewloka@mnd.thm.de

Katharina Dort

Justus Liebig Universität Gießen

Ludwigstraße 23, 35390 Gießen

katharina.dort@physik.uni-giessen.de 


\title{
iScalelT - Automatische Skalierung von Webservern in der Cloud
}

\author{
Stefan Beigel, Jens Kohler, Thomas Specht
}

\begin{abstract}
Für Unternehmen wird der zeitlich schwankende Zugriff auf Webanwendungen zu einer wirtschaftlichen Herausforderung, da Lastspitzen oder Leerlaufzeiten nicht immer exakt vorausgesagt werden können. So müssen sich beispielsweise neue Webanwendungen erst am Markt durchsetzen oder unterliegen saisonalen Schwankungen. Dies gilt auch für Webanwendungen, die in der Cloud in virtualisierten Serverumgebungen betrieben werden. Einerseits steigt bei hohen Zugriffszahlen die Antwortzeit, was im schlimmsten Fall dazu führt, dass die Anwendung nicht mehr verfügbar ist. Die steigende Antwortzeit hat somit einen direkten Einfluss auf die Zufriedenheit der Nutzer. Auf der anderen Seite werden bei geringen Zugriffszahlen unnötig Ressourcen verschwendet.

Genau hier setzt diese Arbeit an und entwickelt eine automatisierte und dynamische Skalierungslösung für Cloud-Umgebungen. So werden bei hoher Auslastung neue virtuelle Server zur Cloud hinzugefügt und bei geringer Auslastung Server wieder entfernt. Die dynamische Skalierungslösung dieser Arbeit setzt dabei nicht auf die Analyse von Vergangenheitswerten, sondern überwacht die Auslastung der bestehenden Cloud-Infrastruktur, und sobald ein definierter Schwellwert überschritten ist, wird automatisch hoch- bzw. herunterskaliert.

Diese Arbeit skizziert also die prototypische Entwicklung einer Managementsoftware zum automatischen Skalieren von Webservern in einer Cloud-Umgebung. Durch automatisiertes Hinzufügen und Entfernen von Webserver-Instanzen werden so dynamisch Lastschwankungen abgefangen. Zur Evaluation wird die entwickelte Lösung (iScale/T) durch einen Lasttest evaluiert. Dieser Lasttest richtet sich gegen eine Webanwendung, die einen einfachen Web-Shop simuliert. Dabei legen Nutzer ihre Waren in einen Warenkorb, der in Sessions auf den Webserver-Instanzen zwischengespeichert wird. Diese Sessions erfordern bei der dynamischen Skalierung besondere Aufmerksamkeit, da sie insbesondere beim Herunterskalieren nicht verloren gehen dürfen. Die Bewertung und anschließende Diskussion der Skalierungslösung erfolgt schließlich anhand der Antwortzeit und der Auslastung der Webserver.

Stichworte: Cloud-Computing; automatische, dynamische Webserverskalierung; Session Management
\end{abstract}

\section{$1 \quad$ Einleitung}

Für Unternehmen spielt in Zeiten von Datenklau und Wirtschaftsspionage die Sicherheit von Daten eine große Rolle. Dabei ist es für viele Unternehmen einfach zu riskant, Daten in öffentlichen oder hybriden Clouds zu speichern. Als Lösung bieten sich private Cloud-Umgebungen, beispielsweise auf laaS-Ebene (Infrastructure as a Service) an, bei denen der komplette Software-Stack individuell verwaltet werden kann ${ }^{18}$. Einerseits führt dies dazu,

\footnotetext{
${ }^{18}$ Die vorliegende Arbeit fokussiert primär die laaS-Ebene, allerdings kann die entwickelte Skalierungslösung transparent in allen Cloud-Servicemodellen (PaaS und SaaS) Anwendung finden.
} 
dass Unternehmen sich selbst um den Aufbau und die Wartung der Cloud-Infrastruktur kümmern müssen. Andererseits dürfen nicht zu viele Ressourcen in die Cloud investiert werden, um die Wirtschaftlichkeit zu wahren. Das bedeutet, dass beispielsweise eine minimale Anzahl von Rechnern für die Cloud-Infrastruktur genutzt werden sollte. Im Gegenzug allerdings, darf die Performance für die Nutzer zu keinem Zeitpunkt eingeschränkt werden, da sich die Kundenzufriedenheit unmittelbar auf den Umsatz bzw. Gewinn eines Unternehmens auswirken kann.

Dies ist insbesondere für Webapplikationen, die unvorhersehbaren und dynamischen Lastschwankungen unterliegen, ein Problem. Um hier eine geeignete Lösung zu finden, werden Webserver-Instanzen auf mehreren Rechnern gestartet und als Cluster zusammengefasst. Problematisch dabei ist allerdings wieder, dass das Cluster nur in Stoßzeiten ausgelastet ist und zu Zeiten mit wenig Kundenandrang trotzdem Ressourcen wie Wartung, Strom, etc. verbraucht.

Eine Lösung für diese Problemstellung ist das automatische Skalieren der Webserver auf Basis einer virtualisierten Cloud-Infrastruktur. Bei einer Lasterhöhung werden neue Webserver-Instanzen automatisiert hochgefahren (hochskaliert), um niedrige Antwortzeiten für die Kunden zu realisieren. Hingegen werden bei einer Lastverringerung Webserver-Instanzen heruntergefahren (herunterskaliert), um Betriebs- und Wartungskosten zu reduzieren.

\section{Problemstellung}

Die Cloud mit ihren fünf wesentlichen Merkmalen rapid elasticity, broad network access, resource pooling, measured services und on-demand self-service (Vgl. [MeGr11]) bietet für Unternehmen neue Möglichkeiten, aber auch Herausforderungen. Dabei ist der größte Vorteil der Cloud die flexible Nutzung von Ressourcen (rapid elasicity) und das dynamische Skalieren und Zusammenfassen der Ressourcen (resource pooling). Die Flexibilität des automatischen und dynamischen Skalierens kann auch für ein Webserver-Cluster genutzt werden. Allerdings muss bei einem Webserver-Cluster und der darauf laufenden Webapplikation eine weitere Herausforderung beachtet werden: die der Stateful-Webapplications. Eine Stateful-Webappliaction speichert Nutzerdaten in einer sogenannten Session auf dem Server, und genau diese Session-Daten müssen beim Skalieren beachtet werden und dürfen (insbesondere beim Herunterskalieren) nicht verloren gehen. Mit dieser Problemstellung beschäftigt sich die vorliegende Arbeit und erarbeitet, implementiert und evaluiert eine Skalierungslösung (iScale/T), die es trotz der Session-Daten ermöglicht, Webserver-Instanzen in einem Cluster automatisiert und dynamisch zu skalieren. Die Methodik zur Problemlösung orientiert sich am Design Science Research Paradigma (Vgl. [HeCh10]) und schafft mit iScale/T einen sog. Artefakt, der als Grundlage für weitere Entwicklungen (z.B. den Transfer in verschiedene Clouds) dient.

\section{Verwandte Arbeiten}

Die oben skizzierte Skalierungsproblematik rückt durch die Cloud erneut in den Fokus weiterer wissenschaftlicher Arbeiten. So stellen (Vgl. [SuGo14]) einen umfangreichen und vielseitigen Artikel, der die Skalierungsproblematik insbesondere auf der laaS-Ebene beleuchtet, 
dar. Es werden verschiedene Metriken (CPU, Speicher, Sensoren, etc.), die als Grundlage der Skalierung dienen, sehr detailliert erörtert. Außerdem werden verschiedene Skalierungsalgorithmen (z.B. netzwerkbasiert, SLA-basiert, spieltheoretische Ansätze) herausgearbeitet und deren Eignung für verschiedene Anwendungsszenarien analysiert. Diese Ansätze nutzen externe Kriterien wie Netzwerklast, Antwortzeit der Server oder Heuristiken. Im Gegensatz dazu verwendet diese Arbeit die konkrete CPU-Auslastung der (virtuellen) Server in einer Cloud, also Cloud-interne Messkriterien, zur Skalierung. Ein vergleichbarer Ansatz ist in (Vgl. [Lloy+14]) gegeben. Hier werden allerdings CPU-, Festplatten- und Netzwerkauslastung der physischen Cloud-Hardware betrachtet. Der Fokus liegt dabei weniger auf der schnellen Skalierungsmöglichkeit, sondern mehr auf der effizienten Platzierung von neuen virtuellen Maschinen auf wenig ausgelasteter physischer Hardware.

Ein weiterer wichtiger Aspekt, der eng mit der Skalierung zusammenhängt ist das Überwachen der Cloud-Ressourcen, das sog. Cloud-Monitoring. Hier geben (Vgl. [Mon+13]) einen detaillierten Überblick über verschiedene Möglichkeiten des Monitorings, auch wenn mehrere verschiedene Clouds im Einsatz sind. Eine Herausforderung dabei ist die Heterogenität der angebotenen Cloud-Dienste, was sich in den unterschiedlichen Service-Modellen (IaaS, PaaS und SaaS) wiederspiegelt. Hier erarbeiten (Vgl. [Mon+13]) eine generische Überwachungslösung, die dann zur Kontrolle (für Anbieter und Nutzer), aber auch zu Skalierungszwecken eingesetzt werden kann.

\section{$4 \quad$ Konzept und Implementierung}

\subsection{Architektur}

Die grundlegende Architektur besteht aus einem Webserver-Cluster, welches in einer laaSCloud läuft. Im Cluster ist den Webserver-Instanzen ein Loadbalancer vorgeschaltet, die Clientanfragen auf die einzelnen Webserver-Instanzen verteilt (Abbildung 1). Hier sind verschiedene Lastverteilungsalgorithmen denkbar, für iScalelT wurde hier auf den Least Connection Algorithmus zurückgegriffen, der eine Clientanfrage derjenigen Webserver-Instanz mit den wenigsten HTTP-Verbindungen zuweist ${ }^{19}$. Dadurch werden einerseits neue Instanzen nach dem Hochskalieren direkt in die Gesamtauslastung des Clusters einbezogen und andererseits können nicht ausgelastete Instanzen für das Herunterskalieren einfach identifiziert werden.

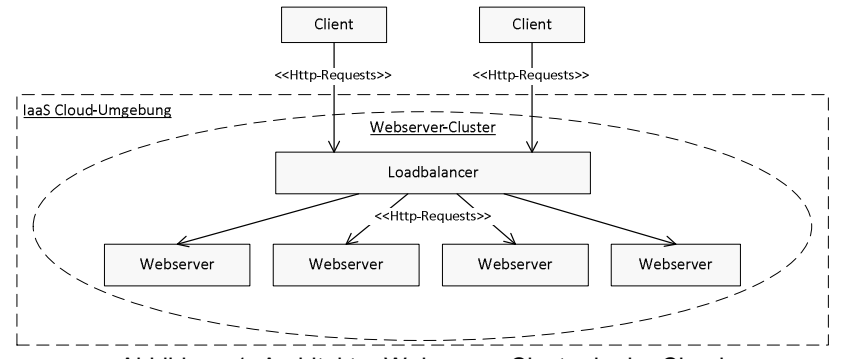

Abbildung 1: Architektur Webserver-Cluster in der Cloud

\footnotetext{
${ }^{19}$ Falls Webserver-Instanzen gleich wenige Verbindungen haben, wird das Round Robin Verfahren verwendet.
} 


\subsection{Session-Management}

Die größte Herausforderung beim Betreiben des Webserver-Clusters ist nun das Verwenden von Sessions, die aktuelle Clientzustände (z.B. einen Warenkorb) speichern. Die SessionDaten werden üblicherweise im Arbeitsspeicher des Webservers vorgehalten. Das führt dazu, dass bei einem Ausfall eines Webservers alle gespeicherten Session-Daten verloren gehen. Dies gilt auch für das automatische Skalieren des Webserver-Clusters, da das Entfernen einer Webserver-Instanz vergleichbar mit dem Ausfall des Webservers ist ${ }^{20}$. Als Lösung für iScalelT wurden zwei verschiedene Architekturen, die die Sicherung der SessionDaten anbieten, analysiert: die Session-Replikation und die zentrale Speicherung der Session-Daten (Abbildung 2).
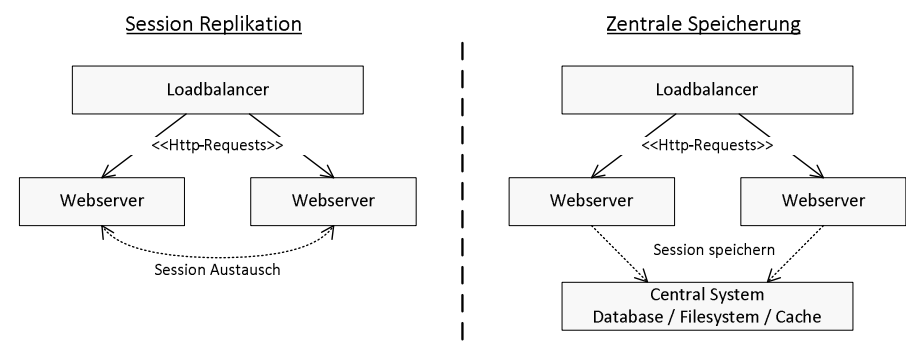

Abbildung 2: Vergleich Session Replikation und Speicherung in zentralem System

Bei der Session-Replikation werden Session-Daten serialisiert und zu einer oder mehreren Webserver-Instanzen im Cluster repliziert. Bei einem Ausfall oder beim Entfernen einer Instanz, sind die Session-Daten immer noch im Cluster verfügbar, allerdings auf einer anderen Instanz.

Eine weitere Möglichkeit ist das Speichern der Session-Daten in einem zentralen Datenspeicher (Datenbank, Filesystem, Cache, etc., vgl. Abbildung 2). Dabei werden die SessionDaten an den Datenspeicher geschickt und können von diesem auch wieder angefordert werden. Um den Vergleich mit der Session-Replikation zu gewährleisten, wird hierfür der InMemory-Cache memcached (Vgl. [Memc17]) verwendet, da die Session-Daten hier im Arbeitsspeicher (als Schlüssel-Wert Paare) vorgehalten werden ${ }^{21}$.

\subsection{Skalierung des Webserver-Clusters}

Generell bezeichnet Skalierung das Hinzufügen von Webserver-Instanzen aus einem Pool in das Cluster bzw. das Entfernen aus dem Cluster wieder zurück in den Pool (Abbildung 3). Dabei laufen eine oder mehrere Instanzen auf einem physischen Rechner (Node). Aus messtechnischen Gründen und aus Gründen der besseren Vergleichbarkeit (z.B. mit späteren Arbeiten und anderen Cloud-Infrastrukturen) wird im Rahmen dieser Arbeit immer nur eine virtuelle Maschine (mit einer Webserver-Instanz) auf einem physischen Rechner betrieben. Das Skalieren (Hoch- bzw. Herunterfahren) einer Webserver-Instanz bedeutet somit auch das Skalieren eines physischen Rechners im Cluster.

\footnotetext{
${ }^{20}$ Mit dem Unterschied, dass das Entfernen einer Webserver-Instanz eine gewollte Aktion ist und davor entsprechende Sicherungsmaßnahmen für die Sessions durchgeführt werden können.

${ }^{21}$ Bei der Session-Replikation werden die Session-Daten ebenfalls im Arbeitsspeicher der jeweiligen Webserver-Instanz gehalten.
} 


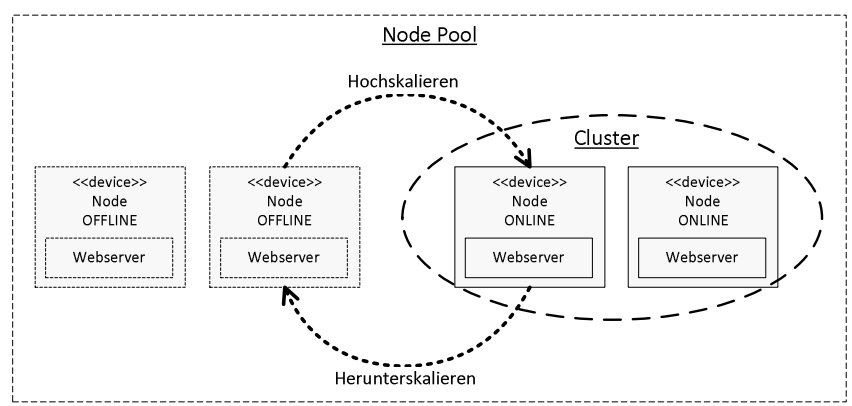

Abbildung 3: Skalierung des Webserver-Clusters

\section{Hochskalieren}

Beim Hochskalieren wird eine zusätzliche virtuelle Maschine, in der dann eine weitere Webserver-Instanz gestartet wird, hochgefahren. Um die virtuelle Maschine zu starten, wird mit der Cloud-Schnittstelle kommuniziert. Sobald die virtuelle Maschine und damit auch die Webserver-Instanz gestartet sind, können Clientanfragen vom Loadbalancer an die neue Webserver-Instanz weitergeleitet werden. Es besteht hierbei die Möglichkeit, dass der Loadbalancer selbstständig erkennt, sobald eine neue Webserver-Instanz verfügbar ist. In dieser Arbeit wird jedoch der Ansatz verfolgt, dem Loadbalancer den neuen Webserver aktiv mitzuteilen. Dies ermöglicht eine bessere Kontrolle und ein besseres Monitoring des Loadbalancers. Sobald die Webserver-Instanz zum Loadbalancer hinzugefügt wurde, ist das Hochskalieren beendet und die restlichen Webserver-Instanzen des Clusters werden durch die neue Instanz entlastet.

Die Webserver-Instanzen müssen für das Verwalten der Session-Daten beim Hochskalieren entsprechend konfiguriert werden. D.h. bei einer Clientanfrage in der Session-Replikation, frägt die Webserver-Instanz, die die Anfrage erhält, bei den anderen Instanzen nach, ob die Anfrage schon Teil einer Session ist. Sollte dies der Fall sein, wird die zugehörige Session auf die anfragende Instanz repliziert. Wenn nicht, muss es sich um eine neue Anfrage/Session handeln.

Bei der zentralen Speicherung hingegen, frägt die jeweilige Webserver-Instanz beim Datenspeicher nach, ob die Anfrage schon Teil einer Session ist und erhält die entsprechende Antwort, d.h. entweder lädt sie die entsprechende Session, falls vorhanden, oder eine neue Session wird in der Webserver-Instanz erstellt und in den Datenspeicher geschrieben.

\section{Herunterskalieren}

Für das Herunterskalieren wird eine Webserver-Instanz aus dem Cluster entfernt und zurück in den Pool gestellt. Die Webserver-Instanz wird zunächst vom Loadbalancer entfernt und so bekommt diese keine neuen Clientanfragen mehr zugewiesen. Dann wird die virtuelle Maschine über die Cloud-Schnittstelle heruntergefahren und sobald die virtuelle Maschine beendet ist, ist auch das Herunterskalieren beendet.

Bei der Session-Replikation ist es nun so, dass jede Session zu einer weiteren WebserverInstanz repliziert wird. Die Anzahl der Replikate ist dabei frei konfigurierbar. Um den Replikationsaufwand allerdings minimal zu halten, wurde für iScalelT die Anzahl der Replikate auf maximal zwei gesetzt. Dies bedeutet allerdings auch, dass vor dem Herunterfahren einer Webserver-Instanz, alle ihre Sessions nochmal auf eine andere Instanz repliziert werden müssen. Andernfalls bestünde die Gefahr, dass zwei wenig ausgelastete Instanzen, die 
gegenseitig ihre Sessions repliziert haben, heruntergefahren würden und die Sessions somit verloren gingen.

Da der zentrale Datenspeicher unabhängig von den Webserver-Instanzen ist, kann jede Webserver-Instanz ohne weiteren Aufwand einfach heruntergefahren werden. Kritisch ist allerdings anzumerken, dass der zentrale Datenspeicher einen Single-Point-Of-Failure darstellt, der die Session-Daten im Arbeitsspeicher vorhält. Ggf. wären hier also noch weitere Sicherheitsmechanismen, wie z.B. das Spiegeln des Datenspeichers notwendig, um bei einem Ausfall keine Session-Daten zu verlieren.

\section{4 Überwachung des Webserver-Clusters}

Um zu erkennen, wann das Webserver-Cluster überlastet ist, werden Daten in Form von Zugriffsstatistiken benötigt. Dafür ist es notwendig, das gesamte Cluster zu überwachen. Erreicht wird dies durch die Überwachung der einzelnen Nodes des Clusters. Um die Auslastung eines Nodes zu erkennen, gibt es verschiedene Messwerte oder Metriken. Im Rahmen dieser Arbeit wird als Metrik die LoadAverage (die durchschnittliche Auslastung der CPUs aller Nodes über einen Zeitraum) verwendet. Der große Vorteil ist, dass der LoadAverage zeitbasiert ist und somit nicht mehr der Durchschnitt über die Zeit gebildet werden muss. Der Zeitraum ist dabei flexibel konfigurierbar. Weitere Metriken wären bspw. die Auslastung des Hauptspeichers, des Festplattenspeichers, der Netzwerkverbindung, etc.

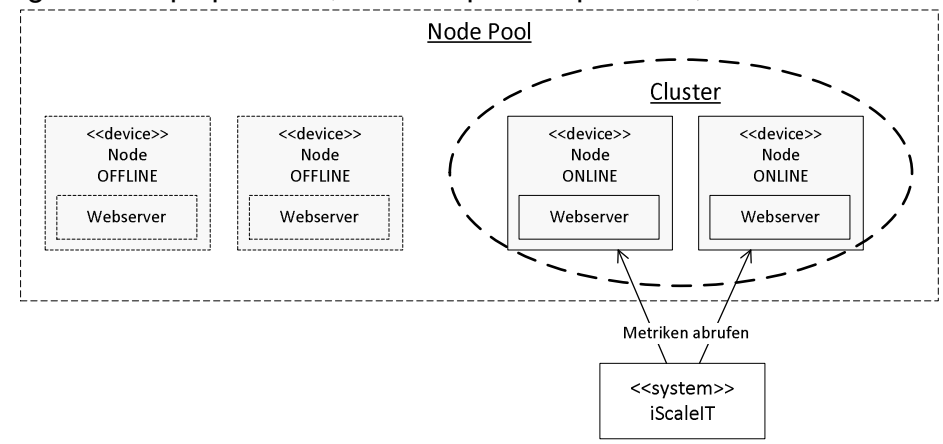

Abbildung 4: Überwachung des Webserver-Clusters

\subsection{Automatische Skalierung}

Zentrales Element der automatischen Skalierung ist das automatische Erkennen von Lastspitzen, bei denen eine Überlastung droht. Dabei dient die zuvor gesammelte Metrik (der LoadAverage) als Input. Um Lastspitzen oder eine zu geringe Auslastung des Clusters zu erkennen, wird eine Schwellwert-Funktion verwendet. Sobald der Messwert eine frei konfigurierbare Schwelle für einen frei definierbaren Zeitraum überschreitet, wird dies als Überlastung des Clusters gewertet. Wird hingegen die Schwelle für einen frei definierbaren Zeitraum unterschritten, wird das Cluster als zu gering ausgelastet gewertet. Entsprechend wird mit einer Hoch- oder Herunterskalierung reagiert.

Um schnell auf die zu hohe Last zu reagieren, wird für die Skalierung der LoadAverage der letzten Minute verwendet. 
Die Evaluation bezieht sich auf die zuvor beschriebenen Konzepte und die dafür entwickelte Implementierung. Mit der Evaluation wird nun geprüft, ob zum richtigen Zeitpunkt Webserver-Instanzen hinzugefügt bzw. wieder aus dem Cluster entfernt werden. Außerdem zeigt die Evaluation, wie lange der eigentliche Skalierungsvorgang dauert. Des Weiteren wird durch die Evaluation festgestellt, welche der beiden Session-Management Architekturen (Session Replikation oder zentrale Speicherung) besser skaliert.

\subsection{Aufbau}

Um den Skalierungsprozess zu analysieren, wird ein Lasttest gegen das Cluster gestartet. Das Cluster wird dabei durch die entwickelte Skalierungssoftware (iScale/T) überwacht und automatisch skaliert. Während des Lasttests soll die automatische Skalierung ausgelöst werden.

Das Cluster besteht aus 5 physischen Rechnern mit je 1 Intel Dual Core Prozessor mit 2,4 GHz Taktrate, 4 GB RAM und 250 GB Festplattenspeicher. Als grundlegende Cloud-Infrastruktur wurde CloudStack 3 (Vgl. [Clou17]) auf Basis von Ubuntu 14.04 verwendet. Jede Webserver-Instanz wird in genau einer virtuellen Maschine betrieben, die je auf $500 \mathrm{MHz}$, 500 MB RAM und 75 GB Festplattenspeicher zugreifen können. Als Webserver kommt in dieser Arbeit Tomcat 8 zum Einsatz. Als Loadbalancer wird HAProxy 1.6 (Vgl. [Hapr17]) verwendet. Der zentrale Datenspeicher wird zudem in einer separaten virtuellen Maschine (s.o.) betrieben und ist als memcached-Instanz (Version 1.3) (Vgl. [Memc17]) implementiert. Der Lasttest wird mit Hilfe von JMeter 3.0 durchgeführt, der die Clientzugriffe in Form von Java Threads simuliert. JMeter wird dabei auf einem separaten physischen Rechner (s.o.) ausgeführt. Für die Evaluation dieser Arbeit wurden zudem folgende Metriken definiert. Als Untergrenze für die minimale Anzahl der Webserver-Instanzen wurden 2 Instanzen definiert, um immer einen Sicherheitsbestand an Instanzen im Cluster vorrätig zu haben. Als Obergrenze gelten 4 Instanzen, um das Einhalten der Grenze durch iScale/T überprüfen zu können. Die Schwelle des LoadAverage, ab welcher hochskaliert wird, beträgt 0,9 (also 90\% Auslastung über die Messzeit von $1 \mathrm{Min}$.) und die Schwelle, ab der herunterskaliert wird, ist 0,2 (also 20\% Auslastung über die Messzeit von 1 Min.).

Der Lasttest richtet sich gegen eine Webapplikation in Form eines Webshops, die auf dem Webserver-Cluster läuft. Mit dem Lasttest werden Clients simuliert, die den Webshop aufrufen und Artikel in ihren Warenkorb legen. Dieser Warenkorb wird in der jeweiligen NutzerSession gespeichert. Um ein Hochskalieren auszulösen, wird die Last (engl. throughput, oder hier die Anzahl der Clients) linear innerhalb von 10 Minuten von 1 auf 500 Clientanfragen pro Sekunde erhöht. Danach laufen die 500 Clientanfragen für 04:30 Min. konstant gegen das Cluster, bis in den letzten 05:30 Min. die Anfragen linear wieder abnehmen und nach 20:00 Min. keine Clientanfrage mehr abgeschickt wird ${ }^{22}$.

\subsection{Messwerte}

Abbildung 5 und Abbildung 6 zeigen nun die Skalierung der Webserver-Instanzen im Cluster unter Verwendung des zentralen Datenspeichers memcached (Abbildung 5) bzw. mit Hilfe der Session-Replikation (Abbildung 6).

\footnotetext{
${ }^{22}$ Das Testszenario wurde experimentell auf Basis der verwendeten Hardware (s.o.) bestimmt und gibt einen Testlauf mit einem bzw. mehrmaligen Hoch- $u$. Herunterskalieren wieder.
} 


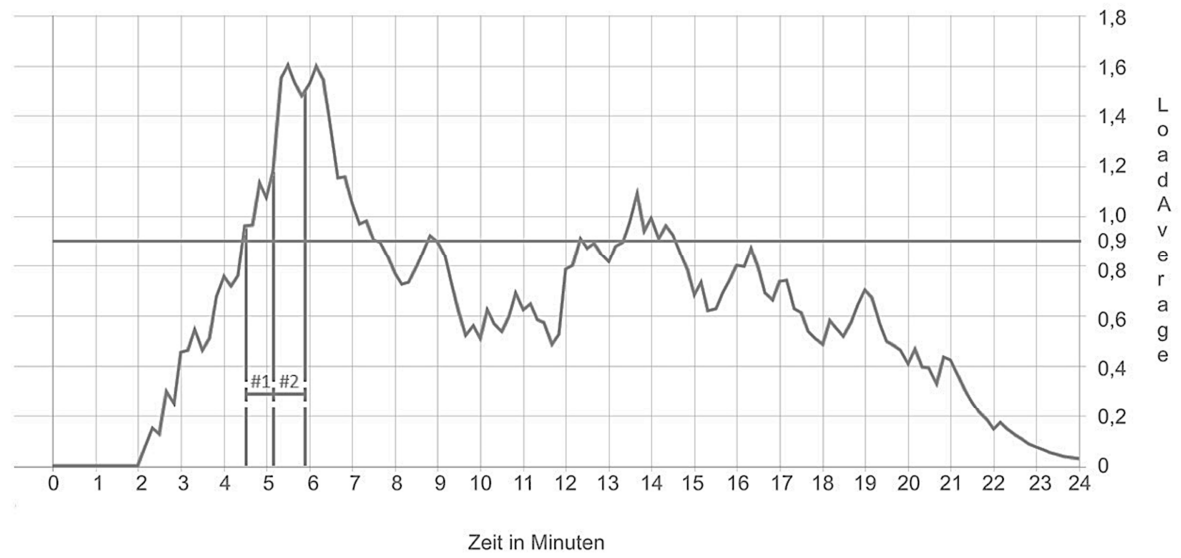

Abbildung 5: Load-Average (Zentraler Datenspeicher)

Session-Replikation

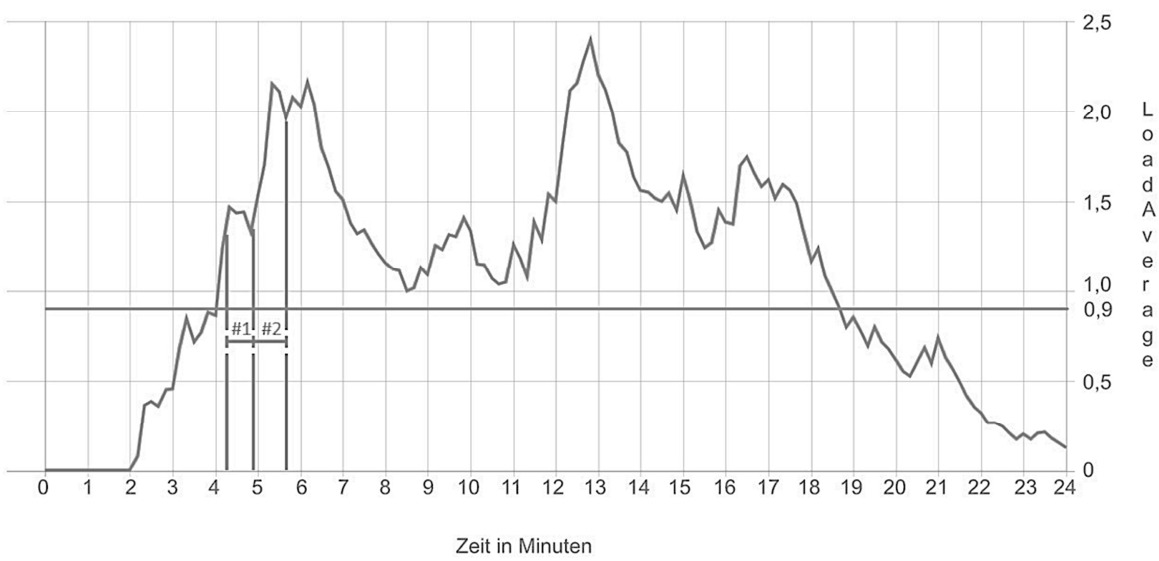

Abbildung 6: Load-Average (Session Replikation)

Beide Abbildungen zeigen ein ähnliches Verhalten: zu Beginn laufen zwei Webserver-Instanzen, bis nach ca. 04:30 Min. die Auslastung (LoadAverage, durchschnittliche Auslastung der letzten Minute) der Instanzen den Schwellwert von 0,9 übersteigt. Das Hochfahren einer weiteren Instanz wird nun initiiert und ist nach durchschnittlich 42 Sek. abgeschlossen (nach ca. 05:05 Min.). Diese erste Skalierung ist in den Abbildungen mit \#1 gekennzeichnet. Da die Last aufgrund der steigenden Clientanfragen allerdings weiter ansteigt, müssen weitere Webserver-Instanzen gestartet werden. Es folgt also ein zweites Hochskalieren (\#2) und eine weitere Webserver-Instanz wird gestartet. Damit ist die maximale Anzahl an Instanzen erreicht, und es können keine weiteren Instanzen hinzugefügt werden. Nach Abschluss des zweiten Hochskalierens fällt die Gesamtauslastung des Clusters bei beiden Ansätzen deutlich ab. In den Abbildungen ist dies ab der ca. 06:00 Min. zu erkennen. Auffällig ist hier allerdings, dass die Last bei der Verwendung des zentralen Datenspeichers memcached unter den definierten Schwellwert von 0,9 fällt, wohingegen die Gesamtauslastung bei der Session-Replikation darüber bleibt. Erst wenn die Clientanfragen wieder 
abnehmen (ab ca. 15:30 Min.) fällt die Last mit einiger Verzögerung wieder unter den Schwellwert. Das Herunterskalieren beginnt beim zentralen Datenspeicher daher ab ca. 15:30 Min. und bei der Session-Replikation ab ca. 19:00 Min.

\section{Fazit}

Die automatische Skalierung des Clusters auf Basis der durchschnittlichen Auslastung funktioniert ordnungsgemäß. Auffällig dabei ist allerdings, dass die Skalierung erst verzögert ihre Wirkung zeigt. Dies ist dem LoadAverage geschuldet, da dieser zeitbasiert ist und sich die zeitliche Verzögerung auf die Skalierung durchschlägt. Ein weiterer Grund ist, dass eine neu hinzugefügte Webserver-Instanz noch nicht warm $^{23}$ gelaufen ist. Damit kann diese direkt nach dem Hochfahren noch nicht so viele Clientanfragen übernehmen. Dies könnte durch eine Aufwärmphase (z.B. mit künstlich generierten Clientanfragen) verbessert werden.

Im Vergleich zwischen Hoch- und Herunterskalierung ist das Hochskalieren deutlich aufwendiger, da hier zunächst die virtuelle Maschine und dann die Webserver-Instanz gestartet werden muss. So dauert das Hochskalieren (bei beiden Ansätzen) mit durchschnittlich 42 Sekunden fast viermal so lange wie das Herunterskalieren mit durchschnittlich 11 Sekunden.

Im Vergleich des Session-Managements, haben die Messwerte ergeben, dass die Speicherung der Session-Daten in der zentralen memcached-Instanz zu einer besseren Performance führt. So bleibt dabei der Verlauf des LoadAverage deutlich unter dem des Replikations-Aufbaus. Der Performance-Nachteil der Session-Replikation kann durch den erhöhten Kommunikations- und Replikationsaufwand erklärt werden. Zudem benötigt iScaleIT bei der Session-Replikation deutlich mehr Arbeitsspeicher, da jede Session auf mindestens zwei Webserver-Instanzen vorgehalten wird. Vorteilhaft hingegen ist bei der Session-Replikation, dass kein zusätzlicher zentraler Datenspeicher wie z.B. memcached benötigt wird und somit kein Single-Point-Of-Failure in der Architektur vorhanden ist.

\section{$7 \quad$ Ausblick}

Die hier gezeigte Skalierungslösung iScalelT ist zunächst auf den beschriebenen TomcatClusteraufbau und auf die laaS-Infrastruktur CloudStack beschränkt. Allerdings sind Schnittstellen für weitere Webserver-Instanzen und Cloud-Infrastrukturen vorbereitet. Derzeit skaliert iScaleIT auf Basis von virtuellen Maschinen in einer CloudStack-Cloud auf laaS-Ebene. Jedoch ist der zeitliche Verzug beim Hochskalieren, bis eine neue Instanz eine entsprechende Menge an Clientanfragen übernehmen kann, problematisch. Fällt die Last nach dem Hochskalieren nicht schnell genug wieder unter den Schwellwert, so wird erneut hochskaliert, obwohl eine weitere Instanz möglicherweise nicht notwendig wäre. Gründe für den zeitlichen Verzug sind das Starten der Webserver-Instanzen, die zugehörige Sessionverwaltung und der Mehraufwand für das Starten der virtuellen Maschinen. Interessant wäre

\footnotetext{
${ }^{23}$ Klassen werden erst beim ersten Aufruf instanziiert und in den Speicher geladen. Daher benötigt eine neue Webserver-Instanz zu Beginn etwas Aufwärmzeit, bis sie Client-Anfragen so schnell bearbeiten kann, wie die anderen Instanzen im Cluster.
} 
es, leichtgewichtige Containerlösungen (z.B. Docker (Vgl. [Dock17]), Kubernetes (Vgl. [Kube17]), etc.) einzusetzen, um diesen Aufwand zu reduzieren.

\section{Literatur}

[Clou17] CloudStack: CloudStack Webseite. 2017. https://cloudstack.apache.org. Abruf am 2017-03-23

[Dock17] Docker: Docker Webseite. 2017. https://www.docker.com. Abruf am 2017-03-23

[Hapr17] HAProxy: HAProxy Webseite. 2017. http://www.haproxy.org. Abruf am 2017-03-23

[HeCh10] Hevner, A., \& Chatterjee, S. (2010). Design Research in Information Systems. Design Research in Information Systems (Vol. 22). Boston, MA: Springer US.

[Kube17] Kubernetes: Kubernetes Webseite. 2017. https://kubernetes.io. Abruf am 2017-03-23

[Lloy+14] Lloyd, W., Pallickara, S., David, O., Arabi, M., \& Rojas, K. (2014). Dynamic Scaling for Service Oriented Applications: Implications of Virtual Machine Placement on laaS Clouds. In: Proceedings of the 2014 IEEE International Conference on Cloud Engineering

[MeGr11] Mell P.; Grance T.: The NIST Definition of Cloud Computing. 2011. http://csrc.nist.gov/publications/nistpubs/800-145/SP800-145.pdf. Abruf am 2017-03-23

[Memc17] Memcached: Memcached Webseite. 2017. https://memcached.org. Abruf am 2017-0323

[Mon+13] Montes, J., Sánchez, A., Memishi, B., Pérez, M. S., \& Antoniu, G. (2013). GMonE: A complete approach to cloud monitoring. Future Generation Computer Systems, 29(8), 2026-2040.

[SuGo14] Sunilkumar M.; Gopal K. S.: Resource management for Infrastructure as a Service (laaS) in cloud computing: A survey. In: Atiquzzaman, M. (Ed.): Journal of Network and Computer Applications 41 (2014) 424-440

\section{Kontakt}

Stefan Beigel, Jens Kohler, Prof. Dr. Thomas Specht

Hochschule Mannheim, Fakultät für Informatik

Mannheimer Wirtschaftsinformatik Institut

Paul-Wittsack-Str. 10, 68163 Mannheim

T +49 621 292-6739, j.kohler@hs-mannheim.de 


\title{
Sicherheitsüberlegungen für spezielle Einsatzbereiche des Internet of Things
}

\author{
Haio Röckle, Frank Thomé, Carsten Dorrhauer
}

\section{Zusammenfassung}

An der Hochschule Ludwigshafen am Rhein wurden in einem Labor des Studienschwerpunkts Wirtschaftsinformatik verschiedene Anwendungsbeispiele auf Basis des „Internet der Dinge“ realisiert. Diese repräsentieren Umsetzungsmöglichkeiten in diesem für Wirtschaftsinformatiker und Wirtschaftsinformatikerinnen sehr interessanten Markt. In der Öffentlichkeit werden allerdings auch regelmäßig Sicherheitsprobleme in Verbindung mit dem „Internet der Dinge“ thematisiert. Der vorliegende Beitrag dient dazu, spezifische Sicherheitsanforderungen und notwendige Sicherheitsmaßnahmen für das „Internet der Dinge“ zu untersuchen und zu systematisieren. Zur praktischen Anwendung werden die durchgeführten Sicherheitsüberlegungen auf eine Smart Home Lösung, die an der Hochschule realisiert wurde, übertragen.

\section{$1 \quad$ Einleitung}

Die Allgegenwärtigkeit elektronischer Komponenten in unserer Gesellschaft nimmt seit vielen Jahren unvermindert zu. Einen starken Bedeutungszuwachs für die Wirtschaftsinformatik erhält diese Entwicklung dadurch, dass zunehmend auch Alltagsgegenstände mit elektronischen Komponenten ausgerüstet und mit dem Internet verbunden werden. Die Gegenstände wandeln sich auf diese Weise zu intelligenten („smarten“) Objekten mit eingebetteter Informations- und Kommunikationstechnik, die mit Hilfe von Sensoren eine Überwachung von Objekt- oder Umgebungszuständen sowie mit Hilfe von Aktoren eine Steuerung der Objekte und nicht zuletzt auch deren Interaktion mit dem Menschen ermöglichen können. Im Zusammenspiel dieser intelligenten Objekte entsteht das sogenannte „Internet der Dinge“ (,Internet of Things“ bzw. „IOT“), wobei "Internet” hierbei stellvertretend für die technische Vernetzung der Objekte auf Basis der TCP/IP-Protokollfamilie steht [AnHä15, S 18; MaFI10, S. 107 f., RaSa17, S. 4]. Dazu gehörende Anwendungskategorien sind beispielsweise „Smart Home“, „Smart Energy“, „Smart Mobility“, „Smart Logistics“ und „Smart Production“.

Anbieter, Beratungsunternehmen und Marktbeobachter überbieten sich in der Darstellung neuer Einsatzbereiche und Business Cases für die genannten Anwendungskategorien und berechnen daraus gewaltige Einsparungen und zukünftige Gewinne [AnHä15, S. 10]. Da es gleichzeitig darum geht, Zukunftsmärkte zu besetzen, besteht hier ein hoher Konkurrenzdruck. Das Innovationstempo steigert sich und es entstehen mit großer Geschwindigkeit Produktfamilien, die nicht kompatibel und schon gar nicht standardisiert sind. Auch die Bezeichnungen unterscheiden sich zwischen den Anbietern, was zu der Uneinheitlichkeit beiträgt. Parallel dazu tauchen in der Öffentlichkeit seit einigen Jahren Meldungen auf [DoMa17], die in mehr oder weniger spektakulärer Weise die Unsicherheit intelligenter und vernetzter elektronischer Komponenten zum Thema haben. Zu den genannten Sicherheitslücken führt außer dem bereits genannten hohen Innovationsdruck aber auch die Tatsache, 
dass Anbieter vernetzter Komponenten in der Regel keine Historie als IT-Experten, sondern eher als Komponenten-Experten besitzen, z.B. Heizungshersteller, Kamerahersteller oder Anlagenbauer. Anders ließe es sich auch nicht erklären, dass viele der gefundenen Sicherheitslücken aus der Sicht erfahrener Informationssicherheitsexperten geradezu trivial sind und durch elementare Sicherheitsmaßnahmen verhindert werden könnten [DoMa17].

Im Labor des Instituts für Wirtschaftsinformatik an der Hochschule Ludwigshafen am Rhein wurden verschiedene loT-Anwendungsbeispiele realisiert. Ziel dieses Beitrags ist es, Sicherheitsüberlegungen für die hierbei zum Einsatz kommenden loT-Komponenten vorzunehmen. Natürlich sind die Komponenten, soweit sie sich innerhalb unseres Labors befinden, in keiner Weise kritisch, allerdings geht es dabei um den Aufbau von Wissen, das im produktiven Umfeld, in dem die Kritikalität eine ganz andere sein wird, genutzt werden soll. Vor diesem Hintergrund geben wir Beispiele, die - nicht an die Ausstattung unseres Labors gebunden - als Anhaltspunkte zur Einschätzung der Kritikalität spezifischer Systeme und Installationen nützlich sein können. Anschließend übertragen wir bewährte Methoden der Informationssicherheit auf die speziellen loT-Gegebenheiten. Die allgemeinen Ergebnisse wenden wir schließlich auf einen in unserem loT-Labor realisierten Anwendungsfall aus dem Bereich "Smart Home“ an, womit wir einerseits ein Sicherheitskonzept für diesen Anwendungsfall erhalten und andererseits einen Proof-of-Concept für die allgemeinen Sicherheitsbetrachtungen.

\section{Allgemeine Sicherheitsüberlegungen}

\subsection{Herausforderungen der loT-Sicherheit}

Aus wissenschaftlich-technischer Sicht ist Informationssicherheit ein gelöstes Problem. Zu spezifischen technischen Herausforderungen gibt es in der Regel passende Lösungen. Auch für die betriebswirtschaftlichen Vorgehensweisen sind Managementstandards verfügbar, allen voran die Standards der ISO 2700x-Familie [ISO13a, ISO13b]. Für abgrenzbare Bereiche der Informationssicherheit wie SAP Sicherheit, Internet Sicherheit, Mobile Security oder Big Data Security hat es sich herausgestellt, dass bewährte Sicherheitsverfahren in Verbindung mit detaillierter Kenntnis der zu schützenden Domäne für angemessenen Schutz ausreichend sind, wenn diese sorgfältig umgesetzt werden. Allerdings ist diese Umsetzung aus drei Gründen nicht einfach: Erstens sind oft längere Checklisten abzuarbeiten, was großen Aufwand verursacht. Zweitens ist hierfür umfangreiches Expertenwissen nötig, da die einzelnen Maßnahmen noch auf die vorhandenen Gegebenheiten vor Ort anzupassen sind und drittens müssen oft Kompromisse zwischen der einfachen Benutzbarkeit und den komplizierteren Sicherheitsmechanismen ausgehandelt und eingegangen werden, was den Aufwand weiter erhöht. Vor diesem Hintergrund bringt loT-Sicherheit aufgrund der großen Zahl unterschiedlicher technischer Komponenten der loT-Systeme besondere Herausforderungen mit sich. Einerseits führt die große Zahl von Komponenten zu einer besonders großen Zahl spezifischer Sicherheitsanforderungen und andererseits ist es aufgrund der Heterogenität der Produkte schwieriger, die erforderlichen Kenntnisse zusammenzuführen. Zudem sind die Komponenten-Hersteller oft unerfahren im Bereich der Informationssicherheit. Hinzu kommt, dass die zu veranschlagenden Kosten für den Sicherheitsaufwand gerade bei kleinen Bauteilen deren Preis vervielfachen können. 


\subsection{Grundsätzliches Vorgehen in Bezug auf loT-Sicherheit}

Um loT-Sicherheit praktisch umzusetzen, können wir uns an den eben genannten Verfahren orientieren. Wir können Checklisten von bekannten Quellen heranziehen [OWAI17] und diese im Rahmen eines Sicherheitsmanagementansatzes nach ISO 2700x abarbeiten. Hier werden anhand von Risikobetrachtungen die angemessenen Sicherheitsmaßnahmen ausgewählt, umgesetzt und kontrolliert, wobei darauf geachtet werden muss, dass das erforderliche Wissen und die erforderlichen Ressourcen zur Verfügung stehen. Um den oben genannten Besonderheiten des loT-Bereichs Rechnung zu tragen, können die folgenden Ansätze dienen. Weder die Ansätze selbst noch deren Zusammenhang mit der loT-Sicherheit sind neu, die Zuordnung zu den oben genannten Herausforderungen scheint uns aber eine besondere Hervorhebung wert:

\begin{tabular}{|l|l|}
\hline Herausforderung & Lösungsansatz \\
\hline $\begin{array}{l}\text { Große Zahl technischer } \\
\text { Komponenten }\end{array}$ & $\begin{array}{l}\text { Berücksichtigung der Informationssicherheit bereits zu } \\
\text { Beginn des Projekts zur Einführung der Komponenten. } \\
\text { Dies gehört zu den unerlässlichen Best Practices der } \\
\text { Informationssicherheit und findet sich z.B. im Abschnitt } \\
6.1 .5 \text { des ISO 27002 von 2013. }\end{array}$ \\
\hline $\begin{array}{l}\text { Erforderliche Kenntnisse zu- } \\
\text { sammenführen }\end{array}$ & $\begin{array}{l}\text { Interdisziplinäres Vorgehen unter Einbeziehung von } \\
\text { Herstellern und Sicherheits-Experten bereits im Einfüh- } \\
\text { rungsprojekt }\end{array}$ \\
\hline $\begin{array}{l}\text { Fehlende IT-Sicherheitserfah- } \\
\text { rung auf Herstellerseite }\end{array}$ & $\begin{array}{l}\text { Interdisziplinäres Vorgehen bereits in der Produktent- } \\
\text { wicklung bei Herstellerunternehmen }\end{array}$ \\
\hline $\begin{array}{l}\text { Sicherheitsaufwand im Ver- } \\
\text { hältnis zum Preis kleiner Bau- } \\
\text { teile }\end{array}$ & Seriöse Kalkulation im Einführungsprojekt \\
\hline
\end{tabular}

Tab. 1: Herausforderungen und Lösungsansätze

Hierbei kann gar nicht oft genug darauf hingewiesen werden, dass mit „Einführungsprojekt“ nicht ein nachgelagertes Projekt zur Einführung von Informationssicherheit gemeint ist, sondern das Projekt, das die zu schützenden „Dinge“ erstmalig auf Anwenderseite implementiert.

Wir gehen davon aus, dass Projekte in den meisten Unternehmen heutzutage professionell abgewickelt werden. Nachlässigkeiten können aber gerade im loT-Bereich schnell auftreten wenn die „Dinge“ nicht im Rahmen geplanter Projekte implementiert werden, sondern zunächst prototypisch ausprobiert und danach ohne Projektplanung in Betrieb genommen werden. Ebenso ist es möglich, dass ein Bauelement oder auch eine größere Anlage zunächst ohne Vernetzung in Betrieb genommen wurde und erst später - wiederum ohne Projektplanung - vernetzt und damit relevant für das „Internet of Things“ wurde. Auch kann ein Mangel an Expertenwissen dazu führen, dass dem Anwender die Vernetzung bei der Inbetriebnahme gar nicht bewusst war, z.B. bei Smart TVs von Privatanwendern.

\subsection{Checklisten zur loT-Sicherheit}

Um ein Gefühl für die Anforderungen der loT-Sicherheit zu vermitteln, zitieren wir an dieser Stelle zwei Aufzählungen aus dem OWASP-Projekt zur loT-Sicherheit. In der Aufzählung möglicher Schwachstellen werden 95 mögliche Schwachstellenbereiche („Vulnerabilities“) ausgeführt, die in 15 Angriffsoberflächenbereiche („Attack Surface Area“) gegliedert sind [OWAl17]. In einer Sicherheitshandreichung [OWAI17] werden 10 Kategorien dargestellt, 
anhand derer die Beteiligten sich hinsichtlich 58 loT-Sicherheitsanforderungen orientieren können. Als Beteiligte gelten dabei Produkthersteller (,Manufacturer“), Softwareentwickler („Developer“) und Anwender („Consumer“). Beide Aufzählungen dienen dazu, die Anforderungen der loT-Sicherheit zu sammeln und zu kategorisieren, stimmen aber aufgrund der etwas unterschiedlichen Herangehensweise nicht vollständig überein. Die folgende Tabelle stellt die jeweiligen Kategorien gegenüber:

\begin{tabular}{|l|l|}
\hline Angriffsoberfläche & Kategorien der Handreichung \\
\hline Ecosystem Access Control & I1: Insecure Web Interface \\
Device Memory & I2: Insufficient Authentication/Authorization \\
Device Physical Interfaces & I3: Insecure Network Services \\
Device Web Interface & 14: Lack of Transport Encryption \\
Device Firmware & I5: Privacy Concerns \\
Device Network Services & I6: Insecure Cloud Interface \\
Administrative Interface & I7: Insecure Mobile Interface \\
Local Data Storage & I8: Insufficient Security Configurability \\
Cloud Web Interface & I9: Insecure Software/Firmware \\
Third-party Backend APIs & I10: Poor Physical Security \\
Update Mechanism & Beteiligte: \\
Mobile Application & Manufacturer \\
Vendor Backend APIs & Developper \\
Ecosystem Communication & Consumer \\
Network Traffic & \\
\hline
\end{tabular}

Tab. 2: Angriffsoberflächen und Kategorien der Handreichung

Wie bei den Grundlagen der Applikationssicherheit [KrSc09] verteilt sich nun auch die Verantwortung für die Sicherheit von loT-Komponenten. Hersteller müssen geeignete Sicherheitsmechanismen implementieren, z.B. die Benutzung von starken Passwörtern oder zertifikatsbasierter Authentisierung ermöglichen, Anwender müssen diese dann aber auch entsprechend des ermittelten Sicherheitsbedarfs einsetzen. Hersteller wiederum sind in der Pflicht, sämtliche sicherheitsrelevanten Optionen sauber zu dokumentieren und diese Dokumentation dem Anwender zur Verfügung zu stellen. So müssen beispielsweise IP-Kameras mit undokumentierten Webservern [DoMa17] oder die Vorschrift zur Einrichtung eines Fernwartungszugangs mit Standardpasswort [Eike13] ausgeschlossen sein.

\subsection{Abgrenzung, Risikoanalyse, Sicherheitsmaßnahmen und Restrisiken}

Anwenderübergreifende Checklisten zur Informationssicherheit müssen vollständig sein, um keine systematischen Lücken zu lassen. Um den dadurch entstehenden Umfang wieder in den Griff zu bekommen, werden Checklisten auf einem relativ hohen Abstraktionsniveau formuliert, das dann für jeden Anwender auf dessen Anwendungsbereich und spezifisches Systemumfeld heruntergebrochen werden muss. Für Überlegungen zur loT-Sicherheit sollte also zunächst das zu schützende Systemumfeld betrachtet und das Zusammenspiel mit verwandten Bereichen festgelegt werden.

Anschließend können Checklisten wie die oben referenzierten genutzt werden, um die Informationssicherheit anhand der bekannten und bewährten Verfahren zu verbessern: Zum Schutz der Sicherheitsgrundwerte Vertraulichkeit, Integrität und Verfügbarkeit sind Risikobetrachtungen durchzuführen, die insbesondere den Business Impact im Schadensfall und die Wahrscheinlichkeit eines Sicherheitsvorfalls berücksichtigen. Größere Risiken werden 
durch Sicherheitsmaßnahmen reduziert, kleinere Risiken können nach Maßgabe des Managements akzeptiert werden. Allerdings erfordert die Akzeptanz von Risiken unbedingt, dass die Risiken vorher bekannt sind, d.h. dies darf nicht als Ausrede dienen, auf die Risikobetrachtungen zu verzichten.

\subsection{Exemplarische Risikobetrachtungen}

In einem öffentlich zugänglichen Umfeld, in dem von einem Sensor die Umgebungstemperatur gemeldet wird, sind diese Daten nicht personenbezogen und auch nicht vertraulich. In einer Smart Home Umgebung hingegen können Temperaturdaten personenbezogen und damit auch vertraulich sein, da sie Rückschlüsse auf die Anwesenheit von Personen geben und damit beispielsweise zur Vorbereitung von Wohnungseinbrüchen genutzt werden können. In Bezug auf die Integrität können fehlerhafte Temperaturdaten in einem Kraftwerk dazu führen, dass dieses fehlerhaft gesteuert wird und womöglich überhitzt. Fehlende Temperaturdaten könnten zu einer Notabschaltung führen, was Auswirkungen auf die Verfügbarkeit des Kraftwerks hat. Aktoren, die zur Steuerung anderer Komponenten dienen, sind offensichtlich anfällig gegen manipulierte Zugriffe. Die konkrete Schadenshöhe solcher Integritätsverletzungen kann sich nur aus der Betrachtung des Umfelds ergeben. Die tatsächliche Risikosituation ergibt sich also erst aus dem konkreten Anwendungsfall und kann damit nicht Bestandteil einer anwenderübergreifenden Checkliste sein.

\subsection{Exemplarische Sicherheitsmaßnahmen}

Wie wir gesehen haben, sollten für Sensordaten die Vertraulichkeit und die Integrität geschützt werden, für Aktordaten mindestens die Integrität. Dies spiegelt sich darin wider, dass die meisten Empfehlungen zur loT-Sicherheit an vorderster Stelle eine möglichst starke Authentisierung sowie den Einsatz kryptografischer Mechanismen zur Verschlüsselung und Integrität fordern [Raeh16]. Wir schließen uns dieser Forderung an. Ein wesentlicher Teil der Untersuchungen der Sicherheitsmöglichkeiten in unserem loT-Labor beschäftigt sich deshalb mit der Frage, ob und inwieweit solche Mechanismen genutzt werden können. Diese Überlegungen betrachten aber nur das Zusammenspiel der Komponenten zur Laufzeit. IoTSicherheitsvorfälle der jüngeren Vergangenheit [DoMa17] beziehen sich aber oft auf die Konfigurationsmechanismen der einzelnen Komponenten, häufig verursacht durch dilettantische Fehler wie offene Ports zur Fernwartung, Standardpasswörter o.ä. Auf die Verhinderung solcher Probleme zielt auch ein großer Teil der in 2.3 dargestellten Kategorien ab, obwohl diese Sachverhalte im engeren Sinne gar nicht neu sind, sondern eben mit dem loT erst wieder kritisch werden. Vor diesem Hintergrund sind deshalb Maßnahmen wie sichere Web- und Mobilanwendungen der Konfigurationskomponente zu sehen. Wenn loT-Komponenten z.B. über eine Weboberfläche konfiguriert werden können, enthalten diese einen Webserver und müssen außerdem Mechanismen der Webserver-Sicherheit erfüllen.

\section{Anwendung der Sicherheitsüberlegungen auf eine Smart Home Lösung}

Abschließend werden die bisher durchgeführten Sicherheitsüberlegungen auf ein ausgewähltes Anwendungsbeispiel übertragen, welches am loT-Labor des Instituts für Wirtschaftsinformatik an der Hochschule Ludwigshafen am Rhein realisiert wurde. Das loT-Labor besteht aktuell aus einer Reihe unterschiedlicher Einplatinencomputer, Sensoren und Aktoren sowie verschiedenen Netzwerkkomponenten. Unter anderem stehen Helligkeits-, 
Feuchtigkeits-, Temperatur- und Luftdruck-Sensoren, Bewegungsmelder, Tür- und FensterKontakte, ein NFC/RFID Lese-/Schreibgerät sowie verschiedene funkbasierte Schaltaktoren und Stellantriebe zur Regelung von Heizkörpern zur Verfügung. Mit diesen Komponenten wurden bislang in verschiedenen Lehr- und Forschungsprojekten Anwendungsbeispiele insbesondere aus den Bereichen Smart Home und Smart Metering, aber auch Smart Logistics und Smart Production realisiert.

\subsection{Technische Ausgangssituation}

Der Anwendungsfall, für den wir nachfolgend die Sicherheitsüberlegungen durchführen werden, ist aus dem Bereich „Smart Home“ gewählt und bewusst einfach gehalten: Es handelt sich um das automatische Aktivieren der Beleuchtung in einem Wohnraum, wenn die Beleuchtungsstärke im Raum einen vorgegebenen Minimalwert (z.B. 500 Lux) unterschreitet sowie, umgekehrt, das automatische Deaktivieren der Beleuchtung, wenn ein vorgegebener Maximalwert (z.B. 1500 Lux) überschritten wird. Die nachstehende Abbildung zeigt ein UML Komponentendiagramm mit der relevanten Systemarchitektur und den von den Komponenten angebotenen Schnittstellen bzw. Ports, ergänzt um die zum Einsatz kommenden Kommunikationsprotokolle und Übertragungswege.

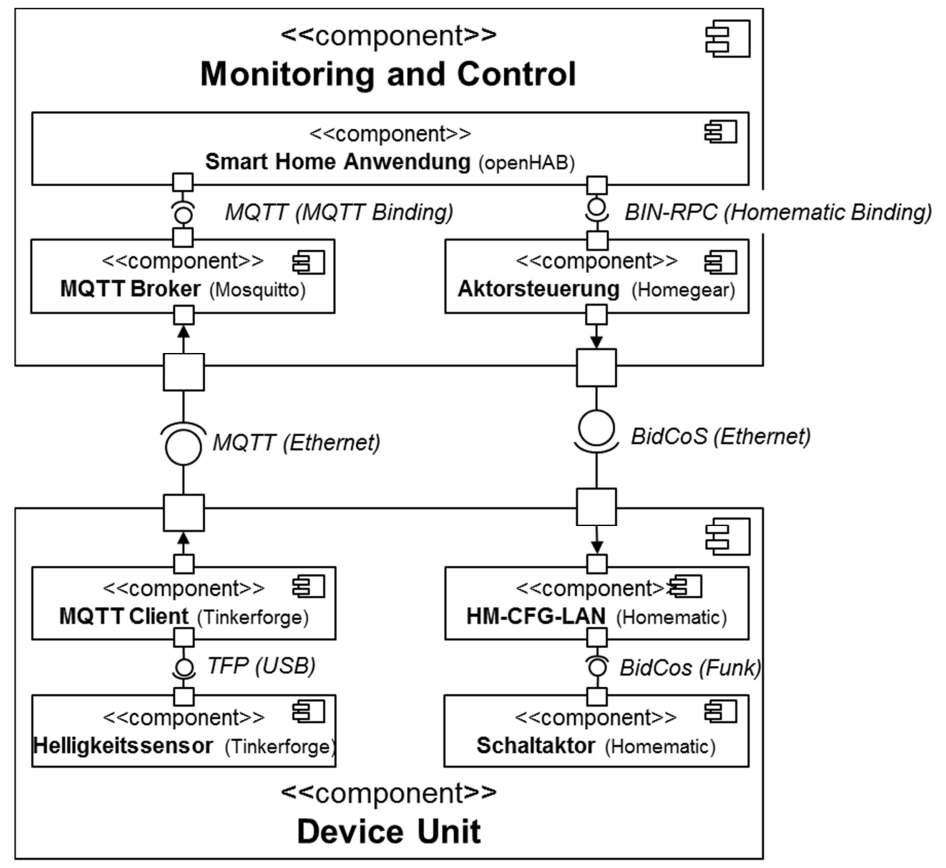

Abb. 1: Anwendungsfallrelevante Systemarchitektur

In der Komponente „Device Unit“ werden alle zum Einsatz kommenden Sensoren und Aktoren zusammengefasst. Im betrachteten Anwendungsfall ist dies ein Helligkeits-Sensor der Marke Tinkerforge sowie ein Funk-Schaltaktor der Marke Homematic, der die Beleuchtung ein- bzw. ausschaltet. Der Tinkerforge-Sensor ist über USB mit einem Einplatinencomputer der Marke Raspberry Pi verbunden und liefert bei jeder Helligkeitsänderung eine entsprechende Information. Mit Hilfe des von der Fa. Tinkerforge bereitgestellten Python-Skripts „Brick MQTT Proxy“ werden diese Sensordaten von dem Tinkerforge TCP/IP Protocol (TFP) 
in das in loT-Lösungen häufig verwendete Kommunikationsprotokoll „Message Queue Telemetry Transport (MQTT)“ umgewandelt und anschließend an den MQTT Broker in der Komponente „Monitoring and Control“ weitergeleitet. Hardwaretechnisch wird diese Komponente ebenfalls durch einen Einplatinencomputer der Marke Raspberry Pi repräsentiert. Für den MQTT Broker wird die Open Source Software "Mosquitto“ eingesetzt. Für die Anzeige der Sensordaten und die Steuerung der Aktoren kommt die Open Source Software „Open Home Automation Bus“ zum Einsatz. Diese stellt ein sogenanntes MQTT Binding zur Verfügung, mit dem eine Weiterleitung der Sensordaten vom MQTT Broker an openHAB ermöglicht wird. Der eingehende Helligkeitswert wird dann über eine Regel in openHAB interpretiert, die bei Unter- bzw. Überschreiten des festgelegten Schwellwertes für die Beleuchtungsstärke im Raum den Homematic Funk-Schaltaktor entsprechend ansteuert. Hierzu wird das Homematic Binding von openHAB in Verbindung mit der Open Source Software Homegear und einem Homematic Konfigurations-Adapter LAN (HM-CFG-LAN) eingesetzt. Zur Übertragung der Signale zwischen openHAB und Homegear wird der „Binary Remote Procedure Call“ (BIN-RPC) eingesetzt, Homegear wiederum sendet die Signale über das Homematic-proprietäre Funkprotokoll „Bidirectional Communication Standard“ (BidCoS) an den Adapter, der diese an den Funk-Schaltaktor weiterleitet.

\subsection{Sicherheitsanforderungen}

Zur genaueren Analyse werden nun die in der Systemarchitektur in Abb. 1 aufgeführten Komponenten mit den zum Einsatz kommenden Übertragungswegen und -protokollen zur jeweils nächsten Komponente einzeln aufgeführt:

\begin{tabular}{|l|l|l|l|}
\hline Nr. & Start-Komponente & Ziel-Komponente & $\begin{array}{l}\text { Übertragungsweg } \\
\text { und -protokoll }\end{array}$ \\
\hline 1 & $\begin{array}{l}\text { Helligkeitssensor (Tinker- } \\
\text { forge) }\end{array}$ & MQTT-Client (Tinkerforge) & $\begin{array}{l}\text { USB-Steckverbindung mit } \\
\text { TFP }\end{array}$ \\
\hline 2 & MQTT-Client (Tinkerforge) & MQTT-Broker (Mosquitto) & $\begin{array}{l}\text { Ethernet-Verbindung mit } \\
\text { MQTT }\end{array}$ \\
\hline 3 & MQTT-Broker (Mosquitto) & $\begin{array}{l}\text { Smart Home Anwendung } \\
\text { (openHAB) }\end{array}$ & $\begin{array}{l}\text { Raspberry Pi interne Ver- } \\
\text { bindung mit MQTT (MQTT } \\
\text { Binding) }\end{array}$ \\
\hline 4 & $\begin{array}{l}\text { Smart Home Application } \\
\text { (openHAB) }\end{array}$ & $\begin{array}{l}\text { Aktorsteuerung (Home- } \\
\text { gear) }\end{array}$ & $\begin{array}{l}\text { Raspberry Pi interne Ver- } \\
\text { bindung mit BIN-RPC } \\
\text { (Homematic Binding) }\end{array}$ \\
\hline 5 & $\begin{array}{l}\text { Aktorsteuerung (Home- } \\
\text { gear) }\end{array}$ & $\begin{array}{l}\text { Homematic Konfigura- } \\
\text { tions-Adapter }\end{array}$ & Ethernet mit BidCoS \\
\hline 6 & $\begin{array}{l}\text { Homematic Konfigura- } \\
\text { tions-Adapter }\end{array}$ & $\begin{array}{l}\text { Homematic-Funk- } \\
\text { Schaltaktor }\end{array}$ & Funk mit BidCoS \\
\hline
\end{tabular}

Tab. 3: Zu schützende Komponenten und Übertragungswege

Generell sind alle Angriffspunkte und Lösungsmechanismen der in Tab. 2 dargestellten Kategorien auf die in Tab. 3 dargestellten Komponenten und Übertragungswege anzuwenden. Hieraus wird ersichtlich, welcher Aufwand mit einer wirklich sorgfältigen Sicherheitsbetrachtung verbunden ist, insbesondere würde eine solche den Rahmen dieser Arbeit bei Weitem sprengen. Die weitere Untersuchung stellt deshalb zunächst nur die Kategorien Netzwerksicherheit, Authentisierung und Verschlüsselung - die sich natürlich überschneiden 
- in den Mittelpunkt der Betrachtung, da hiermit die Schwelle für mögliche Angriffe auf die Vertraulichkeit oder Integrität der Daten sowie eine Manipulation der beteiligten Systeme bereits deutlich erhöht wird. Des Weiteren wird auf eine Betrachtung der Raspberry Pi internen Verbindungen (Nr. 3 und 4 in obiger Tabelle) verzichtet, da eine Manipulation dieser Verbindungen einen physikalischen Zugriff auf den Einplatinencomputer erfordern würde, was sich durch geeignete Zugangsbeschränkungen verhindern lässt. Wesentliche Teile der nachfolgenden Ausführungen wurden in einer von den Autoren betreuten Bachelorarbeit an der Hochschule Ludwigshafen erarbeitet [Feue17].

\subsection{Sicherheitsmaßnahmen}

\section{Authentisierung}

Grundsätzlich sollte Authentisierung auf jedem Übertragungsweg genutzt werden; diese ist dann bei den beteiligten Komponenten zu implementieren. Im oben genannten Szenario gibt es die folgenden Beobachtungen:

- USB-Steckverbindung mit TFP: Mögliche Angriffsszenarien ergeben sich durch den Austausch einer Komponente, z.B. Manipulation des Systems durch Austausch des Helligkeitssensors. Genutzt werden kann Authentisierung mittels Pre-shared Key, was aber nicht standardmäßig aktiviert ist [TinkoJ].

- $\quad$ Ethernet-Verbindung mit MQTT: Grundsätzlich kann jeder MQTT-Client beim MQTTBroker „subscriben“. Eine Authentisierung ist mittels verschiedener Mechanismen möglich (Benutzername/Passwort, X.509-Zertifikate oder verschlüsselt mittels Pre-shared Key) ist aber nicht standard-mäßig aktiviert [MosqoJ].

- $\quad$ Ethernet-Verbindung mit BidCoS: Zwischen Homegear und dem Homematic Konfigurations-Adapter LAN ist eine Authentisierung mittels Pre-shared Key möglich. Um ein Bauteil durch ein bösartiges anderes auszutauschen, müsste also der Schlüssel bekannt sein.

- $\quad$ Funk-Verbindung mit BidCoS: Authentisierung der Aktorsteuerung durch AES-Handshake, also ebenfalls Authentisierung mittels Pre-shared Key.

Zusammengefasst ergibt sich für alle Verbindungen die Möglichkeit der Authentisierung, die jedoch meist nicht standardmäßig aktiviert ist und die eine Problematik des Schlüsselaustauschs mit sich bringt, speziell bei einer größeren Zahl von Kommunikationspartnern. Es ist auch zu beachten, dass nur in die letztgenannte Verbindung mittels Funk relativ einfach eingegriffen werden kann. Eine Manipulation der USB-Verbindung erfordert einen physikalischen Zugriff auf das System und eine Manipulation der Ethernet-Verbindungen einen Zugriff auf das LAN.

\section{Verschlüsselung}

Verschlüsselung dient in erster Linie dem Schutz der Vertraulichkeit, indirekt aber auch dem Schutz der Integrität. Bezogen auf die oben genannten Übertragungswege ergibt sich die folgende Situation:

- USB-Steckverbindung mit TFP: Eine Abhörmöglichkeit besteht hier nur durch physikalischen Eingriff. Die Datenübertragung ist allerdings nicht verschlüsselt.

- $\quad$ Ethernet-Verbindung mit MQTT: Die Daten werden standardmäßig unverschlüsselt übertragen, zur Verschlüsselung stehen aber zwei Mechanismen zur Verfügung:

TLS-Verschlüsselung mit Server-Zertifikat,

- Payload-Verschlüsselung, die einen paarweisen Schlüsselaustausch zwischen

Publisher und allen Subscribern erfordert. 
- Ethernet-Verbindung mit BidCoS: Verschlüsselung der Datenübertragung mittels AES möglich.

- $\quad$ Funk-Verbindung mit BidCoS: Keine Verschlüsselung möglich.

Zusammengefasst sehen wir, dass ausgerechnet die Funkverbindung, die ja potenziell am leichtesten abgehört werden kann, keine herstellerseitige Verschlüsselung erlaubt. Aber auch die anderen Verbindungen werden standardmäßig unverschlüsselt betrieben. Letztlich können diese Mängel aber akzeptiert werden, da auf der Funkverbindung nur Aktordaten übertragen werden, die nicht unbedingt vertraulich sind und ein Abhören der anderen Verbindungen zusätzlich einen Netzwerk- oder physikalischen Zugriff erfordert.

\section{Netzwerksicherheit}

Zur Betrachtung der Netzwerksicherheit verallgemeinern wir die zuvor konkret genannten Übertragungswege zu folgenden generischeren Kategorien:

- $\quad$ Proprietäre Kabelverbindungen, z.B. USB mit TFP

- $\quad$ Standardisierte LAN-Verbindungen, z.B. Ethernet

- $\quad$ Proprietäre Funkverbindungen, z.B. BidCoS

- $\quad$ Standardisierte Funkverbindungen, z.B. WLAN

Dies verdeutlicht zweierlei: Angriffe auf proprietäre Verbindungen erfordern spezifische Zugriffsmöglichkeiten, entweder auf Kabel oder auf den Funkverkehr. Sicherheitsmaßnahmen sind nur möglich, sofern diese herstellerseitig unterstützt werden. Angriffe auf standardisierte Verbindungen sind hingegen nicht loT-spezifisch, sondern können mit den klassischen Mitteln der Netzwerksicherheit bearbeitet werden. Hierzu gehören Verschlüsselung mit WPA2 im WLAN sowie TLS oder VPN im LAN. Auf tieferliegenden Schichten sind z.B. Angriffsformen wie ARP-Poisoning oder MAC-Flooding zu berücksichtigen, die mit Maßnahmen wie VLAN oder Port Security behandelt werden können [Feue17, Kap. 4.1].

\subsection{Erkenntnisse}

Die Übertragung der Sicherheitsüberlegungen auf den Smart Home Anwendungsfall hat gezeigt, dass loT-Lösungen grundsätzlich keine anderen Sicherheitsvorkehrungen erfordern als aus der „klassischen“ IT bekannt sind. Als besondere Herausforderung haben sich allerdings die Vielzahl unterschiedlicher Komponenten und die damit verbundene Komplexität des Gesamtsystems herausgestellt. Dies erfordert eine sehr sorgfältige Analyse der Sicherheitsbedrohungen und -anforderungen und in der Regel auch eine aufwendige Aktivierung unterschiedlicher Sicherheitsmaßnamen, da bei den zum Einsatz kommenden Komponenten häufig standardmäßig keine Sicherheitseinstellungen aktiviert sind. Die Einhaltung des Prinzips ,jedes Projekt soll Informationssicherheit von Anfang an berücksichtigen“ [ISO 27002, Kap. 6.1.5] sowie die Zusammenarbeit von Produktexperten mit einem oder mehreren Sicherheitsexperten sind demnach auch der Schlüssel zur Lösung von Informationssicherheitsproblemen im loT-Umfeld. Es ist zwar verständlich, dass unter Ressourcenknappheit und Innovationsdruck schnell Lösungen entwickelt werden müssen, allerdings darf dies speziell in kritischen Infrastrukturen nicht auf Kosten der Sicherheit gehen. Andernfalls wären die volkswirtschaftlichen Risiken des „Internet of Things“ auch unter Betrachtung des eingangs aufgezeigten Nutzens nicht vertretbar. 


\section{Literaturverzeichnis}

[AnHä15] Andelfinger, Volker P.; Hänisch, Till (Hrsg.): Internet der Dinge - Technik, Trends und Geschäftsmodelle. Springer Wiesbaden 2015.

[DoMa17] Dölle, Mirko; von Malottki, Jonas: Smart Home? Aber sicher! Artikelserie in der c't 8/17, S. 70-75, Heise Verlag, Hannover, 2017

[Eike13] Eikenberg, Roland: Vaillant-Heizungen mit Sicherheits-Leck, https://www.heise.de/ newsticker/meldung/Vaillant-Heizungen-mit-Sicherheits-Leck-1840919.html, abgerufen am 10.05.2017

[Feue17] Feuerstein, Markus: IoT-Security mit praktischer Anwendung, Bachelorarbeit an der Hochschule Ludwigshafen, 2017.

[ISO13a] ISO/IEC 27001: Information technology - Security techniques - Information security management systems - Requirements (ISO/IEC 27001:2013)

[ISO13b] ISO/IEC 27002: Information technology - Security techniques - Code of practice for information security controls (ISO/IEC 27002:2013)

[KrSc09] Kriha, Walter; Schmitz, Roland: Sichere Systeme, Springer Verlag (2009)

[MaFI10] Mattern, Friedemann; Flörkemeier, Christian: Vom Internet der Computer zum Internet der Dinge, Informatik-Spektrum, Bd. 33, Nr. 2, S. 107-121, April 2010.

[MosqoJ] https://mosquitto.org/man/mosquitto-conf-5.html, abgerufen am 10.05.2017

[OWAI17] OWASP loT: https://www.owasp.org/index.php/OWASP_Internet_of_Things_Project und darunter liegende Seiten, insbesondere https://www.owasp.org/index.php/ loT_Security_Guidance und https://www.owasp.org/index.php/loT_Attack_Surface_ Areas, abgerufen am 10.05.2017

[Raeh16] Rähm, Jan: Abwehr von Cyber-Angriffen aus dem Internet der Dinge, http://www.deutschlandfunk.de/sicherheitsluecken-abwehr-von-cyber-angriffen-ausdem.684.de.html?dram:article_id=374190, abgerufen am 10.05.2017

[RaSa17] Rayes, Ammar; Samer, Salam: Internet of Things - From Hype to Reality. Springer Cham 2017

[TinkoJ] https://www.tinkerforge.com/de/doc/Tutorials/Tutorial_Authentication/Tutorial.html\# tutorial-authentication, abgerufen am 10.05.2017

\section{Kontakt}

Prof. Dr. Haio Röckle, Prof. Dr. Frank Thomé, Prof. Dr. Carsten Dorrhauer Institut für Wirtschaftsinformatik an der Hochschule Ludwigshafen

Ernst-Boehe-Str. 4, 67059 Ludwigshafen

Tel.: 0621-5203-160, Sekretariat 0621-5203-150

Prof. Dr. Haio Röckle: haio.roeckle@web.de 


\title{
Qualitative Wertschöpfungsanalyse von Anwendungsfällen des Narrow- band Internet of Things
}

\author{
Dominik Schneider, Frank Wisselink, Christian Czarnecki
}

\section{Zusammenfassung}

Primäre Ziele des Internets der Dinge sind die Steuerung physischer Gegenstände aus der Distanz und das Erfassen von Informationen aus dem Umfeld dieser Gegenstände. Dazu werden Hardwarekomponenten in Gegenstände des täglichen Lebens und die Umwelt integriert. Mithilfe von Informations- und Kommunikationstechnologien entsteht hieraus das Internet der Dinge (Internet of Things, loT). Vor einem Jahr wurde mit Narrowband Internet of Things (NB-loT) eine Technologie standardisiert, die es ermöglicht, Hardwarekomponenten energieeffizient und unmittelbar über das Mobilfunknetz zu vernetzen. Gegenstände werden dadurch über große Reichweiten eigenständig kommunikationsfähig. Das loT steht mit NB-loT vor einem gestiegenen Nutzenpotenzial, da eine zunehmende Anzahl miteinander verbundener Gegenstände und der Austausch größerer Datenmengen realisierbar sind. Damit sind aus wirtschaftlicher Sicht neue, innovative Anwendungsfälle des loT möglich, die auch bereits in der Praxis diskutiert werden. In diesem Beitrag wird anhand eines konkreten Anwendungsfalls untersucht, welche neuen Geschäfts- bzw. Partnermodelle durch die gemeinsame Nutzung von NB-loT-Daten und Big Data-Technologien entstehen und welcher qualitative Mehrwert für die an einem Anwendungsfall beteiligten Stakeholder geschaffen wird. Dazu wird - einem konstruktionsorientierten Forschungsansatz folgend - ein Bewertungsrahmen zur qualitativen Wertschöpfungsanalyse von NB-loT entwickelt, der u.a. auf der Schablone nach Cockburn und dem Business Model Canvas basiert. Anhand dieses Bewertungsrahmens wird ein Anwendungsfall untersucht, der in anonymisierter Form an konkrete Praxisprojekte angelehnt ist. Konkret wird ein Anwendungsfall betrachtet, der einen Fahrradverleih 2.0 basierend auf dem Einsatz von NB-loT vorschlägt. Aus den Untersuchungsergebnissen gehen beispielsweise Erkenntnisse hervor, wie Geschäftsmodelle auf Basis eines unentgeltlichen Informationsaustauschs durch NB-loT gestaltet werden können

\section{$1 \quad$ Einleitung}

In den vergangenen Jahren hat sich weltweit ein wachsendes Interesse an der Fähigkeit entwickelt, über eingebundene Geräte, Sensoren und Aktoren zu kommunizieren [MGMT15]. Schnell entstand die Vision, die physische Welt aus der Distanz wahrzunehmen und zu steuern [MaFl10, BaSe11]. Heute ist eine solche Vorstellung längst keine Vision mehr [LRJN16]. Aus rasanten Technologieentwicklungen ging ein erweitertes Internet hervor, „welches neben klassischen Rechnern und mobilen Endgeräten auch beliebige physische Gegenstände mittels Sensoren und Aktoren in seine Infrastruktur einbindet [...]" [FIWW14]. Das erweiterte Internet wird als Internet der Dinge (englisch: Internet of Things, kurz: loT) bezeichnet.

Aus dem Zusammenspiel von Kommunikation zwischen Gegenständen oder Menschen und der Nutzung der daraus entstehenden Echtzeitdaten gehen bahnbrechende Möglichkeiten 
der Produktgestaltung und des funktionsübergreifenden Zusammenarbeitens hervor, wodurch wiederum Wertschöpfungsketten über mehrere Stakeholder verbessert werden können [Arno15, Busc16]. Auch aufgrund weiterer Potenziale zeichnet sich weltweit ein wachsendes Interesse am loT und an Big Data ab, was sich an der Entwicklung des Suchund Forschungsinteresses erkennen lässt. Der Anwendung "Google Trends" nach war das Suchinteresse für „IoT“ am 9. April 2017 und für „Big Data“ am 21. Februar 2016 im zeitlichen Verlauf der letzten fünf Jahre am höchsten (Stand: 13. April 2017). Auch in [SZGA15] ist die Rede von einem gestiegenen Forschungsinteresse am loT. Dabei sind die beiden Technologien noch jung und aus Marktperspektive schwer zu bewerten. Ein Blick auf den Hype-Zyklus des amerikanischen Marktforschungsunternehmens Gartner [Gart14, Gart15] bestätigt diese Annahme. Denn zwischen 2014 und 2015 erreichten das loT und Big Data das sogenannte „Tal der Enttäuschungen“ [Gart14, Gart15]. Das bedeutet, dass die Technologien bis dahin nicht alle Erwartungen erfüllt haben, die in der vorherigen Hype-Phase entstanden sind.

Ursprünglich wurde das loT u.a. von Kommunikationstechnologien wie Radio Frequency Identification (RFID) oder Bluetooth ermöglicht [PaPa16]. Die genannten Technologien sind insbesondere durch geringe Reichweiten charakterisiert, weshalb eine flächendeckende Vernetzung von Objekten nur schwer möglich ist. Durch die Standardisierung der neuen Schlüsseltechnologie NB-loT wird es nun möglich, wesentlich größere Reichweiten abzudecken und zusätzlich eine höhere Energieeffizienz zu erzielen. Damit lassen sich nahezu alle Gegenstände mit integrierter Hardwarekomponente kommunikationsfähig machen, weshalb in diesem Zusammenhang auch von Massenfähigkeit des loT gesprochen wird [Brey16]. Durch diese Entwicklung ist ein Anstieg der miteinander verbundenen Gegenstände und damit auch der erzeugten Datenmengen zu verzeichnen. Insofern ist ein Nutzen aus dem Einsatz der NB-loT-Technologie eng mit der Möglichkeit verbunden, diese Datenmengen schnell und zielführend zu analysieren. Genau diese Möglichkeit wird durch Big-Data-Technologien bereitgestellt [SZGA15].

Durch die zielgerichtete Bereitstellung von Informationen entlang der Wertschöpfungskette sind damit neue Geschäftsmodelle möglich [Arno15]. Dabei ist zwischen den einzelnen Akteuren der Wertschöpfungskette der Austausch von Gütern bzw. Dienstleistungen, Informationen und Geld in einer Form zu gestalten, die einen Nutzenzuwachs für alle Beteiligten darstellt. Als nutzenstiftendes Element ist der zusätzliche Austausch von Informationen durch NB-loT anzusehen. Dazu wird in diesem Beitrag ein Bewertungsrahmen zur qualitativen Wertschöpfungsanalyse von NB-loT vorgeschlagen. Die Entwicklung dieses Bewertungsrahmens folgt dem gestaltungsorientierten Forschungsparadigma [HMPR04]: (1) Beschreibung der Problemdomäne NB-loT, (2) Entwicklung und Beschreibung des Bewertungsrahmens und (3) Evaluation des Bewertungsrahmens durch Anwendung in einem Praxisbeispiel. Der vorgeschlagene Bewertungsrahmen basiert auf bestehenden Ansätzen der Schablone nach Cockburn [Cock03], dem makroökonomischen Wirtschaftskreislauf [BoDK08], dem Business Model Canvas [OsPi09] und der Methodik von Agile Economics [Wi++16]. Die Bewertung von Geschäftsmodellen des NB-loT ist ein aktuelles Problem aus der Praxis, so dass der vorgeschlagene Bewertungsrahmen als Weiterentwicklung bestehender Lösungen auf ein neues Problemfeld anzusehen ist, was ein originäres Ziel der gestaltungsorientierten Forschung der Wirtschaftsinformatik darstellt [ShHe13].

Der Beitrag ist wie folgt strukturiert. Die Problemdomäne NB-loT wird in Abschnitt 1 erläutert. Abschnitt 2 diskutiert die bestehenden Ansätze, welche in Abschnitt 3 in einen Bewertungsrahmen für NB-loT-Anwendungsfälle überführt werden. Die Anwendung des vorge- 
schlagenen Bewertungsrahmens auf Anwendungsfälle aus der Praxis erfolgt in Abschnitt 4. Mit einem Fazit und Ausblick (siehe Abschnitt 5) schließt der Beitrag.

\section{Bestehende Ansätze}

Da das loT noch vor einem massenfähigen Einsatz in der Praxis steht [Gart14, Gart15], sind gewinnbringende Geschäftsmodelle noch in der Entwicklungsphase. Gerade in der Praxis ist eine zuverlässige Bewertung des Wertschöpfungsbeitrags eine unabdingbare Voraussetzung für einen massenfähigen Einsatz. Als Lösungsvorschlag wird in diesem Beitrag ein Bewertungsrahmen für NB-loT-Anwendungsfälle vorgestellt. Aus genereller Sicht existieren mit der Schablone nach Cockburn [Cock03], dem makroökonomischen Wirtschaftskreislauf [BoDK08], dem Business Model Canvas [OsPi09] und der Methodik von Agile Economics [Wi++16] bereits Ansätze zur Bewertung von Geschäftsmodellen. Diese berücksichtigen jedoch nicht die Besonderheiten der Problemdomäne NB-loT. In diesem Zusammenhang sind reale Gegenstände in einer Form miteinander so zu verknüpfen, dass durch den Datenaustausch in Verbindung mit Güter- bzw. Dienstleistungsflüssen ein Nutzenpotenzial für alle Beteiligten entsteht. Im Folgenden werden die bestehenden Ansätze kurz diskutiert. Auf dieser Grundlage wird dann in Abschnitt 3 der vorgeschlagene Bewertungsrahmen für NBloT entwickelt.

Die Schablone nach Cockburn - benannt nach dem schottischen Informatiker Alistair Cockburn - eignet sich für die Vorstellung verschiedener Anwendungsfälle in einem einheitlichen Format. Cockburn versteht unter einem Anwendungsfall allgemein das Verhalten eines Systems, welches sich aus mehreren Stakeholdern zusammensetzt. Im ersten Schritt ist der Kontext des Systems zu beschreiben, wobei das primäre Ziel des Systems und die auslösende Anfrage des Primärakteurs im Fokus stehen [Cock03]. Als Primärakteur wird der Stakeholder bezeichnet, der den Anwendungsfall durch eine bestimmte Absicht auslöst [Cock03]. Im zweiten Schritt sind der Primärakteur und die weiteren Stakeholder hinsichtlich derer Interessen zu betrachten [Cock03]. Bei einem Anwendungsfall werden die Interessen aller Stakeholder gewahrt, da sonst das Verhalten des Systems nicht aufrecht gehalten werden kann [Cock03]. Die Betrachtung des Systemkontexts und der beteiligten Stakeholder ist eine gängige Methode zur Beschreibung von Anwendungsfällen, die auch nach der Modellierungssprache Unified Modeling Language (UML) Anwendung findet [BoRJ06].

Der einfache makroökonomische Wirtschaftskreislauf ist ein Modell, das die volkswirtschaftlichen Wechselwirkungen zwischen Haushalten und Unternehmen in Form von Geld- und Güter- bzw. Dienstleistungsströmen abbildet. Das Modell ist durch zwei Modelleigenschaften charakterisiert. Als erstes ist die gegenseitige Abhängigkeit der Haushalte und Unternehmen zu nennen [BoDK08]. Damit ist gemeint, dass Haushalte im einfachen Wirtschaftskreislauf keinen Wert ohne Unternehmen schöpfen können, und umgekehrt. Die zweite Modelleigenschaft ist die sogenannte gleichgewichtige Ruhelage, womit gemeint ist, dass jeder Leistung auch eine Gegenleistung mit gleichem Wert gegenüber steht [BoDK08].

Das Business Model Canvas ist ein Werkzeug zur Beschreibung, Analyse und Gestaltung von Geschäftsmodellen [OsPi09]. Für die qualitative Wertschöpfungsanalyse spielt das Business Model Canvas insofern eine Rolle, als dass es dabei hilft, die Geschäftsmodelle der an einem Anwendungsfall beteiligten Stakeholder zu untersuchen und somit die Interessen der Stakeholder zu verstehen [OsPi09]. Grundsätzlich besteht das Business Model 
Canvas aus neun sogenannten Building Blocks, die eine Betrachtung von Geschäftsmodellen anhand verschiedener Kriterien ermöglichen.

Agile Economics ist eine Methodik, die im Innovationscontrolling eingesetzt wird, um Geschäftsmodelle von Innovationen mit erwarteten grundlegenden Veränderungen zu quantifizieren [Wi++14]. Ziel ist es, Geschäftsszenarien mithilfe von Pilotprojekten zu verstehen und Wissen darüber zu sammeln, um das Risiko und die Unsicherheit der getroffenen Annahmen zu minimieren [Wi++14]. Mit Agile Economics kann gleichermaßen die Wertschöpfung validiert werden, über die an dieser Stelle die Brücke zur Analyse der Wertschöpfung durch Big Data geschlagen wird. Der wirtschaftliche Mehrwert von Big Data liegt im Informationswert [Wi++16]. Ein Informationswert entsteht für eine Organisation, wenn sie einen bestimmten Sachverhalt besser und schneller erkennt [Wi++16].

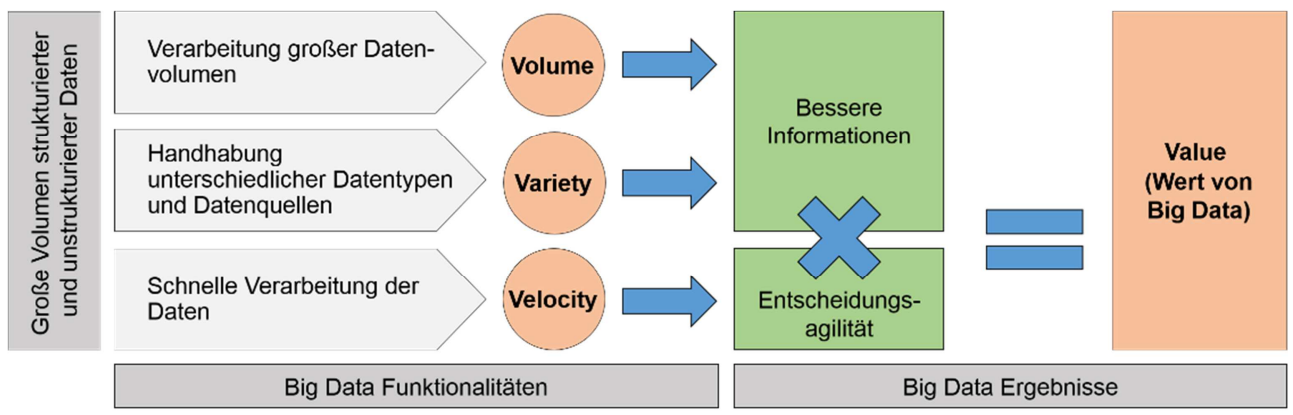

Abbildung 1: Erzeugung von Mehrwert durch Big Data [WI++16]

Die Abbildung 1 veranschaulicht, wie mithilfe von Big Data Mehrwert für Organisationen generiert werden kann. Zum einen können mit Big Data bessere Informationen zur Verfügung gestellt werden, indem große Datenvolumen (Volume) und unterschiedliche Datentypen/-quellen (Variety) verarbeitet werden. Zum anderen wird Entscheidungsagiliät geschaffen, wenn diese Daten in einer angemessenen Zeit (Velocity) verarbeitet und verwendet werden. Wenn bessere Informationen zu agilem Handeln führen, entsteht durch Big Data Mehrwert.

\section{Entwicklung eines Bewertungsrahmens für NB-loT-Anwendungsfälle}

Im vorherigen Abschnitt 2 wurden generische Modelle beschrieben, die im Folgenden zu einem Bewertungsrahmen für die qualitative Wertschöpfungsanalyse eines NB-loT-Anwendungsfalls zusammengeführt und erweitert werden. In der qualitativen Wertschöpfungsanalyse gilt es, den Anwendungsfall vorzustellen, die Geschäftsbeziehungen zwischen den beteiligten Stakeholdern abzubilden, die Geschäftsmodelle der einzelnen Stakeholder zu beschreiben und anschließend den Mehrwert aus der Sicht der Beteiligten entlang der gesamten Wertschöpfungskette zu analysieren.

Die Vorstellung des Anwendungsfalls erfolgt auf Basis der Schablone nach Cockburn. Das zentrale Element der vier Analyseschritte ist jedoch die Abbildung der Geschäftsbeziehungen zwischen den beteiligten Stakeholdern, da hierbei die gesamte Ende-zu-Ende-Wertschöpfungskette analysiert wird. Eine hohe Bedeutung ist einer Ende-zu-Ende-Analyse zuzurechnen, weil ein Anwendungsfall nur dann einen Wert erzielen kann, wenn ein expliziter Endkundennutzen erzeugt wird und der Wert für den Kunden konsequent im Fokus des 
Anwendungsfalls steht [Arno15]. Dazu bietet es sich an, die Art und Weise der Visualisierung und die Modelleigenschaften des einfachen makroökonomischen Wirtschaftskreislaufs zu nutzen. Für die Wertschöpfungsanalyse spielen die volkswirtschaftlichen Zusammenhänge zwischen Haushalten und Unternehmen jedoch keine Rolle, weshalb das Modell für die Untersuchung unterschiedlicher Anwendungsfälle generalisiert wird. Da bei Anwendungsfällen des NB-IoT vor allem Daten und Informationen als wichtige Wirtschaftsgüter zu betrachten sind, wird das Modell des Wirtschaftskreislaufs außerdem um Daten- und Informationsströme ergänzt.
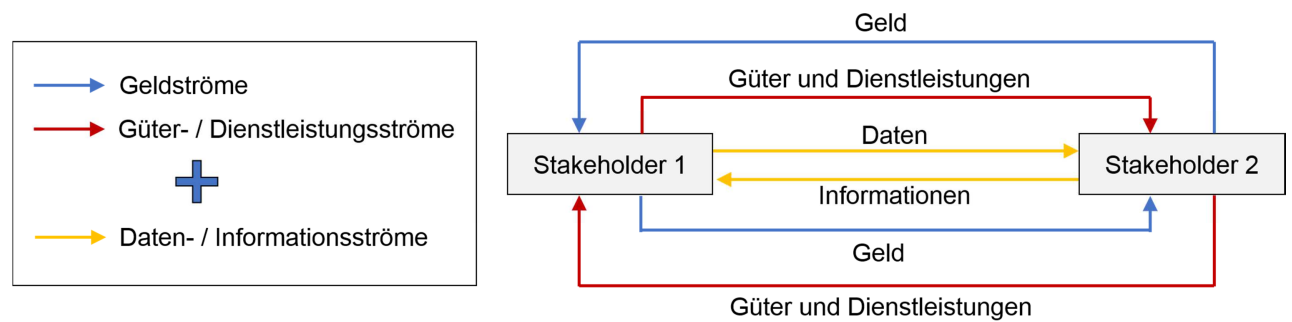

Abbildung 2: Generalisierung des Wirtschaftskreislaufs und Ergänzung der Daten- und Informationsströme

Diese Modifikation des Wirtschaftskreislaufs ermöglicht es, die Geld-, Güter- und Dienstleistungs-, sowie Daten- und Informationsströme zwischen verschiedenen Stakeholdern abzubilden. In Abbildung 2 stellt z.B. der Stakeholder 1 dem Stakeholder 2 Daten zur Verfügung, der als Gegenleistung Informationen von Stakeholder 2 erhält. In Hinblick auf die Analyse der Wertschöpfung durch NB-loT kann Stakeholder 2 als Big Data-Nutzer verstanden werden, da dieser die Daten von Stakeholder 1 auswertet, mit anderen Datenquellen anreichert und in Form von Informationen zurückliefert. Stakeholder 1 kann die Informationen für verschiedene Geschäftszwecke oder interne Optimierungsaufgaben verwenden. Die gegenseitige Abhängigkeit der Stakeholder und die gleichgewichtige Ruhelage bleiben in der Abwandlung des Wirtschaftskreislaufs als wichtige Modelleigenschaften bestehen.

Für die Beschreibung der einzelnen Geschäftsmodelle unter Verwendung des Business Model Canvas stehen das Werteangebot, sowie die Schlüsselressourcen, Schlüsselaktivitäten und Schlüsselpartner als relevante Betrachtungsperspektiven im Vordergrund [OsPi09]. Das Werteangebot beschreibt den Wert, der dem Kunden konkret angeboten wird, und die Kundenbedürfnisse, die damit befriedigt werden. Mit den Schlüsselressourcen werden benötigte physische, intellektuelle, menschliche und finanzielle Ressourcen betrachtet. Die Schlüsselaktivitäten beschreiben nach Produktion, Problemlösung und Plattform/Netzwerk kategorisierte Aktivitäten zur Erstellung des Werteangebots. Der Building Block Schlüsselpartner beantwortet die Frage, welche Schlüsselressourcen von welchen Partnern bezogen werden können. Für die Analyse des Mehrwerts durch NB-loT wird anschließend das Modell der Agile Economics Methode aus der Sicht des Big Data-Nutzers verwendet. Dabei gilt es zu untersuchen, welche Daten zu welchen besseren Informationen analysiert werden und wie diese besseren Informationen zu agilem Handeln führen können.

\section{Anwendung des Bewertungsrahmens auf einen Anwendungsfall aus der Praxis}

Der in Abschnitt 3 beschriebene Bewertungsrahmen für NB-loT wird in diesem Abschnitt für die qualitative Wertschöpfungsanalyse eines NB-loT-Anwendungsfalls angewendet. Der 
Anwendungsfall demonstriert, welcher Mehrwert für die Stakeholder eines Anwendungsfalls durch den Einsatz von NB-loT erzielt werden kann und welche neuen Geschäfts- und Partnermodelle entstehen. In Bezug auf das zugrunde liegende gestaltungsorientierte Forschungsparadigma [HMPR04] stellt der in Abschnitt 3 vorgestellte Bewertungsrahmen eine generische Referenz dar, deren Evaluation in diesem Abschnitt durch ein Praxisbeispiel belegt wird. Der Anwendungsfall ist eine zusammengefasste und teilweise anonymisierte Darstellung von konkreten Praxisprojekten. Die Darstellung basiert auf dokumentierten und publizierten ersten Anwendungsfällen von NB-loT, was als valider Ansatz zur Erforschung von Praxisphänomenen angesehen wird [Yin09]. Untersucht wird nachfolgend ein Fahrradverleih 2.0, der auf NB-loT und Big Data basiert.

Beim Fahrradverleih 2.0 geht es um den Verleih von Fahrrädern, die über NB-loT kommunikationsfähig sind und in regelmäßigen Zeitabständen oder auf Nachfrage Daten (z.B. den aktuellen Standort) an die Verleihfirma senden [Eric16]. Die Deutsche Bahn AG bietet mit "Call a Bike" bereits ein ähnliches Fahrradverleihsystem, bei dem die Vernetzung der Fahrräder über Nahfunk- und klassische Mobilfunktechnik erfolgt [Deut17]. NB-loT ist aufgrund der energieeffizienten Kommunikation eine für den Fahrradverleih 2.0 geeignetere Kommunikationstechnik. Weitere Eigenschaften sind die tiefe Gebäudedurchdringung bis in Fahrradkeller und die Abdeckung großer Reichweiten, die aufgrund der hohen Mobilität von Fahrrädern notwendig sind. Mit NB-loT können ferner mehrere tausend bis Millionen Geräte pro Mobilfunkzelle gleichzeitig kleine Datenvolumen übertragen [Eric16]. In Anlehnung an die Schablone nach Cockburn wird der Anwendungsfall dadurch ausgelöst, dass Verleihfirmen häufig von Fahrraddiebstählen betroffen sind und mithilfe von NB-loT verfolgen möchten, wo sich die Fahrräder zu einem bestimmten Zeitpunkt befinden [Huaw16]. Ergänzend zum sogenannten Fahrrad-Tracking können in Anlehnung an „Pay-How-You-Drive“ (ein Anwendungsfall, der häufig von Autoversicherungen angeboten wird) weitere Daten zum Fahrverhalten an die Verleihfirma gesendet werden, sofern der Kunde der Datenfreigabe zustimmt. Die Leihgebühr lässt sich dadurch individuell je nach Fahrverhalten und Materialverschleiß berechnen. Welche Stakeholder für die Realisierung des Fahrradverleihs 2.0 erforderlich sind und wie die Stakeholder miteinander interagieren, zeigt Abbildung 3.

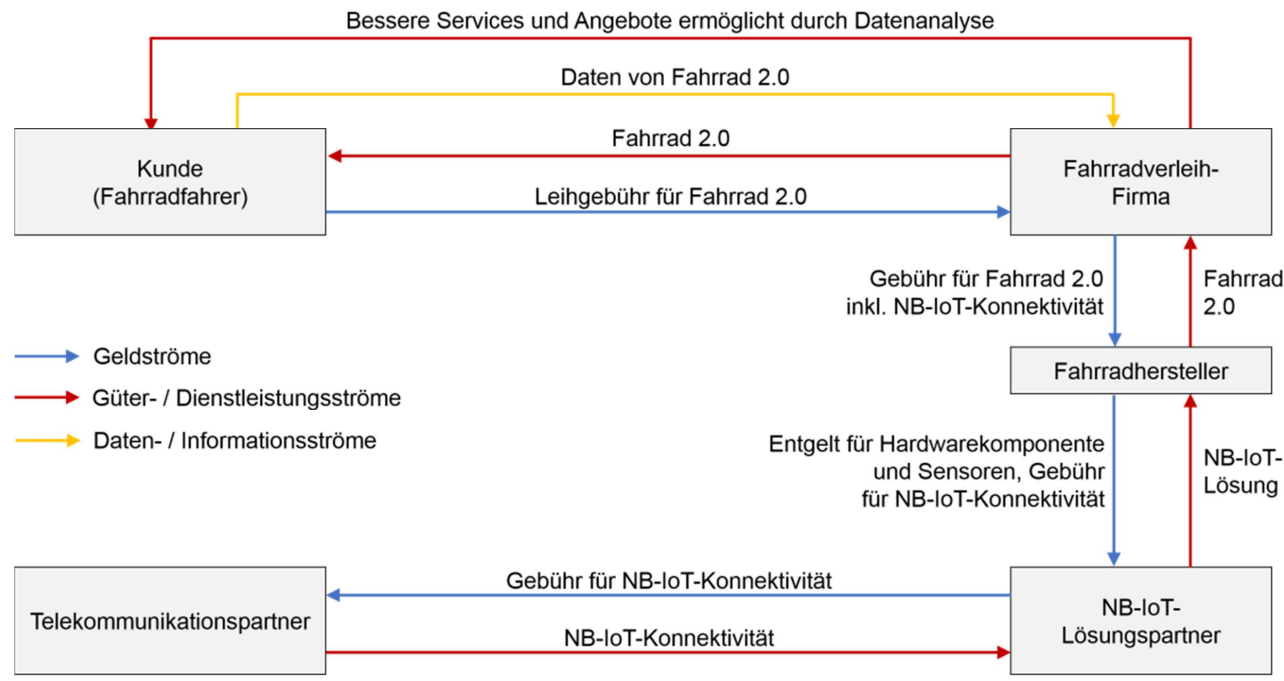

Abbildung 3: Geschäftsbeziehungen zwischen Stakeholdern des Fahrradverleihs 2.0 
Die Wertschöpfungskette des Fahrradverleihs 2.0 beginnt beim Telekommunikationspartner, der dem NB-loT-Lösungspartner NB-loT-Konnektivität verkauft. Der Lösungspartner baut die NB-IoT-Konnektivität mittels eines Chips, der einer SIM-Karte nahe kommt, in die Hardwarekomponente ein. Der Chip ermöglicht die Identifikation der Hardwarekomponente im Mobilfunknetz und damit auch die NB-loT-Nutzung. Der Lösungspartner verkauft dem Fahrradhersteller die kommunikationsfähige Hardwarekomponente und zusätzliche Sensoren als NB-loT-Lösung, der sie in ein Fahrrad zur Erfassung und Übertragung von Daten integriert. An dieser Stelle der Wertschöpfungskette entsteht ein Fahrrad 2.0. Die Verleihfirma kauft Fahrräder 2.0 vom Fahrradhersteller und verleiht sie an Kunden. Sie erhält vom Kunden eine Leihgebühr, sowie Daten, die ein Fahrrad 2.0 regelmäßig sendet. Für das Fahrrad 2.0 zahlt die Verleihfirma an den Fahrradhersteller eine regelmäßige Gebühr, in der auch ein Anteil für die NB-loT-Konnektivität enthalten ist. Für die bereitgestellte NB-loT-Konnektivität fordert der Telekommunikationspartner eine Gebühr vom NB-loT-Lösungspartner, der die Gebühr vom Fahrradhersteller erhält. Die Analyse der entstehenden Daten bietet der Verleihfirma bessere Informationen, die sie für optimierte Services und Angebote einsetzen kann. Davon kann der Kunde profitieren, der das Ende der Wertschöpfungskette darstellt.

Aus der Summe vieler kleiner Datenmengen von Fahrrädern 2.0 entstehen schlussendlich große Datenmengen. Zusätzlich können für die Datenanalyse auch Daten aus sozialen Netzwerken, Kundendaten, Veranstaltungsdaten, Detailinformationen über Fahrräder, Herstellerinformationen und weitere Datenquellen verwendet werden. Dadurch fallen zum einen noch größere Datenmengen an, zum anderen entsteht eine hohe Varianz in den Datenmengen. Mithilfe von Big Data kann die Verleihfirma aus den großen Mengen strukturierter und unstrukturierter Daten bessere Informationen gewinnen. Zu diesen Informationen zählt u.a. der Standort eines Fahrrads, der für die Absicht des Primärakteurs eine sehr hohe Relevanz aufweist. Neben Standortdaten erhält die Verleihfirma außerdem Informationen über die Nutzung des Fahrrads. Beispielsweise kann festgestellt werden, ob ein Kunde sehr schnell beschleunigt und viel bremst oder ob ein Kunde ausgeglichen und konstant fährt. Das Fahrverhalten gibt wiederum Auskunft über den Materialverschleiß eines Fahrrads. Zusätzlich zu den Informationen, die aus den Daten der Hardwarekomponenten gewonnen werden, können auch Informationen aus der Kombination anderer Quellen mit den Mess- und Standortdaten abgeleitet werden. Zum Beispiel lassen sich Interessen eines Kunden herausfinden, indem die Standortdaten des Fahrrads mit Veranstaltungsorten, die aus Daten sozialer Netzwerke hervorgehen, abgeglichen werden. Es entsteht durch den Einsatz von Big Data also eine Menge zusätzlicher, besserer Informationen, die für die Verleihfirma wertschöpfend sein können, wenn sie für zielgerichtetes, agiles Handeln eingesetzt werden.

Beispiele dafür sind, gestohlene Fahrräder schnell wieder zu finden oder eine Preisgestaltung basierend auf der Auswertung der Standortdaten und des Fahrverhaltens anzubieten. Darüber hinaus kann auch die Berechnung des Diebstahlrisikos in Abhängigkeit zum Standort oder zu einer Veranstaltung als agiles Handeln gesehen werden, ebenso wie Predictive Maintenance, also eine vorausschauende Instandhaltung. Materialverschleiß an einem Fahrrad kann anhand der Informationen über das Nutzungsverhalten rechtzeitig repariert werden, bevor ein größerer Schaden entsteht. Eine weitere agile Handlungsmöglichkeit ist das Cross- oder Upselling. Die Verleihfirma kann z.B. versuchen, dem Kunden ein hochwertigeres Fahrrad entsprechend seines Fahrniveaus zu verleihen. Weitere Nutzung der Informationen ist denkbar, so dass die hier vorgestellten Nutzenpotenziale für agiles Handeln als beispielhaft zu verstehen sind. 
Aufgrund der zunehmenden Verbreitung des loT - was auch durch die technischen Möglichkeiten des NB-loT begünstigt wird - entstehen neue Geschäftsmodelle. Dabei ist NB-loT als neue Datenquelle anzusehen, aus deren zielführender Analyse durch Einsatz von Big Data-Ansätzen Wertschöpfungspotenziale entstehen können. Dabei ist die Frage, wie NBIoT in neuen Geschäfts- und Partnermodellen so genutzt werden kann, dass ein Nutzen für alle Beteiligten entlang der Wertschöpfungskette entsteht. So sind z.B. Hardware-Konnektivitäts-Partnerschaften zwischen Hardwareherstellern und Telekommunikationsanbietern zur Herstellung von obligatorischen NB-IoT-Lösungen oder aber auch Daten-Informations-Partnerschaften zwischen Big Data-Plattform-Betreibern und Daten- bzw. Informations-Nutzern zur Generierung von qualitativen Mehrwerten denkbar. Der vorliegende Beitrag schlägt in diesem Zusammenhang einen Bewertungsrahmen zur qualitativen Analyse von NB-loTAnwendungsfällen vor. Seine Anwendung wird anhand eines Praxisbeispiels erläutert, was als eine erste Validierung anzusehen ist. Somit wird mit dem Bewertungsrahmen ein Lösungsansatz geliefert, der bei der aktuellen Diskussion in der Praxis als Referenz genutzt werden kann.

Anhand des Praxisbeispiels Fahrradverleih 2.0 ist zu sehen, dass NB-loT zu neuen Geschäftsmodellen führen kann, wie z.B. der datenbasierten Optimierung herkömmlicher Produkte und Dienstleistungen sowie dem Verkauf oder der unentgeltlichen Bereitstellung von Daten oder Informationen unter Beachtung der Datenschutz-Richtlinien und -Gesetze. Die unentgeltliche Bereitstellung von Daten oder Informationen, wie sie beim Fahrradverleih 2.0 zwischen dem Kunden und der Verleihfirma stattfindet, wird nach der Durchführung der qualitativen Wertschöpfungsanalyse als künftig bedeutsames Geschäftsmodell angesehen. Der Mehrwert bei diesem Geschäftsmodell entsteht nicht zwingend und unmittelbar durch eine finanzielle oder materielle Gegenleistung, sondern durch Wertschöpfungsaktivitäten anderer Stakeholder, die dem unentgeltlichen Datenlieferanten einen mittelbaren Mehrwert verschaffen. Beim Fahrradverleih 2.0 erhält die Verleihfirma z.B. unentgeltlich Daten zur Fahrradnutzung. Durch Big Data-Analysen kann die Verleihfirma optimierte Dienstleistungen anbieten oder die Daten und Informationen unentgeltlich an Dritte weitergeben, um selber Gebrauch von optimierten Produkten oder Dienstleitungen zu machen. Aus den ermittelten neuen Geschäftsmodellen lassen sich qualitative Mehrwerte ableiten. Dazu zählen vor allem Effizienz- und Produktivitätssteigerungen, Produktoptimierungen, sowie ein besseres Kundenverständnis und damit einhergehend auch die Möglichkeit einer genaueren Kundensegmentierung und Produktausrichtung. Ein besseres Kundenverständnis kann zur Erhöhung der Kundenzufriedenheit und des Umsatzes genutzt werden.

Aufgrund der vielfältigen Analysemöglichkeiten vorhandener Daten gibt es eine Vielzahl weiterer qualitativer Nutzenpotenziale, die durch NB-loT und Big Data entstehen können. Die Analyse weiterer Anwendungsfälle anhand des vorgestellten Bewertungsrahmens ist somit möglich. Eine quantitative Bewertung der ermittelten qualitativen Mehrwerte wurde in diesem Beitrag nicht durchgeführt. Abschätzungen über konkrete Umsatzerwartungen oder Kosteneinsparpotenziale für Unternehmen stellen daher ein zukünftiges Forschungsthema in Ausblick. In diesem Beitrag wurde ferner nicht auf Datenschutz-Richtlinien und -Gesetze im Rahmen von Big Data-Analysen eingegangen, die jedoch für jedes Unternehmen, das Datenanalysen betreibt, eine hohe Relevanz haben. Die Untersuchung der Konformität von Datenschutz-Richtlinien und -Gesetzen und der Datensammlung, Datenspeicherung und 
Datenverarbeitung im Rahmen von Big Data-Analysen ist demnach ein weiteres Forschungsthema, das basierend auf diesem Beitrag angegangen werden kann.

\section{Literaturverzeichnis}

[Arno15] Arnold, H. M.: Zum Geleit: Datability und Digitalisierung. In: Linnhoff-Popien, C.; Zaddach, M.; Grahl, A. (Hrsg.): Marktplätze im Umbruch. Springer, Berlin Heidelberg, 2015, S. 705 - 712.

[BaSe11] Bandyopadhyay, D.; Sen, J.: Internet of Things - Applications and Challenges in Technology and Standardization. In: Wireless Personal Communications. Springer US, 2011, S. $49-69$.

[BoDK08] Bonrath, T.; Dinkela, A.; Kührt, P.: Volkswirtschaftslehre für Fachoberschulen und Berufsoberschulen in Bayern. Bildungsverlag EINS GmbH, Troisdorf, 2008.

[BoRJ06] Booch, G.; Rumbaugh, J.; Jacobson, I.: Das UML Benutzerhandbuch. Addison-Wesley, Boston, 2006.

[Brey16] Breyer-Mayländer, T.: Management 4.0 - Den digitalen Wandel erfolgreich meistern. Carl Hanser Verlag GmbH \& Co. KG, 2016.

[Busc16] Buschbacher, F.: Wertschöpfung mit Big Data Analytics. In: Schäffer, U.; Weber, J. (Hrsg.): Controlling \& Management Review Sonderheft 1-2016. Springer Fachmedien, Wiesbaden, 2016, S. 40 - 45.

[Cock03] Cockburn, A.: Use Cases effektiv erstellen. Mitp Verlags GmbH \& Co. KG, 2003.

[Deut17] Deutsche Bahn Connect GmbH: Call a Bike Technik. 2017, https://www.deutschebahnconnect.com/de/mobilitaetskonzepte/fvs/call-a-bike/cabtechnik. Abruf am: 2017-05-04.

[Eric16] Ericsson: Cellular networks for Massive loT - enabling low power wide area applications. 2016, https://www.ericsson.com/publications/white-papers/cellular-networks-formassive-iot--enabling-low-power-wide-area-applications. Abruf am: 2017-03-29.

[FIWW14] Fleisch, E.; Weinberger, M.; Wortmann, F.: Geschäftsmodelle im Internet der Dinge. 2014, http://www.enzyklopaedie-der-wirtschaftsinformatik.de/lexikon/technologien-methoden/Rechnernetz/Internet/Internet-der-Dinge. Abruf am 2016-07-21.

[Gart14] Gartner, Inc.: Gartner's 2014 Hype Cycle for Emerging Technologies Maps the Journey to Digital Business. 2014, http://www.gartner.com/newsroom/id/2819918. Abruf am 2016-07-22.

[Gart15] Gartner, Inc.: Gartner's 2015 Hype Cycle for Emerging Technologies Identifies the Computing Innovations That Organizations Should Monitor. 2015, http://www.gartner.com/newsroom/id/3114217. Abruf am 2016-08-18.

[HMPR04] Hevner, A. R.; March, S. T.; Park, J.; Ram, S.: Design Science in Information Systems Research. In: MIS Quarterly 28. Institute for Operations Research and the Management Sciences, 2004, S. 75 -105.

[Huaw16] Huawei Technologies Co., Ltd.: NarrowBand IoT: Wide Range of Opportunities (MWC16). 2016, http://www.huawei.com/en/events/mwc/2016/summit/global-nbiot/narrowband-iot-wide-range-of-opportunities. Abruf am 2017-03-12. 
[LRJN16] Lin, C.; Ramakrishnan, K. K.; Liu, J.; Ngai, E.: Guest Editorial - Special Issue on Cloud Computing for loT. In: IEEE Internet of Things Journal. 2016, S. 254 - 256.

[MaFI10] Mattern, F.; Flörkemeier, C.: Vom Internet der Computer zum Internet der Dinge. In: Informatik-Spektrum. Springer Berlin Heidelberg, 2010, S. 107 - 121.

[MGMT15] Mehmood, Y.; Görg, C.; Muehleisen, M.; Timm-Giel, A.: Mobile M2M communication architectures, upcoming challenges, applications, and future directions. In: EURASIP Journal on Wireless Communications and Networking. Springer International Publishing, 2015, S. 1 - 37.

[OsPi09] Osterwalder, A.; Pigneur, Y.: Business Model Generation: A Handbook for Visionaries, Game Changers, and Challengers. John Wiley \& Sons, New Jersey, 2009.

[PaPa16] Pande, P.; Padwalkar, A. R.: Internet of Things - A Future of Internet: A Survey. In: International Journal of Advance Research in Computer Science and Management Studies, 2016, S. 354 - 361.

[ShHe13] Shirley, G.; Hevner, A. R.: Positioning and Presenting Design Science Research for Maximum Impact. In: MIS Quarterly 37. Institute for Operations Research and the Management Sciences, 2013, S. 337 - 356.

[SZGA15] Strohbach, M.; Ziekow, H.; Gazis, V.; Akiva, N.: Towards a Big Data Analytics Framework for loT and Smart City Applications. In: Xhafa, F.; Barolli, L.; Barolli, A.; Papajorgji, P. (Hrsg.): Modeling and Processing for Next-Generation Big-Data Technologies. Springer International Publishing, 2015, S. 257 - 282.

[Wi++14] Wisselink, F.; Klöppel, B.; Horn, T.; Ujhelyiová, D.; Obeloer, J.: Agile Economics: A robust method for economic evaluation \& controlling of game changing innovations like Big Data. Detecon International GmbH, Köln, 2014.

[Wi++16] Wisselink, F.; Horn, T.; Meinberg, R.; Obeloer, J.; Ujhelyiová, D.: The Value of Big Data for a Telco: Treasure Trove or Pandora's Box?. In: Detecon International GmbH (Hrsg.): Future Telco Reloaded: Strategies for Successful Positioning in Competition. Köln, 2016, S. $151-161$.

[Yin09] Yin, R. K.: Case Study Research: Design and Methods. Sage Publications 4th Edition, Los Angeles, 2009.

\section{Kontakt}

B. Sc. Dominik Schneider

Dr. ir. Frank Wisselink BSc (Hons.)

Detecon International $\mathrm{GmbH}$

Sternengasse 14-16, 50676 Köln

Dominik.Schneider@detecon.com,Frank.Wisselink@detecon.com

Prof. Dr.-Ing. Christian Czarnecki

Hochschule für Telekommunikation Leipzig

Gustav-Freytag-Straße 43-45, 04277 Leipzig

Czarnecki@hft-leipzig.de 


\section{Wissensmanagement - Wissenstransfer}




\title{
Kompetenzerwerb in angewandter Wissensmodellierung durch Lernen aus Fehlern
}

\author{
Vera G. Meister
}

\section{Zusammenfassung}

Die Fähigkeit fachliche Abläufe und Strukturen zu modellieren, gehört zu den Kernkompetenzen von Wirtschaftsinformatikern. Darüber hinaus hat insbesondere die Modellierung von Geschäftsprozessen breiten Eingang in betriebswirtschaftliche und kaufmännische Curricula sowohl in der akademischen als auch in der beruflichen Bildung gefunden. Neben der Kenntnis von Modellierungsparadigmen, -notationen und -werkzeugen, erfordert ein effektiver Kompetenzerwerb auf diesem Gebiet auch das tiefere Verständnis einer fachlichen Domäne, möglichst aus Sicht eines Leistungserbringers oder -anbieters. Übungen, die nur auf die Analyse bestehender Prozessmodelle bzw. auf die Umsetzung rein textueller Vorgangsbeschreibungen setzen, reichen nicht aus, um ein tieferes Verständnis der Materie sowie einen effektiven Kompetenzerwerb herzustellen. Der Beitrag will zeigen, wie ein didaktisches Konzept, das vorhandenes Domänenwissen aufgreift und in der Umsetzung explizit auf eine positive Fehlerkultur setzt, den Kompetenzerwerb positiv beeinflusst.

\section{$1 \quad$ Einführung}

Die Überbrückung des „Grabens" zwischen den Fachanwendern und -entscheidern in Unternehmen und Organisationen auf der einen Seite und den IT-Entwicklern und -Implementierens auf der anderen Seite kann als vornehmste Aufgabe des Fachgebiets Wirtschaftsinformatik angesehen werden (vgl. z. B. [RaPW15]). Traditionell wird vom Business-IT-Alignement gesprochen, während die neueren Entwicklungen in der Informations- und Kommunikationstechnologie eher den Charakter des IT-Business-Enabling annehmen.

Wenn in dieser Arbeit von zu modellierendem fachlichen Wissen die Rede ist, dann soll dem die folgende Definition von Davenport und Prusak zugrunde gelegt werden: „Knowledge is a fluid mix of framed experience, values, contextual information, and expert insight ... It originates and is applied in the minds of knowers. In organizations, it often becomes embedded not only in documents or repositories but also in organizational routines, processes, practices, and norms. [DaPr00] (S. 5)"

Fachliches Wissen in Unternehmen und Organisationen findet sich demnach als implizites Wissen in den Köpfen der Mitarbeiter bzw. in gelebten betrieblichen Abläufen, kann aber auch explizit in Form von Beschreibungen, Modellen und/oder Programmen vorliegen. Es ist typischerweise stark fragmentiert und in hohem Grade veränderlich; es ist über Abteilungen, Gruppen und Personen verteilt und häufig ist es nur durch menschliche Interpretation zugänglich (vgl. [PrRR10], S. 65 ff.). Den sprichwörtlichen Graben zwischen Business und IT zu schließen bedeutet demnach, dieses Wissen formal so zu modellieren, dass es für Menschen verständlich bleibt (oder dadurch erst wird) und von Maschinen verarbeitet bzw. ausgewertet werden kann. Nach [StBF98] (S. 3) ist genau das ein zentraler Gegenstand des Knowledge Engineering, also der Wissensmodellierung. 
In den letzten zehn Jahren hat sich ein beeindruckendes Portfolio an Brückentechnologien zur (semi-)formalen Modellierung fachlichen Wissens etabliert, die zunehmend zu LehrLern-Gegenständen in den Studiengängen Wirtschaftsinformatik, Betriebswirtschaftslehre und Informatik werden. Die vorliegende Fallstudie fokussiert zwei ähnlich aufgebaute Wahlpflichtmodule, die im Fachbereich Wirtschaft einer Fachhochschule im 4. Semester des Bachelorstudiengangs Wirtschaftsinformatik und im 3. Semester des Masterstudiengangs Betriebswirtschaftslehre angeboten werden. Beide Studierendengruppen haben im Laufe ihres Studiums bereits Basiswissen über Modellierungsmethoden und -notationen, grundlegende Fertigkeiten im Einsatz von Werkzeugen und Anwendungen sowie Grundkompetenzen der Systemanalyse, der Abstraktion und Reflexion erworben.

Gegenstand der genannten Lehrveranstaltungen ist ein Bündel standardspezifizierter, praxisrelevanter Modellierungstechnologien, die im Rahmen individueller Praxisprojekte angewandt und dokumentiert werden sollen. Studierende an Fachhochschulen der genannten mittleren und höheren Jahrgänge verfügen in der Mehrzahl bereits über berufspraktische Erfahrungen aus Praktika oder aus früherer bzw. studienbegleitender Erwerbsarbeit, sodass von einer ausreichend tiefen Expertise in einer exemplarischen fachlichen Domäne ausgegangen werden kann. Der Titel „Angewandte Wissensmodellierung“ wurde gewählt, um eine Abgrenzung zu grundlegenden theoretischen bzw. stark technischen Aspekten der Wissensmodellierung deutlich zu machen.

Der folgende Abschnitt thematisiert zunächst die Lehrveranstaltungen zur angewandten Wissensmodellierung als Forschungsgegenstand und erläutert in diesem Kontext die Forschungsfrage sowie das implementierte Forschungsdesign. Zum Einsatz kommt zum einen die Methode der strukturierten Beobachtung und Reflexion im Laufe eines Kurses und zum anderen eine fragebogenbasierte, qualitative Evaluation am Ende des Kurses. Das konkrete Vorgehen und die Ergebnisse werden jeweils in den Abschnitten 3 und 4 dargestellt. Die Arbeit schließt mit einem Fazit und einer kurzen kritischen Betrachtung.

\section{Forschungsgegenstand und Forschungsdesign}

Forschungsgegenstand dieser Arbeit ist eine Reihe von Lehrveranstaltungen zur angewandten Wissensmodellierung. In diesem Abschnitt werden zunächst die inhaltliche Spezifikation sowie das didaktische Konzept dieser Lehrveranstaltungen dargestellt. Zentrales didaktisches Element ist ein komplexes, individuelles Modellierungsprojekt. Ziel der Arbeit ist es nachzuweisen, dass die dabei zwangsläufig auftretenden Fehler und Iterationsschritte den Kompetenzerwerb maßgeblich fördern.

\subsection{Inhaltliches Konzept}

Bei der Auswahl der Brückentechnologien für die Lehrveranstaltungen zur angewandten Wissensmodellierung wurde zunächst auf grafisch konnotierte, standard-spezifizierte Notationen mit großer Nähe zur fachlichen Modellierung Wert gelegt. Ein wichtiges Auswahlkriterium war auch der Verbreitungsgrad und die Relevanz einer Notation für die Wirtschaftsinformatik als Disziplin. Diese Anforderungen erfüllt die kürzlich komplettierte Notationen-Trias der OMG in herausragender Weise: BPMN, CMMN und DMN [OMG11, OMG16a, OMG16b]. Die Buchstabenfolge „MN“ steht dabei jeweils für „Model and Notation“, was deutlich machen soll, dass es sich um formal spezifizierte und zugleich grafisch nutzbare Technologien handelt. Diese Notationen erlauben die Modellierung von Geschäftsprozes- 
sen, wissensintensiver Sacharbeit und von operativen geschäftlichen Entscheidungen. Um das eingesetzte Vokabular im Rahmen der fachlichen Modellierung formal abzubilden und zur Weiterverwendung aufzubereiten, wurde zudem der W3C-Standard SKOS aufgenommen, dem das Standard-Datenmodell der semantischen Modellierung RDF zugrunde liegt ([W3C09, W3C14]). Mit RDF schließlich lassen sich ganz generell semantische Netze und damit auch beliebige fachliche Strukturen und Relationen abbilden.

Tabelle 1 gibt einen Überblick über die genannten Technologien, die jeweils adressierten Modellierungsgegenstände sowie über relevante informationstechnische Anwendungsfelder.

\begin{tabular}{|l|l|l|}
\hline Modellierungsgegenstand & Notation/Spezifikation & IT-Anwendungsfeld \\
\hline Geschäftsprozesse & BPMN 2.0 (OMG) & Digitalisierte Prozesse \\
\hline Wissensintensive Fallarbeit & CMMN 1.1 (OMG) & IT-Support Wissensarbeit \\
\hline Operative Entscheidungen & DMN 1.1 (OMG) & Automatisierte Regeln \\
\hline Vokabulare und Thesauri & SKOS (W3C) & Big-Data-Analysen \\
\hline Strukturen und Relationen & RDF (W3C) & Künstliche Intelligenz \\
\hline
\end{tabular}

Tab. 1: Technologie-Portfolio für die angewandte Wissensmodellierung

\subsection{Didaktisches Konzept}

Die Lernziele der betrachteten Lehrveranstaltungen umfassen - neben der Aneignung von Wissen über die Technologien selbst sowie dem Erwerb von Fertigkeiten in der werkzeuggestützten Modellierung - insbesondere die folgenden Kompetenzziele:

- $\quad$ Probleme und Strukturen der realen Welt analysieren und definieren,

- mit Stakeholdern kommunizieren,

- geeignetes Abstraktionsniveau auswählen,

- Konzepte und Individuen der realen Welt sowie deren Beziehungen formalisieren,

- geeignete Notation oder Spezifikation auswählen,

- Notationen und Spezifikationen syntaktisch korrekt anwenden,

- geeignete Tools auswählen und adäquat einsetzen,

- die eigene Arbeit kritisch reflektieren.

Dafür wurde schrittweise, über mehrere Semester ein didaktisches Setting entwickelt, das für jede Technologie im Portfolio einen Zyklus bereitstellt, der sich aus Wissensinput, strukturiertem Wissenstransfer, individueller Anwendung in der Fachdomäne und mehrkanaligem Coaching zusammensetzt. Abbildung 1 visualisiert dieses Konzept in komprimierter Form.

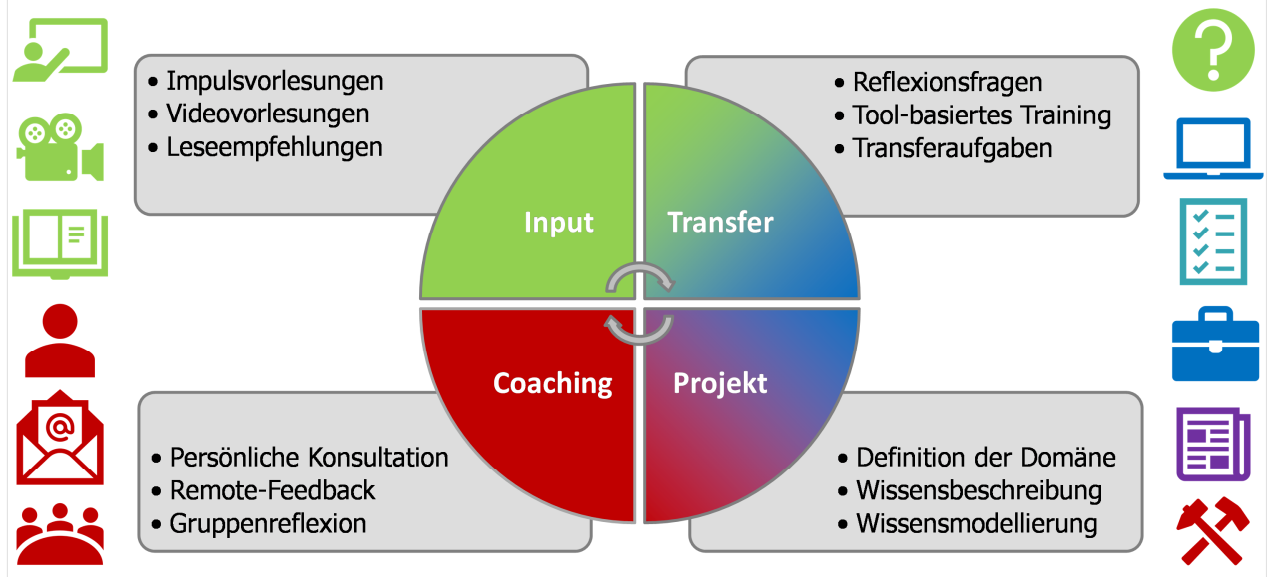

Abb. 1: Didaktisches Konzept der Lehrveranstaltung „Angewandte Wissensmodellierung“ 


\subsection{Lernen aus Fehlern}

Wie [StBF98] ausführen, ist jedes Modell nur eine Annäherung an die Realität, sodass Modellierung potenziell als ein unendlicher Prozess angesehen werden muss. Zudem handelt es sich um einen zyklischen Prozess, da neuentdecktes Wissen zu Verfeinerungen, Veränderungen oder Vervollständigungen führen kann. Schließlich erfordert die Modellierung eine Interpretation der zu modellierenden Realität, was Fehleroptionen immanent einschließt.

Es sei erneut erwähnt, dass die Lehrveranstaltung die Entwicklung von Modellierungskompetenzen zum Ziel hat. Die „objektive“ Qualität der dabei erstellten Modelle kann hingegen als sekundär angesehen werden. Aus diesen Prämissen wurden folgende Implikationen gezogen:

1. Um der Kompetenzentwicklung genügend Raum zu verschaffen, sollte der Prozess des Wissenserwerbs nach [PrRR10] (S. 28) weitestgehend verkürzt werden. Das kann dadurch erreicht werden, dass die zu modellierende Domäne im eigenen Erfahrungsraum verortet wird. Sollten Studierende über keinerlei berufspraktische Erfahrungen verfügen, können auch Freizeitaktivitäten herangezogen werden, die eine Modellierung aus Sicht eines Leistungsanbieters (z. B. Organisator eines Events) erlauben.

2. Fehler im Prozess des Modellierens müssen als Chance für tieferes Verständnis und für die Entwicklung besserer Modelle verstanden werden und nicht als Scheitern an einer Anforderung. Individuelle Modellierung führt zu individuellen Fehlern, Musterlösungen kann es per se nicht geben. Eine positive Fehlerkultur kann dadurch erreicht werden, dass jedem Studierenden - eventuell mehrfach - die Möglichkeit gegeben wird, seine Modellierungsentwürfe kritisch zu reflektieren und kollegial zu diskutieren.

Der in Punkt 2 deklarierte Ansatz wird durch Karl Poppers Theorie des Lernens aus Fehlern gestützt. In [Popp72] (S. 344 f.) führt er aus, dass Fehler (schwerwiegende Probleme) unseren Erwartungshorizont, also unseren Welterklärungsrahmen, wie eine Bombe platzen lassen, sodass wir gehalten sind, diesen neu zu konstruieren. Diese Anstrengungen führen im besten Fall zu einem neuen Welterklärungshorizont auf höherem Niveau, der die problematischen Beobachtungen nun integriert.

[Tür+12] (S. 96) diskutieren im Kontext des Umgangs mit domänenspezifischen Fehlern von Lernenden drei professionelle Kompetenzen von Lehrenden: (i) Kenntnis der möglichen Fehlertypen, (ii) Einsatz geeigneter Maßnahmen bzw. Reaktionen, (iii) Konstruktive Sicht auf Fehler und ihre Nutzung in Lehr-Lernprozessen. Letzteres wurde bereits in Punkt 2 adressiert. Kompetenz (ii) findet ihren Niederschlag im Coaching-Part eines jeden Lernzyklus (vgl. Abb. 1). Auf Fehlertypen wird in Abschnitt 3 eingegangen.

\subsection{Forschungsthese und Forschungsmethoden}

Die im vorigen Abschnitt abgeleiteten und diskutierten Gestaltungsimplikationen im Hinblick auf den Kompetenzerwerb in angewandter Wissensmodellierung sollen nun in einer Forschungsthese formuliert werden: Die Möglichkeit in einer eigenen Domäne zu modellieren und dabei individuelle Fehler zu machen, trägt maßgeblich zum Lernerfolg bei.

Zur Prüfung der These kamen zwei Forschungsmethoden zum Einsatz. Zunächst wurde eine Beobachtung im Laufe der Lehrveranstaltung installiert, welche die Arten der aufgetretenen Fehler und den sichtbaren Kompetenzzuwachs implizit ermittelt. Nähere Ausführungen dazu finden sich in Abschnitt 3. Um die Sicht der Studierenden ebenfalls zu erfassen, wurde in einer konkreten Lehrveranstaltung eine qualitativ-empirische Methode eingesetzt. Alle aktiven Teilnehmer beantworteten eine Reihe überwiegend vorstrukturierter Fragen in schriftlicher Form. Dieses Vorgehen wird in Abschnitt 4 beschrieben. 
Aus der persönlichen, vieljährigen Erfahrung in der Betreuung komplexer, studentischer Modellierungsprojekte ergeben sich folgende Fehlertypen:

- Auswahl eines unpassenden Abstraktionsniveaus,

- unvollständige Analyse und Beschreibung von Strukturen, Abläufen und Beziehungen,

- Vernachlässigung der Restriktionen oder der Supportoptionen von Werkzeugen,

- fehlerhafter Einsatz der Notationssyntax,

- $\quad$ schwache oder fehlerhafte Implementierung der Notationslogik und -semantik,

- $\quad$ Einsatz suboptimaler Muster.

Alle diese Aspekte werden in einführenden Vorlesungen thematisiert und an Beispielen illustriert. Es zeigt sich jedoch wiederholt, dass ein Transfer des Wissens und ein tieferes Verständnis nur durch eigene Praxis und iterative Reflexion erreicht werden kann. Auf diesen Sachverhalt werden die Studierenden wiederholt hingewiesen und ermutigt, sich eine positive Fehlerkultur anzueignen. Den Rahmen dafür bilden im Semester definierte Meilensteine, zu denen die Studierenden Arbeitsstände ihrer Modellierung einreichen. Möglichst zeitnah erhalten sie ein schriftliches oder auch ein mündliches Feedback zu ihren Modellen. Dabei werden die folgenden Feedback-Regeln angewandt:

1. Stilistische Fehler werden im betreffenden Element markiert und auf die einschlägige Stilvorgabe verwiesen.

2. Syntaxfehler betreffen häufig nicht nur ein Element. Hier wird der betreffende Bereich markiert. Es wird auf syntaktische Regeln und/oder auf Implikationen aus dem identifizierten Fehler hingewiesen. Da diese Implikationen häufig der Modellierungsintention zuwiderlaufen, wird die Fehlerursache sichtbar.

3. Für alle weiteren, komplexeren Fehler (in der Logik, dem Abstraktionsniveau, der fachlichen Stringenz etc.) werden die Probleme mehrschichtig, idealerweise domänenspezifisch diskutiert. Ggf. werden Lösungsansätze vorgeschlagen, jedoch nicht vollständig ausgeführt.

Der Methode der Beobachtung soll exemplarisch anhand eines BPMN-Prozessmodells erläutert werden. Abbildung 2 illustriert einen Ausschnitt aus einer frühen studentischen Einreichung mit einer Reihe markierter Fehler und den entsprechenden Kommentaren.

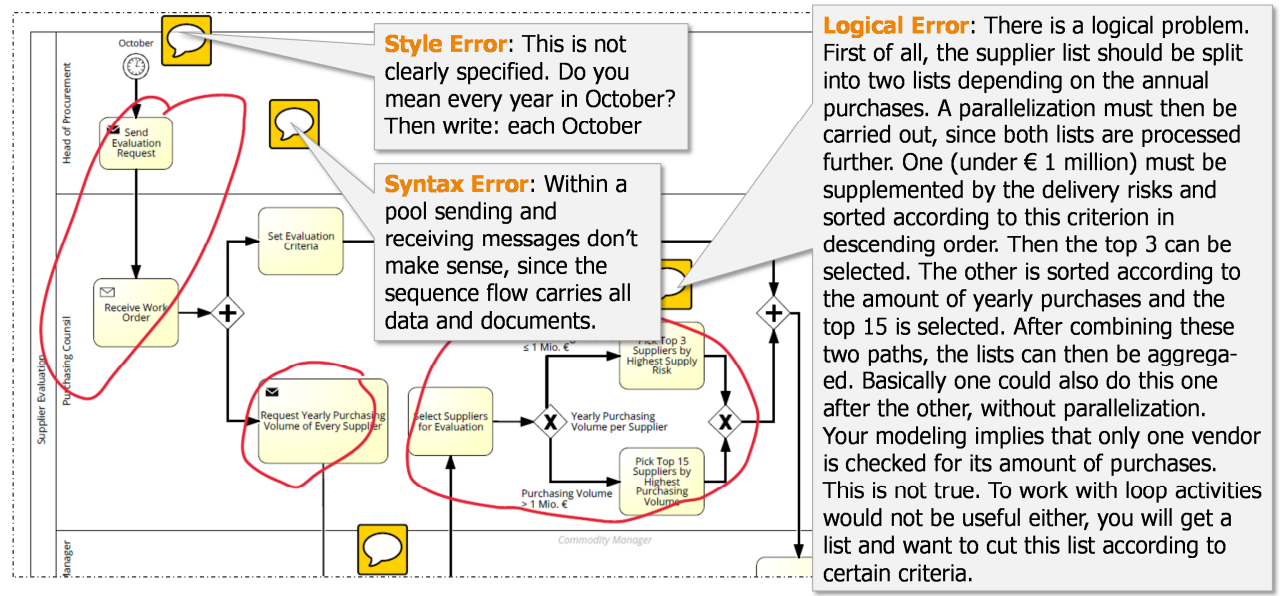

Abb. 2: Studentisches BPMN-Modell mit Fehlern und Kommentaren (Ausschnitt) 
Abbildung 3 zeigt eine verbesserte Einreichung desselben Modellausschnitts mit Visualisierung der jeweils korrigierten bzw. überarbeiteten Aspekte.

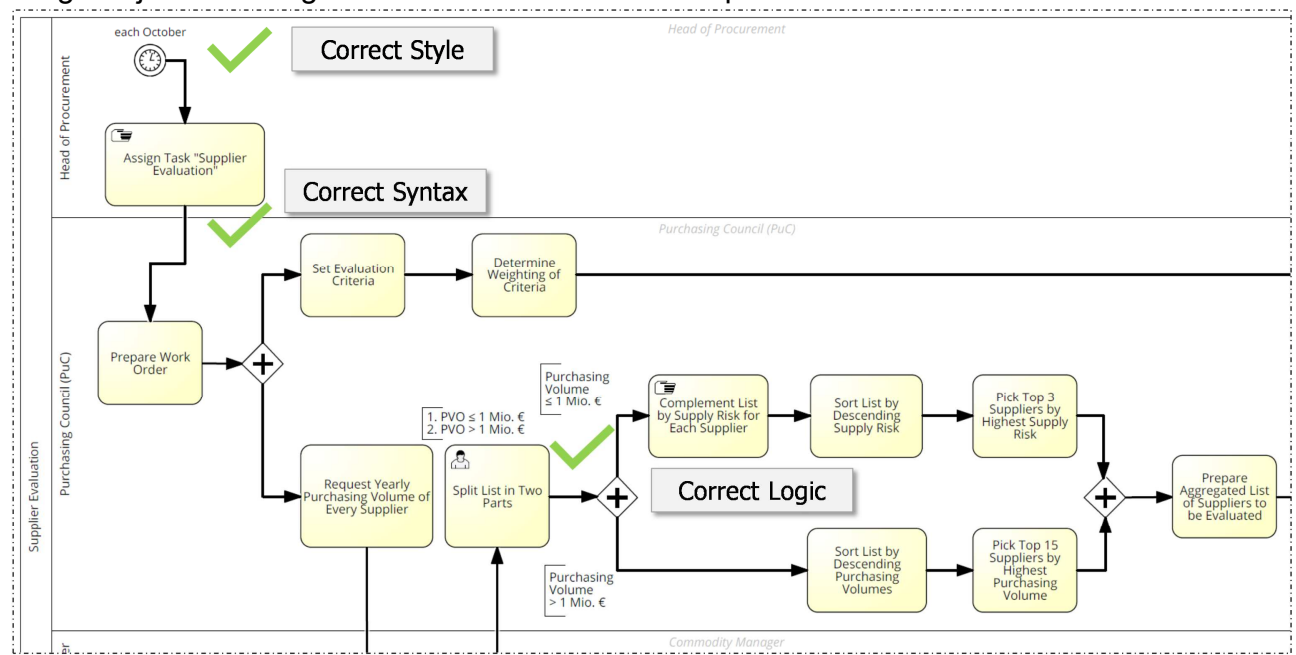

Abb. 3: Finales studentisches BPMN-Modell mit Markierung der korrigierten Aspekte (Ausschnitt)

Es zeigt sich, dass die Korrektur des stilistischen Fehlers keine eigene Kreativität erfordert. Es steht zu bezweifeln, dass ein solcher Fehler im Umfeld einer rein grafischen Modellierung zu einer Neukonstruktion des Erklärungshorizonts nach Karl Popper führt. Anders wäre es, wenn die Prozessmodelle auch technisch implementiert werden müssten. In diesem Fall könnte auch ein solcher Fehler zu Ausführungsstörungen führen. Die Korrektur der Modellierungssyntax erfolgte durch eine Neugestaltung der Aufgabenaufteilung und -spezifikation. Da es sich hier um die grundlegende Frage handelt, wie Teilnehmer innerhalb eines Prozesses kommunizieren, ist davon auszugehen, dass hier signifikante Neuerklärungen konstruiert wurden. Noch deutlicher wird das bei dem logischen Fehler, welcher durch ein komplett neues Modellierungsmuster geheilt wurde.

In Summe kann eingeschätzt werden, dass der/die Studierende damit ein tieferes Verständnis des Prozesses in der eigenen Domäne entwickelt hat, dass sich seine/ihre Modellierungsfertigkeiten allgemein verbessert haben und dass er/sie auch ein umfassenderes Verständnis für die Notation, deren Semantik, Optionen und Restriktionen erworben hat.

\section{Qualitative Evaluation}

Die in dieser Arbeit vorgestellte Forschung hat den Charakter einer Fallstudie, da sie komplexe, schwer abgrenzbare Phänomene in ihrem natürlichen Kontext untersucht (vgl. [WiHe07]). Sie erfolgte direkt im Rahmen aktiver Lehrtätigkeit und ist nicht in ein gesondert ausgewiesenes Forschungsprojekt eingebettet. Die qualitative Evaluation musste somit auf die Teilnehmer einer konkreten Lehrveranstaltung beschränkt werden.

\subsection{Konzept der Umfrage}

Die Umfrage wurde im Februar 2017 im Rahmen der Abschlusspräsentation einer Lehrveranstaltung des Wintersemesters 2016/17 durchgeführt. Der schriftliche, papiergebundene 
Fragebogen wurde von allen 21 aktiven Studierenden des Kurses ausgefüllt. Die Befragung erfolgte anonym. Die Studierenden wurden nicht explizit darüber aufgeklärt, welche Items der besonderen Untersuchung unterliegen. Neben Fragen, die explizit die in Abschnitt 2.4 aufgestellte Forschungsthese adressieren, wurden weitere Items abgefragt. Die Umfrage wurde als Lehrevaluation mit dem Ziel der Qualitätsverbesserung angekündigt.

Tabelle 2 zeigt alle untersuchten Aspekte, ihre Kodierungsstruktur sowie ihren Bezug zur Forschungsthese im Überblick.

\begin{tabular}{|c|c|c|}
\hline Untersuchter Aspekt & Kodierung & Bezug zur These \\
\hline $\begin{array}{l}\text { Selbsteinschätzung des Wissens } \\
\text { und der Fertigkeiten zu Beginn und } \\
\text { zum Ende des Kurses }\end{array}$ & $\begin{array}{l}\text { 6-stufige Skala: } \\
\text { nicht vorhanden bis } \\
\text { ausgezeichnet }\end{array}$ & $\begin{array}{l}\text { kein direkter } \\
\text { Bezug }\end{array}$ \\
\hline $\begin{array}{l}\text { Nutzungshäufigkeit von } 4 \text { verschie- } \\
\text { denen Lernformen bzw. -medien }\end{array}$ & $\begin{array}{l}\text { 4-stufige Skala für jede/s } \\
\text { Form/Medium: nie bis oft }\end{array}$ & kein Bezug \\
\hline $\begin{array}{l}\text { Nützlichkeit der } 4 \text { verschiedenen } \\
\text { Lernformen bzw. -medien }\end{array}$ & Ranking & kein Bezug \\
\hline $\begin{array}{l}\text { Ggf. vermisste alternative Lernfor- } \\
\text { men oder -medien }\end{array}$ & offen & kein Bezug \\
\hline $\begin{array}{l}\text { Persönliches Empfinden im Hinblick } \\
\text { auf den Ansatz "Lernen aus Feh- } \\
\text { lern“ }\end{array}$ & $\begin{array}{l}3 \text { definierte Optionen: unan- } \\
\text { genehm, ungewohnt, her- } \\
\text { ausfordernd; } 1 \text { offene Option }\end{array}$ & mittelbarer Bezug \\
\hline $\begin{array}{l}\text { Zustimmung zu den folgenden Aus- } \\
\text { sagen: } \\
\text { (i) Die Möglichkeit, eigene Fehler zu } \\
\text { machen und aus diesen zu lernen, } \\
\text { hat maßgeblich zu meinem Lerner- } \\
\text { folg beigetragen. } \\
\text { (ii) Die Möglichkeit, in meiner eige- } \\
\text { nen Fachdomäne zu modellieren, } \\
\text { hat maßgeblich zu meinem Lerner- } \\
\text { folg beigetragen. }\end{array}$ & $\begin{array}{c}\text { jeweils } 6 \text {-stufige Skala: } \\
\text { stimme nicht zu bis } \\
\text { stimme zu }\end{array}$ & direkter Bezug \\
\hline
\end{tabular}

Tab. 2: Konzeptionelle Details zur Umfrage

\subsection{Auswertung der Ergebnisse ohne Bezug zur Forschungsthese}

Die erfragten Aspekte ohne direkten Bezug zur Forschungsthese sind dennoch im betrachteten Kontext von Interesse. Zum einen illustrieren sie die Selbsteinschätzung der Studierenden im Hinblick auf ihr fachliches Wissen und ihre Fertigkeiten in der angewandten Wissensmodellierung. Zum anderen leisten sie einen Beitrag zur allgemeinen Evaluation des didaktischen Konzeptes, das in Abschnitt 2.2 vorgestellt wurde. Die Frage nach ggf. vermissten alternativen Lernformen oder -medien wurde nur von fünf Studierenden einschlägig beantwortet. Jeweils einmal genannt wurden: Wiki ohne HTML, Miniübungen, Werkzeugtutorials, zwei Nennungen entfielen auf Frontalpräsentationen eines Modellierungsprozesses, der sich dann auf eigene Anforderungen „ohne Schwierigkeiten“ transferieren lässt.

Bei der Beurteilung der Nutzungshäufigkeit sowie der Nützlichkeit der verschiedenen angebotenen Lernformen bzw. -medien, konzentrierte sich die Umfrage auf die wichtigsten Elemente: Präsenzvorlesung, Videovorlesungen, E-Mail-Feedback und individuelle Konsultationen. Interessant ist, dass die Präsenzvorlesung am häufigsten genutzt wurden (Median: oft) und auch in der Nützlichkeit (Median: am nützlichsten) hervorragend abschnitten. Sowohl nach Nutzungsrate als auch nach Nützlichkeit folgt als nächstes das E-Mail-Feedback. Abbildung 4 zeigt eine gemittelte Darstellung der Antworten im Diagramm. 


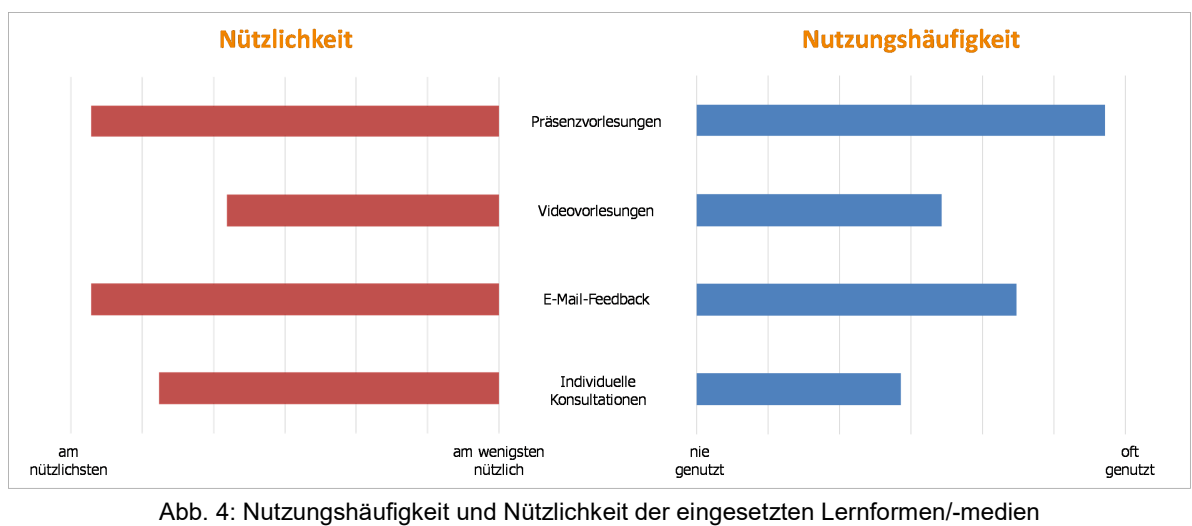

Die Selbsteinschätzung von Modellierungswissen und -fertigkeiten wurde differenziert nach Geschäftsprozessen, fachlichen Entscheidungen und Geschäftsvokabularen ermittelt. Die Ergebnisse illustrieren die Tatsache, dass die Studierenden bereits Vorkenntnisse in der Modellierung von Geschäftsprozessen aus vorangegangen Lehrveranstaltungen mitbringen. In allen Aspekten erkennen die Studierenden eine signifikante Zunahme ihres Wissens und ihrer Fertigkeiten. In der Median-Betrachtung steigen die Werte bei den Geschäftsprozessen um zwei Stufen, bei der Entscheidungsmodellierung um vier Stufen, bei der Modellierung von Vokabularen um drei Stufen. Abbildung 5 zeigt wieder die Ergebnisse in einer gemittelten Darstellung.

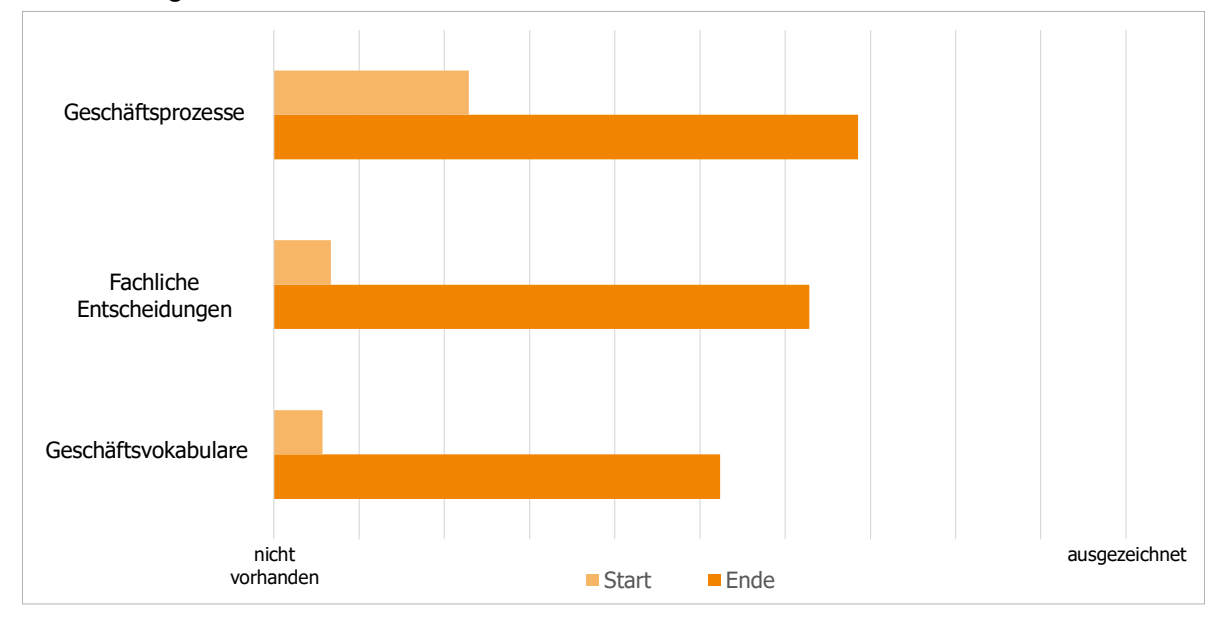

Abb. 5: Selbsteinschätzung der Studierenden ihres Wissens und ihrer Fertigkeiten

\subsection{Auswertung der Ergebnisse mit Bezug zur Forschungsthese}

Für die Frage nach dem persönlichen Empfinden in Bezug auf das "Lernen aus Fehlern“ wurden den Teilnehmern der Studie drei fest kodierte Antwortoptionen vorgelegt: (i) unangenehm, (ii) ungewohnt, (iii) herausfordernd. Als vierte Option wurde ein Freitextfeld bereitgestellt. Letzteres wurde nur von einem Teilnehmer genutzt und als „zu aufwändig“ gekennzeichnet. Unter Annahme, dass Studierende durchaus herausfordernde und auch ungewohnte Lernsettings als positiv einschätzen (je acht Nennungen), wurde diese Frage von $75 \%$ der Teilnehmer positiv beantwortet. 
Den direktesten Bezug zur Forschungsthese weisen die Fragen nach der Zustimmung zu den Aussagen nach dem Beitrag der Aspekte (i) Modellierung in individueller Domäne und (ii) Lernen aus eigenen Fehlern auf den Lernerfolg. Wenn man in der sechsstufigen Skala die drei oberen Werte als Zustimmung und die drei unteren Werte als Ablehnung (jeweils differenziert nach schwach, mittel und stark) interpretiert, dann liegt die Zustimmungsrate zu beiden Aussagen bei $67 \%$. Die Abbildungen 6 und 7 zeigen die Verteilungen im Detail.

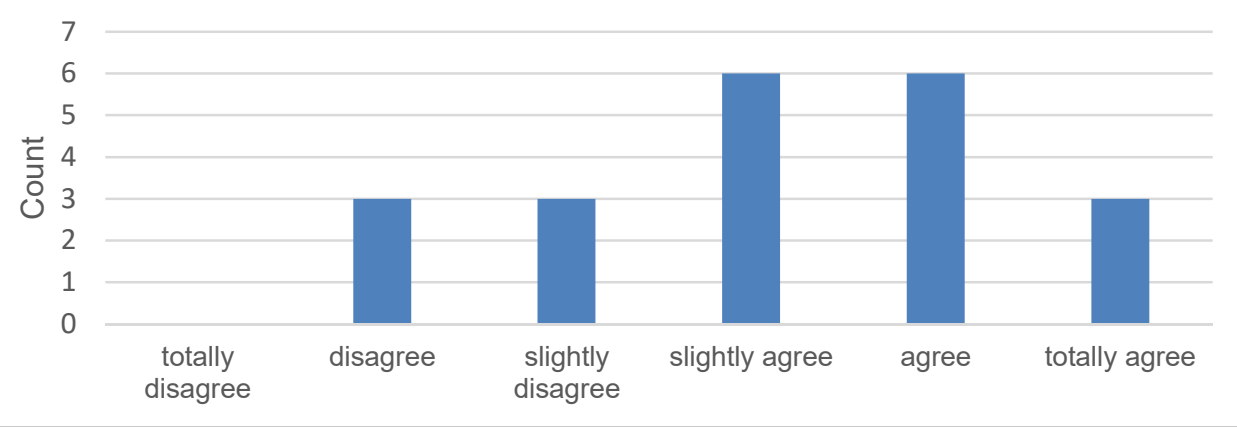

Abb. 6: Zustimmung zur Aussage: Lernen aus eigenen Fehlern hat maßgeblich zum eigenen Lernerfolg beigetragen

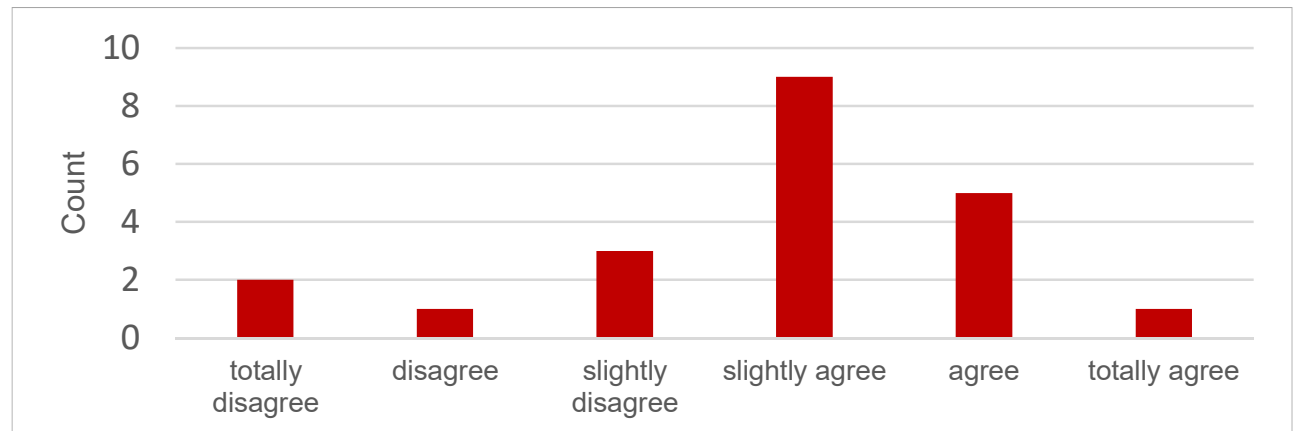

Abb. 7: Zustimmung zur Aussage: Modellierung in der eigenen Domäne hat maßgeblich zum Lernerfolg beigetragen

\section{$5 \quad$ Fazit und Reflexion}

Für Studierende der Betriebswirtschaftslehre ist ein Lernsetting der beschriebenen Art ungewohnt. Sie beurteilten den Kurs als herausfordernd, einige auch als zu hart. Alle aktiven Studierenden des Kurses erzielten mindestens gute Ergebnisse. In einer mündlichen Feedback-Runde berichteten alle über Motivationszuwächse, resultierend aus der persönlichen Expertise in der Fachdomäne und dem intensiven Coaching. Der Kompetenzerwerb in diesem kurzen Zeitraum kann als überdurchschnittlich eingeschätzt werden, was primär auf die umfangreiche individuelle Modellierungspraxis zurückzuführen ist. Dasselbe gilt für den Aufwand der Lektorin, um dieses differenzierte Feedback zu einer Vielzahl von Modellen und Entwicklungsschritten zu geben.

Beide Forschungsansätze, sowohl die strukturierte Beobachtung und Interaktion im Prozess als auch die fragebogenbasierte qualitative Evaluation zum Ende des Kurses stützen die These von einem positiven Effekt der konzeptionellen Ansätze: „Lernen aus Fehlern“ und „Modellierung in einer eigenen Domäne“ auf den gewünschten Lernerfolg. Wegen der gerin- 
gen Anzahl der Untersuchungsteilnehmer und der eingeschränkten Objektivität der Autorin als zugleich verantwortliche Gestalterin der Lehrveranstaltung sind die Ergebnisse möglicherweise nicht ohne Weiteres übertragbar. Andererseits sprechen ergänzende informelle Beobachtungen, z. B. aus persönlichen Gesprächen und nicht systematisch dokumentierten Feedbackrunden, eher für eine Bestätigung der These und damit für einen weiteren Einsatz dieses didaktischen Konzeptes.

\section{Literaturverzeichnis}

[DaPr00] Davenport, Thomas H.; Prusak, Laurence: Working Knowledge: How Organizations Manage What They Know. Harvard Business Press, 2000.

[OMG11] Object Management Group: Business Process Model and Notation 2.0. 2011, http://www.omg.org/spec/BPMN/2.0/, Abruf am 2017-05-09.

[OMG16a] Object Management Group: Case Management Model and Notation 1.1. 2016, http://www.omg.org/spec/CMMN/1.1/, Abruf am 2017-05-09.

[OMG16b] Object Management Group: Decision Model and Notation 1.1. 2016, http://www.omg.org/spec/CMMN/1.1/, Abruf am 2017-05-09.

[Popp72] Popper, Karl: Objective Knowledge - An Evolutionary Approach. Clarendon Press, Oxford, 1972.

[PrRR10] Probst, Gilbert; Raub, Steffen; Romhardt, Kai: Wissen managen. 6. Auflage, Gabler, Wiesbaden, 2010.

[RaPW15] Rainer, R. Kelly; Prince, Brad; Watson, Hugh J.: Management Information Systems. $3^{\text {rd }}$ Ed., Wiley Publishing, 2015.

[StBF98] Studer, Rudi; Benjamins, Richard; Fensel, Dieter: Knowledge Engineering - Principles and Methods. In: Data \& knowledge engineering 25.1-2 (1998): 161-197.

[Tür+12] Türling, Janosch M. e. a.: Teachers' Knowledge about Domain Specific Student Error. In: Wuttke, Eveline; Seifried, Jürgen (eds.): Learning from Errors at School and at Work. Budrich, Opladen, 2012.

[W3C09] World Wide Web Consortium: SKOS Simple Knowledge Organization System - Reference. 2009, https://www.w3.org/TR/skos-reference/, Abruf am 2017-05-09.

[W3C14] World Wide Web Consortium: RDF 1.1 Concepts and Abstract Syntax - Recommendation. 2014, https://www.w3.org/TR/skos-reference/, Abruf am 2017-05-09.

[WiHe07] Wilde, Thomas; Hess, Thomas: Forschungsmethoden der Wirtschaftsinformatik. In: WIRTSCHAFTSINFORMATIK 49 (2007) 4, S. 280-287.

\section{Kontakt}

Prof. Dr. Vera G. Meister

Technische Hochschule Brandenburg

Magdeburger Str. 50, 14770 Brandenburg a.d.H.

T +49 3381 355-297, vera.meister@th-brandenburg.de 


\title{
Elemente eines Referenzmodells für Wissenstransfereinrichtungen - ein Meta-Wissenstransfer?
}

$\mu \varepsilon T \alpha$ !

\author{
Georg Rainer Hofmann
}

\section{Zusammenfassung}

Der aktive Wissenstransfer ist für Hochschulen, Universitäten und Forschungseinrichtungen eine zentrale Aufgabe. Für den Wissenstransfer werden durchaus spezielle Einrichtungen gegründet, als Institute, Vereine, Gesellschaften, und dergleichen mehr. Der Beitrag geht der Frage nach, ob sich Gemeinsamkeiten solcher Einrichtungen identifizieren lassen, um daraus ein Referenzmodell für die - selbstredend erfolgreiche - Gestaltung vergleichbarer neuer, oder auch zu reformierender, Wissenstransfereinrichtungen zu gewinnen. Die Arbeiten wurden im Jahr 2016 veranlasst von der Planung des „Leistungszentrum Digitale Vernetzung (LZDV)“ als Kooperation von vier Fraunhofer-Instituten am Standort Berlin. Es wird im Rahmen einer Case-based-Evidence-Betrachtung eine Reihe von Analogiequellen analysiert, ein Synoptisches Modell des "Wissenstransfers für den Wissenstransfer" - Grundlage für einen Meta-Wissenstransfer? - wird skizziert.

\section{Forschungsfrage und Forschungsanlass}

Akademischer Fortschritt soll zur Wahrung und Verbesserung von Wohlstand und Lebensqualität sinnvoll umgesetzt werden. Universitäten und Hochschulen sind aufgerufen, Forschungsergebnisse per Wissenstransfer der gesamten Gesellschaft zu Gute kommen zu lassen. Fragt man Professor(inn)en an den Hochschulen und Universitäten, oder auch Wissenschaftler(inn)en in den Instituten der Angewandten Forschung, ob sie und ihr Institut aktiv Wissenstransfer aus der Akademie in die Praxis betreiben, so dürfte man selbstverständlich eine zustimmende Antwort erhalten. Dies hat einen guten Grund, denn unsere Gesellschaft steht permanent vor der enormen Aufgabe, akademischen Fortschritt verantwortungsvoll umzusetzen. Universitäten und Hochschulen sind aufgerufen, hierfür geeignete offene Kooperationsformen zu finden: Forschungsergebnisse sollen per Transfer der gesamten Gesellschaft zu Gute kommen.

Der Wissenstransfer wird in einem Positionspapier des Wissenschaftsrates [Wiss16] begrifflich präzisiert, seine jeweilige konkrete organisatorische Ausgestaltung in den Instituten erfolgt indes oft planerisch „from the scratch“ und hat situativen Charakter.

Es stellt sich die Frage, ob sich ein Referenzmodell finden lässt, wie „man“ den Wissenstransfer organisatorisch erfolgreich gestalten kann.

Der Anlass für die hier beschriebenen Arbeiten war Mitte des Jahres 2016 die Gründung des "Leistungszentrums Digitale Vernetzung (LZDV)“ als Kooperation von vier FraunhoferInstituten am Standort Berlin. Das LZDV soll per Wissenstransfer Berlin als führenden Standort der digitalen Vernetzung in Deutschland etablieren und ausbauen. 
Im Rahmen einer - Fraunhofer-externen - studentischen Seminararbeit [El++17] am Information Management Institut (IMI) der Hochschule Aschaffenburg wurde die Frage verfolgt, ob man den Wissenstransfer, insbesondere die Marktansprache, des LZDV nach Maßgabe eines Referenzmodells gestalten könnte. Es wäre ja nachgerade widersinnig, für die Planung des LZDV dessen prospektive Strukturen quasi völlig neu erfinden zu wollen - naheliegend hingegen wäre eine Auswertung bestehender erfolgreicher Strukturen auf deren Verwertbarkeit, im vorliegenden Fall der Gestaltung des LZDV.

Die Komponenten des anhand des Falls LZDV zu findenden Referenzmodells sollten im Rahmen eines „Meta-Wissenstransfers“ übertragbar sein auf die Gestaltung künftiger Wissenstransfereinrichtungen in Hochschulen und Instituten.

\section{Der Fall des LZDV der Fraunhofer-Gesellschaft}

Die Fraunhofer-Gesellschaft ist - bekanntermaßen - eine Einrichtung der Angewandten Forschung in Deutschland. Das „Leistungszentrum Digitale Vernetzung (LZDV)“ besteht seit Mitte 2016 und ist eine Kooperation von vier Fraunhofer-Instituten am Standort Berlin [LZDV16]. Diese sind das Fraunhofer-Institut für Offene Kommunikationssysteme (FOKUS), das Fraunhofer Heinrich-Hertz-Institut $(\mathrm{HHI})$, das Fraunhofer-Institut für Zuverlässigkeit und Mikrointegration (IZM) und das Fraunhofer-Institut für Produktionsanlagen und Konstruktionstechnik (IPK). Das „Leistungszentrum Digitale Vernetzung“ (LZDV) arbeitet eng mit der Technischen Universität Berlin, insbesondere mit den Fakultäten II Mathematik und Naturwissenschaften, IV Elektrotechnik und Informatik und V Verkehrs- und Maschinensysteme, zusammen; es besteht eine Zusammenarbeit mit den anderen Berliner Universitäten sowie zur Universität Potsdam mit dem Hasso-Plattner-Institut.

Das Leistungszentrum wird finanziell gefördert: Es wird mit insgesamt mit mehreren Millionen Euro vom Regierenden Bürgermeister von Berlin, Senatskanzlei - Wissenschaft und Forschung, und aus Mitteln des Europäischen Fonds für regionale Entwicklung (EFRE) unterstützt. Es ist das Ziel des LZDV, die Zusammenarbeit zwischen der Industrie und den Berliner Fraunhofer-Instituten zu stärken, um Berlin als führenden Standort der digitalen Vernetzung in Deutschland und auch international zu etablieren und auszubauen.

Im Zentrum der Arbeit stehen Technologien und Lösungen, die der zunehmenden Digitalisierung und Vernetzung aller Lebensbereiche Rechnung tragen. Gearbeitet wird dabei sowohl am Wissenstransfer von Basis- und Querschnittstechnologien als auch an Lösungen für konkrete Anwendungsbereiche.

Für Industriepartner besteht die Möglichkeit, im Rahmen von Forschungsprojekten mit den beteiligten Fraunhofer-Instituten zu kooperieren. Der Vorteil liegt in der Nutzung der vorhandenen technologischen Infrastrukturen der Institute und im direkten Transfer der Forschungsergebnisse in die Praxis.

\section{Ansatz der Case-based Evidence}

Die Case-based Evidence (deutsch etwa: fallbasierter Nachweis) ist eine wissenschaftliche Methode, der die Annahme zu Grunde liegt, dass sich bestimmte menschliche Verhaltensmuster, auch im Sinne von Einstellungen und Grundhaltungen, insbesondere hinsichtlich 
der Akzeptanz von Systemen, technischen Vorrichtungen und Verfahren, von einer Reihe von gegebenen Problemstellungen, den „Analogiequellen“, auf eine andere, aktuelle Problemstellung, dem „Analogieziel“, übertragen lassen.

Die Methode Case-based Evidence basiert auf mehreren Teilschritten [ScHo16]: Analogieschlüsse bilden das Kernstück und liefern als Ergebnis Mechanismen, die aus den Analogiequellen (vermutlich) auf den aktuellen Fall übertragbar sind. Diese Mechanismen werden daraufhin in einem Synoptischen Modell dargestellt und in einer Serie qualifizierter Experteninterviews überprüft.

Um geeignete Analogien finden zu können, müssen zunächst der gegebene Fall - das Analogieziel - und das zu lösende Problem genauer betrachtet werden. Hier gilt es, die Komponenten ausfindig zu machen, die vermutlich den größten Einfluss auf das zu lösende Problem haben. Relevante Analogiekomponenten können in

- den Eigenschaften des zu betrachtenden Falls, der Beziehung von Eigenschaften,

- der Nutzergruppe oder

- der Beziehung der Nutzergruppe zu Komponenten des Falls

zu finden sein. Bislang ist keine algorithmische Lösung bekannt, wie eine tragfähige Analogie treffsicher gefunden werden kann. Eine Analogie wird sich jedoch nur dann als tragfähig erweisen, wenn sie auf relevanten Analogiekomponenten basiert.

Mit Hilfe der gefundenen Analogiekomponenten und der abstrakten Formulierung des Problems kann nach analogen Fällen - den Analogiequellen - gesucht werden. Die Suche nach analogen Fällen kann aus zwei Perspektiven erfolgen:

- Die Strukturanalogie hat ihren Schwerpunkt im Wiederfinden von Eigenschaften und Strukturen der Ausgangssituation in der Analogie. Diese Analogieform ist insbesondere bei bereits bestehenden Produkten und Dienstleistungen oder laufenden Projekten zu bevorzugen. Die für die Akzeptanz als kritisch erachteten Faktoren des Analogieziels stellen hier den Ausgangspunkt der Suche dar. Es sollte also nach Fällen gesucht werden, die eine Ähnlichkeit hinsichtlich der benannten kritischen Faktoren aufweisen und die daraus resultierenden, erwarteten Akzeptanzprobleme bereits gelöst haben. Da die Akzeptanz eines Produktes, einer Dienstleistung oder eines Projektes unter Umständen an mehreren Attributen hängt, ist es empfehlenswert zu den Komponenten eines Falls, die als besonders relevant für die Akzeptanz eingestuft werden, jeweils eine eigene Analogie zu suchen.

- Die Zielanalogie fokussiert darauf, welches Ziel mit dem gegebenen Fall erreicht werden soll. Es wird also nach einer Art „Vorbild“ gesucht, das das Ziel, welches mit dem gegebenen Fall erreicht werden soll, bereits erreicht hat [HoSc12] [HoSc12]. Dieses Ziel sollte in unterschiedlichen Abstraktionshöhen beschrieben werden, so dass 3owohl nahe als auch ferne Analogien adressiert werden können. Diese Analogieform eignet sich besonders für sich in der Planung befindende Projekte oder für die innovative Produktentwicklung.

Die Komponenten der Analogieschlüsse der Case-based-Evidence-Methode können wie folgt illustriert werden: 


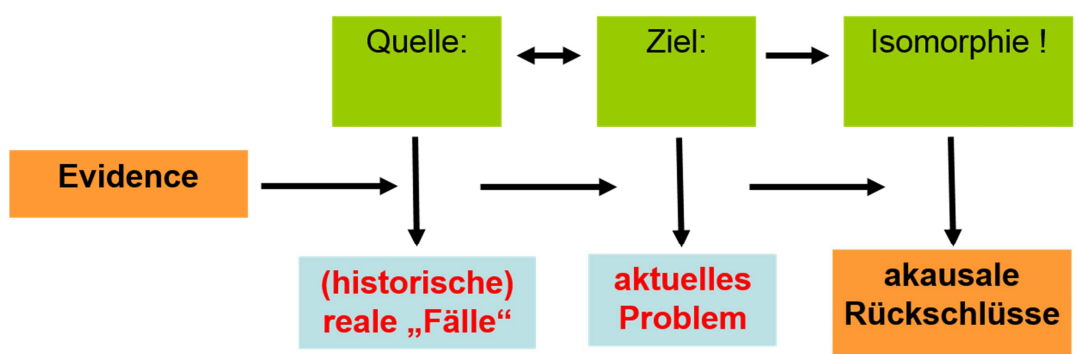

Abbildung 1: Struktur der Case-based Evidence - Analogieschlüsse; nach [ScHo16]

- $\quad$ Aktueller Fall - das Analogieziel. Es wird von einem gegebenen Fall ausgegangen, bei dem die Beweggründe der beteiligten Personen, die zu deren bestimmtem Verhalten führen, nicht eindeutig nachvollzogen werden können, bzw. „offen“ sind und daher die Forschungsfrage darstellen. Zudem ist nicht klar, welche Parameter zu einer Verhaltensänderung, z. B. einer Verbesserung der Akzeptanz führen würden.

- Vergleichsfälle - die Analogiequellen. Es werden strukturähnliche - isomorphe - Fälle identifiziert, die entweder historisch oder auch aktuell in „anderen“ thematischen Bereichen und ökonomischen Branchen zu finden sind. In diesen „Vergleichsfällen“ sind die Parameter, die zu bestimmten kognitiven Prozessen und Verhalten führen, bekannt oder bekannter.

- Isomorphie-Rückschluss. Aus den Erkenntnissen der Analogiequellen werden Rückschlüsse auf das Analogieziel vorgenommen.

Die „Kunst“ besteht darin, eben diese tragfähigen Analogien zu finden und die dort identifizierbaren Einstellungs- und Verhaltensschemata auf die Problemstellung des aktuellen Falls, etwa die Marktakzeptanz eines innovativen IT-Systems, oder eines Dienstleistungsangebots, zu übertragen. Der Zusammenhang zwischen Analogiequelle und Analogieziel ist freilich nicht kausal, da sie „eigentlich“ unabhängig voneinander sind. Es lässt sich jedoch anhand vieler Beispiele beobachten, dass bestimmte Mechanismen, wie Verhaltensmuster von Menschen, von einem Fall auf einen anderen übertragbar sind. Die Fähigkeit, Analogien wahrzunehmen, und gefundene Isomorphie als „Ergebnisse“ von der Analogiequelle zum Analogieziel zu übertragen, erscheint in der kognitiven Psychologie als ein zentraler Prozess, gar als eine wesentliche kulturelle Errungenschaft des Menschen schlechthin. Dieser Umstand wird in der neueren populärwissenschaftlichen Literatur intensiv diskutiert [HoSa14]. Gleichwohl ist zu konstatieren, dass Analogiebildungen - wissenschaftstheoretisch - keinerlei kausal-methodische Grundlage haben.

Das Ursache-Wirkungs-Prinzip tritt hier hinter das Mittel-Zweck-Prinzip zurück.

\section{Die betrachteten Analogiequellen}

Die Auswahl geeigneter Analogiequellen - für den Fall des LZDV als Analogieziel - ist einerseits geleitet von den „passenden Komponenten“, welche einen unterstellten hinreichend großen Einfluss auf das zu lösende Problem haben. Im Sinne einer vermuteten Strukturähnlichkeit. gilt es Eigenschaften und Strukturen des „Zielaufgabe“ in der Analogie wiederzufinden. 
Zum anderen gewährleistet eine gewisse geografische Verteilung der Analogiequellen, dass die Recherche nicht auf eine einzelne Region beschränkt ist (gleichwohl lag ein Schwerpunkt im Bereich der Wissenschaftsstadt Darmstadt). Zum dritten ist die Verfügbarkeit von Informationen und Ansprechpartnern zu den Analogiequellen eine selbstverständliche Voraussetzung für eine sinnvolle Untersuchung.

Die Analogiequellen wurden bezüglich der folgenden Aspekte analysiert:

- Marktstruktur und Marktansprache

- Formen der Leistungsbereitstellung

- Formen der Zusammenarbeit

- Kooperationspartner

- Produkte und Dienstleistungen

Die Analogiequellen weisen zum LZDV eine Ähnlichkeit bezüglich der genannten Kriterien auf und haben die zu erwarteten Akzeptanzprobleme des LZDV bereits in einem hohen Maße gelöst.

Besondere Aufmerksamkeit wurde der Möglichkeit der Rekursivität des Wissenstransfers gewidmet. Gemeint ist damit, dass Forschungsergebnisse eine Resonanz in Gesellschaft und Ökonomie finden und zu neuen Forschungsthemen führen. Rekursive Strukturen dieser Art sind in der Praxis der gewerblichen Wirtschaft vielfältig erprobt. Insbesondere die Hersteller qualitativ hochwertiger Produktlinien haben ein hohes Interesse daran, dass ihre Kunden über Weiterbildung und Aufmerksamkeitsmanagement zu einem qualifizierten Käufer (,informed buyer“) werden.

Die Analogiequellen werden nachstehend kurz vorgestellt. Es ist an dieser Stelle natürlich aus Platzgründen -unmöglich, alle Analogiekomponenten der betrachteten Einrichtungen im Detail darzustellen; insofern muss es bei einer kurzen Beschreibung der angetroffenen Strukturen bleiben.

\subsection{Center for Advanced Security Research Darmstadt (CASED) und Center for Research in Security and Privacy (CRISP), Darmstadt}

Im Jahr 2008 wurde das Center for Advanced Security Research Darmstadt (CASED) von der Technischen Universität (TU) Darmstadt, dem Fraunhofer-Institut für sichere Informationstechnologie (SIT) und der Hochschule Darmstadt gegründet. CASED diente hierbei als Zentrale der drei Institute und koordinierte deren Zusammenarbeit. Das Ziel von CASED war es zum einen, neue Sicherheitslösungen für die stärksten Wachstumssegmente der IT zu erforschen und zu entwickeln, um Manipulationen, Wirtschaftsspionage und Produktfälschungen entgegen zu wirken. Zum anderen sollten neue Internetdienste und Techniken von Nutzern und Anbietern, zuverlässig und sicher eingesetzt werden können.

Seit Juli 2016 ist CASED in das Center for Security and Privacy (CRISP) überführt worden. Im Fokus der Forschung steht hierbei das Thema „Security at Large“. Dabei soll die „Sicherheit für große Systeme, von den Komponenten bis zu ihrem Zusammenspiel in umfassenden Sicherheitslösungen“ ergründet werden. CRISP bildet durch die Gründungsmitglieder von CASED sowie einer weiteren Partnerschaft mit dem Fraunhofer-Institut für Graphische Datenverarbeitung (IGD), die größte Allianz von Forschungseinrichtungen im Bereich Cybersicherheit in Europa. Gefördert und finanziert wird dies vom Hessischen Ministerium für Wissenschaft und Kunst und durch das Bundesministerium für Bildung und Forschung sowie durch Industriepartner. Zurzeit arbeiten ca. 450 Wissenschaftlerinnen und Wissenschaftlern an den verschiedenen Forschungsthemen. 
Bei CRISP gibt es keine Mitgliedschaft, sondern alle Professoren und Forschungsmitglieder der involvierten Institute sind dazu angehalten zu „kooperieren“. Der Beitritt solcher Art zum CRISP ist kostenlos und jeder Teilnehmer ist gleichgestellt. Sie liefern Leistungen und erhalten seitens der Fördergeber direkt Zuwendungen, somit bilden sie ein Netzwerk.

\subsection{Competence Center for Applied Security Technology (CAST), Darmstadt}

Das Forum des Competence Center for Applied Security Technology (CAST) ist als Verein eingetragen und organisiert. Er wurde zum zu Beginn des Jahres 2004 gegründet. Das CAST Forum beschäftigt sich mit Themen aus dem Sicherheitsbereich von Informationstechnologien. Die Zielsetzung des Forums ist die Erlangung und Weiterentwicklung von Kompetenz auf diesem Gebiet um die Wirtschaft und öffentliche Verwaltung zu unterstützen. Außerdem soll der Verein die Zusammenarbeit von Forschern, Anbietern und Nutzern fördern. Aufgrund des langen zeitlichen Bestands des Forums konnten viele Mitglieder gewonnen werden; es sind ca. 260 Mitglieder im CAST Forum vertreten, von denen der Großteil Unternehmen aus dem Mittelstand ausmacht. Die Mitgliedschaft ist an keine bestimmte Gruppenzugehörigkeit gebunden. Kooperationen bestehen unter anderem mit dem Fraunhofer-Institut für Graphische Datenverarbeitung (IGD), dem Fraunhofer-Institut für Sichere Informationstechnologie (SIT), der TU Darmstadt, CRISP und der Gesellschaft für Informatik e.V.

Das CAST Forum bietet diverse Angebote, hauptsächlich Dienstleistungen als „Anlaufstelle für alle Fragen der IT-Sicherheit“. In diesem Zusammenhang werden im Rahmen des Netzwerks auch Beratungsleistungen in Bezug auf Nutzbarkeit, Eignung und Qualität von ITSicherheitstechnologien angeboten. Das CAST Forum organisiert ebenfalls Seminare und andere Informationsveranstaltungen sowie die Vorführung von Demonstrationen im Bereich der Sicherheit der Informationstechnik.

\subsection{InGeoForum, Darmstadt}

Die Gründungsmitglieder des InGeoForum e.V. entschlossen sich im Jahr 1996 eine Plattform für Kooperation, Kommunikation und Information für Beteiligte aus dem Geoinformationsmarkt, der Forschung, Wirtschaft und Verwaltung zu schaffen. Das InGeoForum entstand „als erstes institutionelles Informations- und Kooperationsforum für Geodaten in Deutschland“. Durch das Bereitstellen von Methoden und Tools eines Netzwerkes gelingt es das Geodatenpotenzial zu bündeln und zu strukturieren, um es für die Entscheider aus der Politik und Wirtschaft nutzbar zu machen. In der Projektberatung bietet das InGeoForum, auf Basis der Interessen der Partner, über eine neutrale Plattform einen geschützten Austausch über Projektideen, sowie deren Unterstützung und Begleitung bis zur Marktfähigkeit. Die Mitgliedschaft hat für Anwender und Anbieter von Geodaten unterschiedlichen Nutzen; Anwender können direkt Einfluss auf die Daten-, System- und Dienstleistungsangebote im Geoinformationssystemmarkt nehmen und das Know-how der Plattform zur objektiven Auswahl von Systemen, Dienstleistungen und Geodaten für ihre Projekte nutzen. Zum Jahreswechsel 2016/17 hat das InGeoForum 43 Mitglieder, wobei lediglich eine Firmen- oder Behördenmitgliedschaft angeboten wird. Die Mitglieder profitieren von Informationsveranstaltungen, Netzwerkgesprächen, Broschüren und Dokumentationen aus den verschiedenen Themenreihen. Des Weiteren bietet das Forum einen sogenannten „Round Table“ an. Dabei werden die Mitglieder eingeladen, bspw. mit einem Anwenderverband zu diskutieren, um Gemeinsamkeiten zu finden und Projekte zu generieren. 


\subsection{Initiative D21, Berlin}

Die Initiative D21 hat ihren Sitz in Berlin und ist ein gemeinnütziger Verein, welcher 1999 mit der Aufgabe gegründet wurde, die "Digitale Spaltung in Deutschland“ zu verhindern. Sie beschreibt sich als „Deutschlands größte Partnerschaft von Politik und Wirtschaft für die Informationsgesellschaft“. Die rund 200 Mitgliedsunternehmen und -organisationen aus verschiedensten Branchen sowie politische Partner bilden das Netzwerk. Es werden praxisnahe Non-Profit-Projekte zu den Themen Bildung, Standort und Vertrauen vorangetrieben Finanziert wird dies hauptsächlich durch Mitgliedsbeiträgen und Spenden.

Das Netzwerk bietet seinen Mitgliedern eine neutrale Arbeitsplattform zum Austausch von Perspektiven und Argumenten in Arbeitsgruppen, Forschungsprojekten und Events. Somit kann ein vorwettbewerblicher Austausch und Kompetenztransfer erfolgen. Über die Homepage der Initiative D21 können auch Nicht-Mitglieder einen Newsletter abonnieren. Dieser enthält Neuigkeiten und Termine für die Digitale Gesellschaft. Des Weiteren werden über die Internetseite Flyer, Broschüren, Studien, Interviews und Artikel für jedermann zugänglich gemacht. Mitglieder haben die Chance, die Projekte aktiv mitzugestalten und somit einen Beitrag zur Gestaltung der digitalen Gesellschaft zu leisten. Die Mitgliedschaft untergliedert sich in eine Vollmitgliedschaft und eine Fördermitgliedschaft.

\subsection{Initiative Deutschland Digital, Köln}

Die Initiative Deutschland Digital (IDD) liegt federführend in der Hand der neuland $\mathrm{GmbH}$ \& Co.KG, Köln. Die Initiative wurde im Juli 2016 der Öffentlichkeit vorgestellt. Finanziert wird die Initiative unter anderem durch Beiträge der Mitglieder. Ziel der Initiative ist es primär die Digitale Transformation am Standort Deutschland voranzutreiben. Die IDD sieht sich als eine Art Plattform, um die digitale Kompetenz von Unternehmen und damit auch deren Wettbewerbsfähigkeit zu stärken. Gründungspartner waren unter anderem die (Technologie-)Unternehmen HP und Fujitsu. Mittlerweile sind noch weitere Namhafte Referenzpartner hinzugekommen. Einige Beispiele sind TÜV Rheinland, Avantum oder der eco-Verband.

Bei den Angeboten muss unterschieden werden zwischen Angeboten, die allen zugänglich sind sowie Angeboten, die nur Mitgliedern vorbehalten sind. Nicht-Mitglieder können an verschiedenen Konferenzen, Roadshows oder Workshops teilnehmen. Diese sind teilweise kostenpflichtig oder kostenfrei. Sofern Veranstaltungen kostenpflichtig sind, erhalten IDDMitglieder Rabatte. Zahlreiche Angebote sind nur Mitgliedern vorbehalten. Hierzu gehören die Online-Kurse der IDD-Academy sowie Veranstaltungen, die ausschließlich für Mitglieder zugänglich sind. Außerdem erhalten Mitglieder Rabatte beim Besuch der Veranstaltungen und Zugriff auf verschiedene Erhebungen und Reports zu Trends.

\subsection{Wissenstransfer und Messewesen (WIMES) TU München}

Der Name Wissenstransfer und Messewesen (WIMES) ist Programm; es ist an der Technischen Universität in München (TUM) angesiedelt, die Mitarbeiter sind auch Festangestellte der TU München. Es handelt sich um ein Hochschulreferat der TU München. Die Einrichtung sieht sich als Informationsvermittler zwischen der Wirtschaft und der Wissenschaft. Das Hauptaufgabengebiet des WIMES liegt in Messebeteiligungen. Diese werden für alle bayerischen Hochschulen sowie zugehörige Einrichtungen angeboten. Messebeteiligungen bieten die Möglichkeit, universitäre Leistungen und Kompetenz vorzustellen sowie Kontakte zu möglichen Projektpartnern oder weiteren Universitäten zu knüpfen. Seit mehr als 25 Jahren bietet sich die Möglichkeit einer Beteiligung an den Gemeinschaftsständen der Bayerischen Hochschulen. Die Aufgabe des WIMES findet sich in der Koordination dieser Angebote. 
In das weitere Aufgabengebiet fällt die interne Weiterbildung der Mitarbeiter der TU München. Es werden Seminarreihen zu verschiedenen Themengebieten angeboten. Weiterbildungen sind unter anderem im Bereich der Persönlichkeitsentwicklung oder der Kommunikationsfähigkeit möglich. Auch fachspezifische Weiterbildungen werden angeboten.

\subsection{House of Finance, Frankfurt}

Das House of Finance gehört zur Goethe-Universität, Frankfurt am Main. Gegründet wurde das House of Finance im Jahr 2008. Das House of Finance vertritt Kompetenzen im Bereich des Finanzrechts sowie der Finanzwirtschaft. Das Ziel ist es, ein offenes Forum für Kooperationen und Begegnungen von Politik und Wirtschaft zu bieten. Das House of Finance zählt zu den größten Forschergruppen im finanzwirtschaftlichen Bereich. Dies wird durch die Kooperation von verschiedenen Instituten, welche in der Goethe-Universität angesiedelt sind, erreicht.

Der Schwerpunkt der Angebote des House of Finance liegt hauptsächlich in der Forschung. In den verschiedenen Instituten wird Forschung zu verschiedenen Bereichen der Finanzwirtschaft betrieben. Durch ständige Kommunikation mit den öffentlichen Stellen soll ein optimales ordnungspolitisches Gerüst in Bezug auf aktuelle, für den Finanzmarkt relevante Themen, geschaffen werden. Eine weitere Säule des House of Finance bietet die Aus- und Weiterbildung. Es werden verschiedene Angebote an der Goethe Business School sowie dem Institute for Law and Finance in Frankfurt angeboten. Das House of Finance bietet weiterhin noch verschiedene Veranstaltungen zur Weiterbildung an. Bspw. sind Seminare, Vorträge oder Konferenzen im Programm des House of Finance enthalten.

\section{Experteninterviews}

Um die desktop-research-basierten Erkenntnisse aus den gegebenen Analogiequellen zu ergänzen, wurden mit einigen ausgewählten Vertretern der Einrichtungen zusätzlich Experteninterviews durchgeführt. Die Experten stellten einiges weitergehendes Wissen zu den Analogiequellen zur Verfügung, adressiert wurden die folgenden Positionen, in Stichpunkten:

\section{Organisation}

Aufbau als Offene Gemeinschaft, oder als (eingetragener) Verein - die Rolle der (auch verschiedenen) Mitglieder und der Mitgliederversammlungen, Vorstand und Beisitzern. Entscheidungsgremium, Geschäftsführung, Beirat, Steering Board, Aufsichtsrat. Gemeinnützigkeit.

\section{Mitgliedschaft}

Offen oder institutionell - verschiedene Mitgliedschaften für Behörden, Unternehmen, Forschungseinrichtungen. Ehrenmitglieder. Fluktuationsrate, Stabilität des Mitgliederbestands. Höhe der Mitgliedsbeiträge. Unterschiedliche Formen der Mitgliedschaft.

Mitgliederakquise

Mitglieder durch direkte Ansprache und aktive Mitgliederwerbung. Werbung durch Internetauftritt, Veranstaltungen und Vortragsreihen, zu denen auch Nicht-Mitglieder eingeladen werden. Peer-2-Peer-Propaganda. 


\begin{abstract}
Angebote
Demonstratoren und Technologiemuster. Digitale Vernetzung. „Versicherung gegen verpasste Innovationen“. Dialoge, Round Table, Informations- und Netzwerkveranstaltungen. Broschüren, In-House Schulungen, Konferenzen.

Projekte

Arrangement und „People Management“ für themenspezifische Projekte. Informationen zu aktuellen Ausschreibungen und Förderprogrammen.

Sonstiges

Phasen der Euphorie, der Resignation, der Stagnation und des Weiteraufbaus. Mitarbeiter arbeiten ehren-, neben- oder hauptamtlich. Haupteinnahmequelle Mitgliedsbeiträge und Leistungen, wie Workshops. Kostenstruktur der Geschäftsstelle. Grundfinanzierung über Bund und Länder, über Industriepartner, nationale und internationale Unternehmen.
\end{abstract}

\title{
6 Darstellung der Ergebnisse auf der Ebene von Handlungsempfehlungen
}

Durch die Recherche und die Experteninterviews und auch die Besprechungen mit dem LZDV konnten einige Handlungsempfehlungen erstellt werden die im Folgenden näher vorgestellt werden.

\subsection{Marktstruktur und Marktansprache}

Trivialerweise ist es sinnvoll, eine Zielgruppe für den Wissenstransfer zu definieren, um Angebote und Dienstleistungen an diese anzupassen. Die Zielgruppe kann anhand verschiedener thematischer Ansätze und Möglichkeiten eingegrenzt werden. Eine Unterteilung nach dem geografischen Standort, der Branche oder der Unternehmensgröße kann ebenfalls vorgenommen werden.

Für die Gründungspersonen und das Team der Wissenstransfereinrichtung ist eine Vertrauenswürdigkeit essentiell; sie müssen die Problemlagen des Zielpublikums verstehen und dafür ansprechbar sein. Zudem sollten die Gründungspersonen fachlich und sozial kompetent sein; über ein belastbares sozioökonomische Netzwerk sollte verfügt werden können. Dieses stellt für die Interessenten eine Orientierung dar und zeigt die Relevanz des Themas. Die „Philosophie“ stellt einen wichtigen Bestandteil dar; eine Philosophie sollte formuliert werden, die Interessenten zeigt mit was sich das Forum beschäftigt und welche Zwecke und Ziele verfolgt werden.

\subsection{Formen der Zusammenarbeit}

Bestimmte Angebote und Dienstleistungen sollten exklusiv nur für Mitglieder, andere Angebote und Dienstleistungen für alle Interessenten angeboten werden. Durch die solcherart geschlossene und offene Teilnahme ist es möglich, eine Diversität zu erreichen - die Mitgliedschaft sollte sich lohnen. Diese Diversität kann bspw. aufgebaut werden indem bestimmte Inhalte auf der Homepage oder Veranstaltungen nur für Mitglieder zugänglich gemacht werden.

Die Beitrittsformalitäten bilden einen Zielkonflikt. Einerseits sollte der Beitritt kostengünstig, juristisch einfach und unproblematisch sein damit möglichst viele Interessenten Mitglied werden können, andererseits sollte die Mitgliedschaft von den Mitgliedern ein gewisse fi- 
nanzielle Verpflichtung, Einsatz und Leistungsbereitschaft, auch fachliche Beiträge enthalten.

Für die Form der Zusammenarbeit ist es wichtig ein sozio-ökonomisches Netzwerk aufzubauen. Von diesem können alle Seiten profitieren, da neue Ideen für viele Mitglieder von Vorteil sein können und ein Austausch unter den Mitgliedern helfen kann, neue Projekte entstehen zu lassen oder Geschäftsbeziehungen aufzubauen.

\subsection{Formen der Leistungsbereitstellung}

Leistungen und Inhalte sind auf Abruf „on Demand“ zur Verfügung zu stellen. Die On-Demand Inhalte sollten differenziert Mitgliedern zur Verfügung gestellt werden. Dies ist unter anderem über einen Mitgliederbereich auf der Homepage möglich. Bestimmte allgemeine Inhalte sollten zudem für jedermann online abrufbar sein. Dies trifft auf Veranstaltungspläne, Angebote und Informationen über die Einrichtung zu.

Außerdem sollten sowohl aktive als auch passive Angebote bereitgestellt werden, die entweder Mitglieder, Nicht-Mitglieder oder beide Interessensgruppen adressieren können.

Unter Angebote mit passiver Präsenz werden Seminare und Vorträge verstanden bei denen der Teilnehmer lediglich Informationen aufnimmt. Angebote mit aktiver Präsenz sind unter anderem Workshops, Diskussionsrunden oder Projekte. Hierbei sollten sich die Teilnehmer aktiv an den Diskussionen beteiligen und eigene Erfahrungen oder Meinungen einbringen. Angebote ohne Präsenz sind bspw. die oben angesprochenen On-Demand-Inhalte, das beinhaltet den Download von Studien, Ergebnissen oder Broadcast von überall, zu jeder Zeit.

Eine weitere, sehr interaktive Form der Leistungsbereitstellung wird durch die Einrichtung von Showrooms erreicht. Diese sind Veranstaltungen oder Räume bei denen Interessenten direkt mit Produkten oder Themen in Berührung kommen. Interessente können sich einige neue Techniken und Geräte anschauen und diese aktiv ausprobieren.

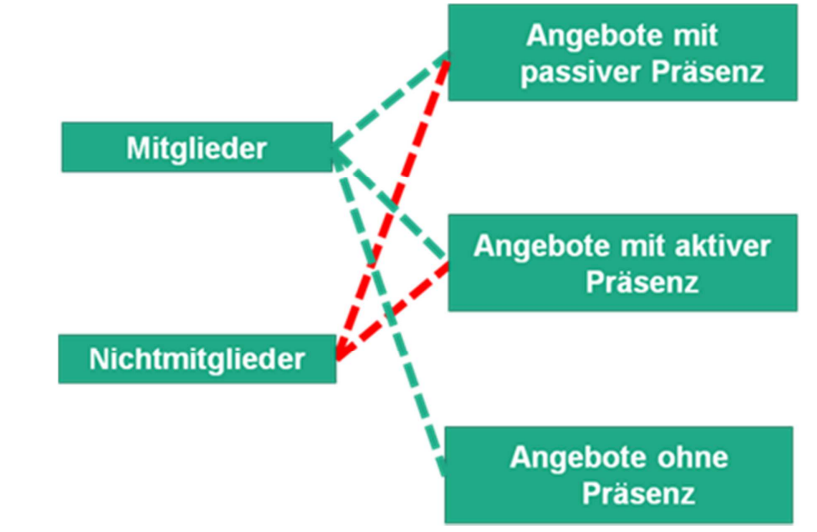

Abbildung 2: Aktive und Passive Angebote vs. Mitglieder und Nichtmitglieder

\subsection{Angebote und Dienstleistungen}

Über offene Veranstaltungen -für Mitglieder und Nicht-Mitglieder - können Interessenten mit der Thematik und der „Atmosphäre“ der Wissenstransfereinrichtung Industrieforums vertraut werden. Nächste Schritte bieten gezielt bei konkreten Herausforderungen Unterstützung, auch Beratung.

Ein mögliches Angebot ist das Durchführen oder Vermitteln von Aus- und Weiterbildungen. Zertifikate im Bereich der Kooperation mit den Universitäten und Hochschulen erscheinen 
attraktiv. Wichtig ist, dass vorher definiert wird, welche Vorbedingungen erfüllt werden müssen, um die Weiterbildungsangebote besser ausrichten zu können. Alle öffentlichen und internen Angebote sollten über die Homepage oder andere Informationskanäle publik gemacht werden. Dadurch wird Interessenten die Möglichkeit gegeben, sich über das Industrieforum zu informieren.

\subsection{Kooperationspartner}

Universitäten und Hochschulen treten als die kanonischen Kooperationspartner von Wissenstransfereinrichtungen auf. Anhand von Best Practice Partnern kann praktisch veranschaulicht werden, wie Vorreiter zu Thematiken sich positionieren. Als Unterstützer von Wissenstransfereinrichtungen Verbände auf, auch die Industrie- und Handelskammern (IHK). Je vernetzter die Kooperationspartner mit den Mitgliedern sind, desto einfacher funktioniert die Zusammenarbeit und desto besser die Kommunikationswege und der Wissenstransfer.

\section{Ein Synoptisches Modell - Elemente eines Referenzmodells}

Die in den in der Studie [El++17] aufgezeigten Case-based-Evidence-Analogien können im Sinne eines gestaltungsorientierten Ansatzes ein einem Synoptischen Modell zusammengefasst werden; es umfasst in der hier präsentierten Form die folgenden Punkte:

1. Die Wissenstransfereinrichtung muss „Wissen“ aufbereiten können. Um beim Zielkunden in der Praxis akzeptiert zu werden, sind oftmals Vereinfachungen und konkrete Beispiele von Nöten. Die komplexen Darstellungen in forschungsnahen Fachpublikationen müssen erklärt und vermittelt werden.

2. Das Denken in den fachlichen Zuständigkeiten der Fakultäten, Institute und Lehrstühle ist nicht produktiv; die Kunden des Wissenstransfers erwarten eine Reduktion des Koordinations-Aufwandes für die Lösung ihrer - für sie komplexen - Problemstellungen;

3. Es sind für die Wissenstransfereinrichtung unterschiedliche juristische Organisationen möglich; es muss definierte eine Mitgliedschaft der Wissenstransfereinrichtung geben. Daneben sind lose Informations- oder Probe-Kooperationen für prospektive Mitglieder möglich.

4. Die von der Wissenstransfereinrichtung vertretenen Themenfelder werden ständig mit passender Frequenz den neu entstehenden fachlichen Zielen und adressierten Zielgruppen angepasst.

5. Dem Führungsteam der Wissenstransfereinrichtung - oft mit dem Gründungsteam identisch - kommt eine entscheidende Rolle zu. Vom Team gehen wesentliche Signale der Verbindlichkeit und des Vertrauens aus. Die „Kunden“ erwarten ein hohes Engagement des Teams, aber auch eine hohe Verbindlichkeit seitens der hinter dem Wissenstransfer stehenden Hochschul- und Institutsleitungen.

6. Die Wissenstransfereinrichtung braucht eine dezidierte Philosophie und Leitlinien; diese zeigen den Sinn und Zweck der Wissenstransfereinrichtung und sind zentral für dessen Identität. Es sollten konkrete (nicht-abstrakte) Themenkreise benannt werden können.

7. Der Erfolg von Wissenstransfer wird oft gestützt von etablierten öffentlichen und politischen Partnern, die an einem Gegenstand ein Interesse haben. Zu nennen sind Kammern und Verbände, aber auch Medien- und Publikationspartner. 
Dieses Referenzmodell ergibt quasi automatisch ein übertragbares Modell nach Maßgabe identifizierter Erfolgsfaktoren - es entsteht eine Liste der umzusetzenden Empfehlungen für eine erfolgreiche Gestaltung des Wissenstransfers.

\section{Das Synoptische Modell und Referenzmodell in der Anwendung „LZDV“}

In der Umsetzung des Referenzmodells in ein organisatorisches Referenzmodell LZDV wurden die untenstehenden Aspekte identifiziert.

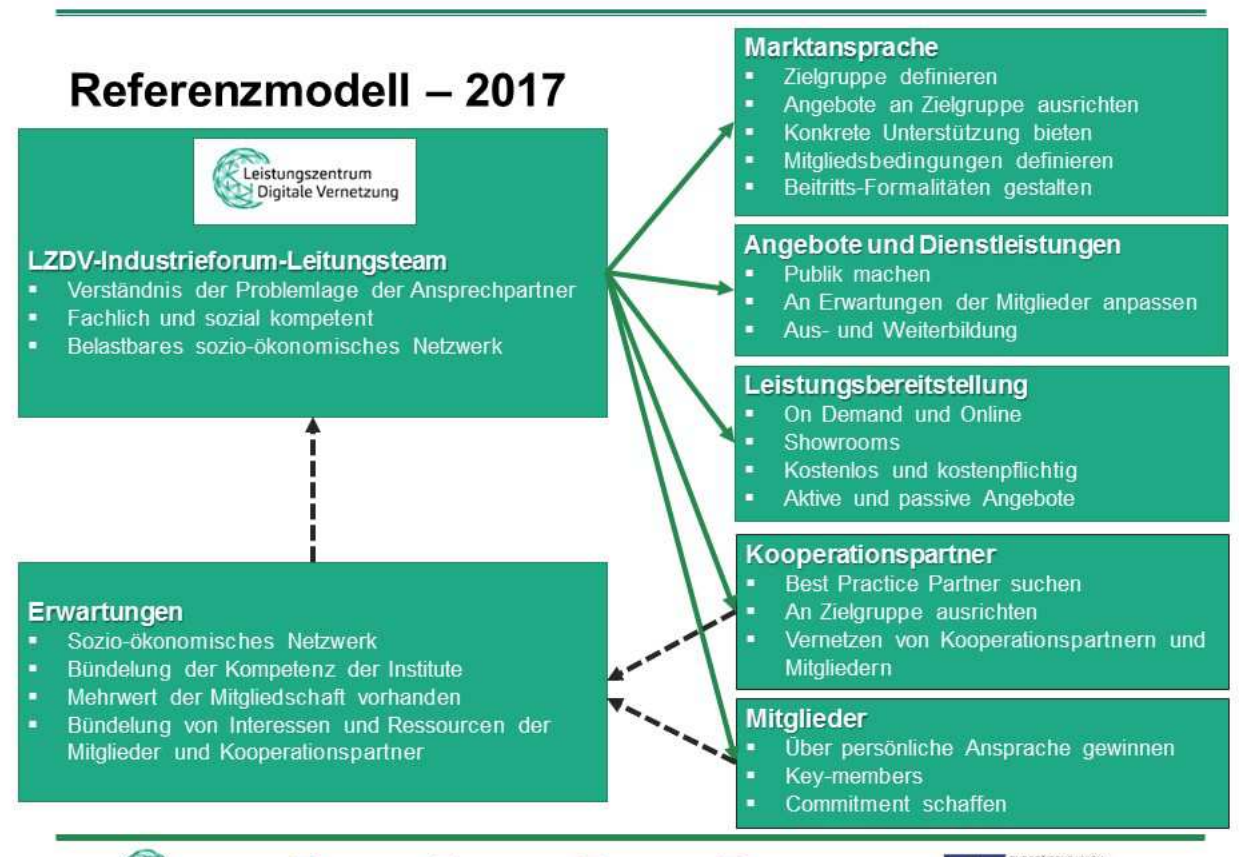

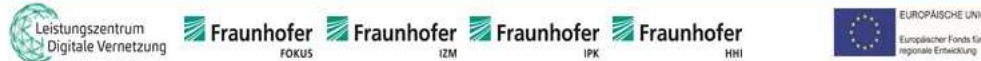

Abbildung 3: Die Umsetzung des Referenzmodells in ein organisatorisches Referenzmodell für das LZDV, Berlin.

Die Komponenten des gefundenen Referenzmodells stellten sich im Rahmen eines MetaWissenstransfers als übertragbar auf die Gestaltung künftiger - hoffentlich nachhaltig erfolgreicher - Wissenstransfereinrichtungen in den Universitäten, Hochschulen und Instituten.

\section{$9 \quad$ Ein Meta-Wissenstransfer?}

Nicht verkannt wird, dass es Ansätze einer „Serienfertigung“ von Wissenstransferzentren durchaus gibt. So etwa im Rahmen der Steinbeis-Stiftung [Stei17], die sich als einer der weltweit erfolgreichsten Dienstleister im Wissens- und Technologietransfer versteht.

Nach Maßgabe der durchgeführten und hier dargestellten Arbeiten und erzielten Ergebnisse kann man zum Schluss kommen, dass ein individuell maßgeschneiderter „Meta-Wissens- 
transfer" als eine - beratungspraktische oder selbsttherapeutische - Umsetzung des hier vorgestellten Referenzmodells in ein operativ umsetzbares organisatorisches Referenzmodell durchaus möglich erscheint.

Im Prinzip könnte jede bereits bestehende Wissenstransfereinrichtung mit Hilfe der oben dargestellten sieben Komponenten des Referenzmodells die „Probe aufs Exempel“ durchführen, inwieweit die identifizierten Erfolgsfaktoren direkt - auch teilweise - umgesetzt worden sind.

\section{Literaturverzeichnis}

[El++17] Elsesser, Eva; Fischer, Annette; Karl, Kay; Löffler, Veronika; Michel, Anna: Marktstruktur und Marktansprache für das Leistungszentrum Digitale Vernetzung - LZDV - in Berlin, Seminararbeit, Aschaffenburg 2017

[HoSc12] Hofmann, Georg Rainer; Schumacher, Meike: „Studie zur Akzeptanz von Cloud Computing“, EuroCloud Deutschland_eco e. V., EuroCloud Austria, 2012, Köln, Wien

[HoSc13] Hofmann, Georg Rainer; Schumacher, Meike: „Abschätzung der Akzeptanz von ITSystemen mittels Methoden der Case-based Evidences und Qualifizierten Experteninterviews - ein Metathema der Integration und Konnexion" in: Integration und Konnexion, Tagungsband zur AKWI 2013, Verlag News und Media, Berlin, 2013.

[HoSa14] Hofstadter, Douglas; Sander, Emmanuel: „Die Analogie: Das Herz des Denkens“, KlettCotta, 2014.

[LZDV16] Das Leistungszentrum Digitale Vernetzung, Geschäftsstelle, Berlin 2016, http://www.digitale-vernetzung.org/, Abruf am 2017-05-20

[ScHo16] Schumacher, Meike; Hofmann, Georg Rainer: „Case-based Evidence - Grundlagen und Anwendung“, Springer Vieweg, 2016.

[Stei17] Steinbeis: Technologie. Transfer. Anwendung. http://www.steinbeis.de/de/, Abruf am 2017-05-20

[Wiss16] Wissenschaftsrat-Drs. 5665-16, „Wissens- und Technologietransfer als Gegenstand institutioneller Strategien“, Oktober 2016, https://www.wissenschaftsrat.de/download/archiv/5665-16.pdf, Abruf am 2017-05-20

\section{Kontakt}

Prof. Dr. Georg Rainer Hofmann

Information Management Institut IMI

Hochschule Aschaffenburg

Würzburger Straße 45, 64743 Aschaffenburg

T +49 6021 4206-700, georg-rainer.hofmann@h-ab.de 


\title{
Datenerhebung für die CDG-Forschung
}

\author{
Niklas Weber, Martin R. Wolf
}

\section{Zusammenfassung}

Die vorliegende wissenschaftliche Ausarbeitung thematisiert die Erhebung von Daten bei Competence Developing Games (CDG). CDG ist ein Überbegriff für alle spielerischen Ansätze zur Wissens- und Fähigkeitenvermittlung, ungeachtet dessen, ob sie computergestützt sind oder nicht. Inkludiert sind beispielsweise Serious Games, Planspiele und Gamification. Ein CDG ist ein Spiel mit ernsten Intentionen, welches die Vermittlung von Wissen und Fähigkeiten in beruflichen und privaten Bereichen zum Ziel hat. In einer Spielsituation werden persönliche, soziale und methodische Fähigkeiten geschult, um somit die berufliche und persönliche Entwicklung des Spielers zu fördern.

Ziel dieser Ausarbeitung ist es, eine geeignete empirische Forschungsmethode für die CDGForschung zu ermitteln. Anhand der erhobenen Daten und deren anschließender Analyse sollen mit den damit resultierenden Informationen Rückschlüsse auf Stärken und Schwächen von CDG gezogen werden. Durch die Beseitigung der Schwächen soll eine Qualitätsverbesserung von $C D G$ angestrebt werden.

Einleitend werden die Begriffe Serious Games, Planspiele, Gamification und CDG erläutert und es wird sich mit den gängigen Methoden der empirischen Forschung auseinandergesetzt. Es werden Anforderungen erarbeitet, die eine empirische Methode erfüllen muss, um für die Datenerhebung bei CDG einsetzbar zu sein. Basierend auf den erarbeiteten Anforderungen werden die gängigen Methoden der empirischen Forschung Befragung, Beobachtung und Inhaltsanalyse einer Analyse unterzogen. Auf Grundlage der erarbeiteten Anforderungen wird eine empirische Forschungsmethode für die CDG-Forschung entwickelt. Abschließend folgt ein Fazit der erarbeiteten Ergebnisse sowie ein kurzer Ausblick auf weitere Forschung in diesem Themengebiet.

\section{$1 \quad$ Einleitung}

In der Lehre und in der Praxis gewinnen spielerische Ansätze zur Wissens- und Fähigkeitenvermittlung immer mehr an Bedeutung und werden als alternative pädagogische Methode angesehen. Durch die Nutzung von spielerischen Ansätzen wird die Motivation bzw. der Kontext einer Spielsituation in eine Lernsituation transferiert. Ein weiterer, nicht unerheblicher Aspekt des spielerischen Ansatzes ist die Tatsache, dass Spieler Strategien und Verhaltensweisen testen können ohne negative Auswirkungen auf sich und ihre reale Umgebung befürchten zu müssen. ([KöWo16], [Sanc11])

Generell wird zwischen verschiedenen Arten von Lehrspielen unterschieden. Serious Games, Planspiele, Gamification, Game-Based Learning oder Edutainment sind nur einige Beispiele. Um zumindest einen kurzen Einblick in die Thematik zu erhalten, werden einzig die Begriffe Serious Games, Planspiel und Gamification näher erläutert. Es wurde sich für diese Begriffe entschieden, da diese zu den weit verbreitetsten und meist eingesetzten Lehrspielen zählen. [KöWo16] 
Im Zeitalter der Digitalisierung sind mit Serious Games computerbasierte Spiele mit einem ausgewogenen Anteil von Spiel- und Bildungsaspekten gemeint. Der Spieler soll im Spiel Wissen und Fähigkeiten erlernen, welche er im wirklichen Leben anwenden kann. ([Marr10], [LaST09], [Hein17])

Ein Planspiel ist ein interaktives Spiel, bei dem Spieler in wechselnden Szenen und Situationen methodisch organisierte Aufgaben durchführen müssen. Planspiele können sowohl computergestützt als auch ohne die Unterstützung technischer Hilfsmittel durchgeführt werden. ([Meye11], [Onpu17], [HeBI00])

Als Gamification wird die Übertragung von spieltypischen Elementen und Prozessen in spielfremde Bereiche bezeichnet. Ziel ist es eine Motivationssteigerung der Nutzer zu erreichen, sich mit einer bestimmten Tätigkeit bzw. einem Objekt intensiver auseinanderzusetzen. ([Spri17], [KoOt12])

Auch wenn Unterschiede zwischen den einzelnen vorher genannten Lehrspielarten bestehen, haben sie alle eines gemeinsam: Ihr Ziel ist es, jemandem etwas beizubringen. Alle Arten basieren auf einer Kombination von motivierenden Spielelementen und didaktischen Lehrmethoden. [KöWo16]

Im Folgenden werden alle spielerischen Ansätze zur Wissens- und Fähigkeitenvermittlung, ungeachtet dessen ob sie computergestützt sind oder nicht, unter dem Begriff „Competence Developing Game“ geführt. Dabei handelt es sich um einen Überbegriff für alle Arten von Spielen mit ernsten Intentionen. ([KVWS17], [KöWo16])

Ein Competence Developing Game (CDG) ist ein Spiel mit ernsten Intentionen, welches die Vermittlung von Wissen und Fähigkeiten in beruflichen und privaten Bereichen zum Ziel hat. In einer Spielsituation werden persönliche, soziale und methodische Fähigkeiten geschult, um somit die berufliche und persönliche Entwicklung des Spielers zu fördern. ([KöWo16], [KVWS17])

In dieser wissenschaftlichen Ausarbeitung wird die Datenerhebung für die CDG-Forschung thematisiert. Ziel ist es, eine geeignete empirische Forschungsmethode für CDG zu finden. Anhand der erhobenen Daten und deren anschließender Analyse soll mit den damit resultierenden Informationen die Qualität von CDG verbessert werden.

\section{Methoden der empirischen Forschung}

Um sich dem Themengebiet der Datenerhebung für die CDG-Forschung zu widmen, werden in diesem Kapitel gängige Methoden der empirischen Forschung erläutert.

Empirische Forschungsmethoden haben das Ziel, die für ein Forschungsprojekt bzw. eine Untersuchung relevanten und interessanten Ausschnitte der Realität möglichst präzise zu beschreiben oder abzubilden. Unterschieden wird zwischen quantitativen und qualitativen Methoden. Bei Nutzung quantitativer Methoden ist die Intention, durch standardisierte Methoden die Daten von möglichst vielen Personen zu erhalten. Bei Nutzung qualitativer Methoden hingegen lautet das Ziel, durch offene Fragestellungen und nicht vorgegebene Antwortmöglichkeiten individuelle Informationen und Meinungen zu erhalten. ([BoDö15], [Sdir17], [BaBl14], [Stie99])

Quantitative und qualitative Methoden sollten nicht als Gegensätze, sondern eher als Ergänzung zueinander angesehen werden. Je nach Forschungskontext können zur Datenerhebung entweder quantitative, qualitative oder auch eine Kombination aus beiden Methoden genutzt werden. $[\mathrm{BaB} \mid 14]$ 
Die Befragung stellt in der Forschungspraxis die wichtigste und meist benutzte Methode dar. Allgemein wird zwischen der mündlichen und der schriftlichen Befragung differenziert. Bei der schriftlichen Befragung werden den Untersuchungsteilnehmern Fragen vorgelegt, welche diese schriftlich beantworten. Mündliche Befragungen (Interviews) lassen sich erneut in verschiedene Varianten differenzieren. Generell gibt es zwei Kategorien der mündlichen Befragung: Die quantitative Befragung und die qualitative Befragung. Bei der quantitativen Befragung wird meist ein vollständig strukturierter Fragebogen verwendet, an welchen sich der Interviewer strikt zu halten hat. Der Gesprächsverlauf ist standardisiert und fest vorgegeben. Wohingegen bei der qualitativen Befragung lediglich ein Leitfaden verwendet wird, der neben einigen wichtigen Fragestellungen eine Auflistung der zu behandelnden Themen enthält. Der Gesprächsverlauf ist offen und nicht an die Vorgabe bestimmter Fragen gebunden. ([BoDö15], [BaBl14], [Stie99], [Lang16])

Beobachtung ist das Sammeln von Erfahrungen und Informationen mittels sämtlicher Wahrnehmungsmöglichkeiten. Beobachtung im wissenschaftlichen Sinne ist zielgerichtet, systematisch und methodisch kontrolliert. ([Laat93], [BoDö15], [Stie99], [Lang16], [BaBl14])

Die Inhaltsanalyse ist eine analytische, empirische Forschungsmethode. Sie ist eine Methode der Datenerhebung und zudem eine Auswertungstechnik. Bei den zu untersuchenden Objekten handelt es sich, anders als bei den beiden oben genannten Methoden, nicht um Personen oder Ereignisse, sondern um Medien. Beispiele dafür sind Dokumente, Computerspiele oder Internetseiten. ([BoDö15], [Stie99], [Lang16])

\section{Anforderungen an eine empirische Forschungsmethode für CDG}

In diesem Kapitel wird auf die Anforderungen eingegangen, die eine empirische Forschungsmethode für die Datenerhebung bei CDG erfüllen muss. Die Methode muss sowohl für ein Serious Game, welches am Computer gespielt wird, als auch für ein Planspiel, welches ohne die Unterstützung technischer Hilfsmittel spielbar ist, anwendbar sein. Somit ist die erste Anforderung an die Methode festzuhalten: 1. Die Methode muss Daten von verschiedenen Arten von Spielen erheben können.

Mit der zu entwickelten Forschungsmethode sollen vor, während und nach dem Spielen eines CDG Daten erhoben werden. Dabei darf der Spieler nicht beim eigentlichen Spielen gestört werden. Infolgedessen ist eine zweite Anforderung festzuhalten: 2. Die Methode muss in den verschiedenen Situationen vor, während und nach dem Spielen die Daten erheben können, ohne dabei den Spieler beim Spielen zu stören.

Die nachfolgende Erarbeitung der Kriterien zur Verbesserung der Qualität von CDG basiert auf der Literatursuche mit den Suchmaschinen "Google“ und „Google Scholar“. Es wurden folgende Suchbegriffe benutzt: „Qualitätskriterien“, „Motivationsförderung“, „Aufgabengestaltung“, „Pädagogische Psychologie“, „Zusammenhang von Interesse und Motivation“, „Motivation and learning“, und „Kleinschrittige Aufgaben“.

Ein Kriterium, mit dem die Qualität gemessen werden kann, ist die Zeit, die ein Spieler zur Lösung einzelner Aufgaben benötigt. Darüber lassen sich Informationen über die Komplexität der Aufgaben ableiten. Lassen sich die Aufgaben zu leicht lösen, können sich die Spieler unterfordert fühlen und die Motivation zum Spielen verlieren. Generell kann ein Motivationsverlust beispielsweise dazu führen, dass der Spieler dem Geschehen während des Spiels unbegeistert und unaufmerksam folgt oder gar das Spielen des CDG beendet. Motivationsverlust kann aber ebenfalls bei zu komplizierten bzw. komplexen Aufgaben auftreten; 
dadurch kann der Spieler sich überfordert fühlen. [Kupk06] Ebenso ist es von Bedeutung zu erfahren, wie lange ein Spieler benötigt bis er die Bedienung bzw. die Regeln des CDG verstanden hat. Wenn ein Spieler zu viel Zeit in die Einarbeitung investieren muss, kann dieser ebenso die Motivation verlieren sich mit dem Spiel weiter auseinanderzusetzen. Ein weiteres Kriterium, welches durch die Messung der Zeit ermittelt werden kann, ist die Dauer, die ein Spieler investieren muss, um das CDG komplett durchzuspielen. Ist die Dauer zu hoch angesetzt, kann es einen Spieler abschrecken sich mit diesem Spiel auseinanderzusetzen. ([Kupk06], [GaAD02])

Zusammenfassend für die drei angeführten Informationen bezogen auf die Ermittlung der Zeit ist eine weitere Anforderung an die Methode festzuhalten: 3. Die Methode muss während des Spielvorgangs eines CDG eine Zeitmessung durchführen können, ohne dabei den Spieler zu stören.

Eine weitere wichtige zu ermittelnde Information ist die Bewertung der Spielstory durch die Spieler. Finden Spieler die Spielstory nicht interessant oder spannend, ist die Wahrscheinlichkeit für einen Motivationsverlust hoch. [Krap92]

Eine ebenso wichtige Information ist die Bewertung der adäquaten Aufteilung von Spiel- und Lehranteilen durch die Spieler. Ein zu geringer Lehranteil schmälert den Lernerfolg der Spieler, ein zu hoher Lehranteil hingegen kann wiederum zu einem Motivationsverlust des Spielers führen. [LaST09]

Zudem sollte ermittelt werden, wie die Spieler die Vielfältigkeit der zu erledigenden Aufgaben bewerten. Bei hoher Eintönigkeit der Aufgaben kann ein Motivationsverlust entstehen, da die Spieler von der Gleichartigkeit der Aufgaben gelangweilt sein könnten. Ebenso kann ein Motivationsverlust bei zu hoher Variation der Aufgabentypen entstehen. [Kupk06] Die Spieler könnten dadurch überfordert sein, dass die Art der Aufgaben gewechselt wird, obwohl sie sich gerade erst daran gewöhnt haben.

Wichtig ist zudem, den eigentlichen Ablauf bzw. die Durchführung des Spiels durch die Spieler bewerten zu lassen. Unter diesen Punkt sollte auch fallen, wie fachkundig, organisiert, hilfsbereit und motivierend die Spielleitung war.

Zusammenfassend für die Informationen, die anhand der Bewertungen durch die Spieler erfolgen, ist folgende Anforderung festzuhalten: 4. Die Methode muss vor und nach dem Spielen eines CDG Befragungen der Spieler durchführen können.

Ebenso ist die Anzahl durchzuführender Arbeitsschritte zur Absolvierung einer Aufgabe wissenswert. Sind für einzelne Aufgaben zu viele Arbeitsschritte notwendig, könnte es zu Motivationsverlust führen, da die Spieler zu lange auf das Gefühl der Zufriedenheit für das Lösen einer Aufgabe warten müssen. ([Kupk06], [Lank12]) Daraus lässt sich eine weitere Anforderung an die Methode formulieren. 5. Die Methode muss während des Spielvorgangs eines CDG Daten durch das Zählen bestimmter Aktionen erheben können, ohne dabei den Spieler zu stören.

Wissenswerte Informationen sind zudem die Reaktionen der Spieler während verschiedener Spielszenarien und Situationen des Spielvorgangs. Gemeint sind damit äußerliche Erscheinungen wie zum Beispiel emotionale Reaktionen, Körperhaltung oder Mimik. Dadurch lässt sich beispielsweise die Zufriedenheit der Spieler bezogen auf verschiedene Spielszenarien bzw. auf das ganze Spiel erschließen. Basierend auf diesen Daten könnten die jeweiligen Spielszenarien bzw. der generelle Ablauf verbessert werden. Somit ist eine weitere Anforderung an die Methode festzuhalten: 6. Die Methode muss während des Spielvorgangs durch Beobachtung der Spieler Informationen erheben können, ohne diese dabei zu stören. 
Wichtig sind auch die Informationen, die durch die Analyse der generellen Spielinhalte wie Unterlagen oder Spielszenarien gewonnen werden können. Infolgedessen lässt sich eine weitere Anforderung an die Methode definieren: 7. Die Methode muss durch die Analyse der CDG-Inhalte Informationen erheben können.

An dieser Stelle wird die Erarbeitung der zu erhebenden Daten abgeschlossen. Es wird aber ausdrücklich darauf hingewiesen, dass dies nicht alle relevanten zu erhebenden Daten sind und dieser Bereich weiter erforscht werden muss.

Abschließend folgt eine Auflistung der erarbeitenden Anforderungen.

1. Die Methode muss Daten von verschiedenen Arten von Spielen erheben können.

2. Die Methode muss in den verschiedenen Situationen vor, während und nach dem Spielen die Daten erheben können, ohne dabei den Spieler beim Spielen zu stören.

3. Die Methode muss während des Spielvorgangs eines CDG eine Zeitmessung durchführen können, ohne dabei den Spieler zu stören.

4. Die Methode muss vor und nach dem Spielen eines CDG Befragungen der Spieler durchführen können.

5. Die Methode muss während des Spielvorgangs eines CDG Daten durch das Zählen bestimmter Aktionen erheben können, ohne dabei den Spieler zu stören.

6. Die Methode muss während des Spielvorgangs durch Beobachtung der Spieler Informationen erheben können, ohne diese dabei zu stören.

7. Die Methode muss durch die Analyse der CDG-Inhalte Informationen erheben können.

\section{Analyse der empirischen Forschungsmethoden}

In diesem Kapitel wird anhand der im Kapitel 3 erarbeitenden Anforderungen eine Analyse der Forschungsmethoden Befragung, Beobachtung und Inhaltsanalyse durchgeführt.

Anforderung 1: Mit der Befragung lassen sich Daten von verschiedenen Arten von Spielen erheben, da bei der Befragung eine Interaktion mit einer Person stattfindet und somit die Art des Spieles irrelevant ist. ([BaBl14], [BoDö15], [Stie99]) Die Anforderung wird somit erfüllt. Mit der Beobachtung lassen sich Daten von verschiedenen Arten von Spielen erheben, da bei der Beobachtung die Spieler im Fokus sind und somit die Art des Spieles irrelevant ist. ([BaB|14], [BoDö15], [Stie99]) Die Anforderung wird somit erfüllt. Mit der Inhaltsanalyse lassen sich Daten von verschiedenen Arten von Spielen erheben, da bei der Inhaltsanalyse verschiedenste Arten von Medien im Fokus der Untersuchung sind. ([Stie99], [Lang16]) Die Anforderung wird somit erfüllt.

Anforderung 2: Mit der Befragung lassen sich die Daten während des Spielens nicht erheben, ohne dabei den Spieler zu stören. ([BoDö15], [BaBl14], [Stie99]) Infolgedessen wird die Anforderung nicht erfüllt. Mit der Beobachtung lassen sich die Daten vor, während und nach dem Spielen erheben, ohne dabei den Spieler beim Spielen zu stören. ([Stie99], [BaBI14], [BoDö15]) Somit wird die Anforderung erfüllt. Mit der Inhaltsanalyse lassen sich die Daten vor, während und nach dem Spielen erheben, ohne dabei den Spieler beim Spielen zu stören. Bei der Inhaltsanalyse sind die Medien im Fokus der Erhebung und der Spieler wird dadurch nicht gestört. ([Lang16], [Stie99]) Die Anforderung wird somit erfüllt.

Anforderung 3: Mit der Befragung lässt sich während des Spielvorgangs keine Zeitmessung durchführen. Die Befragung dient einzig allein der Interaktion mit dem zu Befragenden und ist daher auch nicht für eine Zeitmessung anwendbar. ([Stie99], [BaBl14], [BoDö15]) Infolgedessen wird die Anforderung nicht erfüllt. Mit der Beobachtung lässt sich unter zu Hilfen- 
ahme eines Zeitmessinstruments während des Spielvorgangs eine Zeitmessung durchführen, ohne dabei den Spieler zu stören. ([BaBl14], [BoDö15], [Stie99]) Die Anforderung wird somit erfüllt. Mit der Inhaltsanalyse lässt sich während des Spielvorgangs keine Zeitmessung durchführen. Bei der Inhaltsanalyse sind die Medien im Fokus der Erhebung und die Methode ist nicht zur Messung der Zeit geeignet. ([Stie99], [Lang16]) Infolgedessen wird die Anforderung nicht erfüllt.

Anforderung 4: Mit der Befragung lassen sich vor und nach dem Spielen eines CDG Befragungen der Spieler durchführen, da die Methode ausschließlich der Interaktion zwischen Befragten und Befragendem dient. ([BaBl14], [BoDö15], [Stie99]) Die Anforderung wird somit erfüllt. Mit der Beobachtung lassen sich keine Befragungen der Spieler durchführen, da die Methode nicht zur Befragung ausgelegt ist. ([Stie99], [BaBl14], [BoDö15]) Infolgedessen wird die Anforderung nicht erfüllt. Mit der Inhaltsanalyse lassen sich keine Befragungen der Spieler durchführen. ([Lang16], [Stie99]) Infolgedessen wird die Anforderung nicht erfüllt. Anforderung 5: Mit der Befragung lassen sich während des Spielvorgangs eines CDG durch das Zählen bestimmter Aktionen keine Daten erheben, ohne dabei den Spieler zu stören. Die Methode dient ausschließlich der Interaktion zwischen Befragten und Befragendem und daher wird durch die Interaktion eine Störung verursacht. ([Stie99], [BaB|14], [BoDö15]) Infolgedessen wird die Anforderung nicht erfüllt. Mit der Beobachtung lassen sich unter zu Hilfenahme eines Zählinstruments, während des Spielvorgangs eines CDG durch das Zählen bestimmter Aktionen Daten erheben. ([BaBl14], [BoDö15], [Stie99]) Die Anforderung wird somit erfüllt. Mit der Inhaltsanalyse lassen sich während des Spielvorgangs eines CDG durch das Zählen bestimmter Aktionen keine Daten erheben, ohne dabei den Spieler zu stören. ([Stie99], [Lang16]) Infolgedessen wird die Anforderung nicht erfüllt.

Anforderung 6: Mit der Befragung lassen sich während des Spielvorgangs durch Beobachtung der Spieler keine Informationen erheben, ohne diese dabei zu stören. ([Stie99], [BaBI14], [BoDö15]) Infolgedessen wird die Anforderung nicht erfüllt. Mit der Beobachtung lassen sich während des Spielvorgangs durch beobachten der Spieler Informationen erheben, ohne diese dabei zu stören. ([BaBl14], [BoDö15], [Stie99]) Die Anforderung wird somit erfüllt. Mit der Inhaltsanalyse lassen sich während des Spielvorgangs durch Beobachtung der Spieler keine Informationen erheben, ohne diese dabei zu stören. ([Stie99], [Lang16]) Infolgedessen wird die Anforderung nicht erfüllt.

Anforderung 7: Mit der Befragung lassen sich durch die Analyse der CDG-Inhalte keine Informationen erheben. Bei der Analyse der Inhalte sind Medien und Objekte im Fokus der Untersuchung. Mit der Befragung können aber nur Personen befragt werden. ([Lang16], [Stie99], [BaBI14], [BoDö15]) Infolgedessen wird die Anforderung nicht erfüllt. Mit der Beobachtung lassen sich durch die Analyse der CDG-Inhalte keine Informationen erheben. Das reine beobachten, von beispielsweise eines Dokumentes, liefert nicht die nötigen Informationen. Da es beim Beobachten meist um veränderliche Zustände geht die es zu erfassen gilt, wird das Beobachten eines Dokumentes nicht zielführend sein. ([BaB|14], [BoDö15], [Stie99]) Infolgedessen wird die Anforderung nicht erfüllt. Mit der Inhaltsanalyse lassen sich durch die Analyse der CDG-Inhalte Informationen erheben. Bei der Inhaltsanalyse sind Medien und Objekte im Fokus der Untersuchung. ([Stie99], [Lang16]) Die Anforderung wird somit erfüllt. Nachfolgend werden die Ergebnisse der Analyse in Tabelle $1 \mathrm{zu}-$ sammengefasst.

Wie aus der Analyse ersichtlich wird, erfüllt keine der analysierten Methoden alle Anforderungen. Aus diesem Grund wird im nächsten Kapitel der erste Schritt in die Entwicklung einer empirischen Forschungsmethode für die CDG Forschung getätigt. 


\begin{tabular}{|l|l|l|l|}
\hline & Befragung & Beobachtung & Inhaltsanalyse \\
\hline Anforderung 1 & Erfüllt & Erfüllt & Erfüllt \\
\hline Anforderung 2 & Nicht erfüllt & Erfüllt & Erfüllt \\
\hline Anforderung 3 & Nicht erfüllt & Erfüllt & Nicht erfüllt \\
\hline Anforderung 4 & Erfüllt & Nicht erfüllt & Nicht erfüllt \\
\hline Anforderung 5 & Nicht erfüllt & Erfüllt & Nicht erfüllt \\
\hline Anforderung 6 & Nicht erfüllt & Erfüllt & Nicht erfüllt \\
\hline Anforderung 7 & Nicht erfüllt & Nicht erfüllt & Erfüllt \\
\hline
\end{tabular}

Tabelle 1: Analyse der Methoden

\section{Die CDG-Methode}

In diesem Kapitel wird eine empirische Forschungsmethode für die CDG-Forschung entwickelt. Im Rahmen dieser Ausarbeitung wird die Entwicklung der Methode nur relativ kurz und oberflächlich sein. Im weiteren Verlauf wird die entwickelte Methode als „CDG-Methode“ bezeichnet.

Begonnen wird mit der Durchführung einer Inhaltsanalyse durch einen Experten. Dieser soll die zum CDG gehörigen Inhalte wie Unterlagen und Spielszenarien analysieren, um daraus Informationen zur Verbesserung des CDG zu gewinnen. Anschließend werden quantitative Befragungen mit Spielern durchgeführt. Dabei sollen unter anderem die Erwartungen der Spieler an das CDG erhoben werden. Während des Spielvorgangs werden die Spieler beobachtet. Dadurch sollen deren Verhaltensweisen und äußerliche Erscheinungen, wie zum Beispiel emotionale Reaktionen, Körperhaltung oder Mimik erfasst werden. Durch die Kombination des Verhaltens bzw. der äußerlichen Erscheinung mit den aktuellen Spielszenarien lassen sich so Rückschlüsse auf die jeweilige Akzeptanz der Szenarien bilden. Zudem werden während des Spielvorgangs Zeitmessungen für das Erledigen einzelner Aufgaben und das Durchspielen des Spiels vorgenommen. Abhängig davon, ob das CDG computergestützt gespielt wird oder nicht, wird die Zeit entweder automatisiert durch den Computer oder ansonsten durch Beobachtung und unter zu Hilfenahme eines Zeitmessinstruments erfolgen. Die Zeit, die ein Spieler für das Einarbeiten des CDG benötigt, wird durch eine Mischung aus Beobachtung mit Hilfe eines Zeitinstruments und einer Befragung erfolgen. Hier muss der Spieler vorher darauf hingewiesen werden, dass er aufzeigen soll, sobald er die Bedienung und den generellen Ablauf des CDG verstanden hat. Ist dies geschehen, folgt eine qualitative Befragung, in der der Spieler zu Stärken und Schwächen der Bedienbarkeit bzw. des Ablaufs befragt wird. Abhängig davon, ob das CDG computergestützt gespielt wird oder nicht, wird die Zählung der benötigten Arbeitsschritte zur Durchführung einer Aufgabe entweder automatisiert durch den Computer oder ansonsten durch Beobachtung unter zu Hilfenahme eines Zählinstruments erfolgen. Durch die Methode des Eye-Trackings werden zudem Daten darüber erfasst, wo der Aufmerksamkeitsfokus des Spielenden liegt. Dazu werden ausgewählte Spieler während des Spielvorgangs mit Eye-Tracking Brillen ausgestattet. Daraus kann beispielsweise geschlussfolgert werden, wie bei der Durchführung eines Planspiels die Räumlichkeiten oder Platzierungen von Objekten geändert werden könnten. Bezogen auf ein computergestütztes Spiel, könnte zu einer Verbesserung der Usability das Design oder die Navigation angepasst werden.

Nach dem Spielen des CDG werden durch eine Kombination aus quantitativer und qualitativer Befragung der Spieler Informationen über die Bewertungen des CDG erhoben. Darin 
inkludiert sind unter anderem: Spielszenarien, Aufteilung von Spiel- und Lehranteilen, Variation der Aufgaben und die organisatorische Durchführung. Unter der Kombination von quantitativer und qualitativer Befragung ist ein teilstandardisiertes Leitfadeninterview mit Option auf eine nicht standardisierte Befragung zu verstehen. Dies verfolgt den Zweck, dass der Interviewer bei interessanten, neuartigen oder informativen Antworten gezielter nachfragen kann. Somit können Fragen außerhalb des Leitfadens und innerhalb eines bestimmten relevanten Themengebiets gestellt werden.

\section{$6 \quad$ Fazit und Ausblick}

Ziel der vorliegenden Ausarbeitung ist es, eine geeignete empirische Forschungsmethode für CDG zu finden. Die Erarbeitung der Anforderungen erfolgte unter der Fragestellung, welche Daten erhoben werden müssen, um die Qualität von CDG zu verbessern. Das Ergebnis der Analyse ergab, dass keine der betrachteten Methoden alle Anforderungen erfüllt. Infolgedessen wurde eine eigene empirische Forschungsmethode für CDG entwickelt, die CDG-Methode. Die Entwicklung der CDG-Methode konnte im Rahmen dieser Ausarbeitung nur relativ kurz und oberflächlich erfolgen. Bei der CDG-Methode handelt es sich um keine wissenschaftlich erprobte Methode, sondern um ein rein theoretisches Konstrukt. Ein weiterer Kritikpunkt an der CDG-Methode ergibt sich aus der ersten Anforderung: Die Methode muss Daten von verschiedenen Arten von Spielen erheben können. Dadurch dass die CDGMethode relativ unspezifisch und somit für eine breite Masse an Lehrspielen anwendbar ist, könnte sie Einbußen an Wirksamkeit haben. Würde die CDG-Methode bereits an dieser Stelle auf computergestützte oder nicht computergestützte Lehrspiele spezialisiert werden, würden sich wiederum weitere spezifiziertere Anforderungen an die Methode ergeben. Diese wiederum ermöglichen eine gezieltere Qualitätsverbesserung der jeweiligen CDG. Weitere Anforderungen könnten unter Betrachtung anderer Gesichtspunkte als die Qualität, wie zum Beispiel gesetzliche Vorgaben oder zur Verfügung stehende Ressourcen, ermittelt werden. Der nächste Schritt wäre eine Anwendungserprobung innerhalb eines Experiments. Weitere Forschung in diesem Themengebiet könnte durch die Untersuchung aller in CDG vorkommenden Spielarten erfolgen. Generell müsste für jede Spielart untersucht werden, welche Daten zu erheben sind um die Qualität des Spiels zu verbessern. Anschließend müssten diese Anforderungen analysiert und zu einem großen Anforderungskatalog für CDG erarbeitet werden.

\section{Literaturverzeichnis}

[BaBI14] Baur, Nina; Blasius, Jörg: Handbuch Methoden der empirischen Sozialforschung. Springer Fachmedien Wiesbaden, Wiesbaden, 2014

[BoDö15] Bortz, Jürgen; Döring, Nicola: Forschungsmethoden und Evaluation: Für Human- und Sozialwissenschaftler. 5. Aufl. (Springer-Lehrbuch). Springer, Berlin, 2015

[GaAD02] Garris, R.; Ahlers, R.; Driskell, J. E.: Games, Motivation, and Learning: A Research and Practice Model. In: Simulation \& Gaming 33(4), 2002, S. 441-467. 
[Hein17] Heinz, Daniel: Serious Games - spielend Lernen. URL: https://www.Imz-bw.de/seriousgames.html. Abruf am 2017-01-03.

[HeBI00] Herz, D.; Blätte, A.: Simulation und Planspiel in den Sozialwissenschaften: eine Bestandsaufnahme der internationalen Diskussion. Lit, 2000

[KoOt12] Koch, Michael; Ott, Florian: Gamification - Steigerung der Nutzungsmotivation durch Spielkonzepte, 2012. URL: http://www.soziotech.org/gamification-steigerung-der-nutzungsmotivation-durch-spielkonzepte/. Abruf am 2017-01-03.

[KVWS17] König, Johannes; Völker, Veronika; Wolf, Martin; Schuba, Marko: Gamified Hacking Offence Simulation-based Training (GHOST): Ein Projekt der FH Aachen. URL: http://crisis-prevention.de/bos-katastrophenschutz/gamified-hacking-offence-simulationbased-training-ghost. Abruf am 2017-01-03.

[KöWo16] König, Johannes A.; Wolf, Martin R.: The Pyramid Assessment Framework for 'Competence Developing Games'. In: Stephanidis, Constantine (Hrsg.): HCl International 2016 - Posters' Extended Abstracts. Springer International Publishing, Cham, 2016, S. 232237.

[Krap92] Krapp, Andreas: Interesse, Lernen und Leistung: Neue Forschungsansätze in der Pädagogischen Psychologie. In: Zeitschrift für Pädagogik 1992(38 (5)), S. 747-770.

[Kupk06] Kupke, Marion: Instrumente zur motivationsfordernden Gestaltung von Arbeitsaufgaben. Grin Verlag, [Place of publication not identified], 2006

[Laat93] Laatz, Wilfried: Empirische Methoden: Ein Lehrbuch für Sozialwissenschaftler. Deutsch, Thun, 1993

[LaST09] Lampert, Claudia; Schwinge, Christiane; Tolks, Daniel: Der gespielte Ernst des Lebens: Bestandsaufnahme und Potenziale von Serious Games (for Health). In: MedienPädagogik - Zeitschrift für Theorie und Praxis der Medienbildung(15/16), 2009

[Lang16] Lang, Sabine: Empirische Forschungsmethoden. URL: https://www.unitrier.de/fileadmin/fb1/prof/PAD/SP2/Allgemein/Lang_Skript_komplett.pdf. Abruf am 2016-12-29.

[Lank12] Lankes, Eva-Maria: Kompetent durch Aufgaben?: Die Rolle von Lernaufgaben bei der Entwicklung von Kompetenzen. In: SchulVerwaltung Bayern, 2012, S. 214-216.

[Marr10] Marr, A. C.: Serious Games für die Informations-und Wissensvermittlung - Bibliotheken auf neuen Wegen. Dinges \& Frick, 2010

[Meye11] Meyer, Hilbert: Unterrichtsmethoden. 14. Aufl. Cornelsen Scriptor, Berlin, 2011

[Onpu17] Onpulson: Planspiel. URL: http://www.onpulson.de/lexikon/planspiel/. Abruf am 201701-03.

[Sanc11] Sanchez, Eric: Key criteria for Game Design: A Framework, 2011. URL: archives.reseaucerta.org/meet/Key_criteria_for_Game_Design_v2.pdf. Abruf am 2016-1213.

[Sdir17] SDI-Research: Methodische Formen der Befragung. URL: http://www.sdi-research.at/lexikon/befragung.html. Abruf am 2017-01-03.

[Spri17] Springer Gabler Verlag: Gamification. URL: http://wirtschaftslexikon.gabler.de/Archiv/688938796/gamification-v4.html. Abruf am 2017-01-03. 
[Step16] Stephanidis, Constantine (Hrsg.): HCl International 2016 - Posters' Extended Abstracts, Cham. Springer International Publishing, 2016. (Communications in Computer and Information Science).

[Stie99] Stier, Winfried: Empirische Forschungsmethoden. Zweite, verbesserte Auflage. (Springer-Lehrbuch). Springer, Berlin, Heidelberg, 1999

\section{Kontakt}

Niklas Weber, Martin R. Wolf

Labor für IT Organisation und Management

Fachhochschule Aachen

Eupener Str. 70, 52066 Aachen

Niklas.Weber@alumni.fh-aachen.de

M.Wolf@fh-aachen.de 
Didaktik 


\section{Der Arbeitsmarkt für Wirtschaftsinformatiker - Berufsbilder und Kompe- tenzen im Spannungsfeld der digitalen Transformation}

Frank Bensberg, Gandalf Buscher, Andreas Schmidt

\section{Zusammenfassung}

IT-Zukunftsthemen wie Industrie 4.0, Internet der Dinge, Big Data, Data Science und Cloud Computing geben Impulse zur strukturellen Weiterentwicklung von Wirtschaftssektoren und zur digitalen Transformation von Unternehmen. Der vorliegende Beitrag geht der Frage nach, welche Bedeutung diese IT-Zukunftsthemen derzeit am Arbeitsmarkt für Wirtschaftsinformatiker besitzen. Diese Fragestellung wird mithilfe einer explorativen, großzahligen Stellenanzeigenanalyse beantwortet, die darüber hinaus auch zentrale, arbeitsmarktrelevante Berufsbilder für Wirtschaftsinformatiker transparent macht.

\section{Problemstellung}

Unter dem Begriff der Transformation ist die bewusste Umsetzung von tiefgreifenden Veränderungsmaßnahmen innerhalb gegebener Rahmenbedingungen in einem Unternehmen oder einer Organisation zu verstehen, die über Anpassungsprozesse und kontinuierliche Entwicklungen deutlich hinausgehen [MBHF10]. Die stärkste Triebkraft für die Transformation geht aktuell von Informationstechnologien aus, deren Innovationen sich nicht nur zur Erzielung von Rationalisierungseffekten eignen, sondern vielmehr auch die Erschließung neuer Geschäftsmodelle ermöglichen [Venk05]. Insbesondere die IT-Zukunftsthemen Industrie 4.0, Internet der Dinge, Big Data, Data Science und Cloud Computing forcieren die Digitalisierung von Unternehmensprozessen, Wertschöpfungsketten und Märkten.

Im Zuge dieser Entwicklung bilden sich neue Berufsbilder für Wirtschaftsinformatiker heraus, die wirtschaftliche und informationstechnische Kompetenzen bündeln [ScWi16]. Dabei lässt sich auch die Evolution solcher Jobprofile beobachten, die sich dediziert mit der digitalen Transformation in Unternehmen und der öffentlichen Verwaltung beschäftigen (z. B. Chief Digital Officer, Disruption Officer, Process Transformation Manager). Damit wird die Fragestellung aufgeworfen, welche Berufsbilder und Kompetenzen für Wirtschaftsinformatiker derzeit am Arbeitsmarkt von Bedeutung sind und in welchem Umfang sich bereits spezifische Anforderungen der genannten IT-Zukunftsthemen substantiieren lassen.

Zur Beantwortung dieser Forschungsfrage stellt dieser Beitrag ausgewählte Ergebnisse einer Arbeitsmarktstudie vor, die an der Hochschule Osnabrück durchgeführt wurde und auf der Analyse von Stellenanzeigen für Wirtschaftsinformatiker aus Jobportalen basiert. Im Folgenden wird zunächst das Konzept des Job Mining als analytischer Bezugsrahmen zur Stellenanzeigenanalyse vorgestellt. Darauf aufbauend findet eine Darstellung der empirischen Basis und Analysemethodik statt, an welche sich die Darstellung zentraler Ergebnisse anschließt. Abschließend werden die Resultate reflektiert und Handlungsempfehlungen formuliert. 


\section{Job Mining als analytischer Bezugsrahmen zur Stellenanzeigenanalyse}

Zur analytischen Erschließung von Stellenanzeigen wird der Prozess des Job Mining zugrunde gelegt, der in Abb. 1 im Überblick dargestellt wird.

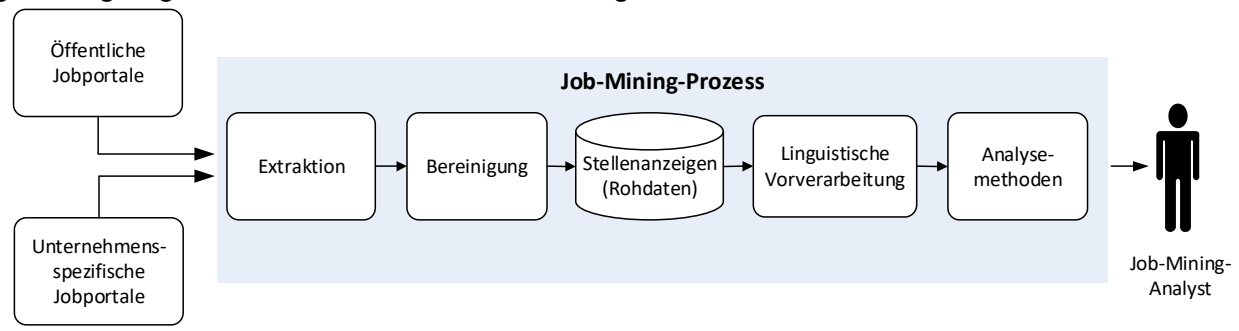

Abb. 1: Job-Mining-Prozess im Überblick [BeBu17]

Ausgangspunkt des Job-Mining-Prozesses bilden öffentliche oder unternehmensspezifische Jobportale als Datenquellen. In der Phase der Extraktion werden die Stellenanzeigen aus Jobportale ausgelesen und in einem anschließenden Schritt bereinigt. Dabei ist z. B. dafür zu sorgen, dass sämtliche Attribute einheitlich codiert sind und keine fehlenden Werte (Missing Values) auftreten. Tab. 1 zeigt die zentralen inhaltsbezogenen Attribute von Stellenanzeigen anhand eines Beispiels.

\begin{tabular}{|c|c|c|}
\hline Attributname & Beschreibung & Exemplarischer Inhalt \\
\hline JobTitle & $\begin{array}{l}\text { Stellen- } \\
\text { bezeichnung }\end{array}$ & $\begin{array}{l}\text { IT Prozessmanager }(\mathrm{m} / \mathrm{w}) \text { für den Controllingbereich } \\
{[\ldots]}\end{array}$ \\
\hline $\begin{array}{l}\text { Employment } \\
\text { Type }\end{array}$ & $\begin{array}{l}\text { Beschäftigungs- } \\
\text { verhältnis }\end{array}$ & Vollzeit \\
\hline $\begin{array}{l}\text { Hiring } \\
\text { Organization }\end{array}$ & $\begin{array}{l}\text { Einstellende } \\
\text { Institution }\end{array}$ & Siemens AG Recruiting Center Talent Acquisition \\
\hline JobLocation & $\begin{array}{l}\text { Beschäftigungs- } \\
\text { ort }\end{array}$ & 91056 Erlangen, Bayern, Deutschland \\
\hline Spider & $\begin{array}{l}\text { Ausgelesenes } \\
\text { Jobportal }\end{array}$ & Bundesagentur für Arbeit \\
\hline $\begin{array}{l}\text { JobDescrip- } \\
\text { tion }\end{array}$ & $\begin{array}{l}\text { Stellenbeschrei- } \\
\text { bung im Lang- } \\
\text { text mit Aufga- } \\
\text { ben und Anfor- } \\
\text { derungen }\end{array}$ & $\begin{array}{l}\text { [...] Werden Sie jetzt Teil unseres Teams von Sie- } \\
\text { mens Healthineers als IT Prozessmanager (m/w) für } \\
\text { den Controllingbereich in Erlangen. Sie unterstützen } \\
\text { bei der Entwicklung neuer harmonisierter "Best Prac- } \\
\text { tice" Prozesse im Controlling Umfeld mit unseren } \\
\text { Business Partnern, den Demand Managern und wei- } \\
\text { teren Implementierungsteams. Sie kümmern sich um } \\
\text { das Change Request Design und die Implementie- } \\
\text { rung aller Anforderungen in den Controlling Prozes- } \\
\text { sen unserer Business-Kollegen. [...] Sie haben erfolg- } \\
\text { reich ein Hochschulstudium der Fachrichtung Be- } \\
\text { triebswirtschaft, Wirtschaftsinformatik oder eines } \\
\text { vergleichbaren Fachbereichs abgeschlossen [...] }\end{array}$ \\
\hline
\end{tabular}

Tab. 1: Inhaltsbezogene Attribute von Stellenanzeigen am Beispiel

In diesem Beispiel wird eine Vakanz für einen IT-Prozessmanager (JobTitle) bei der Siemens AG (HiringOrganziation) in Erlangen (JobLocation) in Vollzeitbeschäftigung (EmploymentType) ausgeschrieben. Diese Stellenanzeige verfügt über eine detaillierte Beschreibung (JobDescription) und wurde aus dem Jobportal der Bundesagentur für Arbeit (Spider) ausgelesen. 
Aus dem skizzierten Beispiel wird deutlich, dass Stellenanzeigen überwiegend aus Textdaten bestehen. Infolgedessen kommt der Vorverarbeitung mithilfe von Techniken der Computerlinguistik zentrale Bedeutung zu. Gängige Verfahren zur Vorbereitung von Textdaten sind die Zerlegung in grundlegende Einheiten (Tokenizing), die Bestimmung einzelner Wortarten (Part-of-Speech-Tagging), sowie die Zerlegung zusammengesetzter Wörter [HeQW12].

Die so vorbereitete Datenbasis kann anschließend mit Verfahren der Textanalyse untersucht werden. Forschungsmethodisch stehen dabei zwei unterschiedliche Herangehensweisen zur Verfügung. Traditionell werden Stellenanzeigen mithilfe der quantitativen Inhaltsanalyse erschlossen ([Harp12], [Sail09]). Dabei wird mit a priori definierten Kategoriensystemen und Wörterbüchern gearbeitet, um die Texteinheiten theoriegeleitet klassifizieren zu können. Daraus entsteht allerdings die Problematik, dass strukturelle Entwicklungen in der Datenbasis - wie etwa das Auftreten neuer, bislang unbekannter Zusammenhänge - nicht erkannt werden. Demgegenüber gestattet ein lexikometrischer, korpusgetriebener Forschungsansatz, bislang unbekannte sprachliche Strukturen und Zusammenhänge in Texten aufzudecken. Zu diesem Zweck stehen folgende Analysemethoden zur Verfügung [DGMS09]:

- Mithilfe von Frequenzanalysen kann die absolute oder relative Häufigkeit des Auftretens von Wörtern oder Wortfolgen in Texten ermittelt werden.

- Die Untersuchung von Kookkurrenzen zeigt auf, welche Wörter oder Wortfolgen in dem zugrundeliegenden Textkorpus häufig gemeinsam auftreten.

- Mithilfe der Teilkorpusanalyse und der Korrelationsanalyse können Aussagen darüber abgeleitet werden, welche Wörter oder Wortfolgen in einer Teilmenge von Texten (Subgruppe) im Vergleich zum Gesamtkorpus über- bzw. unterrepräsentiert sind.

Aufbauend auf dem hier skizzierten Bezugsrahmen des Job Mining werden im Folgenden die empirische Basis und die Analysemethodik der Arbeitsmarktstudie erörtert.

\section{Empirische Basis und Analysemethodik}

Zur Generierung der Datenbasis sind zwischen Juni 2014 und November 2016 mithilfe von Web Crawlern insgesamt 5,69 Mio. Stellenanzeigen gesammelt worden. Diese Stellenanzeigen stammen aus öffentlichen Jobportalen (z. B. Jobserve, LinkedIn, Arbeitsagentur) und unternehmensspezifischen Jobportalen (z. B. Amazon, SAP, Microsoft, IBM). Der Schwerpunkt der Datenextraktion liegt auf Jobportalen und Unternehmen des IT-Sektors.

Aus diesen Stellenanzeigen sind diejenigen extrahiert worden, die in der Stellenbeschreibung den Begriff Wirtschaftsinformatik oder (Wirtschafts-)Informatik aufweisen. Auf diese Weise konnten 129.047 Stellenanzeigen identifiziert werden, die anschließend einer Duplikaterkennung unterzogen worden sind. Dabei wurden 44.332 Duplikate eliminiert (Duplikatquote $34,35 \%$ ). Von den verbleibenden 84.715 Stellenanzeigen sind 99,27\% als deutschsprachig und $0,73 \%$ als englischsprachig klassifiziert worden. Somit wird die empirische Basis durch $n=84.100$ deutschsprachige Stellenanzeigen gebildet.

Um einschlägige Berufsfelder für Wirtschaftsinformatiker zu identifizieren, ist dieses Set anschließend in Bezug auf die verwendeten Stellenbezeichnungen (Attribut JobTitle) untersucht worden. Dieser Analyseschritt basiert auf der informationsökonomisch begründeten Hypothese, dass Stellenbezeichnungen von Unternehmen so gewählt werden, dass rele- 
vante Tätigkeitsfelder prägnant signalisiert und somit die Screening-Prozesse potenzieller Interessenten unterstützt werden [Post15].

Hierauf aufbauend sind sämtliche Stellenbezeichnungen einer Frequenz- und Kookkurrenzanalyse auf Wortebene unterzogen worden. Auf diese Weise wurden zunächst häufige Begriffe und Begriffspaare identifiziert, die die Arbeitsmarktnachfrage nach Wirtschaftsinformatikern thematisch prägen. Aus der Analyse resultieren die in der folgenden Tabelle dargestellten Berufsbilder.

\begin{tabular}{|c|c|c|c|}
\hline $\mathrm{Nr}$ & Berufsbild & Abfragemuster für die Stellenbezeichnung & $\begin{array}{r}\text { relative } \\
\text { Häufigkeit }\end{array}$ \\
\hline 1 & Berater & jobtitle:*berat" ${ }^{*}$ OR jobtitle:*consult* & $26,7 \%$ \\
\hline 2 & Entwickler & $\begin{array}{l}\text { (jobtitle:*entwickl* OR (jobtitle:*software* AND (job- } \\
\left.\text { title:*engineer* OR jobtitle:*develop }{ }^{*}\right) \text { )) }\end{array}$ & $19,1 \%$ \\
\hline 3 & $\begin{array}{l}\text { Projekt- } \\
\text { manager }\end{array}$ & $\begin{array}{l}\text { jobtitle:*projektleiter }{ }^{*} \text { OR jobtitle:* projektmanager* } \\
\text { OR jobtitle:"*project manag*" }\end{array}$ & $6,9 \%$ \\
\hline 4 & Administrator & jobtitle:*administrator* & $4,8 \%$ \\
\hline 5 & Architekt & jobtitle:*architect" OR jobtitle:*architekt ${ }^{*}$ & $3,7 \%$ \\
\hline 6 & Analyst & jobtitle:*analyst* & $3,1 \%$ \\
\hline 7 & Spezialist & jobtitle:"spezialist ${ }^{*}$ & $3,0 \%$ \\
\hline 8 & $\begin{array}{l}\text { Prozess-, } \\
\text { Service-, } \\
\text { Application- } \\
\text { Manager }\end{array}$ & $\begin{array}{l}\text { jobtitle:*manager* AND (jobtitle:*applikation* OR } \\
\text { jobtitle:*service* OR jobtitle:*application* OR } \\
\text { jobtitle:*prozess* OR jobtitle:*process }{ }^{*} \text { OR } \\
\text { jobtitle:*anwendung*) }\end{array}$ & $2,8 \%$ \\
\hline 9 & $\begin{array}{l}\text { Leitungs- } \\
\text { funktion }\end{array}$ & jobtitle:*teamleit" OR "*IT-Leiter*" OR "Leiter IT" & $2,1 \%$ \\
\hline
\end{tabular}

Tab. 2: Berufsbilder für Wirtschaftsinformatiker

Aus Tab. 2 wird deutlich, dass insbesondere die Berufsbilder des Beraters, Entwicklers und Projektmanagers nachgefragt werden. Diese repräsentieren insgesamt 52,7 \% der untersuchten Stellenanzeigen. Infolgedessen werden im Folgenden ausgewählte, charakteristische Anforderungskriterien dieser drei Berufsbilder vorgestellt.

\section{$4 \quad$ Zentrale Berufsbilder für Wirtschaftsinformatiker}

\subsection{Berater}

Der Analyse dieses Berufsbilds liegen die Stellenanzeigen für Berater ( $n=22.450)$ zugrunde. Mithilfe einer Frequenzanalyse über die Stellenbezeichnungen konnte festgestellt werden, dass ein deutlicher Anteil der Fachkräftenachfrage nach Beratern auf konkrete Softwareprodukte der Marke SAP gerichtet ist. So nennen 52,1 \% sämtlicher Beratervakanzen den Begriff SAP in der Stellenbezeichnung. Häufige kookkurrierende Begriffe sind die konkreten Softwareprodukte bzw. Module der SAP-Anwendungslandschaft. Die Top 10 der genannten SAP-Module zeigt Abb. 2.

Aus der Darstellung wird deutlich, dass in 14,1 \% der SAP-spezifischen Vakanzen das SDModul (Sales \& Distribution) gefragt wird. Eine ähnlich hohe Bedeutung weist das Modul zur Materialwirtschaft (MM, 14,0 \%) auf. Anzumerken ist dabei, dass in Stellenbezeichnungen Mehrfachnennungen gängig sind, wie z. B. SAP SD/MM Consultant oder SAP FI/CO Berater. Interessanterweise sind Nennungen der SAP-Technologieplattform HANA in den Stellenbezeichnungen für SAP-Berater nur in geringem Ausmaß vorhanden (1,2 \%). 


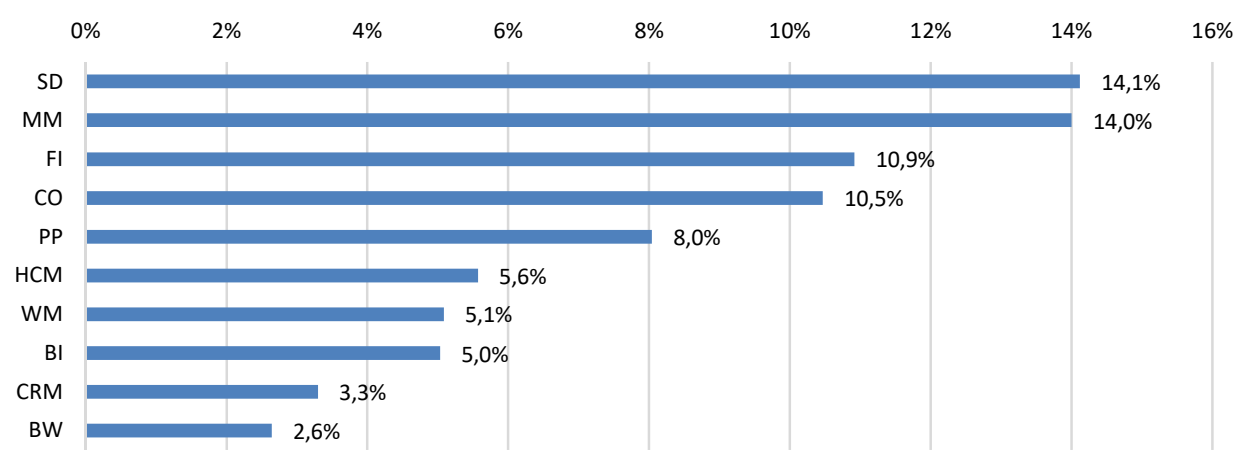

Abb. 2: SAP-Module in Stellenbezeichnungen von SAP-Beratervakanzen nach relativer Häufigkeit (Top 10)

Weitere relevante Themenfelder der Beratervakanzen werden durch generische Anwendungssystemklassen oder Softwareprodukte etablierter Softwarehersteller gebildet. So beziehen sich 7,3\% der Beratervakanzen auf Business Intelligence $(\mathrm{BI})$ und 4,3\% auf Enterprise Resource Planning (ERP). Weitere Schwerpunkte bilden die Programmiersprache Java $(1,4 \%)$, sowie die Produkte Microsoft Sharepoint $(1,1 \%)$ und Microsoft Dynamics $(1,2 \%)$.

\subsection{Entwickler}

Im Datensatz sind 16.047 Stellenanzeigen für Entwickler identifizierbar, die detaillierte Informationen über die geforderten Programmiersprachen liefern. Die folgende Abbildung umfasst die führenden Sprachen (Top 10) nach Häufigkeit in den Entwicklervakanzen.

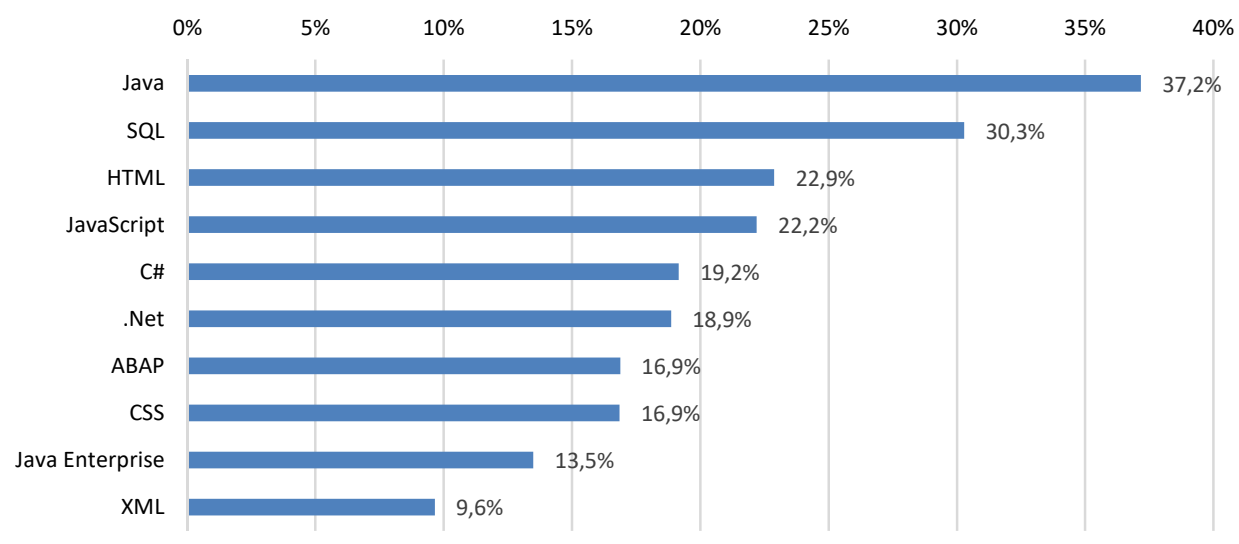

Abb. 3: Programmiersprachen in Stellenanzeigen für Entwickler nach relativer Häufigkeit (Top 10)

Die Abbildung zeigt deutlich, dass Java als Programmiersprache dominiert. Dieses Ergebnis deckt sich mit dem aktuellen TIOBE-Index (Mai 2017) [TIOB17], in dem Java als beliebteste Programmiersprache geführt wird. An zweiter Stelle folgt SQL als Datenabfragesprache. Mit deutlichem Abstand werden HTML und JavaScript verlangt, die insbesondere im Umfeld von Web Applikationen von hoher Bedeutung sind, wie auch CSS/XML. Dabei ist hervorzuheben, dass Skriptsprachen wie Python und Ruby - entgegen dem TIOBE-Index - in den Stellenanzeigen keine größere Bedeutung zukommt. 
Darüber hinaus werden in den Stellenanzeigen nahezu regelmäßig Soft Skills als überfachliche Qualifikationsanforderungen artikuliert. Mithilfe eines Wörterbuchs sind gängige Soft Skills in den Entwicklervakanzen untersucht worden (Abb. 4).

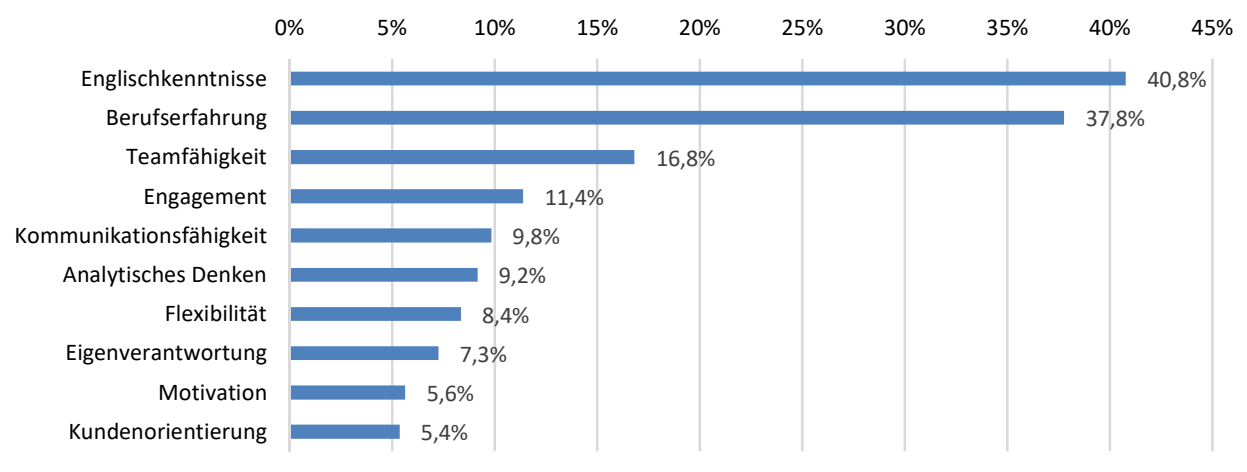

Abb. 4: Soft Skills für das Berufsbild Entwickler (Top 10)

Bei Entwicklertätigkeiten dominieren Englischkenntnisse und Berufserfahrung, während Teamfähigkeit, Engagement und auch Kommunikationsfähigkeit ein deutlich niedrigeres Bedeutungsniveau besitzen. Die führende Bedeutung von Englischkenntnissen kann als Indikator dafür gesehen werden, dass Entwickler häufig in internationalen, räumlich verteilten Teams agieren, in denen Englisch die Lingua franca darstellt.

\subsection{Projektmanager}

Das Berufsbild des Projektmanagers bzw. -leiters ist in insgesamt 5.814 Stellenanzeigen artikuliert worden. Mithilfe einer Korrelationsanalyse wurden folgende typische Tätigkeiten für Projektmanager identifiziert:

- Erstellung von Pflichten- und Lastenheften,

- Planung, Steuerung und Koordination von Projekten mit formalen Planungstechniken,

- Management und Steuerung des Projektbudgets,

- Bewertung der Projektrisiken und Begleitung des Risikomanagements,

- Erfüllung des projektbezogenen Berichtswesens und

- Konzeption, Leitung und Durchführung von Schulungen.

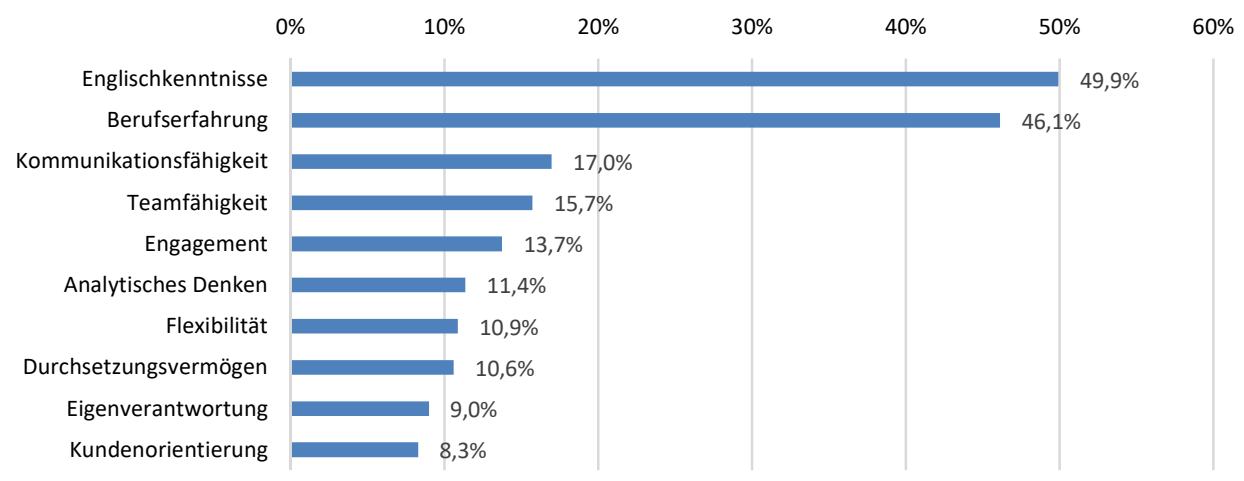

Abb. 5: Soft Skills für das Berufsbild Projektmanager (Top 10) 
Zur Ausübung dieser Tätigkeiten werden in den Stellenanzeigen bisweilen auch Zertifizierungen gefordert, wobei insbesondere PMI (9,5\%), PRINCE2 (8,1\%), ITIL (6,6\%) und GPM $(2,4 \%)$ von Bedeutung sind. Neben den fachlichen Qualifikationen werden auch überfachliche Qualifikationsanforderungen gestellt, die in Abbildung 5 dargestellt werden.

Auch bei diesem Berufsbild dominieren Englischkenntnisse und Berufserfahrung, allerdings werden diese deutlich häufiger genannt als bei Entwicklern. Zudem besitzt die Kommunikationsfähigkeit einen höheren Stellenwert.

\section{$5 \quad$ Bedeutung von IT-Zukunftsthemen}

Zur Analyse von IT-Zukunftsthemen sind Suchanfragen mit entsprechenden Schlagwörtern formuliert worden, die sowohl über die Stellenbezeichnung als auch Stellenbeschreibung ausgeführt wurden. Die Ergebnisse dieser Suchanfragen sind in Verbindung mit den entsprechenden Suchbegriffen in Tabelle 3 abgebildet.

\begin{tabular}{|l|l|l|r|r|r|r|}
\hline Nr. & Zukunftsthema & Suchbegriffe & \multicolumn{2}{|c|}{$\begin{array}{c}\text { in Stellen- } \\
\text { bezeichnung }\end{array}$} & \multicolumn{2}{|c|}{$\begin{array}{c}\text { in Stellen- } \\
\text { beschreibung }\end{array}$} \\
\cline { 3 - 6 } & & & Anzahl & Anteil & Anzahl & Anteil \\
\hline 1 & Cloud Computing & $\begin{array}{l}\text { Cloud, laaS, } \\
\text { PaaS, SaaS }\end{array}$ & 488 & $0,58 \%$ & 3.509 & $4,17 \%$ \\
\hline 2 & Big Data & Big Data, Bigdata & 338 & $0,40 \%$ & 1.771 & $2,11 \%$ \\
\hline 3 & Data Science & $\begin{array}{l}\text { Data Science, } \\
\text { Data Scientist }\end{array}$ & 237 & $0,28 \%$ & 385 & $0,46 \%$ \\
\hline 4 & Internet of Things & $\begin{array}{l}\text { Internet of Things, } \\
\text { loT, M2M, Internet } \\
\text { der Dinge }\end{array}$ & 112 & $0,13 \%$ & 1.368 & $1,63 \%$ \\
\hline 5 & Industrie 4.0 & $\begin{array}{l}\text { Industrie 4.0, } \\
\text { Industrie4.0 }\end{array}$ & 102 & $0,12 \%$ & 575 & $0,68 \%$ \\
\hline
\end{tabular}

Tab. 3: Frequenzanalyse für IT-Zukunftsthemen

Der Tabelle ist zu entnehmen, dass von den hier untersuchten Zukunftsthemen Cloud Computing bislang den höchsten Verbreitungsgrad erreicht hat. Zwar beziehen sich nur etwa $0,58 \%$ der Stellenbezeichnungen auf das Thema Cloud oder die damit verknüpften Dienste (laaS, PaaS, SaaS), allerdings wird dieses Thema in 4,17 \% der Stellenbeschreibungen genannt, sodass ein gewisser Reifegrad dieser Technologie zu konstatieren ist. Als zweitrangiges Zukunftsthema ist Big Data zu verorten, während Data Science und das Internet der Dinge sowie Industrie 4.0 deutlich geringere Verbreitung besitzen.

Für die einzelnen Zukunftsthemen sind weitere Charakteristika identifiziert worden, indem die fünf Teilkorpora in Bezug auf ihre prägenden, lexikalischen Merkmale untersucht worden sind. Zu diesem Zweck wurden mithilfe einer Korrelationsanalyse Substantive bzw. Substantivfolgen ermittelt, die für die Stellenanzeigen des jeweiligen Zukunftsthemas (selektiert nach Stellenbezeichnung) typisch sind. Die Ergebnisse werden in Abb. 6 dargestellt. 


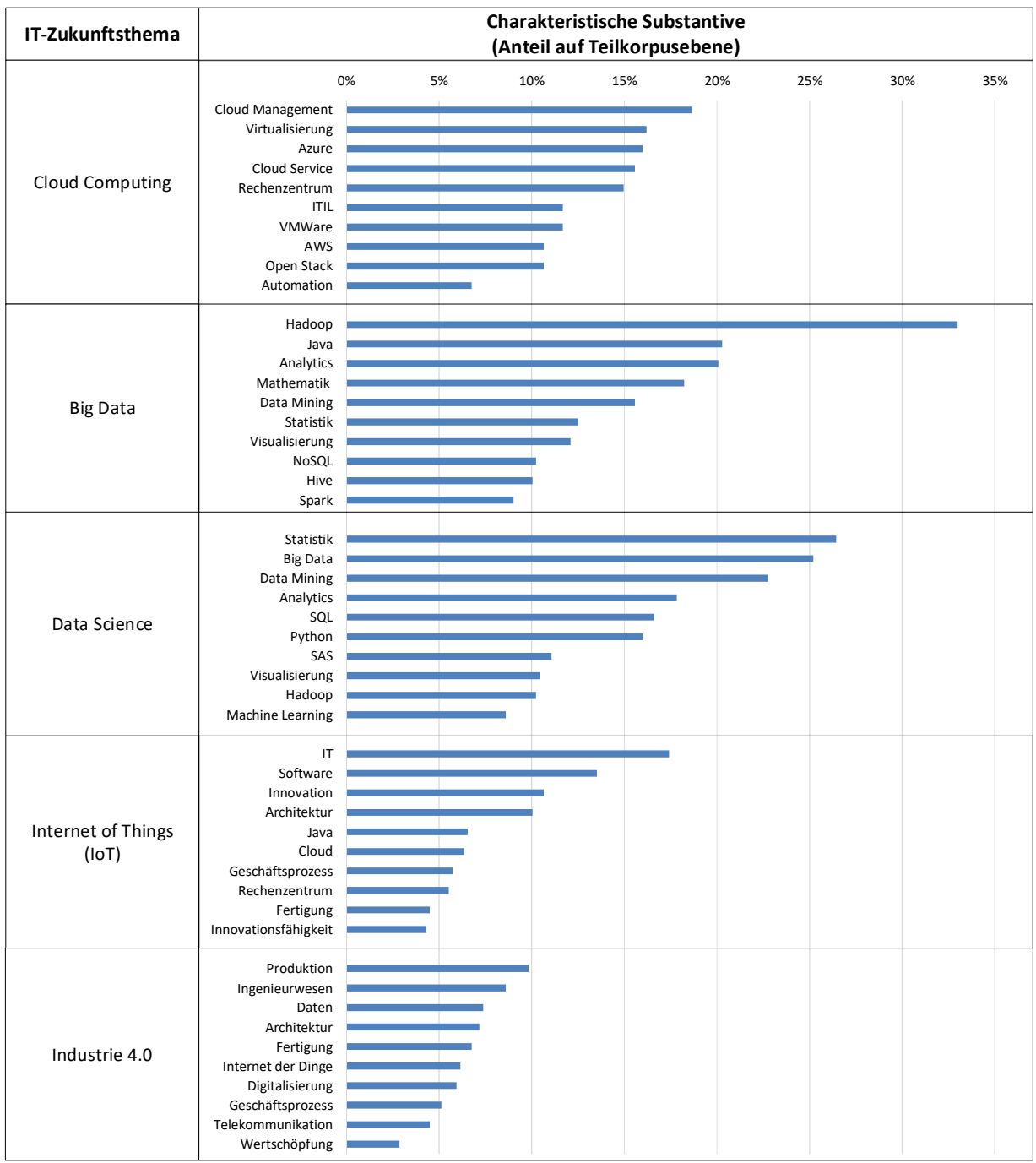

Abb. 6: Charakteristische Substantive in Stellenanzeigen für IT-Zukunftsthemen (Top 10)

Die identifizierten Substantive geben Anhaltspunkte dafür, welche Kompetenzen und welches Produktwissen für Wirtschaftsinformatiker in dem jeweiligen fachlichen Umfeld als relevant signalisiert werden:

- Im Zukunftsthema Cloud Computing scheint sich Arbeitsmarkt auf die Produkte Azure, VMWare, Amazon Web Services (AWS) und OpenStack zu fokussieren, wobei außerdem Kompetenzen in den Gebieten des Cloud Management und der Virtualisierung und charakteristisch sind.

- $\quad$ Eine deutlich stärkere thematische Konzentration ist in den analytischen Themenfeldern Big Data und Data Science erkennbar. Im Big Data-Umfeld werden insbesondere Hadoop, Hive und Spark als konkrete Softwareprodukte nachgefragt, die in Verbindung 
mit Java als Programmiersprache stehen. Data Scientists sollten hingegen vertiefte Kompetenzen im Umfeld von Data Mining und Statistik aufweisen, sowie die Skriptsprache Python beherrschen. Auch proprietäre Analysesysteme wie SAS sind dabei von Bedeutung.

- Das Zukunftsthema Internet der Dinge ist indes durch eine starke Betonung von Innovation bzw. Innovationsfähigkeit geprägt. Relevanz besitzt auch der Einsatz von Cloud Computing im Umfeld von Geschäftsprozessen.

- Die Industrie 4.0 besitzt in den Stellenanzeigen nur ein schwach konturiertes Profil. Der Kontext ist durch die funktionalen Schwerpunkte der Produktion und Fertigung gekennzeichnet, die in Bezug zur Telekommunikation und Wertschöpfung gesetzt werden. Klar erkennbar ist in den Stellenanzeigen auch der ingenieurwissenschaftliche Bezug (Ingenieurwesen).

Diese Ergebnisse deuten darauf hin, dass mit den skizzierten IT-Zukunftsthemen im Arbeitsmarkt konkrete Themenfelder und Produkte verknüpft sind, die für die Forschung und Praxis der Wirtschaftsinformatik von Relevanz sind. Aus quantitativer Perspektive ist indes zu konstatieren, dass IT-Zukunftsthemen - mit Ausnahme des Cloud Computing - in den untersuchten Stellenanzeigen für Wirtschaftsinformatiker nur eine geringe Bedeutung beizumessen ist.

\section{Fazit}

Auf Basis der untersuchten Stellenanzeigen wurde nachgewiesen, dass sich die Nachfrage nach Wirtschaftsinformatikern in den untersuchten Jobportalen auf klassische Berufsbilder konzentriert, die überwiegend den IT-Kernberufen zuzurechnen sind. In diesem Beitrag wurden charakteristische Qualifikationsanforderungen für die Berufsbilder des Beraters, des Entwicklers und des Projektmanagers expliziert. Dabei konnte gezeigt werden, dass sich die Nachfrage nach Beratern insbesondere auf Fachkräfte für betriebliche Anwendungssysteme - insbesondere für SAP-Module zur Unterstützung betrieblicher Funktionsbereiche - konzentriert.

Die anschließende Untersuchung der IT-Zukunftsthemen lässt den Befund zu, dass diese im Arbeitsmarkt für den untersuchten Zeithorizont nur eine untergeordnete Rolle spielen. Lediglich das Thema Cloud Computing scheint eine größere Bedeutung zu besitzen, wie die Nennungen in den Stellenbeschreibungen nachweisen (vgl. Tab. 3). Dieser Befund ist allerdings in Verbindung mit internationalen Arbeitsmarktstudien zu interpretieren ([BeBu16], [BeBu17]), die - für bestimmte Berufsbilder - eine höhere Relevanz cloud-basierter Technologien diagnostizieren.

Die hier vorgelegten Ergebnisse sind für Akteure von Interesse, die an Hochschulen mit der Gestaltung und Qualitätssicherung von Aus- und Weiterbildungsangeboten im Umfeld der Wirtschaftsinformatik betraut sind. Um eine hohe Beschäftigungsbefähigung von BachelorAbsolventen der Wirtschaftsinformatik am Arbeitsmarkt zu gewährleisten, sollten Kompetenzen zur Softwareentwicklung und zur Gestaltung betrieblicher ERP-Systeme fokussiert werden. Dabei sind auch Projektmanagementkompetenzen zu schärfen, die zur Begleitung der notwendigen Entwicklungs- und Betriebsprozesse erforderlich sind. 


\section{Literaturverzeichnis}

[BeBu16] Bensberg, F., Buscher, G.: Digitale Transformation und IT-Zukunftsthemen im Spiegel des Arbeitsmarkts für IT-Berater - Ergebnisse einer explorativen StellenanzeigenanaIyse. In: Nissen, V., Stelzer, D., Straßburger, S., Fischer, D. (Hrsg.): Tagungsband zur Multikonferenz Wirtschaftsinformatik (MKWI) 2016, TU Illmenau, Illmenau, 2016.

[BeBu17] Bensberg, F., Buscher, G.: DevOps im Spiegel des Arbeitsmarkts - Tätigkeitsfelder, Berufsbilder und Kompetenzen. In: HMD, 54: 275. DOI 10.1365/s40702-017-0290-9.

[DGMS09] Dzudzek, I., Glasze, G., Mattissek, A., Schirmel, H.: Verfahren der lexikometrischen Analyse von Textkorpora.In: Glasze G, Mattissek A (Hrsg) Handbuch Diskurs und Raum: Theorien und Methoden für die Humangeographie sowie die sozial- und kulturwissenschaftliche Raumforschung, 2. Aufl., Transcript, Bielefeld, S. 233-260.

[Harp12] Harper, R.: The Collection and Analysis of Job Advertisements - a Review of Research Methodology. In: Library and Information Research 36 (112), S. 29-54.

[HeQW12] Heyer, G., Quasthoff, U., Wittig, T.: Text Mining - Wissensrohstoff Text. W3L Verlag, Herdecke 2012.

[LilA12] Litecky, C., Igou, A.J., Aken, A.: Skills in the management oriented IS and enterprise system job markets. In: Adya, M., Horton, R., Huang, H., Quesenberry, J. (Hrsg.), Proceedings of the 50th annual conference on Computers and People Research (SIGMISCPR '12), New York.

[MBHF10] Mohr, N., Büning, N., Hess, U., Fröbel A. M.: Herausforderung Transformation. Springer Berlin 2010.

[Post15] Posthumus, J.: Use of market data in the recruitment of high potentials: Segmentation and targeting in human resources in the pharmaceutical industry. Springer Gabler, Wiesbaden 2015.

[Sail09] Sailer, M.: Anforderungsprofile und akademischer Arbeitsmarkt - Die Stellenanzeigenanalyse als Methode der empirischen Bildungs- und Qualifikationsforschung. Waxmann, Münster, 2009.

[ScWi16] Schabel, F., Wippich, O.: Digitale Transformation: Wie die IT neue Berufsbilder formt. In: Wirtschaftsinformatik \& Management, 5(8):28-35.

[TIOB17] TIOBE Index for May 2017. https://www.tiobe.com/tiobe-index/ [Abruf am 13-05-2017].

[Venk05] Venkatraman, N.: IT-Enabled Business Transformation: From Automation to Business Scope Redefinition. Operations management: A Strategic Approach:145-163.

\section{Kontakt}

Prof. Dr. Frank Bensberg

Hochschule Osnabrück

Caprivistrasse 30a, 49076 Osnabrück

T +49541969 3264, F.Bensberg@hs-osnabrueck.de 
Gandalf Buscher

Hochschule für Telekommunikation Leipzig (HfTL)

Gustav-Freytag-Str. 43-45, 04277 Leipzig

Gandalf.Buscher@hft-leipzig.de

Prof. Dr. Andreas Schmidt

Hochschule Osnabrück

Caprivistrasse 30a, 49076 Osnabrück

T +49 541969 3820, A.Schmidt@hs-osnabrueck.de 


\title{
Terminologiearbeit als Inhalt und Methode der Wirtschaftsinformatik
}

\author{
Johannes Busse
}

\section{Zusammenfassung}

Wie jede andere Design Science auch erzeugt die Wirtschaftsinformatik Artefakte, wozu nach [Hev04] außer Softwareinstanzen insbesondere auch Terminologien gehören. In der Tat ist ohne Terminologiearbeit wissenschaftliche Modellbildung in soziotechnischen Systemen kaum denkbar. Der Aufsatz diskutiert, wie Terminologiearbeit in einer Erstsemesterveranstaltung „Einführung in die Wirtschaftsinformatik“ gleichzeitig als Inhalt und als Methode implementiert und damit früher und nachhaltiger in einem Studiengang Wirtschaftsinformatik verankert werden kann. Der Aufsatz endet mit dem Vorschlag, digitale Terminologiearbeit auch fächerübergreifend als eine innovative Form von E-Learning zu etablieren.

\section{Begriffe des Titels}

Der Kontext dieses Aufsatzes sei im Folgenden anhand der im Aufsatztitel vorkommenden Begriffe näher dargestellt.

\subsection{Terminologiearbeit}

Begriffe und ihre Beziehungen untereinander kann man auf verschiedenen Komplexitätsstufen modellieren. Wenn man in einer Liste von frei generierbaren Wörtern (folksonomy) oder sachsystematisch vorgegebenen Begriffen (Schlagwörter) die Einträge zusätzlich erläutert, entsteht ein Glossar. Dieses lässt sich technisch leicht als Wiki implementieren, in welchem man bekanntlich die Erklärungstexte selbst durch Links in Beziehung setzen kann. Typisiert man solche Beziehungen zwischen Begriffen, entsteht ein semantisches Netz. Verwendet man dabei Beziehungen wie z.B. broader term (BT), related term (RT) oder use synonym ( $S Y N$ ), modelliert das semantische Netz einen Thesaurus; unterscheidet man weiterhin zwischen Klassen und Instanzen, entsteht eine Ontologie.

Mit einem Thesaurus lassen sich auf einer mittleren Komplexitätsstufe schon ganz interessante Begriffssysteme vergleichsweise differenziert modellieren. Das Konzept ist insbesondere im Bibliothekswesen mit verschiedenen Normen (z.B. ISO 25964) bestens eingeführt. Wir verwenden in der Lehre unserer Terminologiearbeit das Begriffsthesaurus-Schema Simple Knowledge Organization System (SKOS) ([MiBe09]), auf dem z.B. auch der multilinguale EU-Thesaurus EuroVoc ([EuroVoc]) basiert.

Ein Thesaurus ist gerade auch in der digitalen Informationsflut ein grundlegendes sachlogisches Mittel zur Strukturierung von Inhalten. Darüber hinausgehend stellt Terminologiearbeit im Allgemeinen und speziell mit SKOS eine gute Möglichkeit dar, Studierende in eine Vielzahl von grundlegenden Ideen der Informationswissenschaften einzuführen, unter anderem Unterscheidungen zwischen (a) Wort (term) und Begriff (concept), und damit auch zwischen (b) Wort- vs. Begriffsthesaurus; (c) Unterscheidung zwischen Intension und Extension eines Begriffs; (d) Unterscheidung von subclass (Klassifikation, Taxonomie) und part-of (Baugruppenzerlegung, Meronomy) u.a. 


\section{$1.2 \quad$ Inhalt und Methode}

Obwohl Inhalt und Didaktik höchst unterschiedliche Begriffe sind, wollen wir sie nicht als konkurrierende Pole, sondern als unabhängige Achsen eines Koordinatensystems behandeln. Auch diese Modellbildung hindert uns nicht daran, extreme Standpunkte einzunehmen: In rein allgemeindidaktischen Überlegungen sehen wir von spezifischen Inhalten ab und diskutieren Methoden, Medien, Sozialformen etc. Demgegenüber sehen wir in rein inhaltlichen Diskursen üblicherweise von der Didaktik ab. Oft repräsentieren wir hier Inhalte lediglich als Themenlisten, wie sie z.B. auch in den aktuellen Empfehlungen der Gesellschaft für Informatik e.V. zur Wirtschaftsinformatik ([GI2017]) enthalten sind.

Verbinden wir Inhalt und Didaktik zu einer Fachdidaktik, wollen wir zumeist, dass das Ganze mehr sei als die Summe seiner Teile. Doch worin besteht dieses Mehr? Erste Anhaltspunkte gibt uns ein systematischer Perspektivenwechsel:

- $\quad$ Perspektive 1 blickt vom Fach heraus in die Didaktik hinein und versucht, Elemente der Didaktik als Thema des eigenen Faches zu erkennen. In den Gl-Empfehlungen zur Wirtschaftsinformatik hat eine solche Inkorporation beispielsweise dort stattgefunden, wo als integraler Bestandteil von Wissensmanagement explizit auch E-Learning aufgeführt wird.

- $\quad$ Perspektive 2 blickt von der Didaktik heraus auf die Fächer und versucht von diesen zu profitieren; eine solche Inkorporation ist etwa für didaktische Innovationen im Kielwasser technischer Entwicklungen - Stichwort E-Learning - ganz typisch.

Solch ein Perspektivenwechsel ist erhellend, auch wenn ihm ein gewisser Imperialismus anhaftet: Solange man Perspektiven lediglich wechselt, erkennt man nichts Neues, sondern lediglich das Andere als etwas Eigenes wieder. Neues verspricht nicht ein Wechsel, sondern eine Integration von Perspektiven. Wir versuchen eine solche Integration im letzten Abschnitt unserer Argumentation durchzuführen, wo wir digitale Terminologiearbeit als eine innovative Form des E-Learnings interpretieren.

\subsection{Wirtschaftsinformatik}

Die Wirtschaftsinformatik kann als Fach einerseits in ihrem Umfang (also extensional) durch eine umfangreiche Liste von Inhalten charakterisiert werden, wie dies z.B. in den GI-Empfehlungen zur Wirtschaftsinformatik durchgeführt wird. Mit dem Titel unseres Artikels befinden wir uns inhaltlich etwa in Abschnitt 5.10: „Wissensmanagement und kollaborative Technologien f) Wissensakquisition und Wissensverteilung: [...] individuelles und organisationales Lernen; E-Learning; [...] g) Methoden des Wissensmanagements in schwachstrukturierten Datenbeständen: Wissensmodellierung und -visualisierung (u. a. Ontologien, Semantic Web, Topic Maps, Taxonomien)“ ([GI2017], S.21).

Komplementär kann die Wirtschaftsinformatik durch die Methodologie charakterisiert werden, mit der hier gearbeitet wird. Aus professionstheoretischer Sicht ein guter Ausgangspunkt für wissenschaftspraktische Diskurse ist [Hev04] sowie das „Memorandum Wirtschaftsinformatik" ([Öe++10]). Wir wollen diese beiden Texte als Intension, d.h. als konsensfähige Sammlung von charakteristischen Merkmalen des Faches Wirtschaftsinformatik betrachten ([Zele07]). Konsequenterweise ziehen wir diese beiden Texte auch zur Lektüre in unserer Erstsemesterveranstaltung „Grundlagen der Wirtschaftsinformatik“ heran. 


\section{Unser fachdidaktisches Lehr-Lern-Arrangement}

In unserer Erstsemesterveranstaltung "Grundlagen der Wirtschaftsinformatik“ sollen die Studierenden eine Einführung erhalten (a) in Umfang und Charakteristik der Wirtschaftsinformatik als Wissenschaft, (b) in die Terminologiearbeit als Grundlage jeder wissenschaftlichen Beschäftigung mit Inhalten, sowie (c) in die aktive selbstgesteuerte Auseinandersetzung auch mit ungewohnten - hier: wissenschaftlichen - Textsorten. Diese drei Themen verbinden wir wie folgt:

- Im ersten Studienmonat lernen die Studierenden die Grundidee des Begriffsmodells "Thesaurus“ als eine Anwendung der Wissensrepräsentation „Semantisches Netz" und die Kernideen der Wissensrepräsentation „Thesaurus“ kennen. Die Vorlesung hierzu vermittelt die nötigen Kenntnisse; in der Übung erwerben die Studierenden Fertigkeiten im Umgang mit der Open-Source-Mindmap-Software www.freemind.org oder www.freeplane.org. Es hat sich als hilfreich erwiesen, hier bis in die Niederungen der praktischen Handhabung zu gehen, incl. Auswendiglernen von rund einem Dutzend Tastaturkürzeln, damit die Studierenden einen Produktivitätsfortschritt aufgrund digitaler Wissensrepräsentationen erleben können.

- Im zweiten Studienmonat erwerben und habitualisieren die Studierenden die Fertigkeit, in Prosatexten enthaltenes Wissen als explizite semiformale Wissensrepräsentation höher strukturiert darzustellen, indem sie es als Mindmap und Thesaurus digital repräsentieren. Wir üben dies zunächst auf Basis strukturell unproblematischer Wissenstypen wie der Terminologie auch aus dem Themenbereich „Thesaurus“ selbst (vgl. Abb. 1). Natürlich kann dann auch in der Abschlussklausur die Kompetenz, einen Thesaurus zu entwickeln, eingefordert werden - womit man auch gleich ein innovatives Prüfungsformat an der Hand hat.

- Im dritten Studienmonat wenden die Studierenden die so erworbenen Kenntnisse und Fertigkeiten auf den Lehrinhalt "Grundlagen der Design Science“ selbst an. In der Vorlesung führen wir in den Aufsatz von [Hev04] ein und kommentieren die zugehörige Diskussion. In begleitenden Lerngruppen im Rahmen der Übung erarbeiten sich die Studierenden die Lektüre in größerer Tiefe und sichern die Ergebnisse in Form einer digitalen Mindmap und/oder eines Thesaurus.

Im Zeitablauf wird hier ein Dreischritt vollzogen, in welchem der Inhalt „Konstruktion des Artefakt-Typs Terminologie“ zuerst theoretisch und sodann praktisch grundgelegt und zuletzt auf den Grundlagenaufsatz [Hev04] selbst angewandt wird. In der Didaktik nennt man solche Arrangements, in denen ein methodischer Inhalt durch die Methode selbst repräsentiert wird, einen didaktischen Doppeldecker. Innerhalb unserer Einführung in die Wirtschaftsinformatik entpuppt sich dieses didaktische Pattern als fachlicher Inhalt, da das sogenannte Hidden Curriculum der Methode „Modellierung von Begriffssystemen in Mindmaps“ dem Inhaltsaspekt „Artefakte der Design Science“ als anschauliches Beispiel dient. Indem wir die Konstruktion eines Design-Science-Artefakt-Typs gleichzeitig als Inhalt lehren und als Methode des digitalen Lernens einsetzen, verfügen wir über ein kompetenzorientiertes Bootstrap-Verfahren, mit dem Studierende vom ersten Semester an präzise wissenschaftliche Begriffsarbeit üben können. 


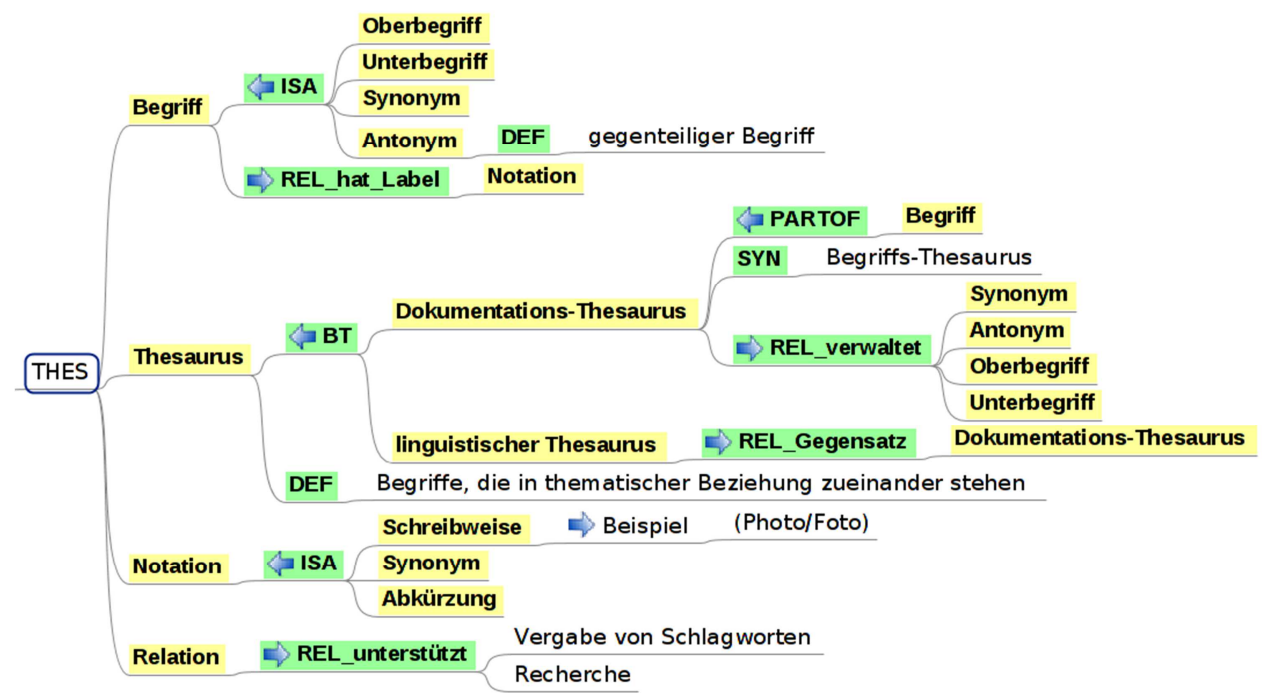

Abbildung 1: Die Aufgabenstellung lautete: „Markieren Sie im Text die wichtigsten Fachbegriffe und erstellen Sie einen Thesaurus in Mindmap-Form. Verwenden Sie jede der Relationen bt, isa, partof, rel, syn, def mindestens einmal. Achten Sie auch auf eine lesefreundliche Darstellung!"

Zugrundeliegender Text (JB, auf Basis von Wikipedia>Thesaurus): „Im Gegensatz zu einem linguistischen Thesaurus besteht ein Dokumentations-Thesaurus aus Begriffen, die in thematischer Beziehung zueinander stehen. Es werden in erster Linie Synonyme, aber auch Ober- und Unterbegriffe verwaltet. Oft werden jedoch keine Antonyme (gegenteilige Begriffe) aufgeführt. Relationen zwischen den einzelnen Begriffen unterstützen die Vergabe von Schlagworten und die Recherche. Notationen wie z.B. unterschiedliche Schreibweisen (Photo/Foto), Synonyme oder Abkürzungen etc. werden einem Begriff durch eine Label-Relation zugeordnet".

\section{Didaktische Innovation durch Digitalisierung}

Im vorangehenden ersten Abschnitt haben wir das Thema Terminologiearbeit curricular strukturiert; im zweiten Abschnitt haben wir aufgezeigt, wie man Inhalt und Methode des grundlegenden allgemeinwissenschaftlichen Themas Terminologiearbeit in der Lehre der Wirtschaftsinformatik fachdidaktisch begründen und implementieren kann. Im vorliegenden dritten Abschnitt werden wir zeigen, dass in dem dargestellten Lehr-Lern-Arrangement eine weitere - hier aus der Wirtschaftsinformatik kommende - allgemeindidaktische Innovation enthalten ist. Dazu betrachten wir den Begriff E-Learning wieder aus der Perspektive der Design Science und interpretieren inn nun wie folgt:

\section{E-Learning $=$ Lernen durch die Arbeit an digitalen Artefakten}

Für Informatiker ist die Arbeit mit digitalen Artefakten im Modus der klassischen Programmierung und Softwareerstellung tägliches Brot; aus Sicht der Design Science im Allgemeinen sowie des vorliegenden Aufsatzthemas handelt es sich hierbei zunächst lediglich um Datenmodellierung, also ebenfalls nichts Neues. Programmieren ist eine der ältesten und am weitesten verbreiteten Methoden digitalen Lernens, auch wenn diese Methode bisher nicht ganz leicht auf andere Fächer übertragbar war. Gleichzeitig hat sich in den vergangenen zwei Jahrzehnten nicht nur ein technologischer und digitaler Fortschritt, sondern auch ein Fortschritt im Wissensbestand der Informatik, insbesondere in der Entwicklung Semantischer Technologien als Theoriehintergrund für unsere Terminologiearbeit ereignet, der sich 
auch als didaktischer Fortschritt erweisen könnte - namentlich indem sich Terminologiearbeit als Methode digitalen Lernens fächerübergreifend etabliert.

Dass Studierende besser lernen, wenn sie Wissensrepräsentationen selbst aktiv herstellen, ist weithin bekannt. Doch woraus resultieren der spezifische Mehrwert - und möglicherweise ein weiterer Innovationsschritt - digitaler Wissensrepräsentationen fürs Lernen?

Allzu oft werden die Möglichkeiten des Digitalen auf Lagerung, Transport oder Verteilung von Lernmedien beschränkt. Wenn ein klassisches Buch im pdf-Format zur Verfügung gestellt wird oder eine klassische Vorlesung als Video bereitgestellt wird, ist das nach unserem Verständnis noch lange kein E-Learning. Mehr noch: Wenn die sog. Neuen Medien dazu verwendet werden, überkommene dysfunktionale Strukturen virtuell zu replizieren, bekommen sie reaktionären Charakter ([BuKK98]).

Demgegenüber suchen wir nach einer Spezifik des Digitalen für Lernen und Prüfen, die sich von solchen traditionellen Nutzungsweisen abhebt und stattdessen das Alleinstellungsmerkmal des Digitalen betont. Unseres Erachtens besteht dieses Alleinstellungsmerkmal in der formalen Struktur und der maschinellen Interpretierbarkeit von digitalem Text, welche die gesuchte didaktische Relevanz des Digitalen erzeugen.

Indem wir Terminologiearbeit als Einstiegstechnologie der semantischen Modellbildung nicht nur als Inhalt, sondern auch als Methode und Werkzeug studentischen Lernens einsetzen, verfügen wir über eine Methode, die sich von traditionellen E-Learning-Ansätzen abhebt. Die Fertigkeit, natürlichsprachliche Texte sowie die darin enthaltenen logisch-begrifflichen Zusammenhänge formal in einem Thesaurus oder anderen semantischen Strukturen zu modellieren, sollte zur grundlegenden wissenschaftlichen Ausbildung in allen Fächer gehören. Die didaktische Praxis zeigt, dass sich eine solche Terminologiearbeit als Inhalt und als Methode passgenau in die Spezifik der digitalen Transformation einfügt.

\section{Literaturverzeichnis}

[BuKK98] Busse Johannes; Krause Detlev; Klaeren Herbert: Verbundlehre als Synthese von Ferne und Präsenz. 1998. In: Didaktisches Design 3/1998

[Bu++14] Busse Johannes: Semantische Modelle mit Mindmaps. In: S. A. Keller, R. Schneider, B. Volk (Hrsg.): Wissensorganisation und -repräsentation mit digitalen Technologien. Walter DeGruyter 2014.

[Bu++15] Busse, J.; Humm B.; Lübbert C.; Moelter F.; Reibold A., Rewald M., Schlüter V.; Seiler B.; Tegtmeier E.; , Zeh T.: Actually, what does "ontology" mean? Journal of Computing and Information Technology (CIT), Vol 23, No 1 (2015), pp. 29-41.

[EuroVoc] EuroVoc, mehrsprachiger Thesaurus der Europäischen Union. http://eurovoc.europa.eu/drupal/?q=de

[Hev04] Hevner Alan R.; March Salvatore T.; Park Jinsoo; Ram Sudha: Design Science in Information Systems Research. MIS Quarterly Vol. 28 No. 1, pp. 75-105/March 2004

[GI2017] Gesellschaft für Informatik e.V. (GI): Rahmenempfehlung für die Ausbildung in Wirt$\begin{array}{llll}\text { schaftsinformatik an Hochschulen, } & \text { März } & \end{array}$ https://www.gi.de/service/publikationen/empfehlungen.html > https://www.gi.de/fileadmin/redaktion/empfehlungen/Empfehlung-Wirtschaftsinformatik2017.pdf 
[MiBe09] Miles Alistair; Bechhofer Sean (ed.): SKOS Simple Knowledge Organization System Reference. W3C Recommendation 18 August 2009. https://www.w3.org/TR/skos-reference/

[Öe++10] Österle Hubert u.a.: Memorandum zur gestaltungsorientierten Wirtschaftsinformatik. In: Zeitschrift für betriebswirtschaftliche Forschung zfbf 62 (2010) 6, S. 66-679.

[Zele07] Kann Wissenschaftstheorie behilflich für die Publikationspraxis sein? in: Franz Lehner, Stephan Zelewski, St. (Hrsg.): Wissenschaftstheoretische Fundierung und wissenschaftliche Orientierung der Wirtschaftsinformatik. GITO Verlag, 2007, S. 71-120

\section{Kontakt}

Prof. Dr. Johannes Busse

HAW Landshut

Am Lurzenhof 1, 84036 Landshut

busse@haw-landshut.de, http://www.jbusse.de 


\title{
Steigerung der Attraktivität von Studiengängen der Wirtschaftsinformatik für Frauen und Männer durch geschlechtersensitives Branding
}

\author{
Dörte Resch, Iris Graf, Anke Dreiling, Jonas Konrad
}

\section{Zusammenfassung}

Der Frauenanteil in ICT-Studiengängen ist tief; auch in dem Bachelorstudiengang Wirtschaftsinformatik der hiesigen Hochschule, wo er nur 5\% beträgt - und dies, obwohl der betriebswirtschaftliche Anteil des Studiums durchaus auch Frauen ansprechen könnte. Gleichzeitig steigt der Bedarf an WirtschaftsinformatikernInnen und somit der Druck, Zielgruppen anzusprechen, die sich trotz Eignung bisher weniger für die Wirtschaftsinformatik interessiert haben. Wie Forschungsergebnisse zeigen, ist in der Phase der Berufs- und Studienwahl die Identitätskonstruktion von jungen Frauen und Männern eher von traditionellen Geschlechterrollen geprägt. Widersprechen die in den Studiengängen verankerten Identitäten diesen Selbstbildern, wird ein Studiengang für die jeweilige Anspruchsgruppe eher unattraktiv. Hieraus ergibt sich die Forschungsfrage, wie diskursiv erzeugte Identitäten der Wirtschaftsinformatik so rekonstruiert werden können, dass diese auch für «untypische» junge Frauen und Männer attraktiv werden. Es wurde diskursanalytisch ausgewertet, welche Identitätsdiskurse die Studiengänge mehr oder weniger attraktiv für diese Zielgruppe erscheinen lassen. Es zeigt sich, dass Ausbildungen als besonders unattraktiv wahrgenommen werden, wenn sich verschiedene Diskurse gegenseitig konfundieren. Der Beitrag zeigt, dass an Konstruktionen wie «ICT als Dienstleistung» und «Lernbarkeit» angeschlossen werden sollte, da sie junge Frauen und Männer gleichermaßen ansprechen. Auf Basis der Ergebnisse wurde mit den Stakeholdern der Studiengänge erarbeitet, wie eine neue Markenidentität entstehen kann, die auch bisherige Zielgruppen nicht ausschließt.

\section{Wie kann der Frauenanteil in ICT-Ausbildungen und Studiengängen erhöht wer- den?}

Die Verbände der Informations- und Kommunikationstechnologie der Schweiz wie auch Economiesuisse weisen stetig auf die Bedeutung der Informations- und KommunikationsBranche (ICT) für die Schweizerische Wirtschaft hin. Diese ist nunmehr mit bereits $5.6 \%$ der nationalen Wertschöpfung die fünft-bedeutendste der Schweiz (s. [Econ13], S. 7). Die Anzahl der ICT-Arbeitsplätze stieg in der Schweiz in den Jahren 2003 bis 2011 um fast 20\% auf $177^{\prime} 000$. Diesem steigenden Bedarf nach Fachkräften steht eine nicht ausreichende Anzahl an jungen nachrückenden Fachkräften gegenüber [BrLG16]. Der bereits bestehende und zunehmende Mangel an qualifizierten ICT-Fachkräften besteht von der Berufslehre bis zum Hochschulstudium.

Die Branche hat erkannt, dass aktuell ein ungenutztes Potential bei weiblichen Fachkräften besteht, welches für eine wirksame Bekämpfung des Fachkräftemangels genutzt werden sollte. Eine Schlüsselfrage hierzu ist, warum vergleichsweise wenige Frauen eine Ausbildung in diesem Bereich aufnehmen und wie dies wiederum positiv beeinflusst werden kann [Bebb02]. In der Geschlechterforschung wird dieses Phänomen der geschlechterspezifi- 
schen Prägungen als "gendering" bezeichnet [WeZi91]. Wenn diese als kulturell geprägt verstanden wird, hat das den Vorteil, dass sich diese kulturellen Prägungen analysieren und verändern lassen. Die Veränderung kultureller Prägungen ist aber kein einfacher Vorgang, da diese Verständnisse oftmals so selbstverständlich sind, dass sie einer Reflexion nicht zugänglich sind. Daher müssen diese "Selbstverständlichkeiten" zunächst nachvollziehbar analysiert werden, was als De-Konstruktion bezeichnet wird [Kela10], um dann in einem zweiten Schritt einer Veränderung zugänglich gemacht zu werden, in dem eine neue Zusammensetzung der Verständnisse erarbeitet wird. Dieser zweite Schritt der Re-Konstruktion erlaubt es, dass Verständnisse der ICT-Berufe und Studiengänge entwickelt werden, die sowohl für junge Frauen, wie auch junge Männer attraktiv sind.

Die Forschung im Rahmen eines Doing Gender untersucht, wie sich diese gegenderten Identitäten erzeugen [AiBB14]. Immer mehr wird aber auch im Rahmen des Un-doing Gender untersucht, wie geschlechterspezifische Bedeutungen und Identitäten aufgelöst werden können [Kela10] und somit erforscht, wie Berufe und Berufsfelder für beide Geschlechter attraktiv werden können. Hier gilt es in den Fokus zu nehmen, wie die geschlechtergeprägte Identität der Berufe diese eher attraktiv - oder unattraktiv - für junge Frauen oder Männer macht. Im Sinne von Martin Parker [Park04] stellte sich hier die Frage: Mit wem oder was identifizieren wir uns und wie kann eine positive und erstrebenswerte Identifikation mit ICTBerufen auch für Frauen möglich werden? Diese Frage ist wichtig, um dem Fachkräftemangel in den ICT-Berufen zu begegnen; aber auch, weil sich die Anforderungen an ICT-Fachkräfte im Wandel befinden. Rein technische Programmiertätigkeiten werden immer stärker in das Ausland verlagert, wohingegen Kompetenzen der Beratung und Anwendung wichtiger werden [swis13].

\section{Forschungsverständnis und Forschungsfragen}

\subsection{Forschungsverständnis: Sozialer Konstruktionismus und Diskursanalyse}

Diskursanalyse bezieht sich auf eine Forschungstradition, die als Basisannahme davon ausgeht, dass Realitäten sprachlich erzeugt werden. Sprache wird nicht mehr als Spiegel von Realität gesehen, sondern die Realität wird als durch Sprache erzeugt verstanden [Burr03]. "Sprache" wird in diesem Ansatz sehr breit verstanden und schließt auch Handlungen und die Zuschreibung der jeweiligen Bedeutungen mit ein. Demnach müssen, wenn Bedeutungen und Verständnisse verändert werden sollten, Sprechakte analysiert werden. Durch diese Analyse wird der Prozess der Bedeutungsgenerierung wieder einem Bewusstsein und damit Veränderung zugänglich. "Diskurs" bezeichnet in diesem Zusammenhang nicht nur eine Art Unterhaltung, sondern ein Set an Bedeutungen, Metaphern, Geschichten und Statements, die zusammen eine bestimmte Version eines Events produzieren [Burr03]. Somit wird das jeweilige Verständnis der Geschlechter aktiv hergestellt und so im Umkehrschluss auch einer Veränderung zugänglich gemacht [WeZi91].

\subsection{Identitäten analysieren: Doing und Un-Doing von Gender}

Insgesamt fokussiert ein gewichtiger Anteil der Forschung zur Gendered Segregation of Work stark auf die Aspekte des Doing Gender, d.h. zu verstehen, wie in Situationen Sinnhaftigkeit erzeugt wird und wie sich aktuelle Ungleichheiten reproduzieren [LLSS14]. Weniger im Fokus ist, wie im Sinne eines Un-doing Gender ein Image des Berufes entstehen kann, welches für beide Geschlechter attraktiv ist. Wichtig ist beim Thema der Re-Konstruk- 
tion, dass damit nicht gemeint ist, einfach "coole Mädchen" auf Broschüren abzubilden (z.B. kritisiert von [IT-D14]). Dies reicht nicht aus, um sich vom vorherrschenden Klischee des männlichen "Nerds" zu verabschieden. Es ist wichtig, grundlegend die Identität der Profession zu analysieren und dann neu zu entwickeln. Identifizierungen sind immer multikontextuell geprägt [Park07], weswegen man sich nicht nur auf das Thema der Geschlechter beschränken sollte. Es gilt die relevanten Bezüge der Identitätskonstruktion in der Untersuchung hinsichtlich des Phänomens aufeinander zu beziehen [BaLa15]. So kann untersucht werden, wie diese Ebenen so interagieren, dass die Persistenz der geschlechterspezifischen Segregation verstanden und verändert werden kann.

\subsection{Zentrale Forschungsfragen}

Im Rahmen dieses Projektes wird sich auf Identität im Foucault'schen Verständnis bezogen, wonach Individuen bestimmte Subjektpositionen, d.h. Wege sich selbst zu verstehen, einnehmen. Diese sind durch "große Diskurse" bestimmt, die bestimmte Subjektpositionen in Relation zum Diskurs als sinnvoll erscheinen lassen. Identitäten ergeben nur Sinn und damit Anschlussfähigkeit, wenn sie sich an breitere Bedeutungszuschreibungen anschließen. Identität entsteht niemals "aus sich selbst heraus", sondern ist im Rahmen von sozialen und historisch geprägten Kontexten zu verstehen. Mittels einer Analyse kann verstanden werden, wie Diskurse die jeweiligen Subjektpositionen von jungen Frauen und Männern im Sinne des Interesses für einen Beruf im ICT-Bereich steuern [KeWW11]. Der Fokus liegt darauf, wie Individuen die gegenderte Identität produzieren und reproduzieren [AIBi09]. Die Fragestellung zur Analyse im Sinne der De-Konstruktion lautet:

Welche (gegenderten) Identitätskonstruktionen sind bei den vorhandenen Professionsidentitäten der ICT zu finden?

Der zweite Schritt der Untersuchung, die Re-Konstruktion der Identitätsdiskurse und Auflösung der horizontalen Segregation, muss die Diskurse in eine neue Relation zueinander setzen. Der Bereich, der sich stark mit einer Re-Konstruktion im Sinne einer Identitätsbildung beschäftigt hat, ist das so genannte Branding. Im Rahmen dieses Projektes soll "Branding" so verstanden werden, dass der Brand (d.h. die "Marke") die verschiedenen Stakeholder an den Beruf bindet [HaSc08]. Die Re-Konstruktion muss daher zu einer eindeutigen und geschlechterintegrativen "Marke" werden. Branding wird als Prozess verstanden, der Vision, Kultur, Strategie und deren Bilder durch einen Branding-Prozess miteinander verbindet und integriert an Zielen ausrichtet. Es geht somit darum, die jeweiligen neuen Bedeutungen und Werte so zusammenzusetzen, dass sie für die Zielgruppen ansprechend sind [AMRS12]. Die Leitfrage des zweiten Teils lautet:

Wie können die (gegenderten) Identitäten von Frauen (und anderen "Untypischen") mittels eines aktiven Branding-Prozesses der ICT-Berufe (Studium und Branche) in eine positive Relation gebracht werden?

Wichtig ist, dass der Branding-Prozess nur als ein erster Schritt zu sehen ist, um Identifizierungsprozesse von Frauen und Männern mit ICT-Berufen zu ermöglichen. Das Leben von Individuen und deren komplexe Verknüpfungen mit gesellschaftlichen Diskursen und institutionellen Rahmenbedingungen wirken als Begrenzung der Strahlkraft des Brandings einer Profession [Simm11]. 
Das Ziel ist, auf Basis der ermittelten "Brand-Identitäten", Identitäten der Professionen vorzuschlagen, die anschlussfähig an die Identitätskonstruktionen von Frauen sind und diese dann mit einem "Brand Image" (Markenidentität) zu verbinden [BrPP11]. Diese Vorschläge bieten eine fundierte Basis, anhand derer sich Studiengänge und Berufsverbände ausrichten können, um den Beruf für Frauen und Männer attraktiv zu machen. Wichtig ist aber auch, dass das Versprechen, das mit dem "Image" des Brands abgegeben wird, eingehalten wird. Nur so kann auch für Frauen eine längerfristige Identifizierung mit dem Beruf ermöglicht werden.

\section{Forschungsmethoden und Gütekriterien}

Die Erhebungs- und Auswertungsmethoden werden im folgenden Abschnitt genauer dargestellt. Da diese Studie als eine explorative angelegt war, wurde eine sozialkonstruktionistische Perspektive [Gerg99] im Rahmen eines poststrukturalistischen Paradigmas (nach [Deet96]) eingenommen. Das Sampling wurde im Sinne eines theoretischen Samplings durchgeführt [Flic10], um eine maximale Variation im Sample zu erhalten und gleichzeitig möglichst "typische" Fälle zu erheben.

Die verfügbaren Dokumente, die die ICT-Berufe darstellen und als reichhaltige Quelle so genannter festgeschriebener Positionierungen verstanden werden können (s. [Czar11], S. 86), werden hinsichtlich des im- und expliziten Genderings der Darstellung der Profession untersucht. Insgesamt wurden 49 Dokumente analysiert.

Als Interviewmethode wurde das Problemzentrierte Interview (nach [Witz00]) verwendet, da es für eine Erhebung von Identitäten notwendige narrative Elemente mit thematisch fokussierten Elementen verbindet. Ergänzt werden die Interviews mit Gruppendiskussionen. Insgesamt wurden 39 Interviews und Gruppendiskussionen mit total 84 Personen durchgeführt. Bei der Transkription der Interviewdaten und Gruppendiskussionen wurde sich an Wetherell [Weth01] und deren Transkriptionsregeln orientiert. Sämtliche Gespräche wurden bei der Transkription anonymisiert.

Als Auswertungsmethode wurde eine integrative Diskursanalyse durchgeführt. Es wurde erstens analysiert, welche Diskurse wie zu einer stabilisierenden Verknüpfung gerenderter Identitäten der ICT-Profession beitragen. Und zweitens, da Realitäten niemals eindeutig sind und sich Situationen verändern, welche Variationen die jeweiligen dominanten Diskurse in ihrer Nutzung erfahren. Ziel der Diskursanalyse war u.a. zu verstehen, welche sprachlichen Strategien von Individuen verwendet werden, um Widersprüchlichkeit gerecht zu werden und diese aufzugreifen [Chia00].

Die Güte der Untersuchung wurde in einem iterativen Prozess im Laufe der Untersuchung, aber insbesondere im Rahmen der Erhebung und Auswertung reflektiert und die passenden Gütekriterien entwickelt. Dabei wurde sich zum einen an den Gütekriterien für qualitative Forschung allgemein orientiert [Hopf05].

\section{$4 \quad$ Ergebnisse}

ICT und Computer erscheinen als untrennbar miteinander verknüpft: Diese Grundannahme liegt allen Diskursen zugrunde, die in den folgenden Kapiteln beschrieben werden. In den 
durchgeführten Interviews und Gruppendiskussionen fungierte der Computer als das Symbol für ICT schlechthin.

Dies bedeutet, dass aus Laienperspektive ICT unhinterfragt eng mit dem Gerät verknüpft ist, obwohl in der Praxis sehr viele interaktive Elemente integriert und diese immer bedeutender werden. Die Diskurse zur ICT-Profession bauen allesamt auf dieser grundlegenden Annahme auf. Die Konsequenz ist, dass Abbildungen von ICT-Fachpersonen mit einem PCBildschirm das Image des "Nerds" sehr viel mehr verstärken, als wenn andere Fachpersonen bei der Bildschirmarbeit abgebildet werden.

Der Diskurs «Technik als Basis» wird von Fachpersonen und Laien getragen, die ICT häufig direkt mit Programmieren oder Hardware verbinden. Aber auch Berufsbeschreibungen und Informationen zu Ausbildungen fokussieren meist auf technische Aspekte: Als Haupttätigkeiten von Informatikerinnen und Informatikern erscheint Programmieren, Installieren und der Umgang mit Hardware und Software. Diese "technische Basis" von ICT-Fachpersonen wird als notwendige Grundlage konstruiert, über die Informatikerinnen und Informatiker verfügen müssen. Weitere Kompetenzen - z.B. im sozialkommunikativen Bereich - sind bei ICT-Fachpersonen ebenfalls erwünscht, doch erscheinen sie im Rahmen dieses Diskurses nur als "Add-On". Mit dieser Konstruktion von ICT geht außerdem einher, dass Technik als besonders schwierig positioniert wird. Sie erscheint als Bereich, der hohe Anforderungen stellt, die nur von besonders begabten oder geeigneten Personen erfüllt werden können. Technikkompetenz wird damit zu etwas, das nicht jeder Person zugänglich ist und das nicht "nebenher" erlernt werden kann. Dies steht im Gegensatz zu Anforderungen in anderen Bereichen wie Wirtschaft, Soziales oder Kommunikation, die als einfacher zugänglich dargestellt werden. Besonders gut lässt sich das im Bereich Wirtschaftsinformatik beobachten: obwohl die Fächergruppen der Wirtschaft integral im Studium verankert sind, werden ausschließlich Informatikfächer als schwierig und relevant gesehen. Die Konsequenzen dieses Diskurses sind, dass dem Fachbereich Prestige und Expertenstatus zugeschrieben wird. Gerade gegenüber Laien genügt ein vergleichsweise geringer Wissensvorsprung und somit erscheinen die Profession und der Beruf als schwierig.

Prestige erhöht die Attraktivität, hat aber auch ausschließende Effekt, weil sich untypische interessierte Jugendliche den Einstieg nicht zutrauen.

Parallel zum Diskurs «Technik als Basis» existiert die diskursive Konstruktion von Dienstleistung als Schwerpunkt der ICT-Profession. Hier wird als wichtigstes Ziel der ICT nicht technische Lösungen, sondern die Dienstleistung an Kundinnen und Kunden beschrieben. Am Anfang jeder technischen Entwicklung steht die Frage, welche Bedürfnisse und Probleme Kund/innen haben und wie diese befriedigt werden können. In diesem Diskurs wird auch der Nutzen der ICT allgemein in den Fokus gerückt: die Informatik bietet Lösungen und Innovationen für Bedürfnisse, die in einem bestimmten Wirtschaftskontext aktuell werden.

Dieser Diskurs knüpft an den Wandel der ICT in den letzten Jahren an. In Interviews und Gruppendiskussionen wurde oft hervorgehoben, wie sehr dieser Wandel aktuell und besonders zukünftig ICT-Berufe und die geforderten Qualifikationen beeinflussen würde. Dies wird damit begründet, dass zunehmend mit agilen Methoden gearbeitet werde, die es erlaubten, schnell Produkte und Dienstleistungen zu entwickeln und während der Entwicklung ständig auf sich ändernde Anforderungen zu reagieren.

Eine Konsequenz dieses Diskurses ist, dass Technik zum Mittel zum Zweck und nicht als Selbstzweck positioniert wird. Die Bedürfnisse der Kundlnnen werden in den Vordergrund gerückt und dadurch, dass diese ermittelt werden müssen, werden soziale und kommunika- 
tive Aspekte zentraler. Eine unerwünschte Konsequenz ist, dass sich im Rahmen dieser diskursiven Logik das Prestige der Profession reduziert, da diese Aspekte als einfacher erlernbar gesetzt werden. Gleichzeitig erhöht sich aber die Zugänglichkeit des Berufs.

Die Diskurse zur Lernbarkeit von Fähigkeiten und Interessen nehmen eine zentrale Rolle ein. Wenn die fachlichen Fähigkeiten und das Interesse am Fach als natürlich gegeben gesetzt werden, wird dies als Voraussetzung für Studium und Ausbildung gesetzt. Dies impliziert, dass Fähigkeiten und Interessen nicht oder nur teilweise erlernt werden können und von Geburt an oder im Kindesalter festgelegt werden. Die Verteilung von Fähigkeiten über Individuen wird als unterschiedlich angenommen. Dies lässt den Schluss zu, dass sich gewisse Individuen für ICT-Berufe eignen, andere nicht. Vorwissen wird als Indikator für "echtes" Interesse gedeutet. Allerdings werden an einer kaufmännischen Lehre interessierte Personen kaum gefragt, ob sie sich in ihrer Freizeit bereits mit Buchhaltung beschäftigt haben.

Die Konsequenzen für die Zugänglichkeit von ICT-Berufen sind, dass eine Auswahllogik für Lernende und Studierende konstruiert wird, die davon ausgeht, dass all jene geeignet seien, welche den Beweis für ihr Vorwissen erbringen können. Eine Lernbarkeit der Inhalte wird tendenziell ausgeschlossen. Es wird hervorgehoben, dass sich geeignete Personen «schon immer» für den ICT-Bereich interessiert haben. Diese «schon immer-Narration» schließt sich an den Naturalisierungsdiskurs an und verstärkt inn. Es zeigt sich auch, dass Interviewpersonen, die nicht berichten können, sich «schon immer» für den ICT-Bereich interessiert zu haben, große identitätsstiftende narrative Anstrengungen unternehmen, um sich als für den Beruf geeignet zu positionieren. So wird zum Beispiel eine Erstausbildung in Physiotherapie als technische Ausbildung positioniert, da der Mensch ja auch ein technisches Wesen sei. Des Weiteren ist die gegenderte Zuschreibung von Fähigkeiten und Interesse eine Konsequenz. Als natürlich gesetzte technische Fähigkeiten und Interessewerden viel eher Männern als Frauen zugeschrieben: Umgekehrt werden Frauen eher natürliche sozialkommunikative Kompetenzen zugeschrieben. Dies kann als Erklärung genutzt werden, weshalb Frauen sich weniger für ICT-Berufe interessieren und warum sie dafür schlechter geeignet sein könnten. Anhand dessen wird nun auch deutlich, warum der "Nerd" - die als typisch wahrgenommene ICT-Fachperson - als männliche Person konstruiert wird. Wenn Männern stärkeres Technikinteresse zugeschrieben wird als Frauen, dann wird Männern auch eher zugetraut, sich einseitig, vertieft und auch in ihrer Freizeit mit technischen Fragen zu befassen - wie das dem Nerd auch zugeschrieben wird. Außerdem gilt der Nerd als unsozial und unkommunikativ, was im Gegensatz zu den Zuschreibungen zu Frauen steht. Das hat eine ambivalente Wirkung auf den Zugang von Frauen zu ICT-Berufen: Sie erscheinen als weniger passend und geeignet, allerdings dürfte es Frauen leichter gelingen, sich vom Nerd-Image zu distanzieren.

Wenn Fähigkeiten und Interesse hingegen als Entwicklungsfähig konstruiert werden, treten einige dieser unerwünschten Konsequenzen weniger auf. Wenn beide als entwicklungsfähig positioniert werden, erscheint ein Studienerfolg mit hinreichendem Engagement als möglich: Es kommt in dieser narrativen Verknüpfung darauf an, dass das jeweils individuelle Engagement für Thema und für das Fach stimmen müsse. Eine Konsequenz ist, dass die Bedeutung von Lehr- und Lernkontexten steigt, es wird wichtiger, dass diese lernförderlich gestaltet sein müssen. Gleichzeitig erhöht sich die Schwierigkeit eine Auswahl der geeigneten KandidatInnen zu treffen und die Zuschreibung der Verantwortung für Erfolg und Misserfolg ist weniger eindeutig. 


\section{$5 \quad$ Erhöhung der Zugänglichkeit durch Re-Branding der Studiengänge der Wirt- schaftsinformatik}

Beim Re-Branding geht es darum, die Erkenntnisse aus der Analysephase gezielt zu nutzen, um "untypischen Personen" die Identifikation mit den ICT-Professionsidentitäten zu erleichtern und dadurch den Zugang zu ICT-Ausbildungen zu verbessern. Im Zentrum des Prozesses steht die Erarbeitung und Etablierung eines Brands, mit der sich potentielle Studieninteressierte identifizieren können. Der Markenkern soll dabei die Besonderheiten und die Einzigartigkeit der Studiengänge in Abgrenzung zu möglichen Konkurrenzangeboten beschreiben. Die Identität des Studiengangs, die dahinterliegende Wertestruktur und die verfolgten strategischen Ziele müssen im Verlauf des Prozesses reflektiert, hinterfragt und neu ausgehandelt werden. Der gezielte partizipative Einbezug von Stakeholdern in den Prozess soll dabei gewährleisten, dass die überarbeitete Markenidentität breit abgestützt und mitgetragen wird. Es wurden mit den beteiligten Studiengängen jeweils drei Workshops durchgeführt, die sich am Branding Zyklus nach Trost (vgl. [Tros12], S. 54) orientiert haben. Es hat sich in den jeweiligen Workshops gezeigt, dass die Ergebnisse bei den beteiligten Stakeholdern des Studienganges sehr anschlussfähig waren und auf dieser Basis sehr gut jeweils eine neue Markenidentität des Studiengangs sowie neue Value Propositions für neue Studierende erarbeitet werden konnten.

Das entwickelte Modell zeigt auf, dass Wirtschaftsinformatik nicht nur als Schnittstelle zwischen Business und Informatik beschrieben wird, wie dies in den Interviews noch häufig formuliert wurde. Vielmehr wurde die Kommunikation als wesentlicher Bestandteil der Wirtschaftsinformatik identifiziert. Die Wirtschaftsinformatik im Spannungsfeld zwischen Mensch, Technik und Wirtschaft anzusiedeln, erlaubte, die Ziele und Spezifika der Wirtschaftsinformatik konkreter zu fassen und auszuformulieren. So konnte eine zielgruppenorientierte, positiv formulierte Markenidentität definiert werden, die über die Metapher der Wirtschaftsinformatik als Schnittstelle zwischen Business und Informatik hinausgeht.

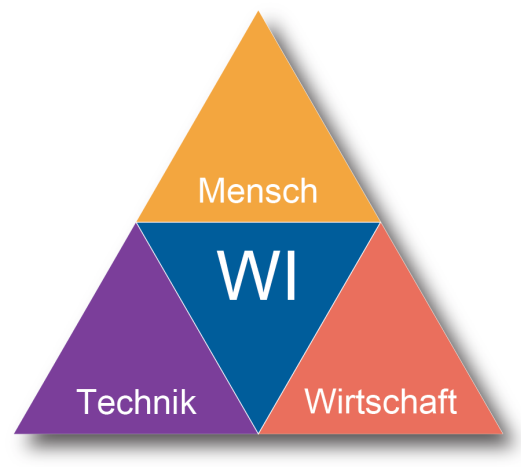

Abbildung 1: entwickeltes Modell der Markenidentität der Wirtschaftsinformatik-Studiengänge der Fachhochschule Nordwestschweiz

Insgesamt lassen sich aus den Ergebnissen der Diskursanalyse und der Auswertung der Workshops die folgenden allgemeinen Empfehlungen für die Erhöhung der Attraktivität von ICT-Berufen und Ausbildungen für untypische Personen geben:

Eine an die Zielgruppen anschlussfähige Kommunikation gewährleisten. Dies geschieht insbesondere durch die Vermeidung von ICT-spezifischen Fachbegriffen in Broschüren und Webseiten, die über Ausbildungen und Studiengänge informieren. Außerdem ist es wichtig, 
dass konkrete Beispiele verwendet werden, nur so können sich Interessierte, die sich noch nicht vertieft mit dem Beruf auseinandergesetzt haben, etwas darunter vorstellen. Gerade für Jugendliche ist es besonders wichtig, dass positive Emotionen in die Beschreibung integriert werden. Sie wollen sehen, dass ihnen eine Ausbildung Spaß machen kann. Im Bildmaterial sollte vermieden werden, zu viele Personen vor Bildschirmen zu zeigen. Stattdessen sollten vermehrt Menschen in solchen Interaktionssituationen gezeigt werden, wie sie täglich in den heutigen Berufen der ICT vorkommen.

Ausgeglichene Darstellung des Kompetenz- und Tätigkeitsportfolios. In der ICT geht es nicht mehr nur darum, zu programmieren oder PCs auseinanderzunehmen - viele Laien glauben das aber weiterhin. Wenn sich diejenigen für ICT-Ausbildungen interessieren sollen, die ein breiteres Interessensspektrum haben, dann müssen die verschiedenen Kompetenz- und Tätigkeitsfelder auch in der Beschreibung gleich gewichtet werden. Dafür sollten die relevanten sozial-kommunikativen Kompetenzen definiert und als herausfordernd beschrieben werden.

Technik als lernbar und zielgerichtet positionieren. Selbstverständlich sind auch weiterhin technische Kompetenzen und Tätigkeiten wichtig für ICT-Berufe. Allerdings dürfen diese, damit auch bisher wenig affine Zielgruppen sich dafür interessieren, nicht als Selbstzweck dargestellt werden. Der Nutzen, den die Technik im jeweiligen Kontext entfaltet, muss klar beschrieben werden. Gleichzeitig muss klarwerden, dass die notwendigen Fähigkeiten im Laufe von Studium und Ausbildung erworben werden können.

Die berufliche Zukunft konkret beschreiben. ICT-Berufe sind die Berufe, die im Rahmen der Digitalisierung der Gesellschaft die Zukunft derselben mitgestalten. Es sind die Berufe, bei denen die Chancen auf dem Arbeitsmarkt auf Basis einer fundierten Ausbildung bei konstanter Weiterbildung hoch bleiben werden. Auf diese Zukunftsfähigkeit sollte in den Beschreibungen der Berufe und der Profession geachtet werden.

\section{$6 \quad$ Fazit}

Insgesamt gilt es also, für eine Steigerung der Attraktivität von ICT-Berufen für Frauen und Männer nicht nur darauf zu achten, dass eine geschlechtergerechte Sprache verwendet und auf Bildern auch Frauen gezeigt werden, sondern darum, das männlich geprägte Image der Profession durch verschiedene Ansätze aufzulösen. Die Studie konnte aufzeigen, dass diese Prägung durch vielfältige Verknüpfungen gesellschaftlich und kulturell verankerter Diskurse geschieht. Somit geht es nicht darum, dem Beruf ein weibliches Image zu verpassen, sondern das implizit männlich geprägte Image durch Auflösung verschiedener Diskurse wie zum Beispiel "Technik als Basis" auch für Frauen und andere, die sich bisher nicht dafür interessiert haben, zugänglich zu machen. Dafür braucht es allerdings mehr als einzelne Maßnahmen, nämlich einen integrativen Zugang. Gerade Ausbildungen und Studiengänge, die sich nachhaltig verändern wollen, müssen eine grundlegende Reflexion ihres Images durchführen.

\section{Literatur}

[AiBB14] Ainsworth, S., Batty, A., \& Burchielli, R. (2014). Women Constructing Masculinity in Vol 
[AMRS12] Ashcraft, K. L., Muhr, S. L., Rennstam, J., \& Sullivan, K. (2012). Professionalization as a Branding Activity: Occupational Identity and the Dialectic of Inclusivity-Exclusivity. Gender, Work \& Organization, 19(5), 467-488.

[BaLa15] Barbour, J. B., \& Lammers, J. C. (2015). Measuring professional identity: a review of the literature and a multilevel confirmatory factor analysis of professional identity constructs. Journal of Professions and Organization, 2(1), 38-60. doi:10.1093/jpo/jou009

[Bebb02] Bebbington, D. (2002). Women in Science, Engeneering and Technology: A Review of The Issues. Higher Education Quarterly, 56(4), 360-375.

[BrPP11] Brannan, M. J., Parsons, E., \& Priola, V. (2011). Placing Branding within Organization Theory. In M. J. Brannan, E. Parsons, \& V. Priola (Eds.), Branded Lives - The Production and Consumption of Meaning at Work. Cheltenham: Edward Elgar.

[BrLG16] Braun-Dubler, N., Langhart, M., \& Gmünder, M. (2016). ICT-Fachkräftesituation Bedarfsprognose 2024. ICT-Berufsbildung Schweiz, Basel.

[Burr03] Burr, V. (2003). Social Constructionism. London: Routledge.

[Chia00] Chia, R. (2000). Discourse Analysis as Organizational Analysis. Organization, 7(3), 513518.

[CFNG12] Cipriano, B., Funk, J. L., Niederberger, G., \& Graf, U. (2012). Empfehlungen für eine Kultur der Chancengleichheit - Gesammeltes Wissen aus zehn Jahren Bundesprogramm Chancengleichheit an Fachhochschulen. Bern: BBT Retrieved from http://www.sbfi.admin.ch/fh/02141/02152/index.html?lang=de.

[Czar11] Czarniawska, B. (2011). Ho to Study Gender Inequality in Organizations? In E. L. Jeanes, D. Knights, \& P. Yancey Martin (Eds.), Handbook of Gender, Work \& Organization. Chichester: Wiley.

[Deet96] Deetz, S. (1996). Describing Differences in Approaches to Organization Science: Rethinking Burell and Morgan and Their Legacy. Organization Science, 7(2), 191-207.

[Econ14] Econlab. (2014). ICT-Fachkräftesituation: Bedarfsprognose 2022. Schlussbericht. ICTBerufsbildung Schweiz, Bern.

[Econ13] Economiesuisse. (2013). Digitale agenda 2.0 - auf dem Weg zu „smart switzerland“. Retrieved from http://www.economiesuisse.ch/de/PDF\%20Download\%20Files/STUDIE_DigitaleAgenda _20130604.pdf

[Flic10] Flick, U. (2010). Qualitative Sozialforschung - Eine Einführung. Reinbek bei Hamburg: Rowohlt Taschenbuch Verlag.

[Gerg99] Gergen, K. J. (1999). An Invitation to Social Construction. London: Sage.

[HaSc08] Hatch, M. J., \& Schultz, M. (2008). Taking brand initiative: How companies can align strategy, culture, and identity through corporate branding. San Francisco: Wiley.

[Hepb04] Hepburn, A. (2004). Crying: Notes on Description, Transcription and Interaction. Research on Language and Social Interaction, 37(3), 251-290.

[Hopf05] Hopf, C. (2005). Forschungsethik und qualitative Forschung. In U. Flick, E. Von Kardorff, \& I. Steinke (Eds.), Qualitative Forschung - Ein Handbuch (pp. 589-600). Reinbek bei Hamburg: Rowohlt Taschenbuch Verlag.

[IT-D14] IT-Dreamjobs. (2014). IT Dreamjobs Retrieved from http://it-dreamjobs.ch/ 
[Kela10] Kelan, E. K. (2010). Gender Logic and (Un)doing Gender at Work. Gender, Work \& Organization, 17(2), 174-194. doi:10.1111/j.1468-0432.2009.00459.x

[KeWW11] Kenny, K., Whittle, A., \& Willmoth, H. (2011). Understanding Identity \& Organizations. London: Sage.

[LLSS14] Liebig, B., Levy, R., Sauer, B., \& Sousa-Poza, A. (2014). Gender Equality Policies in Switzerland: Institutional Factors of Success and Failure. Introduction to the Special Issue. Schweizerische Zeitschrift für Soziologie, 40(2), 169-174.

[OsPK04] Oswick, C., Putnam, L. L., \& Keenoy, T. (2004). Tropes, Discourse and Organizing. In D. Grant, C. Hardy, C. Oswick, \& L. L. Putnam (Eds.), The Sage Handbook of Organizational Discourse. London: Sage.

[Park04] Parker, M. (2004). Becoming Manager: Or, the Werewolf Looks Anxiously in the Mirror, Checking for Unusual Facial Hair. Management Learning, 35(1), 45-59. doi:10.1177/1350507604041164

[Park07] Parker, M. (2007). Identification: Organizations and Structuralisms. In A. Pullen, N. Beech, \& D. Sims (Eds.), Exploring Identity: Concepts and Methods (pp. 61-82). Basingstoke: Palgrave Macmillan.

[Simm11] Simms, M. (2011). Appropriating the Brand: Union Organizing in Front-Line Service Work. In M. J. Brannan, E. Parsons, \& V. Priola (Eds.), Branded Lives. Cheltenham: Edward Elgar.

[swis13] swissICT. (2013). Berufe der ICT - 42 Informatik-Berufsbilder und die notwendigen Kompetenzen. Zürich: vdf Hochschulverlag.

[Tros12] Trost, A. (2012). Talent Relationship Management - Personalgewinnung in Zeiten des Fachkräftemangels. Berlin: Springer.

[UmRü13] Umbach-Daniel, A., \& Rütter, H. (2013). «Transformlng» - Analyse der Rekrutierungsund Förderpraktiken von Unternehmen hinsichtlich genderspezifischer kultureller Hemmnisse und Förderfaktoren. Schweizerischer Nationalfonds, NFP 60, Bern

[WeZi91] West, C., \& Zimmerman, D. H. (1991). Doing Gender. In J. Lorber \& S. A. Farrell (Eds.), The Social Construction of Gender (pp. 13 - 37). Newbury Park, London, New Delhi: SAGE Publications.

[Weth01] Wetherell, M. (2001). Discourse as Data: A Guide for Analysis. London: Sage.

[WeTa10] Wetherell, M., \& Talpade Mohanty, C. (Eds.). (2010). The Sage Handbook of Identites. London: Sage.

[WeTY01] Wetherell, M., Taylor, S., \& Yates, S. J. (Eds.). (2001). Discourse Theory and Practice: A Reader. London: Sage.

[Witz00] Witzel, A. (2000). Das problemzentrierte Interview. FQS, 1(1).

\section{Kontakt:}

Prof. Dr. Dörte Resch, Iris Graf, Anke Dreiling, Jonas Konrad

Fachhochschule Nordwestschweiz (FHNW)

Hochschule für Wirtschaft $(\mathrm{H}, \mathrm{SW})$

Riggenbachstrasse 16, CH-4600 Olten

Tel: +41 62957 2536, doerte.resch@fhnw.ch 


\section{Industrie 4.0}




\title{
Industrie-4.0-Reifegradmodell
}

\author{
Jörg Puchan, Eva Kastl, Alexander Zeifang
}

\section{Zusammenfassung}

Die Fortschritte im Bereich der Informationstechnologien, welche im Kontext zu Industrie 4.0 stehen, verzeichnen immer mehr Auswirkungen auf die industrielle Produktion. Die Möglichkeit, Maschinen, Produkte, Dienste und Systeme mit „Intelligenz" auszustatten, wird Wertschöpfungsketten und Strukturen von produzierenden Unternehmen in Zukunft wettbewerbsentscheidend verändern.

Industrieunternehmen stehen aktuell vor der Herausforderung die aufkommenden Chancen der Digitalisierung zu nutzen und die Transformation ihres bisherigen Geschäftsmodells hin zu einer Smart Factory erfolgreich zu meistern. Trotz der steigenden Bedeutung dieser Thematik mangelt es bisher an wissenschaftlich fundierten Instrumenten, welche Unternehmen bei dem bevorstehenden Wandel unterstützen und innen den individuellen Stand in den entscheidenden Handlungsfeldern aufzeigen.

Im Rahmen dieses Beitrags wird ein wissenschaftlich fundiertes Reifegradmodell als Instrument zur individuellen Bestimmung des Industrie-4.0-Reifegrads vorgeschlagen. Dabei sollen nicht nur die Entwicklungsstände der unterschiedlichen Handlungsfelder bewertet werden, sondern auch eine Hilfestellung für das Ableiten von Maßnahmen für die erfolgreiche Implementierung von Industrie 4.0 erfolgen.

Ziel dieses Beitrages ist es, den aktuellen Entwicklungsstand des Industrie-4.0-Reifegradmodells der Hochschule München vorzustellen. Dabei wird insbesondere der Modellaufbau und die Herleitung der Modellinhalte ausführlich erklärt. Anhand eines praktisch validierten Beispiels wird eine mögliche Anwendung des Reifegradmodells erläutert und abschließend ein Ausblick auf die nächsten Schritte gegeben.

\section{$1 \quad$ Einleitung}

Die Bundesregierung hat im Jahr 2011 das Zukunftsprojekt „Industrie 4.0“ (14.0) gestartet und somit einen Begriff für intelligente und vernetzte Produktionssysteme eingeführt. Unter dieser Bezeichnung sollen entsprechende Themen zentral gebündelt und Umsetzungsempfehlungen für Unternehmen erarbeitet werden. (s. [KaWH13]) Aufgrund der steigenden Anzahl an Publikationen und Forschungsprojekten wird es für Unternehmen immer schwieriger den Überblick in diesem Themenfeld zu bewahren und strategisch relevante Informationen herauszufiltern.

An dieser Stelle bietet ein wissenschaftlich fundiertes Reifegradmodell eine Möglichkeit, die Industrie-4.0-Themen sinnvoll zu strukturieren und ein Rahmenwerk für eine systematische und definierte Vorgehensweise für produzierende Unternehmen zu schaffen. (s. [Fels15], s. S. 46 in [Mett10] und s. [Ab++15])

Als Nachweis der Problemrelevanz erfolgte eine Recherche nach bereits existierenden Industrie-4.0-Reifegradmodellen. Die auf dem Markt vorhandenen Reifegradmodelle, welche von Institutionen, Organisationen und Beratungsunternehmen veröffentlicht wurden, dienen ausschließlich zur groben Einschätzung oder enthalten eher Teilaspekte des Umfeldes 
Industrie 4.0. Es lässt sich zusammenfassend aussagen, dass es auf dem Markt aktuell kein wissenschaftlich fundiertes Instrument gibt, welches produzierende Unternehmen bei dem bevorstehenden Wandel tatsächlich unterstützt.

Mithilfe des Reifegradmodells der Hochschule München soll den Anwendern ermöglicht werden, ihre Industrie-4.0-Fähigkeiten zu beurteilen, Stärken und Schwächen zu benennen und konkrete Verbesserungsmaßnahmen für einzelne Unternehmensbereiche abzuleiten.

In der vorliegenden Arbeit wird der aktuelle Entwicklungstand erstmalig veröffentlicht. Der Aufbau des Beitrages setzt sich wie folgt zusammen: Zuerst wird die Forschungsmethodik erläutert (vgl. Kapitel 2) und anschließend ein Überblick über die Herleitung der Modellinhalte gegeben (vgl. Kapitel 3). Zusätzlich wird der Modellaufbau des Industrie-4.0-Reifegradmodells der Hochschule München vorgestellt und anhand einer Fallstudie die mögliche Anwendung des Modells erklärt (vgl. Kapitel 4). Abschließend wird ein Ausblick auf die nächsten Entwicklungsschritte gegeben.

\section{Methodik}

Nach [Mett09] (s. S. 6) ist die Gestaltung von Reifegradmodellen der gestaltungsorientierten Forschung zuzuordnen. [PTRC07] beschreiben ein Vorgehensmodell für eine gestaltungsorientierte Forschungsarbeit. Aufgrund der Nachvollziehbarkeit wird dieses Modell als Basis für das gesamte Forschungsprojekt verwendet. Das Vorgehensmodell umfasst die fünf Phasen Problemidentifikation und Motivation, Definition der Ziele, Entwurf und Entwicklung, Demonstration und Evaluation sowie Kommunikation. Im vorliegenden Beitrag wird ein Einblick in die Phase Entwurf und Entwicklung gegeben. [Mett10] betrachtet die Entwicklung und die Anwendung eines Reifegrademodells nicht als eigenständige Prozesse, sondern als ineinander verkettete Zyklen. Aus diesem Grund soll einerseits bei der Modellentwicklung die Art der praktischen Anwendung des Reifegradmodells berücksichtigt werden. Andererseits ist es notwendig bei der Vorbereitung der Modellanwendung dafür zu sorgen, dass das erforderliche Wissen und die Hilfsmittel vorhanden sind. Dies wird bei der vorliegenden Arbeit berücksichtigt. Im Folgenden wird die methodische Vorgehensweise für diesen Beitrag beschrieben.

\subsection{Iterative Reifegradmodellentwicklung}

Für den Konstruktionsprozess des Reifegradmodells wird das Vorgehensmodell nach [BeKP09b] als Grundlage verwendet. Dieses beruht auf die von [HMPR04] empfohlenen Richtlinien für die Durchführung einer gestaltungsorientierten Forschungsarbeit. Das Modell unterscheidet insgesamt acht Phasen: Die Problemdefinition, der Vergleich bestehender Modelle, die Festlegung der Entwicklungsstrategie, die iterative Modellentwicklung, die Konzeption von Transfer und Evaluation, die Implementierung der Transfermittel, die Durchführung der Evaluation sowie ggf. ein Verwerfen des Reifegradmodells. Im Rahmen des vorliegenden Beitrages soll der aktuelle Stand der iterativen Modellentwicklung vorgestellt werden. Als Grundlage dient die in der vorherigen Phase formulierte Entwicklungsstrategie: Es werden zum einen Modellinhalte aus bereits identifizierten Reifegradmodellen (vgl. Kapitel 3) übernommen. Diese decken jeweils Teilaspekte von Industrie 4.0 ab und liefern somit einerseits Ideen für die Definition der Reifegradstufen des Reifegradmodells sowie auch für die Inhalte der adressierten Problemstellung. Zum anderen wird durch eine Literaturanalyse nach weiteren Modellinhalten gesucht. 


\subsection{Literaturrecherche und -analyse}

Als erster Schritt der iterativen Reifegradmodellentwicklung wurde eine systematische Literaturrecherche durchgeführt. Die Zielsetzung lautete, einen Überblick über die notwendigen Inhalte für das Reifegradmodell zu erhalten. Dabei wurde nach Studien und weiteren Publikationen im Umfeld Industrie 4.0, Internet der Dinge und Dienste, Digitalisierung und Smart Factory gesucht. Bei der Literaturanalyse wurden die einzelnen Veröffentlichungen anhand der folgenden Kriterien ausgewählt:

Zum einen müssen die Untersuchungen einen expliziten Bezug zu Industrie 4.0 haben, zum anderen sollen die Erkenntnisse empirisch fundiert sein, sowie einen starken Praxisbezug demonstrieren. Die Thematik soll dabei entweder aus einem betriebswirtschaftlichen, organisatorischen und/oder aus einem technologischen Blickwinkel betrachtet werden. Des Weiteren wird die Auswahl weitestgehend auf den deutschsprachigen Raum begrenzt, um sicherzustellen, dass die Erkenntnisse auch auf Unternehmen in Deutschland übertragbar sind. Die hierbei identifizierten Quellen wurden nach Industrie-4.0-Indikatoren, -Fähigkeiten und -Kriterien hin analysiert. Die Literaturrecherche wurde im Zeitraum November 2015 bis Januar 2016 durchgeführt.

\section{Literaturanalyse}

Im Rahmen der Literaturanalyse wurden acht Publikationen ausgewählt und näher betrachtet. (s. [Bisc15], [Ab++15], [Ba++14], [Em++15], [Sp++13], [Li++15], [PuSM15] und [Bu++15]) Jede Quelle wurde unter Berücksichtigung der Fragestellung „Welche Voraussetzungen und Fähigkeiten müssen für die erfolgreiche Umsetzung von Industrie 4.0 in produzierenden Unternehmen geschaffen werden?" untersucht.

Als Ergebnis entstand eine Liste an verschiedenen Industrie-4.0-Indikatoren, -Fähigkeiten und -Kriterien. Die Literaturanalyse zeigte wie umfangreich das Themengebiet Industrie 4.0 ist und wie schwer es ist dieses komplexe Thema in einem einfachen und übersichtlichen Modell darzustellen. Industrie 4.0 ist kein rein technisches, produktionsbezogenes oder wertschöpfungskettenbezogenes Vorhaben, sondern schließt alle Aspekte der Organisations-, Personal- und Technikentwicklung mit ein. Es hat somit Auswirkungen auf alle Bereiche in einem Unternehmen und ist ein sehr breit gefächertes Handlungsfeld. Bei der Umsetzung ist deshalb davon auszugehen, dass sich in der betrieblichen Praxis eine schrittweise Vorgehensweise durchsetzen wird, welches Reifegradmodelle unterstützen.

Zusätzlich wurden in der Forschungsphase „Vergleich bestehender Reifegradmodelle“ Reifegradmodelle im Umfeld Industrie 4.0 identifiziert. Deren Dokumentationen sind, wie bereits bei der Entwicklungsstrategie (vgl. Kapitel 2.1) erläutert, zusätzlich analysiert worden. (s. [Az++14], [SRVW15], [Ab++15], [Schö09], [Bu++15], [Li++15], [MaBü15], [PoHe14] und [An++15]) Zusammenfassend wurde festgestellt, dass in den Modellen entweder eher technische, oder eher strategische und organisationale Fragestellungen behandelt werden. Die Modellinhalte sind dabei meistens zu oberflächlich beschrieben. Sie dienen häufig einer guten ersten Einschätzung, wo sich das Unternehmen in der Umsetzung von Industrie 4.0 grob befindet. Insbesondere eine unterstützende Ableitung von Entwicklungspfaden ist bei keinem der Modelle möglich. Wobei dies ein wichtiges Kriterium für die Weiterentwicklung der Fähigkeiten in einem Unternehmen ist.

Im nächsten Kapitel wird die Weiterverarbeitung der Ergebnisse der Literaturanalyse vorgestellt. 
Nachdem das vorhergehende Kapitel die Herleitung der Modellinhalte aufführt, wird nun der aktuelle Entwicklungsstand des Industrie-4.0-Reifegradmodells der Hochschule München vorgestellt. Dabei wird in diesem Beitrag die erste Version des Reifegradmodells veröffentlicht. Es werden die gewählte Modellarchitektur, die erarbeiteten Modellinhalte und eine mögliche Modellanwendung erläutert. Das Modell wurde gemeinsam mit einem Industriepartner entwickelt und in mehreren Workshops an Anforderungen und Erkenntnissen der Praxis validiert.

\subsection{Modellaufbau}

Das Reifegradmodell beinhaltet fünf Reifegradstufen (RG-Stufe). Jede Stufe beschreibt einen bestimmten Leistungszustand eines Handlungselementes. Die Reifegradstufe 0 ist dabei die niedrigste Stufe „Unternehmen beschäftigen sich noch nicht bzw. lediglich gering mit 14.0-Umsetzungen. " und die Reifegradstufe 4 ist die höchste Stufe „Unternehmen ist ein 14.0-Vorreiter. Alle im Reifegradmodell identifizierten Maßnahmen sind nahezu vollständig umgesetzt.".

Die Reife der höchsten Stufe soll für die Unternehmen in der heutigen Zeit nur sehr schwer zu erreichen sein. Damit sollen die Vision der kontinuierlichen Verbesserung und der technische Fortschritt aufgegriffen werden. Die Organisationen sollen sich beim Erreichen der nächsthöheren Stufe nicht ausruhen. Für die einzelnen Bereiche oder das Unternehmen als Ganzes ist es nicht immer hilfreich, sofort die höchste Reifegradstufe als Ziel zu definieren. Wichtiger ist es, in kurzfristigen Schritten zu denken. Dabei macht es häufig Sinn, aufbauend auf die Ausgangssituation Zwischen- und Abschlussziele zu fixieren. Hierfür sind die festgelegten Reifegradstufen geeignet. Die einzelnen Handlungsfelder und -elemente können somit separat betrachtet und jeweils ein eigener Ziel-Reifegrad festgelegt werden.

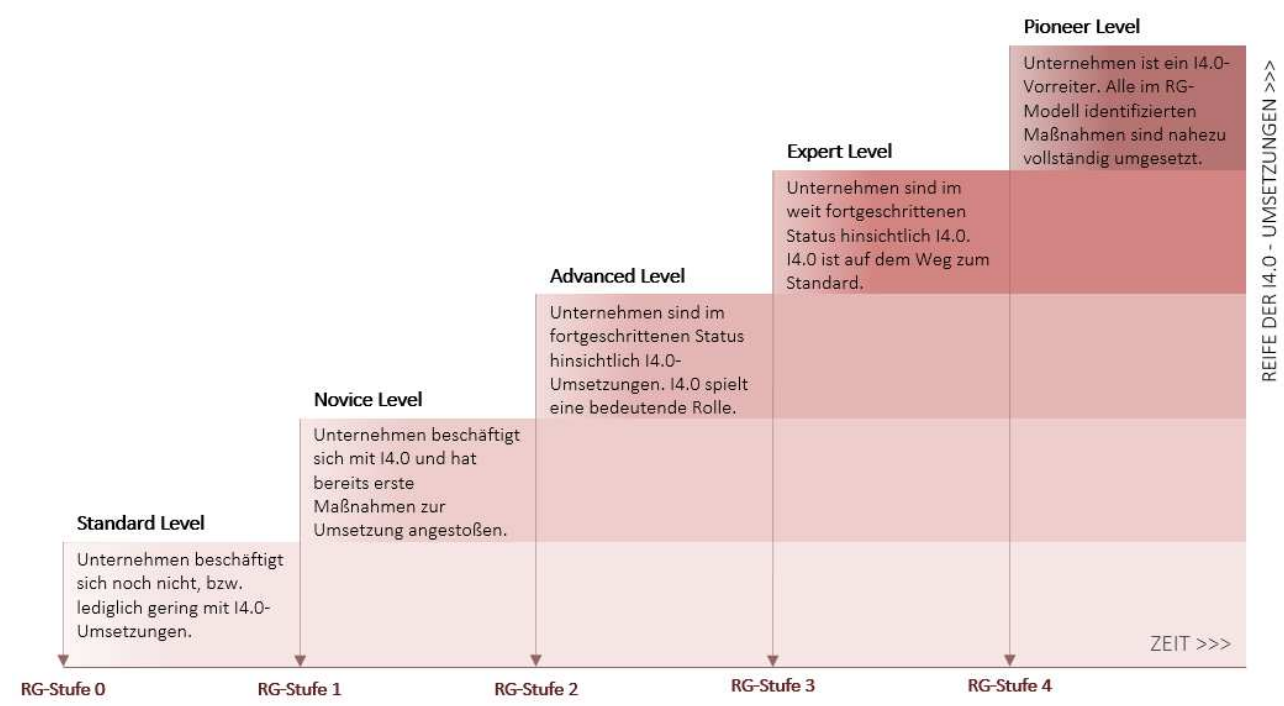

Abbildung 1: Modellaufbau 


\subsection{Modellinhalt}

Der Modellinhalt wird in Handlungsfelder (HF) und Handlungselemente (HE) strukturiert. Dabei wird jedes Handlungselement einem Handlungsfeld zugeordnet. Bei der Auswahl der Handlungsfelder wurde angestrebt, das Unternehmen als Ganzes zu betrachten. Die Handlungsfelder sollen auf jeden Teilbereich der Unternehmensstruktur angewendet werden können. Als Ergebnis entstanden insgesamt fünf Handlungsfelder: Schlüsselfaktoren, Mitarbeiter, Organisation, Produkt und Produktion. Im Folgenden werden die einzelnen Handlungsfelder des Industrie-4.0-Reifegradmodells beschrieben:

- Schlüsselfaktoren. Ein Unternehmen muss erst über bestimmte Kompetenzen in verschiedenen Bereichen verfügen, um an der Umsetzung von Industrie 4.0 überhaupt zu arbeiten. Eine hohe Reife der Schlüsselfaktoren ist somit die Voraussetzung für das Angehen der anderen Handlungsfelder.

- Mitarbeiter. Das Handlungsfeld Mitarbeiter fasst alle Veränderungen zusammen, welche den Menschen in einem Unternehmen betreffen.

- Organisation. Das Handlungsfeld Organisation beinhaltet alle Strukturen außerhalb der Produktion in einem Unternehmen. Hier werden alle organisationalen Veränderungen zusammengefasst.

- $\quad$ Produkt. Das Handlungsfeld Produkt bündelt alle notwendigen Kompetenzen im Bereich der Produktgestaltung, um die Vision der intelligenten und vernetzten Produkte zu realisieren. Hierbei liegt eher die externe Betrachtung des Unternehmens im Fokus (Geschäftsmodelle).

- Produktion. Das Handlungsfeld Produktion fasst alle Handlungselemente zusammen, die notwendig sind, um die Fertigung in eine Smart Factory zu verwandeln.

Diesen fünf Handlungsfeldern sind insgesamt 29 Handlungselemente zugeordnet (siehe Abbildung 2). Im Folgenden werden beispielhaft die einzelnen Handlungselemente des Handlungsfeldes Mitarbeiter definiert:

- Assistenzsysteme. Intelligente Assistenzsysteme mit bedienungsfreundlichen Benutzerschnittstellen unterstützen die Mitarbeiter individuell in ihrer Arbeit.

- $\quad$ Arbeitsstrukturen. Für eine erfolgreiche Veränderung müssen den Mitarbeitern neue Organisations- und Gestaltungsmodelle zur Verfügung stehen. Diese sollen dem Menschen das selbstverantwortliche Handeln ermöglichen und kombiniert sein mit dezentralen Führungs- und Steuerungsformen. Leistungs- und Entwicklungsfähigkeit, Wohlbefinden und Gesundheit der arbeitenden Menschen mit der Möglichkeit zur Belastungsregulation sind einzubeziehen.

- 14.0-Engagement. Grundlegendes Verständnis der Mitarbeiter für Industrie 4.0 als soziotechnisches System, in dem Technik, Organisation und Personal systematisch aufeinander abgestimmt werden müssen. Ohne die Unterstützung der Mitarbeiter ist eine Transformation des Unternehmens nicht möglich.

- Informationssysteme. Zukünftig werden den Mitarbeitern die benötigten Informationen nutzerorientiert angeboten. Der bisherige Anwendungscharakter wird in Zukunft verschwinden und der Nutzer erhält ein auf inn zugeschnittenes Informationsumfeld.

- 14.0-Kompetenz. Die Mitarbeiter müssen für den Weg zu einer Smart Factory über ausreichend Wissen und Fähigkeiten im Industrie-4.0-Umfeld verfügen. Im Laufe der Transformation werden immer umfangreichere und breiter gefächerte Kompetenzen benötigt. 


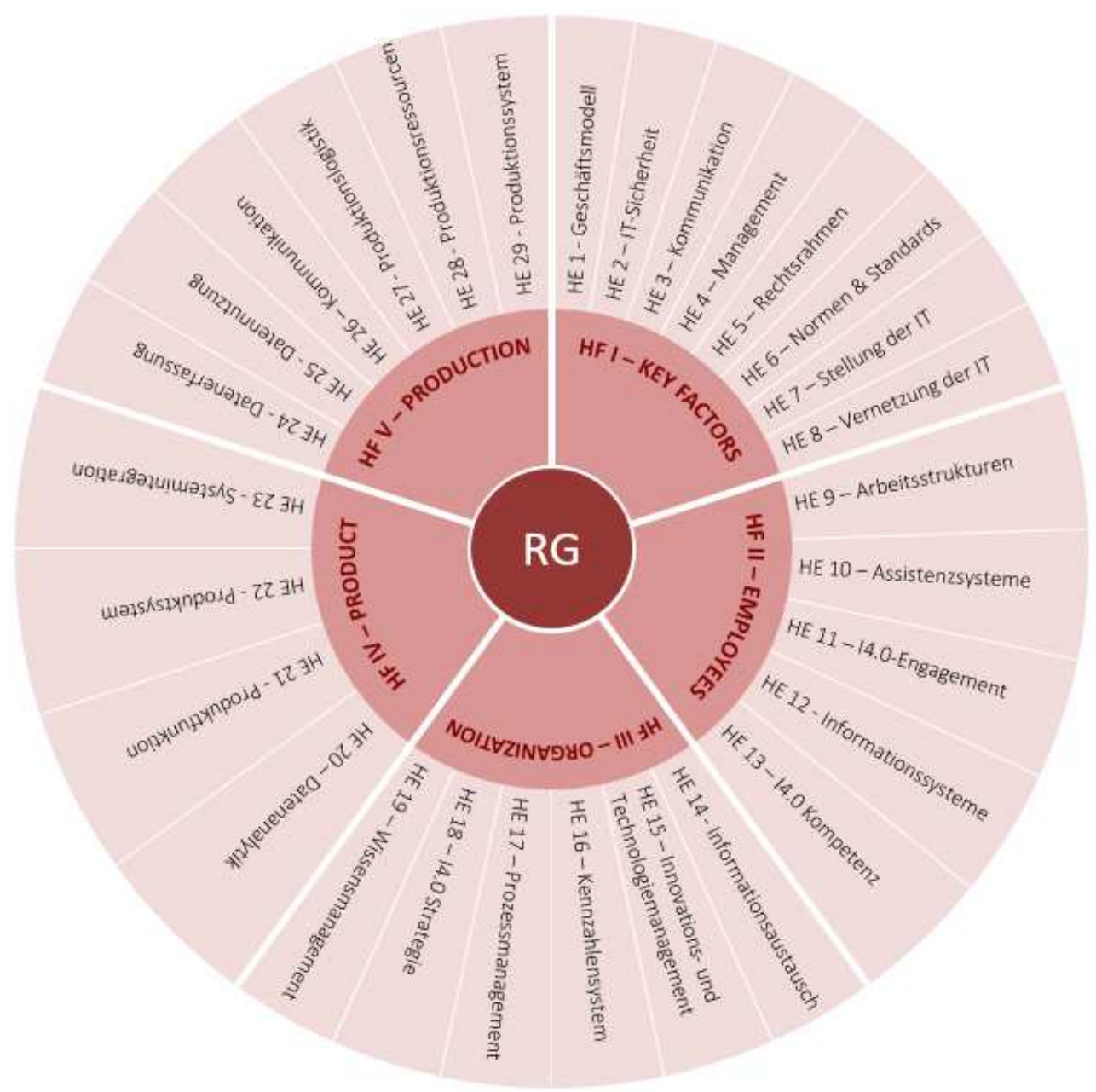

Abbildung 2: Modellinhalt

\subsection{Modellanwendung}

Im Rahmen des vorliegenden Beitrages soll die Modellanwendung anhand eines praktisch validierten Beispiels vorgestellt werden. In Zusammenarbeit mit einem produzierenden Unternehmen aus der Baumaschinenbranche wurde der Prototyp des Modells getestet. Hierfür wurde als Methode eine unterstützende Selbstbewertung, auch Assessment genannt, gewählt. Der Erfolg von Reifegradmodellen ist sehr stark von der Qualität der Leistungsbewertung abhängig. (s. [Link07]) Auch deshalb ist die enge Verzahnung von Konstruktion und Anwendung von Reifegradmodellen so wichtig. Um eine hohe Qualität des Assessments zur erreichen, werden definierte Anforderungen an die Durchführung von Assessments aus der Literatur beim Vorgehen berücksichtigt. (s. [Hölt12], s. [Mett10], s. [DINI11]) Die Leistungsbewertung soll für die verschiedensten Unternehmensbereiche (z.B. einzelne Abteilungen, Unternehmen als Ganzes oder auch Projekte) angewendet werden können. Die Datenerhebung erfolgte dabei mit Hilfe eines Excel-Tools, welches in Anlehnung an das PEM-Modell (s. [Hamm07]) konstruiert wurde. Hierbei wird bei der Leistungsbewertung für jedes Hand- 
lungselement ein Ziel- und Ist-Reifegrad festgelegt. Zeitgleich wird anhand einer Ampeldarstellung der jeweilige Erfüllungsgrad visualisiert. Die Ergebnisse werden zudem in Netzdiagrammen graphisch zusammengefasst dargestellt. Die folgende Abbildung fasst die Vorgehensweise der Modellanwendung zusammen:
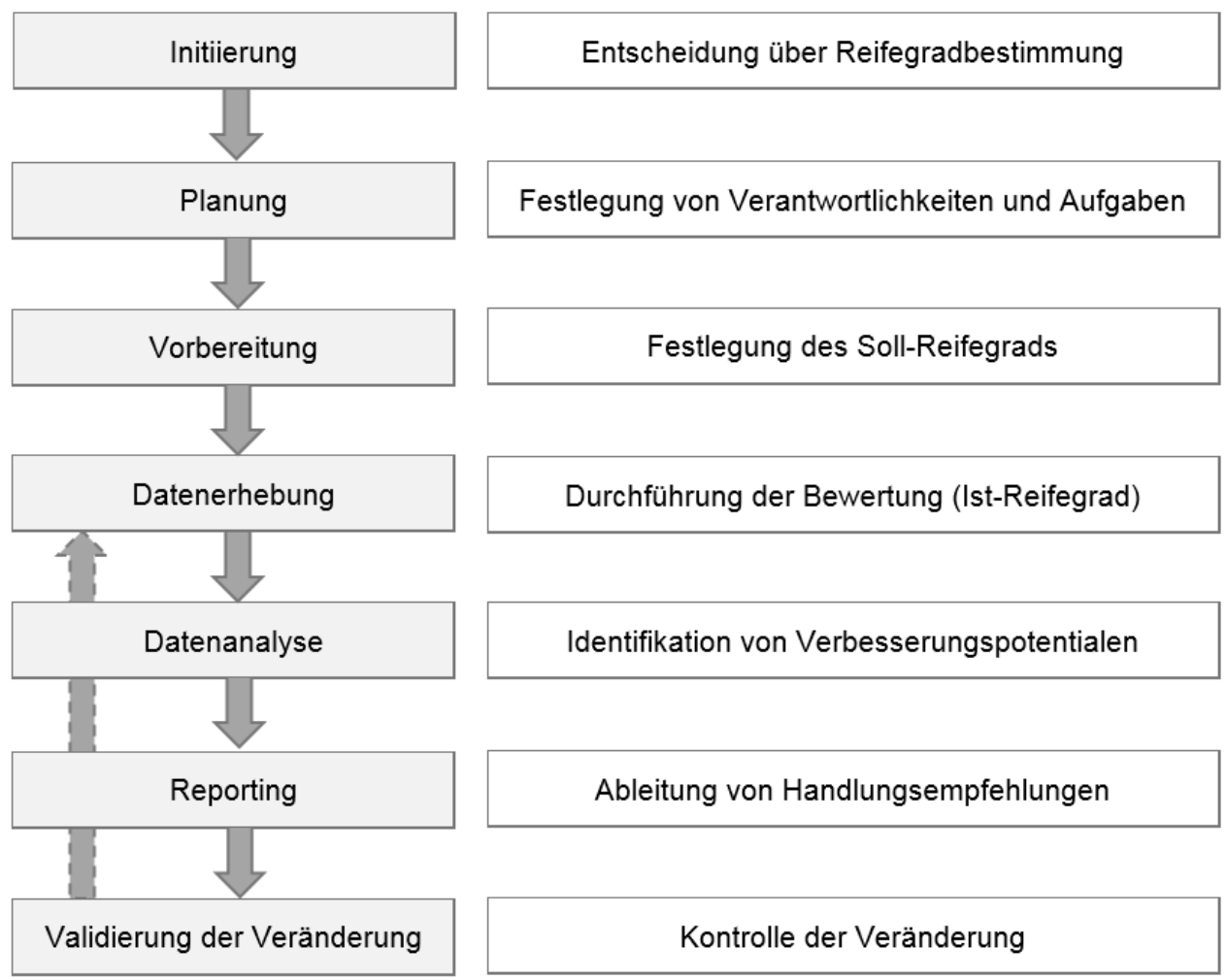

Abbildung 3: Modellanwendung

Konkret wurde in der Fallstudie das Assessment für ein Industrie-4.0-Projekt durchgeführt. Als Ergebnis sollte der Nutzen des Projektes für das Unternehmen aufgezeigt werden. Hierfür wurde zu Beginn des Projektes zusammen mit dem Projektleiter überlegt, welcher Soll-Reifegrad bei den einzelnen Handlungselementen notwendig ist, um das Vorhaben realisieren zu können. Anschließend folgte die Angabe des Ist-Zustandes der Handlungselemente. Die grafische Darstellung der Ist-/Soll-Differenz veranschaulicht sehr gut, wie umfangreich die notwendigen Schritte zur Erreichung der Projektziele sind (Vergleich Aufwand - Nutzen). Als Nächstes wurde auf Basis der Handlungsempfehlungen des Reifegradmodells die Arbeitspakete des Projektes geplant und die Fortschritte regelmäßig überprüft.

\section{Ausblick}

Zukünftig soll das Industrie-4.0-Reifegradmodell der Hochschule München inhaltlich weiterentwickelt werden. Zum einen fließen die Ergebnisse und Erfahrungen der praktischen An- 
wendung des Industrie-4.0-Reifegradmodells in die Überarbeitung mit ein. Zum anderen soll das Reifegradmodell durch Interviews mit Unternehmen weiter verbessert werden. Zudem ist das Industrie-4.0-Reifegradmodell die Basis für die Durchführung einer Studie zur Reife von produzierenden Unternehmen.

In den nächsten Schritten sollte neben der inhaltlichen Weiterentwicklung auch die Modellanwendung verbessert werden. Zentrales Ziel der Validierung des Reifegradmodells war es, die generelle Eignung der Modellarchitektur und der Assessmentmethode zu überprüfen. Das Feedback lautete zusammenfassend, dass das Industrie-4.0-Reifegradmodell eine gute Hilfestellung bei der Erkennung der eigenen Stärken und Schwächen ist sowie bei der Planung, Kommunikation und Analyse von Veränderungen. Die Ableitung von konkreten Maßnahmen sollte noch besser unterstützt werden, um den praktischen Nutzen des Modells zu steigern. Hilfreich wäre auch die Möglichkeit der Anpassung des Modells an die individuellen Anforderungen der Modellnutzer.

\section{$6 \quad$ Zusammenfassung}

Zentrales Ziel dieses Beitrages ist die Vorstellung des aktuellen Entwicklungsstandes des Industrie-4.0-Reifegradmodells der Hochschule München. Hierfür wurde zuerst die ausführliche Herleitung der Modellinhalte erläutert. Die Literaturanalyse bestätigte die Relevanz der Themenstellung. Den Unternehmen fehlt es an einer effizienten Hilfestellung für den Umgang mit Industrie 4.0. Ein Großteil der Publikationen behandelt das Thema oberflächlich. Aufbauend auf die Ergebnisse der Literaturrecherche wurde ein Kriterienkatalog erarbeitet. Diese Liste enthält eine Vielzahl an Voraussetzungen und Fähigkeiten für die Transformation eines Unternehmens zu einem Industrie-4.0-Unternehmen und ist die Basis für die Definition der Modellinhalte. Anschließend wurden die gewählte Modellarchitektur, die erarbeiteten Modellinhalte und eine mögliche Modellanwendung vorgestellt. Die durchgeführte Pilotanwendung des Reifegradmodells zeigt, dass produzierende Unternehmen in der Identifikation ihrer Reife (Soll- und Ist-Reifegrad) und der Ableitung von Maßnahmen für die erfolgreiche Umsetzung von Industrie 4.0 unterstützt werden.

\section{Literaturverzeichnis}

[Ab++15] Abele, E., Anderl, R., Metternich, J., Arndt, A., Wank, A., Anokhin, O., Meudt, T., Sauer, M.: Industrie 4.0 - Potentiale, Nutzen und Good-Practice-Beispiele für die hessische Industrie. Meisenbach Verlag, Bamberg, 2015.

[An++15] Anderl, R., Picard, A., Wang, Y., Fleischer, J., Dosch, S., Klee, B., Bauer, J.: Leitfaden Industrie 4.0. Orientierungshilfe zur Einführung in den Mittelstand. VDMA Verlag, Frankfurt, 2015.

[Az++14] Azhari, P., Faraby, N., Rossmann, A., Steimel, B., Wichmann, K.S.: Digital Transformation Report. neuland GmbH \& Co. KG, Köln, 2014.

[BSMG14] Bauer, W., Schlund, S., Marrenbach, D., Ganschar, O. (Hrsg.): Industrie 4.0 - Volkswirtschaftliches Potential für Deutschland. Studie, BITKOM, Berlin, 2014. 
[BeKP09] Becker, J., Knackstedt, R., Pöppelbuß, J.: Entwicklung von Reifegradmodellen für das IT-Management. Vorgehensmodell und praktische Anwendung. Westfälische WilhelmsUniversität Münster, Institut für Wirtschaftsinformatik, 2009.

[Bisc15] Bischoff, Jürgen (Hrsg.): Erschließen der Potenziale der Anwendung von ,Industrie 4.0` im Mittelstand. Studie, BMWi, Mühlheim an der Ruhr, 2015.

[BI++15] Block, C., Freith, S., Kreggenfeld, N., Morlock, F., Prinz, C., Kreimeier, D., Kuhlenkötter, B.: Industrie 4.0 als soziotechnisches Spannungsfeld. In: Zeitschrift für wirtschaftlichen Fabrikbetrieb, Ausgabe 10/2015, S. 657-660, 2015.

[Bund15] Bundesministerium für Wirtschaft und Energie (Hrsg.): Industrie 4.0 und Digitale Wirtschaft. Berlin, 2015.

[Bu++15] Busch, J., Soukup, A., Dutzler, H., Loinig, M., Gorholt, A.: Industrie 4.0. Österreichs Industrie im Wandel. Studie, PwC Österreich GmbH, 2015.

[DINI11] DIN ISO/IEC 15504-3:2011-07: Informationstechnik - Prozess-Assessment - Teil 3: Richtlinien für die Durchführung von Assessments. Beuth Verlag, Berlin, 2011.

[Em++15] Emmrich, V., Döbele, M., Bauernhansl, T., Paulus-Rohmer, D., Schatz, A., Weskamp, M.: Geschäftsmodell-Innovation durch Industrie 4.0. Chancen und Risiken für den Maschinen- und Anlagenbau. Studie, Dr. Wieselhuber \& Partner GmbH, München, 2015.

[Fels15] Felser, W.: Ein Rahmenwerk, um Industrie 4.0 jetzt auch für den Mittelstand greifbarer zu machen!. In: Competence Book, Industrie 4.0 Kompakt I, S. 48-55, 2015.

[Hamm07] Hammer, M.: The Process Audit. Harvard Business Review, 2007.

[HeCh10] Hevner, A.R., Chatterjee, S.: Design Science Research in Information Systems. Theory and Practise. Springer US, 2010.

[HMPR04] Hevner, A.R., March, S.T., Park, J., Ram, S.: Design science in information systems research. Management Information Systems Quarterly Vol. 28 No. 1, S. 75-105, 2004.

[Hölt12] Höltz, N.: Lean Logistics Maturity Model. Ein Reifegradmodell zur Bewertung schlanker intralogistischer Unternehmensstrukturen. Dissertation, Brandenburgische Technische Universität Cottbus, Cottbus, 2012.

[IIPu13] Illes, C., Puchan J.: Die Self Assessment Method Munich. In: e-Journal of Practical Business Research, Ausgabe 15 (4/2014). Berlin, 2013.

[KaWH13] Kagermann, H., Wahlster, W., Helbig, J. (Hrsg.): Umsetzungsempfehlungen für das Zukunftsprojekt Industrie 4.0. Abschlussbericht des Arbeitskreises Industrie 4.0. Frankfurt a.M., 2013.

[KnPB09] Knackstedt, R., Pöppelbuß, J., Becker, J.: Vorgehensmodell zur Entwicklung von Reifegradmodellen. In: Business Services: Konzepte, Technologien, Anwendungen, S. 535544, 2009.

[Li++15] Lichtblau, K., Stich, V., Bertenrath, R., Blum, M., Bleider, M., Millack, A., Schmitt, K., Schmitz, E., Schröter, M.: INDUSTRIE 4.0-READINESS. Studie, VDMA, 2015.

[Link07] Link, S.: Definition und Anwendung eines Prozess-Assessment-Modells zur Testprozessverbesserung. Hagen, 2007.

[MaBü15] Mangiapane, M., Büchler R.P.: Modernes IT-Management. Methodische Kombination von IT-Strategie und IT-Reifegradmodell. Springer Vieweg, Wiesbaden, 2015. 
[Mett09] Mettler, R.: A Design Science Research Perspective on Maturity Models in Information Systems. Arbeitsbericht Nr. BE IWI/HNE/03, Institute of Information Management, Universität St. Gallen, 2009.

[Mett10] Mettler, T.: Supply Management im Krankenhaus. Konstruktion und Evaluation eines konfigurierbaren Reifegradmodells zur zielgerichteten Gestaltung. Dissertation, Universität St. Gallen, Sierke, 2010.

[PTRC07] Peffers, K., Tuunanen, T., Rothenberger, M. A., Chatterjee, S.: A design science research methodology for information systems research. In: Journal of Management Information Systems, Volume 24 Issue 3, S. 45-78, 2007.

[PoHe14] Porter, M.E., Heppelmann, J.E.: Wie smarte Produkte den Wettbewerb verändern. In: Harvard Business Manager, Sonderdruck aus Heft 12/2014, 2014.

[PoHe15] Porter, M.E., Heppelmann, J.E.: Wie smarte Produkte Unternehmen verändern. In: Harvard Business Manager, Sonderdruck aus Heft 12/2015, 2015.

[PuSM15] Puchan, J., Seif, H., Mayer, D.: Bestimmung des Stands deutscher produzierender Unternehmen auf dem Weg zu Industrie 4.0 und Verwendung der Ergebnisse für ein Industrie 4.0 Reifegradmodell. In: Prozesse, Technologie, Anwendungen, Systeme und Management 2015, Angewandte Forschung in der Wirtschaftsinformatik, S. 58-68. mana-Buch, München, 2015.

[SRVW15] Schäfer, D., Rossmann, A., Vogel, R., Wichmann, K.S.: Digital Transformation Report 2015. WirtschaftWoche \& neuland GmbH \& Co. KG, Köln, 2015.

[Schö09] Schönefeld, F.: Praxisleitfaden Enterprise 2.0. Carl Hanser Verlag, München, 2009.

[Sp++13] Spath, D. (Hrsg.), Ganschar, O., Gerlach, S., Hämmerle, M., Krause, T., Schlund, S.: Produktionsarbeit der Zukunft - Industrie 4.0. Fraunhofer Verlag, Stuttgart, 2013.

\title{
Kontakt
}

Prof. Dr. Jörg Puchan

Fakultät für Wirtschaftsingenieurwesen

Hochschule München / Munich University of Applied Sciences

Lothstr. 64, 80335 München

T +49 89 1265-3937, joerg.puchan@hm.edu

\section{Eva Kastl}

Fakultät für Wirtschaftsingenieurwesen

Hochschule München / Munich University of Applied Sciences

Lothstr. 64, 80335 München

T +49 89 1265-3982, eva.kastl@hm.edu

\author{
Alexander Zeifang \\ Fakultät für Wirtschaftsingenieurwesen \\ Hochschule München / Munich University of Applied Sciences \\ Lothstr. 64, 80335 München \\ T +49 170 3446314, zeifang@hm.edu
}




\title{
Schlüsselkompetenzen für die Entwicklung digital-vernetzter Lösungen
}

\author{
Steffen Kinkel, Johanna Rahn, Bernhard Rieder
}

\section{Zusammenfassung}

Der deutsche Maschinen- und Anlagenbau spielt eine Schlüsselrolle als Anbieter und Anwender digital-vernetzter Produkte, Prozesse und Geschäftsmodelle für die Industrie 4.0. Bislang wurde aber noch nicht empirisch untersucht, in welchem Umfang seine Unternehmen dazu in der Lage sind, selbständig digital-vernetzte Produkte und Services zu entwickeln und welche Fähigkeit sie dazu intern vorhalten müssen. Eine Onlinebefragung von 335 deutschen Maschinenbauunternehmen und 15 vertiefende Experteninterviews zeigen, dass sie vor allem digitalisierte Dienste wie Predictive Maintenance oder Condition Monitoring als Kernkompetenzen betrachten und im eigenen Haus entwickeln. Agile und designorientierte Entwicklungsmethoden können die Fähigkeiten der Unternehmen zur eigenen Entwicklung digitaler Produkte und Services deutlich verbessern. Zukünftig immer wichtiger wird die interdisziplinäre Zusammenarbeit, da digital-vernetzte Innovationen zunehmend an den Grenzflächen verschiedener Disziplinen stattfinden.

\section{$1 \quad$ Einleitung}

Die Digitalisierung durchdringt und vernetzt immer mehr Industriebereiche und Geschäftsprozesse [DwZa14, KRRL16]. Die erwarteten Effekte sind vielfältig und bisweilen radikal, so dass vielfach von einer vierten industriellen Revolution gesprochen wird (,Industrie 4.0“). Diese basiert auf der intelligenten, in Echtzeit stattfindenden, horizontalen und vertikalen Vernetzung von Wertschöpfungsprozessen und Geschäftsmodellen [Klet13, Baue14]. Im Kern sollen die Voraussetzungen für eine hochflexible und gleichzeitig hocheffiziente Produktion geschaffen werden, die es ermöglicht, hochindividualisierte Produkte unter den wirtschaftlichen Rahmenbedingungen eines Massenherstellers herzustellen [LiSt15].

Eine Schlüsselrolle spielt hier der deutsche Maschinen- und Anlagenbau als Anbieter und Anwender digital-vernetzter Produkte, Prozesse und Geschäftsmodelle. Die digitale Transformation in Richtung 14.0 bietet ein großes Potenzial für diesen Sektor als weltweit führender Produktionsausrüster [KaWH13]. Darüber hinaus verfügt Deutschland auch über ein umfangreiches Know-how in der industriellen Informationstechnologie (IT), Automatisierungstechnik, eingebetteten Systemen und intelligenten Netzwerken [PKKK17]. Eine Studie beziffert das zusätzliche Wertschöpfungspotenzial für die Branche bis zum Jahr 2025 auf 23 Milliarden Euro und das entsprechende, jährliche Wertschöpfungswachstum durch Industrie 4.0-Technologien auf 2,2 \% [BiFh14]. Herausforderungen entstehen insbesondere dadurch, dass klassische Branchengrenzen zwischen Maschinen- und Anlagenbau einerseits und IT andererseits verschoben werden und es zu Regelbrüchen und Markteintritten von Dritten kommen kann [EDBP15]. Das prognostizierte, hohe Potenzial ergibt sich aus der spezifischen Möglichkeit der Unternehmen, als Anbieter und Anwender von digital-vernetzten Lösungen zu profitieren: 
- $\quad$ Aus Anbietersicht ergeben sich für den Maschinen- und Anlagenbau große Chancen und Differenzierungsmöglichkeiten durch innovative, digitalisierte Produkte und Dienstleistungen sowie neue, datenbasierte Geschäftsmodelle.

- Aus Anwendersicht kann der Maschinen- und Anlagenbau wie andere Industriebranchen auch von optimierten, hochflexiblen und gleichzeitig ressourceneffizienten Produktionsprozessen und Wertschöpfungsnetzwerken und den sich daraus ergebenden Effizienzgewinnen profitieren.

Die Industrie 4.0 wird auch die Art und Weise beeinflussen, wie wir zukünftig arbeiten werden [BMAS15]. Disruptive Technologien für Kommunikation und Zusammenarbeit werden Prozesse radikal verändern, die Arbeit deutlich vernetzter, interdisziplinärer und komplexer [Zinn15]. Dies reicht von veränderten Prozessen in Einkauf, Fertigung, Vertrieb oder Instandhaltung bis hin zu weitreichenden Auswirkungen auf Geschäftsmodelle und Arbeitsorganisation [KaWH13]. Diese Transformation wird industrielle Berufsprofile verändern und erfordert Mitarbeiter - oder Teams - mit einem breiten Spektrum an Kompetenzen [KaWH13, acat16, SKMC16]. Bislang wurde aber noch nicht empirisch untersucht, in welchem Umfang die Unternehmen des Maschinen- und Anlagenbaus dazu in der Lage sind, selbständig digital-vernetzte Produkte und Services zu entwickeln und welche Fähigkeit sie dazu intern vorhalten müssen. Zu dieser Lücke will dieser Artikel einen Beitrag leisten.

\section{Verständnis von digital-vernetzten Innovationen}

Die digitale Transformation wird große Teile der Produkte, Geschäftsprozesse, Vertriebskanäle und Lieferketten des Maschinen- und Analagenbaus verändern. Folglich reicht sie weit über die Grenzen des einzelnen Unternehmens hinaus in das externe Ökosystem. Im Ergebnis können neue Formen von Interaktionen und ganzen Geschäftsmodellen entstehen [DoNu13], die eine klare Digitalisierungs- und Transformationsstrategie benötigen, um die vielen unabhängigen Aktivitäten und Fäden erfolgreich zu koordinieren [MaHB15]. Die "Digitalisierungsstrategie" ist in den meisten Unternehmen aber vor allem eine IT-Strategie, die sich auf das Management von IT im Unternehmen konzentriert und kaum Innovationen in der Produkt- und Geschäftsmodellentwicklung vorantreibt. Im Gegensatz dazu würde eine „echte“ Digitalisierungs- und Transformationsstrategie eine ganzheitliche Sicht auf neue Produkte, Dienstleistungen, Prozesse und organisatorische Aspekte, die durch digitale Technologien ermöglicht werden, anlegen [MaHB15].

Erfolgversprechende Digitalisierungs- und Vernetzungslösungen sind als soziotechnische Systeme zu verstehen. Sie umfassen neben physischen Maschinen und Produkten auch die Interaktion und Verbindung mit den Menschen und der Organisation sowie die virtuelle Abbildung der physischen Dimensionen in entsprechenden IT-Systemen. Damit spannt sich eine Vier-Felder-Matrix auf, die in einer Dimension zwischen Produkt-/Anbietersicht und Prozess-/Anwendersicht sowie in der anderen Dimension zwischen physischen und virtuellen Elementen im Innovationssystem eines Unternehmens differenziert. Diese Matrix verbindet die vier Felder eines ganzheitlichen Innovationsverständnisses (innovative Produkte, innovative Services, innovative Prozesstechnik, innovative Organisation, KiLW05) symbiotisch mit den vier Feldern neuer Industrie-4.0-Lösungen (Smart Products, Data-driven Services, Smart Factory, Smart Operations; LiSt15) und stellt den konzeptionellen Rahmen für die folgenden Analysen dar. 


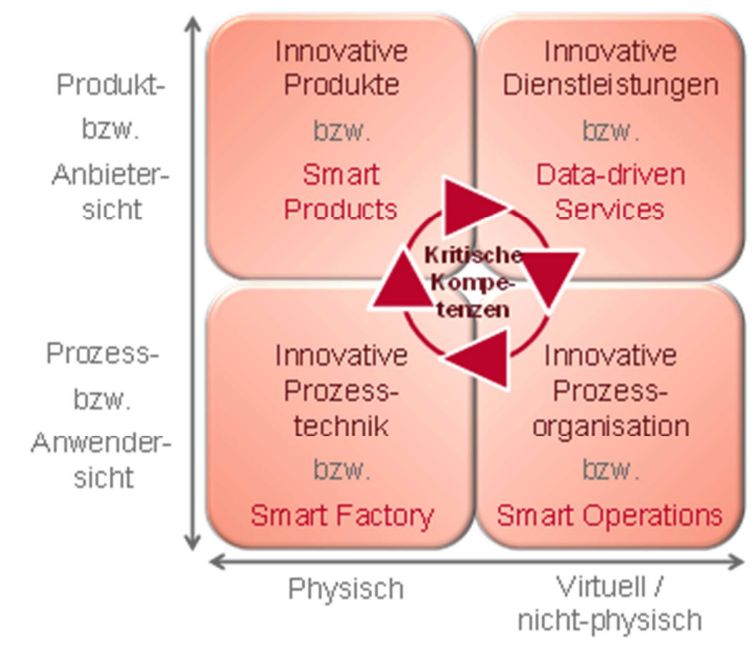

Abbildung 1: Ganzheitliches Verständnis von Industrie-4.0-Innovationen und dazu benötigten Kompetenzen (in Anlehnung an KiLW05; LiSt15)

Die einzelnen Felder lassen sich für den Maschinen- und Anlagenbau wie folgt verstehen:

- $\quad$ Smart Products verfügen über die Fähigkeit, Daten zum eigenen Herstellungs- und Betriebsprozess während der Fertigungs- und Nutzungsphase zu sammeln und zu kommunizieren. Dies ermöglicht das digitale Abbild der Herstellungsprozesse und das Angebot von datenbasierten Dienstleistungen für den Kunden in der Nutzungsphase.

- Data-driven Services zielen auf die Steigerung des Kundennutzens durch neue, datenbasierte Geschäftsmodelle ab, die auf der Auswertung aufgenommener und gesendeter Daten aus der Betriebs- und Nutzungsphase der "Smart Products" basieren.

- Smart Factory beschreibt eine dezentrale und hochautomatisierte Produktionsumgebung, in der sich intelligente Werkstücke, Fertigungsanlagen sowie Logistiksysteme weitgehend selbst organisieren. Die Grundlage hierfür sind digital-vernetzte Systeme, die Maschinen und Ressourcen digital abbilden und über das „Internet der Dinge“ eine Kommunikation in Echtzeit ermöglichen.

- Smart Operations umfassen die digitalen Methoden für grundlegend neue Formen der Produktionsplanung und -steuerung (PPS) und des Supply-Chain-Managements (SCM) zur Realisierung der Vision des sich selbst steuernden Werkstückes.

Aus strategischer Sicht ist es wichtig, eine "Digitalisierungsstrategie" in enger Abstimmung mit der IT-Strategie, der F\&E-Strategie, der Produktions- und Supply-Chain-Strategie und allen anderen organisatorischen und funktionalen Strategien zu entwickeln [MaHB15, HeVe93] und sie zu einer umfassenden "digitalen Geschäftsstrategie" zu konsolidieren [BEPV13]. Für eine erfolgreiche digitale Transformation sind auch neue Kompetenzen der Mitarbeiter erforderlich. Da Arbeit und Prozesse in 14.0 mehr und mehr vernetzt ablaufen, werden Kompetenzen wie interdisziplinäre Kooperation, Kommunikation oder Teamarbeit eine besondere Rolle spielen [PKKK17]. Hieraus ergeben sich wesentliche Herausforderungen und Kernfragen wie beispielsweise: Welche der neu benötigten Kompetenzen können intern entwickelt werden oder müssen extern bezogen werden? Welche werden als Kernkompetenzen betrachtet, die das Unternehmen auf- oder ausbauen bzw. „insourcen“ will? Wie und wo kann man Fachkräfte mit den notwendigen Kompetenzen und technischen Fähigkeiten in der IT- und Softwareentwicklung, IT-Sicherheit, Datenanalyse etc. gewinnen? 
Dies wird für kleine und mittlere Unternehmen (KMU) mit einem wenig bekannten Namen, Image oder an wenig attraktiven Standorten besonders schwierig. Und wie kann man die Erfahrung und das Anwendungswissen der aktuellen Mitarbeiter halten und fruchtbar mit der neuen, möglicherweise stärker durch Digitalisierungstechnologien geprägten Denkweise der jüngeren Generationen kombinieren?

Studien zeigen, dass eine qualifizierte Belegschaft mit den erforderlichen Digitalisierungskompetenzen ein wichtiger Erfolgsfaktor zur Erreichung der ambitionierten Ziele digital-vernetzter Prozesse und Geschäftsmodelle ist. Laut der Readiness Studie der IMPULS-Stiftung geben etwa $30 \%$ der befragten Unternehmen an, betriebsintern keine entsprechenden Kompetenzen zu besitzen, um die bevorstehenden Anforderungen bewältigen zu können [LiSt15]. Die wachsende Digitalisierung und Vernetzung der Produkte und Dienstleitungen stellt den Maschinenbau damit auch vor personelle Herausforderungen. Der aktuellen Trendstudie des VDMA-Forums IT@Automation zufolge ist die Personalverfügbarkeit für zwei Drittel der befragten Unternehmen nach wie vor das größte Hemmnis bei der Entwicklung und Konstruktion von IT und Automatisierungstechnik [VDMA15]. Andererseits ist es für viele mittelständische Industrieunternehmen noch weitgehend unklar, welche (sozio)technischen Kompetenzen sie für den anstehenden, durch die digitale Vernetzung induzierten Strukturwandel benötigen und wie sie mit ihren verfügbaren Kompetenzen dafür gerüstet sind [ZaSB14]. Insgesamt weist die bisherige Forschung eindeutig auf die Bedeutung der Mitarbeiterkompetenzen für eine erfolgreiche, digitale Transformation hin und fordert eine klare Definition der dafür benötigten Kompetenzen [Jasc14].

\section{Forschungsfragen und Methoden}

Vor diesem Hintergrund versucht dieser Beitrag folgende Forschungsfragen zu beantworten:

- Welche digitalisierten Produkte und Services entwickeln die Unternehmen des deutschen Maschinen- und Anlagenbaus selbst und erachten sie als Kernkompetenzen?

- Welches sind die zentralen Erfolgsfaktoren, um die Eigenentwicklungs- und Innovationsfähigkeit bei diesen digital-vernetzten Angeboten sicherstellen zu können.

Basierend auf dem oben dargestellten, konzeptionellen Verständnis wurden diese beiden Forschungsfragen mit einem Mixed-Methods-Ansatz aus den folgenden zwei Elementen untersucht, deren Kombination einen gleichzeitig umfassenden und vertiefenden Einblick ermöglicht:

- $\quad$ Eine spezifische Onlinebefragung von insgesamt 335 Unternehmen des deutschen Maschinen- und Anlagenbaus,

- 15 Experteninterviews mit Vertretern von Unternehmen, von denen acht als „Vorreiter“ und sieben als „Einsteiger“ in die Digitalisierung ihrer Produkte und Services charakterisiert werden können.

An der Online Umfrage haben zwischen Februar und April 2016 insgesamt 335 Personen aus der Geschäftsführung und der IT-Leitung von VDMA-Mitgliedunternehmen teilgenommen. Aufgrund unvollständig ausgefüllter Fragebögen haben 164 Befragte schließlich Angaben zu der Größe ihres Unternehmens gemacht: $23 \%$ kommen aus kleinen Unternehmen (< 100 Beschäftigte), $41 \%$ aus kleinen bis mittleren Unternehmen (100 - 499 Beschäftigte), $20 \%$ aus mittleren bis großen Unternehmen (500 - 1999 Beschäftigte) und $17 \%$ aus großen Unternehmen ( $\geq 2000$ Beschäftigte). Hier zeigt sich eine typische überproportionale Teilnahme von mittleren und großen Unternehmen [LiSt15], die auf eine allgemein höhere 
Antwortbereitschaft von Vertretern dieser Unternehmen zurückzuführen ist. Laut Statistischem Bundesamt (2016) haben nur 6 \% der Maschinenbauunternehmen in Deutschland 500 oder mehr Beschäftigte. Gleichwohl lässt die vorliegende Datenbasis valide Aussagen zu den Digitalisierungsstrategien und -aktivitäten aller Unternehmensgrößenklassen zu.

Die 15 Experteninterviews wurden basierend auf einem teilstandardisierten Leitfaden zwischen Februar und Juni 2016 mit Entwicklungsleitern, Innovationsleitern, Technischen Geschäftsführern oder Geschäftsführern geführt. Die 15 Unternehmen (U1 bis U15) kommen aus verschiedenen Bereichen des Maschinen- und Anlagenbaus (z.B. komplette Fertigungssysteme oder einzelne Komponenten wie Pumpen und Armaturen). Die Gespräche wurden transkribiert und mit Hilfe der Textanalysesoftware MAXQDA von zwei Kodierern analysiert.

\section{$4 \quad$ Zentrale Ergebnisse}

Wie die Studie zeigt, ist die Digitalisierung als strategisches Zukunftsthema im deutschen Maschinen- und Anlagenbau angekommen. Die meisten Unternehmen halten die digitale Transformation für äußerst relevant, um weiterhin wettbewerbsfähig zu bleiben. „Wir könnten es lassen und dann werden wir irgendwann von Fernost überholt, ganz bestimmt" (U9). Ein Drittel der 335 online befragten Unternehmen hat bereits eine dezidierte Digitalisierungsstrategie, ein weiteres Viertel will in den nächsten drei Jahren eine Digitalisierungsstrategie einführen. Die Personalentwicklung beschäftigt sich dagegen bislang nur in etwa jedem fünften Unternehmen strategisch mit den Herausforderungen der Digitalisierung. Dabei sind bestimmte Digitalisierungskompetenzen der Belegschaft eine zentrale Voraussetzung, um erfolgreich eigene digitale Produkte und Services entwickeln und anbieten zu können. Die Experten empfehlen daher in den Interviews, ausreichend Ressourcen einzusetzen, um Industrie 4.0 für das eigene Unternehmen konkret zu definieren und eine Strategie zu fixieren. Dadurch werden auch unterschiedliche Erwartungshaltungen klar und die Notwendigkeit und der Nutzen können den Beschäftigten besser kommuniziert werden (U9, U11).

Hinsichtlich der Frage der Eigenentwicklung (Make) oder des Zukaufs (Buy) zeigen die Analysen, dass die befragten Maschinenbauunternehmen insbesondere digitalisierte Serviceangebote wie Predictive Maintenance, Condition Monitoring oder Onlineschulungen sowie Angebote zur M2M-Kommunikation, zur Echtzeitdatenanalyse und modulare Apps selbst entwickeln (Abbildung 2). Hier scheinen die Unternehmen mehrheitlich Kernkompetenzen zu sehen, mit denen sie sich vom (internationalen) Wettbewerb differenzieren und zusätzlichen Nutzen für ihre Kunden schaffen können. Dagegen werden vor allem bei mobilen Geräten für die Programmierung und Bedienung der Anlagen sowie bei Cloud-Diensten und Simulationssoftware eher vorhandene Technologien „von der Stange“ zugekauft. Hier scheinen die befragten Unternehmen weniger Differenzierungsmöglichkeiten durch eigene Lösungen zu sehen. In etwa ausgeglichen ist die Quote zwischen Eigenentwicklung und Zukauf bei Remote Services und datenbasierten Dienstleistungen auf Basis komplexer Datenanalysen. Bei datenbasierten Diensten, die vielfach als wichtiger Treiber für das Angebot neuer Geschäftsmodelle erachtet werden [ArKV16, LeKY14], verfügen sie möglicherweise auch (noch) nicht über ausreichend eigene Fähigkeiten, um diese selbstständig entwickeln zu können. 


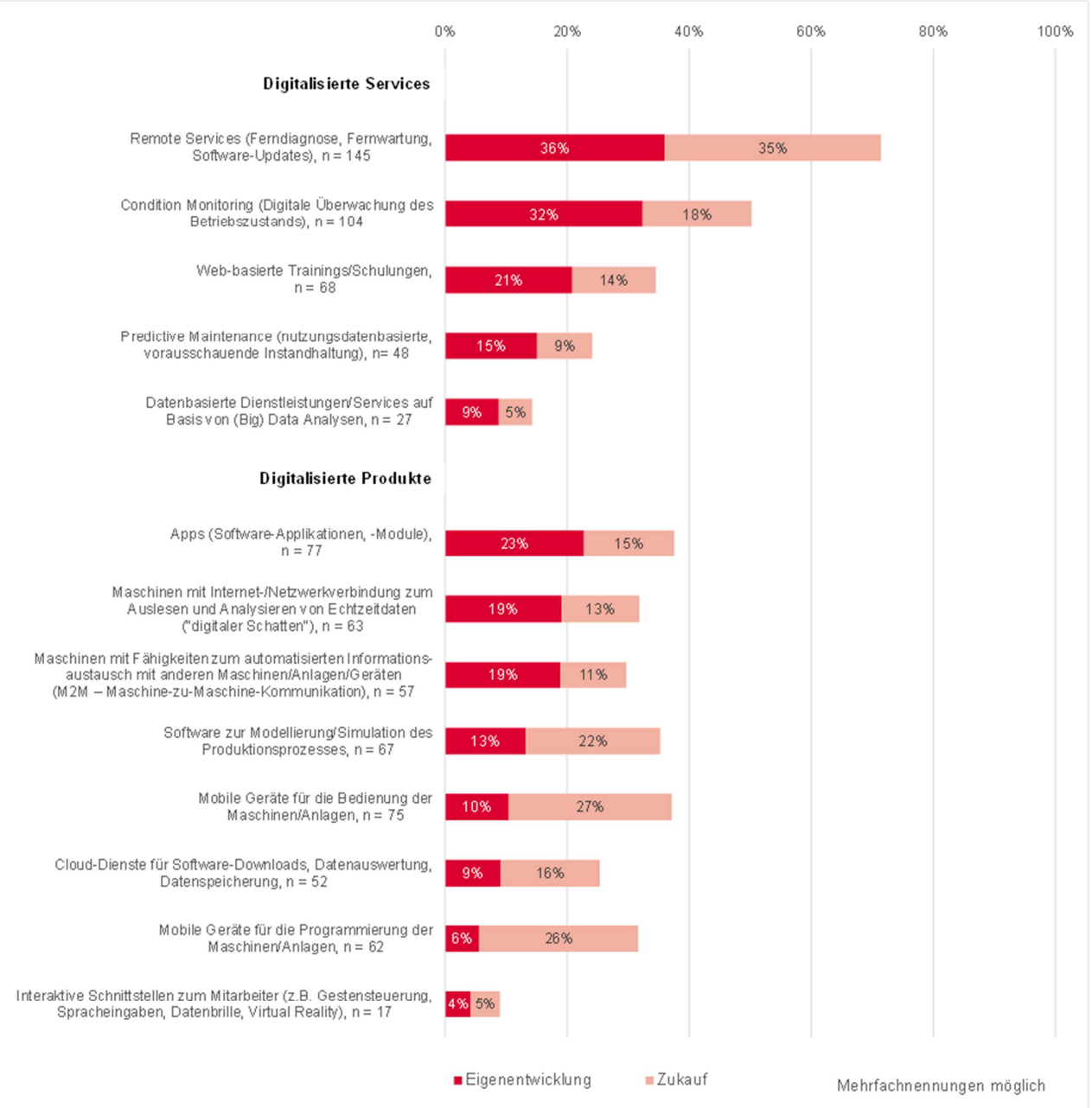

Abbildung 2: Make or buy digitaler Technologien für die eigenen Produkte und Dienstleistungen

Zur Gesamteinschätzung der Eigenentwicklungsinitiativen und der daraus resultierenden Kompetenzanforderungen wurden drei Aktivitätstypen gebildet. Die Unternehmen werden als Außenstehende klassifiziert, wenn sie keine der Technologien selbst entwickelt haben. Einsteiger haben jeweils ein bis zwei der abgefragten digitalisierten Produkte bzw. Services eigenständig entwickelt. Fortgeschrittene zeichnen sich durch Eigenentwicklungen von jeweils mindestens drei der abgefragten Technologien (Produkte bzw. Services) aus.

Für eine schnelle und kundenorientierte Eigenentwicklung digitalisierter Produkte und Services ist es von besonderer Relevanz, leistungsfähige Entwicklungsmethoden für die Software- und IT-Entwicklung einzusetzen. Es zeigt sich, dass es signifikante Unterschiede in der Anwendung von agilen Entwicklungsmethoden, designorientierten Entwicklungsmethoden und IT-Styleguides bei Unternehmen gibt, die erfolgreich digitalisierte Produkte selbst entwickeln (Abbildung 3). Unternehmen, die in der Eigenentwicklung digitalisierter Produkte „fortgeschritten“ sind, also mindestens drei der abgefragten Technologien für digital-vernetzte Produkte selbst entwickeln, nutzen etwa acht Mal häufiger agile Entwicklungsmetho- 
den als die Gruppe der „Außenstehenden“. Designorientierte Entwicklungsmethoden werden etwa sechs bis sieben Mal häufiger angewendet und IT-Styleguides etwa drei Mal so häufig. Insgesamt zeigt sich damit, dass agile und designorientierte Entwicklungsmethoden die Eigenentwicklungs- und Innovationsfähigkeit der Unternehmen bei digitalisierten Produkten signifikant befruchten können.

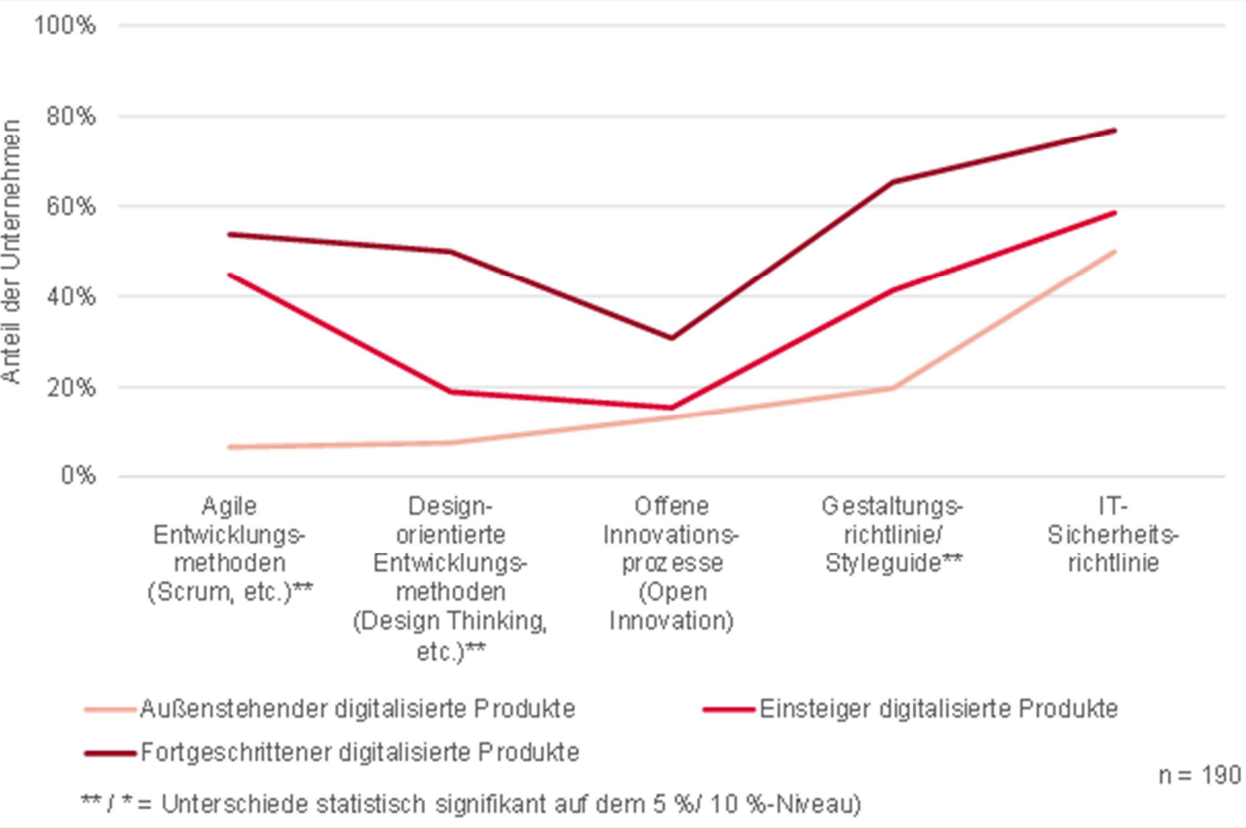

Abbildung 3: Methodeneinsatz nach Aktivitätstypen der Eigenentwicklung digitalisierter Produkte

Die interne Fähigkeit der Unternehmen, eigenständig digital-vernetzte Produkte und Services entwickeln zu können, hängt zudem in starkem Maße davon ab, ob und in welchem Umfang sie eigene Mitarbeiter haben, die über die notwendigen Digitalisierungskompetenzen verfügen [ArKV16, KRRL16]. So weisen Unternehmen, die in der Eigenentwicklung digitalisierter Produkte „fortgeschritten“ sind, signifikant überdurchschnittliche Mitarbeiteranteile mit Kompetenzen in der Programmierung der Maschinen- und Anlagensteuerungen sowie in der Softwareentwicklung für modulare Applikationen (Apps) in den eigenen Produkte auf (Abbildung 4). Gleiches gilt für den internen IT-Betrieb und für das benutzerorientierte IT-Design. Die entsprechenden Mitarbeiteranteile mit diesen Kompetenzen sind etwa doppelt so hoch wie bei „Einsteigern“ und bis zu viermal höher als bei den „Außenstehenden" bei der Eigenentwicklung digitalisierter Produkte.

Dies belegt, dass interne Kompetenzen bei der Programmierung und Softwareentwicklung eine wichtige Voraussetzung für die Fähigkeit sind, eigenständig digitalisierte Produkte entwickeln und anbieten zu können. Außerdem sind Fähigkeiten zur kundenorientierten Gestaltung benutzerfreundlicher IT-Oberflächen eine wesentliche Voraussetzung, um digitalisierte Lösungen erfolgreich in den Markt einführen und an den Kunden bringen zu können. Auch Kompetenzen bei der IT- und Datensicherheit sind in diesem Kontext wichtig. Allerdings sollte man hier nicht, wie leider viele deutsche Unternehmen, wie das Kaninchen auf die Schlange starren und auf Lösungen von außen warten, sondern „lieber selbst oder in 
Kollaboration mit anderen de facto Standards für die Vernetzung schaffen und sich an der Diskussion um Standards und Sicherheit beteiligen" (U15).

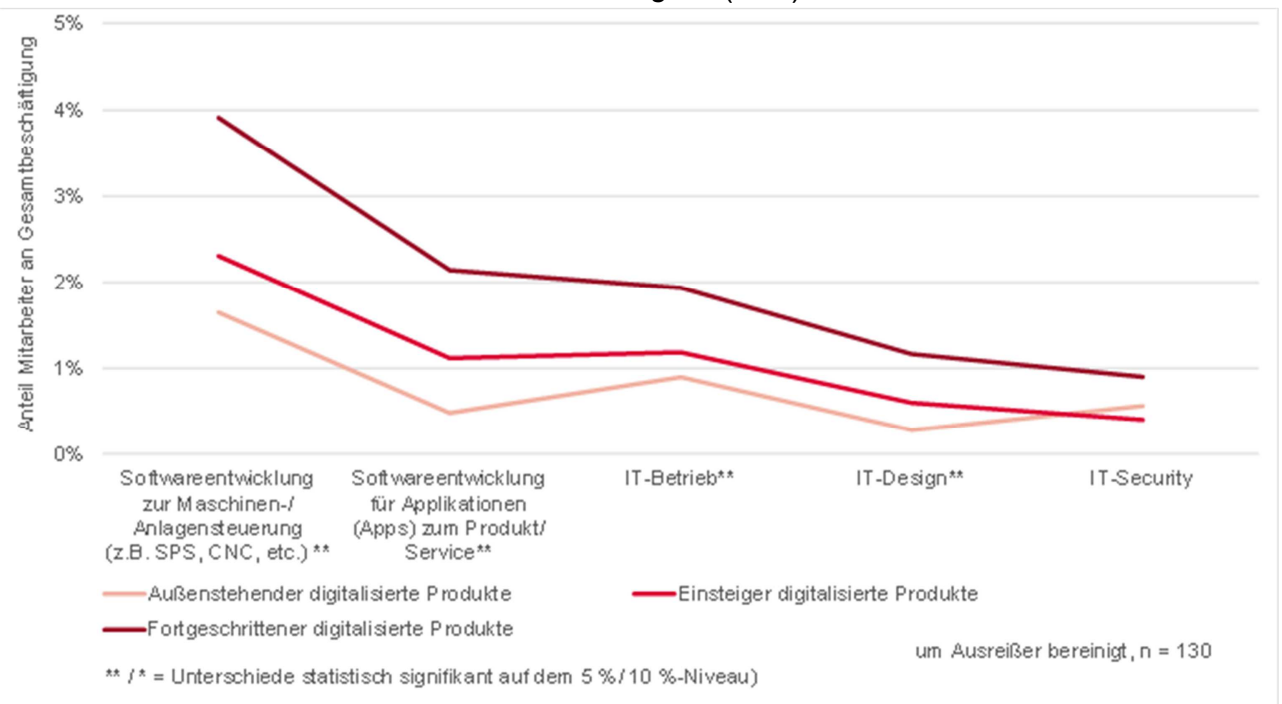

Abbildung 4: Anteile der Mitarbeiter mit ausgewählten Digitalisierungskompetenzen an der Gesamtbeschäftigung nach Aktivitätstypen der Eigenentwicklung digitalisierter Produkte

Wie vertiefte Analysen zeigen, liegen weitere Schlüsselkompetenzen für die digitale Vernetzung in der Fähigkeit zur Analyse komplexer Daten und im Verständnis der Kundenprobleme. Zukünftig immer wichtiger wird ein interdisziplinäres Verständnis, da digital-vernetzte Innovationen zunehmend im Grenzbereich verschiedener Disziplinen stattfinden. Daher sollten Mitarbeiter der verschiedenen Ingenieur- und IT-Disziplinen bei konkreten Projekten konsequent zusammenarbeiten, um die Sichtweisen der verschiedenen Welten besser verstehen zu lernen.

In den Experteninterviews wurde angeregt, Mitarbeiter mit interdisziplinären Ausbildungshintergründen zu suchen, die Informatik und Produktionstechnik vereinen, oder entsprechende Teams zu bilden, in denen diese Kompetenzträger eng zusammen arbeiten (U8). Ein Experte meinte dazu: „Ich glaube, dass die Zusammenarbeit im interdisziplinären Team ein Thema ist, welches ... unterschätzt wird. Aber die Potenziale die daraus erwachsen ... werden immer wichtiger. Da als Unternehmen die Rahmenbedingungen und die kulturellen Voraussetzungen zu schaffen, damit man so bereichsübergreifend zusammenarbeiten kann, halte ich für ein sehr wichtiges Thema" (U1).

Die betrieblichen Maßnahmen zum Auf- und Ausbau der notwendigen Digitalisierungskompetenzen umfassen den klassischen Kanon von internem Wissensmanagement $(83 \%$ der Betriebe), Weiterbildung (77\%), Zusammenarbeit mit IT-Unternehmen (74 \%) und Einstellungen $(71 \%)$. Neueinstellungen zur Rekrutierung entsprechender IT- und Softwarespezialisten sind für kleine Unternehmen schwieriger und werden von diesen deutlich seltener betrieben (44\%). Sie betreiben daher häufiger eine Auslagerung der Softwareentwicklung an externe Dienstleister (82 \%). Dabei gilt es allerdings darauf zu achten, die zentralen Kompetenzen auch im eigenen Haus vorzuhalten. Sehr hilfreich kann es dafür sein, „einzelne Mitarbeiter bei deren eigenen Digitalisierungsprojekten zu unterstützen, langfristig kann das ganze Unternehmen davon profitierten" (U9). 
Eine weitere strategische Option ist die Ausgründung eigener IT- und Softwareunternehmen, die ein erstaunlich hoher Anteil (20\%) der befragten Unternehmen anwendet. Aus den Experteninterviews werden die zentralen Gründe hierfür ersichtlich. Dies sind die organisatorische Trennung zur Unterstützung der „zwei Entwicklungsgeschwindigkeiten“ von traditionellem Maschinenbau und agiler Softwareentwicklung, der bessere Zugang zu neuen Branchen und Geschäftsmodellen sowie die höhere Anziehungskraft eines eigenständigen, kleinen Softwareunternehmens für die Gewinnung qualifizierten IT-Personals (U1, U2, U8, U9). Zudem denken einige Unternehmen (u.a. U3, U14) verstärkt über strategische Kooperationen nach, wenn es um die Entwicklung von digitalisierten Services und Produkten geht, „weil Industrie 4.0 nur funktionieren wird, wenn die Unternehmen zum Wohle der Kunden miteinander arbeiten." (U3).

\section{$5 \quad$ Beitrag zur Forschung zu Digitalisierungskompetenzen}

Die Ergebnisse zu Schlüsselkompetenzen, die für die Entwicklung von digitalen Produkten und Dienstleistungen deutscher Maschinenbauunternehmen entscheidend sind, stehen im Einklang mit der bestehenden Forschung zu wichtigen Kompetenzen für den digitalen Wandel von Industrieunternehmen. Die identifizierten, kritischen Kompetenzen werden im Folgenden den vier Kategorien von [ErRo07] zugewiesen, die eines der bekanntesten Ordnungsmodelle für Kompetenzen darstellen: Personale Kompetenzen, sozialkommunikative Kompetenzen, aktivitäts- und umsetzungsorientierte Kompetenzen und fachlich methodische Kompetenzen.

Die kritischen Kompetenzen in der Programmierung, Softwareentwicklung und in der IT- und Datensicherheit sind im Einklang mit vorliegenden Forschungsergebnissen zu wichtigen fachlichen Kompetenzen von Absolventen der Fachrichtungen Informatik und Wirtschaftsinformatik. Dazu zählen etwa Modellierung und Programmierung [EJHS16, BCG15, VDIA15, KASR13], Cloud Computing und Cloud Architekturen [acat16, HKOW15, PKKK17] oder Datensicherheit [PKKK17]. Die kritische Kompetenz im kundenorientierten Design von anwenderfreundlichen IT-Oberflächen werden bislang eher selten diskutiert, vor allem als fachliche Kompetenzen von Absolventen der Informatik, Wirtschaftsinformatik und des Wirtschaftsingenieurwesens in den Bereichen Serviceorientierung und Produkt-Service-Systemen [PKKK17; acat16, Zinn15, HKOW15]. Die kritische Kompetenz bei der Analyse komplexer Daten, um daraus Mehrwerte für Kunden oder das eigene Geschäft zu generieren, wird durch die Forschung zur Bedeutung von fachlichen Kompetenzen von Absolventen in den Bereichen Big Data, Datenbanken, Datenanalyse und -interpretation [acat16, Zinn15, EJHS16, BCG15, VDIA15, RBSC14, HKOW15, Capg15, DwZa14, LeKY14, TaZh16] sowie allgemeine analytische Fähigkeiten [EJHS16, BCG15] bestätigt. Sie wird zudem auch eindeutig als Kernkompetenz für eine erfolgreiche digitale Transformation von Unternehmen angesehen [KRRL16, DwZa14, PKKK17].

Die kritische Kompetenz zur schnellen Erfassung und Verständnis von Kundenproblemen und Kundengeschäftsmodellen wird vor allem als fachliche Kompetenz im Bereich der Geschäftsprozessmodellierung [acat16, Zinn15, EJHS16, VDIA15, RBSC14, PKKK17], der Geschäftsmodelltransformation [Zinn15, RBSC14] und des Entrepreneurship [KiWo15, PKKK17] diskutiert. Es wird angenommen, dass diese Kompetenzen für die Industrie 4.0 eine besondere Rolle spielen werden, da sie wichtige Grundlagen für den Wandel zu digitalen Geschäftsmodelle darstellen [PKKK17]. Wichtig in diesem Zusammenhang sind zudem 
soziale Kompetenzen bei der Problemlösung [acat16, GeGN15, SKMC16, EJHS16, Windelband, 2014, KiWo15; SVCS16, DwZa14], Zusammenarbeit und Teamarbeit [acat16, EJHS16, VDIA15, RBSC14, KiWo15, GrKo15] sowie Kompromiss- und Verhandlungsfähigkeit [EJHS16], da sie eine wichtige Voraussetzung für erfolgreiche Kundenorientierung, Kundenbeziehungen und -netzwerke sind [acat16; Guo15; HKOW15].

Die kritische Kompetenz eines interdisziplinären Verständnisses, um verschiedene Disziplinen integrieren zu können, wird durch die Forschung zur Bedeutung sozialer Kompetenzen wie Flexibilität und Anpassungsfähigkeit in interdisziplinären Umgebungen bestätigt [acat16, GeGN15, BCG15, VDIA15, GrKo15, RBSC14]. Hinzu kommen fachliche Kompetenzen bei der Integration heterogener Technologien [GrKo15], aber auch Systemkenntnisse über internetbasierte Netzwerktechnologien, M2M-Kommunikation, Robotik oder künstliche Intelligenz [acat16, BCG15, Zinn15, GeGN15, EJHS16, RBSC14]. Diese interdisziplinären Kompetenzen werden immer wichtiger, da immer mehr Innovationen für die digitale Transformation an den Grenzflächen verschiedener Disziplinen entstehen. Die Entwicklung solcher interdisziplinärer Kompetenzen ist eine lange Reise und braucht einfache praktische Ansätze (,hands on“), um früh beginnen und lernen zu können. Es wird empfohlen, dass Unternehmen konkrete Projekte starten, in denen Mitarbeiter der verschiedenen Ingenieur- und IT-Disziplinen konsequent zusammenarbeiten, um die Sichtweisen der verschiedenen Welten besser zu verstehen [KRRL16, PKKK17].

Wie die Analyse zeigt, konzentriert sich der Großteil der Forschung zu kritischen Kompetenzen für 14.0 oder die digitale Transformation entweder auf fachliche Kompetenzen von Absolventen der Informatik und Wirtschaftsinformatik oder auf sehr breit definierte soziale Kompetenzen wie "Problemlösung", " Kommunikation "oder" Zusammenarbeit ". Die beiden anderen Dimensionen von [ErRo07], personale und aktivitäts- bzw. umsetzungsorientierte Kompetenzen, fehlen zumeist. Um in diese Richtung zu wirken, wäre ein gründlicheres Verständnis der konkreten Kompetenzbedürfnisse von Unternehmen, die sich der digitalen Transformation stellen, erforderlich. So sind beispielsweise hierfür nicht nur Kompetenzen in der Analyse komplexer Daten erforderlich, sondern auch die Fähigkeiten, daraus einen Mehrwert für die Kunden oder das eigene Geschäft zu generieren. Auch ist es nicht ausreichend, Fähigkeiten zur Lösung eines Kundenproblems zu haben; manche Mitarbeiter müssen auch in der Lage sein, schnell die Auswirkungen auf das eigene und das Geschäftsmodell des Kunden zu erfassen.

Die bisherige Forschung konzentriert sich vor allem auf Wissen und Fähigkeiten, die von den Absolventen der relevanten Disziplinen -Informatik, Wirtschaftsinformatik, Ingenieurwesen, Wirtschaftsingenieurwesen - in die Unternehmen "gebracht" werden. Diese Studie hat gezeigt, dass es wichtigere Maßnahmen und Aktivitäten von Industrieunternehmen gibt, um die notwendigen Digitalisierungskompetenzen aufzubauen, als nur die Rekrutierung der relevanten Spezialisten - insbesondere für KMU, für die es immer schwieriger wird, Mitarbeiter mit diesen Engpassqualifikationen zu bekommen. Daher sollten zunehmend auch erfolgversprechende firmeninterne Maßnahmen zur Kompetenzentwicklung für die digitale Transformation wie etwa systematische Weiterbildung, Vernetzung von Schlüsselpersonal, die Unterstützung einzelner Engagierter bei "ihren" Digitalisierungsprojekten oder auch Kooperationen mit anderen Unternehmen in die Analyse mit einbezogen werden. Eine solche Sichtweise könnte den Blickwinkel auf kritische Kompetenzen für die digitale Transformation und vielversprechende Maßnahmen zu einer adäquaten Kompetenzentwicklung hilfreich erweitern. 
Agile Methoden (wie z.B. Scrum) oder designorientierte Entwicklungsprozesse (wie z.B. Design Thinking) können die Fähigkeit zur eigenen Entwicklung digitaler Produkte und Services signifikant befruchten. Sie werden bislang aber nur von wenigen Unternehmen eingesetzt. Doch auch eine Förderung einzelner Mitarbeiter mit Affinität zum Thema Digitalisierung kann sich auszahlen. Unternehmen, die sich eine systematische Übersicht über die Fähigkeiten und Ideen ihrer Beschäftigten verschaffen und ihnen Freiräume geben, diese weiterzuentwickeln, können von neuen Produkten, Prozessen oder Services profitieren, die im eigenen Haus entwickelt wurden. Als Schlüsselkompetenzen für die erfolgreiche Eigenentwicklung digital-vernetzter Lösungen wurden identifiziert:

- Kompetenzen in der Softwareentwicklung von modularen Anwendungen (Apps),

- Kompetenzen bei der Programmierung von Maschinen- und Anlagensteuerungen,

- Kompetenzen in der IT- und Datensicherheit und im nutzerorientierten IT-Design,

- Kompetenzen bei der Analyse komplexer Daten und der Fähigkeit, daraus einen Mehrwert für die Kunden oder das eigene Geschäft zu generieren,

- Kompetenzen beim Erfassen und Verstehen von Kundenproblemen und Kundengeschäftsmodellen,

- $\quad$ interdisziplinäre Kompetenzen, um verschiedene Disziplinen integrieren zu können.

Zunehmend wichtig wird auch die Bereitschaft der Unternehmen zum raschen Experimentieren, Testen und Lernen. Für weitreichende Wandlungsprozesse wie die digitale Transformation ist eine positive Fehlerkultur unumgänglich, um Dinge pragmatisch ausprobieren zu können und aus den gemachten Erfahrungen schnell zu lernen: „be brave and fail - and learn - fast"! Die Ausgliederung eigener IT- und Softwareunternehmen kann eine interessante strategische Option für Unternehmen sein, die versuchen, digital-vernetzte Lösungen im eigenen Haus zu entwickeln. Die Hauptgründe für diese Strategie sind die organisatorische Trennung zur Unterstützung der „Zwei Entwicklungsgeschwindigkeiten“ von traditionellem Maschinenbau und agiler Softwareentwicklung, der Zugang zu neuen Branchen und Geschäftsmodellen sowie die höhere Anziehungskraft eines eigenständigen, kleinen Softwareunternehmens für die Gewinnung qualifizierten IT-Personals. Der letzte Punkt wird insbesondere für KMU immer wichtiger, für die es immer schwieriger wird, Mitarbeiter mit diesen Engpassqualifikationen zu bekommen. 


\section{Literatur}

[acat16] acatech, Fraunhofer Institut für Materialfluss und Logistik, equeo GmbH: Kompetenzentwicklungsstudie Industrie 4.0. Report (2016)

[ArKV16] Arnold, C.; Kiel, D., and Voigt, K.I. (2016): How the Industrial Internet of Things changes business models in different manufacturing industries. In: International Journal of Innovation Management, December 2016, Vol. 20, No. 08, doi: 10.1142/S1363919616400156

[Baue14] Bauernhansl, T. (2014): Die Vierte Industrielle Revolution - Der Weg in ein wertschaffendes Produktionsparadigma, in: Bauernhansl, T./ten Hompel, M./Nogel-Heuser, B. (Hrsg.): Industrie 4.0 in Produktion, Automatisierung und Logistik. Wiesbaden: Springer, S. 5-35.

[BEPV13] Bharadwaj, A.; El Sawy, O.A.; Pavlou, P.A.; Venkatraman, N. (2013): Digital Business Strategy: Toward a Next Generation of Insights. In: MIS Quart 37 (2), 471-482

[BiFh14] Bitkom/Fraunhofer IAO (2014): Industrie 4.0 - Volkswirtschaftliches Potenzial für Deutschland. URL: https://www.bitkom.org/Publikationen/2014/Studien/Studie-Industrie-40-Volkswirtschaftliches-Potenzial-fuer-Deutschland/Studie-Industrie-40.pdf Abgerufen: 17.06.2016

[BMAS15] Bundesministerium für Arbeit und Soziales (BMAS).: Arbeiten 4.0. Report, BAS (2015)

[Capg15] Capgemini Consulting: Industry 4.0. Report, Capgemini Consulting (2015)

[DoNu13] Downes, L.; Nunes, P.F. (2013): Big-Bang Disruption. In: Harvard Bus Rev 91 (3), $44-56$

[DwZa14] Dworschak, B.; Zaiser, H. (2014): Competences for cyber-physical systems in manufacturing - first findings and scenarios. In: Procedia CIRP 25 (2014), pp. 345 - 350

[EDBP15] Emmrich, V.; Döbele, M.; Bauernhansl, T.; Paulus-Rohmer, D.; Schatz, A., Weskamp, M. (2015): Geschäftsmodell-Innovation durch Industrie 4.0. Chancen und Risiken für den Maschinen- und Anlagenbau. Hg.: Dr. Wieselhuber \& Partner GmbH München. Dr. Wieselhuber \& Partner GmbH München, Fraunhofer IPA.

[EJHS16] Erol, S., Jäger, A., Hold, P., Sihn, W.: Tangible Industry 4.0: a scenario-based approach to learning for the future of production. In: Conf. on Lear. Fact. Gjøvik, pp. 1-6. Norway (2016)

[ErRo07] Erpenbeck, J., Rosenstiel, L.: Handbuch Kompetenzmessung. Schäffer Poeschel (2007)

[GeGN15] Gebhardt, J., Grimm, A., Neugebauer, L.M.: Developments 4.0 Prospects on future requirements and impacts on work and vocational education JOTED 3, 117-133 (2015)

[GrKo15] Grega, W., Kornecki, A.J.: Real-Time Cyber-Physical Systems-Transatlantic Engineering Curricula Framework. In: Conf. on Comp. Sc. and Inf. Sys., pp. 755-762. Gdansk (2015)

[GrTö14] Grimheden, M.E., Törgren, M.: Towards curricula for Cyber-Physical Systems. In: Workshop on Embedded and Cyber-Physical Systems Education, New Delhi, India (2014)

[Guo15] Guo, Q.: Learning in a Mixed Reality System in the Context of ,Industrie 4.0'.JOTED 3, 92-115 (2015)

[HeVe93] Henderson, J.C.; Venkatraman, N. (1993): Strategic Alignment: Leveraging Information Technology for Transforming Organizations. In: IBM Syst J 32 (1), 4-16

[HKOW15] Hoberg, P., Krcmar; H., Oswald, G., Welz, B.: Skills for Digital Transformation. $\operatorname{TUM}(2015)$

[Jasc14] Jaschke, S.: Mobile Learning Applications for Technical Vocational and Engineering Education. In: Int. Conf. on Interactive Collaborative Learning, pp. 603-608. Dubai (2014) 
[KaWH13] Kagermann, H., Wahlster, W., Helbig, J.: Recommendations for implementing the strategic initiative Industrie 4.0. Report, Industry 4.0 Working Group (2013)

[KiWo15] Kiesel, M., Wolpers, M.: Educational challenges for employees in project-based Industry 4.0 scenarios. i-KNOW, Graz, Austria (2015)

[KRRL16] Kinkel, S., Rahn, J., Rieder, B., Lerch, C., Jäger, A. (2016): Digital-vernetztes Denken in der Produktion. Studie für die IMPULS-Stiftung des VDMA, Karlsruhe, November 2016

[KiLW05] Kinkel, S.; Lay, G.; Wengel, J. (2005): Innovation: Mehr als Forschung und Entwicklung. In: Industrie-Management 21 (2005), 3, S. 54-58.

[Klet13] Kletti, J. (2013): Das MES der Zukunft. MES 4.0 unterstützt Industrie 4.0, in: Productivity Management $18(2)$, S. 17-20.

[KASR13] Kortuem, G., Arosha K., Smith, N., Richards, M. Petre, M.: Educating the Internet-ofThings generation. Computer 46, 53-61 (2013)

[LeKY14] Lee, J.; Kao, H.-A.; Yang, S. (2014): Service innovation and smart analytics for Industry 4.0 and big data environment. In: Procedia CIRP 16 (2014), pp. 3-8

[LiSt15] Lichtblau, K.; Stich, V.; Bertenrath, R.; Blum, M.; Bleider, M.; Millack, A. et al. (2015): Industrie 4.0-Readiness. Hg.: IMPULS-Stiftung.

[MaHB15] Matt, C.; Hess, T.; Benlian, A. (2015): Digital Transformation Strategies. In: Business and Information Systems Engineering, 57(5), 339-343

[PKKK17] Prifti, L.; Knigge, M.; Kienegger, H. Krcmar, H. (2017): A Competency Model for "Industrie 4.0" Employees. In: Proceeding of the 13th International Conference on Wirtschaftsinformatik, February 12-15, 2017, St. Gallen, Switzerland

[RBSC14] Roland Berger Strategy Consultants: Industry 4.0. Report, Roland Berger (2014)

[SVCS16] Schneckenberg, D.; Velamuri, V.K.; Comberg, C.; Spieth, P. (2016): Business model innovation and decision making: uncovering mechanisms for coping with uncertainty. In: R\&D Management, January 2016, DOI: 10.1111/radm.12205

[SKMC16] Smit, J., Kreutzer, S., Möller, C., Carlberg, M.: Industry4.0.Report. European Parliament (2016)

[TaZh16] Tan, K.H. and Zhan, Y. (2016): Improving new product development using big data: a case study of an electronics company. In: R\&D Management, November 2016, DOI: 10.1111/radm. 12242

[BCG15] The Boston Consulting Group: Man and Machine in Industry 4.0. Report, BCG (2015)

[VDIA15] VDI \& ASME: Industry 4.0. Report, VDI \& ASME (2015)

[VDMA15] VDMA (2015): IT und Automation im Maschinenbau. VDMA-Trendstudie 2015, Frankfurt.

[ZaSB14] Zanker, C., Som. O., Buschak, D. (2014): Industrieller Mittelstand: Spitzenstellung in Gefahr? Analyse der Innovationsfähigkeit kleiner und mittlerer Unternehmen in der Metropolregion Stuttgart. IHK Stuttgart (Hrsg.), Stuttgart 2014.

[Zinn15] Zinn, B.: Conditional variables of 'Ausbildung 4.0'. JOTED 3, 1-9 (2015) 


\section{Kontakt}

Prof. Dr. Steffen Kinkel

Leiter ILIN Institut für Lernen und Innovation in Netzwerken (www.ilin.eu)

Fakultät für Informatik und Wirtschaftsinformatik

Hochschule Karlsruhe - Technik und Wirtschaft

Moltkestr. 30, 76133 Karlsruhe

Tel.: 0721 925-2915, steffen.kinkel@hs-karlsruhe.de

Dr. Johanna Rahn

Leibniz-Institut für Wissensmedien (IWM)

Schleichstraße 6, 72076 Tübingen

Tel: +49 7071 979-310, j.rahn@iwm-tuebingen.de

Bernhard Rieder

Eduard-Baumgartner-Straße 5, A-5020 Salzburg

Mobil: +43664 4454946 


\title{
Industrie-4.0-Reifegradbestimmung produzierender Unternehmen im deutschsprachigen Raum und Identifikation bedeutender Industrie-4.0- Einflussfaktoren
}

\author{
Jörg Puchan, Denis Heideker, Sascha Hertkorn
}

\section{Zusammenfassung}

Diese empirische Studie untersucht den aktuellen Umsetzungsstand (2016) von Industrie 4.0 in produzierenden Unternehmen im deutschsprachigen Raum und identifiziert bedeutende Industrie-4.0-Einflussfaktoren anhand von Hypothesenprüfungen. Mithilfe eines fünfstufigen Industrie-4.0-Reifegradmodells der Hochschule München (HM-Reifegradmodell) werden Unternehmen in unterschiedlichen Handlungsfeldern bewertet. Das HM-Reifegradmodell umfasst neben seinen fünf Handlungsfeldern weitere 29 untergeordnete Handlungselemente. Diese spiegeln die Voraussetzungen von Industrie 4.0 wider und sind daher für eine erfolgreiche digitale Transformation unumgänglich. Das Design und die Methode der wissenschaftlichen Studie werden erläutert. Die 107 befragten Unternehmen repräsentieren ein ausgewogenes Mischverhältnis von großen Konzernen sowie kleinen und mittelständischen Unternehmen. Zudem können mehr als die Hälfte der Befragten der oberen oder mittleren Führungsebene zugeordnet werden. Es lässt sich festhalten, dass die deutsche Industrie noch am Anfang der vierten industriellen Revolution steht. Acht von zehn Umfrageteilnehmer sind der niedrigsten Reifegradstufe (Standard Level) zuzuordnen und weitere zwei von zehn Unternehmen erreichen lediglich das nächsthöhere Reifegradlevel (Novice Level). Nach derzeitigem Umsetzungsstand nehmen vor allem Großunternehmen eine Vorreiterrolle bei der Adaption von Industrie 4.0 ein. Das Handlungsfeld Schlüsselfaktoren wurde mit seinen definierten Grundvoraussetzungen für Industrie 4.0 zudem als bedeutender Einflussfaktor für die Industrie-4.0-Reife ermittelt. Vor allem in Bezug auf die Produktion wurden die Datennutzung und die Vernetzung von IT-Systemen als weitere bedeutende Einflussfaktoren identifiziert. Unternehmen, die auch in Zukunft eine führende Rolle in der Industrie anstreben, sollten zudem ganzheitliche Produktlösungen inklusive digitaler Serviceleistungen anbieten.

\section{$1 \quad$ Einleitung}

Die zunehmende Digitalisierung verändert das klassische Modell der industriellen Produktion immer mehr und ist daher das prägende Thema in der Industrie. Sie beeinflusst unsere Gesellschaft auf unterschiedlichste Weise und führt zu Veränderungen in annähernd allen Lebensbereichen unseres Alltags (s. [HeEK16] S. 13; [Brüh15] S. V). Um die Wettbewerbsfähigkeit des Standortes Deutschland auch weiterhin zu erhalten, sind die großen Herausforderungen der vierten industriellen Revolution zu meistern und die damit verbundenen notwendigen Veränderungen erfolgreich und schnell umzusetzen (s. [HeEK16] S. 13). Wegweisend ist hierbei, zu erkennen, dass diese Umgestaltung nicht nur technische Neuerungen erfordert, sondern auch einen Wandel organisationaler und kultureller Natur mit sich bringt (s. [Bund15] S. 3). Um den Weg zur vollständigen Umsetzung von Industrie 4.0 
nachverfolgbar zu machen und den Fortschritt der Industrie aufzuzeigen, muss eine entsprechende Bewertung durchgeführt werden. Es fehlt derzeit jedoch noch der Einsatz eines ausgereiften Hilfsmittels zur Identifizierung der Industrie-4.0-Gesamtreife, sowie der Stärken und Schwächen in einzelnen Handlungsfeldern der produzierenden Unternehmen in der D-A-CH-Region (s. [Ab++15] S. 2). Diese Studie erforscht die Fragestellung nach dem derzeitigen Umsetzungsstand (2016) der vierten industriellen Revolution in produzierenden Unternehmen im deutschsprachigen Raum und identifiziert zudem bedeutende Industrie4.0-Einflussfaktoren anhand von Hypothesenprüfungen. In diesem Zusammenhang wird der Begriff Industrie 4.0 in Anlehnung an die von Plattform Industrie 4.0 getroffene Begriffsdefinition verwendet. Diese besagt, dass die vierte industrielle Revolution zu unternehmensübergreifenden Wertschöpfungsnetzwerken und insbesondere intelligenten Fabriken (sog. Smart Factories) führt (s. [Bund16]).

\section{Industrie-4.0-Reifegradmodell der Hochschule München}

Das Industrie-4.0-Reifegradmodell der Hochschule München (HM-Reifegradmodell) (s. [Kast16]) dient der vorliegenden Studie als Strukturierungsmethode zur Bewertung der erarbeiteten Hypothesen. Dieses theoretische Modell bildet die Basis für die Leistungsbewertung sowie für den Leistungsvergleich der teilnehmenden Unternehmen. Das HM-Reifegradmodell bezieht alle wichtigen Industrie-4.0-Elemente ein und wurde auf Basis einer ausführlichen Literaturrecherche und wissenschaftlich fundierter Arbeitsweise entwickelt. Durch den Einsatz des Modells kann der Umsetzungsstand von Industrie 4.0 in einem einzelnen Unternehmen und der Grundgesamtheit bestimmt werden.

\subsection{Industrie-4.0-Handlungsfelder (HF) und Industrie-4.0-Handlungselemente (HE)}

Um die in einem komplexen Konstrukt enthaltenen latenten Merkmale zu messen werden psychometrische Skalen eingesetzt. Das HM-Reifegradmodell ist als eine mehrdimensionale psychometrische Skala zu sehen, bei der aus jeweils einem Teil der Items Subskalenwerte (Reifegrade der Handlungselemente) gebildet werden, welche die Ausprägung von Unteraspekten (Reifegrade der Handlungsfelder) des gemessenen Merkmals (Industrie-4.0-Reifegrad) repräsentieren (s. [DöBo16] S. 268f.). Es werden also mehrere ähnliche Indikatoren (Skalen-Items) verwendet und zu einem Gesamtwert zusammengefasst, was den Vorteil einer präziseren Erfassung gegenüber einem Einzelindikator mit sich bringt (s. [DöBo16] S. 267f.). Die im Einzelnen identifizierten Handlungsfelder und -elemente können der Ergebnisdarstellung in Absatz 4 entnommen werden.

\subsection{Reifegradberechnung}

Um die Handlungsfelder und Handlungselemente messbar zu machen, wurde das HM-Reifegradmodell in einen standardisierten Fragebogen zur Datenerhebung überführt. Die daraus gewonnenen Messwerte wurden anschließend nach einer definierten Berechnungssystematik verrechnet und resultieren in exakten Reifegradwerten der einzelnen Handlungselemente (z.B. 2,66). Bei den Reifegradberechnungen der Handlungselemente entscheiden jedoch nicht nur einzelne Fragen. Stattdessen setzt sich der RG aus mehreren Items zusammen. Dies verbessert die Qualität der Ergebnisse. Alle HE-Reifegrade eines Handlungsfeldes ergeben letztlich den Reifegrad des HF. Hierbei werden alle Handlungselemente gleich gewichtet. Für die Berechnung des Gesamtreifegrades wird nach identischem 
Schema vorgegangen. Alle Handlungsfelder sind für die Umsetzung von Industrie 4.0 von gleich großer Bedeutung und werden daher nicht spezifisch gewichtet.

Die Reifegradberechnung für die Studienteilnehmer erfolgt in Anlehnung an die von [Kast16] definierte Vorgehensweise. Hierbei wird das für das Process and Enterprise Maturity Model (PEMM) eingesetzte Bewertungsverfahren vorgeschlagen. Während ein Erfüllungsgrad von größer oder gleich $80 \%$ das Erreichen des nächsthöheren Reifegrades bedeutet, wird bei einem Erfüllungsgrad von weniger als $80 \%$ der RG als nicht erreicht gewertet (s. [Kast16] S. 47; [GaPI14] S. 326). Zu beachten ist, dass der jeweils niedrigste Einzelwert der Handlungselemente den HF-Reifegrad bestimmt. Wird Beispielsweise im HF „Organisation“ dreimal die RG-Stufe 4, zweimal die RG-Stufe 3 und einmal die RG-Stufe 1 erreicht, so ist der RG dieses Handlungsfeldes 1. Hinsichtlich der Industrie-4.0-Gesamtreife wird dieselbe Einordnungsmethode unter Betrachtung der HF-Ergebnisse eingesetzt. Für die statistischen Berechnungen sowie die Hypothesenprüfungen werden jedoch die exakt berechneten Reifegradwerte verwendet. Hierdurch wird erreicht, dass das Studienergebnis und damit auch der Einfluss nicht durch Auf- oder Abrunden zur nächsten RG-Stufen verfälscht werden.

\subsection{Reifegradstufen (RG-Stufen)}

Für die Beschreibung der Gesamtreife eines Unternehmens werden die in Tabelle 1 gegebenen Definitionen herangezogen. Die RG-Stufe 4, beschreibt hierbei die höchste Industrie4.0-Reife.

\begin{tabular}{|c|l|l|}
\hline RG-Stufe & Bezeichnung & $\begin{array}{l}\text { Beschreibung } \\
\text { Das Standard Level beschreibt den I4.0-Reifegrad eines Unternehmens, } \\
\text { welches sich aktuell noch nicht oder lediglich in geringem Maße mit der } \\
\text { Umsetzung von I4.0 beschäftigt. }\end{array}$ \\
\hline 1 & Standard Level & $\begin{array}{l}\text { Novice Level } \\
\text { Unternehmen mit dem Novice Level beschäftigen sich mit I4.0 und haben } \\
\text { bereits erste Maßnahmen zur Umsetzung angestoßen. }\end{array}$ \\
\hline 2 & Advanced Level & $\begin{array}{l}\text { Das Advanced Level beschreibt ein Unternehmen, welches sich in einem } \\
\text { fortgeschrittenen Status hinsichtlich der I4.0-Umsetzung befindet. Es } \\
\text { wurden bereits konkrete Maßnahmen eingeleitet. 14.0 spielt zudem eine } \\
\text { bedeutende Rolle im Unternehmen. }\end{array}$ \\
\hline 3 & Expert Level & $\begin{array}{l}\text { Unternehmen die sich auf dem Expert Level befinden gehören hinsichtlich } \\
\text { I4.0 zu den weit fortgeschrittenen Unternehmen. I4.0 ist im Unternehmen } \\
\text { auf dem Weg zum Standard. }\end{array}$ \\
\hline 4 & Pioneer Level & $\begin{array}{l}\text { Als 14.0-Vorreiter werden die Unternehmen mit dem Pioneer Level bezeich- } \\
\text { net. Diese höchste Stufe wird durch die nahezu vollständige Umsetzung } \\
\text { aller im Reifegradmodell identifizierter 14.0-Maßnahmen erreicht. }\end{array}$ \\
\hline
\end{tabular}

Tabelle 1: Industrie-4.0-Reifegradstufen der Gesamtreife

Weiterhin gibt das nachfolgende Schaubild einen Überblick über die RG-Stufen der HF. 


\begin{tabular}{|c|c|c|c|c|c|}
\hline$H F$ & O Standard Level & 1 Novice Level & 2 Advanced Level & 3 Expert Level & 4 Pioneer Level \\
\hline 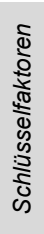 & $\begin{array}{l}\text { Das Management } \\
\text { treibt die notwen- } \\
\text { digen Grundvoraus- } \\
\text { setzungen für } 14.0 \\
\text { nicht ausreichend } \\
\text { voran oder ist an } \\
\text { einer Veränderung } \\
\text { nicht interessiert. }\end{array}$ & $\begin{array}{l}\text { Es bestehen erste } \\
\text { I4.0 Pilotinitiativen } \\
\text { und es wird an den } \\
\text { notwendigen } \\
\text { Grundvoraussetzun- } \\
\text { gen gearbeitet. }\end{array}$ & $\begin{array}{l}\text { Die Bedeutung und } \\
\text { Möglichkeiten von } \\
14.0 \text { sind im Unter- } \\
\text { nehmen klar. } \\
\text { Grundvoraussetzun- } \\
\text { gen sind bereits } \\
\text { teilweise umgesetzt. }\end{array}$ & $\begin{array}{l}\text { 14.0-Grundvoraus- } \\
\text { setzungen sind } \\
\text { umfassend umge- } \\
\text { setzt und verbes- } \\
\text { sern die betriebliche } \\
\text { Abläufe. }\end{array}$ & $\begin{array}{l}\text { Alle } 14.0-\text {-Grund- } \\
\text { voraussetzungen } \\
\text { sind umfassend } \\
\text { umgesetzt und } \\
\text { werden stetig } \\
\text { optimiert. }\end{array}$ \\
\hline 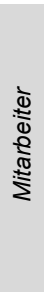 & $\begin{array}{l}\text { Neben technischen } \\
\text { und strukturellen } \\
\text { Voraussetzungen } \\
\text { sind keine oder } \\
\text { lediglich äußerst ge- } \\
\text { ringe I4.0-Kompe- } \\
\text { tenzen vorhanden. } \\
\text { Zudem herrscht } \\
\text { allgemeine Unklar- } \\
\text { heit bezüglich 14.0. }\end{array}$ & $\begin{array}{l}\text { Das Bewusstsein } \\
\text { für } 14.0 \text { wird ge- } \\
\text { schaffen und es } \\
\text { existiert erste digi- } \\
\text { tale Mitarbeiterun- } \\
\text { terstützung. }\end{array}$ & $\begin{array}{l}\text { Mitarbeiter akzeptie- } \\
\text { ren den Wandel zu } \\
14.0 \text { und erhalten } \\
\text { Zugang zu relevan- } \\
\text { ten Informationen } \\
\text { sowie digitale } \\
\text { Unterstützung. }\end{array}$ & $\begin{array}{l}\text { Mitarbeiter erhalten } \\
\text { umfassende Unter- } \\
\text { stützung für den mit } \\
14.0 \text { verbundenen } \\
\text { Wandel. }\end{array}$ & $\begin{array}{l}\text { Veränderungen die } \\
14.0 \text { für den Men- } \\
\text { schen mitbringen } \\
\text { werden proaktiv und } \\
\text { mit den Mitarbeitern } \\
\text { gemeinsam umge- } \\
\text { setzt. Mitarbeiter } \\
\text { wirken an weiteren } \\
\text { Verbesserungen } \\
\text { aktiv mit. }\end{array}$ \\
\hline 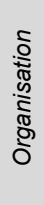 & $\begin{array}{l}\text { Es wird keine 14.0- } \\
\text { Strategie verfolgt } \\
\text { und die notwendige } \\
\text { Prozess- und IT- } \\
\text { Integration fehlt. }\end{array}$ & $\begin{array}{l}\text { Innovation wird im } \\
\text { Unternehmen als } \\
\text { wichtig angesehen } \\
\text { und es findet teil- } \\
\text { weise ein systemin- } \\
\text { tegrierter Informati- } \\
\text { onsaustausch statt. }\end{array}$ & $\begin{array}{l}\text { Die Organisation } \\
\text { arbeitet zielgerichtet } \\
\text { auf die Umsetzung } \\
\text { einer } 14.0 \text {-Strategie } \\
\text { hin und schafft not- } \\
\text { wendige strukturelle } \\
\text { Voraussetzungen. }\end{array}$ & $\begin{array}{l}\text { Eine 14.0-Strategie } \\
\text { befindet sich in der } \\
\text { Umsetzung und das } \\
\text { Unternehmen bleibt } \\
\text { bezüglich neuer } \\
\text { 14.0-Anforderungen } \\
\text { up to date. }\end{array}$ & $\begin{array}{l}\text { Alle organisatio- } \\
\text { nalen Verände- } \\
\text { rungen sind gere- } \\
\text { gelt, umgesetzt und } \\
\text { werden stetig } \\
\text { weiterentwickelt. }\end{array}$ \\
\hline $\begin{array}{l}\frac{7}{3} \\
\frac{0}{0} \\
0\end{array}$ & $\begin{array}{l}\text { Produkte sind rein } \\
\text { physisch vorhanden } \\
\text { und enthalten keine } \\
\text { digitalen Features. } \\
\text { Systemintegration } \\
\text { ist nicht möglich. }\end{array}$ & $\begin{array}{l}\text { Physische Produkte } \\
\text { werden um erste } \\
\text { 14.0-Komponenten } \\
\text { ergänzt und ermög- } \\
\text { lichen somit ansatz- } \\
\text { weise Datenana- } \\
\text { lytik. }\end{array}$ & $\begin{array}{l}\text { Es werden erste } \\
\text { Ergebnisse der Da- } \\
\text { tenanalytik zur Pro- } \\
\text { dukt- und Service- } \\
\text { optimierung ausge- } \\
\text { wertet. Systemin- } \\
\text { tegration ist teil- } \\
\text { weise möglich. }\end{array}$ & $\begin{array}{l}\text { Das Unternehmen } \\
\text { nutzt intelligente } \\
\text { Produkte und } \\
\text { Datenanalytik um } \\
\text { digitale Produkt- } \\
\text { funktionen zu } \\
\text { implementieren. }\end{array}$ & $\begin{array}{l}\text { Im Rahmen von } \\
\text { neuen Geschäfts- } \\
\text { modellen werden } \\
\text { komplexe Pro- } \\
\text { duktsysteme in- } \\
\text { klusive digitalen } \\
\text { Services angebo- } \\
\text { ten. }\end{array}$ \\
\hline $\begin{array}{l}\frac{5}{2} \\
\frac{1}{2} \\
\frac{0}{2} \\
0\end{array}$ & $\begin{array}{l}\text { Die Produktion läuft } \\
\text { konventionell ab } \\
\text { und verfügt nicht } \\
\text { über die notwen- } \\
\text { digen Vorausset- } \\
\text { zungen für 14.0. }\end{array}$ & $\begin{array}{l}\text { Die Produktion mit } \\
\text { den relevanten } \\
\text { Objekten, Daten } \\
\text { und Systemen wird } \\
\text { ansatzweise digital } \\
\text { aufgezeichnet. }\end{array}$ & $\begin{array}{l}\text { Die automatisierte } \\
\text { Produktion ist teil- } \\
\text { weise vernetzt und } \\
\text { über IT ansteuerbar. } \\
\text { Es werden verstärkt } \\
\text { Produktionsdaten } \\
\text { erfasst und zur Wer- } \\
\text { tegenerierung } \\
\text { genutzt. }\end{array}$ & $\begin{array}{l}\text { Die Produktion lässt } \\
\text { sich digital und in } \\
\text { Echtzeit nachver- } \\
\text { folgen. Die Daten- } \\
\text { erfassung und } \\
\text {-nutzung ist von er- } \\
\text { heblicher Bedeu- } \\
\text { tung. }\end{array}$ & $\begin{array}{l}\text { Die Produktion er- } \\
\text { folgt in einer Smart } \\
\text { Factory, welche } \\
\text { durch ein wandel- } \\
\text { bares Produktions- } \\
\text { system und einem } \\
\text { digitalen Abbild der } \\
\text { Fabrik maximale } \\
\text { Effizienz ermöglicht. }\end{array}$ \\
\hline
\end{tabular}

Tabelle 2: Industrie-4.0-Reifegradstufen je Handlungsfeld

\section{Untersuchungsmethode}

Für die Beschreibung der methodischen Vorgehensweise zur Studiendurchführung wird nachfolgend die Untersuchungsmethode, inklusive des -designs und der -planung erläutert.

\subsection{Untersuchungsdesign}

Hinsichtlich des Untersuchungsdesigns werden die von Döring und Bortz beschriebenen neun Klassifikationskriterien herangezogen (s. [DöBo16] S. 182). Als wissenschaftstheoretischer Ansatz der Studie wurde aufgrund von bereits erforschten Sachverhalte auf den qualitativen Forschungsansatz zurückgegriffen. Während es sich beim Erkenntnisziel der Studie um eine anwendungswissenschaftliche Studie handelt, gilt als Gegenstand der Studie der empirische Ansatz. Im Hinblick auf die Art der Datenerhebung wurde eine Primäranalyse, 
also eine erstmalige Datenerhebung und -analyse durchgeführt. Da es sich um die Überprüfung von vorher aufgestellter Hypothesen handelt, ist die vorliegende Studie von explanativer Art. Im Zusammenhang mit Untersuchungsgruppen und dem Anstreben von einer hohen Validität wurde ein experimentelles Studiendesign gewählt. Um auch eine möglichst hohe externe Validität zu gewährleisten wurde eine Felduntersuchung durchgeführt. Aufgrund des (vorläufigen) Ziels der Reifegradbestimmung zu einem einzelnen Zeitpunkt wurde keine Messwiederholung angestrebt. Mithilfe einer Gruppenstudie wurde zudem eine Stichprobe von Untersuchungseinheiten aus der interessierenden Grundgesamtheit gebildet. Damit werden Rückschlüsse auf die Grundgesamtheit ermöglicht.

\section{$3.2 \quad$ Untersuchungsplanung}

\subsubsection{Stichprobenkonstruktion}

Im Hinblick auf die Zielpopulation wurde diese in einem ersten Schritt auf Mitarbeiter von produzierenden Unternehmen in der D-A-CH-Region eingegrenzt. Weiterhin erfolgte eine Spezifizierung der Zielgruppe auf Unternehmensvertreter, welche sich mit dem Thema Industrie 4.0 beschäftigen und über entsprechendes Wissen innerhalb der Firma verfügen. Auf Grund der Komplexität des Themas sowie des entwickelten Fragebogens sind Vorkenntnisse in diesem Umfeld zwingend erforderlich. Während hinsichtlich der Stichprobenart auf die nicht-zufälligen Methoden zurückgegriffen wurde, galt ein Stichprobenumfang von mindestens $\geq 30$ Teilnehmer.

\subsubsection{Rekrutierung von Untersuchungspersonen}

Die Anwerbung der Teilnehmer erfolgte grundsätzlich über drei verschiedene Wege. Neben der direkten und persönlichen Ansprache von Ausstellern auf der Hannover Messe Industries 2016, dem Versand von personalisierten E-Mails an Unternehmen, welche bereits Industrie 4.0 Anwendungen einsetzen und im persönlichen Bekanntenkreis der Autoren sowie einem allgemeinen Teilnehmeraufruf. Dieser erfolgte über verschiedene Newsletter, Rundmails und der Nutzung von Gruppen auf Social-Media-Plattformen zum Thema Industrie 4.0.

\subsubsection{Stichprobenerhebung}

Die Untersuchungsdurchführung fand online mittels eines Befragungstools von LimeSurvey statt. Hierbei wurde ein standardisierter Fragebogen zu Grunde gelegt. Durch die Vergabe von Teilnehmer-IDs konnte die Umfrage unkompliziert anonymisiert werden. Der Umfragezeitraum erstreckte sich über sieben Wochen, vom 5. Juli 2016 bis zum 21. August 2016.

\subsubsection{Datenauswertung}

Die bei der quantitativen Datenanalyse eingesetzten statistischen Verfahren lassen sich in zwei große Bereiche gliedern - in die deskriptive und die induktive Statistik. Jede quantitative empirische Studie bedient sich zunächst deskriptiv-statistischer Methoden um die Stichprobendaten anhand zentraler soziodemografischer Merkmale zu beschreiben. Beispielsweise lassen sich die Stichprobenverhältnisse durch Grafiken, Tabellen, Variablen und Variablenrelationen (z.B. Reifegrad oder einzelne Fragen) anschaulich darstellen. Bei der darauffolgenden induktiven Statistik wurden die Forschungshypothesen anhand der empirischen Daten in Relation zu den Variablen geprüft. Die Ergebnisse werden in Absatz 4 dargestellt. 
Die Studienergebnisse geben einen Überblick über den Umsetzungsstand von Industrie 4.0 in produzierenden Unternehmen der D-A-CH-Region und zeigen somit Stärken und Schwächen der Unternehmen auf. Dadurch können erste Muster für die Einführung und Umsetzung von Industrie 4.0 identifiziert werden. Dem vorausgehend erfolgt eine Beschreibung der untersuchten Stichprobe, welche die Basis für die inhaltlichen Ergebnisse bildet.

\subsection{Stichprobenbeschreibung}

Die 107 befragten Unternehmen repräsentieren ein ausgewogenes Mischverhältnis von großen Konzernen sowie kleinen und mittelständischen Unternehmen. Zudem können mehr als die Hälfte der Befragten der oberen oder mittleren Führungsebene zugeordnet werden. Hinsichtlich der teilnehmenden Branchen ergeben sich vier große Gruppen sowie die ergänzende Gruppe Sonstige. Hierbei bilden der Maschinen- und Anlagenbau (24\%) sowie die Elektrotechnik- und Elektronikindustrie (20\%) die beiden stärksten Gruppen. Zusammen mit den Automobilherstellern (16\%) und den Automobilzulieferern (15\%) stellen diese vier Branchen drei Viertel aller Befragten.

\subsection{Industrie-4.0-Umsetzungsstand}

Der Industrie-4.0-Umsetzungsstand von produzierenden Unternehmen in der D-A-CH-Region wird anhand des HM-Reifegradmodells bestimmt. Nachfolgend wird auf den Gesamtreifegrad sowie die Reifegradverteilung in den Industrie-4.0-Handlungsfeldern eingegangen.

\subsubsection{Verteilung der Industrie-4.0-Gesamtreife}

Die Betrachtung der Gesamtreife über alle Studienteilnehmer und Branchen hinweg zeigt, dass die Umsetzung von Industrie 4.0 insgesamt noch sehr schwach ausgeprägt ist. Während auf Basis der ermittelten Daten acht von zehn Unternehmen dem Standard Level (RGStufe 0) zuzuordnen sind, erreichen $20 \%$ das Novice Level (RG-Stufe 1), welche sich durch das Anstoßen von ersten Industrie-4.0-Maßnahmen auszeichnen. Zudem erreicht ein Unternehmen das Advanced Level und befindet sich damit bereits im fortgeschrittenen Status bei der Umsetzung von Industrie 4.0. Da das Pioneer Level (RG-Stufe 4) die Zielvision und einen nahezu unerreichbaren Zustand beschreibt, verwundert es nicht, dass kein Unternehmen diesen höchsten Reifegrad erreicht.

Die geringen Werte sind unter anderem auf die Bewertungssystematik des sehr umfassenden HM-Reifegradmodells zurückzuführen, wodurch bei ca. einem Viertel der Unternehmen (28), der niedrige Reifegrad aufgrund von nur einem am schwächsten ausgeprägten HF bestimmt wird (z.B. ist die RG-Stufe 0 nur einmal vertreten). Abbildung 1 veranschaulicht eine mögliche Entwicklung, welche sich durch die Verbesserung dieser 28 Unternehmen ergibt. Steigern diese Unternehmen Ihren Reifegrad in dem jeweils am geringsten fortgeschrittenen HF um eine RG-Stufe, so gehören bereits $5 \%$ der befragten Unternehmen dem Advanced Level an. Der Anteil in RG-Stufe 1 würde sich mit einem Wachstum von 18\% nahezu verdoppeln und in RG-Stufe 0 um $22 \%$ reduzieren. Für den durchschnittlichen Reifegrad hätte diese Veränderung eine Verbesserung um mehr als das Doppelte zur Folge (von 0,21 auf 0,47 ). 


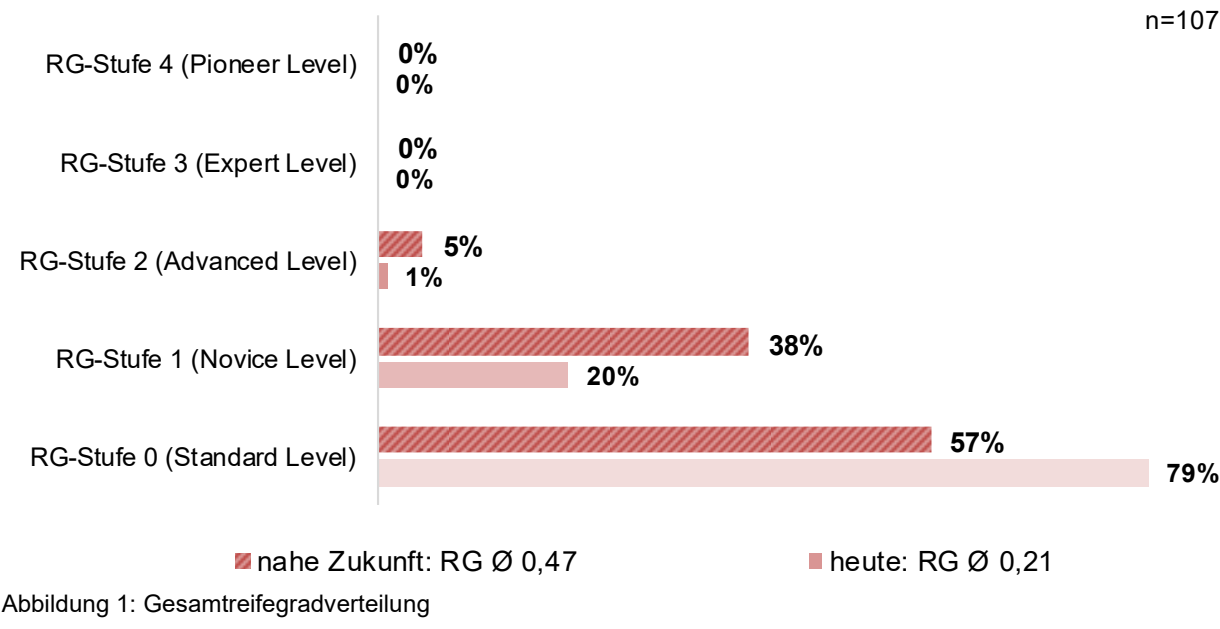

Bei näherer Betrachtung des Gesamtreifegrades anhand der einzelnen Handlungsfelder, ergibt sich das in Abbildung 2 veranschaulichte Diagramm. Hierbei befinden sich die meisten Unternehmen über alle Handlungsfelder hinweg auf den zwei niedrigsten RG-Stufen.

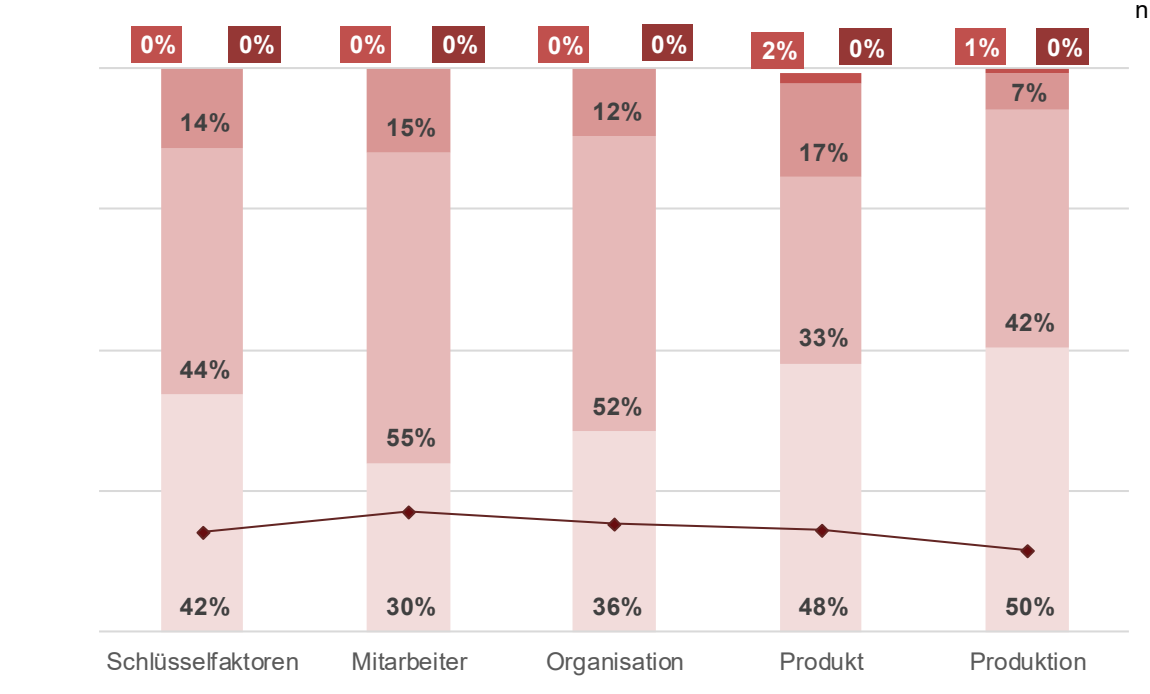

$\mathrm{n}=107$

3

2

1

0

RG-Stufe 0 RG-Stufe $1 \backsim$ RG-Stufe $2 \backsim$ RG-Stufe $3 \backsim$ RG-Stufe $4 \multimap \varnothing$ RG

Abbildung 2: Übersicht über die Industrie-4.0-HF-Reifegradmessung

\subsubsection{HF Schlüsselfaktoren}

Die Mittelwerte der Handlungselemente im HF Schlüsselfaktoren schwanken zwischen 1,50 (Stellung der IT im Unternehmen und Vernetzung der IT-Systeme) und 2,07 (Management), wodurch sich eine hauptsächliche Verteilung der Untersuchungseinheiten auf die RG-Stufen 1 und 2 abzeichnet (vgl. Abbildung 3). Das HE Management sticht mit einem durchschnittlichen Reifegrad von 2,07 positiv heraus. Das Management beteiligt sich demnach, in allen teilnehmenden Unternehmen aktiv an der Umsetzung der Industrie-4.0-Strategie. Die interne und über die Unternehmensgrenzen hinausgehende Vernetzung der IT-Systeme gilt 
als eine der wichtigsten Voraussetzungen für Industrie 4.0. Es fällt auf, dass in diesem HE die Unternehmen mit einem durchschnittlichen Reifegrad von 1,50 besonders schlecht abschneiden.

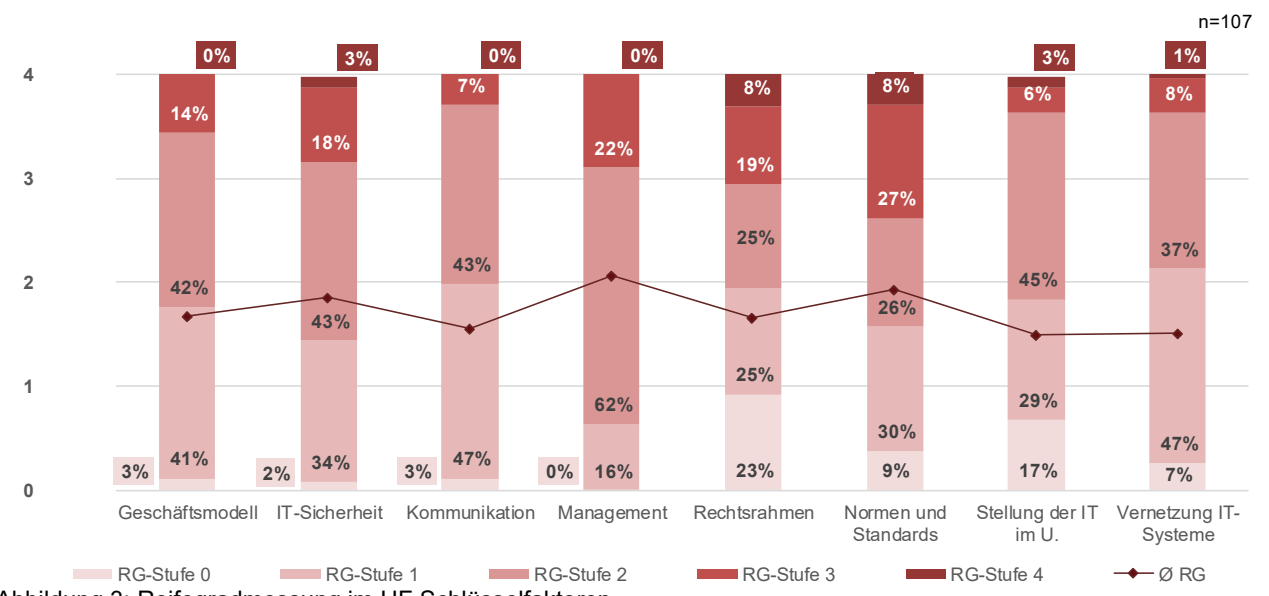

Abbildung 3: Reifegradmessung im HF Schlüsselfaktoren

\subsubsection{HF Mitarbeiter}

Das HF Mitarbeiter (vgl. Abbildung 4) schneidet mit einem durchschnittlichen Reifegrad zwischen 1,27 für Assistenzsysteme und 1,87 bei Arbeitsstrukturen, äußerst stark zwischen den Stufen 1 und 2 verteilt ab. Das HE Arbeitsstrukturen schneidet hierbei besonders gut ab. Mit einem durchschnittlichen Reifegrad von 1,34 und sechs von zehn Befragten in der RG-Stufe 1 ist der Aufbau von Industrie-4.0-Kompetenzen eine der größten Hürden in diesem HF.

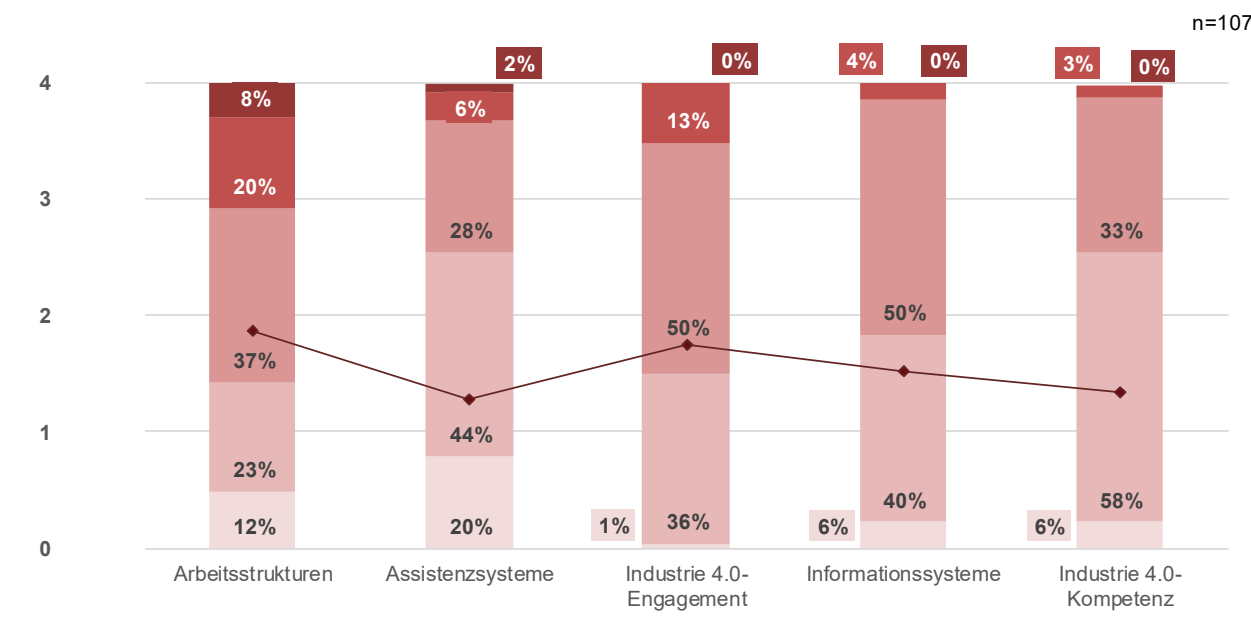

RG-Stufe 0
Abbildung 4: Reifegradmessung im HF Mitarbeiter

\subsubsection{HF Organisation}

Im HF Organisation (vgl. Abbildung 5) schwanken die Mittelwerte der Handlungselemente zwischen 1,16 für das HE Kennzahlensystem und 1,91 für die Industrie-4.0-Strategie. Mit einem Anteil von 22\% im HE Prozessmanagement, können ca. ein Viertel der Befragten der 
RG-Stufe 3 zugeordnet werden. Eine große Herausforderung stellt das Thema Wissensmanagement dar. Ungefähr ein Viertel der teilnehmenden Unternehmen geben an, dass sie kein systematisches Wissensmanagement einsetzen und mit sog. „Wissenshamstern“ zu kämpfen haben. Dies ist ein grundlegendes Problem, welches vor allem für die Adaption von Industrie 4.0 angegangen werden sollte.

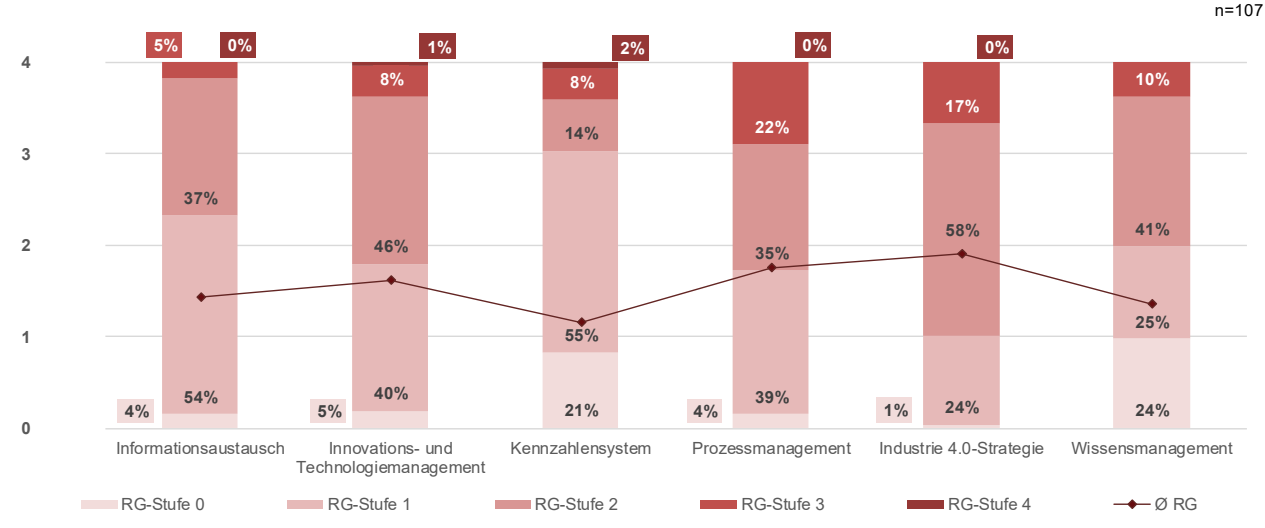

Abbildung 5: Reifegradmessung im HF-Organisation

\subsubsection{HF Produkt}

Im HF Produkt wurden die Handlungselemente Datenanalytik, Produktfunktion, Produktsystem und Systemintegration untersucht. Diese Handlungselemente hängen sehr eng miteinander zusammen. Der durchschnittliche Reifegrad liegt hierbei mit Werten zwischen 0,93 für die Datenanalytik und 1,39 im HE Produktsystem im unteren Bereich (vgl. Abbildung 6). Dies deutet bereits darauf hin, dass Industrie 4.0 auf die Produkte bezogen noch am Anfang steht.

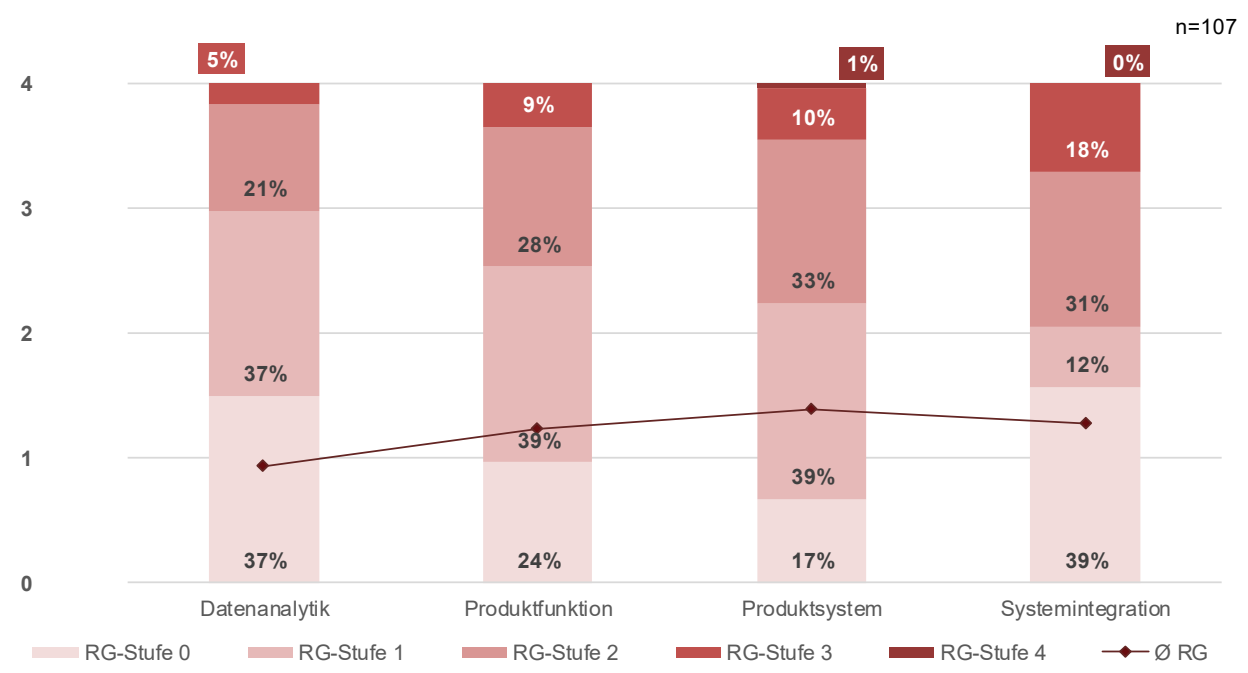

Abbildung 6: Reifegradmessung im HF Produkt

\subsubsection{HF Produktion}

Mit Blick auf Abbildung 2 zeigt sich, dass die Produktion insgesamt das bisher am schlechtesten vorangeschrittene HF ist. Der durchschnittliche Reifegrad der Handlungselemente 
schwankt hierbei zwischen 0,98 bei der Datennutzung und 1,45 für die Produktionsressourcen (vgl. Abbildung 7).

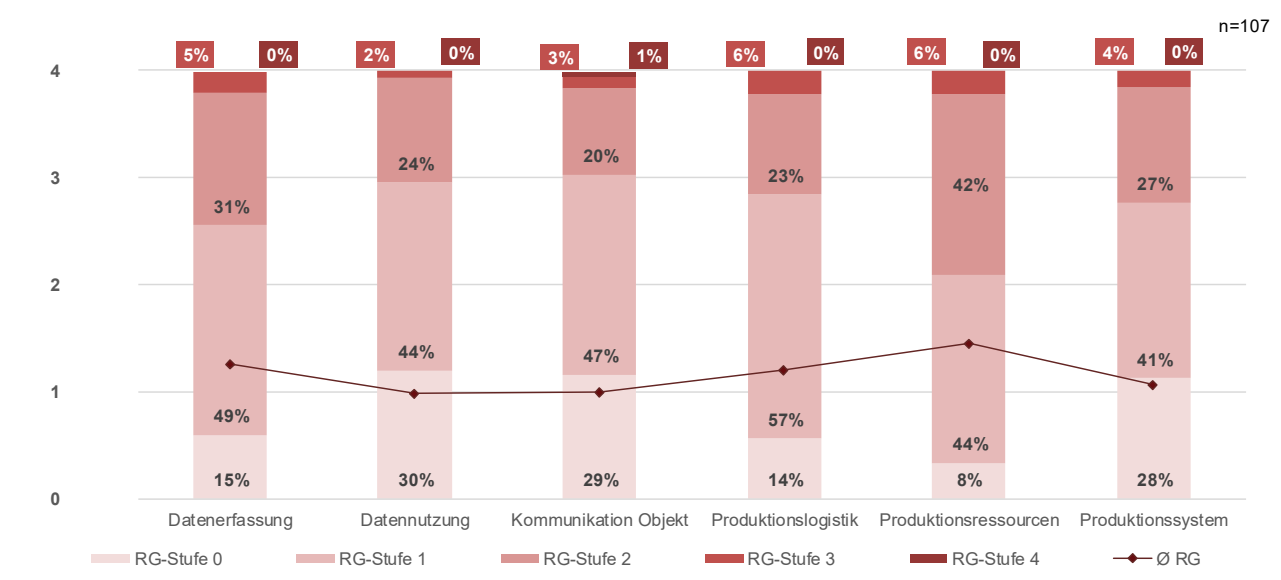

Abbildung 7: Reifegradmessung im HF Produktion

\subsection{Bedeutende Industrie-4.0-Einflussfaktoren}

Neben der Bestimmung des Industrie-4.0-Umsetzungsstandes stellt die empirische Studie bedeutende Industrie-4.0-Einflussfaktoren anhand von Hypothesenprüfungen heraus. Für das Testen von hypothesenprüfenden (explanativen) Studien steht eine Vielzahl möglicher Analyseverfahren der induktiven Statistik zur Verfügung. Die in der quantitativen Forschung wohl gängigste und auch in der vorliegenden Arbeit angewendete Methode, ist der klassische Signifikanztest. Mittels dieses Testverfahrens lässt sich eine Entscheidung über die Bestätigung oder nicht Bestätigung einer Hypothese herbeiführen. Nach der Auswahl der geeigneten Zusammenhangsmaße wurde der bivariate Zusammenhang mittels des Rangkorrelationskoeffizienten $r_{s}$ nach Spearman berechnet (s. [BoSc10] S. 178). Ergibt sich ein statistisch signifikantes Ergebnis, ist außerdem eine Aussage über die Effektgröße der Alternativhypothese und bezüglich der theoretischen und / oder praktischen Relevanz erforderlich. Für die bivariate Korrelationen haben sich hierbei standardisierte Effektgrößenmaße nach Jacob Cohen (1988) etabliert. Die Klassifikation der Effektgröße basiert auf dem Korrelationskoeffizienten und wird in klein $(0,10)$, mittel $(0,30)$ und groß $(0,50)$ unterteilt (s. [DöBo16] S. 668f.; 821; 826).

\subsubsection{Großunternehmen als Industrie-4.0-Vorreiter}

H1: Je größer Unternehmen sind, umso höher ist ihr jeweiliges Industrie-4.0-Reifegradlevel. Zur Untersuchung welcher Zusammenhang zwischen der Unternehmensgröße und dem Umsetzungsstand von Industrie 4.0 besteht, wurden zwei Subhypothesen für Hypothese H1 formuliert. Diese prüfen die Unternehmensgröße anhand praxisüblicher Merkmale (Unternehmensumsatz und Anzahl der Mitarbeiter) mit dem Industrie-4.0-Reifegrad.

Die Unternehmensgröße gemessen an dem erzielten Jahresgesamtumsatz (SH1.1) und der Anzahl der beschäftigten Mitarbeiter (SH1.2) korreliert höchst signifikant mit dem Industrie4.0-Gesamtreifegrad. Dabei handelt es sich um einen mittleren Effekt. $\mathrm{H} 1$ ist anhand der bestätigten Subhypothesen $\mathrm{SH} 1.1$ und $\mathrm{SH} 1.2$ bestätigt. 


\subsubsection{HF-I-Schlüsselfaktoren als Grundvoraussetzung für die Adaption von Industrie 4.0}

H5: Je weiter Unternehmen hinsichtlich der Industrie-4.0-Schlüsselfaktoren vorangeschritten sind, umso weiter sind diese auch in allen anderen wichtigen Industrie-4.0-HF fortgeschritten. Zur Untersuchung des Zusammenhangs zwischen Industrie-4.0-Schlüsselfaktoren und weiteren, für die erfolgreiche Umsetzung von Industrie 4.0 entscheidenden Faktoren (HF II bis HF V), wurde Hypothese H5 in vier Subhypothesen (SH5.1 bis SH5.4) untergliedert.

Das HF Schlüsselfaktoren ist auch nach der statistischen Untersuchung als grundlegendes HF für alle weiteren Industrie-4.0-Faktoren zu sehen. Die Prüfung ergibt in drei von vier Fällen einen großen Korrelationseffekt mit den Industrie-4.0-Handlungsfeldern. In der Korrelationsanalyse mit dem HF Produkt wurde zudem ein mittlerer Effekt ermittelt. Das Ergebnis kann mit höchster Signifikanz auf die Gesamtpopulation übertragen werden. H5 ist damit bestätigt.

\subsubsection{Vernetzung der IT-Systeme}

H6: Je stärker die IT-Systeme von Unternehmen vernetzt sind, umso weiter fortgeschritten sind diese hinsichtlich Industrie 4.0.

Die Vernetzung der IT-Systeme von Unternehmen korreliert höchst signifikant mit der Industrie-4.0-Gesamtreife. Dabei handelt es sich um einen großen Effekt. H6 ist damit bestätigt. Da die Vernetzung von IT-Systemen eine der wichtigsten Voraussetzungen von Industrie 4.0 darstellt, ist dieses Ergebnis wenig überraschend. Besonders in Bezug auf eine zukünftige Smart Factory sind vernetzte Systeme und Anlagen unumgänglich.

\subsubsection{HF V Produktion}

Speziell die Themen Datennutzung und der Einsatz eines Fertigungsmanagementsystems sind für einen hohen Industrie-4.0-Reifegrad im HF V Produktion wichtige Voraussetzungen. Anhand von zwei Hypothesen ( $\mathrm{H} 14$ und $\mathrm{H} 15)$ wird der Zusammenhang mit der Industrie-4.0Gesamtreife untersucht.

H14: Unternehmen die im Hinblick auf eine Smart Factory bereits weit entwickelt sind, sind auch im Bereich der Datennutzung weiter fortgeschritten.

Der Industrie-4.0-Gesamtreifegrad korreliert höchst signifikant mit dem HE Datennutzung. Dabei handelt es sich um einen großen Effekt. $\mathrm{H} 14$ ist damit bestätigt.

H15: Je umfangreicher Unternehmen ein Fertigungsmanagementsystem (z.B. ein MES) in ihre Produktionsplanung und -steuerung integriert haben, umso weiter sind diese zum Thema Industrie 4.0 vorangeschritten.

Der Einsatz eines Fertigungsmanagementsystems zur Produktionsplanung und -steuerung korreliert höchst signifikant mit dem Industrie-4.0-Gesamtreifegrad. Dabei handelt es sich um einen großen Effekt. H15 ist damit bestätigt.

\section{$5 \quad$ Diskussion und Ausblick}

Die Studie zum Thema Industrie-4.0-Reifegradbestimmung produzierender Unternehmen im deutschsprachigen Raum stellt entscheidende Industrie-4.0-Einflussfaktoren anhand eines wissenschaftlich fundierten Reifegradmodells heraus. Diese Einflussfaktoren können Unternehmen zur Verbesserung ihrer Industrie-4.0-Reife heranziehen. Mit dem ganzheitlichen Lösungsansatz des HM-Reifegradmodells wird der Industrie-4.0-Reifegrad bestimmt. Jedoch sind 92\% der Teilnehmer dem Standort Deutschland zuzuordnen. Die Studienergeb- 
nisse tragen unter Einbeziehung der technischen Voraussetzungen für Industrie 4.0 zur Erweiterung des derzeitigen Forschungsstandes bei. Es ist zudem festzuhalten, dass das von der HM entwickelte Industrie-4.0-Reifegradmodell als geeignetes Instrument zur Ermittlung des Industrie-4.0-Umsetzungsstandes in produzierenden Unternehmen bestätigt wird. Die im Modell definierten Handlungsfelder und Handlungselemente bieten eine umfassende Möglichkeit zur Standortbestimmung anhand unterschiedlichster Industrie-4.0-Einflussfaktoren. Die Stichprobengröße und -verteilung können als weitere Stärken der Studie angesehen werden. Mit einer Teilnehmerzahl von 107 Untersuchungseinheiten und der Verteilung auf fünf große Branchengruppen können aussagekräftige Ergebnisse für die produzierende Industrie in der Zielregion präsentiert werden. Des Weiteren konnten die vorab definierten Forschungshypothesen auf Zusammenhänge, Effektstärke und Signifikanz überprüft werden.

Die repräsentativen Studienergebnisse bilden eine gute Grundlage für zukünftige Vergleichsstudien. Solche wären beispielsweise innerhalb der D-A-CH-Region möglich oder könnten durch das Heranziehen von anderen Ländern weltweit ausgeweitet werden. Nachfolgend werden mögliche Vergleichsstudien erläutert.

- Vergleich Süddeutschland mit Norddeutschland. Da die befragten Unternehmen der vorliegenden Studie hauptsächlich im süddeutschen Raum ansässig sind, bietet eine Datenerhebung in Norddeutschland eine interessante Vergleichsmöglichkeit.

- Internationalisierung der Studie durch einen Vergleich mit der sog. Greater China Area, als aufstrebender asiatischer Markt.

- Internationalisierung der Studie durch einen Vergleich mit dem Industrie-4.0-Umsetzungsstand in den USA. Hier wird das Thema Digitalisierung beispielsweise durch das Industrial Internet Consortium (IIC) vorangetrieben.

Eine weitere Möglichkeit für ein zukünftiges Forschungsprojekt stellt der Ausbau zu einem kontinuierlichen Benchmarking-Angebot dar. Hierbei könnten mittels Veränderungshypothesen zukünftige Entwicklungen der Industrie 4.0-Reife nachverfolgt und überprüft werden.

\section{Literaturverzeichnis}

[Ab++15] Abele, E.; Anderl, R.; Metternich, J.; Arndt, A.; Wank, A.: Industrie 4.0 - Potentiale, Nutzen und Good-Practice-Beispiele für die hessische Industrie - Zwischenbericht zum Projekt Effiziente Fabrik 4.0. Meisenbach Verlag, Bamberg, 2015.

[BoSc10] Bortz, J.; Schuster, C.: Statistik für Human- und Sozialwissenschaftler. Springer-Verlag, Berlin, 2010.

[Brüh15] Brühl, V.: Wirtschaft des 21. Jahrhunderts - Herausforderungen in der Hightech-Ökonomie. Springer-Verlag, Wiesbaden, 2015.

[Bund15] Bundesministerium für Wirtschaft und Energie (Hrsg.): Industrie 4.0 und Digitale Wirtschaft - Impulse für Wachstum, Beschäftigung und Innovation. 2015, http://www.bmwi.de/Redaktion/DE/Downloads///industrie-4-0-und-digitale-wirtschaft.pdf?__blob=publicationFile\&v=3. Abruf am 2016-05-10.

[Bund16] Bundesministerium für Wirtschaft und Energie: Was ist Industrie 4.0?. 2016, http://www.plattform-i40.de//40/Navigation/DE/Industrie40/WasIndustrie40/was-ist-industrie-40.html. Abruf am 2016-06-10. 
[DöBo16] Döring, N.; Bortz, J.: Forschungsmethoden und Evaluation in den Sozial und Humanwissenschaften. Springer-Verlag, Berlin, 2016.

[GaPI14] Gausemeier, J.; Plass, C.: Zukunftsorientierte Unternehmensgestaltung (2. Auflage). Carl Hanser Verlag, München, 2014.

[HeEK16] Heilmann, D.; Eickmeyer, L.; Kleibring, J.: Industrie 4.0 im internationalen Vergleich. 2016, http://www.huawei-studie.de/downloads/handelblattsresearchinstitute_Huawei_ Studie_Industrie4_0_im_internationalen_Vergleich.pdf. Abruf am 2016-07-14.

[Kast16] Kastl, E. M.: Industrie 4.0 Reifegradmodell. Bachelorarbeit, Hochschule München, München, 2016.

\section{Kontakt}

Prof. Dr. Jörg Puchan

Hochschule München, Fakultät für Wirtschaftsingenieurwesen

Lothstraße 64, 80335 München

T +49 891265 3937, joerg.puchan@hm.edu

Denis Heideker

Hochschule München, Fakultät für Wirtschaftsingenieurwesen

Lothstraße 64, 80335 München

T+49 152 22804695, denis.heideker@gmx.de

\section{Sascha Hertkorn}

Hochschule München, Fakultät für Wirtschaftsingenieurwesen

Lothstraße 64, 80335 München

T+49176 83185340, saschahertkorn@googlemail.com 


\section{Autoren}

Prof. Dr. Gunnar Auth

Hochschule für Telekommunikation Leipzig

Gustav-Freytag-Str. 43-45, 04277 Leipzig

T +49 3413062 235, auth@hft-leipzig.de

Prof. Dr. Thomas Barton

Hochschule Worms

Erenburgerstr. 19, 67549 Worms

barton@hs-worms.de

\section{Stefan Beigel}

Hochschule Mannheim, Fakultät für Informatik Mannheimer Wirtschaftsinformatik Institut

Paul-Wittsack-Str. 10, 68163 Mannheim

Prof. Dr. Frank Bensberg

Hochschule Osnabrück

Caprivistrasse 30a, 49076 Osnabrück

T +49541969 3264

f.bensberg@hs-osnabrueck.de

Prof. Dr. Lars Brehm

Hochschule München

Fakultät für Betriebswirtschaftslehre

Am Stadtpark 20, 81243 München

lars.brehm@hm.edu

\section{Gandalf Buscher}

Hochschule für Telekommunikation Leipzig

Gustav-Freytag-Str. 43-45, 04277 Leipzig

Gandalf.Buscher@hft-leipzig.de

\section{Prof. Dr. Johannes Busse}

HAW Landshut

Am Lurzenhof 1, 84036 Landshut

busse@haw-landshut.de

Prof. Dr. Christian Czarnecki

Hochschule für Telekommunikation Leipzig

Gustav-Freytag-Str. 43-45, 04277 Leipzig

T +49 3413062 227, czarnecki@hft-leipzig.de

\section{Julian Dörndorfer}

Hochschule Landshut

Am Lurzenhof 1, 84036 Landshut

T.: +49 (0) 871506731

julian.doerndorfer@haw-landshut.de
Prof. Dr. Carsten Dorrhauer

Institut für Wirtschaftsinformatik

an der Hochschule Ludwigshafen

Ernst-Boehe-Str. 4, 67059 Ludwigshafen

$\mathrm{T}+49$ 621-5203-160, +49 621-5203-150

\section{Katharina Dort}

Justus Liebig Universität Gießen

Ludwigstraße 23, 35390 Gießen

katharina.dort@physik.uni-giessen.de

\section{Anke Dreiling}

Fachhochschule Nordwestschweiz

Hochschule für Wirtschaft

Riggenbachstrasse $16, \mathrm{CH}-4600$ Olten

Prof. Dr. Dorothee Feldmüller

Hochschule Bochum

Campus Velbert/Heiligenhaus

Kettwiger Straße 20, 42579 Heiligenhaus

dorothee.feldmueller@hs-bochum.de

\section{Iris Graf}

Fachhochschule Nordwestschweiz

Hochschule für Wirtschaft

Riggenbachstrasse 16, CH-4600 Olten

\section{Marco Graf}

Hochschule Worms

Erenburgerstr. 19, 67549 Worms

graf@hs-worms.de

\section{Prof. Dr. Michael Guckert}

Kompetenzzentrum für

Informationstechnologie

Technische Hochschule Mittelhessen

Fachbereich MND

Wilhelm-Leuschner-Str. 13, 61169 Friedberg

T +49 6031 604-4751, -452

michael.guckert\}@mnd.thm.de

\section{Denis Heideker}

Hochschule München

Fakultät für Wirtschaftsingenieurwesen

Lothstraße 64, 80335 München

T +49 152 22804695, denis.heideker@gmx.de 
Sascha Hertkorn

Hochschule München

Fakultät für Wirtschaftsingenieurwesen

Lothstraße 64, 80335 München

T +49 17683185340

saschahertkorn@googlemail.com

\section{Prof. Dr. Georg Rainer Hofmann}

Information Management Institut

Hochschule Aschaffenburg

Würzburger Straße 45, 64743 Aschaffenburg

T +49 6021 4206-700

georg-rainer.hofmann@h-ab.de

\section{Eva Kastl}

Fakultät für Wirtschaftsingenieurwesen

Hochschule München

Lothstr. 64, 80335 München

T +49 89 1265-3982, eva.kast|@hm.edu

\section{Prof. Dr. Steffen Kinkel}

Institut für Lernen und Innovation in

Netzwerken

Fakultät für Informatik und

Wirtschaftsinformatik

Hochschule Karlsruhe

Moltkestr. 30, 76133 Karlsruhe

Tel.: 0721 925-2915

steffen.kinkel@hs-karlsruhe.de

\section{Holger Kloetzner}

GFFT Technologies GmbH

Niddastraße 6, 61118 Bad Vilbel

holger.kloetzner@gfft-technologies.de

\section{Jens Kohler}

Hochschule Mannheim, Fakultät für Informatik Mannheimer Wirtschaftsinformatik Institut Paul-Wittsack-Str. 10, 68163 Mannheim

T +49 621 292-6739

j.kohler@hs-mannheim.de

\section{Jonas Konrad}

Fachhochschule Nordwestschweiz

Hochschule für Wirtschaft

Riggenbachstrasse 16, CH-4600 Olten

\section{Katja Kubitta}

Technische Hochschule Mittelhessen

Campus Gießen, Fachbereich MNI

Wiesenstraße 14, 35390 Gießen

\section{Marcel Lang}

Kompetenzzentrum für

Informationstechnologie

Technische Hochschule Mittelhessen

Wilhelm-Leuschner-Str. 13, 61169 Friedberg

marcel.lang@mnd.thm.de

\section{Jan-Felix Mehret}

ProSeS BDE GmbH

Richard-Wagner-Allee 10c, 75179 Pforzheim

T +49 723114737 - 64, j.mehret@proses.de

\section{Prof. Dr. Vera G. Meister}

Technische Hochschule Brandenburg

Magdeburger Str. 50, 14770 Brandenburg

T +49 $3381355-297$

vera.meister@th-brandenburg.de

\section{Prof. Dr. Frank Morelli}

HS Pforzheim

Tiefenbronnerstr. 65, 75175 Pforzheim

T +49 7231 28-6697

frank.morelli@hs-pforzheim.de

\section{Natascha Nitzl}

Kompetenzzentrum für

Informationstechnologie

Technische Hochschule Mittelhessen

Fachbereich MND

Wilhelm-Leuschner-Str. 13, 61169 Friedberg

T +49 6031 604-4751, -452

natascha.nitzl@mnd.thm.de

\section{Martin Przewloka}

Technische Hochschule Mittelhessen

Wiesenstraße 14, 35390 Giessen

martin.przewloka@mnd.thm.de

\section{Prof. Dr. Jörg Puchan}

Fakultät für Wirtschaftsingenieurwesen

Hochschule München

Lothstr. 64, 80335 München

T +49 89 1265-3937, joerg.puchan@hm.edu

\section{Dr. Johanna Rahn}

Leibniz-Institut für Wissensmedien Schleichstraße 6, 72076 Tübingen

Tel: +49 7071 979-310

j.rahn@iwm-tuebingen.de 
Prof. Dr. Dörte Resch

Fachhochschule Nordwestschweiz

Hochschule für Wirtschaft

Riggenbachstrasse 16, CH-4600 Olten

Tel: +41 62957 2536, doerte.resch@fhnw.ch

\section{Marco Richter}

Technische Hochschule Mittelhessen

Kompetenzzentrum für

Informationstechnologie

Wilhelm-Leuschner-Str. 13, 61169 Friedberg

$\mathrm{T}+496031$ 604-4783

marco.richter@mnd.thm.de

\section{Bernhard Rieder}

Eduard-Baumgartner-Str. 5, A-5020 Salzburg

Mobil: +43664 4454946

\section{Prof. Dr. Tobias Rieke}

FH Münster

Institut für Technische Betriebswirtschaft

Bismarckstr. 11, 48565 Steinfurt

tobias.rieke@fh-muenster.de

Prof. Dr. Harald Ritz

Technische Hochschule Mittelhessen

Campus Gießen, Fachbereich MNI

Wiesenstraße 14, 35390 Gießen

T+49 641 309-2431, harald.ritz@mni.thm.de

\section{Prof. Dr. Haio Röckle}

Institut für Wirtschaftsinformatik

an der Hochschule Ludwigshafen

Ernst-Boehe-Str. 4, 67059 Ludwigshafen

T +49 621-5203-160, +49 621-5203-150

haio.roeckle@web.de

Prof. Dr. Andreas Schmidt

Hochschule Osnabrück

Caprivistrasse 30a, 49076 Osnabrück

T +495419693820

A.Schmidt@hs-osnabrueck.de

\section{Dominik Schneider}

Detecon International $\mathrm{GmbH}$

Sternengasse 14-16, 50676 Köln

Dominik.Schneider@detecon.com
Prof. Dr. Christian Schulze

Kompetenzzentrum für

Informationstechnologie

Technische Hochschule Mittelhessen

Fachbereich MND

Wilhelm-Leuschner-Str. 13, 61169 Friedberg

$\mathrm{T}+496031$ 604-4751, -452

christian.schulze@mnd.thm.de

\section{Johanna Schwab}

inconso AG

In der Hub 1-8, 61231 Bad Nauheim

T +49 6032 348-3017, jschwab@inconso.de

Prof. Dr. Christian Seel

Hochschule Landshut

Institut für Projektmanagement und

Informationsmodellierung

Am Lurzenhof 1, 84036 Landshut

christian.seel@haw-landshut.de

\section{Prof. Dr. Carlo Simon}

Hochschule Worms

Erenburgerstraße 19, 67549 Worms

T +49 6241 509-369, simon@hs-worms.de

\section{Prof. Dr. Thomas Specht}

Hochschule Mannheim, Fakultät für Informatik Mannheimer Wirtschaftsinformatik Institut

Paul-Wittsack-Str. 10, 68163 Mannheim

\section{Prof. Dr. Frank Thomé}

Institut für Wirtschaftsinformatik

an der Hochschule Ludwigshafen

Ernst-Boehe-Str. 4, 67059 Ludwigshafen

$T$ +49 621-5203-160, +49 621-5203-150

\section{Tristan Eberhard Thomé}

Hochschule für Telekommunikation Leipzig

Gustav-Freytag-Str. 43-45, 04277 Leipzig

T+49 228181 12769, s139109@hft-leipzig.de

\section{Prof. Dr. Holger Timinger}

Hochschule Landshut

Institut für Projektmanagement und

Informationsmodellierung

Am Lurzenhof 1, 84036 Landshut

holger.timinger@haw-landshut.de 
Melanie Vanderpuye

StudiumPlus

Charlotte-Bamberg-Straße 3, 35578 Wetzlar

$\mathrm{T}+496441$ 2041-223

melanie.vanderpuye@zdh.thm.de

\section{Niklas Weber}

Labor für IT Organisation und Management

Fachhochschule Aachen

Eupener Str. 70, 52066 Aachen

Niklas.Weber@alumni.fh-aachen.de

\section{Thorsten Weidt}

BridgingIT GmbH

Marienstr. 17, 70178 Stuttgart

T +4915152669398

thorsten.weidt@bridging-it.de

\section{Matthias Willems}

Kompetenzzentrum für

Informationstechnologie

Technische Hochschule Mittelhessen

Wilhelm-Leuschner-Str. 13, 61169 Friedberg

matthias.willems@mnd.thm.de
Dr. ir. Frank Wisselink

Detecon International $\mathrm{GmbH}$

Sternengasse 14-16, 50676 Köln

Frank.Wisselink@detecon.com

\section{Martin R. Wolf}

Labor für IT Organisation und Management

Fachhochschule Aachen

Eupener Str. 70, 52066 Aachen

M.Wolf@fh-aachen.de

\section{Alexander Zeifang}

Fakultät für Wirtschaftsingenieurwesen

Hochschule München

Lothstr. 64, 80335 München

T +49 170 3446314, zeifang@hm.edu

\section{Lucas Zitterbart}

HS Pforzheim

Tiefenbronnerstr. 65, 75175 Pforzheim

T +49 7231 28-6697

zitterba@hs-pforzheim.de 
Beiträge der Fachtagung „Angewandte Forschung in der Wirtschaftsinformatik Prozesse, Technologie, Anwendungen, Systeme und Management“ im Rahmen der 30. Jahrestagung des Arbeitskreises Wirtschaftsinformatik an Fachhochschulen (AKWI) vom 17. bis 20.09.2017 an der Hochschule Aschaffenburg

Autoren: Gunnar Auth, Thomas Barton, Stefan Beigel, Frank Bensberg, Lars Brehm, Gandalf Buscher, Johannes Busse, Christian Czarnecki, Julian Dörndorfer, Carsten Dorrhauer, Katharina Dort, Anke Dreiling, Dorothee Feldmüller, Iris Graf, Marco Graf, Michael Guckert, Denis Heideker, Sascha Hertkorn, Georg Rainer Hofmann, Eva Kastl, Steffen Kinkel, Holger Klötzner, Jens Kohler, Jonas Konrad, Katja Kubitta, Marcel Lang, Jan-Felix Mehret, Vera G. Meister, Frank Morelli, Natascha Nitzl, Martin Przewloka, Jörg Puchan, Johanna Rahn, Dörte Resch, Marco Richter, Bernhard Rieder, Tobias Rieke, Harald Ritz, Haio Röckle, Andreas Schmidt, Dominik Schneider, Christian Schulze, Johanna Schwab, Christian Seel, Carlo Simon, Thomas Specht, Frank Thomé, Tristan Eberhard Thomé, Holger Timinger, Melanie Vanderpuye, Niklas Weber, Thorsten Weidt, Matthias Willems, Frank Wisselink, Martin R. Wolf, Alexander Zeifang, Lucas Zitterbart 